


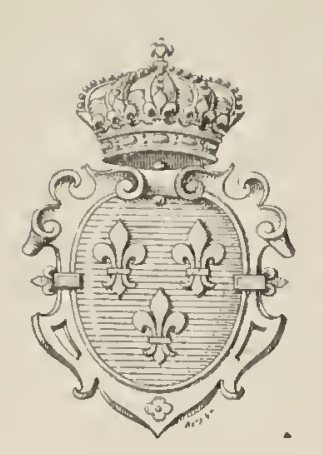

\section{DUC D'ORLEANS}

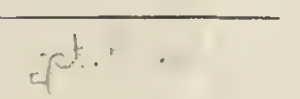

\section{Campagne arCTiQue DE 1907}

\section{MICROPLANKTON}

\section{des Mers de Barents et de Kara}

PAR le Dr Alph. MEUNIER

Professeur à l'Université de Louvain

37 PLANCHES HORS TEXTE

IMPRIMERIE SCIENTTFIQUE

Charles B ULENS, EDiteur

75, RUE TERRE-NEUVE, 75

BRUXELI,ES

1910 


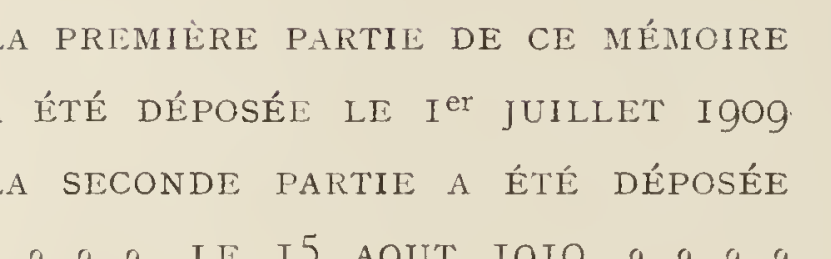

508,3
$0.5 c$ 


\section{TABLE SYSTEMATIQUE DES MATIERES}

INTRODUCTION

I. Définition de lobjet.

II. Caractère de l'étude.

III. ICONographie de L'objet.

IV. Caractere biotaxique de l'objet.

V. Caractère géograpingle de l'objet.

\section{TABLEAUX SYNOPTIQUES DES RÉCOLTES}

I. Tableau des stations planktonigues. . . . . . . . . . . . . . . . I4

II. Tableau des pèchles de plankton de surface et des rícoltes (ilaciaires í

\section{CARTES}

I. Itinéraire de la belgica dans les Mers de Barents et de Kara.

II. Ithéraire de la Belgica Autour de l'Ile Sud de la Nouvelle-Zenible.

\section{PREMIERE PARTIE}

\section{PROTISTES AUtRES QUE lEs DIATOMACÉS}

$$
\text { Groupe I. - PÉRIDINIACÉES }
$$

Genre Peridinim Ehrenberg. 
Séric B. - Carozontes

Section 111. - Sinistrogyres . . . . . . . . . . . . . . . . 38

Genre Glenodinim Stein . . . . . . . . . . . . . . . . . . . . . 44

Genre Heterocapsa Stein . . . . . . . . . . . . . . . . . . . . . . $4^{5}$

Genre Diplopsalopsis g. n. . . . . . . . . . . . . . . . . . . . . $t^{6} 6$

Genre Diplopsalis Bergh . . . . . . . . . . . . . . . . . . . . . ${ }_{4} 6$

Genre Nephrodinium g. n. . . . . . . . . . . . . . . . . . . . . ${ }_{4} \delta$

Genre Protoceratim Bergh . . . . . . . . . . . . . . . . . . . . 49

Genre Amylax g. n. . . . . . . . . . . . . . . . . . . . . . . 5г

Genre Gonyaular Diesing. . . . . . . . . . . . . . . . . . . $5_{4}$

Genre Orytorm Stein. . . . . . . . . . . . . . . . . . . . . . . . 55

Genre Ceratim Schrank . . . . . . . . . . . . . . . . . . . . 56

Genre Dinophysis Ehrenberg. . . . . . . . . . . . . . . . . . 58

Genre Gymnodinimm Stein. . . . . . . . . . . . . . . . . . . . 62

Genre Spirodinium Stein . . . . . . . . . . . . . . . . . . 62

\section{Groupe II. - MICROPHYTES DES NEIGES COLOREES}

Caractère spécial de l'objet. $\quad . \quad$. . . . . . . . . . . . . . . . . . . . . . . . 64

Origine des matériaut détude. . . . . . . . . . . . . . . . . . . . 65

EXamen Des formes principales. . . . . . . . . . . . . . . . . . 65

Genre Diamylon Meunier. . . . . . . . . . . . . . . . . . . . . 65

Genre Echimum g. n. . . . . . . . . . . . . . . . . . . 70

Genre Folliculus g. n. . . . . . . . . . . . . . . . . . . . . 72

Microphytes incerte sedis. . . . . . . . . . . . . . . . . . . . . . . 73

Formes polyédriques . . . . . . . . . . . . . . . . . . . 74

Formes sphériques nues. . . . . . . . . . . . . . . . . . 75

Formes sphériques hérissées de soies . . . . . . . . . . . . . . 76

Formes variées à membrane structurée . . . . . . . . . . . . . . 77

Formes algologiques frustes trouvées en milieu marin . . . . . . . . 77

\section{Groupe III. - CRYPTONONADACERS}

Genre Dinobryon Ehrenberg. . . . . . . . . . . . . . . . 78

Genre Corbicula g. n. . . . . . . . . . . . . . . . . . . . . . 79

Genre Phococystis Lagerheim. . . . . . . . . . . . . . . . . . . SI

Hydrun penicillatus Agardh . . . . . . . . . . . . . . . . . S I $_{\text {S }}$ 


\section{Groupe IV. - SILICOFL, IGELLATES}

Genre Distephanus Stöhr . . . . . . . . . . . . . . . . . . . . . 82

Genre Gymnaster Schuitt?. . . . . . . . . . . . . . . . . . . . . 82

Genre Diaster g. n. . . . . . . . . . . . . . . . . . . . . . 83

Groupe T. - ORGANISMIES ÉNIGMATIQUES

Genre Halosphara Schmitz . . . . . . . . . . . . . . . . . . . . 86

Genre Polyasterias g. n. . . . . . . . . . . . . . . . . . . . . . 87

Genre Pterosperma Pouchet . . . . . . . . . . . . . . . . . . . SS

Genre Chitonosperma g. n. . . . . . . . . . . . . . . . . . . 93

Genre Glyphosperma g. n. . . . . . . . . . . . . . . . . . . . . 94

Genre Radiosperma g. n. . . . . . . . . . . . . . . . . . . . . 95

Genre Amplicephalosperma g. n. . . . . . . . . . . . . . . . . . . 96

Genre Setosperma g. n. . . . . . . . . . . . . . . . . . . $99^{6}$

Série des Papulifères . . . . . . . . . . . . . . . . . . . . 97

Genre Fusopsis g. n. . . . . . . . . . . . . . . . . . . . . . . 98

Genre Piropsis g. n. . . . . . . . . . . . . . . . . . . . . 102

Genre Sphceropsis g. 11. . . . . . . . . . . . . . . . . 103

\section{Groupe VI. - PROTOZOAIRES LORIOUÉS}

SECTION I. - TINTINNIDES. . . . . . . . . . . . . . . . . . IO7

Genre Cyttarocylis Fol. . . . . . . . . . . . . . . . . . . . . . 109

Genre Ptychocylis Brandt. . . . . . . . . . . . . . . . . . . . . I2I

Genre Amphorella Daday. . . . . . . . . . . . . . . . . . . . . I2S

Genre Tintinnus (Schrank) Daday. . . . . . . . . . . . . . . . . . 133

Genre Tintinnopsis Stein . . . . . . . . . . . . . . . . . . . . . I36

Série I. - Les tubuleuses. . . . . . . . . . . . . . . . . i3S

Série II. - Les orthostomes . . . . . . . . . . . . . . . . I4 I

Série III. - Les sténostomes . . . . . . . . . . . . . . . . I $_{4}^{3}$

Série IV. - Les Fauciféres. . . . . . . . . . . . . . . . . I44

Genre Conocylis g. n. . . . . . . . . . . . . . . . . . . . . . 146

Genre Strombidium Claparède et Lachmann . . . . . . . . . . . . . . I4 8 
SECTION H. - VORTICELLIDES. . . . . . . . . . . . . . . . . . . . I 49 .

Genre Cothumia Ehrenberg. . . . . . . . . . . . . . . . . . . . I 49

Genre Vaginicola Lamarck, ou Thuricola Saville-Kent. . . . . . . . . . . I50.

SECTION III. - RHIZOPODES AMGBiENS . . . . . . . . . . . . . I50

\section{Groupe VII. - INFUSOIRES NON LORIQUES}

SECTION I. - HYPOTRICHIDES . . . . . . . . . . . . . . . I52

Genre Euplotes Ehrenberg . . . . . . . . . . . . . . . . . . I52

SECTION II. - HOLOTRICHIDES . . . . . . . . . . . . . . I54

Genre Didinim Stein. . . . . . . . . . . . . . . . . . . . . . 154

Genre Cyclotrichinm g. n. . . . . . . . . . . . . . . . . . . . . ${ }_{1} 64$

Genre Proboscidium g. n. . . . . . . . . . . . . . . . . . . . . I68

Genre Prorodon Ehrenberg . . . . . . . . . . . . . . . . . . . . I7I

Genre Cephalotrichim g. n. . . . . . . . . . . . . . . . . . . . . 172

Genre Zonotrichizm g. n. . . . . . . . . . . . . . . . . . . . . 173

Genre Condylostoma Dujardin?. . . . . . . . . . . . . . . . . . . I7t

Genre Climacostomum Stein?. . . . . . . . . . . . . . . . . . . . 175

Genre Trachelius Claparède et Lachmann? . . . . . . . . . . . . . . 176

Genre Lacrymaria Ehrenberg? . . . . . . . . . . . . . . . . . . . 177

Genre Enchelys Ehrenberg? . . . . . . . . . . . . . . . . . 177

Infusoires incerte sedis . . . . . . . . . . . . . . . . . . . 177

Genre Stappersia g. n. . . . . . . . . . . . . . . . . . . . . . 179

Genre Gymnozoum g. n. . . . . . . . . . . . . . . . . . . . IÉ

Section ili. - infusolres tentaCulifères ou SUCEURS . . . . . igh

Groupe VIII. - RHIZOPODES

Section I. - RADIOlaires . . . . . . . . . . . . . . Ig6

A. Ordre des Monopylaires . . . . . . . . . . . . . . 197

B. Ordre des Acanthaires. . . . . . . . . . . . . . . . 200

C. Formes incerte sedis. . . . . . . . . . . . . . 203. 
SECTION 11 - FORAMINIFliRES.

SECTION III. - TAXOPODES.

SHCTON III. TAXOPODES.

Genre Stricholonche Hertwig.

\section{SECONDE PARTIE}

Groupe IX. - DIATOMACEES

Généralités

Division .

DESCRIPTION DES ESPECES.

\section{SOUS-FAMILLE I. - ARAPHIDÉES}

Genre Chatoceros Ehrenberg. . . . . . . . . . . . . . . . . 2II

Sous-gente Pifaoceras Gran. . . . . . . . . . . . . . . 2 I2

Section I. - Atlantice Ostenfeld . . . . . . . . . . . . $2 \mathrm{I2}$

Section II. - Boreales Ostenfeld. . . . . . . . . . 2 It

Sous-genre Hyalochate Gran. . . . . . . . . . . . . . . . . 2 I9

Groupe A. Phanerolencite . . . . . . . . . . . . 2 I9

Groupe B. Cryptoleucita. . . . . . . . . . . . . . 230

Genre Rhizosolcnia (Ehrenberg) Brightwell. . . . . . . . . . . . . . . 253

Genre Eucampia Ehrenberg. . . . . . . . . . . . . . . . . . . . 258

Genre Leptocylindrus Cleve . . . . . . . . . . . . . . . . . . . . 258

Genre Skcletonema Greville . . . . . . . . . . . . . . . . . . . . 259

Genre Thalassiosira Cleve. . . . . . . . . . . . . . . . . . . . . 259

Genre Lauderia Cleve. . . . . . . . . . . . . . . . . . . . . . 269

Genre Bacterosira Gran . . . . . . . . . . . . . . . . . . . . . 270

Genre Melosira Agrardh . . . . . . . . . . . . . . . . . . . . . 27 I

Genre Paralia Heiberg . . . . . . . . . . . . . . . . . . . . . $27+$

Genre Coscinodiscus Ehrenberg . . . . . . . . . . . . . . . . . . 274 
Genre Stephanodiscus (Ehrenberg) Grunow . . . . . . . . . . . . . . . 280

Genre Coscinosira Gran . . . . . . . . . . . . . . . . . . . . . 28o

Genre Phacodiscus g. n. . . . . . . . . . . . . . . . . . . . . . . . 28I

Genre Hyalodiscus Ehrenberg . . . . . . . . . . . . . . . . . . . 282

Genre Isthmia Agardh . . . . . . . . . . . . . . . . . . . . . . . . . 283

Genre Biddulphia Gray . . . . . . . . . . . . . . . . . . . . . 284

SOUS-FAMILIES II et III. - PSEUDO-RAPHIDÉES ET RAPHIDÉES

Genre Amphiprora Ehrenberg . . . . . . . . . . . . . . . . . . . 289

Genre Orthotropis Cleve . . . . . . . . . . . . . . . . . . . $29 \mathrm{I}$

Genre Donkinia Ralfs . . . . . . . . . . . . . . . . . . . . 292

Genre Campylodiscus Ehrenberg. . . . . . . . . . . . . . . . . . 292

Genre Surrella Turpin . . . . . . . . . . . . . . . . . . . . . 293

Genre Cocconcis Grunow . . . . . . . . . . . . . . . . . . . . 294

Genre Raphoncis Ehrenberg . . . . . . . . . . . . . . . . . 295

Genre Cymbella Agardh . . . . . . . . . . . . . . . . . . . . . 296

Genre Encyonema Kützing . . . . . . . . . . . . . . . . . . . . 297

Genre Amphora Ehrenberg . . . . . . . . . . . . . . . . . . . . 297

Genre Epithemia Brébisson . . . . . . . . . . . . . . . . . . . . 30I

Genre Grammatophora Ehrenberg . . . . . . . . . . . . . . 303

Genre Plagiogramma Greville . . . . . . . . . . . . . . . . . $30+$

Genre Rhabdonema Küitzing . . . . . . . . . . . . . . . . . . . 30t

Genre Striatella Agardh . . . . . . . . . . . . . . . . . . . . 306

Genre Denticula Küutzing . . . . . . . . . . . . . . . . . . . . 306

Genre Meridion Agardh . . . . . . . . . . . . . . . . . . . . . 306

Genre Diatoma de Candolle. . . . . . . . . . . . . . . . . . . . 307

Genre Gomphonema Agardh . . . . . . . . . . . . . . . . . . 307

Genre Licmophora Agardh . . . . . . . . . . . . . . . . . . . . 308

Genre Eunotia Ehrenberg. . . . . . . . . . . . . . . . . . . 309

Genre Thalassiothrix Cleve et Grunow . . . . . . . . . . . . . . 309

Genre Synedra Ehrenberg . . . . . . . . . . . . . . . . . . . . 3ro

Genre Fragilaria Lyngbye . . . . . . . . . . . . . . . . . . . . 3ir

Genre Stauropsis g. n. . . . . . . . . . . . . . . . . . . 3is

Genre Achnanthes Bory . . . . . . . . . . . . . . . . . . . . . 326

Genre Achanthidium Grunow . . . . . . . . . . . . . . . . . . . 328

Genre Hantzschic Grunow . . . . . . . . . . . . . . . . . . . . 328

Genre Nitsschia Hassal . . . . . . . . . . . . . . . . . . . . . 330 
TABLE SYSTÉMATIOLIE DES MATIËRS

111

Genre Nitzschiella Rabenhorst

Genre Homoocladia Agardh

Genre Schisoncma Agardh.

Genre Pleurosigna W. Smith

I. - Formes à stries décussées.

II. - Formes à stries perpendiculaires

Genre Rhoicosigma Grunow

Genre Scoliopleura Grunow

Genre Rhoiconeis Grunow.

Genre Navicula Bory .

Genre Mastogloia Thwaites . . . . . . . . . . . . . . . . . . . . 355

Note finale . . . . . . . . . . . . . . . . . . . . . 355 



\title{
INDEX ALPHABÉTIQUE
}

\author{
DES GROUPES, GENRES ET ESPÈCES CITÉS
}

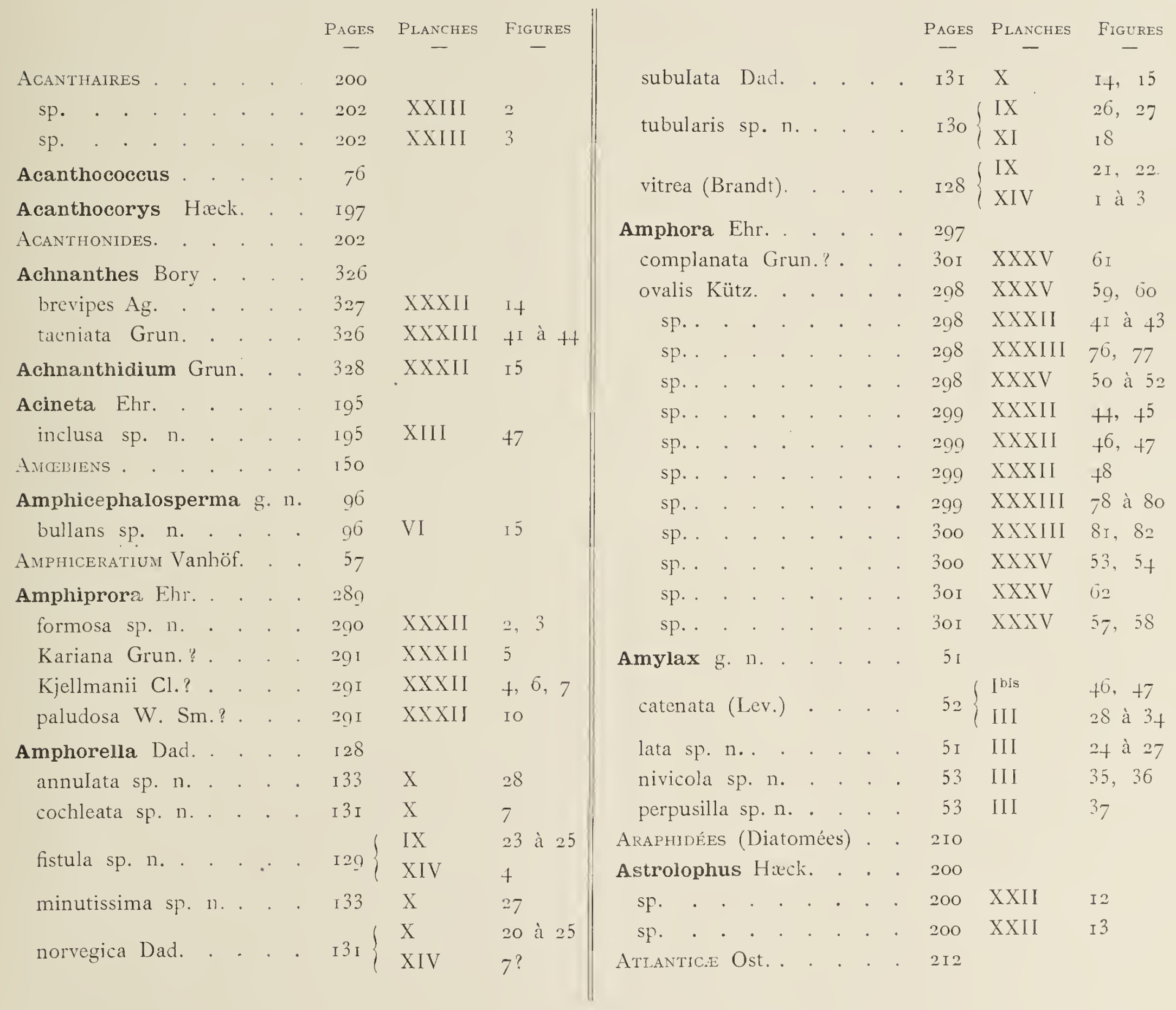




\begin{tabular}{|c|c|c|c|c|c|c|c|c|c|}
\hline & & $\begin{array}{c}\text { Pages } \\
-\end{array}$ & $\begin{array}{l}\text { Planches } \\
-\end{array}$ & $\begin{array}{c}\text { FigURES } \\
-\end{array}$ & & & $\begin{array}{l}\text { PAGES } \\
-\end{array}$ & Planches & FigURES \\
\hline Bacterosira Gran. & - & 270 & & & f. curvens (Cl.) & & 216 & XXIV & 8 \\
\hline fragilis (Gran). . . & . & 270 & $\mathrm{XXX}$ & 10 ì 15 & f. polans (Schütt.) & & $21 \overline{0}$ & XXIV & 8 \\
\hline Biceraticm Vanhöf. . & . & 37 & & & debile $\mathrm{Cl}$. . . & . & 242 & XXVII & 19 à 23 \\
\hline Biddulphia Gray . . & . & 284 & & & & & & $\mathrm{XXV}$ & I 2 à I $\overline{0}$ \\
\hline arctica Boyer. . & . & 284 & & & decipiens $\mathrm{Cl}$. & . & 210 & XXVII & 45 \\
\hline f. balana. . . . & - & 285 & XXXI & $10, \quad$ I I & & & & XXVIII & I \\
\hline f. typica . . . . & · & 285\{ & $\begin{array}{l}\text { XXXI } \\
\text { XXXII }\end{array}$ & 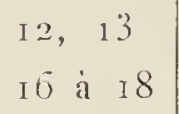 & $\begin{array}{l}f . \text { princeps. . . . } \\
\text { densum Cl. . . . }\end{array}$ & . & $\begin{array}{l}222 \\
217\end{array}$ & $\begin{array}{l}\text { XXVIII } \\
\text { XXIV }\end{array}$ & I $5, \quad$ I 6 \\
\hline aurita Bréb. . . & . & 286 & $\mathrm{XXXI}$ & $I+\dot{a} \quad I Q$ & diadema Ehr. & . & 232 & XXVI & I 5 à 23 \\
\hline granulata Rop. . . & . & 287 & XXXI & 20 à 23 & filiforme sp. n. . & . & 252 & XXVII & $3 \mathrm{I}$ \\
\hline rhombus W. Sm. & . & 288 & XXXI & 24 à 26 & fragile sp. n. . & . & $2+4$ & XXVII & 27 à 29 \\
\hline BOREales Ost. . . & . & $2 I+$ & & & furellatum Rail & & & XXVII & I I à I 6 \\
\hline Botryopyle Hack. & . & 197 & & & rurcenatum Бан. & & & XXVIII & 2,3 \\
\hline setosa Cl.?. . . & . & 197 & XXII & t. 6 & glaciale sp. n. . & . & $25 \mathrm{I}$ & XXVII & 38 à $+t$ \\
\hline stenostoma sp. n. & & $\lg 8$ & XXII & 5 & Ingolfianum Ost. . & . & $\cdot 230$ & XXVI & 2 à I 4 \\
\hline & & & & & Karianum Grun.. . & . & 246 & XXVIII & 5 à I 2 \\
\hline & & & & & laciniosum Schuitt. & . & 235 & XXVI & 24 à 3I \\
\hline Campylodiscus Ehr. . & . & 292 & & & longisetum Cl. . & 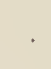 & $2+2$ & XXVII & I 8 \\
\hline angularis Greg. . . & . & 293 & XXXII & $2 \mathrm{I}$ & medium Schütt . . & . & 229 & XXV & I7 \\
\hline Thuretii Bréb. . . & - & 293 & XXXII & $19, \quad 20$ & mitra Bail. . . & & 223 & $X X V$ & I à 7 \\
\hline Cayozones (Peridinium & & 38 & & & radians Schü̈t. . . & . & 247 & XXVII & $3=$ \\
\hline Centric.玉. . . . & · & 210 & & & Schüttii Cl. . . & . & 236 & XXVI & 32 à 35 \\
\hline Cephalotrichium g. n. & . & 172 & & & simile $\mathrm{CL}$. & & & XXVII & 37 \\
\hline tonsuratum sp. $\mathrm{n}$. & · $\quad \cdot$ & 172 & $\mathrm{X} I \mathrm{X}$ & 7 à 12 & . & & $2+3$ & XXVIII & 4 \\
\hline Ceratium Schr. . & . & 56 & & & sociale Laud. . . & . & $2+9$ & XXVII & 33 à 36 \\
\hline fusus Clap. et Lach & hm. & 57 & II & $5 \mathrm{I}$ & f. flabelliformis . & . & 249 & XXVII & 36 \\
\hline longipes Gran. . & . & 56 & & & f. solitaria. . . . & . & 250 & XXVII & $3+, 35$ \\
\hline var. amputata. & . & 57 & I I & 50 & subtile Cl. . . . . & 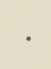 & 242 & XXVII & I 8 \\
\hline var. arctica (Cl.). & . & 57 & $\begin{array}{l}\text { II } \\
\text { III }\end{array}$ & $\begin{array}{l}+9 \\
50\end{array}$ & $\begin{array}{l}\text { teres Cl. . . . } \\
\text { Wighami Bright. }\end{array}$ & . & $\begin{array}{l}226 \\
244\end{array}$ & $\begin{array}{l}\text { XXV } \\
\text { XXVII }\end{array}$ & $\begin{array}{l}8 \dot{a} \text { I I } \\
26\end{array}$ \\
\hline var. Baltica Ost.? & . & 56 & Ibis & 30 & sp. . . . & • & 237 & XXVII & I à 6 \\
\hline var. Oceanica Ost.?. & . & 56 & II & $+7,+8$ & sp. . . & . & 239 & XXVII & $7 \dot{a} \quad 10$ \\
\hline var. lentricosa Ost. . & · & 56 & $\begin{array}{l}I^{\text {hls }} \\
\text { II I }\end{array}$ & $\begin{array}{l}40 \\
23\end{array}$ & $\begin{array}{ccc}\text { sp.. } & \cdot & \cdot \\
\text { Chitonosperma } & \text { g. } & \text { n. }\end{array}$ & . & $\begin{array}{r}241 \\
03\end{array}$ & XXVII & 17 \\
\hline Ceratoneis! . & . & 309 & & & coronatum sp. n. & . & 03 & $\mathrm{~V}$ & 33,34 \\
\hline Chætoceros Ehr. . & . & 211 & & & umbilicatum sp. n.. & . & 93 & $\mathrm{~V}$ & 31,32 \\
\hline atlanticum Cl. . & . & 212 & XXIV & I $\dot{a} 3$ & sp....$\quad \cdot \quad \cdot \quad$. & · & 94 & VI & I I, I 2 \\
\hline baculites sp. 11. & . & $25 \mathrm{I}$ & XXVII & 30 & Chlorococcum Fries. & . & 77 & IV & 79 \\
\hline boreale Bail. . . & - & $2 \mathrm{I} 4$ & XXIV & $+\dot{a} 7$ & Climacostomum Stein? & 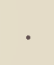 & 175 & & \\
\hline f. solitaria CI. . . & . & 2 I 5 & XXIV & + & gigas sp. n. . . & . & I 75 & XVII & I I \\
\hline convolutum Cast. & . & 2 I 8 & XXIV & I7 à I9 & Cocconeis Grun. . & . & 294 & & \\
\hline contortum Schütt. & - & 227 & $\begin{array}{l}X X V \\
X X V I\end{array}$ & ${ }_{\text {I }} 7 \dot{a} \quad 27$ & $\begin{array}{l}\text { dirupta Greg.? } \\
\text { scutellum Ehr.? }\end{array}$ & . & $\begin{array}{l}294 \\
294\end{array}$ & $\begin{array}{l}\text { XXXII } \\
\text { XXXII }\end{array}$ & $\begin{array}{l}25 \\
29\end{array}$ \\
\hline crinitum Schütt. . & . & 243 & XXVII & $2+, \quad 25$ & sp. . . . . . & . & $29+$ & XXXII & 24 \\
\hline criophilum Cast. . & . & 215 & XXIV & 8 à If & $\cdot \cdot \cdot$ & . & 204 & XXXII & 26 \\
\hline
\end{tabular}


Pages Planches Figuies

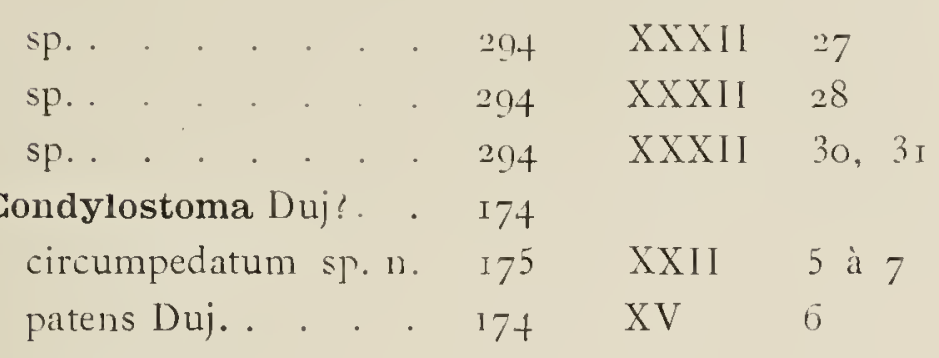

Conocylis g. 11. . . I 16

constricta sp n. . . I $47 \quad \mathrm{X} \quad 36,37$

helis sp.n... . . It7 $\quad$ X 32 à 35

$\begin{array}{llll} & & \end{array}$

striata sp.n. . I $48 \begin{cases}\text { XI } & 22 \\ \text { XXIII } & \text { I 3 }\end{cases}$

Corbicula g. п. . . . 79

socialis sp.n. . . 79

Coscinodiscus Ehr. . . 274

concinnus W. Sm. . 278

curvatulus Grun. . . 279$\}$

excentricus Ehr. . 277

Kützingii A. Schm. . 278

lacustris Grun. . $\quad 274$

nobilis Grun. . . . 279

oculus iridis Ehr. . 275

Coscinosira Gran . . 280 polychorda (Gran). . 280

Cosmarium sp. . . 74

Cothurnia Ehr. . . I 49 curva Stein.? . . I50 imberbis Ehr.? . I +9 nodosa Clap. et Lachm ? 149

Cryptoleucite . . 230

Cryptomonadacées . $\quad 7^{8}$

Gyclotrichium g.n. . $\mathrm{I}_{4}$ cyclokaryon sp. 11. . $\quad$ I05 $\begin{cases}\text { XVI } & 2,4, \text { I } 3 \text { à I T } \\ \text { XVII } & 0,7 \\ \text { XVIII } & 7\end{cases}$

Cymbella Ag. * . . 296 amphicephala Nieg. . 296 recurva $\mathrm{sp} . \mathrm{n}$. . . $\quad 296$

XXXV 63 à 65

XXXII

Cyrtoïdes . · · · $\quad$. 997

Cyttarocylis Fol. . . I cuspidata sp.n. . I I $\begin{cases}\text { VIII } & \text { I } 2 \text { à I } 7 \\ \text { X } & 8 \text { à I } 2 \\ \text { XI } & \text { I } 3 \text { à I4 }\end{cases}$

$\begin{array}{ll}\text { XXXI } & 2 \text { à } \overline{6} \\ \text { IV } & 80 \\ \text { XIV } & \text { Io } \\ \text { X } & 3 \text { I } \\ \text { XIV } & 9\end{array}$

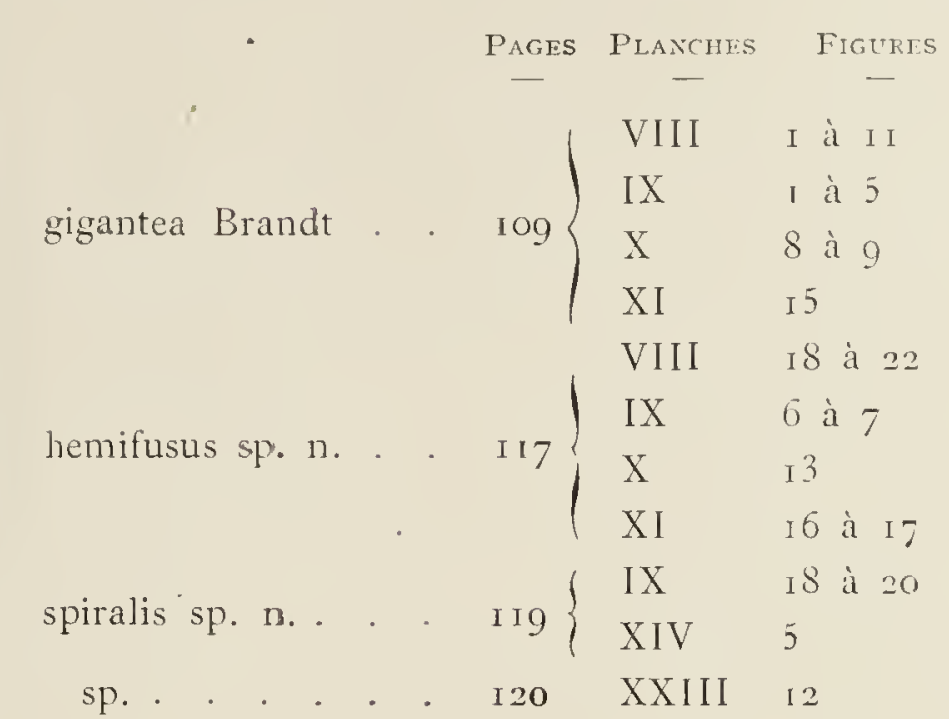

Denticula kütz . . . 306 sp. . . . 306

Diamylon Meunier . 65 nivale Meunier. . . 65

Diaster g. n. . . . 83 macrokaryon sp.n. . 83

Diatoma de Cand. . 307 vulgare Bory . . 307 Diatomacées . . . 207

Dictyocha Ehr. . . 82

Didinium Stein . . $\quad$ I 54

Didinium Stein

Gargantua sp. n. . $\quad 15+\frac{1}{4}$

$\begin{array}{ll}\text { XV } & 8 \text { à } 15 \\ \text { XVI } & \text { I, 3, } 5 \text { à } 12 \\ \text { XVII } & \text { I à } 5 \\ \text { XVIII } & 1,2,4,6,8,10,11 \\ \text { XIX } & \text { I, } 16 \text { à I } 8 \\ \text { XXII } & \text { I I } \\ \text { XX } & 4,5\end{array}$

nasutum Müll.! . I I $\dot{y}_{+} \mathrm{XX} \quad+, 5$

Difflugia Lecl. . . . I 50 nivalis sp. n.. I 50 XIII 3 I à 40 spharica sp. n. . 15 I XIII 30

sp. ? . . I 5 I XIII $4 \mathrm{I}, 4^{2}$

sp. . . . I 5 I XIII $4^{3}$ à $t^{5}$

DiFflugines . . . 150

Dileptus . . . . 176

Dinobryon Ehr. . $7^{8}$

Belgica sp. n. . . $79 \quad \mathrm{~V} \quad 3$ pellucidum Lev. . $\quad 78 \mathrm{~V} \quad$ I, 2 balticum (Schütt.). $\quad 7^{8} \mathrm{~V} \quad$ I, 2 Sertularia Ehr. . . 79

Dinodendron Schütt. . $\quad 7^{8}$ baIticum Schütt. . . 78 
Dinophysis Ehr. . . . jo

apiculata sp. n. . . . 62

cunciformis sp. n. . . . bo

norregica Clap. et Lachm.

rotundata Clap. et Lachm.

semen sp. $\mathrm{n}$.

vertex sp. n.

Diplopsalis Bergh.

lenticula Bergh.

spharica sp. n.

Diplopsalopsis g. n. .

orbiculare (Pauls.)? .

Distephanus Stöhr.

speculum Hiek. .

Donkinia Ralfs .

recta (Donk.) Grun.? . . 20

Echinum g. 1.. . . . 70

majus sp. n. . . . . 70

micraster sp. n. . . . . 71

minus sp. n. . . . . 7 I

nudum sp. n. . . . . $7^{1}$

sphæricum sp. n. . . . 71

Enchelys Ehr.?. . . . . 177

sp.? . . . . . . 177

Encyonema Kütz . . . . 297

sp.. . . . . . . 297

Epithemia Bréb. . . . 30

sp...... . 301 301

sp. . . . . . 302

sp. . . . . . 302

sp....... . 302

Eucampia Ehr. . . . . 258

groenlandica $\mathrm{Cl}$. . . . 258

Euceratiun Gran . . . 50

Eunotia Ehr. . . . . 300 arcus Ehr.. . . . . 300 dolium sp. n. . . . 300

Euplotes Ehr. . . . . . 152

caudata sp. 11. . . . 152$\}$

truncata sp. n. . . I53 XX 8 il 14

XXXV 66,67

XXXY 55,56

XXXlY 50, 0

XXXlV oj a $\sigma_{4}$

XXYIV ó

XXVIII 20, 30

XXXIII 74,75

XXXIII 72,73
Pages Planches Figures

FAUCifères (Tintinnopsis). . 144

Folliculus g. n. . . . . $7^{2}$

flagellifer. . . . . . 73

Foraminteleres. . . . . 204

Fragilaria Lyngb . . . 3 3 I

groenlandica sp. n. . . 3 316

islandica Grun. . . . 3 I 1

mollis sp. n. . . . $3 \mathrm{I}_{2}$

oceanica Cl. . . . 3,3

virescens Ralfs. . . . 318

IV

I 7 à 2 I

usopsis g. n. . . . . 08

elongata sp. n. . . . 98

Hagrifera sp. n. . . . IOO

pauperata sp.n. . . I I00

polyedra sp. n. . . . $10 \mathrm{I}$

spiralis sp. n. . . . . 99

umbracula sp. n.

99

NXXIll Io ì I 3

XXXIll I à 6

XXXIIl 7 à 9

XXXIll It à 24

XXXIII 25

$\mathrm{VI}$

2 I

VI $\quad 7,8$

VI 24, 25

VI 22, 23

VII 6

VI I9. 20

Glenodinium Stein. . . 44

bipes Pauls.?. . . . $44^{5}$ III

danicum Pauls. $\quad+4 \begin{cases}\text { II } & \text { to à } 42 \\ \text { XIV } & \text { Io, }\end{cases}$

inflatum sp. n. . . . 45 II

Glœocystis Naeg. . . . . 77

sp. . . . . . . 77

Glyphosperma g. n. . . . $9+$

flagelliferum sp. n. . . . 94

nervosum sp. n. . . . 95

Golenkinia Chod. . . . 76

Gomphonema Ag. . . . 307

greminatum Ag. . . . 30-

sp. . . . . . 307

sp. . . . . . 308

sp. . . . . . 308

Gonyaulax Dies. . . . 54

polygramma Stein.

spinifera Dies. . . . 54

Grammatophora Ehr. . . 303

arctica Cl. . . . . 304 XXXIV 57,58

islandica Ehr.. . . 303 XXXIl 55 à 57

serpentina Ehr. . . . 303 XXXIV 55, 56

Gymnodinium Stein . . . 62 


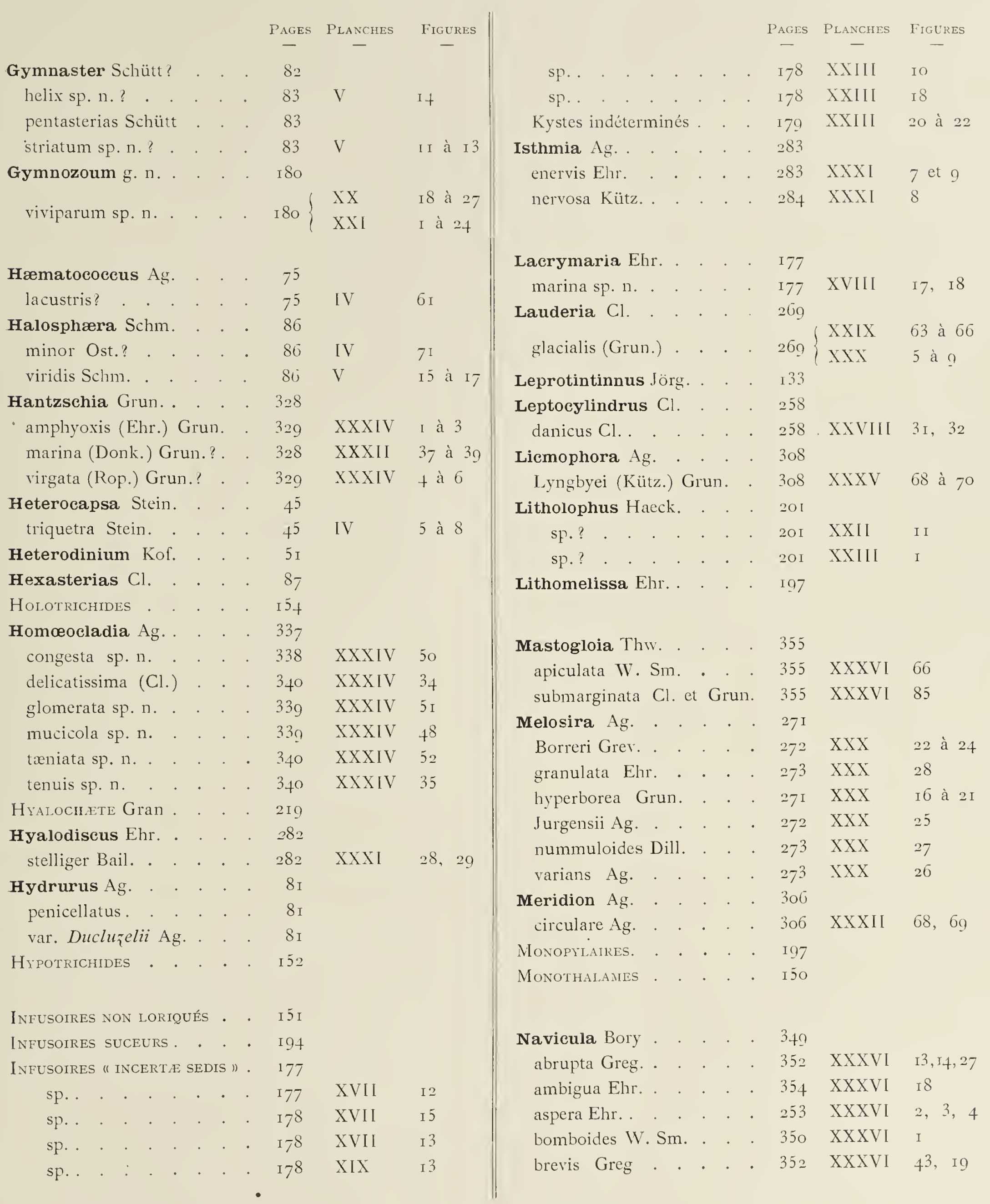




\begin{tabular}{|c|c|c|c|}
\hline & $\begin{array}{c}\text { Pages } \\
-\end{array}$ & $\begin{array}{c}\text { Plaxchis } \\
-\end{array}$ & Figures \\
\hline cancellata Donk. & 354 & XXXVI & 37.38 \\
\hline chersonensis Grun.? & 350 & XXXVI & 53.54 \\
\hline clepsydra Donk. . & 353 & XXXVI & $4^{6,}+7$ \\
\hline crabro Ehr.? & 350 & XXXV & 38,30 \\
\hline cruciformis Donk. & $35+$ & XXXVI & 57,$58 ; 51,5$ \\
\hline cuspidata Kütz. & 352 & XXXVI & 6I \\
\hline didyma Ehr. & $35 \mathrm{I}$ & XXXVI & 22 \\
\hline elliptica Kütz. & $35 \mathrm{I}$ & XXXVI & 23,24 \\
\hline forcipata Grev. . & & & $+2,43$ \\
\hline fusiformis Grun.? & $35+$ & XXXVI & $30,-2,73,83,8+$ \\
\hline glacialis Cl. . . & 340 & XXXVI & 7 a $9 ; 10$, II; $50, c$ \\
\hline Kariana sp. n. . & 354 & $\begin{array}{l}\text { XXXVI } \\
\text { XXXV }\end{array}$ & 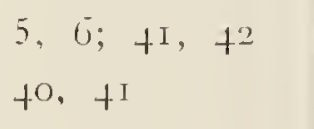 \\
\hline lanceolata Kütz. . & $35+$ & XXXVI & 17 \\
\hline var. arenaria Kïitz. & 354 & XXXVI & 17 \\
\hline littoralis Donk. . & $35 \mathrm{I}$ & XXXVI & $8 I$ \\
\hline Jya Ehr. . & 35 I & XXXVI & 15, 16 \\
\hline oculata Bréb. . . & $35 \mathrm{I}$ & XXXVI & 80 et 82 \\
\hline palpebralis Bréb. & 352 & XXXVI & 33 \\
\hline peregrina Kütz. . & 354 & XXXVI & $t^{5}$ \\
\hline pinnularia Cl. . . & 353 & XXXVI & $3 \mathrm{I}, \quad 32 ; 04, \quad 63$ \\
\hline quadratarea A. Schm. & 353 & XXXVI & 31,32 \\
\hline sandriana Grun. . & $35 \mathrm{I}$ & XXXVI & 50 \\
\hline scutellum O’Meara & $35 I$ & XXXVI & 78 \\
\hline Smithii Bréb. . & $35 I$ & XXXVI & $2 I$ \\
\hline var, clongata var. n. & $35 \mathrm{I}$ & XXXVI & 79 \\
\hline tridens sp. 11. . . & 353 & XXXVI & $70,7^{1}$ \\
\hline viridis Kütz. . . & 353 & XXXVI & $67,6 \mathrm{~S}$ \\
\hline sp. . . . . . & 353 & XXXVI & 30,40 \\
\hline $\mathrm{sp} \cdot \cdot \cdot \cdot \cdot \cdot$ & 354 & XXXVI & $48,+9$ \\
\hline sp. $\cdot \quad \cdot \quad \cdot \quad \cdot$. & $35+$ & XXXVI & $35, \quad 36$ \\
\hline sp. $\cdot \cdot \cdot \cdot$. & $35+$ & XXXVI & 76,77 \\
\hline $\mathrm{sp} \cdot \cdot \cdot \cdot \cdot \cdot$ & $35+$ & XXXVI & 74 \\
\hline Formes indéterminées & $35+$ & XXXVI & $62,63,75$ \\
\hline Formes indéterminées & 354 & XXXVI & I $2,20,3+$ \\
\hline ephrodinium g. $n$. & 48 & & \\
\hline Karianum sp. n. & $4 ?$ & IV & I5, 16 \\
\hline nivale sp. n. . & +8 & II & $\begin{array}{l}4^{1} \text { a } \text { t }^{3} \\
7,8\end{array}$ \\
\hline itzschia Hass. . . & 330 & & \\
\hline angularis $\mathrm{W}$. Sm. & 332 & XXXIV & 17 \\
\hline bilobata WV. Sm. . & 332 & IXXIV & I 5, If \\
\hline
\end{tabular}

\begin{tabular}{|c|c|c|c|}
\hline & $\begin{array}{c}\text { Pages } \\
-\end{array}$ & Planches & $\begin{array}{c}\text { FIGURES } \\
-\end{array}$ \\
\hline frigida Grun. . . . & 335 & XXXIV & 36 \\
\hline scalaris $W$. Sm.! & 333 & XXXIV & $2 I, \quad 22$ \\
\hline seriata $\mathrm{Cl}$. & $33+$ & XXXIV & 38 à 40 \\
\hline sigma W. Sm. . & 333 & XXXIV & 28 \\
\hline sigmoidea W. Sm. . & 333 & XXXIV & $20, \quad 30$ \\
\hline spathulata Bréb. . & 332 & XXXIV & $16, \quad 20$ \\
\hline spectabilis Ralfs & 333 & XXXIV & 20 \\
\hline Tryblionella Hant & 333 & XXXIV & 33 \\
\hline sp. & $33 \mathrm{I}$ & XXXIV & 7,8 \\
\hline sp. . . . & $33 \mathrm{I}$ & XXXIV & 9 \\
\hline sp. . . . & $33 \mathrm{I}$ & XXXIV & IO, I I \\
\hline sp. . & 332 & XXXIV & $\mathrm{I} 2$ \\
\hline sp. . . . . & 332 & XXXIV & I3, It \\
\hline Ezschiella Rab. & 336 & & \\
\hline acicularis WV. Sm. & 337 & XXXIV & +7 \\
\hline longissima (Brúb.) Rab. & 337 & XXXIV & $f^{\mathrm{I}}$ à fú et \\
\hline var. typica & 337 & XXXIV & +3 \\
\hline var. parra V. $\mathrm{H}$. & 337 & XXXIV & $4 \mathrm{~T}$ \\
\hline var. Closterium $\mathrm{W}^{\top} \mathrm{Sm}$. & 337 & XXXIV & H. $4^{5}, 4^{\circ}$ \\
\hline var. fasciculata var. $n$. & 337 & XXXIV & 65 \\
\hline var. reversa IV. Sm. & 337 & XXXIV & $t^{2}$ \\
\hline
\end{tabular}

Olpidium . . . . passim

phycophagum sp. n. $222 \mathrm{XXV}$, etc. 16,27 , etc.

Oocystis Næg. . . . 77 spherica sp. n. . . 77

OrThostomes(Tintinnop.) I I

Orthotropis Cl. . . $29 \mathrm{I}$ lepidoptera (Gres.) Cl. $20 \mathrm{I}$ maxima (Greg.) Cl. . 292

Oxytoxum Stein. . 55

Belgicx sp. 11. . . 55 XIV 38 à +1

Papulatekes . . . 97

Paralia Heib. . . $27 t$ sulcata Ehr. . . $27+$ XXX 29 à 32

Pennatiz . . . . 288

Pérjdintacées . . . 19

Peridinium Ehr. . 2 I

anceps sp. 1... . 3t I 32,23

bulla sp. n. . . $4^{3}$ I bis 32 a 34 


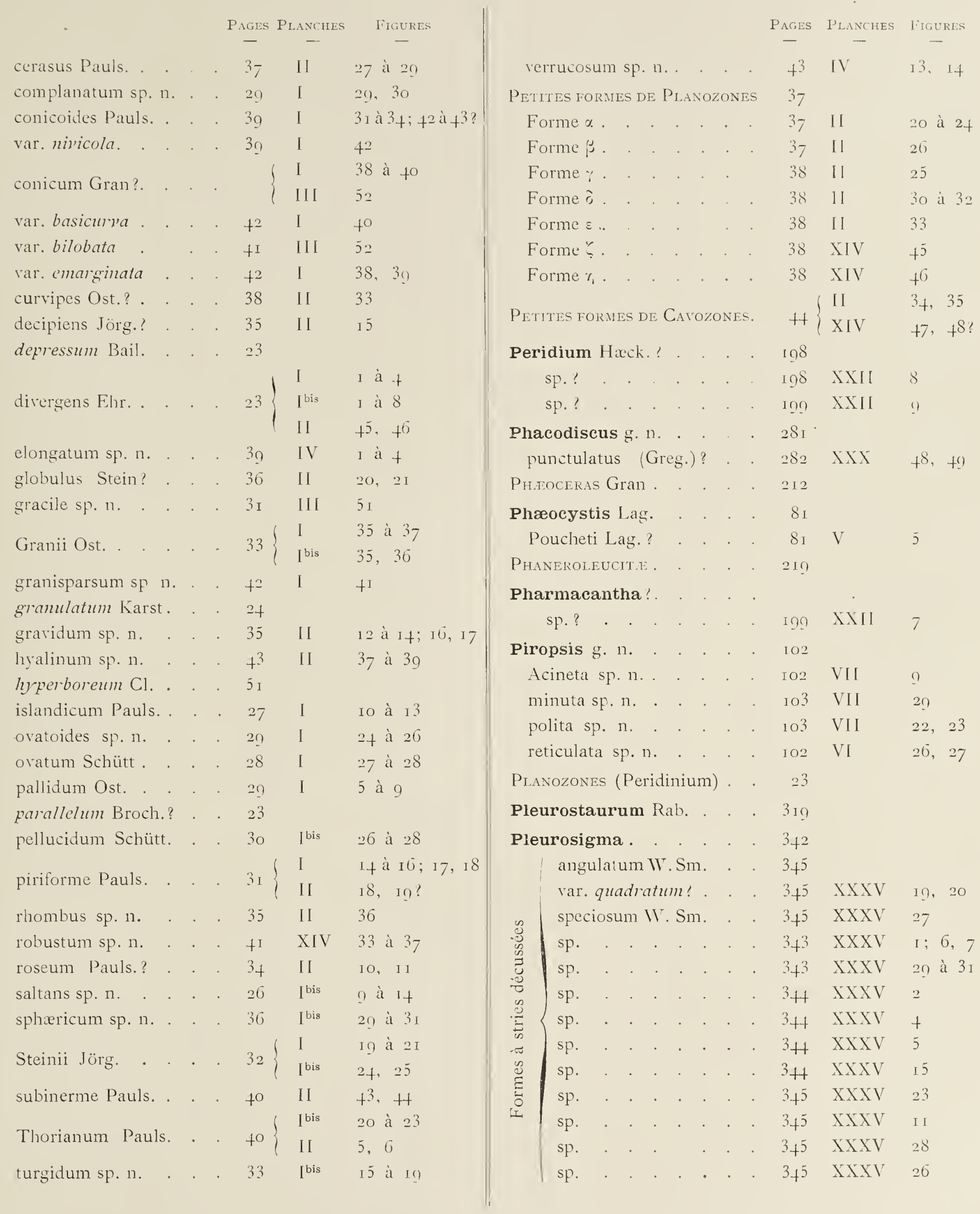




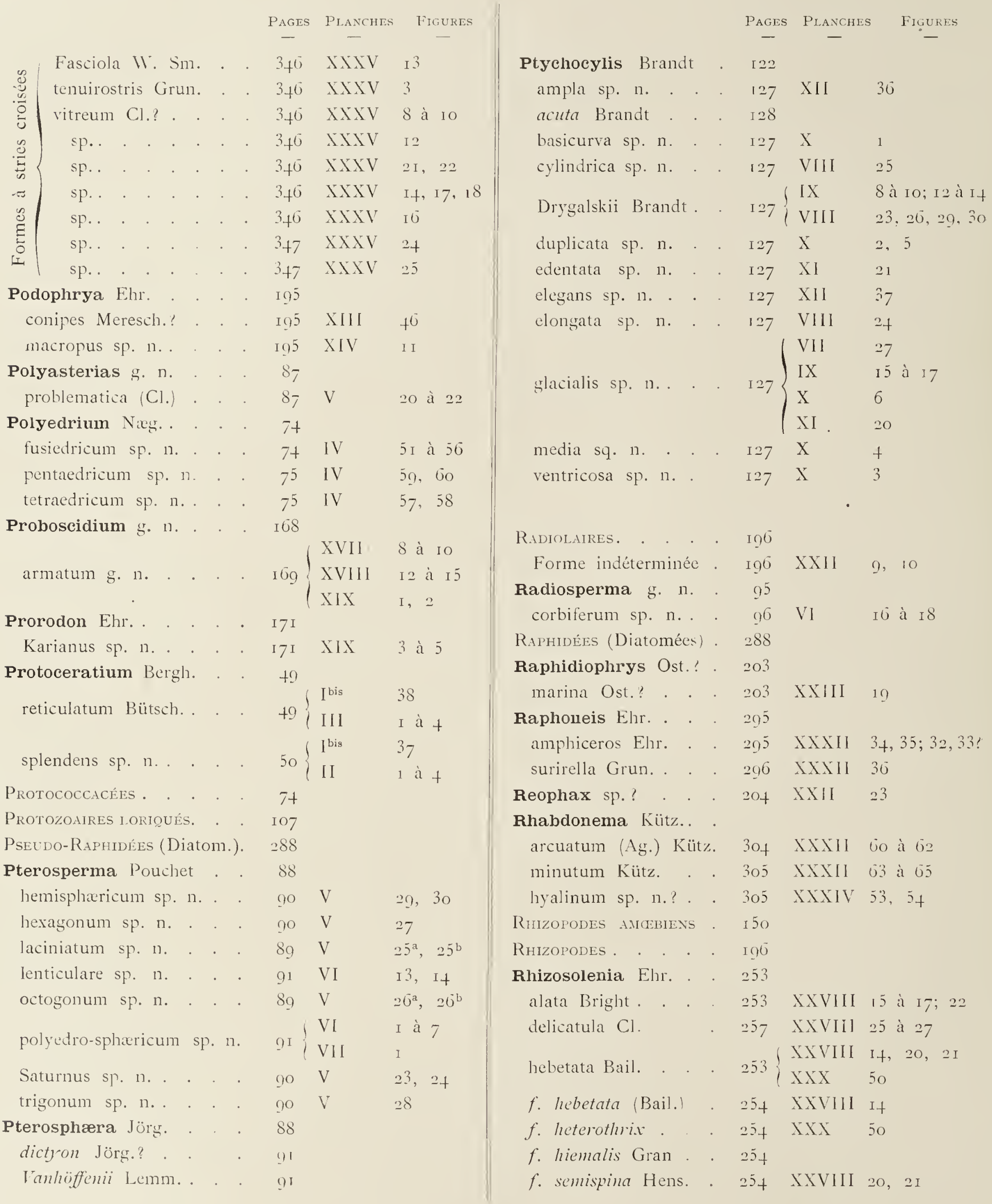


obtusa Hens.

setigera Bright.

Shrubsolei Cl. .

Stolterfothii Per.

styliformis Bright.

Rhoiconeis Grun. sp. .

Rhoicosigma Grun. sp. .

Schizonema $\mathrm{Ag}$.

$$
\text { sp. . }
$$$$
\text { sp. }
$$

Scoliopleura Grun latestriata (Bréb.) Grun. sp. .

Setopilium Hack.

sp. .

Setosperma g. 11 .

equatorale sp. $n$.

sp.?

SIIICOFLAGELLATES

Skeletonema (Grev.) costatum Grev.

Sphæropsis g. n

brevisetosa sp. n

echinata sp. n.

elliptica sp. 11.

heterosetosa sp. n.

levigata sp. $n$. .

longisetosa sp. $\mathrm{n}$.

nimbata sp. 11 .

nivalis $\mathrm{sp} . \mathrm{n}$.

spumosa $\mathrm{sp}$. $\mathrm{n}$

Spirodinium Stein

fusus sp. 11.

grave sp. $\mathrm{n}$.

lacryma sp. $n$.

Stappersia g. 11 . fusus sp.n. .

Stauroneis Ehr.

Stauropsis g. 17 acuta sp. n

\begin{tabular}{|c|c|c|}
\hline $\begin{array}{c}\text { Pages } \\
-\end{array}$ & $\begin{array}{c}\text { Planciles } \\
-\end{array}$ & $\begin{array}{c}\text { Figures } \\
-\end{array}$ \\
\hline 255 & XXVIII & I 5 à I 7 \\
\hline 253 & XXVIII & I 3 \\
\hline 256 & XXVIII & 13,24 \\
\hline 257 & XXVIII & 28 \\
\hline 254 & XXVIII & I 8, I 9 \\
\hline $3+8$ & & \\
\hline $3+8$ & XXXIII & 50,60 \\
\hline $3+7$ & & \\
\hline 347 & XIXV & 32 à 35 \\
\hline
\end{tabular}

$3+\mathrm{I}$

$3+\mathrm{I}$

$34 \mathrm{I}$

$3+8$

$3+8 \quad \mathrm{XXXV} 36,37$

$3+8$ XXXVI 86,87

199

I99

97

98

98

82

250

250

I 03

I 04

I 05

I 05

I 03

I 06

IOH

IOH

IO4

I 05

62

6.

6.

0

03

I 70

3 i 9

3 I 8

325
IXXIII 47

SxXII +9

XXIII +

VII 3, +

VII 5

XXVIII 35, 36

VII I 2

VII I6

XXIIl It

VII IO, II

VII 24, 25

VII It, I 5

VII 2 I

VII I 3

VII I 7 à 20

XIV 23 à 20

$\mathrm{XIV} 27, \quad 28$

XIV 2I, 22

XVIII 2I

XXXIII 50
Pages Planches Figures

amphicephala sp. n. . . 325 XXXII 5r

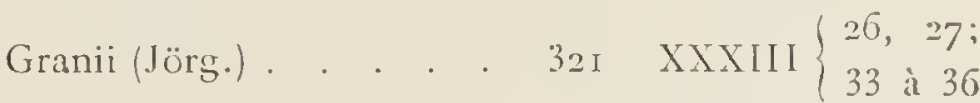

majuscula sp.n. . . . $325\left\{\begin{array}{l}\text { XXXIII 52, } 53 \\ \text { XXXV }\end{array}\right.$

membranacea (Cl.) . . 3 I9 XXXIII 37 à 40

pelagica (Cl.) . . . . 323 XXXII $4^{8}$

septentrionalis (Grun.) • . 322 XXXIII 28 à 32

Vanhöffenii (Gran)? . . 322 XXXIII 46

sp. . . . . 322 XXXIII $t^{5}$

Stenostomes (Tintinnopsis). It 3

Stephanodiscus (Ehr.) Grun. 280

densus sp.n. . . . . 280

Sticholonche Hertw. . . . 204 ventricosa sp. n. . . . 204

Zanclea Hertw. . . . . 204

Striatella Ag. . . . . . 306 unipunctata Ag. . . . . 306

Strombidium Cl. et Lach. . I 48 longipes sp. n. . . . . $\mathrm{I}_{4} 8$ sp. ? . . . . . . I 49

Suceurs (Infusoires) . . . 195

Surirella Turp. . . . . . 293

ovalis Bréb. . . . . 293

Synedra Ehr. . . . . 3 зо acus Grun. . . . . . 3 IO affinis Kütz. . . . . 3 1 Gallioni Ehr. . . . . 3 Iо pulchella Kütz. . . . . 3II ulna Ehr.

310\{

XXXI 27

XXII I à 3

XXXII 61 à 63

XIV 8

XVIII 20

XXXII 22,23

XXXIII 58

XXXII 54

XXXIII 54,55

XXXII 53

XXXII 50 à 52

XXXIlI 56,57

Tabellaria Ehr. . . . 305

Thxopones H. Fol.? •. . 204

Tentaculifieres (Infusoires). $\quad$ I9t

Tetraspora Lintz . . . . $\delta_{I}$

Poucheti Hariot . . . $8_{\text {I }}$

Thalassiosira Cl. . . . 250

bioculata Grun. . . . : 200

fallax sp. n. . . . . 268

gravida Cl. . . . . 259

XXIX $\quad t^{5}$ à 62

XXX I à 4

Nordenskiöldii Cl. . . . 265

XXIX I à 32

rotula sp.n. . . . . 264 XXIX 67 à 70 


$$
\text { Pages Plancines Figurits }
$$

Thalassiothrix CI. et Grun. 309 longissima $\mathrm{Cl}$. et Grun. . 300 Thuricola S. Kent . . . I 50 sp.! . . . . . 150

Tintindiles . . . . 107

Tintinnopsis Stein. . . 136 angusta sp. n. . . . If anmulata Dad. . . . I 1 o beroidea Stein! . . . I fo campanula Ehr. . . . I I 3 cylindrica sp. n. . . . I fo fusus sp. n. . . . . IfI incurvata sp. 1n.?. . . If6 lata sp.n. . . . . $1+1$ Lobiancoi Dad.? . . . 138 macropus sp.n. . . . $\quad$ I38 major sp. n. . . . . 138 nivalis sp. n. . . . . $\mathrm{I}_{4}{ }^{3}$

oliva sp. 11 . I 44\{ patula sp. n.

rapa $\mathrm{sp} . \mathrm{n}$. . I +4

sinuata Brandt I +5 tubulosa Lev. . . . 138 tubulosoides sp. n. . . . 159 Undella sp.n. . . . . $\quad$ I +6 urnula sp. n.

$\mathrm{I}+5$
ventricosoides sp. $n$. I +2

IXXII 49

$\mathrm{X} \quad 20,30$

XIII I7

XII 23, 24

$\mathrm{XIl} \mathrm{I}_{4}$ à $\mathrm{I} 8$

XII I2 à is

XII 25 à 28

XIII 20

XII In à 22

XII 5 à ?

XII 2 à 4

XII I

XIII 26, 27

XIII 9 à I 2

XIV 6

XIII I8, IO

XII 29 à 35

XIII It à I6?

XIII 20

XII 5 à ?

XII IO, II

$\mathrm{XIII} 28$

XIII 2 I

I 43 XIII I à 8
Tintinuus (Schr.) I)ad.

PAGES

Planches FIGURES acuminatus Clap, et Lachm. I 33 botnicus Nordq. . . . . 136 pellucidus CI. . . . . . I 3 t

$13+x$

X I6 à IO XI I I à I 2

Trachelius Clap. et Lachm. I 70 sp. . . . . . . 170

Trachelomonas. . . . 73

Triceratium Ehr. . . . 285 arcticum Bright. . . 285

Trigonum Cl. . . . . 285

arcticum Cl. . . . . 285

Trochiscia Lenm. . . . 76-กI dictyon Lemm. . . . 9I Vanhöfenii Lenm. . . . ดा

Tubuleuses (Tintinnides). . I 38 .

Vorticellides . . . . I I ?

Vaginicola Lam. . . I I sp.? . . . . . I 50

$\mathrm{X} \quad 20,30$

Xanthidium Cl. . . . $8 n$

Zonotrichium g. 11. . . I 73 discoides sp. n. . . . . 173

Zygoceros Ehr. . . . . 285 balana Ehr. . . . . 285
XIX 6 




\title{
MICROPLANKTON
}

\section{DES MERS DE BARENTS ET DE KARA}

\author{
par M. Alph. MEUNiER \\ Professeur à l'Université de Lone'ain
}

\section{INTRODUC'TION}

\section{I \\ DÉIIINITION DE L'OBJET}

Dans les pages qui vont suivre, nous nous proposons de faire l'étude quelque peu détaillée du Microplankton recucilli au cours de la croisière arctique de Mgr le Duc D'OrléANs, à bord de la Belgica, accomplie en Igo7, dans les parages de la Nouvelle-Zemble.

On sait que le terme de Plankton a été créé par HENSEN en I887, à l'époque où l'extension des recherches océanographiques rendit opportune la création d'une terminologie nouvelle, pour désigner des groupements d'organismes que ces études faisaient envisager à des points de vue nouveaux.

Dans son acception la plus large, ce terme s'applique à l'ensemble dés organismes qui flottent librement dans l'eau douce, saumàtre ou salée, sans moyens de locomotion propres suffisants pour les rendre indépendants des courants.

Ces organismes, d'ordre inférieur généralement, errent dans l'eau, à des profondeurs variables, à la merci des circonstances physico-chimiques qui modifient leur milieu naturel. 
C'est tout un monde d'êtres, de très petite taille pour la plupart, qui pullulent presque partout et dont les innombrables légions ne signalent leur présence que par de légères modifications dans la teinte et la transparence de l'eau.

Entre eux existent les àpres rapports de mangeurs et de mangés.

Ceux qui se sont repus des plus faibles deviennent à leur tour la proie d'autres mieux outillés ou plus forts, d'après la loi de subordination alimentaire des espèces, qui garde sa rigueur à tous les échelons de l'échelle du monde animé, jusqu'aux termes les plus élevés de la série animale. C'est ce que traduit vulgairement l'adage courant : les gros mangent les petits.

Le terme initial de cette hiérarchie alimentaire, qui a en réalité l'homme omnivore pour terme final, est représenté, en milieu aquatique, par des Algués pigmentées que leur chromophylle met à mème de s'alimenter aux dépens des substances minérales dissoutes dans l'eau, dont elles réalisent la synthèse organique, avec le concours de la radiation lumineuse.

Les Diatomacées sont évidemment celles qui jouent le rôle à la fois le plus important et le plus extensif. Ce sont elles que l'on peut le plus raisonnablement comparer aux graminées des prairies sur la terre ferme. Elles constituent, par leurs innombrables espèces, la prairie flottante des eaux : provende mondiale dont s'alimentent en dernière analyse, de ricochet en ricochet, tous les représentants de la taune aquatique tant d'eau douce que marine.

On peut subdiviser le Plankton en Microplankton et Macroplankton, en partant de l'inégalité des dimensions de ces organismes. Ceux qui peuvent passer au crible d'un tamis fin, dont la dimension des mailles est fixée arbitrairement, constituent le Microplankton; ceux que le tamis retient forment le Macroplankton.

C'est là une division toute artificielle, résultat d'un simple blutage, effectué en milieu liquide, des individus souvent innombrables qu'une pèche planktonique, exécutée au moyen d'un filet en fine étamine de soie, a réunis pèle-mêle, au hasard des circonstances.

Ce n'est pas ce que nous voulons; car, dans le microplankton ainsi entendu se coudoient les représentants des groupes organiques les plus disparates qui n'ont de commun que leurs dimensions très réduites: Protophytes et Protozoaires, Cœlentérés larvaires ou de petites formes, Echinodermes larvaires ou très jeunes, Vers larvaires, petits Nématodes, Desmocolécides, Chétognates, Rotateurs, Gastrotriches, petits Crustacés de toutes sortes, sous forme d'œufs, de larves ou d'adultes, Acariens, Mollusques larvaires ou très jeunes, œufs de Poissons, etc., etc.

Tout cela fait, en effet, partie du plankton microscopique et son étude est réellement du ressort des micrographes; car c'est du domaine des invisibles.

Mais nous devons limiter davantage l'objet de notre étude.

Par microplankton nous entendons, non pas le produit d'un triage par ordre de grandeur dont il vient d'ètre question, mais uniquement l'ensemble des espèces 
planktoniques unicellulaires, Protophytes ou Protozoaires, sans urger plus qu'il ne convient la distinction entre ces deux groupes d'organismes élémentaires ou Protoorganismes.

Nous estimons, en effet, que chez eux la distinction entre végétaux et animaux manque souvent de fondement réel et que l'on n'est pas même d'accord sur un critcrium distinctif conventionnel.

Il y a cependant les exigences de la biotaxie et nous reconnaissons qu'il est bon d'avoir le souci de s'y plier et de rechercher les affinités naturelles des microorganismes, pour les grouper harmonieusement, plutôt que d'entretenir délibérément la confusion que l'imprécision des caractères distinctifs rend difficile à dissiper.

On comprendra que nous voulions laisser de côté tous les organismes réellement multicellulaires : Métaphytes ou Métazoaires, quelles que soient leur simplicité relative, leur infériorité réelle ou apparente et même parfois leurs infimes dimensions, qu'il n'est pas rare de voir descendre en dessous de celles de beaucoup de Protistes.

C'est sous ce dernier vocable que nous voudrions ranger tous les organismes unicellulaires proprement dits, sans égard à leur nature végétale ou animale, suivant les vues de chacun.

Nous le reprenons ainsi, non plus au sens restreint que HaEckel y attachait et qui tombe en désuétude, dans cette acception, parce qu'il manque d'objectivité réelle bien définie; mais dans le sens de Protoorganismes, - vocable trop long celui-ci - pour désigner l'ensemble des organismes unicellulaires vivant isolément ou groupés en colonies homogènes, sans différenciation morphologique des individus ni division du travail entre eux, circonstance qui ne leur enlève pas leur caractère d'organismes unicellulaires vrais.

Par contre, nous excluons de ce cadre, quand leur nature est connue, bien entendu, les cellules libres, temporairement représentatives de Métaorganismes, à titre de phase initiale de reproduction agame ou sexuée : spores ou œufs.

Pour d'autres raisons, nous laisserons de còté les Bactériacées que leurs caractères morphologiques trop frustes sont presque toujours insuffisants à distinguer utilement et dont le rôle en milieu marin semble fort limité.

\section{CARACTÉRE DE L'ÉTUDE}

Dans ce travail nous comptons nous écarter délibérément des chemins battus par la plupart des microplanktonistes.

Généralement ceux-ci se contentent de consigner le résultat de leurs recherches sous la forme de listes des espèces reconnues, au cours de leurs observations, sur des matériaux donnés, dont ils se bornent à faire le dépouillement. 
Les espèces acquises déjà à la Science antérieurement sont simplement rappelées, avec référence à l'auteur, qui en a créé ce que l'on pourrait appeler l'état civil, avec signalement, en figure ou en diagnose.

Eventuellement les espèces inédites, chose peu rare dans ce domaine d'exploration récente, sont décrites, dénommées et classées.

C'est souvent la seule chose qui intéresse le micrographe et qui retienne son attention, surtout si les objets sont décrits ou figurés avec un soin suffisant pour donner une impression de la réalité (I).

C'est, on le voit, un travail de systématique pure, dont la valeur est incontestable et qui s'impose, lors de la prise de possession d'un nouveau domaine scientifique : le recensement, au fur et à mesure des progrès de l'exploration.

Les statisticiens de la science puiseront dans ces archives les matériaux de leurs études sur l'aire d'habitation des espèces, la composition des flores et faunes marines locales, l'influence, sur ces groupements, des circonstances du milieu : température, salinité, profondeur, courants, voisinage des fleuves, proximité ou éloignement des còtes, etc., etc.

Mais il faut bien convenir que ces listes n'intéressent que le petit nombre des spécialistes que leur aridité ne rebute pas et qu'atteignent les publications à faible tirage où elles paraissent : Bulletins académiques, Revues périodiques d'institutions scientifiques, Publications relatives à des recherches océanographiques éditées récemment dans toutes les langues de l'Europe septentrionale, etc.

La littérature française, par contre, est restée très pauvre en mémoires sur ce sujet et c'est une des raisons pour lesquelles les résultats acquis jusqu'ici sont loin de s'être vulgarisés même dans le monde scientifique de langue trançaise.

Il y aurait possibilité cependant d'intéresser un plus grand nombre de lecteurs à ces recherches modernes, qui nous paraissent dignes de fixer l'attention parce qu'elles portent en germe la matière d'une science jeune, qui sortira bientòt de sa phase chrysalidaire, pendant laquelle un travail intense mais obscur s'est effectué, pour créer et harmoniser ses parties constitutives, en préparant son éclosion.

Il y a pour cela à vaincre une difficulté qui est inhérente à la petitesse des organismes du microplankton, qui les rend insaisissables à l'œil nu.

(I) On peut regretter qu'il ne soit pas toujours satisfait à ce " desideratum », à propos d'organismes de certains groupes homogènes, pour lesquels la précision minutieuse des notes spécifiques a d'autant plus d'importance que les caractères génériques sont plus simples et plus fixes.

Certains travaux sont, sous ce rapport, d'une médiocrité déploralıle. Une publication récente: Nordisches Plankton, dètient actuellement un record de mauvais goùt, qui, nous l'espérons, ne lui sera jamais disputé. 11 est fàcheux que cette synthèse des travaux si méritants des planktonistes du Nord ait été illustrée avec une négligrence qui en amoindrit beaucoup la valeur taxinomique. 
Cette difficulté ne comporte, à notre avis, d'autre solution que la figuration des espèces telles que l'usage du microscope les fait voir, pour en noter non seulement les caractères morphologiques spécifiques, ce à quoi la plupart des auteurs se sont bornés jusqu'ici, mais encore et plutôt les caractères physiologiques et sociaux, qui permettraient de prendre contact plus intimement avec tout ce petit monde d'invisibles. Je veux dire : leur reproduction, leurs phases évolutives, leur régime alimentaire, leurs exigences vis-à-vis de l'ambiance, leur adaptation à un milieu donné, l'harmonie de leurs groupements, leurs rapports de sociabilité, de commensalisme, de symbiose, de parasitisme, toutes choses qui permettraient de s'intéresser à leur vie sociale.

On s'est occupé trop exclusivement de faire le dénombrement de cette population variée.

Il y a lieu d'en rechercher les principaux traits de l'histoire naturelle et d'en pénétrer davantage la vie individuelle et sociale, sans négliger le recensement des acquisitions nouvelles.

Le rôle de cette poussière vivante dans l'économie de la nature est à peine aperçu. Il réserve évidemment bien des surprises. C'est en en urgeant l'étude qu'on peut espérer trouver la solution de problèmes qui se posent depuis longtemps et qui intéressent l'importante industrie de la pêche à laquelle se trouve liée la prospérité de certaines populations littorales.

Dans ce milieu aquatique, plus homogène, plus uniforme que le milieu terrestre, la solidarité des organismes est plus étroite, leur enchainement est plus rigoureux.

L'équilibre vital y est plus stable; mais on conçoit que celui-ci dépende surtout du point d'appui : la florule planktonique, créatrice des principes organiques les plus simples, mais aussi les plus difficiles à réaliser, dont dépendent d'une façon plus ou moins immédiate tous les représentants de la faune aquatique, quelle que soit leur position dans la série animale.

C'est dans cet esprit que nous nous sommes livré à l'étude des matériaux microplanktoniques recueillis dans les parages de la Nouvelle-Zemble, par le personnel scientifique de la Belgica.

Ecartant a priori la présomption vaine de vider les questions que le sujet comporte, nous nous sommes efforcé tout au moins de consigner les faits d'observation que les matériaux nous ont présentés, en vue de fournir quelques éléments, - ceux que le hasard des prises d'échantillons a permis de surprendre — à l'histoire naturelle des Protistes de ces régions.

La nature de ces matériaux, très limités dans le temps et dans l'espace, ne permet évidemment pas la reconstitution intégrale des espèces et des groupements d'espèces, aux points de vue morphologique, physiologique et social. On n'en peut tirer que des faits occasionnels qui, venant comme appoint à d'autres déjà connus ou à connaitre ultérieurement, contribueront, comme autant d'anneaux d'abord épars, à constituer la chaine des traits saillants de l'histoire naturelle de ces organismes. 
La rareté et la brièveté des prises de contact avec ceux des parages peu explorés rendent forcément leur connaissance fragmentaire et lentement perfectible. C'est une raison de plus pour ne rien négliger de ce que les rares matériaux recueillis peuvent présenter d'intéressant à quelque point de vue que ce soit.

Il n'est pas douteux qu'il resterait déjà actuellement bien peu à ajouter utilement à la connaissance de beaucoup d'organismes, si l'on avait toujours eu le souci de tirer des produits de pêche tous les renseignements qu'on pouvait y puiser. Mais beaucoup ont été sacrifiés à des vues plus restreintes; par exemple, l'étude exclusive des frustules bien silicifiés des Diatomacées, par les procédés en usage chez les diatomistes. On sait que ces moyens violents, qui visent l'élimination de toute trace de protoplasme des tests siliceux, sont destructifs des caractères physiologiques des Diatomacées elles-mêmes et entrainent la perte complète de presque toutes les autres formes planktoniques, qui peuvent s'y trouver associées.

\section{ICONOGRAPHIE DE L'OBJET}

Conscient de la difficulté qu'il y a pour le lecteur, même versé dans la connaissance des Protistes, de recourir aux sources pour prendre contact avec les figures originales relatives aux organismes planktoniques d'une région qui lui est moins familière, nous avons voulu lui faciliter la tàche, en figurant nous-mème, non seulement les formes que nous considérons comme nouvelles, mais mème celles qui sont déjà connues et dont il importait cependant de montrer le facies particulier qu'elles présentent dans nos matériaux.

Aussi bien, beaucoup de figures publiées hâtivement, aux débuts des recherches planktoniques dans les mers du Nord, n'ont pas toujours l'exactitude ou tout au moins la précision des détails suffisante pour assurer la distinction des formes envisagées d'abord d'avec celles qui ne devaient être connues que plus tard. Ce manque de précision est cause, en partie, de l'état d'indivision dans lequel sont restées des séries d'organismes, fort homogènes d'ailleurs (I), que l'uniformité des caractères généraux a fait ranger sous une étiquette commune. C'est d'une étude plus minutieuse des

\footnotetext{
(1) Les Formenkreis des auteurs de langue allemande.
} 
caractères tant morphologiques que physiologiques qu'il faut attendre le démembrement de ces séries de formes en espèces distinctes, s'il y a un fondement réel à cette opération.

Pour assurer la plus grande fidélité possible à nos reproductions; nous nous sommes astreint à ne graver les figures de nos planches qu'avec l'objet sous nos yeux, au microscope, et non d'après des croquis exécutés auparavant avec la précision moindre que l'on apporte à une simple ébauche exécutée cursivement au crayon.

Dans le mème but, nous avons préalablement fait subir aux objets les manipulations qui, suivant les cas, nous ont paru les plus propres à en laisser percevoir les détails de structure qu'ils comportent et les phénomènes physiologiques qui s'y produisaient, au moment où ils ont été figés dans leur attitude, par les réactifs fixateurs.

Enfin, sauf de très rares exceptions dont mention sera faite dans le texte, nous avons adopté une échelle uniforme d'agrandissement pour toutes les figures, seul moyen pratique de permettre la comparaison d'organismes dont les dimensions réelles, toujours imperceptibles, ne sauraient ètre utilement traduites à l'imagination, en données métriques.

L'agrandissement à 500 diamètres environ nous a paru le meilleur, comme terme transactionnel entre les plus grands et les plus petits des Protistes, permettant la figuration suffisante des détails, sans exagérer les difficultés de la gravure, sans exiger trop de place et sans abuser de l'attention du lecteur. Ce grossissement est, en outre, celui qui nous a paru convenir le mieux aux besoins usuels de ce genre de recherches.

A se servir d'un système optique plus faible on se condamne à négliger beaucoup d'espèces que leur petitesse rendrait indiscernables ou dont on ne pourrait saisir les particularités intéressantes.

Par contre, l'usage d'un système de lentilles plus puissant, outre qu'il prendrait énormément plus de temps et entrainerait une fatigue insoutenable, ne serait pas compatible avec des recherches courantes de longure durée, dans ce domaine des petits où les difficultés inhérentes à la nature même des choses doivent ètre réduites à des limites acceptables, dans la pratique intensive des observations.

C'est pour ces raisons aussi que nous nous sommes défendu de reproduire des détails visibles seulement avec des combinaisons optiques d'objectifs à immersion, d'un pouvoir amplifiant de I, Ooo à I, 500 diamètres.

Ces détails trouveraient leur place dans une étude monographique d'une espèce ou d'un petit groupe d'espèces, où rien ne serait à négliger, suivant le précepte : Non multa, sed multum. Ils manquent de valeur pratique dans une étude générale de grande extension, où les caractères d'ordre moyen sont seuls compatibles avec l'étendue du sujet et où doit s'appliquer le principe : Non multunn, sed multa. 


\section{CARACTÉRE BIOTAXIQUE DE L'OBJET}

Les groupes d'organismes constitutifs du Microplankton marin, entendu comme il a été dit plus haut, sont loin de se présenter dans l'ordre d'importance relative que leur assigne la biotaxie générale. La préséance y revient souvent à des groupes dont le ròle, ailleurs, est très effacé ou mème nul.

Dans ce monde très mélangé où des espèces tenues pour végétales en coudoient d'autres considérées comme animales celles-ci n'occupent pas toujours les premiers rangs sociaux, ni par le nombre, ni par la taille, ni par la physionomie qu'elles impriment à l'ensemble.

Cette importance relative des catégories de Protistes varie du reste tellement avec le temps, le lieu, les mille circonstances de l'ambiance, qu'il serait difficile, en thèse générale, de formuler à cet égard quelque chose de précis.

Il n'est pas douteux toutefois que les Diatomacées et, parmi elles, les Chétocérées particulièrement y détiennent presque toujours une place de choix.

Logiquement, nous devrions donc nous y attacher d'abord.

Mais la délicatesse de leur structure et la minutie déconcertante de leurs caractères distinctifs exigent des procédés d'étude qui ne seraient pas de mise pour les formes qui leur sont communément associées.

De plus, leur membrane siliceuse, réfractaire aux agents chimiques les plus énergiques et même à la combustion, autorise à leur égard une technique spéciale que les autres groupes de Protistes ne réclament pas et dont ils ne sauraient mème aucunement s'accommoder.

Ce sont les raisons pour lesquelles nous en réserverons l'étude pour la fin, après que nous aurons tiré parti de nos matériaux pour l'examen des autres formes microplanktoniques, sans nous exposer à en rien gaspiller, au préjudice de celles-ci; surtout lorsqu'il s'agit d'échantillons peu copieux.

Ces autres formes sont très diverses et ne se laissent pas toujours aisément distribuer dans les casiers préétablis des systèmes de classification dont les cadres varient, du reste, selon les vues personnelles des auteurs.

Les unes, pourvues de chromophylle, auraient au moins le caractère de plasmadomes ou constructeurs de plasma, que l'on est assez d'accord pour considérer comme propre au monde végétal, si l'action combinée de la lumière et du milieu conservateur n’avait généralement détruit tout pigment de ce genre. 
La disparition de ce caractère n'a pas d'inconvénient lorsqu'il reste d'autres raisons pour confirmer cette attribution, comme il en est chez les Péridiniacées et certaines Cryptomonadacées, par exemple, dont les traits de famille subsistent à travers toutes les causes de défiguration.

D'autres se révèlent, de toute évidence, comme des plasmophages et on n'hésite pas à les rapporter à des familles bien connues du monde animal : les Tintinnides, par exemple, qui forment l'élite des Infusoires dans le microcosme planktonique.

Mais combien n'en est-il pas d'autres dont les affinités sont douteuses ou mème dont on ne saurait pas dire actuellement s'ils sont bien des organismes autonomes plutôt que des formes unicellulaires représentatives d'organismes plus complexes, à défaut d'avoir surpris jusqu'ici le secret de leur origine ou de leur évolution!

Ces dernières constituent provisoirement des énigmes dont la science trouvera tòt ou tard la solution, à la condition de les imposer à l'attention des chercheurs que les circonstances aideront à établir des rapprochements encore insoupçonnés. Ainsi se réduira peu à peu le nombre encore considérable des organismes incertae sedis.

La nature de nos matériaux d'étude nous a fait adopter la distribution suivante :

I $^{\circ}$ Péridiniacées et organismes connexes;

$2^{\circ}$ Microphytes des neiges colorées;

3o Gryptomonadacées ;

$4^{\circ}$ Silicoflagellates ;

$5^{\circ}$ Organismes énigmatiques;

60 Protozoaires loriqués ;

$7^{\circ}$ Protozoaires non loriqués;

8o Rhizopodes - Radiolaires, principalement;

$9^{\circ}$ Diatomacées, de toute origine, qui formeront la matière de la seconde partie de ce mémoire.

Nous en ferons autant de GROUPES, objets de chapitres distincts.

Dans cette classification, certains groupes bien connus d'organismes s'offrent comme points d'appui pour d'autres, moins importants ou moins bien définis, qui ne méritent pas une mention spéciale.

Il ne faut voir dans cet arrangement que des raisons de convenance occasionnelle déterminée : $\mathrm{I}^{\circ}$, par la nature du matériel d'étude; $2^{\circ}$, par les exigences des procédés de recherche; $3^{\circ}$, par les difficultés de l'agencement plus ou moins systématique des figures dans les planches annexées.

Nous ne le reconnaissons pas comme autrement fondé. Il nous sera, du reste, difficile de nous y asservir strictement; car la nature ne se prète pas, avec toute la bonne volonté que certains voudraient lui voir, à toutes nos découpures artificielles de cette admirable unité organique : vaste champ où il n'y a pas d'autres clòtures que celles que nous essayons d'y planter. 
Quoi qu'on fasse, le parcage systématique des organismes est toujours artificiel de quelque còté et le meilleur, dans un cas donné, est celui qui répond le mieux aux besoins de la situation.

C'est d'ailleurs cette difficulté de délimiter nettement les groupes qui nous a induit à les traiter tous ensemble, plutôt que d'en faire des monographies distinctes qui nous en auraient fait préjuger les limites.

\section{CARACTÉRE GÉOGRAPHIQUE DE L'OBJET}

L'examen des matériaux recueillis par la Belgica, dans les parages de la NouvelleZemble, nous a mis en présence d'associations étranges qui ne peuvent s'expliquer que par le mélange de formes continentales et de formes océaniques, mélange que les circonstances du lieu favorisent d'une manière exceptionnelle.

Dans les régions côtières où la Belgica s'est presque toujours maintenue, pendant sa croisière scientifique, les eaux continentales provenant de la fusion des neiges et des glaces sont largement tributaires de la mer, non seulement au voisinage des cours d'eau, mais, d'une manière plus générale, sur tous les points où s'opère la fonte des glaces flottantes : c'est-à-dire sur toute la surface d'une mer peu ouverte, comme l'est la Mer de Kara, où les glaçons de provenance diverse, qui y ont conflué comme dans un carrefour, subissent une fonte plus ou moins complète, pendant leur débâcle estivale vers la Mer Mourmane.

Or, les neiges et les glaces qui, à première vue, sembleraient absolument stériles, constituent un champ de culture pour des microorganismes d'espèces, plus variées qu'on ne pourrait le supposer, et représentées par des légions d'individus dont le nombre prodigieux supplée à la petitesse des dimensions.

La fusion de leur milieu originel, de leur habitat normal, les précipite dans le milieu marin et leur présence donne au planliton de ces parages un caractère hybride, à la fois continental et marin, dont on n'a des exemples analogues qu'au voisinage plus ou moins immédiat des fleuves, dans les régions tempérées ou chaudes.

D'autre part, si les apports continentaux fournissent au plankton de la mer un contingent d'espèces originairement étrangères à ce milieu, la réciproque s'exerce du côté des formes marines, que les vents et les vagues peuvent jeter sur les glaces flottant au large ou fixées à la còte. Mais il n'y a pas compensation pour la quantité et, de plus, il ne s'agit jamais ici que d'un prèt à court terme. 
Il importe donc de débrouiller les associations d'espèces, qui font l'objet de ces échanges, entre milieux différents et d'en rapporter les formes chacune à son habitat propre.

C'est un còté spécial des recherches planktoniques qui n'a guère fixé l'attention jusqu'ici, à défaut sans doute de matériaux de contrôle.

Heureusement, M. Louis Stappers, le naturaliste de l'Expédition, pressentant sans doute cette solidarité plus étroite entre tous les organismes des régions polaires, n'a pas négligé de faire des prélèvements d'échantillons de produits continentaux, toutes les fois que des circonstances spéciales appelaient son attention sur l'existence probable d'ètres animés dans ces produits.

Il se fait, en effet, que plusieurs d'entre eux sont extrèmement instructifs et ont une grande valeur interprétative, à l'endroit de l'économie du monde animé de ces régions.

Cette circonstance, d'une importance spéciale pour la Mer de Kara, nous inviterait à jeter d'abord un coup d'œil sur les microorganismes continentaux de son voisinage qui deviennent ainsi tributaires du plankton marin et lui impriment ce caractère ambigu dont nos matériaux d'étude fournissent des exemples plus frappants que ceux d'autres expéditions à caractère plus pélagique; mais ce serait anticiper sur des considérations générales de distribution géographique des espèces, que nous ne pourrons convenablement traiter qu'après avoir pris connaissance des formes elles-mêmes et d'en avoir fixé les cadres systématiques.

La partie analytique de notre étude portera donc sur l'ensemble des Protistes recueillis, sans tenir compte de la diversité d'habitat.

La diversité géographique de l'objet se reflète dans les tableaux joints ci-après des prises d'échantillons, où sont consignées, avec leur numéro d'ordre, les circonstances de temps, de lieu, de profondeur, de procédés de pèche, etc., qui constituent pour chacune un certificat d'origine.

Le premier Tableau donne des détails sur les vingt-deux stations de pèches verticales de plankton. Les stations I à í sont situées dans la Mer de Kara, les stations I7 et IS dans le détroit de Matotchkine, les stations ig à 22 dans l'Océan Glacial, au nord de la Mer de Barents.

Dans le second TABleau sont consignées les pèches horizontales de plankton de surface et d'autres récoltes relatives à la florule glaciaire.

La carte du théàtre des opérations auxquelles s'est livré le personnel scientifique de l'Expédition constitue un supplément de documentation, auquel le lecteur ne manquera pas de recourir. On a eu soin de pointer, sur l'itinéraire suivi, d'une part, les pêches verticales qui ont été effectuées dans vingt-deux stations et, d'autre part, les pèches horizontales effectuées pendant la marche du navire. Celles-ci sont 
marquées d'un trait épaissi, dont la longueur est proportionnelle à la distance parcourue pendant le traînage du filet. Les stations sont marquées d'un gros point rond, dont le numéro d'ordre, traduit en chiffres gras, embrasse les plus ou moins nombreuses opérations réalisées en chacun de ces endroits.

Une deuxième carte, à échelle plus grande, mais n'embrassant que l'ìle sud de la Nouvelle-Zemble, donne au lecteur une intuition plus claire du lieu géographique des seize premières stations, que leur rapprochement rend difficiles à distinguer dans la carte d'ensemble.

Dans la pratique, il suffira généralement de rapporter les espèces observées à certaines catégories d'échantillons, dont les unes ont un fondement géographique, tandis que d'autres n'ont de caractéristiques que les circonstances locales du milieu. Ces dernières se rattachent presque toutes, géographiquement, à la Mer de Kara et aux terres de la Nouvelle-Zemble.

Les voici, avec le libellé sous lequel nous comptons les désigner dans le corps du travail :

I Région sud de la Mer de Barents, depuis les còtes norvégiennes jusqu'à la hauteur du Détroit de Matotchkine.

$2^{\circ}$ Région nord de la Mer de Barents, à partir du Détroit de Matotchkine jusqu'au $7^{\mathrm{e}}$ parallèle.

3o Région de l'Océan Glacial.

$4^{\circ}$ Région du Détroit de Matotchkine.

50 Région de la Mer de Kara. Eaux marines.

$6^{\circ}$ Eaux douces de la Mer de Kara provenant de la fusion superficielle, sur place, des neiges et des glaces et déterminant des sortes de petits lacs, dans les dépressions des champs glacés.

\section{$7^{\circ}$ Rivière Matotchka. \\ $8^{\circ}$ Neiges colorées. \\ $9^{\circ}$ Glaces. \\ IO ${ }^{\circ}$ Dépôts glaciaires. \\ I ${ }^{\circ}$ Boues marines de fond.}

On voit, par cette énumération, que les matériaux recueillis ne sont pas tous planktoniques; mais ceux qui n'ont pas ce caractère n'en sont pas moins intéressants par la place qu'ils tiennent dans la florule des régions arctiques. 


\section{TABLEAUX (1)}

INDIQUANT LES LOCALITÉS OÙ ONT ÉTÉ RECUEILLIS

\section{LES ÉCHANTILLONS DE PLANKTON}

AINSI QUE

LE TEMPS ET LES PROCÉDÉS DE RÉCOLTE

(I) Dressés par MM. A. DE Gerlache et L. Stappers. 
(PÂCHES

\begin{tabular}{|c|c|c|c|c|c|c|c|c|}
\hline \multirow{2}{*}{$\begin{array}{l}\text { NuMERo } \\
\text { DE LA } \\
\text { Station }\end{array}$} & \multirow{2}{*}{ DATE } & \multicolumn{2}{|c|}{ POSITION } & \multirow{2}{*}{ 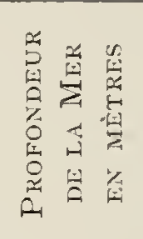 } & \multicolumn{2}{|c|}{ 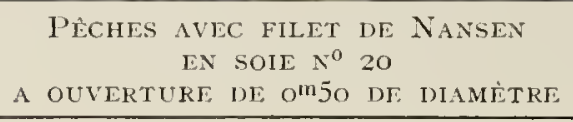 } & \multicolumn{2}{|c|}{$\begin{array}{r}\text { PÉches AVEC FILET DE NANSLN } \\
\text { EN SOIE } N^{\circ} 3 \\
\text { A OUVERTURE DE I MIETRE DE DIAMĖTRE }\end{array}$} \\
\hline & & Latitude $N$ & Longitude E & & Niveau & $\begin{array}{l}N^{0} \text { DU JournaL } \\
\text { DE P'tiche }\end{array}$ & Nive,au & $\begin{array}{l}N^{0} \text { DU JOURNaL } \\
\text { DE PretiIE }\end{array}$ \\
\hline 1 & $\begin{array}{l}1907 \\
17 \text { juillet }\end{array}$ & $72^{n} 39^{\prime}$ & $56^{\circ} 18^{\prime}$ & 162 & $160-0$ & 30 & $160-0$ & 29 \\
\hline 2 & $17 "$ & $72^{\circ} 35^{\prime}$ & $56^{\prime \prime} 10^{\prime}$ & 65 & $65-0$ & 34 & $\begin{array}{l}65-30 \\
65-0\end{array}$ & $\begin{array}{l}35 \\
36\end{array}$ \\
\hline 3 & $22 \quad 11$ & $71^{\circ} 59^{\prime}$ & $55^{\prime \prime} 53^{\prime}$ & 122 & $\begin{array}{l}122-0 \\
35-0\end{array}$ & $\begin{array}{r}43 \\
44\end{array}$ & $\begin{array}{c}122-30 \\
30-0 \\
30-0\end{array}$ & $\begin{array}{l}45 \\
46 \\
47\end{array}$ \\
\hline 4 & 23 & $71^{\circ} 58^{\prime}$ & $55^{\prime \prime} 49^{\prime}$ & 111 & $\begin{array}{l}110-30 \\
30-0\end{array}$ & $\begin{array}{l}48 \\
49\end{array}$ & $\begin{array}{c}105-75 \\
75-30 \\
30-0\end{array}$ & $\begin{array}{l}50 \\
51 \\
52\end{array}$ \\
\hline 5 & 25 & $71^{\circ} 52^{\prime}$ & $55^{\circ} 52^{\prime}$ & 151 & $\begin{array}{c}150-35 \\
35-0\end{array}$ & $\begin{array}{l}54 \\
55\end{array}$ & $\begin{array}{c}105-35 \\
35-15 \\
35-0 \\
15-0\end{array}$ & $\begin{array}{l}56 \\
57 \\
58 \\
59\end{array}$ \\
\hline 6 & 27 & $71^{\circ} 49^{\prime}$ & $55^{\circ} 52^{\prime}$ & 124 & $\begin{array}{c}120-35 \\
35-0\end{array}$ & $\begin{array}{l}63 \\
64\end{array}$ & $\begin{array}{c}120-35 \\
35-0\end{array}$ & $\begin{array}{l}65 \\
66\end{array}$ \\
\hline 7 & 29 & $71^{\circ} 44^{\prime}$ & $55^{\circ} 56^{\prime}$ & 115 & $\begin{array}{l}110-: 35 \\
35-0\end{array}$ & $\begin{array}{l}70 \\
71\end{array}$ & $\begin{array}{l}110-35 \\
35-0\end{array}$ & $\begin{array}{l}68 \\
69\end{array}$ \\
\hline 8 & 30 & $71^{\circ} 42^{\prime}$ & $55^{\circ} 58^{\prime}$ & 127 & $\begin{array}{l}125-40 \\
40-0\end{array}$ & $\begin{array}{l}76 \\
77\end{array}$ & $\begin{array}{c}125-40 \\
40-15 \\
15-0\end{array}$ & $\begin{array}{l}73 \\
74 \\
75\end{array}$ \\
\hline 9 & 31 & $\tau 1^{\circ} 39^{\prime} 30^{\prime \prime}$ & $56^{\circ} 12^{\prime}$ & 134 & - & - & $132-0$ & 80 \\
\hline 10 & 6 août & $71^{\circ} 34^{\prime}$ & $57^{\circ} 15^{\prime}$ & $2: 06$ & $225-0$ & 101 & $225-150$ & 100 \\
\hline
\end{tabular}


(PÊCHES HORIZONTALES

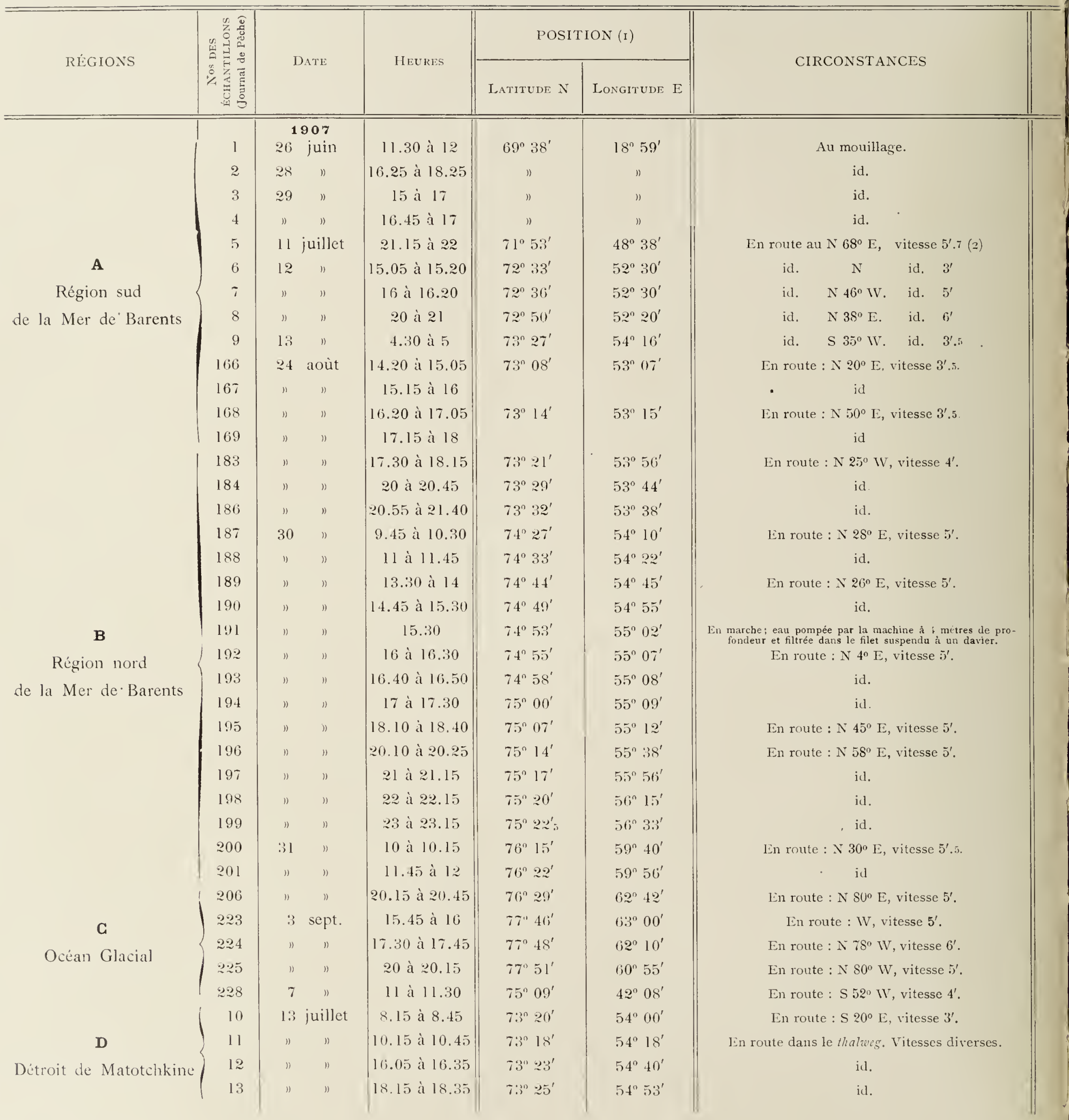




\section{DE SURFACE}

ET FLORULE DES GLACES)

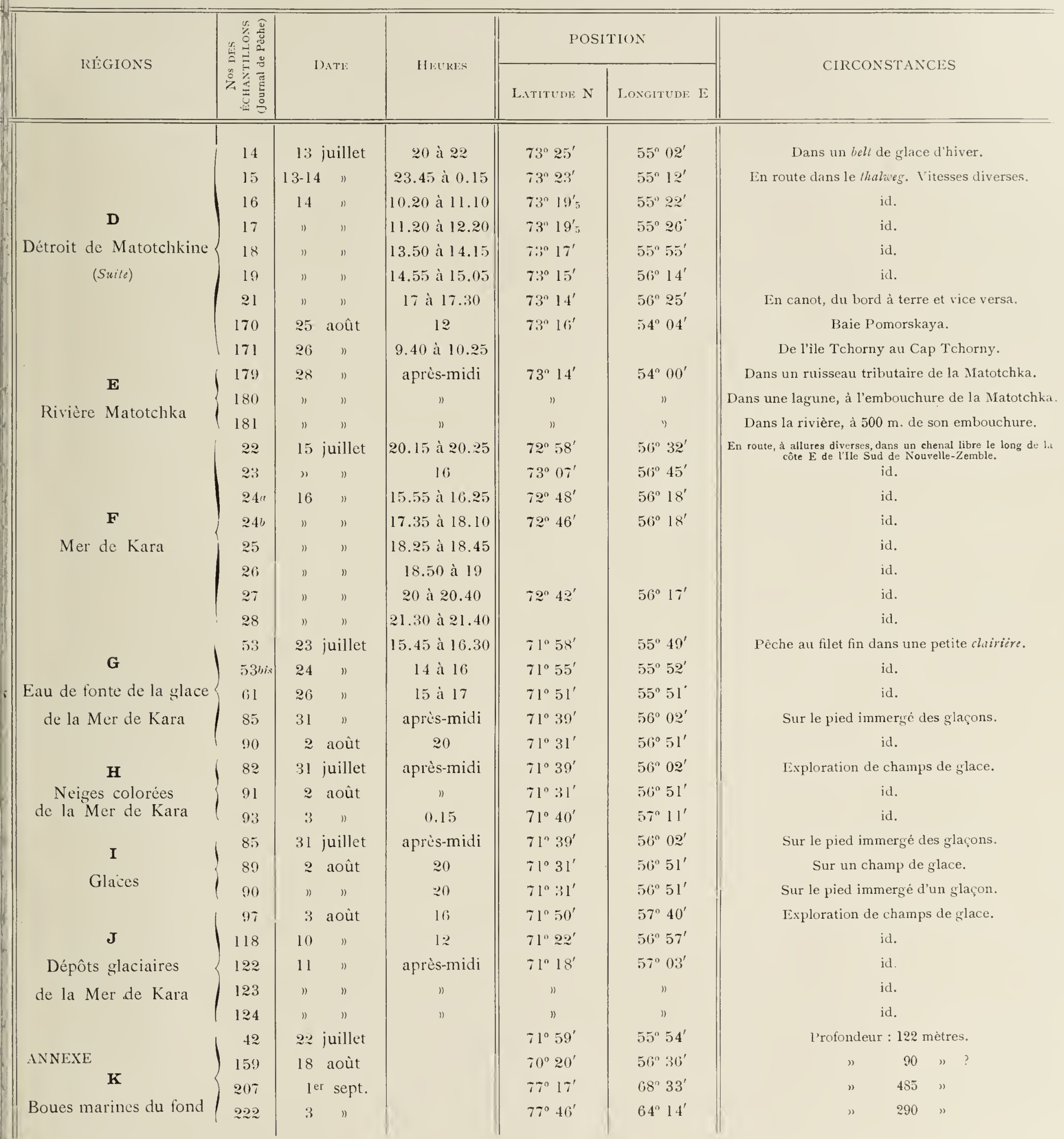


LIBRARY

OF THE

IINIVRSITY OF ILLINOIS 


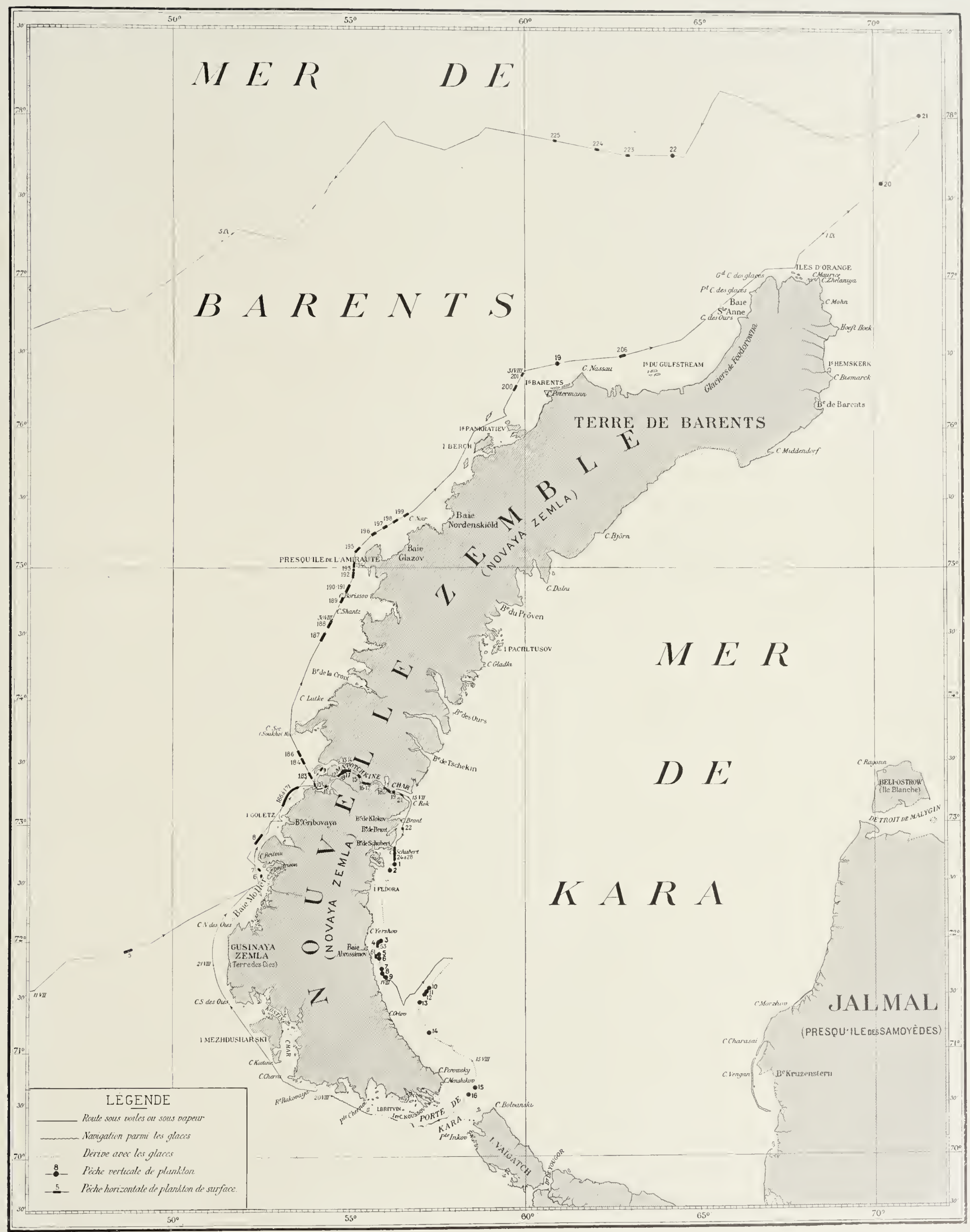

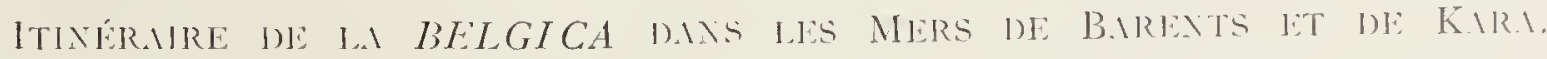


OF THE

IINIVERSITY OF ILLINOIS 


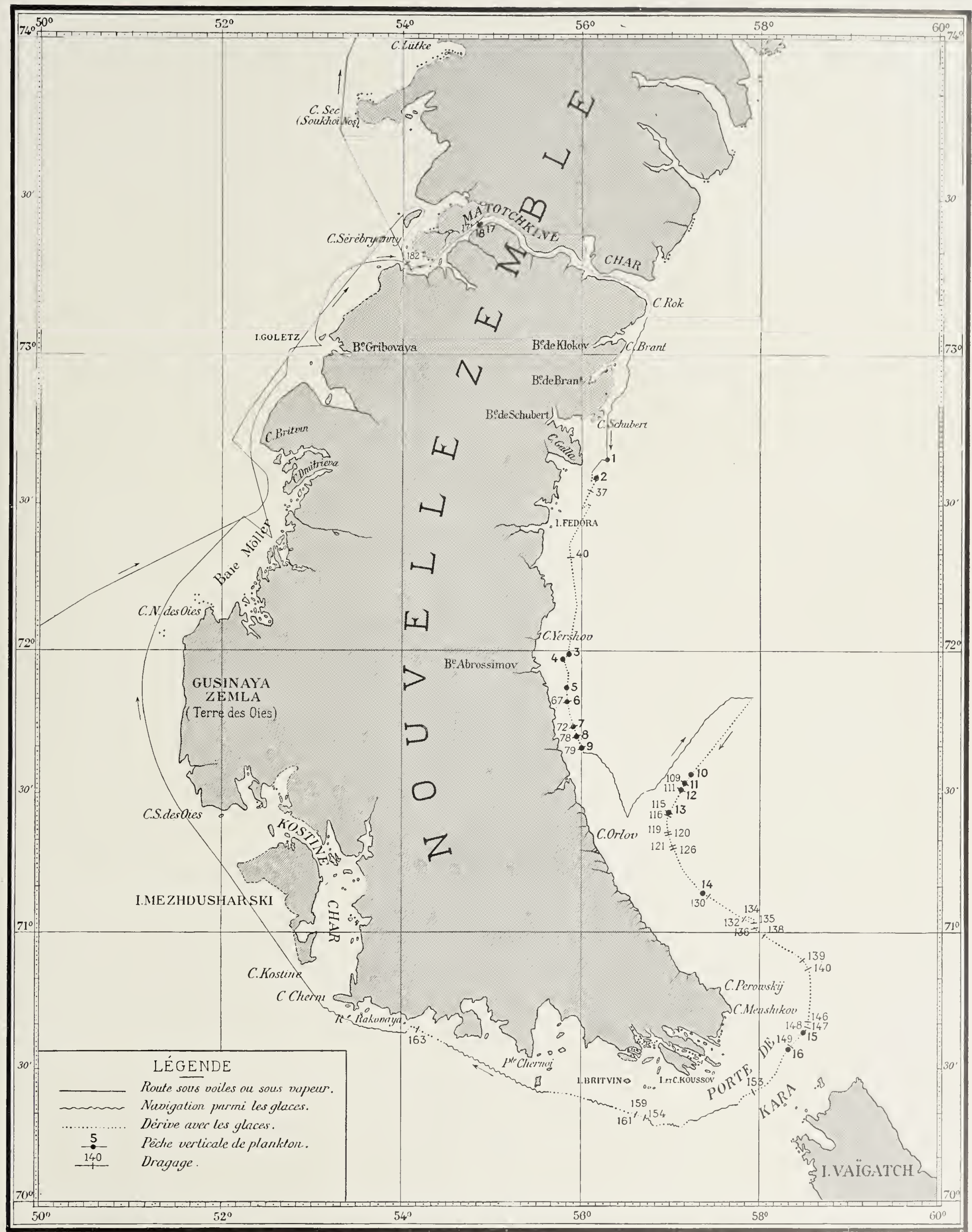

ITINERARE DE IA BELGICA AUTOUR DE L'ILE SUI DE LA NOUTELle-ZEMible. 
LIBRARY

OF THE

UNIVERSITY OF ILLINOIS 


\title{
PREMIERE PARTIE
}

\section{PRO'TISTES AU'TRES QUE LES DIA'TOMACÉES}

\author{
Groupe I. - PÉRIDINIACÉES
}

Nous tenons les Péridiniacées pour une famille de la classe des Phéophycées: microphytes unicellulaires porteurs de deux longs cils vibratiles ou flagellums attachés latéralement et dirigés l'un en bas, dans un sillon dit longitudinal ou ventral, l'autre en travers, dans un sillon transversal, la ceinture, qui fait le tour du corps.

Ces faibles moyens de locomotion en font des organismes essentiellement microplanktoniques.

La plupart sont pourvus d'une membrane cellulosique assez épaisse et rigide constituant une enveloppe capsulaire formée de plaques distinctes, en nombre variable, que l'on peut aisément dissocier. Ces plaques, soudées d'abord suivant des lignes de suture - sutures simples - peuvent glisser plus tard l'une sur l'autre sans perdre leur adhérence et permettent l'accroissement de la capsule, en donnant des bandes intercalaires - sutures doubles. Ils forment la sous-famille des Péridiniées.

Ceux qui n'ont qu'une membrane mince et lisse se rangent dans la sous-famille de Glénodiniées.

On peut aussi, suivant Delage (I), considérer les Péridiniens comme une sous-classe des Flagellés, les Dinoflagellés qui se subdiviseraient en trois ordres : les Dinoférides, pourvus des deux sillons typiques mentionnés plus haut, les Polydinides, ornés de plusieurs sillons transversaux, et les Adinides, dépourvus de sillons.

Ces organismes ont des caractères de famille très nets; mais on n'en peut dire autant des caractères génériques et spécifiques, sur lesquels l'accord des planktonistes est loin d'être fait.

(I) Y. Delage et En. Hórouard : Traité de Zoologie concrite, t. I, Paris, 1896. 
Sur ce thème très simple d'un organisme unicellulaire, libre, à membrane capsulaire, à endochrome brun et à deux cils locomoteurs, la nature s'est ingéniée, semblet-il, à moduler des variations sans nombre, que la délicatesse des nuances rend difficiles à saisir.

Aussi faut-il convenir que, au point de vue taxinomique, ce petit monde sort à peine de l'état chaotique que les premières signalisations ont créé, que les découvertes subséquentes ont entretenu et que les premières ébauches de classification n'ont pas réussi à dissiper.

Cela tient à des causes multiples :

Io Au défaut d'éléments de comparaison à la portée des auteurs, qui ont dù décrire et dénommer des formes dont ils ne pouvaient apprécier les rapports avec d'autres voisines ou mème spécifiquement identiques;

$2^{\circ}$ A l'absence d'entente sur des caractères, au moins conventionnels, dont on pourrait faire état dans les diagnoses;

3o Au défaut d'attention suffisante dans l'observation des caractères, qui auraient pu guider ceux qui ont tenté des travaux synthétiques sur cette curieuse famille;

$4^{\circ} \mathrm{A}$ l'imperfection des dessins prétendument figuratifs des espèces observées, dessins purement schématiques parfois ou exécutés, avec une large marge d'approximation, par des auteurs peu familiers avec l'art du portraitiste. Ce terme est ici de circonstance; car la physionomie de ces petits ètres ou, mieux, l'expression de leur physionomic est, à notre avis, la seule note caractéristique de beaucoup d'espèces de certains groupes très homogènes de cette famille. Une diagnose, si précise soit-elle, ne réussirait pas à les faire distinguer. Ce qu'il faut c'est un portrait de face et de profil.

Bien que chacun doive sentir maintenant le besoin d'une grande précision des détails dans la description de formes si homogènes, on ne s'y astreint pas toujours assez dans les publications sur la matière en question. On connait des ouvrages, ne datant pas de deux ans, dans lesquels aucune espèce de Peridinium expressément figurée, par exemple, n'est réellement identifiable, sans des concessions excessives faites à la défectuosité des procédés de figuration.

Pour ces raisons, nous nous abstiendrons de faire des références à des ouvrages dont le défaut capital est de projeter de l'ombre là où il faudrait de la lumière.

A notre avis, ces organismes à structure dorso-ventrale et antéro-postérieure devraient toujours être figurés dans des poses conventionnelles, facilement repérables parce que fondées sur l'orientation des axes et les conditions de symétrie de leur corps, des poses classiques:

I $^{\circ}$ Vue de face antérieure, normale aux deux axes longitudinal et latéral;

$2^{\circ}$ Vue postérieure, orientée de mème, souvent inutile;

3o Vue latérale droite ou gauche, l'une valant l'autre généralement; c'est la vue de profil; 
polaire ;

$4^{\circ}$ Vue apicale, ou vue d'en haut, dans la direction de l'axe longitudinal ou

5. Vue antapicale, ou vue d'en bas, dans la même direction (I).

Toutes les autres vues, plus ou moins obliques, devraient être laissées, pour ne pas introduire des éléments de confusion, si ce n'est dans les cas exceptionnels où il y aura lieu de noter une particularité importante qui ne serait pas atteinte par l'une ou l'autre des vues normales susmentionnées.

Dans l'exposé analytique qui va suivre, nous nous attacherons non pas uniquement à l'ordre taxinomique qui s'imposerait dans un travail de synthèse, mais aussi et plutôt à l'ordre économique, si l'on peut dire, résultant du rôle que les organismes jouent dans les milieux où les pêches ont été effectuées. C'est un point de vue spécial ayant sa légitimation dans ce travail où nous nous proposons surtout de mettre en relief les conditions sociales des espèces planktoniques, dans des endroits déterminés où le hasard des captures en a révélé la coexistence et les rapports de dépendance.

\section{Genre Peridinium Ehrenberg}

Nous estimons qu'il n'y a lieu de maintenir dans ce genre que les espèces présentant vingt et une plaques capsulaires, dont quatorze du côté apical ou supérieur et sept du còté antapical, sans compter les deux sillons transversal et ventral.

Cela est nécessaire pour conserver l'unité dans ce genre, d'ailleurs très riche en espèces, et maintenir entre celles-ci au moins un caractère commun.

Ce qui nous paraît contribuer le plus à imprimer aux espèces une physionomie spéciale c'est :

I $^{\circ}$ La direction droite ou gauche de la ceinture, quand elle est réellement spirale et non rigoureusement circulaire, comme il arrive rarement;

(I) Les termes : ventral et dorsul, antérieur et postérieur, s'expliquent d'eux-mêmes, par la position de l'orifice buccal dans le sillon longitudinal, qui fait placer celui-ci sur le côté ventral ou antérieur.

Quant aux termes : haut et bas, apext et antapex, cône apical et cône antapical, ils n'ont qu'une portée conventionnelle.

L'usage a prévalu, en effet, de firurer ces organismes tels qu'on les voit au microscope, en inage renversée consé(1uemment, et dans une position qui satisfait mieux notre tendance instinctive à nous représenter les objets dans un état aussi conforme que possible aux lois de l'équilibre. Pour satisfaire à ce besoin naturel, les espéces tricuspides doivent prendre assiette sur le còne qui porte les deux cornes ou pointes gèminèes.

Peu importe l'attitude rraic de ces organismes nageurs dans leur milieu naturel, à l'état vivant. A vouloir en tenir compte, en toutes circonstances, on ajouterait une grande difficulté à toutes celles inhérentes au sujet. 
$2^{\circ}$ La forme de la ceinture qui est tantòt plate ou peu déprimée, maintenue à peu près au niveau du reste du corps et bordée de deux ailettes parallèles généralement bien visibles : fig. $4, .8$, IO, I7, 24, etc., pl. I, tantôt déprimée, creusée en sillon ou en gouttière à section semi-cylindrique et alors exempte d'ailettes distinctes sur les bords : fig. 32, 38, 40, etc., pl. I.

Beaucoup d'auteurs n'ont pas accordé assez d'attention à l'expression fidèle de cette particularité dans les croquis des espèces qu'ils ont silhouettées, exagérant à plaisir la dépression du sillon transversal, quand elle est à peine indiquée, ou lui attribuant l'effet produit par les ailettes latérales et parallèles de la ceinture, qu'ils ne traduisent pas suffisamment.

Il en résulte une modification dans la physionomie, suffisante pour rendre douteux les rapprochements que l'on tenterait de faire avec des formes ainsi figurées si on ne les connaissait autrement pour les avoir observées soi-mème.

Bon nombre de figures de Nordisches Plankton, XVIII, Peridiniales, qui, pour la plupart, sont la reproduction telle quelle des dessins originaux des auteurs, sont entachées de ce défaut.

Nous attachons néanmoins à cette particularité une valeur suffisante pour en faire le pivot de la classification que nous proposons ici, bien qu'on ne doive généralement pas en trouver l'expression adéquate dans les dessins de nos prédécesseurs.

L'application de ces caractères les plus généraux nous fait distinguer les trois sections suivantes, qui se ramènent elles-mèmes à deux séries :

\section{Série A. -.. PLANOZONES}

Section I. - Espèces à ceinture plate, ailée, sinistrogyre.

Section II. - Espèces à ceinture plate, ailée, dextrogyre.

\section{Série B. - CAVOZONES}

Section III. - Espèces à ceinture creuse, non ailée, sinistrogyre plus ou moins manifestement; sinon, circulaire (I).

(I) On reconnaitra que nos sections I et III correspondent assez bien au sous-genre Euperidinium Gran, qui englobe les espèces sinistrogyres; notre section II au sous-genre Protoperidinium Bergh, qui embrasse les espèces dextrogyres. 


\section{Série A. - PIANOZONES}

\section{Section I. - Sinistrogyres}

\section{Peridinium divergens Ehrenberg, var. reniforme.}

Planche I, figures I à 4 . Planche Ibis, figures I à 8. Planche II, figures $4^{5}$ et 46 .

Synonyme. - P. parallelum Broch? P. divergens var. granulatum Karsten, igo6; non $P$. depressum Bailey, etc., etc.

Voir à ce sujet : Nordisches Plankton. Achte Lieferung, XVIII, page 53. Kiel und Leipzig, Igo8 (I), où l'espèce en question est désignée, à tort selon nous, sous le nom de $P$. depressum Bailey.

Nous croyons devoir conserver le nom de divergens, sans plus, à cette belle espèce que de bonnes figures de Schütт (2), de Pouchet (3) et d'autres ont vulgarisée, sous cette étiquette, malgré l'incertitude, peut-être, de son attribution primordiale.

Ses grandes dimensions, sa large distribution géographique, ses caractères bien accusés, sa physionomie si spéciale auraient dû la tenir à l'abri du danger de la synonymie. Loin d'y échapper cependant, elle y a été sujette autant et plus que toute autre et ce fait donne la mesure de l'arbitraire qui règne dans le domaine des Péridiniacées et qui tient aux causes multiples sur lesquelles nous avons appelé l'attention ci-devant.

Nous ne connaissons aucune bonne raison pour considérer cette belle forme, que l'on pourrait dire classique, comme une variété d'un type autre qui aurait des titres mieux fondés au rang de chef de lignée phylogénique.

$\mathrm{Au}$ contraire, nos observations nous en fournissent d'excellentes, pour la tenir comme un type spécifique de premier ordre et l'admettre à la justification tardive de ses titres trop longtemps méconnus.

C'est plus qu'une réhabilitation qu'il faut, car son état civil a été vicié dès son baptême, et ses nombreux transferts dans les registres de la synonymie n'en ont pas corrigé le vice originel.

Il n'est pas trop tard pour le redresser. Pour ce faire, il faut distraire du dossier les pièces erronées, douteuses ou inutiles et jeter ainsi un peu de lest syno-

(1) Pour tout ce qui concerne la synonymie et la bibliographie relatives aux Péridiniens, nous renvoyons le lecteur à cette publication récente, où ces organisınes ont été traités, dans la huitième livraison, par Ove Paulsen, avec toutes les références synonymiques désirables. Il serait fastidieux de représenter celles-ci à peu près dans les mêmes conditions.

(2) Die Peridineen der Plankton-Expedition, I, Tafel XIII, fig. $43_{22}$ et $43_{24}$. Kiel und Leipzig, 1895 .

(3) Contribution à l'histoire des cilio-flagellés, foumal de lanatomie et de la physiologie, tome XIX, I883, pl. XX et XXI, fig. $24,25,26,27$. 
nymique, pour tendre à se dégager de l'imbroglio des fausses appréciations d'une époque de tàtonnements, où les meilleures volontés étaient mal servies et désorientées par la nouveauté, l'imprévu et le décousu des premières constatations.

C'est, croyons-nous, le plus grand des Peridinimm connus.

Corps très excentrique, fortement renflé au milieu (fig. 4, pl. I, vue de profil gauche). Sillon transversal à fond plat, sinistrogyre et très oblique sur l'axe longitudinal ou polaire. Cône apical déprimé et prolongé en pyramide à base élargie (fig. I, vue oblique du haut). Còne antapical prolongé en deux cornes habituellement creuses (fig. 2, vue oblique du bas). Section transversale, au niveau du sillon transversal, largement réniforme (fig. 3, vue apicale). Ailettes de la ceinture larges et manifestement striées radialement (fig. I, 2, 3, 4). Capsule irrégulièrement réticulée en relief, avec renforcements aux points d'anastomose des trabécules du réseau (mèmes figures). Sutures simples (fig. 2 et 4) ou doubles (fig. I et 3), suivant l'état de développement de l'individu.

Les figures I à 8 de la planche $\mathrm{I}^{\text {bis }}$ complètent ce signalement, en montrant de face (fig. I, 3, 5, 7) et de profil droit (fig. 2, 4, 6, 8), quatre variantes du type dont les modalités plus ou moins tranchées sont cependant impuissantes à cacher les caractères si fortement imprimés de l'espèce.

Des auteurs ont fait état de ces modalités pour démembrer l'espèce. D'autres ont voulu y voir des variétés du type, ce qui serait plus acceptable s'ils avaient pu déterminer les causes efficientes de ces variétés et la constance de leurs caractères, dans toutes les conditions où ces causes sont opérantes. Mais cela n'a pas été fait et la chose nous parait d'autant moins probable que nous avons trouvé ces variantes plus fusionnées, dans les mèmes coups de filet planktoniques.

La forme figurée I et 2 , pl. I ${ }^{\text {bis }}$, est plus allongée et d'un port plus raide, les cornes antapicales sont plus droites et atténuées en pointe plus aiguë, bien que creuses jusqu'au bout.

On pourrait y voir, peut-ètre, ce que Karsten a dénommé $P$. divergens var, granulatum. Taf. XXIII, fig. $17^{\mathrm{a}}$ et $\mathrm{I} 7^{\mathrm{e}}$ ? (I).

La forme figurée 3 et 4 , pl. I ${ }^{\text {bis }}$, est plus ramassée, plus courte; son corps est plus arrondi; ses cornes antapicales sont plus projetées en avant, plus courtes, plus étroites et pleines dans leur partie terminale atténuée en pointe émoussée. Ce nous parait être ce dont Broch (2) a fait son P. parallelum.

(I) Wissenschaftliche Ergebnisse der Deutschen Tiegsee-Expedition, Iena, 1906.

Mais nous ne saurions garantir le bien-fondè du rapprochement; car les figures de l'auteur, très artistement exécutées du reste, nous paraissent trop artificielles pour fournir des élèments d'appréciation, mème approximatifs. Cette remarque s’applique aussi aux autres figures de la même planche relatives aux Péridiniens.

(2) Broch, HJ. : Bemerkungen üher den Formenkreis von Peridinium depressum s lat. Nyt Magazin for Naturvidensk. Kristiania, I 906. 
La forme des figures 5 et 6 de la planche $I^{\text {bis }}$ a le còne antapical plus dévié encore en avant; les cornes sont très courtes, creuses, aiguës à l'extrémité et convergentes l'une vers l'autre, en forme de pince de crabe (fig. 5), ce qui en modifie profondément le facies.

Les figures 7 et 8 de la planche $I^{\text {bis }}$ reproduisent, dans leur plein épanouissement, tous les caractères qui nous paraissent les plus propres à l'espèce, représentée ici par un spécimen de taille maxima, qui offre le développement harmonieux de toutes ses parties organiques.

Ces quelques modalités choisies entre beaucoup d'autres, que l'examen de nos matériaux nous a permis de rencontrer, suffiront à montrer l'étendue du champ des variations de cette espèce.

Nous y rattachons encore la forme représentée de face (fig. 46), et de profil droit (fig. 45 de la pl. II), bien que les dimensions soient plus petites, le còne apical moins déprimé - ce qui rend le corps plus piriforme - les cornes antapicales plus divergentes et surtout le sillon transversal beaucoup moins oblique.

Nous ne l'avons observée que rarement. Serait-ce une forme jeune? Serait-elle liée à un mode particulier de reproduction de l'espèce? Faut-il plutôt lui reconnaitre des affinités avec l'espèce que nous allons décrire ci-après? Le lecteur appréciera.

Remarque. - Nous nous garderons bien de suivre certains auteurs dans la subdivision de ces formes en variétés, dont la nomenclature pèse lourdement et sans profit sur la littérature de la science planktonique.

C'est une complication aussi inutile que nuisible; car elle a créé un chaos synonymique, dont il faudrait chercher à sortir au plus tôt pour le bien de la science. L'espèce en question, la plus franche peut-être de toutes les Péridiniées, fournit un exemple frappant de cet abus de la subdivision en variétés. A force d'en créer, parfois mème en sortant des limites vraisemblables du type spécifique, on a perdu de vue celui-ci, à tel point qu'on se demande quel il est (I).

Qu'on laisse donc ces entités vagues et fugitives qui n'apportent que des éléments de confusion dans une matière où l'identification des types, mème les mieux modelés, présente déjà des difficultés bien grandes!

Qu'importe du reste la notion de variété dans un monde d'organismes, où les notions de genre et d'espèce ne répondent elles-mèmes généralement qu'à des conceptions purement subjectives?

A notre avis, la création de variétés, de sous-variétés, etc., bien loin d'avancer la science, n'en a fait que retarder le progrès, en détournant les chercheurs de ce qui aurait dù solliciter davantage leur attention : la recherche du simple dans le complexe, de l'unité dans la variété et la multiplicité.

(1) Par exemple : les variétés pentagonum Gran, pallidum Ostenfeld, obtusum, pyramidale, excãuatum Karsten, rapportées par ce dernier auteur à $P$. divergens, nous paraissent manifestement étrangères à l'orbite de cette espèce. 
A ce travers, il y a une excuse : l'étude souvent exclusive de produits planktoniques locaux et saisonniers et, conséquemment, l'absence d'éléments suffisants de comparaison entre les mains de ceux qui ont touché à ces matières difficiles.

Aussi appelons-nous de tous nos vœux un travail synthétique sur le sujet, dont l'auteur fera table rase de tous les travaux antérieurs, pour ne tenir compte que de ses observations personnelles, sur des matériaux de provenance mondiale, qu'il aura su se procurer.

Distribution géographique. - Peridinimm divergens, entendu comme il vient d'être dit, est l'espèce la plus constante dans les pêches de la Belgica. Commune tout le long des còtes ouest des deux îles de la Nouvelle-Zemble, plus rare dans le détroit de Matotchkine, elle s'observe encore dans la Mer de Kara, au voisinage de ce détroit; mais elle semble faire défaut dans le sud de cette Mer, pour reparaitre à la Porte de Kara et dans la Mer Mourmane.

C'est toutefois dans les hautes latitudes, vers l'Océan Glacial, qu'elle acquiert les plus grandes dimensions, en même temps qu'elle présente les modalités les plus diverses en mélange.

Elle est particulièrement abondante dans les nos 188 à 206, où elle constitue parfois l'élément dominant des produits de pêche au filet fin.

\section{Peridinium saltans sp. n.}

Planche I ${ }^{\text {bis, }}$, figures 9 à 14 .

Nous désignons ainsi une espèce évidemment voisine de la précédente, dont elle rappelle l'allure générảle; mais en réduction.

Elle présente aussi une excentricité bien marquée des deux moitiés supérieure et inférieure sur l'axe polaire. Le sillon transversal est plat, ailé, sinistrogyre; le cône apical est fortement défléchi; le cône antapical est prolongé en deux longues cornes creuses, aiguës et divergentes.

Mais elle est beaucoup plus petite; la section transversale (fig. I2, vue apicale) est plus étroitement réniforme. Les extrémités des deux cornes antapicales plus sveltes, plus arquées et très divergentes prennent une direction opposée : l'une, la gauche, en avant; l'autre, la droite, en arrière; ce qui se voit très bien dans les figures de profil 9 et I4. Ces silhouettes de l'espèce sont évocatrices du nom que nous lui donnons.

La capsule examinée en milieu liquide paraît exempte de sculptures; mais observée à sec elle se montre finement réticulée.

Les figures 9 à $I 2$ se rapportent à un même spécimen, vu de profil droit, 9 ; de dos, io; de face, II; d'en haut, I2. Les figures I3 et I4 sont d'un second individu.

Distribution géographique. - Rencontré sur les cotes ouest de la NouvelleZemble, dans d'assez nombreux échantillons, mais toujours en spécimens clairsemés. 
Remarque. - Il ne sera pas inutile de faire observer que nos matériaux arctiques ne nous ont pas fourni un seul spécimen d'un autre type spécifique qui, autrement, devrait trouver sa place dans cette section. Nous voulons dire : celui dont Vanhöffen (I) a fait Peridinimm occanicum et qui reste pour nous, Pcridinimm depressum Bailey; attribution que nous avons toujours faite de cette forme, dans les listes planktoniques de la Mer Flamande (2), où elle n'est pas rare.

Bien que nous n'ayions pas à nous occuper ici de cette espèce, cette observation est nécessaire pour expliquer la divergence de vues à son sujet, que le lecteur ne manquera pas de constater entre ces notes et l'ouvrage synthétique de Ove Paulsen, dans Nordisches Plankton, auquel nous faisons de fréquents renvois.

Il faut se garder aussi de confondre cette espèce, étrangère aux matériaux qui font l'objet de cette étude, avec $P$. saltans nobis, présenté ci-avant. L'une et l'autre de ces deux formes nous étant familières, nous n'hésitons pas à les distinguer spécifiquement, malgré certaines analogies de forme incontestables, non pas avec la variété typica ou clegans de certains auteurs, mais plutòt avec la variété oblongum d'autres planktonistes.

Nous ne parlerons pas de $P$. claudicans Paulsen, qui fait aussi défaut dans nos produits de pêche.

\section{Section II. - Dextrogyres}

Dans l'analyse des assez nombreuses formes de cette section, nous tiendrons compte de la longueur relative des axes et aussi de l'obliquité éventuelle de l'axe polaire sur le plan de la ceinture.

io Formes a ane polaire plus court que l'axe latéral

A. Axe polaire très oblique

\section{Peridinium islandicum Paulsen.}

Planche 1 , figures io à 13 .

Synonyme. - Voir Nordisches Plankton, page 5o.

Espèce à caractères bien distincts, dont la vue latérale (fig. I3) est la plus suggestive, car elle montre bien l'obliquité de l'axe polaire sur la ceinture.

(1) VAnhöFrix, E. : 1)ie Fauna und Flora Grönlands, Grönland Fixp. der Gesellsch. für Erdkunde zu Birlin, I897.

(2) Bulletin trimestriel du Conseil permanent international pour l'Exploration de la Mes. Copenhague, Igoz-IgIo. 
Ceinture dextrogyre, plate, bordée d'ailettes larges et fortement striées radialement (fig. II et I2, vues antapicale et apicale); cône apical déjeté en arrière, cône antapical ramené en avant et terminé par deux épines courtes, d'égale valeur, incurvées aussi en avant; membranelle orale très saillante (fig. Io, vue de face; fig. I3, vue de profil).

La section transversale, au niveau de la ceinture, est arrondie, à peine réniforme (fig. II et I2).

Le spécimen figuré est visiblement réticulé et présente des sutures doubles.

Distribution Géographigue. - Fréquemment rencontré sur les côtes ouest de la Nouvelle-Zemble, mais toujours peu abondant.

\section{B. Axe polaive droit}

Peridinium ovatum Schütt.

Planche I, figures 27 et 28 .

Synonyme. - Voir Nordisches Plankton, page 44.

Il est fàcheux que le nom de cette espèce, commune dans la Mer du Nord, ne soit pas plus en harmonie avec sa forme, qui ne présente qu'une analogie très lointaine avec celle d'un ouf d'oiseau.

Elle est à ceinture dextrogyre, parfois un peu déprimée, comme dans le spécimen de la figure 27, mais plus souvent plate et bordée d'ailettes bien striées (I). L'axe polaire est notablement plus court que les deux autres latéral et dorso-ventral. Ceux-ci sont sensiblement égaux et rendent la section transversale arrondie, légèrement réniforme (fig. 28, vue apicale). Le còne antapical très surbaissé, comme le còne apical, et même quelque peu aplati porte deux spinules plus ou moins ailées, longues, un peu inégales, à peu près parallèles entre elles et à l'axe polaire.

La sculpture finement réticulée de la capsule est toujours bien manifeste.

Le spécimen figuré est à sutures simples, cas le plus habituel.

Distribution géographique. - Observé un peu partout; mais jamais abondant.

(1) Ce caractère est frappant. Il permet toujours de distinguer á premiẻre vue cette espèce, l'une des mieux connues et des plus répandues, d'avec Diplopsalis lenticula Bergh, qui en épouse assez bien la forme; mais dont les ailettes sont lépourvues de striation radiale.

Quand ces espèces sont associées dans les produits de pêche, ce qui n'est pas rare dans la Mer Flamande que nous avons spécialement étudiée, au point de vue microplanktonique, l'observation de ce caractère dispense d'un examen plus minutieux, dans les recherches cursives. 


\section{Peridinium ovatoüdes sp. n.}

Planche I, figures 24 et 26 .

Cette espèce, très affine à la précédente dont elle n'est peut-être qu'une variété, en diffère par ses deux spinules ailées, longues, égales, divergentes (fig. 24, vue de face) et arquées vers l'avant (fig. 25, vue latérale gauche).

La ceinture est dextrogyre, plate et bordée d'ailettes très saillantes à striation radiale.

La section transversale est plus réniforme que dans $P$. ovatum (fig. 26, vue apicale).

Le spécimen reproduit est à sutures doubles.

Distribution géographique. - Assez rare, en mélange avec d'autres espèces dans des échantillons riches en Péridiniacées, spécialement dans le no 30 du Journal de pêche.

\section{Peridinium complanatum sp. n.}

Planche I, figures 29 et 30 .

Nous désignons ainsi une espèce à axe polaire très raccourci. Le corps prend ainsi la forme d'un disque aplati, légèrement relevé du còté supérieur par le sommet du cône apical très court. Le còne antapical, très surbaissé aussi, est porteur d'une longue spinule insérée sur le còté droit du sillon ventral.

La spinule symétrique du còté gauche est inexistante ou très réduite et rapprochée de la membranelle orale peu développée également (fig. 27, vue ventrale).

La ceinture n'est que légèrement dextrogyre, à cause de l'aplatissement exagéré du corps. Les ailettes de la ceinture ne sont pas striées (fig. 3o, vue antapicale). La capsule est visiblement réticulée sur toute sa surface, sauf sur le sommet du cône antapical où des ponctuations isolées se substituent aux trabécules du réticulum.

Vue de face, elle se distingue aisément de toute autre espèce.

Distribution géographique. - Toujours assez rare, région sud de la Mer de Barents et Détroit de Matotchkine surtout.

\section{$2^{\circ}$ Formes a AXE POLAIRE PLUS LONG QUE L'AXE LATÉRAL}

A. Ceinture oblique sur l'axe polaire

\section{Peridinium pallidum Ostenfeld.}

Planche I, figures 5 à 9 .

Synonyme. - Voir Nordisches Plankton, page 48.

C'est aussi une bonne espèce, facile à reconnaitre surtout si on la voit de profil (fig. 7 et 9), position qui traduit très bien l'obliquité de la ceinture sur l'axe polaire. 
L'axe dorso-ventral est beaucoup plus court que les deux autres, ce qui rend la vue apicale étroitement réniforme (fig. 6).

La vue ventrale (fig. 5 et 8) montre les deux spinules égales et ailées qui terminent le còne antapical, la membranelle orale assez ample, la direction dextrogyre de la ceinture bordée d'ailettes larges et striées.

La capsule est réticulée.

Les figures 5, 6 et 7 reproduisent les vues classiques d'un individu plus rebondi latéralement et à sutures simples. Les figures 8 et 9 sont d'un autre individu, plus svelte, dont les spinules antapicales sont plus longues, plus divergentes et plus projetées en avant; les sutures y sont doubles.

Distribution Géographique. - C'est, dans nos échantillons, la forme la plus constante, après Peridinium divergens. On la rencontre plus ou moins abondamment dans presque tous les numéros où il y a des Péridiniens.

\section{B. Ceinture normale à l'axe}

\section{Peridinium pellucidum Schütt.}

Planche I ${ }^{\text {bis, }}$, figures $26,27,28$.

Synonymie. - Voir Nordisches Plankton, page 49.

Nous pensons qu'il y a lieu de retenir sous ce nom une forme qui, vue de face (fig. 47), a de l'analogie avec la précédente, mais qui en diffère par ses dimensions habituelles un peu moindres, sa forme plus svelte due à la dépression du cône apical et surtout par la position de la ceinture qui est perpendiculaire à l'axe polaire (fig. 26, vue latérale). La longueur de l'axe dorso-ventral se rapproche de celle de l'axe latéral : ce qui donne à la vue apicale (fig. 28) la forme d'un rein plus arrondi.

L'allure des spinules antapicales rappelle celle de Peridinium pallidum.

La sculpture réticulée de la capsule est généralement très peu accusée et visible seulement dans des conditions spéciales, à sec particulièrement.

Remarque. - Dans la forme que nous avons en vue et que nous connaissons aussi de la Mer Flamande, la ceinture dextrogyre est plane et bordée d'ailettes striées, ce qui est conforme aux figures d'Ostenfeld, et non déprimée en sillon creux et exempte d'ailettes, comme dans les figures de BERGH.

Nous devons dire aussi que nous ne retrouvons aucunement la physionomie de notre espèce dans les figures données sous ce nom par ScHütt dans Peridineen der Plankton-Expedition. Taf. I4, figure 45 (I895). 
La différence des procédés de figuration devrait ètre insuffisante à expliquer d'aussi grands écarts dans la reproduction des caractères d'un même type organique. Nous croyons plutòt à une confusion de formes distinctes sous une même dénomination, chose malheureusement trop fréquente dans la matière qui nous occupe.

Distribution géographique. - Assez rare ; çà et là seulement, dans quelques pêches disséminées de la Mer de Barents.

\section{Peridinium gracile sp. n.}

Planche III, figure $5 \mathrm{I}$.

Jolie petite forme, d'une coupe élégante, dont là seule vue de face suffit à faire saisir les caractères spécifiques.

Corps svelte, allongé, élégamment piriforme, dextrogyre, à ceinture plate, bordée d'ailettes striées, perpendiculaire à l'axe polaire.

Section transversale arrondie, légèrement réniforme. Còne antapical atténué au sommet et prolongé en deux spinules courtes, plutòt convergentes. Capsule très finement réticulée, à sec.

Distribution géographique. - Rare, dans la partie du nord de la Mer de Barents.

\section{Peridinium piriforme Paulsen.}

Planche I, figures 14 à 16 , et peut-être aussi 17 et 18 . Planche II, figures 18 et 19 .

Synonymie. - Voir Nordisches Plankton, page $4^{6 .}$

Espèce à axe longitudinal plus long que les deux autres qui sont presque égaux.

La section transversale en est donc arrondie, légèrement réniforme (fig. I5, pl. I, vue antapicale).

Ceinture dextrogyre, plane ou très légèrement creuse, bordée d'ailettes étroites et striées (fig. I4, vue ventrale).

Cône antapical très arrondi (fig. I6, vue latérale droite).

Sillon ventral n'atteignant pas le sommet du còne antapical, ce qui laisse les deux spinules ailées sur la face antérieure, où elles sont un peu convergentes et dirigées en avant. Membranelle orale saillante. Capsule réticulée.

Cette dernière est parfois à parois très épaisses. Nous reproduisons (pl. II, fig. IS et I9) un individu où cette particularité est remarquablement accusée.

Si l'on tient pour caractéristiques de cette espèce la position antérieure du sommet antapical, la projection en avant des spinules et la forme rebondie de la moitié infé- 
rieure de la capsule, on peut encore y rattacher le spécimen reproduit planche I, figures I7 et I8, où ces caractères se retrouvent encore, malgré la forme un peu plus ramassée du corps.

L'étude des caractères plus intimes du protoplasme, du noyau, des chromoleucites, etc., permettrait de s'assurer si cette assimilation est fondée; mais le grand nombre d'échantillons à dépouiller ne nous a pas permis de nous attarder à dissiper nos doutes à cet égard.

Quoi qu'il en soit, sous sa forme type, Peridinizm piriforme nous parait être une bonne espèce, que Paulsen a bien fait de retirer des formes confusément désignées sous le nom de Peridinim Steinii.

Distribution géographique. - Assez rare dans la Mer de Kara, rencontrée dans le $n^{\circ} 22$ surtout.

Peridinium Steinii Jörgensen.

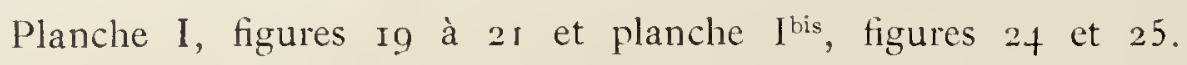

Synonyme. - Voir Nordisches Plankton, page 47.

- Espèce assez petite, qui rappelle $P$. piriforme, mais qui en diffère, selon nous, par la position terminale des spinules antapicales. Ces spinules, souvent pourvues de membranelles assez larges, sont dans la direction de l'axe polaire, quoique un peu incurvées en avant (fig. 20, pl. I, vue latérale droite).

La vue ventrale est elliptique (fig. I9). La ceinture dextrogyre est plane et bordée d'ailettes étroites et striées (fig. 2I, vue apicale).

La capsule est vigoureusement réticulée, parfois même jusque sur les membranelles des spinules.

A ce type, nous rattachons la forme reproduite planche $I^{\text {bis }}$, figure 24 , d'un spécimen un peu différent et provenant des régions les plus boréales atteintes par la Belgica.

La forme de la figure 25, planche $I^{\text {bis }}$, malgré son apparence un peu plus trapue, nous semble aussi devoir s'y rattacher. La capsule est ici remarquablement épaisse.

Remarque. - Nous n'ignorons pas que cette forme a reçu les dénominations les plus diverses et que réciproquement le nom spécifique de Steinii a été attribué à des formes non moins variées, preuve nouvelle de l'imprécision de la diagnose originale et de l'inconvénient qu'il y a généralement à attribuer aux espècès des noms d'auteurs ou de lieux qui ne sont suggestifs d'aucun caractère propre à guider la détermination.

Distribution GÉographigue. - Assez rare; nord de la Mer de Barents. 
Peridinium lurgidum sp. n.

Planche $\left[^{\text {bis }}\right.$, figures 15 à 19 .

Corps assez grand, piriforme (fig. I7, vue latérale droite), rebondi, turgescent, à parois capsulaires minces.

Ceinture dextrogyre, normale à l'axe polaire, plate, bordée d'ailettes assez étroites et peu striées (fig. I5, vue ventrale).

Còne antapical renflé, hémisphérique, à sillon ventral n'atteignant pas le sommet (fig. I8, vue antapicale), et orné de deux spinules non ailées, assez distantes, peu divergentes, insérées : l'une, la gauche, $\iota$, sur le flanc antérieur au bord du sillon, et l'autre, la droite, $e$ ', au sommet de la courbure antapicale et à une certaine distance du sillon.

Section transversale circulaire, à peine un peu réniforme (fig. Ig, vue apicale).

La figure i6 est la vue dorsale du même individu.

La réticulation peu apparente ne s'observe bien qu'à sec.

Distribution GÉographigue. - Observée seulement dans l'Océan Glacial.

\section{3o Formes a axe polaire Égal a l'axe latéral}

\section{Peridinium Granii Ostenfeld.}

Planche I, figures 35 à 37 , et planche $1^{\text {bis }}$, figures 35 et 36 .

Synonyme. - Voir Nordisches Plankton, page 52.

Corps à trois axes sensiblement égaux. Ceinture plane, circulaire ou légèrement spirale-dextrogyre, bordée d'ailettes à striation peu visible (fig. 35, vue ventrale, et 36, vue apicale).

Còne apical déprimé en avant et sur les côtés, arrondi par derrière, à sommet obtus et légèrement spinulé par l'extrémité des sutures des plaques (fig. 37, vue latérale gauche). Cette vue est la plus interprétative.

Còne antapical terminé par deux prolongements droits, coniques, creux, pointus au sommet.

La capsule est réticulée, bien que ce caractère n'apparaisse pas toujours nettement en milieu liquide.

Les figures 35 et 36 de la planche. I bis montrent l'organisme à l'état d'enkystement. La spore, sp, est arrondie, à membrane épaisse et brune.

Nous n'avons pas figuré le réticulum, peu visible ici du reste, pour ne pas cacher la vue de la spore (fig. 35, vue de face; fig. 36, vue latérale gauche). 
C'est une très bonne espèce, facile à identifier, et que nous connaissons depuis longtemps de la Mer Flamande.

Distribution géographique. - Mer de Barents; toujours à l'état très disséminé, en société de beaucoup d'autres formes.

Peridinium anceps sp. n.

Planche $I$, figures 22 et 23 .

Nous sommes forcé de donner un nom à cette torme, que nous ne savons rattacher à aucune des espèces décrites des régions boréales, peut-être à cause de l'imprécision des figures qu'on en a données, si toutefois on l'a eue en vue.

Elle n'a pas la physionomie de $P$. curvipes que nous connaissons de la Mer Flamande où cette espèce est peut-ètre la plus constante bien qu'elle s'en rapproche par certains caractères, entre autres par le grand développement de la membranelle orale. Mais les deux spinules antapicales ont ici une grande longueur et la gauche est nettement séparée de la membranelle (fig. 22, vue ventrale). La vue apicale (fig. 23) est brièvement réniforme et montre la striation radiale des ailettes étroites de la ceinture qui est souvent un peu creusée en sillon.

La capsule est réticulée.

Distribution géographigue. - Neige jaune et çà et là, dans la Mer de Kara; mais toujours très rare ici où elle semble n'avoir passé que par fusion des neiges. Nous la tenons pour une espèce nivicole.

\section{Peridinium roseum Paulsen?}

Planche II, figures io et II.

Synonymie. - Voir Nordisches Plankton, page 44.

Ce n'est qu'avec hésitation que nous tentons le rapprochement de cette forme de celle décrite par Paulsen qui emprunte son nom à un caractère de coloration disparaissant dans le milieu conservateur des produits de pêche.

Ce qui nous y porte, néanmoins, c'est sa forme, équilatérale à peu près, et ses deux spinules assez longues, placées sur le flanc antérieur, à une notable distance du sommet antapical; particulièrement la gauche, qui se trouve sur le bord du sillon ventral (fig IO). L'autre, la droite, moins distante du sommet, est très écartée de la membranelle orale; caractère sur lequel PAulsen insiste dans sa diagnose mais qui ne se traduit pas bien dans ses figures. 
Notre forme est à ceinture plane, dextrogyre, bordée d'ailettes peu larges et obscurément radiées (fig. II). La capsule parait lisse dans les conditions habituelles d'observation et 'même à sec.

Distribution géographioue. - Trouvée surtout dans la Mer de Kara, au voisinage du Détroit de Matotchkine; beaucoup plus rare dans la Mer de Barents.

\section{Peridinium decipiens Jörgensen?}

Planche II, figure 15 .

Synonyme. - Voir Nordisches Plankton, page 5o.

La forme assez écrasée du corps, surtout du còté antapical, et l'absence de spinules visibles sur le sillon ventral, où se projette seule la membranelle orale, sont les circonstances qui établissent une certaine analogie entre l'organisme que nous figurons de face (fig. 25) et l'espèce assez vaguement établie par Jörgensen sous le nom de $P$. decipiens.

Notre forme est plutôt isodiamétrale qu'aplatie en forme de couque.

$\mathrm{Ne}$ serait-ce pas une forme jeune de la suivante?

\section{Peridinium gravidum sp. n.}

Planche II, figures I2 à I4 et figures I6 et i7.

Forme massive, isodiamétrale, à contours arrondis, rebondis et d'aspect un peu pentagonal en vue de face (fig. I3 et I6). Vue antapicale presque circulaire (fig. I2 et I7). Sommet apical à peine émergeant. Ceinture plane, dextrogyre, bordée d'ailettes peu larges. Membrane capsulaire souvent épaisse (fig. I2 et I3) et lisse.

Les spinules antapicales, $c$, sont souvent très courtes, insérées sur le flanc antérieur et dirigées en avant, presque perpendiculairement à l'axe polaire (fig. I4, vue latérale).

La droite est, comme toujours, plus rapprochée du sommet que la gauche.

Distribution géographique. - Cette forme facilement identifiable n'est pas rare dans les échantillons de pêche des deux mers de Barents et de Kara qui sont assez riches en Péridiniens.

\section{Peridinium rhombus sp. n.}

Planche II, figure 36.

Corps losangique en coupe optique longitudinale, à ceinture plane, dextrogyre, à spinules antapicales courtes, terminales et peu distantes. 
Ajoutons, d'après le spécimen figuré, que la membrane est double, comme si une nouvelle membrane capsulaire se formait à l'intérieur de l'autre par un phénomène de régénération cellulaire.

La membrane extérieure, mince et délicate, recouvre comme d'un voile toutes les parties en saillie de la capsule proprement dite.

Quelle est la signification de cet organe exceptionnel?

Nous avons eu trop peu d'exemples sous les yeux pour pouvoir donner une interprétation fondée de cette particularité de structure.

N'est-ce qu'un caractère accidentel? N'est-ce pas plutòt un indice d'une phase évolutive de l'organisme? Quoi qu'il en soit, la rareté du fait le rend digne de remarque.

Distribution géographique. - Très rare; nord de la Mer de Barents.

Peridinium sphaericum sp. n. (non Murray and Witting, I899) (I).

Planche I ${ }^{\text {bis, }}$, figures 29 à 31 .

Les notes spécifiques de cette forme consistent : Io dans sa forme sphérique à peine déformée par les sommets des deux cònes polaires dont l'apical est brièvement acuminé et l'autre terminé par deux spinules peu distantes et très légèrement incurvées en avant; $2^{\circ}$ dans les mouchetures saillantes qui garnissent la coque, au lieu d'un réticulum.

La ceinture est dextrogyre, plane, bordée d'ailettes nettement striées (fig. 29, pl. I ${ }^{\text {bis }}$, vue de face; fig. 30, vue latérale gauche; fig. 3I, vue antapicale).

Distribution géographique. - Assez rare, Mer de Barents.

\section{Peridinium globulus Stein?}

Planche II, figures 20 et $2 \mathrm{I}$.

Synonyme. - Voir Nordisches Plankton, page 43.

Nous ne saurions garantir que la forme ici figurée soit celle à laquelle revient légitimement ce nom, vu les écarts de diagnose des auteurs et l'impossibilité de concilier les figures qu'ils en donnent.

Notre forme est globuleuse, dextrogyre, à ailettes zonales fortement striées (fig. 2I, vue antapicale) et dépourvue de spinules.

Seule la membranelle orale émerge, sur la vue de profil gauche (fig. 20).

(I) Murray and Witting: New Peridiniaceae from the Atlantic, Transact. of the Linnean Soc. of London. 
On a décrit sous ce nom des formes à spinules et d'autres sans spinules.

Où est la vérité?

Ceux qui s'occupent de ces petits organismes ne s'étonneront pas de ces doutes embarrassants qu'entretient une connaissance encore trop imparfaite des Péridiniens dans leurs variations mondiales.

Les vues d'ensemble font encore défaut, l'analyse des Planktons locaux ayant seule occupé jusqu'ici les spécialistes.

\section{Peridinium cerasus Paulsen?}

Planche II, figures 27 à 29 .

Notre embarras reparaît au sujet de cette forme de dimensions plus réduites encore, dont nous reproduisons les vues classiques (fig. 29, de face; fig. 28, de profil droit; fig. 27, du sommet apical).

Qui nous dira que notre rapprochement est fondé? Tant il est vrai que dans ce domaine d'organismes très petits, très simples et fort similaires au fond, la moindre incorrection du dessin fausse complètement la physionomie des objets représentés!

L'identification tentée ici ne va pas sans beaucoup de bonne volonté; mais nous ne la ménageons pas, dans le but de réduire la synonymie.

\section{Petites formes}

Dans l'incertitude où nous nous trouvons d'avoir affaire ou à des formes adultes et spécifiquement distinctes, quoique de très petites dimensions, ou à des formes jeunes, phases évolutives d'espèces plus grandes, nous nous bornerons à signaler ces formes, sans chercher à les élever au rang d'espèces, ni à les rattacher à d'autres espèces connues, à titre érentuel d'étapes de développement.

\section{Forme a}

Planche II, tigures 22,23 et 24 .

Corps piriforme, còne apical assez longuement atténué au sommet, còne antapical bien arrondi et porteur de deux spinules longues, ailées, peu divergentes et arquées en avant.

\section{Forme $\beta$}

Planche II, figure 26.

Corps piriforme plus allongé, spinules antapicales longues, ailées et divergentes. 


\section{Forme $\gamma$}

Planche II, figure 25.

Corps piriforme plus court, spinules antapicales courtes et convergentes.

\section{Forme o}

Planche II, figures 30,31 et 32 .

Corps encore piriforme, mais plus ramassé; spinules antapicales moins longues, moins fortes, à peine ailées; membrane orale très saillante.

\section{Forme $\varepsilon$}

Planche II, figure 33.

Forme analogue à la précédente, mais un peu plus forte, à silhouette évocatrice de $P$. curvipes Ostenfeld.

\section{Forme $\zeta$}

Planche XIV, figure $4^{5}$.

Corps plus petit, plus grèle, plus arrondi, à spinules ténues, placées en avant du còne antapical dont le sommet est arrondi.

\section{Forme $\eta_{0}$}

Planche XIV, figure 46 .

Mème forme à peu près, mais avec spinules terminales et sommet antapical déprimé et comme émarginé par l'extension du sillon ventral.

Nous groupons ici toutes ces petites formes, eu égard à l'aspect de la ceinture, plane, dextrogyre, bordée d'ailettes, qui est leur caractère commun en mème temps qu'il les rapproche des espèces décrites ci-devant.

\section{Série B. - CAVOZONES}

\section{Section III. - Espèces sinistrogyres a CEINTURe EN SILlon CREUX}

Nous rangerons ici les formes observées, chez lesquelles la note dominante nous paraît être la forme de la ceinture, qui est déprimée en gouttière et constitue ainsi un vrai sillon transversal, non bordé d'ailettes saillantes distinctes. Ce sillon est 
toujours ou bien circulaire ou bien spirale et légèrement sinistrogyre. Ce double caractère imprime à ces formes une physionomie spéciale, qui garde toute sa valeur taxinomique, si l'on ne tient comme afférentes à cette section que les espèces trapues, sensiblement isodiamétrales et dépourvues de longs prolongements antapicaux.

\section{Peridinium elongatum sp. n.}

Planche IV, figures I à $t$.

C'est une espèce à physionomie bien spéciale.

L'axe polaire du corps est notablement plus long que les deux autres.

La face ventrale est déprimée, aplatie (fig. I); la face dorsale est convexe, arrondie (fig. 3), ce qui ramène sur le devant le sommet du cône antapical, comme on le voit sur la vue latérale gauche (fig. 2).

La section transversale au niveau de la ceinture est légèrement trilobée, comme il apparait sur la vue antapicale (fig. 4).

Le sillon ventral est très large et va même s'élargissant vers la base du corps, où sa limite est marquée par deux spinules très courtes, visibles seulement dans une position propice (fig. I et 2 ).

La ceinture est creuse, dépourvue d'ailettes et circulaire, si ce n'est qu'elle s'abaisse d'une façon égale des deux còtés vers le sillon ventral.

La membrane est couverte partout, en dehors des sillons, de très fines pointes qui en hérissent la surface.

Distribution GÉographique. - Mer de Barents.

\section{Peridinium conicoïdes Paulsen.}

Planche I, figures $3 \mathrm{I}, 32,33,34$, et subsidiairement 42 et $4^{3}$.

C'est à cette espèce que nous pensons pouvoir rapporter la forme que nous figurons ici et qui nous semble synthétiser le mieux l'ensemble des caractères de la section des Cavozones.

Le corps est isodiamétral, comme il résulte du simple examen des figures 3 I à 34 , qui reproduisent les vues classiques de cette jolie forme.

On remarquera les deux spinules courtes qui terminent le sillon ventral.

La ceinture est circulaire. La surface de la capsule est réticulée dans nos spécimens, bien que PAulsen suppose son espèce exempte de sculptures.

La forme plus petite, reproduite figure $4^{3}$, devrait sans doute s'y rattacher à défaut de caractères distinctifs suffisants pour l'en détacher. Ce n'en est peut-être qu'une forme plus jeune et moins différenciée. 
Nous y rattachons également la forme de la figure 42 , qui ne s'en distingue que par l'étroitesse du sillon ventral; particularité peut-ètre simplement accidentelle et tenant au milieu spécial, la neige jaune, d'où nous tenons ce spécimen.

Distribution géographique. - Nord de la Mer de Barents surtout. Rare.

Peridinium subinerme Paulsen.

Planche II, figures $4^{3}$ et +4 .

Synonyme. - Voir Nordisches Plankton, page 6o.

Forme isodiamétrale, biconique, arrondie comme la précédente; mais s'en distinguant aisément par l'aspect du sillon ventral qui, étroit dans la première partie de son parcours à partir de la ceinture, s'élargit brusquement dans sa partie inférieure où il forme une ample excavation que limitent deux petites pointes distancées, au sommet du cône antapical (fig. 43, vue de face; fig. 44, vue antapicale).

La membrane capsulaire est réticulée.

Avec une dose suffisante de bonne volonté, on peut trouver une certaine analogie d'allure entre nos figures et celles de PAulsen. Cela nous porte à croire qu'il pourrait y avoir identification malgré les différences de reproduction imputables aux procédés d'observation et d'exécution des figures.

Distribution géographique. - Assez répandu dans nos matériaux d'étude; mais toujours clairsemé.

\section{Peridinium Thorianum Paulsen.}

Planche I Is, figures 20, 2I, 22, 23, et planche 1 , figures 5 et 6 .

C'est une bonne espèce, à caractères bien distincts.

Le corps est isodiamétral, biconique, arrondi, à ceinture creuse, sinistrogyre et marquée de sculptures très apparentes, sous la forme de petites cannelures transverses.

Le sillon ventral est profond, étroit, prolongé jusque vers le sommet du cône antapical, dont la forme est arrondie et qui ne porte aucun appendice.

La membrane est mince, comme finement plissée, ondulée ou creusée de nombreuses petites fossettes, qui en rendent la coupe optique finement sinueuse.

L'examen des figures en dira plus que toute description.

Figure 20 , planche $I^{\text {bis }}$, vue de profil; figure $2 \mathrm{I}$, vue ventrale; figure 22 , vue apicale, avec les I4 plaques classiques; figure 23, vue antapicale, avec les 7 plaques réglementaires. Sutures simples. 
Les figures 5 et 6 de la planche II se rapportent évidemment à la même espèce; mais ici les sutures sont doubles, larges et occupent une grande partie de la surface du corps. Cette particularité en modifie assez bien l'aspect pour ne permettre l'identification qu'à la suite d'un examen attentif.

Distribution géographique. - Sud de la Mer de Barents.

\section{Peridinium robustum sp. n.}

Planche XIV, figures 33 à 37 .

Cette forme n'est pas sans analogie avec la précédente, dont elle se distingue assez peu par les contours. Mais ici la membrane est épaisse, solide et fortement sculptée d'un réticulum à mailles sinueuses arrondies au fond desquelles se trouvent des ponctuations circulaires.

Nous en figurons cinq vues prises suivant les trois axes principaux.

La ceinture est sinistrogyre et creusée en sillon semi-cylindrique : figure 33, vue ventrale, qui montre aussi l'étroitesse du sillon ventral; figure 34, vue dorsale montrant le còne antapical un peu émarginé par le sillon ventral; figure 35, vue latérale droite; figure 36, vue antapicale montrant l'ordonnance des 7 plaques de ce cône ; figure 37, qui montre de face le còne apical avec ses it plaques à sutures doubles, mais étroites et sans striation.

Distribution Géographique. - Nord de la Mer de Barents et Océan Glacial.

\section{Peridinium conicum Gran?}

Planche III, figure 52 ; planche I, figures 38,39 et fo.

Synonymie. - Voir Nordisches Plankton, page 58.

Le signalement vrai de cette espèce anciennement citée nous parait encore problématique, à cause autant des différences de diagnose des auteurs que de nos propres observations, qui nous ont souvent mis en présence de formes qui, par quelque côté, se réclament de ce nom et qui, d'autre part, semblent différer les unes des autres par des caractères qui semblent inconciliables avec une identité de nature spécifique.

$\mathrm{Si}$ nous ne nous trompons, le spécimen reproduit planche III, figure 52, se rapproche le plus de ce que l'on pourrait considérer comme la forme-type, avec sa partie antapicale profondément partagée, en deux cônes aigus et égaux, par une large dépression en rapport avec le sillon ventral.

Nous en ferons provisoirement la forme bilobata pour souligner ce détail. 
Le spécimen reproduit planche I, figure 38, en vue ventrale, et figure 39 en vue apicale, ne montre plus qu'une légère dépression entre les sommets des deux cônes qui surmontent la base antapicale. Les dimensions beaucoup plus grandes ne laissent pas supposer que la faible dépression antapicale soit un caractère de jeunesse.

Nous le signalons comme forme emarginata.

Une troisième forme représentée, figure 40, en vue ventrale, ne montre plus qu'un sillon ventral très étroit à son origine et dilaté plus bas, mais qui n'entame pas le sommet du cône antapical. Celui-ci reste arrondi en vue ventrale normale.

Cette particularité caractériserait, si l'on veut, la forme basicurva.

Ces formes, et d'autres que nous avons négligées parce que ce n'est pas le lieu de nous livrer à une étude monographique de cette énigmatique espèce, ont de commun une grande analogie dans la forme du cône apical et dans l'apparence lisse de la surface de la capsule, dans les conditions ordinaires d'observation, en milieu liquide.

Cette espèce, ou du moins ce qu'on paraît tenir pour telle, nous semblait mieux connue par nous des formes de la Mer Flamande, qui ont une membrane réticulée; mais nous n'avons pas à en tenir compte ici.

D'autres trouveront peut-être une relation entre ces formes et $P$. achromaticum Levander. C'est encore possible; mais nous n'insistons pas, parce que nous manquons d'éléments concrets d'appréciation.

Distribution GÉographigue. - Çà et là, toujours très rare, sous n'importe quel aspect.

\section{Peridinium granisparsum sp. n.}

Planche I, figure 4 I.

La forme ici figurée en vue ventrale, petite, globoïde, à sillon ventral échancrant le sommet du cône antapical, n'a rien de commun, comme on pourrait le penser, avec l'espèce $P$. punctulatum Paulsen que nous connaissions très bien de la Mer Flamande où nous l'avions fréquemment observée avant que PAULsen ne l'ait dénommée en 1907 .

La physionomie en est toute autre; les ponctuations saillantes qui ornent les plaques sont clairsemées et le sillon ventral découpe tout autrement le cône antapical.

Distribution géographique. - Sud de la Mer de Barents. 


\section{Peridinium verrucosum sp. n.}

Planche IV, figures i 3 et It.

Forme à axe polaire court et à cône antapical déprimé, aplati à la base ; figure I3, vue dorsale. Coupe transversale pentagonale arrondie, figure 24, vue apicale. Membrane mince non réticulée, mais relevée de verrues peu nombreuses, distancées.

Cette espèce est très fragile et se déforme aisément dans les réactifs.

Distribution géographique. - Sud de la Mer de Barents et côtes norvégiennes.

\section{Peridinium bulla sp. n.}

Planche $I^{\text {bis }}$, figures 32 à 34 .

Forme isodiamétrale, sphérique, à ceinture creuse, sinistrogyre, à sillon ventral court, n'atteignant pas le sommet du còne antapical, qui reste arrondi (fig. 32, vue ventrale). Le cône apical est également arrondi, hémisphérique et exempt de saillie terminale. La membrane est très finement réticulée. Les plaques caractéristiques des Peridinim s'y observent, bien que les lignes suturales soient peu marquées.

Distribution géographique. - Mer de Kara. Très rare.

\section{Peridinium hyalinum sp. n.}

Planche II, figures 37 à 39 .

Forme globuleuse encore, mais à axe dorso-ventral plus court; ce qui rend réniforme la coupe transversale (fig. 38, vue apicale). Cône apical conique et plus petit; cône antapical plus grand et arrondi à la base (fig. 3g, vue latérale gauche). Sillon ventral très peu développé en longueur et en largeur (fig. 37, vue ventrale). La membrane capsulaire nous a toujours paru exempte de sculptures, même à sec, circonstance où les sutures des plaques deviennent visibles.

A part ce dernier détail, on serait peut-être tenté de rapporter cette forme au genre Glenodinium, si celui-ci était établi sur autre chose qu'un caractère négatif : l'absence de plaques distinctes, qui pourrait bien être plus apparente que réelle.

Distribution Géographique. - Mer de Kara. 


\section{Petites Formes}

Nous ne ferons que signaler les formes minuscules qui, présentant la physionomie des précédentes, n'en sont peut-être que des termes ontogéniques ou encore, certaines d'entre elles, de petites espèces de Glenodinimm sans caractères distinctifs précis.

Telles sont (pl. II, fig. 34 et $35 ;$ pl. XIV, fig. 44) des formes qui sont parfois nombreuses dans les échantillons et qui semblent se rattacher aux Peridinium.

Les formes figurées planche XIV, figures 47 et 48 , se rattacheraient mieux, semble-t-il, aux Glenodinium, si l'on était fixé et sur ce qu'elles sont actuellement et sur ce qu'elles peuvent devenir.

\section{Genre Glenodinium Stein}

L'analogie des formes extérieures des derniers Peridinimm cités ci-devant avec celles plus frustes des Glenodininm nous porte à présenter immédiatement ici les remarques que l'examen de nos échantillons nous a suggérées au sujet des rares représentants de ce genre, réserves faites, comme plus haut, sur le bien fonclé des caractères de celui-ci.

\section{Glenodinium danicum Paulsen.}

Planche XIV, figures I9 et 20 ; planche II, figures 40 , fr $^{\mathrm{r}}$ et $4^{2}$.

Synonyme. - Voir Nordisches Plankton, page 23.

Ce Péridinien globuleux, à ceinture creuse, sinistrogyre et à sillon ventral court, ne laisse pas voir une subdivision de la membrane capsulaire en plaques distinctes malgré sa similitude complète de forme avec d'autres chez lesquels cette particularité s'observe. C'est la seule raison que l'on ait, d'après des matériaux conservés, pour l'exclure du genre Peridinium; base d'appréciation bien fragile, dans l'état d'ignorance où l'on est encore des phases évolutives des Péridiniens.

Ce n'est donc que pour mémoire que nous le signalons en passant, pour noter l'état fréquent d'enkystement de cette forme ou d'une autre très similaire, comme dans la figure 40 de la planche II, où l'on voit la spore globuleuse à l'intérieur de la capsule hyaline. 
Remarque. - Cette petite forme, très sporifère dans certains de nos échantillons, est souvent associée, dans les mêmes milieux, à une autre plus grande, également sporifère (pl. II, fig. 4I), dont la moitié antapicale, tant de la capsule que de la spore, est légèrement déformée dans sa courbure. La déformation de la spore se traduit mème parfois (fig. 42 - spore mise en liberté -) par deux petites cornes pleines, à pointe émoussée..

Distribution géographique. - Mer de Barents, no i68.

\section{Glenodinium bipes Paulsen?}

Planche III, figure I8.

Synonyme. -- Voir Nordisches Plankton, page 25.

Nous n'avons pas de conviction faite, non plus, au sujet de cette forme minuscule observée rarement dans nos échantillons.

La figure I8, qui la reproduit de face, nous la montre dextrogyre et ornée de deux spinules divergentes. sur le cône antapical.

Nous ne sommes pas fixé sur ses affinités réelles.

Distribution GÉographique. - Mer de Barents; très rare.

\section{Glenodinium inflatum sp. n.}

Planche II, figure 9.

C'est encore sur ces caractères frustes et négatifs que nous nous basons pour placer ici la forme représentée, en vue ventrale, et montrant, sur un corps globuleux elliptique, une ceinture circulaire à bords arrondis et conséquemment mal délimités. Le sillon ventral est aussi mal déterminé. La capsule hyaline ne laisse voir que des striations confuses dans la direction des deux pôles; striation difficile à dégager, d'ailleurs, de l'aspect granuleux du protoplasme cellulaire sous-jacent.

Cette forme est peut-être moins rare qu'elle nous a paru; mais comme les réactifs éclaircissants la rendent hyaline et atténuent encore ses caractères morphologiques, elle a pu se dissimuler souvent à l'œil appliqué à la recherche des Péridiniens.

\section{Genre Heterocapsa STeIN}

\section{Heterocapsa triquetra Stein?}

Planche IV, figures 5 à 8 .

Synonyme. - Voir Nordisches Plankton, page 26. 
La mème réserve nous est imposée au sujet de cette toute petite forme de physionomie assez spéciale, mais d'affinités douteuses, que nous n'avons rencontrée que rarement dans nos échantillons. Les vues classiques du spécimen figuré dispensent de toute description.

\section{Genre Diplopsalopsis g. n.}

La création d'un genre nouveau nous parait utile pour retirer du genre Peridinizm l'espèce orbiculare Paulsen, dont le còne apical n'a pas le nombre réglementaire de plaques, exception que rien n'expliquerait dans ce genre déjà si touffu d'espèces et dont on a détaché, pour des raisons non plus péremptoires, le genre Diplopsalis.

Noús proposons ce nom à cause de la confusion facile de la forme qui nous le suggère, avec une espèce sphérique de Diplopsalis dont nous dirons un mot plus loin.

\section{Diplopsalopsis orbiculare (Paulsen)?}

Planche III, figures 14 à 17.

Synonymie. - Voir Nordisches Plankton, page 42.

La forme est sphérique. La ceinture un peu creuse et circulaire est bordée d'ailettes très étroites et non striées. Le sillon ventral est court et exempt de spinules. Par contre, la membranelle est très large, très proéminente. Vue dans toute son ampleur sur la figure de profil gauche (fig. I5), elle se montre en projection sur les vues apicale (fig. I6) et antapicale (fig. I7).

Le cône apical (fig. I6) ne se montre constitué que de onze plaques asyméiriquement distribuées.

Les spécimens rencontrés n'ont, comme celui figuré, que des sutures étroites quoique nettement dédoublées. La capsule parait exempte de sculptures.

A un examen superficiel on confondrait aisément cette forme, soit avec Peridinium globulus, soit avec Diplopsalis sphaerica nobis.

Distribution grógraphigue. - Rare dans la Mer de Barents.

\section{Genre Diplopsalis Bergh}

Ce genre, fondé primitivement sur les caractères de l'espèce lenticula Bergh, nous paraît bien établi pour cette espèce, bien que la figuration en ait été généralement défectueuse. Nous ne sommes pas documenté pour penser de mème au sujet des autres formes qu'on y a rattachées. 


\section{Diplopsalis lenticula Bergh.}

Planche III, figures ig à 22 .

Synonymie. - Voir Nordisches Plankton, page 35.

Le nom suppose une forme lenticulaire, réalisée réellement par le spécimen figuré de face (fig. I9), de profil droit (fig. 20), en vue apicale (fig. 2I) et en vue antapicale (fig. 22).

A part la légère proéminence du còne apical, le corps ne porte aucune spinule. Seules émergent la membrane orale et les ailettes peu proéminentes et exemptes de toute striation de la ceinture, caractère important pour différencier à première vue cette forme d'avec Peridinim ovatum qui, à d'autres égards, présente beaucoup de similitude d'aspect quand on n'en aperçoit pas les spinules antapicales.

La ceinture est circulaire.

Le còne apical comporte onze plaques de grandeur très inégale, mais symétriquement disposées. La plaque losangique antérieure est très étroite; les trois autres terminales sont amples, surtout la postérieure qui couvre une grande partie de l'hémisphère; les plaques marginales sont très étroites.

Le còne antapical (fig. 22) ne comporte que cinq plaques, si l'on considère comme unique celle dans laquelle se trouve encastré le sillon ventral.

Toutes les plaques sont réticulo-ponctuées.

Ces notes ne concordent pas absolument avec les descriptions des auteurs, mais nous les avons expressément vérifiées sur nature, d'après nos matériaux.

Distribution géographique. - Clairsemé, de-ci de-là, dans les deux mers de Barents et de Kara.

\section{Diplopsalis sphaerica sp. n.}

Planche IV, figures 9 à 12 .

Nous avons souvent été étonné, dans l'étude du Microplankton de la Mer Flamande, de rencontrer les caractères de Diplopsalis dans une forme non lenticulaire, mais presque rigoureusement sphérique, ce qui est contradictoire du nom spécifique lenticula donné à la forme précédente.

L'examen du microplankton du voisinage de la Nouvelle-Zemble nous a remis en présence du mème fait.

C'est pourquoi nous proposons le nom spécifique de sphaerica pour cette forme, que nous figurons ici dans quatre positions classiques et chez laquelle l'égalité des trois axes est le caractère le plus apparent.

La capsule est ornée de fortes ponctuations combinées avec un fin réticulum. 
Les sutures larges sont toujours en dépression par rapport aux pans sculptés des plaques.

Au point de vue taxinomique, il nous semble plus simple d'en faire une espèce distincte plutôt qu'une variété sphérique d'une autre espèce lenticulaire, ce qui serait une antonymie.

Distribution GÉographigue. - Même que la précédente.

\section{Genre Nephrodinium g. n.}

Nous proposons ce genre nouveau pour caser deux espèces réniformes trouvées dans un milieu très spécial : la neige jaune rencontrée sur les glaces de la Mer de Kara d'où la fusion du substratum les fait passer accidentellement dans l'eau marine sous-jacente.

La distribution caractéristique des plaques sera décrite ci-après, dans le signalement de l'espèce principale.

Nephrodinium nivale sp. n.

Planche Ibis, figures $4^{\mathrm{I}}$ à $4^{5}$; planche II, figures 7 et 8 .

Le corps est à trois axes inégaux. La vue ventrale est elliptique, à grand axe longitudinal (fig. 4I, pl. I ${ }^{\text {bis }}$ ). La ceinture est un peu dextrogyre et creuse, exempte d'ailettes distinctes. Le sillon ventral est court, peu déprimé et présente un orifice oral de forme particulière, dépourvu de membranelle et variable d'aspect avec l'épaississement de la capsule qui n'est jamais assez accusé, du reste, pour l'empècher d'être très fragile.

En dessous de ce sillon on voit, même sur la face ventrale, une petite plaque supplémentaire, de forme pentagonale, qui porte à huit le nombre de ces parties du cône antapical. La vue antapicale (fig. 44) en montre la distribution. La vue apicale (fig. 43) montre l'ordonnance irrégulière des plaques de ce côté, au nombre de seize ou même dix-sept, si l'on tient compte de la petite pièce terminale, bordée de sutures plus fortes et plus saillantes.

La figure 42 montre l'espèce de profil droit et laisse voir que la ceinture s'abaisse notablement sur l'arrière. L'axe antéro-postérieur est beaucoup plus court, ce qui fait que le corps a la forme d'un ellipsoïde aplati, la forme d'un rein.

La capsule est finement réticulée, sauf sur le sillon ventral.

La figure 45 le montre avec son contenu transformé en spore quiescente, $s p$. Celle-ci est de forme lenticulaire; sa membrane est mince.

Les sutures sont le plus souvent simples.

La figure 8, planche II, les montre doubles. 
La figure 7 , planche II, représente un spécimen jeune chez lequel les sculptures sont encore inapparentes, même à sec.

Ces deux dernières figures ont d'abord été exécutées d'après des spécimens qui nous ont légèrement induit en erreur sur l'allure réelle et le nombre des plaques du cône apical; particularités difficiles à saisir, d'ailleurs, à cause de la forme aplatie latéralement des dòmes terminaux, de la parfaite hyalinité de la membrane et de la finesse habituelle des sutures.

Distribution GÉographique. - Rencontré surtout dans la neige jaune et sur la glace humide de la Mer de Kara; mais ramené aussi, rarement, par le filet planktonique, des eaux marines sous-jacentes où la fusion des glaces l'a fait descendre.

\section{Nephrodinium karianum sp. n.}

Planche IV, figures $\mathrm{i} 5$ et $\mathrm{r} 6$.

Petite espèce réniforme, incurvée en arrière, à ceinture creuse et médiane. Les deux cônes terminaux sont arrondis au sommet et ne portent aucune trace d'appendice. La distribution des plaques est très dissymétrique, les sutures sont faiblement marquées. La capsule, très fragile, nous a toujours paru exempte de sculptures.

Distribution Géographique. - Même habitat spécial que l'espèce précédente.

\section{Genre Protoceratium Bergh}

Le nom donné à ce genre a l'inconvénient, à notre avis, de lui faire supposer des caractères qui le rapprocheraient plus que tout autre du genre Ceratium : la présence, par exemple, de cornes rudimentaires. Or, c'est là précisément ce qui fait complètement défaut dans les espèces qu'on lui attribue.

Nous suivrons néanmoins les auteurs en le maintenant, dans le but de déranger le moins possible la terminologie reçue.

Protoceratium reticulatum Bütschli.

Planche III, figures I à 5 ; planche $I^{\text {bis }}$, figure 38 .

Synonyme. - Voir Nordisches Plankton, page 27.

Le corps irrégulièrement globuleux et excentrique serait assez difficile à décrire sans l'aide de figures le représentant sous des aspects différents. 
La figure I, qui le montre de face, indique l'orientation sinistrogyre de la ceinture qui est creuse et dépourvue d'ailettes. Elle divise le corps en deux parties très inégales, au profit du còne antapical.

Le sillon ventral, où se perdent les deux bouts de la ceinture, qui décrit moins qu'un tour complet de spire, intéresse les deux cònes, sans limites précises ni vers le haut, ni vers le bas.

La figure 2 le présente dè profil latéral gauche et permet de saisir l'excentricité des deux cònes vis-à-vis de l'axe polaire.

La figure 3 montre de face le cône apical, avec une plaque centrale et sept plaques marginales, séparées par des sutures les unes simples, les autres doubles; celles-ci difficiles à repérer dans les mailles irrégulières du réticulum fortement imprimé sur toute la capsule. Les trabécules de ce réticulum sont des crêtes saillantes dont le profil se dessine en pointes sur la coupe optique de toutes les parties du corps. Les mailles polyédriques, toujours très visibles, sont occupées par un réticulum plus fin, que nous dirons de second degré et qui n'est visible qu'avec de bons objectifs sur des objets convenablement préparés.

La figure 4 montre le còne antapical formé, à notre avis fondé sur beaucoup d'observations comparées, de quatre plaques seulement, dont l'antérieure englobe le sillon ventral, sans délimitations respectives bien nettes.

La figure 38 de la planche $I^{\text {bis }}$ en montre un individu passé à l'état de spore quiescente, sp. Celle-ci est de forme ovale, un peu irrégulière.

Distribution géographioue. - Assez commun, dans la Mer de Kara surtout et, aussi, dans des échantillons d'autre provenance particulièrement riches en Péridiniens de petites formes dont elle constitue parfois l'espèce dominante.

\section{Protoceratium splendens sp. n.}

Planche II, figures I à 4 ; planche $I^{\text {bis, }}$ figure 37 .

La comparaison souvent refaite entre la forme précédente et celle dont il est question ici nous a forcé à distinguer spécifiquement celle-ci, que nous représentons : figure I, de face ventrale; figure 2, de profil latéral droit; figure 3, de vue apicale; figure 4 , de vue antapicale.

La forme est plus grande, moins anguleuse, plus arrondie; mais surtout, et c'est cela qui nous la fait tenir pour spécifiquement distincte de l'autre, la sculpture maillée est beaucoup plus dense; les mailles sont plus petites, leurs contours plus sinueux.

La figure 37 , planche $I^{\text {bis }}$, la montre à l'état d'enkystement. Le kyste, encore inclu dans la capsule, est de forme globuleuse; sa membrane est mince.

Distribution GÉographioue. - Fréquente les mèmes parages que la précédente, mais ne l'accompagne pas toujours. Elle est notablement plus rare. 


\section{Genre Amylax g. n.}

Nous proposons ce genre nouveau pour grouper plus harmonieusement des espèces rapportées jusqu'ici à des genres qui ne les revendiquent à aucun titre et douées cependant de caractères communs d'ordre non seulement morphologique, mais aussi physiologique. Nous visons ici la production de la fécule que l'on rencontre toujours en certaine abondance dans leur protoplasme, en grains arrondis et assez volumineux.

Le nom Amylax suggère cette particularité et rappelle, en outre, par sa consonance, le genre Gonyaulax, avec lequel les espèces ici groupées ont certainement leurs plus proches analogies morphologiques.

\section{Amylax lata sp. n.}

Planche III, figures 24 à 27 .

Il n'est pas impossible qu'il s'agisse ici de Gonyaulax triacantha Jörgensen, I899, de Ceratium hyperboreum Cleve, Igoo, et de Heterodinium triacantha Kofoid, Igo6. Mais, à notre avis, ce n'est pas plus un Gonyaulax qu'un Ceratium, et l'espèce n'est pas triacantha, parce qu'elle n'a pas que trois épines ou spinules mais un plus grand nombre, ce qui se rencontre aussi dans les autres espèces affines à celle-ci et que nous groupons dans le même genre.

Nous appelons celle-ci lata, parce que ses grandes dimensions nous la font prendre comme type du groupe.

Le corps est large, vu de face, figure 24, vue ventrale; figure 25, vue dorsale; et aplati dans le sens dorso-ventral, figure 26 , vue latérale gauche, sur laquelle se profile la membranelle orale.

La ceinture creuse, spirale, sinistrogyre, dessine un peu plus d'un tour de spire. Elle divise le corps en deux parties inégales, dont la supérieure s'atténue brusquement en protubérance conique, tandis que l'inférieure est trapézoïdale et plus développée du côté droit. Cette partie porte plusieurs spinules qui sont de valeur inégale et qui sont en rapport avec les sutures des plaques antapicales.

Le sillon ventral, qui va s'élargissant vers le sommet antapical, se prolonge aussi d'autre part, en s'étranglant, dans le cône apical, dont il atteint à peu près le sommet.

La membrane capsulaire est couverte de fines sculptures, sous la forme d'un réticulum irrégulier, dont nous nous sommes efforcé de reproduire le style particulier.

La confusion qui s'établit entre les trabécules de ce réticulum et les sutures des plaques rend difficile le dénombrement de celles-ci. Les sutures, visiblement distinctes, sont marquées dans nos figures avec le degré d'évidence qu'elles présentent suivant les endroits. 
La coupe optique longitudinale (fig. 27) montre la présence dans le protoplasme d'enclaves graisseuses, $h$, et de grains de fécule, $f$, situés à la périphérie et facilement décelables par l'iode qui les colore en bleu violacé.

C'est ce dernier caractère, peu banal dans le monde des Péridiniens, que nous considérons comme prépondérant pour l'établissement du genre Amylax.

Distribution géographique. - Mer de Kara et Détroit de Matotchkine; no 22 surtout, à la sortie du Détroit dans la Mer de Kara.

\section{Amylax catenata (Levander).}

Planche III, figures 28 à 34 ; planche $\left.\right|^{\text {bis, }}+46$ et +7 .

Synonynie. - Peridinium catenatum Levander. Voir Nordisches Plankton, page 63.

Cette curieuse espèce serait certainement dépaysée dans le genre Peridinium ramené aux limites plus étroites qu'il y a lieu, à notre avis, de lui assigner.

Le corps est petit, pentagonal en coupe optique longitudinale, étroitement réniforme en coupe optique transversale : planche III, figure 33, vue ventrale; figure 34, vue dorsale; figure 3I, vue apicale; figure 32, vue antapicale. Individus isolés.

La ceinture creuse et sinistrogyre fait souvent un peu plus d'un tour de spire dans les sujets jeunes. Le sillon ventral s'étend d'un pòle à l'autre; étroit dans le cône apical, très large dans le còne antapical où il se creuse en une vaste dépression se modulant sur le cône apical de l'individu placé en dessous, dans la série en chainette suivant laquelle les sujets restent groupés par quatre, six ou huit, le plus fréquemment. Figure 28, vue ventrale; figure 29, vue oblique; figure 3o, vue dorsale de séries d'individus soudés.

Le bord irrégulier de la dépression antapicale porte de petites spinules qui s'appuient sur le sujet voisin et semblent jouer le ròle d'agrafes, pour le maintenir dans sa position normale et assurer la régularité rectiligne de la colonie.

La capsule est toute converte d'un réticulum, toujours bien visible, mais plus ou moins marqué suivant l'àge du sujet sans doute.

Le nombre exact des plaques est difficile à reconnaitre à cause de la confusion qui s'établit entre les sutures et les trabécules du réticulum.

Sur des sujets très différenciés (pl. I bis, fig. 46), les plaques antapicales semblent réduites à six.

Le cytoplasme est toujours riche en enclaves amylacées : caractère du genre, planche $I^{\text {bis }}$, figure 47 ; coupe longitudinale optique; $f$, fécule; n, noyau.

Distribution géographigue. - Commune dans la Mer de Kara, on l'y trouve même dans la neige jaune et sur la glace. Elle est exceptionnelle dans les produits planktoniques de la Mer de Barents. 


\section{Amylax nivicola sp. n.}

Planche III, figures 35 et 36 .

Corps élancé, de petites dimensions, en forme de poire aplatie dans le sens dorso-ventral. Cône apical longuement conique, à sommet droit ou, plus généralement, recourbé du côté droit. Ceinture large, creuse, sinistrogyre, décrivant un peu moins d'un tour de spire. Sillon ventral très élargi sur la base du cône antapical, qui porte plusieurs fines spinules, et prolongé en fente étroite du côté apical. Figure 35, vue ventrale; figure 36 , vue dorsale.

La capsule est toujours très épaisse et vigoureusement sculptée d'un réseau dont les trabécules rendent indistinctes les sutures des plaques. Elle prend une teinte cuivrée dans le milieu d'enrobage : iode et chloral concentré.

Après macération, elle bleuit franchement sous l'action de l'iode et traduit ainsi sa nature cellulosique.

Le protoplasme est toujours très dense, parfois exempt de réserves amylacées, plus souvent riche en petits grains de fécule, sans préjudice des réserves graisseuses qui ne font jamais défaut.

Le noyau, volumineux, occupe toujours la moitié apicale de la cellule.

Distribution géographioue. - La neige jaune de la Mer de Kara, où il n'est pas rare, semble être son habitat normal. Il est rarement repêché par le filet fin dans les eaux marines des mêmes régions.

\section{Amylax perpusilla sp. n.}

Planche III, figure 37.

Corps extrêmement petit, à physionomie de $A$. catenata, et rencontré dans les mêmes stations que cette dernière (fig. 37 , vue ventrale).

Nous n'avons pas rencontré les individus de cette petite forme agrégés en colonies linéaires; ce qui nous défend de la considérer comme une forme jeune de celle-là, à moins de supposer que les individus d'abord isolés et d'origine encore inconnue s'agrégeraient plus tard, pour se mettre en série et se présenter alors, sous des dimensions amplifiées, comme $A$. catenata. Simple hypothèse, dont nous ne pouvons tenir compte présentement pour passer outre à la petitesse de la forme — seul caractère certain pour nous - et la rattacher sans preuves suffisantes à une autre espèce.

Distribution GÉographioue. - Mer de Kara; parait très rare, à cause peut-être de ses très petites dimensions. 


\section{Genre Gonyaulax Diesing}

\section{Caracteres du Genre. - Voir Nordisches Plankton, page 27.}

Ce genre, élagué des espèces aplaties d'avant en arrière, comme nous l'avons fait plus haut pour constituer le genre Amylax, n'est plus représenté dans nos échantillons que par les formes reproduites planche III, figures 5 à I3, qui, avec des dimensions variables du simple au double, donnent néanmoins l'impression d'une parenté tellement étroite qu'on ne saurait guère y relever des caractères différentiels suffisants pour autoriser leur attribution à des espèces distinctes.

\section{Gonyaulax polygramma Stein.}

Planche III, figures 5 à r 3 .

Synonyme. - Voir Nordisches Plankton, page 29.

Corps globuleux, ellipsoïde, à còne apical brusquement rétréci à quelque distance du sommet, au-dessus des six plaques latérales; cône antapical arrondi à la base, avec cinq plaques latérales qui en circonscrivent une sixième, déprimée, dans laquelle le sillon ventral se prolonge en s'élargissant.

La ceinture, profonde, sinistrogyre, dessine plus d'un tour complet de spire.

Le sillon ventral, très élargi vers le bas, est limité tout autour par une crête qui s'épanouit en lamelle réticulée sur la base du corps.

La capsule, à sutures simples ou dédoublées, suivant les cas, est vigoureusement sculptée, sur toute son étendue, d'un réseau irrégulier dont les trabécules sont parfois renforcés localement au point de donner l'illusion de sutures surnuméraires.

Ces sculptures difficiles à analyser dans leurs détails sont plus difficiles encore à rendre par le dessin. Peut-ètre est-ce là la cause des différences, plus apparentes sans doute que réelles, entre Gonyaulax polygramma Stein et Gonyaulax spinifera Diesing.

Le spécimen, reproduit (fig. 6, 7, 8, 9) en quatre positions normales, présente les caractères moyens du type.

Le spécimen dessiné (fig. 5), en vue ventrale, est plus fort, plus biconique et présente une bifurcation de son prolongement lamellaire antapical.

Les sculptures plus vigoureuses s'y traduisent sous la forme d'un réseau de petites crêtes saillantes. Serait-ce là le caractère du prétendu G. spinifera? A dire vrai, les figures des auteurs traduisent ces détails trop imparfaitement pour que leur comparaison permette d'asseoir un jugement. 
Les figures Ir, vue ventrale, et Io, vue latérale droite, reproduisent une forme beaucoup plus petite, chez laquelle les sutures sont néanmoins dédoublées.

Les figures I2, vue ventrale, et I3, vue antapicale, sont d'un autre spécimen encore, d'aspect quelque peu différent de celui de ses congénères.

La figure i6 de la planche XXIII en montre la division du noyau et du corps protoplasmatique, à l'intérieur de la capsule, en coupe optique; $c p$, capsule; $c$, ceinture; $n d$, noyau en division.

A notre avis, l'unité spécifique existe entre toutes ces formes que l'on trouve du reste associées dans les mèmes milieux.

Distribution géographique. - Peu rare sur les còtes occidentales de la NouvelleZemble, dans la Mer de Barents.

\section{Genre Oxytoxum Stein}

Caractéres du Genre. - Voir Nordisches Plankton, page 68.

Nos matériaux d'étude ne nous ont fourni qu'une seule espèce de ce genre représentée elle-même par de très rares individus. Station I, Mer de Kara.

Nous ne savons la rapporter à aucune des espèces décrites jusqu'ici, à notre connaissance.

\section{Oxytoxım Belgicae sp. n.}

Planche IV, figures 38 à $4^{\mathrm{I}}$.

La forme du corps (pl. XIV, fig. 38, vue ventrale; fig. 39, vue dorsale) rappelle assez bien Oxytoxum tesselatum Schütt (r895, pl. XVII, fig. 52); mais elle en diffère essentiellement par l'ornementation toute différente des plaques.

Celles-ci portent, dans notre espèce, des panneaux très finement ponctués, striés, bordés d'encadrements lisses, dont la largeur se rétrécit, comme les panneaux euxmêmes, vers l'extrémité antapicale.

La figure 40, vue antapicale, montre cinq plaques inégales convergentes vers le sommet.

La figure 4I, vue apicale, laisse voir cinq plaques latérales alternantes avec autant de plaques terminales dont l'antérieure est comme divisée par le prolongement apical du sillon ventral.

L'examen attentif de ces diverses vues d'un même individu fera saisir les autres particularités de forme et de structure qui complètent le signalement de cette jolie espèce.

Distribution GÉographigue. - Station i, Mer de Kara. 


\section{Genre Ceratium Schrank}

Caractères du Genre. - Cfr. Nordisches Plankton, page 7 .

\section{Sous-genre Euceratium Gran}

Les formes référables à ce groupe sont remarquablement homogènes dans nos échantillons microplanktoniques, bien que certains d'entre eux en soient richement pourvus. Comparaison faite entre d'innombrables individus de formes plus ou moins différentes, mais rattachées entre elles par des transitions insensibles, nous devons les verser toutes dans une même espèce.

\section{Ceratium longipes Gran.}

Planche I ${ }^{\text {bis }}$, figures 39 et fo; planche II, figures 47 à 50 ; planche III, figures 23 et 50 .

Synonyme. - Toir Nordisches Plankton, pages 85 à 87.

Les différences existantes entre les quelques individus que nous avons reproduits, pour jalonner en quelque sorte l'étendue des variations de la forme, les feraient prendre pour des représentants d'espèces distinctes si la présence de nombreuses formes intermédiaires ne plaidait victorieusement en faveur de leur unité spécifique.

Cette diversité des aspects a frappé du reste les planktonistes du Nord qui ont cru bon d'y repérer certaines variétés, à titre de découpures utiles pour fixer l'esprit de l'observateur, dans l'examen des formes variées qui constituent cette espèce polymorphe.

A leur suite, nous y distinguerons les variétés suivantes :

$I^{\circ}$ Les formes sveltes, à cornes très longues et peu hérissées de denticules. Planche I Iis, figure 39; var. Baltica? Ostenfeld.

$2^{\circ}$ Les formes trapues, à corps court, à cornes antapicales plus ou moins recourbées vers la corne apicale et plus ou moins denticulées. Planche II, figures 47 et 48 ; var. oceanica? Ostenfeld.

30 Les formes à corps plus allongé, renflé excentriquement du côté gauche ; corne apicale plus grosse à sa naissance près du corps, plus amincie vers son extrémité. Planche $\mathrm{I}^{\mathrm{bis}}$, figure 40 , et planche II, figure 23 ; var. ventricosa Ostenfeld. 
$4^{\circ}$ Les formes plus raides, à cornes antapicales moins recourbées, presque droites parfois, plus fortement hérissées d'ailettes et de denticules. Planche II, figure 49, et planche III, figure 5o; var. arctica nobis.

A rapprocher de Ceratium arcticum Cleve.

Synonyme. - Voir Nordisches Plankton, page 87.

50 Les formes à cornes antapicales très courtes, comme amputées, peu divergentes de l'axe polaire du corps, peu hérissées. Planche II, figure 5o; var. amputata nobis.

Faut-il voir dans les attributs de cette variété des caractères de jeunesse qui se modifieront avec l'àge et les circonstances du milieu pour imprimer aux individus la physionomie plus ou moins tranchée des autres variétés?

Nous répétons que nos observations ne nous permettent pas de voir dans ces formes autre chose que des modalités plus ou moins frappantes d'un polymorphisme exagéré dont la cause ne saurait résider exclusivement dans les conditions du milieu puisqu'elles se coudoient d'habitude dans les produits d'un même coup de filet.

Quelle peut être, d'autre part, sur la physionomie de ces organismes, l'influence de l'àge de l'individu, de son éloignement phylogénique de la souche : spore ou œuf, etc.? Nos observations ne nous ont pas suffisamment documenté sur ces questions de genèse et d'évolution des Péridiniens en général et des Ceratium en particulier pour nous permettre de les élucider ici.

Distribution géographioue. - Répandu presque partout; mais surtout abondant dans le nord de la Mer de Barents, vers l'Océan Glacial.

\section{Sous-genre Biceratium VANHÖFFEN}

Nous ne citons ce sous-genre que pour placer ici une observation négative. Nous voulons dire : l'absence positivement constatée de tout représentant de ce groupe dans les produits pourtant si copieux et si variés des pêches planktoniques exécutées sur tout le parcours de la Belgica pendant sa campagne de 1907.

Ce fait, qui nous parait étrange, mérite d'ètre signalé tout au moins comme circonstance saisonnière de l'époque de l'année, à laquelle les pèches ont été faites : du 20 juin au 7 septembre.

\section{Sous-genre Amphiceratium VANHÖFfEN}

Ceratium fusus Claparède et Lachmann.

Planche II, figure $5 \mathrm{I}$.

Synonyme. - Voir Nordisches Plankton, page 90. 
Cette espèce, trop connue pour qu'il soit utile de la décrire, est seule à représenter ici ce sous-genre. Encore est-elle très rare dans le petit nombre d'échantillons où nous avons pu l'observer.

Nous nous contentons d'en reproduire un spécimen, en vue dorsale, pour noter l'aspect qu'il revêt ici, tant dans sa forme et ses dimensions que dans les détails de structure de sa capsule.

Remargue. - C'est un fait digne de remarque que la pauvreté de nos matériaux en espèces du genre Ceratium. Est-ce dû au milieu ou bien à la saison? Tout ce que nous pouvons dire, c'est que d'autres matériaux originaires des parages assez voisins du Spitsbergen et de l'Océan Arctique nous ont montré une beaucoup plus grande diversité spécifique du genre Ceratinm.

\section{Genre Dinophysis EHRENBERG}

\section{Caractères du Genre. - Voir Nordisches Plankton, page i2.}

Si les caractères génériques de Dinophysis sont bien nets, les caractères spécifiques des formes qu'on range dans ce genre le sont beaucoup moins, si ce n'est pour certaines d'entre elles, D. homunculus Stein, D. miles Cleve, et D. Schiittii Murray et Whitting, par exemple, dont la seule silhouette fournit un critérium d'identification bien suffisant pour les besoins de la systématique. Mais les formes moins excentriques se dérobent davantage aux recherches d'identification spécifique parce que les variations individuelles de grandeur, d'àge, de phase évolutive, etc., peuvent donner le change sur leurs affinités réelles.

Quant à l'identification des formes vues en nature avec les figures y relatives des auteurs, elle est souvent matériellement impossible à cause de l'incorrection ou, tout au moins, de l'insuffisance manifeste de ces figures. Certaines sont tellement frustes qu'il faut de la bonne volonté pour y retrouver ne fùt-ce que la note même du genre Dinophysis.

Pour ces raisons, nous ne garantissons pas le rapprochement que nous tenterons entre les formes observées par nous et celles décrites ailleurs, dont nous emprunterons les noms.

Dinophysis norvegica Claparède et Lachmann?

Planche III, figures 38 à 42 .

Synonymie. - Voir Nordisches Plankton, page i4.

La figure 38 (vue latérale droite) représente, croyons-nous, cette espèce sous sa forme la plus commune dans nos matériaux d'étude. 
La figure 9 (vue latérale droite) bien que présentant des excroissances lamellaires sur l'ourlet longitudinal, ce qui parait ètre un caractère individuel, ne nous semble pas appartenir à une espèce différente.

Le même individu présenté en vue ventrale (fig. 40) et en vue apicale (fig. 4I) en montre les détails de forme et de structure mieux et plus clairement que nous ne pourrions les décrire par des mots, ce qui est vrai communément pour ces tout petits organismes qui rachètent la ténuité de leur taille par le luxe de leur décoration.

Dans la figure 42 , nous présentons un spécimen en voie de division, chez lequel la production de deux valves nouvelles au sein de la cellule mère a préparé la genèse de deux cellules filles dont chacune hérite d'une des deux valves maternelles avec ses attributs : demi-ceinture et demi-ailette ventrale.

Cette figure montre, en effet, que la membranelle ou ailette ventrale est formée de deux parties distinctes : l'une, la supérieure dépendant de la valve gauche, l'autre, l'inférieure, de la valve droite.

Les valves nouvellement produites sont d'abord exemptes de sculptures. Elles s'appuient sur l'intérieur des valves anciennes, en retrait sur les bords de celles-ci, dont une zone d'une certaine largeur est sacrifiée. Cette zone, produite pendant une phase préparatoire à la division, présente des sculptures plus faibles et d'une autre allure.

Sur les jeunes valves, la plaque de la demi-ceinture nouvelle est distincte dès le début; mais les ailettes qui doivent la border n'apparaissent qu'après l'isolement des cellules sœurs, celles-ci restant plus ou moins longtemps associées latéralement dans la position que leur impose leur genèse.

Ces associations génétiques n'ont, on le voit, rien de commun avec les cas de gémination dont on a des exemples très connus dans Dinophysis hommoulus Stein et Histioneis magnifica Stein, où les individus géminés sont accouplés dos à dos.

Ce mode de division que nous avons observé aussi, fréquemment, dans l'espèce qui va suivre rend très étroites les affinités entre les Péridiniacées et les Diatomacées, que rapproche d'autre part la similitude de l'endochrome : la phycophéine.

Distribution Géographioue. - Còtes est et ouest de l'ille sud de la Nouvelle-Zemble.

Dinophysis rolundala Claparède et Lachmann?

Planche III, figures $4^{3}$ à 46 .

Synonyme. - Voir Nordisches Plankton, page I7.

Si, parmi les espèces récoltées par la Belgica, il en est une que l'on peut avec quelque vraisemblance désigner sous ce nom, c'est celle que nous figurons (fig. 43), de profil droit, montrant sa forme arrondie et l'émergence du sommet apical au-dessus de la ceinture. La figure 44 montre, de dos, un individu qui témoigne de sa division prochaine par l'élargissement du corps dù à la production, chez les deux 
valves latérales, d'une large bande hyaline qui côtoie la suture longitudinale. Le corps est ainsi rendu à peu près sphérique.

Que ce soit là l'expression d'une phase évolutive en vue de la cytodiérèse, nous en avons la preuve dans les nombreux individus observés chez lesquels ce phénomène est en cours ou déjà effectué, comme le montre la figure $4^{5}$.

Dans cet exemple, les deux jeunes individus sont englobés dans la capsule maternelle, dont ils garderont chacun une valve, avec perte ultérieure des bandes hyalines à la formation desquelles leur genèse a été subordonnée.

Le cytoderme maternel est encore entier; les zones d'accroissement ont brisé la continuité de la ceinture sur la face antérieure et distendu le sillon ventral, dont les deux parties de l'ailette sont séparées et fort distancées.

Par transparence, on distingue à l'intérieur les deux Dinophysis filles, dont les valves nouvelles, gauche pour l'une, droite pour l'autre, sont encore dépourvues d'ailettes aux bords de la demi-ceinture de nouvelle formation et aussi de la membranelle du sillon ventral, qu'elles n'acquerront que plus tard.

Dans la figure 46 nous montrons, en profil droit, un individu très jeune récemment séparé de sa sœur jumelle et constitué de deux valves inégales : l'une, la droite, nouvellement formée, hyaline, dépourrue encore d'appendices et de sculpture; l'autre, la gauche, cachée en grande partie par la première, ornée de son appendice, sculptée et dépassant, vers le bas, la valve nouvelle.

Remaroue. - Il résulte des phénomènes de cytodiérèse, dont nous avons dit un mot, sur exemples empruntés aux deux espèces décrites ci-devant, que peu après la division, la présence d'une valve jeune à còté d'une plus ancienne modifie l'aspect des deux individus issus d'une mème mère, suivant qu'ils héritent de la valve maternelle gauche ou droite. Entre autres choses, cela modifie la membranelle orale, dont les deux moitiés sont d'âge différent comme les valves qui les portent.

On peut en déduire la fragilité des caractères puisés parfois à l'aspect de cet organe, puisque celui-ci varie dans les individus d'une même série généalogique.

L'accentuation des sculptures est donc, aussi, généralement différente sur les deux valves d'un même individu puisque la plus jeune en est d'abord exempte et n'en reçoit l'empreinte que progressivement.

Distribution Géographioue. - Mer de Kara, près du Détroit de Matotchkine, et Mer de Barents, partie sud.

\section{Dinophysis cuneiformis sp. $\mathrm{n}$.}

Planche XIV, figures $3 i$ et 32 ; planche XXIII, figure 15.

Notre impuissance à rapprocher cette forme de l'une ou l'autre des deux précédentes avec lesquelles seules elle a un peu d'analogie, nous force à la signaler à part, 
à cause principalement de l'aspect tout spécial du contenu cellulaire : protoplasme et noyau, que nous regrettons n'avoir songé à figurer que tardivement (pl. XXIII, fig. I5).

Le corps est atténué brusquement en coin vers le sommet antapical, aussi bien en vue latérale (fig. 3I) qu'en vue ventrale (fig. 32). La ceinture est située très haut, mais la plage apicale qu'elle circonscrit a encore une certaine extension.

Que la membranelle ventrale soit dépourvue de toute sculpture, dans le spécimen figuré, ce n'est qu'un caractère individuel dont il n'y a pas lieu de faire état.

Dans la figure I5, planche XXIII, destinée à montrer l'aspect du contenu cellulaire en coupe longitudinale optique, on remarquera la forme du noyau, $n$, qui est allongé et lobé. Cet organe cellulaire est en outre flanqué de plusieurs groupes de corpuscules réfringents, $x$, dont nous n'avons pu nous attarder à rechercher ni la nature ni la signification.

Distribution gÉographique. - Mer de Barents, vers le Détroit de Matotchkine.

\section{Dinophysis vertex sp. n.}

Planche XIV, figures 29 et 30 .

Autre forme que nous ne saurions rattacher à l'une des espèces décrites du Nord. Le trait saillant en est la position très élevée de la ceinture qui confine au sommet (vertex) du corps et circonscrit une aire apicale très limitée, comme il apparait aussi bien dans la vue de còté (fig. 29) que dans la vue ventrale (fig. 3o).

Le corps est presque arrondi en bas. Les aréoles qui ornent les valves sont distancées. La membranelle orale est généralement peu développée.

Distribution GÉographique. - Océan Glacial; très rare.

\section{Dinophysis semen sp. $\mathrm{n}$.}

Planche III, figures 47 et 48 .

Forme très petite, globuleuse, à base arrondie, à membranelle orale relativement bien développée, comme aussi les ailettes de la ceinture. La sculpture réticulée des valves est très serrée.

Ces caractères sont le contre-pied de ceux attribués à D. arctica. Mereschkowski, qui n'a de commun avec $D$. semen nobis que sa petitesse.

Distribution Géographigue. - Rare, ci et là, dans les deux mers de Barents et de Kara. 


\section{Dinophysis apiculata sp. n. \\ Planche III, figure 49 .}

Forme minuscule présentant un prolongement apiculé, court, sur l'extrémité inférieure du corps (fig. 49, vue latérale droite).

Distribution Géographigue. - Toujours très rare, sans localisation spéciale à noter, car nous avons négligé de tenir compte des numéros où nous l'avons observée.

\section{Genre Gymnodinium SteIN}

Caractères Du Genre. - Voir Nordisches Plankton, page 97.

L'étude des représentants de ce genre sur matériaux fixés est particulièrement pénible, à défaut des caractères physiologiques qui suppléeraient avantageusement à l'imprécision des formes, lorsque celles-ci ne sont relevées par aucun trait capable de retenir l'attention de l'observateur et de lui permettre d'asseoir un diagnose morphologique.

Nous confessons en avoir rencontré plusieurs, que cette imprécision des formes nous a détourné de figurer, jugeant qu'il n'y a guère lieu de faire état de caractères instables que l'action des milieux conservateurs peut modifier profondément.

Leur étude ne peut être utilement faite que par ceux qui ont la faveur de les voir évoluer à l'état vivant et d'en percevoir les caractères d'ordre plus intime.

Peut-être pourrait-on placer ici l'organisme que nous avons signalé plus haut sous le nom de Glenodinium inflatum nobis; si l'on estimait que la membrane cellulaire en est trop mince et trop indistincte pour pouvoir être considérée comme une capsule présentant quelque rigidité.

Il y a là, comme on voit, une question d'appréciation personnelle plutôt qu'un caractère naturel de réelle valeur taxinomique.

Si l'on en juge par les données actuelles sur ce sujet obscur, données nébuleuses, indécises et souvent contradictoires, on peut ajouter que la délimitation entre ce genre et le suivant est encore mal taillée et doit être tenue pour sujette à révision, sur des faits mieux établis.

\section{Genre Spirodinium STEIN}

Caractères du GenRe. - Voir Nordisches Plankton, page ror.

Les remarques qui viennent d'être faites, à propos du genre Gymnodinium, s'appliquent aussi ici. 
La membrane mince, hyaline, lisse, des espèces qu'on rapporte au genre Spirodinium, donne également peu de prise à la figuration des individus et moins encore évidemment à la notification des caractères d'ordre plus extensif, tels que ceux. d'espèce ou de genre.

Le caractère générique emprunté à la disposition fortement spiralée du sillon transversal a cependant une valeur taxinomique qui n'est pas négligeable. C'est en tenant compte de cette particularité que nous réunissons ici certaines formes moins frustes rencontrées exceptionnellement, du reste, dans nos matériaux d'étude.

\section{Spirodinium lacryma sp. n.}

Planche IV, figures 21 et 22 .

Corps assez gros, en massue, en larme, arrondi d’un côté, supposé inférieur, leffilé et terminé en pointe de l'autre côté, supposé supérieur.

Ceinture, s, peu profonde, spiralée, dessinant environ un tour de spire, mais à limites diffuses. L'ectoplasme est chargé de petits corps réfringents, qui rappellent les trichocystes des infusoires et qui y sont régulièrement distribués en séries longitudinales nombreuses.

Le noyau, n, volumineux, central, ellipsoïde, présente un aspect nettement strié suivant son grand axe.

Distribution GÉographique. - Mer de Kara, station 8. Très rare.

\section{Spirodinium fusus sp. n.}

Planche XIV, figures 23 à 26 .

La forme que nous prenons comme type est celle de la figure 25, longuement atténuée aux deux bouts terminés en pointe aiguë.

Les figures 23 et 24 en montrent une variante plus petite, sous deux aspects opposés : devant et derrière.

Le spécimen représenté dans la figure 26 n'en est sans doute non plus qu'une variante bien que, par l'un de ses deux bouts, qui est obtus, elle se rapproche également de l'espèce précédente.

Du reste, la structure de l'ectoplasme est la même; mais dans les formes dont il s'agit ici, on remarque toujours distinctement une sorte de vacuole, $v$, en rapport intime avec le noyau, $n$.

La ceinture spiralée, $s$, est difficile à suivre dans tout son parcours, à cause de la très faible saillie de ses bords. Le sillon ventral est encore moins aisément saisissable.

Distribution géographioue. - Océan Glacial. Assez rare. 
Spirodinium grave sp. $\mathrm{n}$.

Planche XIV, figures 27 et 28 .

Corps ramassé, ellipsoïde, d'aspect très variable, suivant la position qu'il occupe sous le microscope. Vu de face dans la figure 27 , avec sa ceinture, $s$, et son sillon ventral oblique, il montre son còté dorsal dans la figure 28.

L'ectoplasme strié longitudinalement n'offre pas de ces espèces de trichocystes sériés qui donnent aux deux formes précédentes un aspect si particulier.

Le noyau, $n$, est globuleux et présente une structure fibrillaire comme chez les autres espèces.

Distribution Géographique. - Océan Glacial. Très rare.

\section{Groupe II. -- MICROPHYTES DES NEIGES COLORÉES}

\section{Caractère SPÉcial de L'obJet}

Nous groupons ici des microphytes de très petites dimensions trouvées, pour la plupart, dans un milieu très spécial : les neiges et les glaces de la Mer de Kara.

Ces organismes ne sont donc pas planktoniques par nature, puisqu'ils ont leur habitat naturel soit dans les champs de neige que leur présence colore en jaune, soit à la surface de la glace où leur accumulation s'explique aisément par la fusion de la neige.

Ils ne deviennent planktoniques que par accident, à la suite de la fusion des glaces, qui les fait passer dans le milieu liquide, où leur mélange avec les formes réellement marines imprime aux associations planktoniques de ces parages le cachet spécial sur lequel nous aurons à nous expliquer plus loin, dans un coup d'œil synthétique sur les différentes stations de pêche.

Cette circonstance d'origine est la principale raison de leur réunion dans ce groupe. Nous y joignons quelques-uns d'autre provenance, qui se réclament du même groupement, par leur petitesse et aussi par une certaine ambiguité de nature, qu'ils doivent, en partie, aux circonstances de leur récolte et de leur conservation.

La recherche de leurs affinités taxinomiques exigerait des travaux d'un genre peu conciliable avec notre étude analytique actuelle.

Plusieurs de ces formes méritent toutefois de faire l'objet d'un examen un peu spécial, en vue de pénétrer leur nature intime et de les tirer, si possible, de leur état actuel d'indétermination. C'est ce que nous ferons dans une certaine mesure pour ceux d'entre eux dont nos matériaux fournissent des éléments suffisants d'appréciation. 


\section{Origine des matértaux D'Étude}

Le caractère si spécial des échantillons récoltés pendant l'exploration des champs de glace de la Mer de Kara (3i juillet et 2 aoùt I907) nous oblige à entrer ici dans quelques développements à leur sujet, car leur examen nous met en présence d'organismes que nous saisissons ici dans l'habitat normal auquel nous devrons les rapporter quand le hasard des circonstances les présentera dans d'autres milieux qui ne leur sont pas propres.

Les plus intéressants sous ce rapport sont :

I $^{\circ}$ Le $n^{\circ}$ 9I, qui est le résidu de la fusion d'une dizaine de litres d'une neige jaune couvrant de vastes étendues de glace, par $7 \mathrm{I}^{\circ} 3 \mathrm{I}^{\prime}$ Lat. $\mathrm{N}$ et $56^{\circ} 3 \mathrm{I}^{\prime}$ Long. E.

$2^{\circ}$ Les nos $82,85,89$ et 90 renseignés, soit comme échantillons de neige jaune verdâtre disposée en plaques sur la glace, soit comme échantillons des petits amas organiques de couleur brune trouvés dans d'étroites dépressions creusées sur le pied immergé des glaçons.

Ces derniers numéros, 85 et 90 surtout, sont particulièrement riches en Diatomacées et feront plus spécialement l'objet de notre attention dans la seconde partie de ce Mémoire, en même temps que les échantillons de boues glaciaires recensées sous les numéros 93, II 8 et I23.

Nous accorderons présentement une attention plus spéciale à l'échantillon no $9 \mathrm{I}$, qui nous permettra de prendre contact avec les facteurs organiques intervenant dans la coloration des neiges, sans intention cependant de revenir sur les généralités que nous avons exposées à ce sujet dans une notice préliminaire publiée, en annexe, dans La Revanche de la Banquise (I).

\section{EXAMEN DES Formes PRINCIPALES}

\section{Genre Diamylon Meunier}

Microphyte sphérique à pigment jaune, avec deux grains de fécule : caractères génériques confondus jusqu'ici avec ceux de l'espèce unique du genre :

\section{Diamylon nivale Meunier (2).}

Planche IV, figures 35 à 44 .

Cette petite Algue étant l'élément dominant de l'échantillon $\mathrm{n}^{\circ} 9 \mathrm{I}$, nous reproduisons ici à peu près ce que nous en avons dit dans la notice susdite dont l'objectif

(r) Duc D'Ortéans : La Revanche de la Banquise, Paris, 1909. Appendice V. Alph. Meunier : Notice sur la florule des neiges et des glaces de la Mer de Kara.

(2) Loc. cit., p. 277 et sq. 
était la mise en relief du ròle joué par ce microphyte dans la coloration des neiges.

Cet échantillon est finement pulvérulent et reste longtemps en suspension à la suite d'une agitation violente du bocal où il est conservé dans un mélange de solution aqueuse de formol à 5 p. C. et d'alcool.

Macroscopiquement c'est un dépòt de couleur terne et neutre, vaguement gris jaunàtre, décoloré sans doute par la lumière et par le milieu conservateur car, au dire du naturaliste qui a présidé à sa récolte, le produit était d'abord manifestement jaune.

A l'examen microscopique, on le voit composé exclusivement d'organismes, sans aucun mélange de matières minérales : preuve de la pureté de la neige d'où il provient.

Mais une seule chose frappe d'abord, c'est l'abondance des petits globules ronds, libres, incolores, qui couvrent le porte-objet.

Ces microphytes, qui ne peuvent être qu'une Algue à thalle unicellulaire, forment, en effet, la très grande masse de l'échantillon : 99 p. C. au moins.

La forme de l'Algue est rigoureusement sphérique; ses dimensions, peu variables, oscillent entre $\mathrm{I} 2$ et $\mathrm{I} 5$ microns de diamètre.

La membrane est lisse, homogène, assez mince, bien qu'à double contour évident. On n'y distingue pas de couches concentriques; aucune partie ne traduit une tendance à la gélification. Il n'y a pas de cils locomoteurs ni autres; aucun accident de la surface ne garde la trace d'un flagellum ou de cils qui auraient disparu, ce qui nous porte à croire que l'Algue est immobile pendant sa période de végétation active; car l'époque de sa récolte, première moitié d'aoùt, doit ètre celle de sa grande activité physiologique.

Nous n'ignorons pas toutefois que des micro-organismes incontestablement doués de moyens de locomotion de ce genre pendant la vie peuvent très bien n'en garder la moindre trace après la mort. Ce pourrait être le cas ici; mais nous ne pouvons pas préjuger de ce que nous cachent sans doute nos matériaux fixés.

La rigidité de la membrane est moins réelle qu'apparente car la plasmolyse causée par l'alcool, la glycérine, les solutions salines quelque peu concentrées, etc., amène aisément la déformation $d u$ cytoderme qui suit la contraction $d u$ contenu cellulaire (pl. IV, fig. 37).

Elle n'est point de cellulose ordinaire car on ne réussit pas à la colorer en bleu, dans les conditions où la cellulose traduit si bien cette réaction caractéristique : nous voulons dire le traitement par le chloroiodure de zinc ou par l'iode et l'acide sulfurique concentré, alors que d'autres objets, inclus dans les mèmes préparations, témoignent très bien de l'action spécifique de ces réactifs.

Cette résistance ne tient pas non plus à une sorte de cutinisation, car rien de pareil ne se révèle ni par l'aspect, ni par l'action des réactifs appropriés : iode vert de méthyle, rouge de fuchsine, etc. 
Après la macération de Schultze, traitement violent que l'Algue supporte mal, le cytoplasme est enlevé et la membrane, en partie dissoute et flasque, ne se colore qu'en jaune vif sous l'action de l'iode.

Traitée par l'hypochlorite de potasse, eau de Javel concentrée, la membrane, vidée également mais mieux conservée, garde néanmoins son indifférence vis-à-vis des réactifs spécifiques de la cellulose, tandis que, dans les mèmes conditions, les membranes végétales les plus incrustées laissent reconnaitre leur fond cellulosique.

Le traitement par les acides forts la dispose à l'extension, que l'on peut ensuite réaliser par l'action du chloral concentré de manière à doubler, si l'on veut, le diamètre des cellules, sans que le cytoderme traduise une structure appréciable (fig. 43 et 44).

Le contenu cellulaire paraît très grossièrement granuleux dans le milieu conservateur déjà mentionné : alcool formolisé; résultat de l'action déshydratante de l'alcool (fig. 36). Par contre, la glycérine et la potasse diluées en rendent l'aspect presque homogène (fig. 38). Est-il naturellement coloré? La coloration jaune originale de l'échantillon permet de supposer que le cytoplasme présente un pigment soluble de cette teinte; mais, comme nous l'avons dit, ce pigment a dù être détruit dans un milieu, eau et alcool, où il devait rapidement se dissoudre et se dénaturer. De fait, l'Algue est actuellement incolore tant à l'intérieur qu'à l'extérieur.

La réfringence sensiblement égale des granules du protoplasme leur ferait attribuer à première vue une certaine homogénéité de composition. Mais l'apport de la moindre trace d'iode y révèle immédiatement la présence de deux gros grains de fécule, trait essentiel du signalement de ces petits organismes (fig. 40).

Traités ainsi par l'iode, préférablement en solution alcoolique, et éclaircis ensuite par une solution concentrée de chloral où ils restent enrobés, ces microphytes présentent un aspect très suggestif. C'est à ce traitement aussi avantageux qu'expéditif que nous avons eu recours pour l'étude de bon nombre d'échantillons car, outre qu'il présente très bien les objets disparates qui encombrent les préparations, en les éclaircissant dans leurs moindres détails, il révèle immédiatement chez chacun d'eux des caractères très propres à les différencier eux-mèmes les uns des autres.

Notre petite Algue (fig. 35), grossic à plus de 1,500 diamètres, montre alors un cytoplasme réduit à une mince couche périphérique et à de rares cordons et lamelles qui englobent des enclaves de deux sortes : huile et fécule.

Ces éléments du cytoplasme deviennent plus évidents à la suite d'un traitement par l'éther ou le chloroforme destiné à éliminer les matières grasses dont la présence semble être une des conditions nécessaires d'existence, dans les milieux à très basse température, des organismes tant inférieurs que supérieurs.

Ces matières sont facilement identifiées sur place par l'action soit de l'anchusine en solution alcoolique, soit $\mathrm{du}$ bleu de méthylène en solution aqueuse, surtout en présence de l'iode. Elles occupent plusieurs vacuoles ( $v$, fig. 35) de dimensions inégales et baignent en quelque sorte le protoplasme. 
Les cellules déshuilées au préalable et traitées par l'iode montrent, d'une façon plus évidente encore si possible, au milieu du cytoplasme vacuoleux vivement coloré.en jaune d'or, les enclaves féculentes qui, dans le même milieu, prennent une belle coloration bleu violet. Ce sont invariablement deux gros grains réniformes d'égale grandeur, rapprochés par leur partie concave et situés symétriquement l'un à l'autre, en avant ou en arrière, comme on voudra, d'un petit corps brillant qui se distingue, dans presque toutes les positions de la cellule, par sa haute réfringence (n, fig. 35). Celui-ci semble être le noyau de la cellule, mais ses faibles dimensions, deux microns au plus, en rendent indistincts les détails de structure.

Les deux grains de fécule sont assez gros pour occuper souvent la moitié de la capacité cellulaire. Ils possèdent un hile très clair avec un corpuscule central très réfringent qui semble être un pyrénoïde.

Les pyrénoïdes des deux grains accouplés sont eux-mêmes rattachés l'un à l'autre par un cordon de protoplasme qui croise à angle droit la lamelle de même nature qui sépare les deux grains. De là l'aspect présenté, par les cellules convenablement orientées, d'une petite croix dont trois branches sont terminées par un point brillant de matière azotée plus dense : en arrière, le noyau; en avant, à gauche et à droite, les deux pyrénoïdes.

Les grains colorés par l'iode restent néanmoins incolores au centre, aux environs immédiats du pyrénoïde, comme si la matière amylacée y faisait défaut. Ils paraissent ainsi perforés, aspect que traduisent encore mieux les grains expulsés mécaniquement des cellules et retrouvés épars dans le milieu d'enrobage (fig. 4I).

Les acides forts, l'acide chlorhydrique en particulier, les désagrègent partiellement et en expulsent une matière solubilisée qui se dissémine dans le cytoplasme où l'iode la précipite en petits granules colorés en bleu, tandis que la trame squelettique du grain restée en place ne se colore plus qu'en rouge cuivre (fig. 42). Cette dissociation possible en granulose et en amylocellulose les rapproche singulièrement des grains de fécule des végétaux supéricurs.

Les caractères si nets, si constants, de ce micro-organisme le différencient évidemment de Sphaerella nivalis Sommerfeld, autre microphyte auquel on attribue aussi un rôle dans la coloration des neiges.

Nous avons pu étudier également celui-ci sur échantillons de provenances diverses.

La comparaison des deux formes ne permet aucun rapprochement.

C'est pourquoi, laissant toute recherche d'analogie qui n'aboutirait vraisemblablement qu'à obnubiler davantage des notions déjà trop confuses, nous croyons préférable de marquer l'objet si précis que nous avons sous les yeux d'un nom qui, rappelant ses caractères manifestes, soit susceptible de lui créer une sorte d'état civil qui le tire d'une promiscuité avec d'autres formes mal définies dont beaucoup d'Algues inférieures pâtissent encore actuellement. 
Nous l'appelons Diamylon nivale, eu égard à son habitat propre et à la présence dans les cellules de l'Algue, pendant celle de ses phases évolutives qui semble la principale, de deux gros grains de fécule géminés, qui lui créent une physionomie spéciale et permettent de la reconnaitre partout où le hasard des circonstances peut la mener, par exemple dans le filet à Plankton.

En effet, presque tous les échantillons microplanktoniques de la Mer de Kara nous en montrent des individus plus ou moins nombreux, que la fonte des neiges et des glaces a fait passer dans l'eau de mer de cette région.

On constate mème qu'ils y sont fort recherchés par les petits organismes animaux : Tintinnides, Rotateurs, Crustacés, etc., qui trouvent sans doute un aliment de choix dans cette manne que la fusion des neiges leur ménage pendant la saison estivale car on en aperçoit fréquemment dans leur organe digestif et les déjections des Copépodes, petits crottins glaireux bien reconnaissables et toujours très abondants, en sont souvent farcies; la membrane de l'Algue tout au moins étant restée réfractaire à la digestion.

C'est Diamylon nivale qui tient de très loin le record du nombre dans l'échantillon $n^{\circ}$ 9I et son rôle prépondérant dans la coloration de cette neige jaune ne saurait être mis en doute, malgré l'existence dans le même milieu de nombreux organismes évidemment non apparentés à celui-ci, dont l'endochrome également jaune doit constituer néanmoins un appoint à cette coloration du névé. Mais le petit nombre relalif des représentants de chacune des autres espèces ne permet pas de leur attribuer un résultat que leur rare dissémination dans la masse serait impuissante à expliquer.

Comment se propage Diamylon nivale?

Sous la forme décrite tout à l'heure on ne le voit jamais se diviser : aucune tendance à la division ni interne, ni externe ne s'y traduit.

A moins d'admettre que cet organisme ne soit qu'une microspore de macrophyte apportée en immense quantité là où on la trouve, hypothèse que ses caractères ne semblent pas permettre, on doit penser que sa multiplication est due à un processus d'essaimage dont les conditions actuelles du milieu ne favorisent pas l'exercice. De fait, à s'en tenir aux renseignements puisés dans l'examen de ce seul échantillon la question devrait rester sans réponse. L'organisme s'y présente toujours sous la même livrée, au mème stade d'évolution à peu près.

Seules de faibles différences de taille et de résistance aux réactifs témoignent d'une certaine inégalité d'âge. Exemple : figure 39, membrane plus mince, grains de fécule moins gros.

Le reste de l'échantillon, moins d'un centième certainement, est formé d'organismes très variés qui, pour la plupart, lui sont manifestement étrangers.

Les voici, présentés par groupes, dans l'ordre de leur importance numérique:

Groupe A. - Diatomacées, espèces très variées, que nous décrirons avec leurs congénères d'autre origine dans la seconde partie de ce travail. 
Groupe B. - Péridiniacécs vraies que nous avons recensées plus haut, à leur place, avec les espèces d'autre provenance et, en outre, des organismes paraissant avoir avec les Péridiniacées quelque affinité. De ceux-ci nous dirons un mot plus loin.

Groupe C. - Autres microphytes; les uns connus : Silicoflagellates, Desmidiées Confervacées; les autres inconnus dont plusieurs trouveront leur place dans le présent chapitre.

Groupe D. - Organismes animaux : a) Protozoaires : Tintimides et Radiolaires, qui seront signalés plus loin; b) Métazoaires: Acariens, dont nous n'avons pas à nous occuper dans ce Mémoire; c) oufs et organes indéterminés.

Groupe E. - Apports éoliens : pollen de Gymnospermes et d'Angiospermes ; spores rares de Cryptogames vasculaires; spores et thalle de Champignons; fragments macérés de végétaux supérieurs, fibres, cellules scléreuses, cellules subéreuses, de rares grains de fécule, etc.

Ces derniers objets n'ayant rien de commun avec notre étude actuelle, il n'en sera plus question.

Après ce coup d'œil d'ensemble sur les organismes de la neige jaune, à l'occasion de Diamylon nivale, qui est l'élément biologique le plus caractéristique de ce milieu, nous essaierons de recenser ceux d'entre eux qui n'ont pas leur place marquée dans l'une des autres subdivisions prévues de ce Travail.

\section{Genre Echinum g. n.}

Organismes caractérisés par leur membrane cellulosique épaisse et généralement hérissée de piquants, leur forme bicéphalée, réniforme ou sphérique, leur noyau volumineux, leur protoplasme dense et riche en réserves grasses et féculentes.

Echinum majus sp. n.

Planche IV, figures 22 à 26 .

Forme allongée, rétrécie au milieu, renflée aux deux extrémités. Celles-ci sont chargées de fortes épines, expansions de la membrane et de mème nature qu'elle.

Tantòt obtuses (fig. 22 et 23), tantôt aiguës (fig. 24), ces expansions membraneuses sont le plus souvent droites, exceptionnellement recourbées (fig. 26), toujours absentes sur la zone déprimée du milieu du corps.

La figure 23 en reproduit un spécimen en coupe optique, montrant le noyau central à structure filamenteuse, le protoplasme dense et les amas de très petits grains de fécule vers chacun des deux pôles de la cellule.

En spécimens épars, assez rares. 
Echinum nudum sp. n.

Planche IV, figures 29 et 30 .

Forme longuement elliptique, parfois légèrement étranglée au milieu ou même un peu réniforme. Elle est complètement exempte de piquants.

Malgré l'absence de ces appendices nous ne la détachons pas des autres parce que, par les caractères plus intimes du contenu cellulaire, de la structure du noyau, de la nature de la membrane, etc., elle garde avec elles la plus grande analogie:

Figure 29, spécimen réniforme vu de l'extérieur, $(a)$; vu en coupe optique, (b).

Figure 3o, spécimen bicéphalé reproduit de mème.

Individus toujours épars.

\section{Echinum minus sp. n.}

Planche IV, figures 27 et 28 .

Celui-ci est de dimensions plus petites que les précédents et se rapproche de la forme sphérique sans toutefois se montrer jamais rigoureusement isodiamétral. On y distingue un axe polaire plus long et une zone médiane légèrement déprimée. Celle-ci est dépourvue des piquants qui se pressent sur les deux moitiés, aussi nombreux, mais moins puissants que dans Echinum majus.

Même structure et même aspect du contenu cellulaire, vu en coupe optique (fig. 28). On le voit habituellement formant de petites colonies (fig. 27) de plusieurs individus, réunis bien plutôt par l'extrémité adhésive de leurs piquants que par une substance mucilagineuse étrangère. Plus rarement on les rencontre isolés, mais c'est alors sans doute par rupture de leurs adhérences naturelles.

\section{Echinum sphericum sp. n.}

Planche IV, figures $31,32,33$.

Nous assimilons aux précédentes, à cause des mêmes analogies de structure intime, cette forme plus rare, rigoureusement sphérique, toujours libre et régulièrement épineuse sur toute la surface. Le calibre en est variable. Les épines, assez longues et généralement pointues (fig. 32 et 33), sont parfois réduites à de petites protubérances arrondies (fig. 3I).

\section{Echinum micraster sp. n.}

Planche IV, figure 34 .

C'est avec plus d'hésitation que nous rattachons au mème groupe une forme très petite, 4 à 5 microns seulement, absolument sphérique aussi et très finement épineuse sur tout son pourtour. 
Les spécimens en sont rares et isolés dans la neige jaune, mais nombreux dans les eaux marines sous-jacentes et aussi dans certains échantillons de la Mer de Barents où on les rencontre souvent agrégés, non point directement par leurs piquants comme Echinum mimus, mais par l'intermédiaire d'une membrane mince, amorphe, hyaline, de nature gélatineuse ou mucilagineuse sans doute, sur laquelle leur profil étoilé se détache vivement.

Vivraient-ils en symbiose avec certains des organismes au milieu desquels le hasard du coup de filet les réunit? On serait porté à le penser, mais il est difficile de résoudre la question à cause de l'enchevêtrement inextricable d'autres organismes aussi infimes qu'eux qui encombrent les préparations.

Ils semblent constituer un aliment très recherché par les espèces plasmophages, car on les y trouve souvent à l'état d'inclusion ou noyés dans les matières excrémentielles.

Remarque. - L'absence actuelle de pigment dans les espèces signalées ci-dessus et l'absence aussi de phénomènes de multiplication surpris en cours d'exécution doivent dicter la plus grande circonspection au sujet de leur nature. A ne tenir compte que de leur manière de réagir vis-à-vis de l'iode dans le milieu d'enrobage dont nous avons parlé à plusieurs reprises, elles ne sont pas sans ressemblance avec les formes les plus frustes de Péridiniens dont elles n'ont pas toutefois ni la ceinture, ni la structure compliquée de la membrane capsulaire. D’autre part, la singularité du milieu prépare l'esprit à la rencontre de formes qui n'auraient pas leurs similaires ailleurs.

Faut-il les considérer comme des spores, que nous ne saurions d'ailleurs rattacher à aucune espèce pas plus nivicole que marine? C'est aussi possible.

Nous n'ignorons pas, enfin, l'analogie de ces formes avec celles désignées communément sous les noms de Trochiscia et de Xanthidium, si l'on s'en tient au seul aspect extérieur. Celles-ci sont décrites comme marines et leur pigment vert, dit-on, les fait ranger parmi les Chlorophycées, dans des familles diverses suivant le point de vue des auteurs. Mais que sait-on positivement sur leur nature réelle? L'histoire de ces organismes infimes récemment entrés dans le domaine de la science, par des voies diverses, est encore si obscure qu'on pourrait émettre des doutes sur le caractère végétal de plusieurs des formes mentionnées sous cette étiquette, sans autre raison qu'une simple ressemblance extérieure.

\section{Genre Folliculus g. n.}

C'est aussi et uniquement l'aspect du noyau, si semblable à celui des Péridiniens, volumineux, à structure filamenteuse, colorable en jaune rouge par l'iode, qui nous fait placer ici un organisme doué, à l'une des phases de son existence, d'un long et puissant flagellum. 
Folliculus flagellifer sp. n.

Planche IV, figures 17 à 21 .

Ses dimensions atteignent parfois 40 microns, sa forme est celle d'une sphère un peu allongée. Sa membrane lisse, épaisse, rigide, un peu cutinisée extérieurement, présente de ce chef une teinte variant du jaune au brun. Le noyau volumineux occupe un des pôles de la cellule : l'inférieur. Le cytoplasme plus ou moins dense et granuleux présente presque toujours des réserves amylacées en petits grains épars.

A l'autre pôle se trouve fixé le flagellum (fig. I7, coupe optique, et fig. Ig, vue extérieure), dont la longueur atteint cinq à huit fois le grand diamètre de la cellule.

Chez la plupart des individus observés le flagellum a disparu et est remplacé par un petit bouton formé de plusieurs - généralement quatre - granules confluents et d'apparence gommeuse, au-dessus d'une légère irrégularité dans la structure du cytoderme (fig. I8, coupe longitudinale optique, et fig. 2I, vue d'ensemble par transparence).

Sous cette forme il est assez fréquent dans la neige jaune, qui est son habitat normal, pensons-nous, car nous ne l'avons guère rencontré ailleurs.

Très rarement on le voit avec sa membrane gélifiée et distendue (fig. 20).

A certains égards, cet organisme rappelle, par la forme, non par le contenu cellulaire qui est plutòt celui d'un Péridinien, les Palmellacées voisines des Trachelomonas; mais nous nous défions des solutions prématurées dans un sujet où la lumière est loin d'être faite.

Nous formulons les mêmes réserves relativement aux Pterospcrma, auxquels il y aurait lieu de rapporter quelques formes trouvées dans le même milieu de la neige jaune, si la connaissance de ces organismes était mieux assise et si l'on était d'accord aussi bien sur leur nature que sur la place à leur assigner.

Nous en ferons mention plus loin, dans le groupe prévu des organismes énigmatiques auquel nous les rattacherons.

Avant de passer à l'examen de microphytes de la neige jaune mieux définis morphologiquement, il nous parait opportun de signaler un micro-organisme aussi difficile à étudier et à décrire qu'abondant dans les échantillons puisés aux plaques de neige d'un jaune verdâtre qui recouvrent localement les glaces, dans les parages susdits de la Mer de Kara (pl. IV, fig. 45 à 5o).

On le voit sous la forme de petits globules ronds, généralement agglomérés, dépourvus de membrane distincte, incolores actuellement, parce que décolorés sans doute.

Ils sont de dimensions variables, depuis trois ou quatre microns pour les plus petits (fig. 49), jusqu'à une vingtaine de microns pour les plus grands. 
Dans le chloral iodé, ils se colorent uniquement en jaune, ne renfermant aucune trace de fécule.

Les plus petits ont un protoplasme granuleux, avec un corpuscule central qui doit être le noyau. Les moyens présentent une grande vacuole sphérique un peu excentrique et gorgée d'huile ( $h$, fig. 5o). D'autres encore ont un protoplasme spongieux (fig. 46), dans lequel on peut distinguer un petit noyau et souvent aussi deux pyrénoïdes, comme dans Diamylon, mais sans fécule.

Ces organismes, réunis pêle-mêle sous le microscope et souvent agglutinés ensemble, semblent avoir été doués, à une des phases de leur développement, de deux cils jumeaux que l'on peut exceptionnellement reconnaître chez ceux qui sont dégagés (fig. 45).

Ajoutons qu'ils forment souvent des groupements qui suggèrent l'idée d'une genèse commune, par division simultanée d'une matricule (fig. 47 et $4^{8}$ ) en de nombreux individus. Quel aspect auraient ceux-ci si l'alcool ne les avait pas modifiés? Se traduiraient-ils comme des zoospores? Tout le fait croire, mais il resterait encore à établir leur destinée et leurs rapports possibles avec d'autres formes, peut-être avec Diamylon nivale, ce qui exigerait la disposition de matériaux frais.

Parmi les organismes trouvés encore dans le même milieu, il en est un que l'on reconnaît immédiatement pour une Desmidiée, la seule que renferment nos échantillons.

C'est un Cosmarimm (pl. IV, fig. 8o), toujours enkysté, à protoplasme dense, de couleur brune comme la membrane et recouvert d'une enveloppe supplémentaire de couleur claire et de "nature gommeuse.

Le milieu n'a pas l'air de lui être favorable. Il est très rare.

Un autre est évidemment une Confervacée : un Cladophora sans doute, de petite forme, à thalle multicellulaire articulé. Extrêmement rare. Nous ne l'avons pas figuré parce que non unicellulaire.

D'autres encore, assez variés, semblent appartenir aux Protococcacées, bien que la disparition de l'endochrome rende leurs affinités difficiles à saisir.

\section{Formes polyédriques}

Les unes, eu égard à leur forme polyédrique, semblent se réclamer du genre Polyedrium Naegli.

$A$. C'est d'abord un organisme assez répandu dans la neige jaune verdâtre, qui affecte la forme d'un fuseau court et trigone (pl. IV, fig. 5I et 52) dont les trois secteurs sont délimités par autant de côtes méridiennes formant de légères saillies, d'un pôle à l'autre, dans le cytoderme.

De dimensions variables, ses représentants passent de Io à 40 microns environ en gardant la même forme. Le cytoplasme y est rare et vacuoleux. Le noyau est petit 
et de structure indistincte. L'organisme est toujours exempt de fécule. Appelons-le Polyedrium fusiedricum, provisoirement.

Rarement nous l'avons vu (fig. 53) rempli de cellules-filles évidemment formées par division simultanée. Il est probable, conséquemment, qu'on puisse le rattacher à une forme plus petite (fig. 54 et 55), d'allure analogue, légèrement elliptique, aiguë à une extrémité, un peu échancrée à l'autre, où elle aurait pu présenter des cils locomoteurs actuellement invisiblẹs. Dans l'hypothèse, ce seraient des zoospores issues de la forme adulte (fig. 53) et destinées à la reconstituer par accroissement, après la perte des cils (fig. 56).

$B$. Une autre forme, tétraédrique celle-ci (fig. 57 et 58), a un certain air de grain de pollen. La membrane, relevée de six nervures qui séparent les quatre faces du polyèdre sphérique, est exempte de cutinisation; le protoplasme est vacuoleux et dépourvu d'enclaves; le noyau est simple et central. On pourrait en faire Polyedrium tetracdricum.

C. Plus nombreux sont les spécimens de grandeur différente d'un organisme pentaédrique, ayant la forme d'un prisme trigone. Des cinq faces, deux sont triangulaires, les bases du prisme; trois sont rectangulaires ou carrées, les côtés du prisme (fig. 59 et 60 ). De là l'aspect tantôt trigone, tantôt tétragone, tantòt polygone, en coupe optique, de l'organisme, suivant sa position sous le microscope. Le contenu cellulaire - cytoplasme vacuoleux et noyau arrondi - ne présente aucun caractère saillant. On conviendra que le nom de Polyedrium pentaedricum lui revient.

\section{Formes SPHÉRIQUES NUES}

Les microphytes sphériques ayant naturellement la même forme simple fournissent peu de caractères morphologiques distinctifs propres à fixer leur diagnose et, éventuellement aussi, leur comparaison avec les formes bien connues par leurs caractères physiologiques.

L'observateur les distingue aisément, parce que les particularités les plus subtiles qu'il y découvre suffisent à guider son discernement; mais comment traduire à d'autres ces impressions personnelles et fugitives (I)?

Malgré ces difficultés, nous essaierons de donner une idée de ceux qui nous ont paru moins réfractaires à toute diagnose.

A. Planche IV, figure 6I. Cellules sphériques ayant de I5 à 40 microns de diamètre, à cytoderme épais, non cutinisé et insensible à l'action du chloroiodure de zinc, à cytoplasme dense, sans fécule, hébergeant un noyau central qui ne se colore qu'en jaune pâle sous l'action de l'iode. Nous croyons y reconnaitre, malgré la

(I) On sait que les traités spéciaux sur la matière sont pleins d'équivoques. 
destruction du pigment, des spores quiescentes de Hamatococcus lacustris. Les spécimens de diverses grandeurs sont figurés en coupe optique.

B. Planche IV, figure 64. Microphytes d'une trentaine de microns, à membrane peu épaisse, claire, non cutinisée, mais néanmoins rigide, à cytoplasme clair plus ramassé contre la paroi de l'une des deux moitiés de la sphère et riche en petits grains de fécule uniformes qui manquent dans l'autre moitié où le protoplasme plus rare, plus aqueux, était sans doute exempt d'endochrome pendant la vie.

Les grains de fécule, $f$, sont distribués suivant quatre secteurs qui confluent vers la portion de la cellule où le protoplasme est plus dense. Figure 64 , $a$, vue latérale extérieure; $b$, vue en coupe optique perpendiculaire à l'axe de symétrie de la structure cytoplasmatique. Le noyau est petit, central, peu colorable par l'iode.

C. Planche IV, figure 65. Microphytes à membrane peu épaisse, dont la couleur brun foncé rend peu distinct le cytoplasme, au sein duquel on ne peut reconnaitre que des granulations dont nous n'avons pas recherché la nature.

$D$. Planche IV, figure 62. Ici la membrane est cutinisée à l'extérieur et finement mamelonnée. Vue de l'extérieur, en $a$; en coupe optique, en $b$. Le protoplasme est vacuoleux, le noyau central, peu distinct.

E. Planche IV, figure 63. Cellule un peu ovoïde, à membrane recouverte d'une mince cuticule chagrinée, $c$, et plus faible vers l'une des extrémités du grand axe (fig. 63, $a$, vue en coupe longitudinale optique).

Le noyau, $n$, occupe cette partie de la cellule que nous dirons antérieure.

Du côté opposé ou postérieur se voient deux pyrénoïdes, $p$, entourés chacun d'une sphérule formée d'un grand nombre de tout petits grains de fécule, $f$, que l'iode bleuit intensément. En $b$, l'organisme est figuré en coupe optique transversale passant au niveau des deux pyrénoïdes figurés de même.

\section{Formes SPHÉRIQUes hérisséEs DE SOIES}

La présence de soies hérissant toute la surface du corps fait penser tout naturellement aux formes décrites sous les noms de Trochiscia, d'Acanthococcus, de Golenkinia, etc. dont les affinités véritables semblent loin d'être fixées pour toutes.

En l'absence de pigment et de caractères physiologiques, il serait hasardeux de vouloir préciser la nature réelle des formes suivantes rencontrées surtout dans les neiges colorées.

A. Planche IV, figures 66 et 67 . Cellules isolées, sphériques, hérissées de soies très longues mais assez distancées. Figure 66, vue extérieure; figure 67, coupe optique, qui montre le protoplasme chargé d'enclaves de petites dimensions.

$B$. Planche $I V$, figures 68 et 69 . I es soies qui hérissent la membrane sont plus courtes, mais plus nombreuses. Leur distribution sur le corps de la cellule semble 
en rapport avec les mailles du réticulum qui décore extérieurement la membrane. Fig. 68, $a$, vue extérieure d'un petit spécimen reproduit en coupe optique en $b$. Fig. 69, spécimen plus gros, vue extérieure.

C. Planche IV, figure 70. Les soies excessivement serrées sur tout le corps sphérique de l'organisme ne permettent pas de juger de la nature du contenu cellulaire dans l'état présent. Nous doutons, du reste, de son caractère végétal.

\section{Formes VARIÉEs a membrane structurée}

A. Planche IV, figure 73. Coupe optique d'un petit organisme présentant deux membranes concentriques, maintenues à distance l'une de l'autre par des trabécules radiaux nombreux. Dans le cytoplasme homogène apparaissent deux petits corps symétriques qui semblent être des noyaux.

B. Planche IV, figure 74. Micro-organisme dont nous n'avons rencontré que la coque vide qui jaunit sous l'action de l'iode. Cette coque, perforée de petits trous ronds et équidistants, présente antérieurement une sorte de courte tubulure ouverte. Vue extérieure.

C. Planche IV, figure 75. Cellules sphériques dont la membrane assez épaisse laisse voir un large réticulum à mailles pentagonales régulières. Les spécimens observés étaient vides.

\section{Formes algolog1QUes Frustes trouvées en M1L1EU Marin}

A. Planche IV, figure 76. Cellules très petites, sphériques, ovö̈des ou ellipsoïdes, entourées d'une membrane très mince et farcies de minuscules grains de fécule qui réagissent très bien sous l'action de l'iode. Ces microphytes sont généralement groupés et tenus à distance par une substance amorphe qui ne se laisse pas percevoir.

B. Planche IV, figures 77 et 78. Forme de Glaocystis sans doute, assez fréquemment rencontrée, non dans les neiges colorées prises sur place, mais bien dans certains échantillons planktoniques provenant des côtes occidentales de la Nouvelle-Zemble, dans lesquels s'observent aussi d'autres formes qui semblent résulter de la fonte des glaces dans ces parages.

C. Planche IV, figure 79. Microphytes de grosseur inégale, à cytoplasme entouré d'une très épaisse membrane amorphe et parfaitement hyaline. Les individus sont réunis en paquets informes. Ce doit ètre une sorte de Chlorococcum Fries.

D. Planche V, figure I8. Sorte d'Oocystis, sans doute, auquel la forme sphérique des matricules vaudrait le nom de sphacrica. Dans les cas observés, les cellules-mères renfermaient toujours quatre cellules-filles un peu ovoïdes ou ellipsoïdes. 
E. Planche $V$, figure I9. Organismes de très petites dimensions réunis par une gangue amorphe et hyaline, généralement géminés par le fait, sans doute, d'un phénomène de fissiparité qui préside à leur multiplication. Les membranes en sont très réfringentes de même qu'un corpuscule central, seul élément percevable du cytoplasme.

F. Planche XXIII, figure I7. Petites cellules sphériques agrégées latéralement dans un seul plan, en un thalle mince et discontinu, par des soudures tangentielles, de manière à ménager entre elles des espaces libres d'étendue variable et de formes diverses.

Ces délicates membranules ajourées, sans limites définies, devaient flotter dans l'eau de la Mer de Barents en spécimens rares, si nous en jugeons par leur peu de fréquence dans les produits planktoniques de cette provenance.

Leur réfringence remarquable fixe forcément l'attention de l'observateur.

\section{Groupe III. - CRYPTOMONADACÉES}

En dehors de ceux des micro-organismes des neiges et des glaces que l'on pourrait rattacher aux Cryptomonadacées et qui sont peut-être plus nombreux que la disparition de l'endochrome caractéristique ne permet de l'affirmer, il en est peu d'autres dont nous ayons à relever la présence dans les pêches planktoniques de la Belgica.

\section{Genre Dinobryon Ehrenberg}

Grâce à leur capsule hyaline, en forme d'étui, et à leur association en colonies rameuses, les Dinobryon se laissent aisément reconnaître au milieu des récoltes planktoniques dont ils forment parfois l'élément dominant.

Ce sont des Chromulinées, à corps mobile muni antérieurement de deux cils inégaux : l'un, plus grand, qui s'observe encore souvent sur les individus fixés; l'autre, plus petit, généralement indistinct après fixation.

Dinobryon pellucidum Levander.

Planche $\mathrm{V}$, figures $I$ et 2 .

Synonymie et littérature. - Dinodendron balticum Schütt. Cfr. Nordisches Plankton, XXI, Flagellatae, etc., page 4 .

Cette espèce, la plus commune dans nos échantillons, est caractérisée par ses capsules étroites, longuement cylindriques dans leur partie médiane, légèrement évasées 
au sommet, rétrécies en pointe oblique à la base et disposées en colonies volumineuses bi-, tri-, quadrifurquées, dont la figure I ne reproduit qu'un fragment. Cette figure nous semble se rapporter à une partie jeune d'une colonie.

Dans les parties vieilles des colonies, les capsules sont plus allongées, plus sinueuses (fig. 2).

Dans les deux cas, la grande transparence des colonies et l'étroitesse des organismes qui en rend l'observation difficile dans les milieux quelque peu éclaircissants et même dans l'eau, légitiment bien mieux le nom de pellucidum Levander, que celui de balticum Schütt, qui lui ferait supposer une aire géographique peu étendue.

Nos échantillons. nous la présentent sur tout le parcours de l'itinéraire de l'Expédition arctique de I907, mais en particulière abondance dans le no 2I4, au nord de la Mer de Barents.

\section{Dinobryon Belgicae sp. n.}

Planche $\mathrm{V}$, figure 3.

La forme figurée ici nous paraît manifestement distincte de la précédente, sans qu'on puisse davantage la rapporter à l'espèce Sertularia Ehrenberg qui est d'eau douce et qui abonde dans la plupart de nos eaux où nous l'avons mille fois observée.

Dinobryon Belgicae a ses capsules plus courtes, plus larges, plus trapues que l'espèce pellucidum, bien qu'elles soient cylindriques aussi et non renflées au milieu, comme dans l'espèce Sertularia.

Ses colonies sont aussi plus denses, moins hyalines et arrètent davantage l'oil du micrographe, bien qu'elle soit plus rare que la précédente et plus spécialement propre à la Mer de Kara, vers l'entrée de la Mer Mourmane.

\section{Genre Corbicula g. n.}

C'est ici, à còté du genre Dinobryon, que nous parait devoir trouver sa place naturelle un petit organisme que nous n'avons vu signaler nulle part encore et que nous figurons sous le nom de Corbicula sacialis qui rappelle à la fois la forme en corbeille des individus et le groupement de ceux-ci en colonies.

\section{Corbicula socialis sp. n.}

Planche $\mathrm{V}$, figure 4 .

Les individus ont, comme les Dinobryon, un corps mobile pourvu en avant d'un ou peut-être de deux cils. Ils sont logés dans le fond d'une capsule brièvement conique, 
pointue à la base, largement ouverte au sommet et portée sur un pédicelle filiforme très ténu, de longueur variable, atteignant ou dépassant le plus souvent celle de la capsule. Ces pédicelles, réunis entre eux par leur extrémité, groupent les individus en colonies plus ou moins étalées dont il est difficile de saisir la forme naturelle, à cause de l'écrasement qu'elles subissent dans les préparations.

Les éléments de la figure $4, a, b, c$, n'en reproduisent que de petits fragments destinés à montrer les multiples aspects des capsules, suivant la position qu'elles occupent : les unes habitées, les autres vides.

Celles-ci, $a$ et $b$, se prêtent mieux à l'examen de la capsule, qui est formée de deux parties séparées par un étranglement circulaire. La partie inférieure, étroitement conique, est seule occupée par l'organisme contracté par la mort et par l'action des réactifs, en c. La partie supérieure est largement dilatée en forme de corbeille hémisphérique. Les parois en sont formées par des trabécules très minces, uniformes, au nombre de huit à dix, qui partent du fond pour aboutir en divergeant, à égale distance l'un de l'autre, au bord libre de la capsule, qui est circulaire et lisse.

Vraisemblablement ces trabécules sous-tendent une membranule imperceptible.

Dans les milieux liquides ordinaires, ces capsules sont tellement hyalines qu'on les devine plutôt qu'on ne les voit, à la condition même de les avoir préalablement observées dans des conditions d'examen plus favorables, à sec, par exemple.

Nous ne leur avons pas trouvé de colorant spécifique, circonstance qui, jointe à leur fragilité extrême, nous fait croire qu'elles sont de nature gommeuse.

L'iode, le vert de méthyle, la safranine, la fuchsine sont inactifs; le bleu de méthylène en colore légèrement les trabécules; mais cette coloration ne se traduit sensiblement qu'à sec, c'est-à-dire dans des circonstances où cette coloration n'est plus utile.

Dans nos matériaux d'étude, cette forme est très rare et ne se rencontre qu'exceptionnellement dans des produits de pêche de la Mer de Kara, à l'état de colonies bien groupées, mais très clairsemées.

La parfaite hyalinité de la capsule et la petitesse du micro-organisme sont cause sans doute de l'incognito qu'il a pu garder bien qu'il se soit trouvé, fréquemment peut-être, sous les yeux des observateurs.

Nous en avons la preuve dans l'analyse d'un échantillon planktonique provenant de la Mer du Grönland, dans laquelle cet organisme, exceptionnellement abondant et mélangé à des granulations inorganiques très petites, a été pris pour Phaocystis Poucheti Lagerheim et noté très commun comme tel, alors que celui-ci fait complètement défaut. Cette erreur ne s'explique que dans une certaine mesure car, s'il est vrai que les petits thalles protoplasmatiques de Corbicula, seuls visibles dans les milieux liquides, n'apparaissent que comme des corpuscules plus ou moins régulièrement distancés dans une gangue hyaline, leur aspect homogène ne peut jamais donner le change avec celui des Phaocystis, dont le chromatophore bi- ou quadrilobé est un caractère spécifique suffisant dans les matériaux fixés. 
On comprendra aisément qu'à défaut de matériaux frais et vivants nous n'ayons pu nous fixer sûrement sur la nature du pigment de cet organisme, ce qui nous force à faire des réserves sur la légitimité de la place que nous ne lui attribuons que d'après des analogies morphologiques.

\section{Genre Phaocystis Lagerheim}

Les matériaux fixés se prêtent mal à l'étude des Phaocystis que leur délicatesse rend trop sensibles aux effets contractifs et décolorants des milieux conservateurs à base d'alcool ou même de formol.

C'est une des raisons pour lesquelles nous ne saurions avec certitude rapporter la ou les formes dont nos échantillons nous ont assez fréquemment fourni des spécimens, à l'une des deux espèces : Poucheti Lagerheim et globosa Scherffel, connues dans les mers du Nord.

Une autre raison, c'est qu'il ne nous a pas été donné de voir des colonies entières à formes définies, mais seulement des individus épars ou agrégés dans une sorte de gangue hyaline, lamellaire, indéterminée, au milieu d'un enchevêtrement inextricable d'organismes très variés, dont beaucoup de Chatocérées, qui sont, par le fait, indissolublement soudées dans les produits de pêche au filet fin.

\section{Phceocystis Poucheti Lagerheim.}

Planche V, figure 5.

Synonymie et littérature. - Tetraspora Poucheti Hariot, etc. Cfr. Nordisches Plankton, XXI Flagellatae, page I.

Nous croyons que c'est plutôt à cette espèce qu'il y a lieu de rapporter la forme dont nous reproduisons ici un fragment, tel qu'il apparait dans les préparations qui ont été quelque peu éclaircies par le chloral glycériné, comme il est utile de le faire pour aider à débrouiller le magma hétérogène dont ce microphyte est souvent accompagné.

Nous n'insisterons pas sur les caractères de l'Algue mise dans l'état où nous l'avons sous les yeux. Elle est ainsi impropre à traduire ses caractères vrais, ceux-ci étant déjà difficiles à saisir sur le vif.

\section{Hydrurus penicellatus AGARDH}

Nous ne ferons que citer en passant cette Phéophycée bien connue. Elle a été recueillie sur les pierres submergées d'un ruisseau tributaire de la rivière Matotchka, dans l'ile sud de la Nouvelle-Zemble. Au dire de M. le docteur L. Stappers, qui en a prélevé un échantillon, - no I79 - elle y était très abondante.

Il -s'agit ici plus spécialement de la variété Ducluselii Agardh.

Cette Algue n'étant ni unicellulaire, ni planktonique, nous ne l'avons pas figurée. 


\section{Groupe IV. - SILICOFLAGELLATES}

Ce petit groupe est assez pauvrement représenté dans les récoltes de la Belgica igo7.

\section{Genre Distephanus SтӧнR}

\section{Distephanus speculum Haeckel.}

Planche V, figures 6 à Io; planche XXII, figures 14 et 15 .

Synonyme et littérature. - Dictyocha speculum Ehrenberg, etc. Cfr. Nordisches Plankton, XXI, Flagellatae, etc., page 29.

Cette espèce, bien connue et de grande extension géographique, se présente en spécimens clairsemés dans presque tous nos matériaux et conséquemment sur tout l'itinéraire de la Belgica, soit dans sa variété rcgularis Lemmermann (fig. 6, vue latérale d'un test entier et vide; fig. 7, coupe optique latérale de l'organisme entier; fig. 8, vue de face de l'une des deux valves), soit dans sa variété septenaria Jörgensen (pl. XXII, fig. I4 et I5), beaucoup plus rare.

La seule remarque que nous suggèrent nos observations à son sujet, c'est la fréquence, dans l'une et l'autre de ces deux variétés, de spécimens à structure anormale. Les pièces du test offrent souvent un nombre de mailles plus élevé que dans la forme type, soit même une asymétrie qui altère plus ou moins la forme classique de ces pièces squelettiques sur lesquelles les auteurs ont basé leurs diagnoses (pl. V, fig. 9 et Io; pl. XXII, fig. I4 et I5).

\section{Genre Gymnaster Schüтt?}

Est-il possible de rapporter à ce genre la forme représentée planche $V$, figures II, I 2 et I 3 ?

Rencontrée presque exclusivement dans nos échantillons de neige colorée, elle est réduite à son squelette formé de deux pièces étoilées dont les cinq rayons striés perpendiculairement à leur axe (fig. I I) se soudent par leur extrémité à leur symétrique de la plaque jumelle (fig. I2, vue latérale), à moins que les chocs n'aient détruit leurs faibles adhérences (fig. I3, vue de face). 
Pour pouvoir affirmer quelque chose à cet égard, il faudrait d'abord être mieux fixé sur les caractères du genre Gymnaster au sujet duquel la littérature scientifique est encore trop sobre de renseignements, et ensuite il nous aurait fallu avoir sous les yeux des organismes complets.

Ce qui est certain, c'est que notre forme ne reproduit pas les caractères morphologiques de Gymmaster pentasterias de Schüt, dans Die Peridineen der Plankton Expedition (pl. XXVII, fig. Ioo), ni de Ramsay Wright, dans The Plankton of Eastem Nova Scotia Waters, 1907 (pl. I, fig. 2I, $a$ et $b$ ).

Nos spécimens, d'ailleurs très rares et réduits à leurs pièces squelettiques d'apparence siliceuse, ont été trouvés, avons-nous dit, dans les résidus de fusion de la neige colorée. C'est à ce fait, sans doute, de leur présence dans un milieu qui ne leur est probablement pas propre et où les vents ou les vagues ont pu les faire passer accidentellement, qu'ils doivent de ne s'y trouver qu'à l'état d'épaves, privés de leur corps protoplasmatique.

Néanmoins leur charpente squelettique fait repousser toute idée d'identification avec celle de Gymnaster pentasterias Schütt, bien que les pièces du test soient aussi pentaradiées.

Pour permettre d'en fixer la notion, nous la désignerons provisoirement sous le nom de Gymnaster striatum sp. n.

Nous ne saurions que refaire des observations du même genre au sujet du microorganisme auquel appartient le test représenté dans la figure I4 et trouvé dans les mêmes circonstances que le précédent. Nous n'avons rencontré ces pièces squelettiques que dépourvues de toute trace de substance sarcodique et isolées. Cela nous astreint à plus de réserve encore à leur sujet car nous n'avons ici d'autre élément d'appréciation qu'une simple analogie de forme.

Tenant compte du caractère spécifique que l'on pourrait éventuellement trouver dans la courbure spirale des rayons qui sont lisses et pointus, nous dénommerons l'organisme Gymnaster helix, jusqu'à plus ample information.

\section{Genre Diaster g. n.}

La structure bipolaire de la forme énigmatique représentée planche XIV, figures I2 à I8, nous engage à la présenter ici aussi, malgré le doute dans lequel nous sommes au sujet de ses affinités réelles.

Sa constitution complexe nous a paru d'une interprétation difficile à cause :

Io de l'absence de membrane d'une épaisseur appréciable;

$2^{\circ}$ de la densité du corps sarcodique;

3o de l'absence de symétrie, qui lui fait prendre des aspects très variés suivant la position qu'elle a sous l'objectif. 
La partie la plus visible et qui garde dans toutes les positions l'aspect le plus constant, c'est un gros noyau, $n$, de forme ellipsoïde, rempli de grosses granulations, que nous ne pouvons pas affirmer, malgré les apparences, être la coupe optique d'un gros filament unique et contourné formant l'élément chromatique ou nucléinien.

Sur un des flancs du noyau se développe une aire plus claire, à parois striées (fig. I2, I3, IS), qui s'étend parfois également du côté opposé (fig. I5). Nous n'en connaissons pas la signification.

D'un côté s'observe souvent une sphérule hyaline, $x$ (fig. I 2, I 3 , I4, I6), dont la fonction nous échappe aussi.

Aux deux pòles du noyau, - à droite et à gauche dans nos figures - logés dans le protoplasme cellulaire, s'observent normalement deux corps pentaradiés, st ${ }^{l}$ et $s t^{2}$, dont nous ne saurions dire la nature, puisqu'ils n'apparaissent bien que comme des solutions de continuité, en forme d'étoile, dans le sarcoplasme : en coupe : figures I 2 et I3; de face : figure I4, dans une vue polaire de l'organisme.

L'impénétrabilité de l'objet, même après l'action éclaircissante du chloral concentré, nous a amené à en présenter un schéma stéréoscopique (fig. I6) dans lequel, faisant abstraction du cytoplasme, nous ne montrons que les rapports de position des deux corps stellaires, $s t^{1}$ et $s t^{2}$, de la sphérule hyaline, $x$, et du noyau, $n$, pour aider le lecteur à l'interprétation des autres figures, réellement figuratives de l'objet, mais aussi peu explicites naturellement que l'objet lui-même.

La figure I7 montre l'aspect de l'organisme pendant une phase de sa division. Le noyau est divisé et chacune des deux moitiés est flanquée de son aire transparente et striée superficiellement.

La figure IS se rapporte sans doute à un individu issu d'une division récente, car il ne présente encore qu'un seul corps stellaire, st, à gauche.

L'étude de cet organisme a naturellement reporté notre esprit vers la figure IoO ${ }^{\text {bis }}$, planche 27, de Schütт (Die Peridineen der Plankton-Expedition), sans qu'il nous ait été possible d'y voir autre chose qu'une vague analogie, fortuite sans doute, sur laquelle il serait imprudent d'asseoir une opinion fondée.

Je ne m'explique pas bien, du reste, que Schütт ait voulu voir, dans la forme qu'il décrit, un Péridinien à squelette interne. D'autre part, si cet auteur avait eu sous les yeux notre organisme, aurait-il négligé de figurer le noyau qui y tient une place si importante et lui communique le caractère le plus tangible?

Bien qu'il faille faire la part large aux difficultés de reproduction d'organismes de ce genre, nous croyons qu'il serait vain de chercher un rapprochement entre les deux formes.

C'est à une mème conclusion que nous conduit aussi une tentative de rapprochement de notre organisme avec Diplocystis antarctica de Cleve (Öfuersigt af Vet. Ak. Förhandl., Igoo, no S), dont les figures, très frustes, il faut bien le reconnaitre, nous avaient également fait penser à une certaine analogie de structure. 
Bref, des observations ultérieures jetteront sans doute plus de lumière sur les affinités de cet organisme. Qu'il nous suffise pour le présent de le signaler à l'attention des micrographes sous la rubrique Diaster macrokaryon, nom qui tient compte à la fois de l'aspect si caractéristique du noyau et des deux corps pentaradiés logés dans le cytoplasme.

En ce faisant nous n'affirmons pas qu'il s'agisse d'un Silicoflagellate vrai.

Nous en posons le problème.

Distribution géographique. - Nous n'avons guère observé cet organisme que dans les échantillons recueillis sur les côtes occidentales de la partie nord de la Nouvelle-Zemble, dans le no 192 surtout.

\section{Groupe V. - ORGANISMES ÉNIGMATIQUES}

Nous nous proposons ici de jeter un coup d'œil sur un ensemble d'organismes planktoniques variés sur la nature desquels la lumière n'est point suffisamment faite. Certains d'entre eux ont été dùment recensés; d'autres ne l'ont été que d'une façon sujette à caution; il en est enfin qui n'ont pas assez fixé l'attention et au sujet desquels les renseignements sont nuls ou à peu près.

Malgré le caractère ambigu de ces derniers, caractère qui les a fait négliger jusqu'ici par les planktonistes, on conviendra qu'il n'est pas inutile d'en poser le problème aux biologistes, aux zoologistes particulièrement, qui en ont peut-être déjà la solution ignorée des botanistes. Plusieurs de ces formes nous paraissent, en effet, avoir des accointances avec des Métazoaires.

Nous procéderons, dans ce chapitre, des formes plus connues aux moins connues, en passant outre aux espèces algologiques dont il a déjà été fait mention dans l'aperçu sur les organismes des neiges et des glaces, dont nous avons fait le groupe II.

Pour la littérature scientifique nous renvoyons le lecteur au travail systématique sur les Chlorophycées de E. Lemmermann dans Nordisches Plankton, XXI.

Il faut savoir gré à ce dernier d'avoir entrepris la tâche difficile de mettre un peu d'ordre dans ces organismes dont le signalement est éparpillé dans les publications les plus diverses et qui n'ont souvent été figurés que d'une manière trop sommaire pour permettre d'établir des rapprochements et de saisir les affinités naturelles entre des formes dont plusieurs n'ont jamais été observées à l'état vivant, mais plutôt à l'état modifié par les réactifs fixateurs qui en ont dénaturé, sinon toujours l'aspect morphologique, du moins les caractères physiologiques les plus utiles. 


\section{Genre Halosphæra Sснмiтz}

Cellules sphériques, simples, libres, ne se multipliant pas par division, mais seulement par spores d'essaimage, zoospores, et par spores quiescentes, aplanospores.

Halosphara viridis Schmitz.

Planche $\mathrm{V}$, figures $15, \mathrm{I} 6,17$.

Littérature. - Cfr. Nordisches Plankton, XXI, page 20.

Nos observations sur cette espèce s'accommodent très bien de la description qu'en donne Lemmermann.

Le spécimen reproduit figure $\mathrm{I} 7$ est le plus petit qu'il nous ait été donné d'observer dans nos matériaux actuels. Le noyau unique, central, s'y voit aisément, grâce au petit nombre de chromatophores dans le périplasme.

Notre figure 5 en reproduit un spécimen choisi parmi les plus petits chez lesquels s'observe déjà un certain groupement des chromatophores en vue de la formation des spores.

Dans le spécimen de la figure I6, ce groupement est accompli autour d'un grand nombre de petits noyaux issus du noyau primitif.

La membrane y est assez épaisse et traduit le plus souvent sur les bords de la sphère, par suite de la réfraction des rayons lumineux, une belle teinte bleu violacé qui, chez d'autres, est remplacée par une teinte jaune orangé, complémentaire de la première.

Nous n'avons pas eu l'occasion d'observer les phases plus avancées de l'élaboration des spores.

Distribution géographigue. - Cette espèce, réputée propre aux eaux du Gulfstream, se rencontre, en effet, dans ceux de nos échantillons qui proviennent de la partie sud de la Mer de Barents; mais elle est plus constante dans ceux provenant de la Mer de Kara. Est-il établi que les eaux du Gulfstream pénètrent dans cette mer par la Porte de Kara en suivant la còte orientale de la Nouvelle-Zemble? Sinon, la solidarité de cette espèce avec ces eaux semblerait sujette à caution.

\section{Halosphera minor Ostenfeld?}

Planche IV, figure $7 \mathrm{I}$.

Littérature. - Cfr. Nordisches Plankton, XXI, page 22.

Le rapprochement que nous tentons entre la petite forme sphérique que nous figurons ici et celle désignée sous ce nom par Ostenfeld est-il légitime? Il serait téméraire de l'affirmer, malgré les analogies de grandeur et d'aspect. 
Une circonstance qui nous commande la réserve, c'est l'habitat spécial de notre forme que nous n'avons trouvée que dans la neige jaune. Pour cette raison nous aurions pu la mentionner dans le groupe précédent s'il n'avait semblé plus logique de la joindre ici à sa congénère probable.

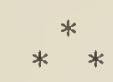

S'il n'est pas douteux que les Halosphara soient des Algues, peut-on affirmer avec la même assurance qu'il en soit de même des organismes désignés sous les noms de Trochiscia, Pterosperma, Hexasterias, etc.?

N'y a-t-il pas lieu plutôt de mettre en doute même leur nature végétale et peutêtre aussi le caractère d'organismes autonomes que beaucoup d'auteurs leur accordent ou tout au moins leur laissent supposer faute de pouvoir les rattacher à d'autres organismes à un titre quelconque, celui de spores quiescentes, par exemple?

Ceci nous paraît réellement plus probable et, si nous suivons Lemmermann dans la classification qu'il fait de ces organismes, dans Nordisches Plankton, c'est avec la réserve expresse de ne pas les tenir toutes indifféremment ni pour des formes autonomes d'Algues, ni même pour des microphytes, avant que la preuve n'en soit faite par l'observation de leur origine et de leurs phases évolutives.

Conséquemment, le groupement méthodique de ces organismes en genres et en espèces ne saurait, à notre avis, être considéré que comme un expédient utile pour donner une satisfaction provisoire à l'esprit dans son besoin légitime de systématique.

\section{Genre Polyasterias g. n.}

\section{Polyasterias problematica (Cleve) pro parte.}

Planche $\mathrm{V}$, figures $20,2 \mathrm{I}, 22$.

Synonyme. - Genre Hexasterias Cleve. Cfr. Nordisches Plankton, XXI, page 20.

Cleve a créé le genre Hexasterias pour un organisme unicellulaire bien connu, à corps circulaire central pourvu, sur son pourtour, d'expansions radiaires équidistantes, qui se sont trouvées être au nombre de six dans le ou les spécimens que le hasard des observations planktoniques lui a mis sous les yeux.

Ne serait-ce pas abuser des termes que de conserver ce nom générique déterminatif du nombre six des rayons, s'il est établi que ce nombre est variable et ne peut conséquemment ètre pris que comme un caractère tout au plus spécifique, si pas seulement individuel? 
A notre avis, ce n'est là qu'un caractère individuel car, ayant eu l'occasion d'en observer un assez grand nombre de spécimens, nous en avons vu à quatre, à cinq, à six, à sept (fig. 22) et à huit rayons (fig. 20).

L'expression Hexasterias octoradiata, par exemple, serait assurément trop paradoxale!

C'est pourquoi nous proposons la substitution du terme Polyasterias, dont peuvent s'accommoder les spécimens présentant un nombre quelconque de rayons. C'est d'autant plus indiqué, à notre point de vue, que la forme à six rayons nous a paru la plus rare, dans les produits de pèche de la Belgica.

Nous ne croyons pas, du reste, qu'il s'agisse ici d'une forme végétale ni même d'un organisme autonome. Nous y verrions plus volontiers une forme unicellulaire représentative d'un organisme plus complexe auquel le hasard des circonstances permettra tôt ou tard de le rattacher.

Quoi qu'il en soit et malgré les différences existant entre nos figures et celle de Cleve (I), différences qui peuvent tenir soit à la phase évolutive de l'objet, soit au mode de préparation, soit aussi au procédé de figuration, il n'est pas douteux qu'il y ait identité de l'objet observé de part et d'autre.

Comme nous l'avons dit plus haut, il présente, dans les limites de nos observations, un nombre de rayons variant de quatre à huit.

Le corps lenticulaire, biconvexe (fig. 2I, vue latérale), est rempli de protoplasme dense et granuleux, qui n'a rien de végétal.

Les expansions radiaires, simples dépendances de la membrane, sont vides, de forme cylindrique au milieu, plus ou moins élargies à leur base et assez largement évasées en pavillon à leur extrémité libre. Là s'épanouissent, en crochets indépendants, les fibrilles qui sillonnent les bras dans toute leur longueur, où ils sont retenus en faisceau creux par une gaine hyaline très mince.

Si l'on en sent le besioin, rien n'empêche d'y distinguer des variétés d'après le nombre des rayons : 4, 5, 6, 7, \& radiata, etc.; mais cette minutie nous semble superflue, si l'on pense comme nous qu'il n'y a là que des variantes individuelles.

Distribution géographioue. - Çà et là, sur tout le trajet de la Belgica, toujours en spécimens épars et rares.

\section{Genre Pterosperma Pouchet}

Nous ne prenons non plus ce terme de Pterosperma que comme une étiquette propre à réunir des formes d'organismes ambigus, dont la nature réelle n'est pas connue

(I) The Plankton of the North Sea, the English Channel and the Skagerak. Kongl. Sv. Vet. Akad. Handl., Bd. XXX11, no 8 , page 22 , figure 6 . 
et au sujet desquels on n'a d'autre donnée d'appréciation que leur forme dans laquelle on s'étudie à trouver au moins un vague caractère commun : celui d'une ou de plusieurs expansions membraneuses faisant saillie de quelque façon, en forme d'ailettes sur le corps.

Etymologiquement, ce te̊rme suggère en outre discrètement l'idée de germe, de spore, qui pourrait bien recéler quelque part de vérité.

Lemmermann emploie le terme de Trochiscia pour réunir, sous un nom commun, les formes ailées et les formes épineuses, les Xanthidium d'autres auteurs qui usurpaient en cela le nom d'un genre de Desmidiées. Ce terme nous paraît moins heureux et ne pourrait ètre opportun que si toutes les formes à couvrir de ce pavillon avaient au moins en commun la forme d'un disque ou d'une roue, que ce nom leur fait supposer.

Mais, en réalité, on aurait tort d'attacher plus d'importance qu'il ne faut, en l'occurrence, à ce qui n'est pour nous qu'une simple question de terminologie dont l'unique valeur dépend de la portée toute conventionnelle qu'on entend lui donner, à l'exclusion de toute signification biotaxique réelle. On désignerait ces organismes par un numéro ou par un signe conventionnel quelconque, que le résultat pratique serait le même, si ce n'était toutefois qu'un numéro n'est pas évocatif d'une image comme un nom.

Nous signalerons en première ligne quelques formes trouvées dans la neige colorée et sur les glaces de la Mer de Kara, formes qui présentent cette particularité commune d'avoir un corps différencié, à deux faces, antérieure et postérieure, diversement bombées et séparées par un étranglement circulaire, visible seulement sur les spécimens vus de profil, position instable dans laquelle on ne réussit que difficilement à les placer et à les maintenir (fig. $25^{\mathrm{b}}$ et $26^{\mathrm{b}}$ ).

\section{Pterosperma laciniatum sp. n.}

Planche V, figure $25^{\mathrm{a}}$, vue de face; figure $25^{\mathrm{b}}$, vue de côté.

Le corps, vu de face, est circulaire, légèrement elliptique, avec une membrane épaisse, un cytoplasme grossièrement granuleux, un noyau relativement gros. Sur la moitié la plus convexe du corps, que nous appellerons dorsale (voir la vue latérale), s'insère, immédiatement au-dessous du sillon circulaire, une membrane très mince, élégamment découpée et soutenue, dans la partie médiane des segments, par des épaississements qui figurent des nervures. Cette membrane frangée, très hyaline, est difficile à percevoir.

\section{Plerosperma octogonum sp. n.}

Planche $\mathrm{V}$, figure $26^{\mathrm{a}}$, vue de face ventrale; figure $26^{\mathrm{b}}$, vue latérale.

Diffère du précédent par la forme octogonale de la vue de face tant du corps que de la membrane. Huit nervures radiales sous-tendent la membrane dans la direction 
des angles de même nombre. La vue de côté montre également le sillon qui sépare la face dorsale bombée de la face antérieure plus aplatie.

Pterosperma hexagonum sp. n.

Planche V, figure 27 , vue de face.

Même structure que le précédent avec la seule différence de la forme hexagonale. Nous n'avons pas reproduit la vue de profil, qui ne se distinguerait pas de celle du précédent.

\section{Pterosperma trigonum sp. n.}

Planche V, figure 28 , vue de face.

Le nom en traduit suffisamment la particularité de structure et d'aspect.

Même observation que ci-dessus au sujet de la vue de profil.

Le lecteur préfère-t-il ne voir dans ces trois dernières formes que des modalités octogone, hexagone, trigone, d'un seul type : polygonmm? Nous n'y voyons pas d'inconvénient.

Ces formes paraissent propres aux neiges et aux glaces.

Les formes qui suivent n'ont plus d'étranglement transversal séparant les deux parties dorsale et ventrale du corps. La plupart sont planktoniques.

\section{Pterosperma Saturnus sp. n.}

Planche V, figure 23 , vue de face, et 24 , vue de profil.

Le corps assez gros, sphérique, est à cytoderme épais, lisse, hyalin et renferme un cytoplasme riche en globules huileux et contracté par la solution fixatrice. L'anneau membraneux qui l'entoure, comme l'anneau de la planète Saturne, est renforcé d'épaississements rayonnants et va s'amincissant vers le bord extérieur. Sa limite périphérique est à peine ondulée. Sa surface est partout finement chagrinée.

Nous l'identifierions volontiers avec Pterosperma Möbii Jörgensen (Cfr. Nordisches Plankton, XXI, page I8) si nous pourions savoir que les différences qui nous arrêtent sont plus inhérentes aux reproductions des objets qu'aux objets eux-mêmes.

Distribution géographique. - Détroit de Matotchkine et Mer de Kara; très rare.

\section{Pterosperma hemispharicum sp. n.}

Planche V, figure 29, vue de face antérieure; figure 30 , vue oblique.

Le corps est hémisphérique, fortement convexe d'un côté, légèrement concave de l'autre. Il renferme un noyau assez volumineux, au milieu d'un cytoplasme huileux 
d'aspect grossièrement granuleux; le tout colorable en jaune par l'iode. Sur le pourtour de la partie antérieure court une membrane assez rigide, aux contours irrégulièrement ondulés, et peu large.

Cette forme nous est surtout connue des neiges colorées et des glaces d'où elle a passé parfois, par fusion de ce milieu, dans l'eau marine sous-jacente de la Mer de Kara.

Nous ne lui reconnaissons aucun caractère végétal.

\section{Plerosperma lenticulare sp. n.}

Planche VI, figure 13 , vue de face; figure 14 , vue de profil.

Le corps est lenticulaire, biconvexe, renflé, aplati sur le milieu des deux faces.

Le cytoplasme, d'aspect grossier, renferme de nombreux globules d'huile et de matières azotées plutôt propres à un organisme animal.

Il porte une aile circulaire comme le précédent, mais plus large et plus manifestement striée concentriquement.

Distribution Géographique. - Trouvé, non dans les neiges, mais en rares spécimens isolés dans la Mer de Kara.

\section{Pterosperma polyedro-spharicum sp. n.}

Planche VI, figures I à 7 ; planche VII, figure I.

Synonyme et Littérature. - Pterosphara Vanhöffenii Jörgensen et Pterosphara dictyon Jörgensen, Trochiscia dictyon Lemmermann, Trochiscia Vanhöffenii Lemmermann, etc. Cfr. Nordisches Plankton, XXI, page I9.

L'examen comparé d'un assez grand nombre de spécimens des formes ci-devant désignées sous des appellations différentes nous a engagé à les réunir sous une dénomination commune, eu égard aux diverses modalités de structure ménageant la transition entre des types qui, au premier abord, sembleraient irréductibles à un schéma commun.

C'est ce que montrera suffisamment, pensons-nous, la comparaison des quelques figures que nous consacrons à cet organisme polymorphe.

Le corps est toujours parfaitement sphérique s'il n'a été déformé soit par pression, soit par l'action des réactifs qui ont pu le contracter ou le distendre (fig. 7).

Le cytoplasme est bien plutòt d'aspect animal, chargé de matières grasses et souvent contracté en boule par l'action plasmolytique des liquides conservateurs.

Les ailettes qui ornent le corps sont parfois très larges et élégamment plissées (fig. I, 2, 3, pl. VI) - Krausenei, de VAnHöfen - d'autres fois plus étroites (fig. 4, 5, 6, 7, pl. VI) - Trochiscia ou Pterosphara dictyon des auteurs. 
Les lignes d'intersection de ces ailettes sur la sphère y déterminent des polygones dont le nombre est variable et indépendant aussi bien de la largeur des ailettes que du volume du corps sphérique.

Ce volume varie beaucoup; comparer la figure I, planche VI, à la figure I, planche VII, en tenant compte du fait que les spécimens figurés ont été choisis parmi ceux à dimensions moyennes.

Nous tenons conséquemment toutes ces variantes pour des modalités d'un mème organisme :

Planche VI, figure I. - Spécimen vide et débarrassé de toute surcharge accidentelle par des manipulations appropriées et montrant les ailettes qui se manifestent comme des franges larges et élégamment froncées, dont les lignes d'insertion déterminent à la surface du corps un tétraèdre sphérique.

Figure 2. - Spécimen analogue, à franges moins larges et à cytoplasme contracté en boule.

Figure 3. - Spécimen à ailettes très larges, froncées, à corps couvert de globules étrangers qu'il serait facile d'éliminer par l'usage des dissolvants des matières grasses.

Figure 4. - Spécimen nettoyé par le procédé indiqué ci-dessus. Le cytoplasme est ramassé en une masse sphérique au centre de la cavité cellulaire. Les ailettes, bien que moins larges, sont encore légèrement froncées et dessinent aussi sur le corps un tétraèdre sphérique.

Figure 5. - Spécimen dont le protoplasme huileux est resté en place, appliqué contre le cytoderme. Les ailettes étroites et non froncées délimitent à la surface du corps un polyèdre irrégulier dont on aperçoit cinq faces.

Figure 6. - Spécimen plus volumineux dont le cytoplasme a abandonné la membrane pour se contracter en une sphérule mal délimitée dans laquelle on aperçoit, comme toujours, de nombreux globules de matière grasse.

Les ailettes étroites et tendues découpent aussi la surface du cytoderme en aires polygonales inégales. Celui-ci montre sa sưface parsemée de nombreux points gélifiés ou gélatinisés.

Figure 7. - Le corps de cet exemplaire s'est distendu sous l'action du chloral et s'est bombé dans les aires délimitées par les ailettes, sans que cette extension ait pu se produire au niveau d'insertion de ces dernières. La forme sphérique en est ainsi artificiellement altérée.

Planche VII, figure I. - Spécimen nettoyé et vidé qui laisse voir, parallèlement aux ailettes très étroites, des rangées de pores rectangulaires qui résultent d'une gélification locale. Cette particularité s'observe plus rarement, comme celle du mème genre de la figure 6, planche VI. La signification ne s'en révèle pas d'elle-mème, mais il nous paraît au moins probable d'y voir une différenciation tardive du cytoderme en rapport avec l'évolution de l'organisme dont les affinités nous paraissent animales, bien que nos devanciers le rangent parmi les Chlorophycées. 
Remarque. - L'exécution peu réussie de la figure de Vanhöffen - Krausenei figure peu explicite d'ailleurs et qui se rapporte probablement à un objet déformé, a sans doute contribué beaucoup à entretenir entre Trochiscia Vanhöffenii Lemmermann et Trochiscia dictyon Lemmermann une distinction qui n'a pas d'autre fondement que la différence occasionnelle des figures originales s'y rapportant.

Distribution géographique. - En spécimens épars dans les produits planktoniques de la Mer de Kara. Beaucoup plus rare dans ceux provenant de la Mer de Barents.

\section{Genre Chitonosperma g. n.}

Sous cette appellation, nous voulons désigner des organismes qui paraissent, à certains égards, analogues aux précédents, mais dont le corps est orné non d'ailettes, mais bien d'une tunique (Xirín, cöros) supplémentaire.

\section{Chilonosperma umbilicalum sp. n.}

Planche V, figures $3 \mathrm{I}$ et 32 .

Le corps central, à peu près sphérique, ne présente qu'une légère dépression à un pôle (fig. 32, vue de profil). Il est bondé de corpuscules arrondis, de grosseur sensiblement égale, dont l'aspect suggère l'idée d'un phénomène de sporulation.

Ce corps est logé dans une membrane extérieure qui n'en épouse pas la forme mais s'en écarte au contraire beaucoup, suivant une zone équatoriale au milieu de laquelle elle dessine un pli saillant en forme de crète circulaire. On s'en rendra compte en comparant la figure 32 avec la figure 3 I qui est une vue de la face antérieure, c'est-à-dire du côté où le corps sphérique central est quelque peu ombiliqué.

La tunique extérieure est très mince, hyaline, légèrement plissée; elle ne cache pas la vue du corps central, dont la membrane propre est plus épaisse.

Distribution Géographigue. - Sortie du Détroit de Matotchkine dans la Mer de Kara.

\section{Chilonosperma coronatum sp. n.}

Planche V, figure 33, vue de face antérieure; figure 34 , vue de profil.

Le corps est ici quelque peu piriforme. Il renferme aussi des corpuscules qui semblent résulter d'une sporulation endogène. Il est doublé d'une tunique hyaline d'aspect granulé qui s'applique étroitement sur la partie inférieure du cytoderme 
proprement dit et s'en détache dans la partie supérieure pour circonscrire autour d'elle un espace annulaire, dont la figure 34 traduit la forme spéciale en vue stéréoscopique latérale. Notre qualificatif prétentieux ne l'exprime qu'avec une très lointaine approximation.

Les renseignements précis tirés de la comparaison des deux figures 33 et 34 suppléeront à l'insuffisance de la description.

Distribution GÉographigue. - Mer de Kara; très rare.

\section{Chitonosperma sp.}

Planche VI, figure II, vue de face antérieure; figure I2, vue de profil.

Nous ne saurions placer ailleurs qu'ici cette forme organique que nous avons rencontrée maintes fois dans le plankton de la Mer de Kara et qui semble réduite à la tunique extérieure, modifiée légèrement dans sa forme, de Chitonosperma umbilicatum décrit plus haut. Pour que cette interprétation fût fondée, il faudrait pouvoir admettre que le corps sphérique central a été dissout après la réalisation du phénomène hypothétique de sporulation dont nous nous sommes borné à émettre l'idée car nous n'en avons pas la preuve.

Pour ces raisons, nous réservons l'appellation spécifique de l'organisme.

\section{Genre Glyphosperma g. n.}

Ce n'est pas le goùt du néologisme, mais le besoin d'un nom nouveau pour désigner une chose nouvelle, qui nous fait proposer ce terme pour désigner des formes organiques rencontrées çà et là dans les produits de pêche planktonique de la Belgica, qui présentent une double membrane, dont l'extérieure est comme burinée (riúpw, je burine) et qui paraissent être ou bien des œufs d'organismes métazoaires inconnus, ou bien des spores quiescentes d'organismes inférieurs dont on ne connaît pas encore les rapports naturels mais dont il paraît utile de poser la question de la nature et des origines.

\section{Glyphosperma flagelliferum sp. n.}

Planche VI, figures 8, 9, Iо.

La forme sphérique du corps, déterminée par la membrane interne (fig. 9, coupe optique longitudinale), est modifiée extérieurement par les replis de la membrane externe, que traduisent des crêtes saillantes symétriquement distribuées vis-à-vis d'un plan passant par l'axe vertical de nos figures et par le point d'insertion du long flagellum, 
dont la position détermine la face que nous appellerons antérieure (fig. 8, vue stéréoscopique antérieure avec flagellum, ou plutòt une sorte de byssus filamenteux, qui pourrait bien servir d'attache de l'objèt sous forme d'amarre).

La figure Io reproduit l'objet en vue latérale gauche. En rapprochant cette vue de la précédente, on se rendra compte de la distribution des crêtes sur le corps, en même temps que des plissements méandrés qui intéressent partout la membrane externe.

La figure 9 en est une coupe longitudinale optique montrant le protoplasme dense, chargé de corpuscules graisseux et protéiques, avec, en outre, un noyau assez volumineux, qui en occupe le centre. L'étude de ces détails exige naturellement l'action préalable de puissants réactifs éclaircissants, tels que le chloral concentré et glycériné.

De quel organisme cet objet pourrait-il être représentatif, à titre d'œuf ou de spore quiescente, par exemple? Nous ne tenterons pas même à ce sujet une hypothèse qui risquerait trop d'être trouvée en défaut, étant données les rares occasions que nous avons eues de l'observer tel quel sans que rien suggérât une idée d'assimilation à d'autres organismes capturés dans les mêmes coups de filet.

Distribution Géographique. - Mer de Kara; très rare.

\section{Glyphosperma nervosum sp. n.}

Planche VII, figure 2.

Cette forme organique a de commun avec la précédente, outre la forme sphérique que donne au corps la membrane interne, l'aspect buriné en sillons méandreux de la membrane externe ou cuticule qui dessine des côtes saillantes en manière de polyèdre sphérique.

L'objet figuré en vue stéréoscopique, sans orientation déterminée, nous a paru dépourvu de symétrie et privé d'un appendice, flagellum, byssus, amarre, analogue à ce qui s'observe chez le précédent.

Distribution géographique. - Mer de Kara; très rare.

\section{Genre Radiosperma g. n.}

Nous avons en vue ici des formes organiques du genre de celles que des auteurs allemands, HENSEN le premier, ont appelées Stemhaarstatoblast ou Stemenhaar-Statoblast, désignation bien évocatrice, dans leur langue, des caractères morphologiques les plus apparents de ces objets, mais dont la nomenclature linnéenne, généralement suivie, même à titre provisoire, pour les organismes incertae sedis, ne peut convenablement s'accommoder. 


\section{Radiosperma corbiferum sp. n.}

Planche VI, figure 16 , vue de face antérieure; figure 17 , vue de profil; figure 18 , vue oblique postérieure latérale.

C'est le seul représentant du genre que nous ayons rencontré dans nos matériaux.

Le corps est en forme de cône à sommet arrondi et à base développée en une sorte de corbeille ajourée, formée de trabécules qui suivent une direction spiralée dès leur origine, pour se terminer en crochet, après s'être anastomosés vers les deux tiers de leur parcours et avoir déterminé vers le bas une sorte de cuvette encerclée.

Le corps protoplasmatique, très réduit par rapport à ce qui semble n'être qu'un appareil flotteur, est grossièrement granuleux et prend une coloration jaune brun sous l'action de l'iode.

Nous renvoyons le lecteur à nos figures pour l'analyse plus détaillée de la structure de l'objet.

Distribution GÉographique. - Çà et là, surtout dans la Mer de Barents, notamment dans les nos i 5 I et ig3.

\section{Genre Amphicephalosperma g. n.}

\section{Amphicephalosperma bullans sp. n.}

Planche VI, figure 15.

Nous proposons cette appellation pour la forme organique que nous reproduisons d'après de rares spécimens observés et que nous ne pouvons verser dans aucune des autres catégories d'organismes énigmatiques qui nous occupent dans ce chapitre. Le corps en est quelque peu allongé, déprimé au milieu et arrondi aux deux extrémités symétriques. Celles-ci sont relevées d'expansions membraneuses simulant des alvéoles.

La nature et les rapports nous en sont inconnus.

Distribution Géographique. - Mer de Kara; très rare.

\section{Genre Setosperma g. n.}

Il s'agit ici probablement d'œufs sphériques ornés de soies raides, mais flexibles, dont les extrémités aboutissent à une membranule concentrique au corps de l'objet. 


\section{Setosperma equatorale sp. $\mathrm{n}$.}

Planche VII, figure 3, vue polaire ou perpendiculaire à l'équateur; figure 4, vue équatoriale.

Le cytoplasme inclus dans la membrane intérieure est chargé d'enclaves volumineuses. La sphère intérieure porte, sur une zone équatoriale assez large, des soies raides qui traversent radialement l'espace vide à l'intérieur d'une autre membranule hyaline externe, qui est ainsi maintenue concentrique à la première (fig. 4). Le même objet (fig. 3), vu de l'un des pôles, laisse voir l'auréole de fibrilles radiales qui se pressent sur la zone équatoriale.

Nous rattachons à la même forme l'objet de la figure 5, qui n'en est sans doute qu'une modalité caractérisée par l'existence d'une seconde zone circulaire de soies perpendiculaire à la première, mais beaucoup plus étroite que celle-ci. De plus, la membranule extérieure a disparu, par dissolution sans doute, car les soies qui devaient y aboutir n'ont pas été dérangées de leur position régulière, circonstance qui ne se concilierait guère avec l'hypothèse d'un arrachement. Nous avons, du reste, eu l'occasion d'observer la membranule extérieure dans cette forme aussi, mais elle y semble toujours extrêmement délicate et sa dissolution éventuelle n'a rien qui doive étonner. Celle-ci caractérise sans doute une phase évolutive de l'organisme.

Distribution géographique. - Mer de Kara. Assez rare; no 34 entre autres.

\section{Série des PAPULIFÈRES}

La désignation de Papulifères n'a d'autre but que de servir d'étiquette à tout un paquet de formes microplanktoniques, qui se réclament d'une certaine analogie de nature et peut-être aussi de parenté, par un caractère commun : la présence à une extrémité du corps d'une sorte de bouton hyalin (papula), en rapport avec un épaississement local très marqué de la membrane.

\section{Que sont ces organismes?}

Nous avions pensé d'abord qu'ils pouvaient être des spermatophores de Crustacés de petites formes, mais nos recherches dans cette voie n'ont pas confirmé cette opinion.

D'autre part, CanU (I) a émis l'opinion qu'on pourrait voir, dans l'un d'entre eux, un cuf de Trématode parasite.

Nous n'y contredirons pas, au contraire, mais nous ne sommes pas plus en mesure que ce biologiste d'en fournir la preuve!

(I) Ann. de la station aquicole de Boulogne-sur .1or, vol. I, part. 2, p. II2, pl. V11, fig. 8 et 9. 
L'ignorance dans laquelle on se trouve encore actuellement de la nature de ces formes énigmatiques est une raison de plus pour présenter au lecteur la collection assez riche de ces sphinx, que les récoltes de la Bclgica nous ont permis de recenser.

En l'occurrence, poser la question ce n'est sùrement pas la résoudre; mais nous estimons néanmoins faire chose utile en la posant sur des bases plus larges qu'on ne l'a fait jusqu'ici, grâce à la richesse exceptionnelle de nos matériaux d'étude.

La solution des questions biologiques de ce genre, il faut l'attendre du hasard des circonstances et des rencontres; mais celles-ci risquent moins de rester inopérantes, si l'attention de l'observateur a été mise en éveil par l'intérêt qui s'attache à tout ce qui regarde l'enchainement des formes vivantes.

Tôt ou tard on finira bien par trouver les affinités naturelles de ces formes curieuses, et dès lors celles-ci pourront s'émanciper du pseudonyme que nous leur donnons et qui ne peut que leur constituer un état civil provisoire.

\section{Genre Fusopsis g. n.}

C'est à ce genre qu'appartient la forme figurée par CANU d'abord (voir plus haut), par Ramsay Wright ensuite ( $\mathrm{I}$ ). Nous y rangeons les formes en fuseau, dont les suivantes :

\section{Fusopsis elongata sp. n.}

Planche VI, figure 2I.

Corps fusiforme, longuement prolongé en pointe d'un côté, terminé de l'autre par un bouton ellipsoïde, hyalin, qui surmonte un épaississement très marqué du cytoderme. Cet épaississement persiste après la disparition de la papule qui est caduque. Cette caducité semble liée à l'une des phases évolutives de l'organisme.

Le cytoplasme est d'aspect variable. Finement ou grossièrement granuleux, plus ou moins concentré autour du noyau qui est central, il entoure toujours celui-ci d'une couche très dense à structure plus fine. Plus rarement on le dirait transformé en une infinité de corpuscules arrondis appelés à jouer un rôle individuellement, après libération. C'est ce qui pouvait donner l'idée d'une individualisation de spermatozoïdes au sein d'un spermatophore. Voir cet aspect dans la figure 20.

(1) The Plankton of Eastern Nova Scotia Waters, pl. V, fig. 4. - Further Contributions to Canadiun Biology, 1902-Igo5 
Ces variantes d'aspect s'expliqueraient peut-être aussi bien dans l'hypothèse d'un œuf dont le vitellus serait l'objet d'un travail de transformation pendant sa maturation. L'avenir dira ce qu'il faut en penser.

Distribution Géographique. - Cette forme est loin d'être spéciale aux mers de Barents et de Kara, où elle se rencontre fréquemment en spécimens isolés. Nous la connaissons depuis longtemps de la Mer Flamande, où nous l'avons observée en spécimens disséminés, pleins ou vides, presque en toute saison, - plus rarement pendant les mois d'hiver — dans les produits des pêches planktoniques saisonnières.

Fusopsis spiralis sp. n.

Planche VII, figure 6.

Synonymie. - Euf de Trématode, d'après Canu; cf. Ramsay Wright. Voir plus haut.

Cette forme ne diffère de la précédente que par ses dimensions plus grandes et les cannelures spiralées qui ornent le corps de la capsule.

Dans le spécimen figuré, le bouton terminal a disparu : preuve d'usure ou d'atrophie, après disparition du contenu cellulaire.

Ces organismes, comme leurs congénères, se montrent, en effet, souvent complètement vidés, sans qu'on puisse voir comment s'est effectuée la déhiscence, s'il est vrai toutefois qu'un phénomène de ce genre doive se réaliser pour la mise en liberté de produits endogènes : spores, gamospores ou embryon.

Dans certains cas cependant on observe une dissolution de l'axe du bouchon terminal, d'où résulte un pore qui pourrait ètre interprété comme un mode de déhiscence, s'il est permis d'y voir autre chose qu'un fait d'usure ou de commencement de décomposition.

Distribution géographique. - Assez rare dans la Mer de Kara; plus rare dans la Mer de Barents. Rencontré aussi dans la Mer Flamande, avec d'autres formes dont nous ne ferons pas mention ici, parce qu'elles sont étrangères aux récoltes de la Belgica.

\section{Fusopsis umbracula sp. n.}

Planche VI, figures i9 et 20.

Cette forme est la moins rare dans nos échantillons, spécialement dans ceux de la Mer de Kara.

Elle a, sous des dimensions un peu plus petites, la forme de Fusopsis elongata, mais elle s'en distingue par un ensemble de soies flexibles disposées en ombelle sur 
l'extrémité atténuée de la capsule, où elles s'insèrent sur le pourtour d'un léger renflement terminal.

La forme de la capsule subit des variantes d'un spécimen à l'autre.

Parfois elle est plus dilatée au milieu du fuseau; parfois aussi la dilatation est moindre que dans les spécimens reproduits et le corps présente une zone cylindrique médiane plus ou moins large.

Dans le spécimen de la figure I9, le cytoplasme est irrégulièrement granuleux et étendu à toute la cavité de la capsule.

Dans celui de la figure 20, le cytoplasme, plus ramassé autour du noyau central, s'y montre composé principalement de globules uniformes que l'on prendrait pour des produits individualisés, dont l'action de l'iode révèle la nature azotée.

Distribution géographique. - Se rencontre en spécimens épars sur tout l'itinéraire de la Belgica.

\section{Fusopsis flagrifera sp. n.}

Planche VII, figures 7 et 8 .

Dans ces formes, le corps, moins svelte, se termine brusquement au pôle opposé à celui que surmonte la papule hyaline, pour y porter un faisceau de soies longues et 'flexibles disposées parallèllement coinme les lanières d'un martinet (flagrum, fig. 7), ou étalées en ombelle (fig. 8), sessiles sur le sommet arrondi (fig. 7) ou brièvement fusiforme (fig. 8).

Nous avons dû nous demander naturellement si nous n'avions pas affaire ici à des spécimens atrophiés et anormaux du type précédent; mais, à défaut d'éléments suffisants d'appréciation, il nous a semblé plus rationnel, dans un travail d'analyse comme celui-ci, de les recenser séparément pour ne rien préjuger d'une question dont il faut attendre la solution d'ailleurs.

Même distribution.

Fusopsis pauperata sp. n.

Planche VI, figures $2+$ et 25 .

Le corps est brièvement fusiforme, sans aucun appendice autre que la papule hyaline terminale, qui se montre toujours réticulée à la surface.

Le cytoplasme, plus vacuoleux, traduit des caractères de jeunesse qui nous auraient fait croire à des phases évolutives de l'un ou l'autre des types ici décrits si nous n'avions pas trouvé ces formes plus frustes exclusivement confinées dans la neige jaune, où elles ne sont pas très rares. 
Dans le spécimen de la figure 24, la membrane est mince et le cytoplasme très vacuoleux est exempt d'enclaves : témoignages d'un développement incomplet.

Dans le spécimen de la figure 25, les enclaves ont fait leur apparition et le noyau central est entouré d'une auréole claire, finement granuleuse.

S'il convient de n'y voir que des étapes de développement d'une forme mieux caractérisée à l'état parfait, c'est apparemment à la forme suivante, rencontrée dans le même milieu, qu'il faudrait les rattacher.

\section{Fusopsis polyedra sp. n.}

Planche VI, figures 22 et 23.

Corps à la fois fusiforme et polyédrique, comme résultant de deux pyramides réunies par leur base, avec alternance entre leurs facettes latérales.

D'un côté, le fuseau se termine en pointe mousse; de l'autre, il se couronne d'un gros bouton hyalin à membrane grillagée, qui surmonte lui-même un bouchon discoïdal résultant d'un fort épaississement local de la membrane capsulaire.

Le nombre des facettes latérales n'est pas constant, comme le montrent les figures des deux spécimens reproduits entre beaucoup d'autres observés.

Les angles solides qu'elles déterminent sont plus ou moins émoussés.

Le contenu capsulaire présente le plus souvent, outre un noyau assez volumineux, un endoplasme dense et finement granuleux et un ectoplasme vacuoleux dans lequel sont disséminés des corpuscules de calibre différent, de nature protéique sans doute (fig. 22).

Dans la figure 23, le cytoplasme n'a pas été représenté pour ne pas surcharger le dessin au détriment des caractères morphologiques extérieurs de l'organisme.

Distribution géographique. - C'est aussi principalement dans les neiges colorées que cet organisme s'est montré avec une certaine fréquence, ce qui nous fait croire que les rares spécimens observés dans les produits des pêches planktoniques y ont passé accidentellement, par fusion de la neige.

Mais que penser de cette station, assurément inattendue, pour un organisme de ce genre? Cette circonstance ne parait-elle pas plus propre à en obscurcir l'histoire qu'à l'éclairer, dans l'hypothèse que ces formes manqueraient d'autonomie et ne seraient que des organes libérés de formes organiques plus élevées dans l'échelle biologique? Comment expliquer que des œufs de Trématodes, vers parasites, ou d'autres espèces animales, soient précisément plus communs dans un milieu d'où les espèces productrices éventuellement de ces œufs semblent devoir ètre exclues? 


\section{Genre Piropsis g. n.}

Le caractère commun des formes groupées sous ce nom générique, c'est leur aspect piriforme.

\section{Piropsis Acinela sp. n.}

Planche VII, figure 9.

Le corps piriforme est chargé de soies raides, implantées perpendiculairement à la surface et assez régulièrement espacées. Ces appendices rappellent quelque peu les suçoirs des Acinètes, avec cette différence toutefois que ceux-ci sont autrement groupés.

Le bouchon membraneux terminal est surmonté d'une papule volumineuse dont la paroi mince est soutenue par des nervures grillagées à larges mailles.

Nous avons cru inutile de figurer le contenu cellulaire dont l'aspect rappelle celui des autres formes similaires.

Distribution géographique. - Çà et là, surtout dans la Mer de Kara. Très rare.

\section{Piropsis reliculata sp. n.}

Planche VI, figures 26 et 27.

Corps piriforme à membrane superficiellement reticulée, porteur autour du pôle basilaire de plusieurs prolongements, souvent trois ou quatre, hyalins, courts, terminés en arrachement, comme s'ils avaient été détachés d'un objet auquel ils auraient d'abord été soudés, à la façon d'organes fixateurs (figg. 26, vue stéréoscopique de còté). Nous n'avons toutefois jamais rencontré l'organisme ainsi fixé.

La structure réticulée de la membrane n'apparait guère à première vue, cachée qu'elle est par la projection optique des granulations du cytoplasme. Elle ne s'impose à l'observateur qu'à la suite de manipulations tendant à l'éclaircissement du contenu protoplasmatique ou mieux encore de son élimination.

La papule - bouton terminal du pòle antérieur - est petite, hémisphérique, hyaline. Elle surmonte, comme chez tous les organismes congénères, un bouchon résultant d'un épaississement local de la membrane.

La figure 27 en reproduit une coupe longitudinale optique, avec la membrane, le. noyau central, l'aire finement granuleuse qui entoure ce dernier, le cytoplasme périphérique chargé d'enclaves et deux des prolongements ou appendices basilaires, dont la structure est fibrillaire et dont la nature n'est pas sans analogie avec l'élastine.

Distribution géographique. - Mer de Kara. Assez rare. 


\section{Piropsis polita sp. n.}

Planche VII, figures 22 et 23 .

Son aspect rappelle tout à fait la forme précédente, à part la réticulation de la membrane, qui fait défaut. On y trouve les mêmes pieds basilaires, à structure fibrillaire, le plus souvent au nombre de trois.

La figure 22 en est une vue stéréoscopique, avec protoplasme visible par transparence de la membrane.

Le spécimen vide de la figure 23 est dépourvu de pieds. Nous croyons qu'il s'agit néanmoins de la même forme privée accidentellement de ces appendices, par usure, après avoir terminé le cycle de ses évolutions.

\section{Piropsis minuta sp. n.}

Planche VII, figure 29 .

Forme de très petites dimensions, que sa petitesse dissimule peut-être plus souvent que toute autre et que nous n'avons guère rencontrée que dans des crottins de Crustacés, si nombreux dans beaucoup d'échantillons planktoniques de la Mer de Kara.

Elle n'a pas d'appendices basilaires. Son contenu, vu par transparence après éclaircissement, est granuleux.

\section{Genre Spharopsis g. n.}

Chez les termes de cette lignée, le corps est le plus souvent sphérique, parfois légèrement ovale ou ellipsoïdal.

\section{Spharopsis heterosetosa sp. n.}

Planche VII, figures ro et ir.

Le corps est petit, sphérique, surmonté d'un bouton allongé et grillagé.

Des soies de deux sortes hérissent toute la surface : les unes plus courtes, filiformes, plus nombreuses; les autres longues, légèrement capitées, plus fortes, largement espacées (fig. Io, vue latérale stéréoscopique; fig. II, coupe longitudinale optique montrant les grosses enclaves du cytoplasme).

Distribution géographigue. - Très rare, dans la Mer de Kara. 


\section{Sphceropsis brevisetosa sp. $\mathrm{n}$.}

Planche VII, figure I2, vue extérieure.

Corps sphérique très volumineux; bouton terminal court et sillonné de faibles nervures; soies courtes, très nombreuses, régulièrement distribuées sur toute la surface.

Distribution Géographique. - Mers de Kara et de Barents. Très rare.

\section{Spharopsis longisetosa sp. n.}

Planche VII, figures 14 et 15.

Corps sphérique surmonté d'un bouton allongé et grillagé; soies longues, mollés, de valeur égale, assez denses; cytoplasme rempli d'enclaves volumineuses vers la périphérie, presque absentes dans l'aire hyaline qui entoure le noyau (fig. I5, coupe longitudinale optique; fig. I4, vue stéréoscopique).

Distribution GÉographique. - Plus fréquente que ses congénères, aussi bien dans la Mer de Barents que dans la Mer de Kara.

\section{Spharopsis nimbata sp. n.}

Planche VII, figure 21.

La coupe longitudinale optique figurée montre que les soies nombreuses insérées radialement, sur toute la surface du corps sphérique, aboutissent à une membranule supplémentaire concentrique à la capsule.

La forme allongée et grillagée du bouton hyalin terminal lui donne, de ce chef, un trait de ressemblance avec la précédente, ce qui nous fait demander si elle n'en serait pas une forme évolutive plus jeune? On pourrait supposer, en effet, que la dissolution de la membranule externe rendrait possible ultérieurement l'allongement des soies. Simple hypothèse, car l'isolement constant de ces formes mortes contrarie l'étude de leurs rapports éventuels.

Distribution Géographigue. - Très rare, dans la Mer de Kara.

\section{Spharopsis nivalis sp. $\mathrm{n}$.}

Planche VII, figure 13, coupe longitudinale optique.

Corps sphérique chargé de soies très courtes, très serrées, agglutinées en petites touffes, coalescentes au sommet. Le spécimen figuré ne présente pas de papule terminale, hyaline, mais dans d'autres on en voit une ébauche peu émergente. 
Le fait de n'avoir rencontré cette forme que dans les neiges colorées de la Mer de Kara légitime la dénomination que nous lui donnons, pour marquer cette station spéciale.

\section{Sphceropsis elliptica sp. n.}

Planche XXIII, figure 14 , vue stéréoscopique.

La forme est celle d'un ellipsoïde allongé. La papule terminale, pa, est assez peu développée et rehaussée de nervures. Les soies courtes et raides qui garnissent le corps sont assez espacées. Le contenu capsulaire, assez clair, vu par transparence dans la figure, présente des enclaves disséminées dans l'ectoplasme. Le noyau, $n$, central se montre constitué de grosses granulations et présente ainsi un aspect exceptionnel.

Distribution Géographique. - Très rare, çà et là, dans la Mer de Kara.

\section{Spharopsis echinata sp. n.}

Planche VII, figure $ı \overline{0}$.

Forme assez grosse, différant à première vue de ses congénères par la substitution aux soies de fortes épines plus clairsemées sur le cytoderme.

Ces épines, un peu renflées vers leur extrémité libre, s'y divisent en plusieurs pointes courtes et acérées.

Le bouton terminal est allongé et grillagé, comme dans plusieurs des formes recensées plus haut.

Distribution géographique. - Très rare; Mer de Kara.

\section{Spharopsis spumosa sp. n.}

Planche VII, figures 17,18 , 19 et 20.

L'aspect extérieur si spécial de cette forme, souvent de grande taille, lui vient de l'épais revêtement alvéolaire qui intéresse tout le corps, à l'exception du bouchon terminal. Ce bouchon hémisphérique n'est pas surmonté d'une papule hyaline, comme dans les représentants de la mème série.

La figure I7 reproduit la coupe optique longitudinale d'un spécimen dont la figure is montre la vue stéréoscopique apicale. On remarquera que la surface de la membrane écumeuse est vallonnée.

Les figures Ig et 20 se rapportent à un spécimen moins volumineux, mais dont le revêtement alvéolaire est plus épais et aussi plus régulier. 
La comparaison des deux coupes optiques (fig. 17 et I9) permettra de se rendre compte des variantes dans l'aspect du cytoplasme et, plus spécialement, dans la grosseur et la distribution des enclaves.

Distribution géographique. - Cette espèce n'est pas rare, mais ne se présente avec une certaine fréquence que dans les produits planktoniques originaires des côtes occidentales de l'île nord de la Nouvelle-Zemble, dans la Mer de Barents.

\section{Spharopsis lavigata sp. n.}

Planche VII, figures 24 et 25 .

Nous nous servons de cette étiquette pour désigner des formes qui, bien que dépourvues de toute surcharge extérieure, témoignent cependant, par leur forme et l'aspect de leur contenu cellulaire, des caractères fondamentaux de notre série des papulifères.

Les caractères négatifs qu'ils présentent vis-à-vis d'autres congénères plus ornẹs sont-ils spécifiques, ou sont-ils le fait accidentel de dégradations subies dans des circonstances inconnues? Nous ne savons.

Les figures 24 et 25 sont la reproduction, en coupe longitudinale optique, de deux exemplaires différents quelque peu par la taille et dépourvus l'un et l'autre de papule. Seul le bouchon membraneux terminal existe, plus ou moins atrophié.

La forme analogue, mais moins différenciée de l'organisme reproduit planche VII, figure 26, nous l'a fait joindre à ceux décrits plus haut, nous demandant s'il ne représentait pas une forme jeune de l'un d'entre eux.

On doit l'augurer de l'aspect vacuoleux et encore pauvre en enclaves de son cytoplasme, aussi bien que de l'absence de la papule terminale qui, dans l'hypothèse, devrait se différencier plus tard dans le prolongement apical du corps.

On ne peut dénier à tous ces organismes un air de famille qui doit leur faire reconnaitre une certaine analogie de nature.

Nous en avons recensé ici un certain nombre; dans la Mer Flamande, nous en avons observé d'autres dont nous nous défendons de parler dans ce travail pour n'en pas déranger l'unité; nous ne doutons pas non plus que les planktonistes en aient rencontré de leur côté, dans d'autres milieux, et de plus curieux peut-être encore, dont ils n'ont pas jugé à propos de relever les formes, pour des raisons que nous n'avons pas à rechercher. 
Leur grand nombre augmente donc l'intérêt que présenterait la connaissance de leur origine et de leur destinée. Si, comme il parait, ce ne sont pas des organismes autonomes, mais bien plutòt des produits organiques que leur aptitude à flotter peut entraìner loin de leur souche, il est peu probable que les microplanktonistes détiennent jamais, dans leurs matériaux d'étude, les éléments nécessaires à la solution des problèmes qu'ils soulèvent. Les organismes producteurs peuvent, en effet, n'être pas planktoniques comme leurs produits et, à cause de leur cantonnement dans d'autres milieux, n'avoir guère de chance conséquemment de se rencontrer dans un même coup de filet.

La lumière nous viendra plutôt des zoologistes, quand des circonstances favorables les mettront en présence des générateurs de ces produits organiques ou, avec plus de bonheur encore, en présence du fait physiologique de leur production elle-même.

En attendant, il parait utile de poser la question pour disposer les chercheurs à la résoudre.

\section{Groupe VI. - PROTOZOAIRES LORIQUÉS}

\section{Section I. - Tintinnides}

Il a fallu l'entreprise des recherches océanographiques et l'usage répandu du filet fin pour révéler l'existence de nombreuses formes de la famille des Tintinnides, à laquelle les zoologistes n'accordaient, jusque dans ces derniers temps, qu'une place imperceptible dans leurs ouvrages de systématique générale et même dans des publications d'un caractère plus spécial, n'ayant que les Protozoaires pour objet.

Actuellement il est déjà reconnu que cette famille autrefois méconnue tient, dans les eaux marines, une place éminente parmi toutes celles de l'embranchement des Protozoaires, tant par la variété des formes que par le grand nombre des individus qui constituent fréquemment l'élément dominant des produits de pêches microplanktoniques.

Le nombre des formes observées s'accrut rapidement et ce fut un sujet de trouble pour les micrographes qui durent prendre le souci de leur créer un état civil scientifique et de les classer clans des cadres systématiques acceptables, c'est-à-dire en harmonie avec les tendances légitimes de la taxinomie moderne, qui vise tout au moins à réfléter les affinités naturelles des organismes.

Il y eut des tàtonnements inévitables, tant à cause de la nouveauté de l'objet que des mauvaises conditions d'observation qui ne mettaient que rarement les objets vivants sous les yeux des observateurs. 
Ceux-ci sont rarement en situation de les observer dans leur milieu naturel, immédiatement après la capture, soit parce qu'ils ne sont pas sur les lieux, soit parce que les travaux de pêche en mer sont peu conciliables, à bord des bateaux, avec les exigences de calme et de durée des observations microscopiques.

D'autre part, ces organismes ne se prêtent ni au transport ni à la conservation, à l'état vivant, en dehors de leur milieu naturel.

Le biologiste ne se trouve guère en présence que de produits de pêche plus ou moins bien fixés, mais qui ne représentent que des cadavres auxquels la momification a enlevé les caractères physiologiques dont la manifestation serait d'un si grand secours pour mettre sur la piste de leurs affinités.

Si les réactifs fixateurs déforment généralement le corps mou et contractile de ces Protozaires, il n'en est pas de même de la capsule qu'ils se sont façonnée et dans laquelle ils trouvent un organe de soutien et de protection.

La plus grande fixité morphologique de ce test l'a tout naturellement imposé à l'attention des micrographes préoccupés de la recherche de leurs caractères distinctifs. Mais l'usage trop exclusif que l'on a fait des particularités morphologiques de cet organe accessoire laisse trop souvent planer des doutes sur la légitimité des espèces qu'on a créées sur cette base insuffisante d'appréciation.

On a beaucoup négligé l'habitant, pour ne se soucier que de l'habitation.

Nous nous efforcerons, de notre côté, de ramener davantage l'attention sur le premier, pour en signaler les faits physiologiques et les phases évolutives dont nos matériaux nous ont permis l'observation.

Toutefois, voulant conserver à ces notes leur caractère analytique et local, nous ne nous préoccuperons que secondairement du point de vue systématique, lequel ne pourrait être abordé plus avantageusement qu'on ne l'a fait qu'à la condition d'embrasser tous les représentants connus de cette famille cosmopolite.

L'analyse doit précéder la synthèse.

La dispersion mondiale de ses membres n'en a pas permis encore ce coup d'œil d'ensemble, mais les tentatives de classification déjà réalisées, pour imparfaites et insuffisantes qu'elles soient, ne constituent pas moins des acquisitions précieuses, très propres à guider les recherches dans les groupes régionaux des formes qui en ont fait l'objet.

Tel est le cas pour l'essai de Daday sur les Tintinnides du golfe de Naples (I), repris par JöRGENSEN, avec modifications (2), pour l'adapter aux formes observées par ce dernier dans les mers du nord.

(I) Daday : Monographie der Familie der Tintinnideen. .Titheilungen aus der Zoologischen Station zu Neapel, vol. VII, I $886-1887$.

(2) Bergens Museums Aarbog, IS89 et 1900. 
Nous y renvoyons le lecteur, sans intention ni de confirmer, ni d'infirmer les vues de ces auteurs, qui ne reposent, comme les nôtres, que sur l'observation de matériaux d'origine trop localisée pour autoriser des formules générales.

\section{Genre Cyttarocylis FoL.}

L'accord semble exister entre les micrographes pour prendre, comme caractéristique de ce genre, la membrane double de la capsule dont les deux lamelles, interne et externe, sont reliées par de minces cloisons disposées perpendiculairement aux premières pour les rattacher, en y déterminant un réseau alvéolaire qui se traduit superficiellement par un aspect nettement réticulé de la capsule.

Beaucoup de nos échantillons de pêche planktonique sont très riches en Cyttarocylis. De l'examen comparé des innombrables spécimens qui nous ont passé sous les yeux, il nous reste cette impression qu'il y a lieu de démembrer Cyttarocylis denticulata, dont le nom, basé sur un caractère commun, couvre tout un ensemble de formes similaires dans lesquelles il faut voir non pas toujours et seulement des modalités d'une espèce unique, mais bien de certains types spécifiques distincts, derrière lesquels disparaît la réalité objective du premier.

Cela résulte moins de l'examen des traits morphologiques de la capsule qui sont certainement d'une grande similitude, que de l'étude des particularités physiologiques dont la valeur taxinomique est bien supérieure.

C'est sans doute pour avoir négligé celles-ci que Jörgensen s'est laissé entrainer à la nomenclature d'un fouillis de variétés et de formes, où la systématique a plus à perdre qu'à gagner. Nous ne le suivrons pas dans ce dédale sans issue.

Dans la mesure de nos observations sur les matériaux de la Belgica, nous distinguerons, parmi les formes à capsule régulièrement réticulée en mailles hexagonales, les types spécifiques suivants :

\section{Cyttarocylis gigantea Brandt.}

Planche VIII, figures I à II; planche IX, figures I à 5 ; planche X, figures 8 et 9 ; planche XI, figure I 5 .

Littérature. - Cfr. Bibliotheca zoologica, Heft. 20, Lfg. 2, IV. K. Brandt : Die Tintinnen. Stuttgart, Igo6.

Ce type spécifique est sujet, comme les autres du reste, à des variantes individuelles dont les caractères mobiles pourraient, à première vue, détourner l'attention au détriment des caractères spécifiques, dont la constance est la seule garantie de valeur taxinomique. 
Nous considérons comme forme idéale, si l'on peut dire, de l'espèce, celle reproduite planche VIII, figure $I$.

La capsule, longuement cylindrique, s'atténue en cône à la base et se termine en un prolongement étroit plus ou moins long, souvent flexueux et toujours sensiblement obtus au sommet.

L'orifice antérieur est brièvement évasé et garni de très nombreuses dents, courtes, obtuses, qui accentuent l'évasement de l'orifice capsulaire.

Le nombre des dents, une cinquantaine au moins (fig. 4, pl. IX), est toujours supérieur à celui des autres' types et pourrait constituer à lui seul un caractère spécifique, s'il ne faisait parfois partiellement ou même complètement défaut par la chute accidentelle ou physiologique, nous ne savons, de ce couronnement.

Il n'est pas rare, en effet, de rencontrer des capsules partiellement dégarnies de ces dents, comme en a (fig. 5, pl. VIII), ou même complètement dépourvues de ces appendices, comme le spécimen de la figure 3, même planche; circonstance fortuite sur laquelle certains auteurs se sont basés pour créer des variétés edentata, dont la valeur taxinomique nous parait nulle.

On assiste, en effet, assez fréquemment à la chute de ce couronnement denté, dont nous avons montré la préparation dans la figure 4, où on le voit déjà détaché, en $a$, sur une grande partie du pourtour de l'orifice. Nous ne saurions dire que la cause de cette avarie soit plutôt mécanique que physiologique; mais elle est réelle, dans des circonstances dont l'indétermination n'enlève rien au caractère accidentel du fait, même pendant la vie de l'organisme.

Nous n'attachons pas plus d'importance à d'autres déformations qui, sans doute, ne sont que le résultat d'un traumatisme dont il n'est pas possible de préciser la cause. Telle, la déformation présentée par le spécimen (fig. I, pl. IX) dont nous n'avons reproduit que la partie inférieure. Il présente, en $t$, des excroissances de la capsule, qui semblent dues à un travail de régénération hypertrophique de parties lésées et trouées, peut-être à la suite d'une irritation parasitaire.

Telle aussi l'avarie causée, en $t$ (fig. 2, pl. IX), qui se traduit par l'atrophie du prolongement terminal et des solutions de continuité dans la région basale de la capsule.

Telle encore la forme anormale de la figure 3, même planche, qui semble se rapporter à un individu vieilli chez lequel se traduisent, du côté antérieur, des caractères de sénilité ou des manifestations pathologiques, qui ont produit un évasement anormal de la capsule et une certaine irrégularité dans la structure alvéolaire. Cette irrégularité se traduit même plus bas, dans le corps de la capsule, par des alvéoles plus grands éparpillés dans la trame réticulée. En arrière on observe une malformation de la base qui s'est déjetée latéralement en prenant une forme irrégulière et spiralée, en dessous d'un trou, $t$, qui résulte évidemment d'une lésion.

Dans les individus normaux la structure alvéolaire présente une grande régularité due à la forme hexagonale des alvéoles et conséquemment de la distribution de ceux-ci 
suivant trois directions qui se coupent sous des angles de $60^{\circ}$ et dont l'une est précisément parallèle à l'axe longitudinal de la capsule, ce qui tient à l'orientation des hexagones.

Mais cette régularité est parfois faussée par la présence d'alvéoles non hexagonaux interposés aux autres dont ils détruisent l'harmonie d'arrangement, comme dans le spécimen reproduit figure 6 , planche VIII. L'aspect est celui d'un réseau à mailles polyédriques inégales. Nous ne pouvons davantage préciser la cause de cette anomalie, qui ne suffit pas à cacher les caractères que nous avons donnés comme spécifiques.

Le spécimen en question a, en outre, la base plus arrondie, comme aussi celui de la figure 7. Le prolongement terminal est plus raccourci, plus émoussé, caractère qui se représente encore dans la figure 2, même planche.

La figure I5, planche XI, reproduit le spécimen le plus petit qu'il nous a été donné d'observer. On aura ainsi une idée des écarts de grandeur que l'espèce peut présenter dans les milieux explorés par la Belgica, si l'on observe que les dimensions de la figure I, planche VIII, ne sont pas extrêmes, mais sont souvent dépassées par d'autres exemplaires que nous n'avons pas voulu figurer, pour rester plutôt dans la note moyenne de l'espèce.

On trouverait difficilement des caractères spécifiques dans le Protozoaire propriétaire de la capsule, à cause de la contraction que les milieux fixateurs lui font subir, en le figeant dans des poses occasionnelles que l'animal présentait au moment où'le réactif l'a saisi.

La figure 2, planche VIII, en montre un exemplaire en état d'épanouissement incomplet, avec sa couronne orale de lamelles fibrillaires, co, son noyau, $n$, le seul visible en l'occurrence, et ses nombreuses inclusions, ic, témoins de son régime alimentaire.

On y voit figurer, entre autres, sous forme bien reconnaissable, un Tintinnide, Amphorella norvegica Daday et deux Diamylon nivale nobis.

Cette petite Algue paraît ètre l'aliment favori non seulement des Cyttarocylis, mais aussi de tous les autres Tintinnides et mème de tous les Protozoaires habitant plus spécialement la Mer de Kara, au moins pendant la saison estivale, celle de la campagne arctique de la Belgica, celle aussi, sans doute, de la plus grande activité physiologique de ces organismes.

\section{Sporulation}

Mais il est un fait qui milite victorieusement en faveur de la spécificité réelle de Cyttarocylis gigantea, s'il est vrai que nous pouvons identifier la forme dont il s'agit ici avec celle ainsi dénommée par BRANDT : ce que nous ne pouvons garantir à cause de la description trop sommaire qu'il en donne. 
Ce fait, c'est un phénomène de sporulation que cette forme est seule à présenter et présente abondamment dans les circonstances de temps et de lieu où les pêches l'ont surprise.

Certains échantillons, notamment le $\mathrm{n}^{\circ} 228$, originaire du large de l'Océan Glacial par $75^{\circ}$ Lat. $\mathrm{N}, 42^{\circ}$ Long. E, 8 septembre, nous la présentent presque exclusivement à cette étape intéressante de son évolution, alors que les spécimens d'autres formes qui s'y trouvent mélangées et dont nous parlerons plus loin, ne présentent pas cette phase évolutive dernière qui prépare l'organisme au repos hivernal.

Comment procède cette sporulation?

Nous en avons vainement cherché les phénomènes précurseurs, nous trouvant d'emblée en présence $d u$ fait accompli. Le corps du Protozoaire est divisé en un nombre variable de spores qui se sont partagé le corps protoplasmatique de l'individu, négligeant des fragments, portions résiduaires du partage $(r$, dans plusieurs figures des planches VIII et IX), qui n'ont pas trouvé leur utilisation dans le processus de fragmentation du protoplasme maternel.

Une pêche faite un peu plus tôt ou à un autre point géodésique de l'Océan Glacial nous aurait sans doute fourni des éléments complémentaires d'étude; mais cette pêche n'a pas été faite et nous ne pouvons que regretter l'absence des précieux matériaux qu'elle aurait pu nous procurer.

En fait, nous voyons ici l'animal transformé en spores quiescentes, sp, dont le nombre varie de un (fig. I, pl. IX) à dix (fig. 2, pl. IX), de grosseur variable et souvent inégale dans le même individu (fig. 6, pl. VIII), dotées d'une membrane épaisse, $m$, pourvues d'un noyau, $n$, d'un gros globule huileux, $h$, et, chez les plus jeunes, de nombreux granules, $f$, que leur coloration en bleu sous l'action de l'iode nous force à considérer comme de nature féculente (fig. II, pl. VIII et mieux fig. 5, pl. IX).

A titre documentaire, comparer :

Figure I, planche IX, avec une seule spore, sp.

Figure 3, planche VIII, avec trois spores, sp, dont la membrane, $m$, le noyau, $n$, le globule huileux, $h$, et le résidu, $r$.

Figure 7 , planche VIII, avec quatre spores; mêmes désignations.

Figure 6, planche VIII, avec six spores de grosseur inégale; mèmes désignations.

Figure 8, planche VIII, avec huit spores figurées en dehors de la capsule.

Figure 9, planche VIII, avec deux spores, dont l'une mùre, $a$, l'autre, $b$, incomplète et comme avortée et un résidu volumineux, $r$, de structure écumeuse, $c$.

Figure Io, planche VIII, avec trois spores encore jeunes où apparaissent le noyau, $n$, des enclaves, $e c$, non encore définitivement modelées et des résidus, $r$, inutilisés.

Figure II, planche VIII, avec trois spores encore jeunes, dans lesquelles, malgré la présence de corpuscules féculents, on aperçoit déjà le globule huileux, $h$, qui pour- 
rait bien provenir de la transformation progressive de la fécule et d'autres produits simples de l'activité du protoplasme sous l'influence des ferments solubles qui contribuent à leur mise en réserve.

Figure 2, planche IX, avec dix spores, plus petites, parce que plus nombreuses sans doute. Le traumatisme de la base de la capsule n'a pas empêché la sporulation.

Figure 5, planche IX, avec deux spores seulement, chez lesquelles l'iode révèle les nombreux corpuscules amylacés dont les produits de transformation iront probablement grossir le globule huileux, $h$, dans la suite, car les spores mùres ne présentent plus d'enclaves féculentes.

On comprendra aisément que c'est par recherche de la simplification que nous nous sommes abstenu de dessiner la capsule dans les dernières figures ci-dessus.

Dans les premières nous avons supposé un arrachement d'une partie de la paroi capsulaire, pour figurer les spores en place sans les cacher sous les détails de structure de cet organe.

Remarque. - Cette espèce ne nous a pas présenté d'exemples de multiplication de l'organisme par fissiparité : phénomène encore fréquent, à cette même époque, chez les autres formes de Tintinnides. La période en est close pour celle-ci. Ce caractère négatif s'ajoute à ceux rappelés plus haut pour confirmer la spécificité de cette forme.

\section{Cyttarocylis cuspidata sp. n.}

Planche VIII, figures I2 à I7; planche X, figures 8 à 12 ; planche XI, figures 13 et 14 .

Ce deuxième type spécifique présente des dimensions habituellement plus grandes encore que le précédent.

Longuement cylindrique, à la façon d'un tube à essai ou d'un manchon de bec Auer, dont elle semble une réduction microscopique, la capsule s'arrondit d'ordinaire brusquement à la base. Du milieu de celle-ci émerge un prolongement conique et pointu, très court le plus souvent (fig. I2, pl. VIII), parfois mème si réduit qu'il n'est pas visible sous certaine orientation (fig. IO, pl. X), mais pouvant aussi se traduire sous un développement plus grand dont les figures I3, I4, I5, i6 marquent la gradation ascendante, d'après un certain nombre de spécimens dont nous n'avons reproduit que la partie inférieure, seule utile en 'l'occurrence.

Quel que soit son développement, cet appendice terminal conserve sa direction axiale et sa pointe acérée (cuspis), dont nous tirons le nom de l'espèce.

Le léger évasement qui s'observe à l'orifice antérieur de la capsule intéresse, non pas la paroi de celle-ci, qui reste sensiblement cylindrique jusqu'au bout, mais les dents qui la couronnent et qui sont plus ou moins déjetées en dehors.

Ces dents, toujours moins nombreuses que dans l'espèce précédente, trente-cinq à quarante seulement, sont beaucoup plus longues, pointues au sommet, élargies à la 
base. Elles fournissent un bon caractère qui, combiné avec celui que fournit la forme si spéciale de la base de la capsule, suffit toujours à diagnostiquer l'espèce.

Il n'est pas rare de rencontrer des capsules dont la couronne dentaire est rendue irrégulière par la présence de dents plus petites, comme en $x$ (fig. 9, pl. $\mathrm{X}$ ), qui semblent résulter d'un processus de régénération, à la suite d'avaries subies antérieurement dans ces appendices fragiles.

La figure I7, planche VIII, montre, derrière un arrachement de la capsule incomplètement dessinée, le Protozoaire contracté au fond de sa loge, avec sa couronne orale, co, de lamelles striées, au milieu de laquelle fait saillie la protubérance centrale; son noyau, $n$; des inclusions alimentaires, $i c$, et une vacuole sphérique, $v$.

Dans la figure 8, planche $\mathrm{X}$, l'organisme a été surpris presque en plein épanouissement, avec sa couronne orale, co, à moitié étalée, sa bouche, $b$, ouverte, son œsophage, ces, dilaté et longuement prolongé dans le corps cytoplasmatique, deux gros noyaux, $n$, des enclaves ou inclusions, $e c$.

Le prolongement du corps vers l'extrémité inférieure du tube permet de s'assurer qu'il en occupait toute la capacité avant que les réactifs fixateurs n'en eussent déterminé la plasmolyse, particulièrement dans cette partie du corps qui est plus vacuolaire péndant la vie.

\section{Division SCISSIPARE}

Cette espèce, particulièrement commune dans les hautes régions de la Mer de Barents, présente de fréquents exemples de division ou de multiplication scissipare, dont les figures I I et I2, planches $X$, et la figure I3, planche XI, suffisent à illustrer le processus qui, à quelques différences près, se retrouve le mème dans la plupart des Tintinnides des pèches de la Belgica.

Dans la figure II, planche $\mathrm{X}$, on voit le corps bien amplifié du Protozoaire muni de deux noyaux, $n$, issus d'une division préalable de cet organe et distancés l'un de l'autre. Entre les deux noyaux, il s'est formé sur les flancs du corps une nouvelle couronne orale, coj, dont les membranules striées sont d'abord insérées perpendiculairement à la paroi du corps, autour d'une dépression qui deviendra l'ouverture buccale du jeune individu. Cette couronne orale, présentée ici de profil, se montre de face dans la figure I2, même planche, empruntée à un autre individu arrivé au même stade.

Plus tard, la dépression, déjà visible dans ces figures, qui se traduit au-dessus de la couronne orale jeune, prépare la séparation des deux individus, filles d'une mère commune, qui s'en partagent les dépouilles et en perpétuent l'existence.

Cette séparation, presque complète dans la figure I3, planche XI, résulte, comme on peut le constater ici, d'un étranglement favorisé par un étirement de la dernière 
attache qui unit encore les deux produits de la division. Un dernier effort donnera la liberté à l'individu supérieur, qui, avec un désintéressement tout fraternel, abandonnera la loge maternelle à son frère jumeau, pour courir les risques et les difficultés d'une installation nouvelle.

On s'imagine aisément comment, chez le jeune individu resté en place, la couronne orale encore excentrique, dans le même exemple, prendra plus tard sa position normale en même temps que se répareront les lésions causées par la scissiparité.

Celle-ci ne semble pas amener le moindre trouble dans les fonctions nutritives de la souche. Les inclusions alimentaires qui peuvent se trouver dans l'organisme mère, au moment critique de la division, deviennent, au petit bonheur, le partage des deux organismes frères. C'est ainsi que dans l'exemple de la figure I3, planche XI, le sujet inférieur a dù hériter des grains de fécule, $f$, qu'on y voit et qu'il n'a pas pu absorber lui-même parce que ses organes propres de préhension n'ont pas encore $\mathrm{pu}$ fonctionner, tandis que le sujet supérieur reste lesté d'un squelette siliceux de Distephanus speculum, $D$, qui ne lui constitue, à coup sủr, qu'une bien maigre provende. Il est vrai, d'autre part, que celui-ci présente toujours, non loin de son sommet, de nombreux microsomes, $m$, d'une nature spéciale, dont il serait intéressant de rechercher la composition et la signification.

Ne serait-ce pas des matériaux déjà préparés en vue de l'édification rapide d'une nouvelle loge que rend nécessaire l'expropriation à laquelle il a dû consentir? C'est un fait digne de remarque que les échantillons, parfois'si riches en individus de cette espèce qui se divisent, n'en présentent pour ainsi dire pas d'exemplaires dépourvus de capsule. N'est-ce pas parce que la reconstitution de cette dernière est rendue facile et rapide par la mise en réserve préalable de produits immédiatement utilisables à cet effet?

Nous ne sommes pas actuellement en mesure de résoudre cette question qui nécessiterait des recherches sur le vif, mais il nous paraît utile de la poser aux observateurs de l'avenir.

\section{Sporulation}

Le phénomène de sporulation si commun en septembre 1907 dans Cyttarocylis gigantea ne s'observe guère dans Cyttarocylis cuspidata rencontré à la même époque, dans les mêmes milieux. C'est, à notre avis, une preuve de plus de la distinction spécifique de ces deux formes.

Nous reproduisons (fig. Io, pl. X) l'un des rares spécimens observés chez lesquels on peut constater la production d'un phénomène analogue. On y voit, en effet, deux groupes de spores géminées, sp, de grosseur inégale, dont la membrane encore très mince semble témoigner de la jeunesse de ces produits. 
Dans le protoplasme de chacun d'eux on remarque un globule huileux, mais le noyau n'apparait pas distinctement. Les rares observations faites à ce sujet nous disposent à penser que cette espèce présente aussi un mode de sporulation analogue à celui dont l'espèce gigantea nous a fourni tant d'exemples, mais dans des conditions qui ne sont pas ici pleinement réalisées.

Remaroue. - Les figures 8 et io, planche $\mathrm{X}$, que nous avons rapprochées à dessein, permettent de saisir les variantes extrèmes de la forme de la loge, exempte de prolongement basal (fig. Io), pourvue d'un long appendice pointu (fig. 8).

Quelle peut ètre la signification du développement relatif de cet appendice?

Faut-il y voir une conséquence de l'àge de l'individu? Nous ne le pensons pas, car les spécimens qui sont en division et qui, pour cette raison, doivent être considérés comme adultes, sont souvent brièvement terminés (fig. II et I2, pl. X).

Nous croyons plutòt qu'il s'agit là d'un caractère individuel contracté par l'organisme dès le début de l'édification de la capsule.

\section{Multiplication endogìne?}

Il nous reste à dire un mot de ce que nous considérons, jusqu'à plus ample information, pour un troisième mode de multiplication de Cyttarocylis cuspidata et qui se traduit, à l'époque des récoltes que nous avons sous la main, comme en figure I4, planche XI, par une déformation complète du corps de l'animal, dont une partie, $r$, semble devoir ètre sacrifiée comme impropre à l'usage, tandis que l'autre s'entoure d'une membrane délicate, $m$, prend la forme d'un boudin incurvé et se segmente en une foule de petits fragments lenticulaires, $z$, dont nous n'avons pu suivre ni la séparation, ni l'évolution subséquente.

Ces petites cellules nucléées, étroitement pressées les unes contre les autres et disposées en spirale comme les écailles d'un strobile, suggèrent l'idée de spores qui prendraient naissance simultanément par voie endogène, en vue d'un phénomène d'essaimage.

Nous ne savons rien de ce qui doit en résulter; bien que nous ayons vu d'assez nombreux exemples de cette curieuse formation, non seulement dans l'espèce actuelle, mais aussi dans celle dont il sera question plus loin.

Il semble donc qu'il y a là l'indice d'une manifestation physiologique commune à plusieurs espèces de Tintinnides et subordonnée à des circonstances qui nous sont inconnues, mais que d'autres observations permettront sans doute de déterminer. 


\section{Cyltarocylis hemifusus sp. n.}

Planche VIII, figures I8 à 22; planche IX, figures 6 et 7 ; planche $\mathrm{X}$, figure 13 ; planche XI, figures 16 et 17 .

Un coup d'œil jeté sur toutes les figures empruntées à cette espèce donnera au lecteur, mieux que toute description, une impression nette de sa physionomie et des limites de ses variations morphologiques.

Sa forme rappelle celle de la grande moitié d'un fuseau qui aurait été coupé transversalement un peu au delà du milieu de sa longueur.

L'orifice antérieur présente, au-dessus d'un faible étranglement local, un léger évasement qu'accentue la couronne dentaire. Celle-ci compte d'ordinaire de 35 à 40 dents longues, étroites du sommet jusqu'à la base, ò̀ elles ne présentent qu'un faible empâtement.

La paroi capsulaire est plus mince que dans les deux types précédents, sa texture alvéolaire plus fine, plus délicate.

La capsule, terminée à la base en fuseau court (fig. I9, pl. VIII), se prolonge plus souvent en une corne effilée, tantôt creuse jusqu'au sommet (fig. I 8 et 22, pl. VIII), tantôt réduite à un rostre très étroit (fig. 20, pl. VIII). Plus rarement la base est tronquée (fig. I6, pl. XI).

Sous ces multiples aspects, ce type spécifique est le plus répandu dans les échantillons provenant de la Mer de Kara et du Détroit de Matotchkine.

Les figures qui s'y rapportent dans les planches VIII, IX, X et XI reproduisent quelques-unes des attitudes les plus communément prises par le Protozoaire sous l'action des agents fixateurs.

Nous les passerons rapidement en revue.

Planche VIII, figure I8, capsule vide de la forme la plus ordinaire.

Planche VIII, figure Ig, capsule terminée inférieurement en fuseau court et montrant, derrière un arrachement artificiel de la paroi, l'animal contracté, avec sa couronne orale, co, ses inclusions alimentaires, ic, et son noyau situé plus bas.

Planche VIII, figure 20, capsule prolongée à la base en un long rostre étroit. Du milieu de la couronne orale contractée, co, émerge la protubérance orale, po.

Le corps renferme aussi des inclusions alimentaires de nature indéterminée, ic.

Le noyau situé plus bas occupe aussi sa place habituelle, dans la région inférieure.

Planche VIII, figure 2I. Individu en expansion presque complète et occupant toute la capacité de la loge. La couronne orale, co, n'est que légèrement contractée, la bouche, $b$, dilatée en forme d'entonnoir communique, par une sorte de rétrécissement œsophagien, avec une grande vacuole digestive dans laquelle se reconnaissent aisément plusieurs Diamylon nivale, D., petite Algue nivicole dont ce Tintinnide fait une grande consommation, aussi bien que ceux de ses congénères qui vivent dans les mêmes eaux. 
Le noyau, n, volumineux montre un filament chromatique pelotonné, qui, par son aspect, témoigne d'une tendance à la division.

Planche VIII, figure 22. Capsule à base creuse longuement atténuée, renfermant un indiridu rajeuni par suite d'un phénomène de division dont nous avons figuré quelques étapes chez l'espèce cuspidata.

La couronne orale, co, de nouvelle formation n'a pas encore pris la position définitive qu'elle devra présenter, lorsque le jeune individu aura réparé la lésion causée par le départ de son frère jumeau. Son corps excentrique en garde encore la trace, sous la forme d'un lobule protoplasmatique dirigé vers l'orifice de la loge. En $n$, deux gros noyaux d'aspect anormal, dont la présence fait présager une nouvelle division.

Planche IX, figure 6. Individu amputé de son train antérieur. De la partie postérieure restante, qui renferme encore un noyau, $n$, émerge un corps de nature énigmatique, $x$, dont le caractère nous échappe, aussi bien que la signification.

Ayant observé cette bizarrerie plusieurs fois, ce qui semble exclure l'idée d'un fait purement accidentel, nous avons cru utile de le signaler, à titre documentaire, en attendant d'autres recherches.

Planche IX, figure 7. Individu présentant un phénomène physiologique du genre de celui dont il a été question plus haut, à propos de l'espèce cuspidata et dont nous avons donné un exemple propre à cette espèce dans la figure I4, planche XI.

Ici aussi, le corps contracté de l'organisme s'est divisé en nombreux segments, z, dont la séparation semble plus prochaine, la fine membrane extérieure commune à l'ensemble ayant déjà disparu.

A rapprocher de la figure $\mathrm{I} 7$, planche XI, qui en reproduit un autre exemple dans la mème espèce. La membrane commune, $m$, s'observe encore ici, malgré son extrème finesse, autour du groupement strobiliforme des produits fragmentaires, $z$, qui se sont partagé la masse protoplasmatique primitive.

Nous en laissons l'interprétation à ceux auxquels le hasard des circonstances en fournira les moyens.

Planche $\mathrm{X}$, figure I3. Individu chez lequel la division du noyau, $n$, a déjà préludé à la division du corps. La couronne orale, co, entoure la bouche infundibuliforme, $b$, qui se prolonge en un osophage dilaté, as.

Planche XI, figure I6. Capsule à base légèrement tronquée et, à cause de cela, de forme quelque peu exceptionnelle. L'animal contracté dans une attitude spéciale laisse voir sa protéburance orale, po, et le prolongement œesophagien, ces, de la bouche. A noter aussi la forme contractée de la couronne orale, co, et l'aspect grossier du noyau, $n$.

Planche XI, figure I7. Phase d'un phénomène de multiplication endogène? Voir plus haut. 
On remarquera que nous ne faisons aucune allusion à la production de spores quiescentes. C'est que cette forme spécifique ne nous a rien offert de semblable, malgré les recherches dirigées expressément dans cette voie, en vue d'apprendre si des différences physiologiques corroboraient la distinction spécifique suggérée par les allures morphologiques. Il ne s'en suit pas qu'il faille refuser à Cyttarocylis hemifusus une aptitude constatée chez ses congénères. On n'en peut inférer qu'une chose : c'est que cette aptitude ne s'exerce éventuellement que dans d'autres circonstances.

Remaroue. - La confusion qu'une vague analogie de forme et de structure a entretenue entre les trois types précédents et d'autres qui sont étrangers à nos matériaux actuels, au profit de Cyttarocylis denticulata, n'a pas pu ètre dissipée, ni mème diminuée par la création de nombreuses variétés fondées sur des caractères aussi futiles qu'arbitraires (I).

Il faut abandonner cette désignation trompeuse qui a donné une fausse sécurité aux planktonistes dans la détermination usuelle des formes qui s'accommodent d'une diagnose aussi simple que peu précise et ne la maintenir, si l'on veut, qu'à titre d'étiquette d'un sous-genre embrassant les formes denticulées, dont la structure capsulaire est très accusée et caractérisée par la forme régulière, hexagonale, des mailles alvéolaires.

\section{Cyttarocylis spiralis sp. n.}

Planche IX, figures 18,19 , 20 ; planche XIV, figure 5.

Cette forme ne rentre évidemment pas dans le groupe des précédentes.

La membrane de la capsule est encore alvéolaire dans l'intervalle des deux lamelles peu distantes qui la constituent; mais le réticulum dessiné à la surface est ici irrégulier, confus et en outre très faiblement marqué. Sa résistance est aussi beaucoup moindre et les plus faibles pressions suffisent à la déformer.

Un sillon spiralé qui part de la base y décrit des tours de spire dont le nombre augmente avec l'accroissement progressif de l'organe. Les bords de l'orifice sont d'habitude finement frangés, mais ne portent pas de dents proprement dites.

Dans nos matériaux, où l'organisme est d'une rareté relative, les loges sont vides le plus souvent (fig. is et 20, pl. IX).

La figure I9, même planche, en montre un spécimen habité par l'animal contracté, avec sa couronne orale, $c o$, sa protubérance orale, po, deux noyaux, $n$, un 
cytoplasme vacuoleux renfermant des inclusions. A la base, un petit pédicelle étroit le rattache au fond de la loge.

La figure 5, planche XIV, en est un autre, avec l'animal moins contracté et montrant mieux la structure de la couronne orale, co, avec les nervures qui la soustendent et qui s'accusent plus fort en se prolongeant plus bas du côté du corps qui correspond au creux cesophagien. On y voit aussi deux noyaux, $n$, de même valeur. La paroi de la capsule, cp, est exceptionnellement mince.

La difficulté d'en percevoir les détails de structure aurait-elle fait prendre cette forme pour une Amphorella par Jörgensen? (I889). Son Amphorella ampla (fig. $4^{\text {a-b }}$, pl. III) le ferait croire.

\section{Cyttarocylis sp.}

Planche XXIIl, figure 12.

Il ne nous parait pas certain qu'on puisse identifier à la forme précédente, plus ou moins courte, un peu ventrue et arrondie à la base, la forme figurée ici, plus longue, atténuée à la base, dont les zones supérieures d'accroissement sont marquées de limites irrégulièrement sinueuses en rapport avec la trame réticulée de la capsule qui est beaucoup mieux dessinée.

Nous en avons rencontré trop peu d'exemplaires, toujours vides du reste, pour pouvoir asseoir un jugement fondé sur la valeur de ces caractères morphologiques différentiels, qui pourraient n'être qu'accidentels.

Nous réservons notre appréciation jusqu'à plus ample information.

Remarque. - Par sa forme en dé à coudre et la maillure irrégulière de sa capsule, Cyttarocylis spiralis, décrit plus haut, semble ménager la transition aux Ptychocylis qui se réclament de caractères similaires sans qu'on puisse dire, dans tous les cas, ceux qu'il convient, en outre, de leur reconnaitre comme exclusivement propres et susceptibles de créer une ligne de démarcation nette entre les deux genres. Dans les travaux des auteurs les preuves abondent de leur indécision à ce sujet.

En fait, cette indécision ne présente d'inconvénient que pour la nomenclature que l'on voudrait voir plus unifiée et s'adapter à des découpures mieux tranchées du monde organique.

Dans l'occurrence, cette difficulté ne se pose pas, puisque les espèces de Ptychocylis que nous avons à présenter légitiment assez leur distinction des Cyttarocylis par leur silhouette si spéciale et la forme si particulière de leur orifice capsulaire rétréci et denticulé. 


\section{Genre Ptychocylis Brandt}

Nos matériaux d'étude sont presque tous très riches en représentants de ce genre, mais, autant il est facile de les reconnaitre comme tels, gràce à la forme classique de la capsule, autant il est difficile d'y trouver les bases d'une distinction spécifique, que 'l'on soupçonne cependant derrière les nombreuses modalités de détails, qu'il semble impossible de rapporter à un unique type spécifique : le Ptychocylis umula Brandt ou le Tintinnus urmula Claparède et Lachmann.

Ou bien il faut accepter, pour celui-ci, l'idée d'un polymorphisme exagéré, dont peu d'entités organiques donnent l'exemple, ou bien il faut chercher à démembrer le groupe des formes rangées longtemps sous une étiquette commune, à défaut d'en avoir fait suffisamment encore l'étude critique comparée.

Celle-ci ne serait du reste possible que si un même micrographe disposait de matériaux de toutes provenances, lui permettant de jeter d'abord un coup d'œil d'ensemble sur toutes les modalités du type et d'y faire ensuite des coupes aussi rationnelles que possible, en se laissant guider par ses impressions personnelles, fruit d'un examen réfléchi des formes nombreuses référables au genre en question.

Ces coupes, fussent-elles purement artificielles, constitueraient tout au moins un précieux instrument d'analyse au service des planktonistes, car nous doutons qu'on puisse réaliser ce desideratum en tablant sur les illustrations des auteurs. Ces figures avec leurs inexactitudes parfois, leur faible degré d'approximation presque toujours, et le facies spécial qu'elles doivent aux différents procédés d'exécution auxquels les auteurs ou les professionnels de la lithographie ont eu recours, sont plus propres à égarer l'imagination qu'à guider l'esprit dans la recherche des affinités réelles, au milieu du dédale de ces formes si semblables apparemment et cependant si diverses réellement.

Chacun sait très bien la difficulté qu'il y a de rapporter sûrement certains organismes microplanktoniques vus en nature à des formes décrites ou mème figurées dans des publications sur la matière.

C'est éminemment le cas pour les Ptychocylis, pour lesquels la création de variétés et de sous-variétés n'a fait germèr aucune idée de coordination synthétique, bien qu'il faille reconnaitre aussi que l'élévation de certaines formes au rang d'espèces n'a pas été suffisamment légitimée.

C'est que l'étude comparée des formes de la capsule seule est incapable de fournir un critérium certain de la distinction des espèces, aussi longtemps que celle-ci ne sera pas corroborée par d'autres caractères plus intimes, des caractères d'ordre physiologique.

Pour ces raisons, nous délaisserons quelque peu le point de vue systématique pour nous attacher plutòt à traduire les caractères physiologiques des organismes dont il sera question ici, à travers celles de leurs phases évolutives que nous avons pu observer. 
Planche VIII, figure 23. Forme la plus commune dans la Mer de Kara.

Capsule plutôt petite, ramassée, presque isodiamétrale, à parois minces, légèrement réticulée, à orifice rétréci et dentelé comme toutes les formes du groupe désigné primitivement sous le nom de Ptychocylis zmmla.

L'animal, à peine contracté, remplit toute la cavité de sa loge et se montre gorgé d'inclusions alimentaires, ic, parmi lesquelles des cellules de Diamylon nivale, $D$, témoignent du ròle prédominant que jouent ces petites Algues nivicoles dans l'alimentation des Tintinnides de ces parages : fait déjà signalé à propos d'autres espèces. Les inclusions susdites cachent le ou les noyaux autres que celui de ce que nous appellerons la souche, s, et que l'on observe d'habitude au tond de la capsule, dans la partie la plus rétrécie de celle-ci.

Planche VIII, figure 24. Mème milieu. Capsule beaucoup plus allongée, plus svelte, avec un long prolongement inférieur en forme de còne tronqué au sommet.

Le corps contracté de l'animal recèle aussi des inclusions alimentaires, ic, avec Diamylon nivale. Le noyau strié, $n$, semble disposé à la division.

Planche VIII, figure 25. Capsule d'aspect plus cylindrique et très rare dans nos matériaux de mème origine. Au fond de la loge on aperçoit un corps protoplasmatique réduit et muni d'une sorte de noyau, s, qui persiste en cet endroit après la vidange de la capsule provoquée par des causes que nous ignorons.

Ce corps, qui d'habitude occupe cette position dans l'organisme normal et qu'un peu d'attention permet d'y découvrir, bien qu'il ne soit pas délimité du cytoplasme voisin, nous semble jouer le ròle de souche, s, capable de reconstituer le Protozoaire disparu. La fréquence des observations auxquelles il donne lieu dans les Ptychocylis du type commun dont il est ici question, nous le fait considérer comme un organe normal chargé d'un rôle physiologique déterminé plutòt que comme le résultat d'une déchirure fortuite du pédicelle de l'organisme.

Planche VIII, figure 26. Spécimen analogue, pour la forme, à celui de la figure 23.

La division scissipare de l'animal vient d'avoir lieu suivant le processus déjà décrit dans les Cyttarocylis (I). L'individu supérieur s'est échappé laissant un arrachement du protoplasme visible en $c$. La couronne orale jeune, coj, de l'individu restant n'a pas encore pris tout son développement et n'a pas encore pu se redresser pour occuper sa position normale.

L'aspect strié du noyau, $n$, présage d'une nouvelle division prochaine.

En $s$, on voit ce que nous appelons la souche.

(1) Voir plus haut, page i14. 
Planche VIII, figure 27. Capsule d'un dessin spécial, plus propre aux eaux du nord de la Mer de Barents. La paroi en est épaisse, très manifestement réticulée, formée de deux lamelles également distancées partout, sauf sur la base où elles forment un épaississement marqué.

L'animal récemment rajeuni, sans doute, par un phénomène de division, montre un corps émacié, presque exempt d'inclusions, avec deux groupes de noyaux géminés, $n, n$, surpris pendant une période d'activité physiologique de caractère inconnu. A l'extrémité du pédicelle, la souche s.

Planche VIII, figure 28. Spécimen dont l'animal fraichement rajeuni par scissiparité s'est rétabli du traumatisme causé par cette circonstance. On y distingue, outre la couronne orale, co, que l'on voit ramassée sur elle-même en mouvement spiralé, deux petits noyaux, $n$, rapprochés qui semblent provenir d'une division récente et des inclusions ic, dont une cellule de Diamylon nivale, $D$.

Cette forme à parois assez minces appartient aussi à la faunule de la Mer de Kara.

Planche VIII, figure 29. Individu en passe de division scissipare, avec la couronne orale primitive, $c o$, et la couronne orale adventive, coj, produite par une sorte de gemniparité au profit d'un nouvel individu. Un seul gros noyau strié est visible, $n$, dans la position sous laquelle l'objet se présente; mais il en existe un autre derrière, que nous n'avons pas pu figurer. On y remarque des inclusions, ic, entre autres : Diamylon nivale, $D$, dont la présence suffit à révéler l'origine de l'organisme : la Mer de Kara.

Planche VIII, figure 3o. Spécimen en division et en complet épanouissement, avec couronne orale primitive, co, couronne orale jeune, coj, des noyaux de deux sortes, $n$ et $n^{\prime}$, des inclusions, dont Diamylon nivale, $D$, et, dans la zone antérieure du corps, des granulations grossières dont bénéficiera, après la division, l'individu qui sera mis en liberté. On se rappelle que nous avons fait remarquer une particularité du même genre dans la division de Cyttarocylis cuspidata (page II4), en émettant l'idée que ces granulations pouvaient ètre des réserves utilisables pour l'édification d'une nouvelle capsule de la part de l'organisme exproprié de sa loge originelle. Mer de Kara.

Planche VIII, figure 3r. Vue apicale du même individu, montrant de face, en projection sur l'ouverture circulaire de la capsule, la couronne orale épanouie, que la figure précédente montre de profil.

On voit manifestement qu'elle est formée, non de cils libres, mais d'une lamelle striée, développée sur un peu plus d'un tour complet de la dépression orale.

Les lobes qui en divisent le bord libre et lui donnent l'aspect d'une corolle gamopétale formée de plus d'une vingtaine de pièces, correspondent à autant de secteurs séparés par des nervures, dont l'accentuation se traduit asymétriquement du côté correspondant au pharynx. 
Cette structure n'est pas spéciale aux Ptychocylis; on la retrouve essentiellement la même, avec de faibles différences d'adaptation, chez les Tintinnides en général. Inutile de dire que les auteurs ont généralement fait erreur en figurant cet organe sous la forme de soies libres.

Planche IX, figure 8. Vue apicale d'un spécimen dont la couronne orale, co, est régulièrement rabattue, par contraction, dans l'orifice de la capsule, cap.

Planche IX, figure 9. Petite forme commune dans la Mer de Kara, vue de profil, dans un état de contraction analogue à celui de la figure 8, ci-devant.

On y voit deux gros noyaux, $n^{\prime}$, qui présideront à la division cellulaire. Un troisième, logé plus bas, dans la partie rétrécie de la capsule, fait partie de la souche. Diamylon nivale, $D$, s'y trouve mêlée à d'autres inclusions.

Planche IX, figure Io. Il y a ici deux groupes de noyaux géminés, $n$, dont l'aspect strié révèle l'activité physiologique.

Ces figures concourent avec d'autres de la planche VIII et IX à donner une idée des mouvements nucléaires qui rappellent ceux dont beaucoup d'infusoires fournissent des exemples bien connus. Il serait certes intéressant d'en reconstituer la série des phases évolutives; mais ce n'est pas le moment de nous arrêter à cette question. Les matériaux morts se prêtent mal à des études d'ordre purement physiologique, parce que les faits qu'ils permettent de repérer ne portent pas suffisamment la marque de leur succession.

Planche IX, figure II. Individu d'un type morphologique un peu différent, à cause du plus grand développement en longueur et en largeur de la région inférieure de la loge.

L'animal, très amoindri par l'exode des individus filles qui ont quitté successivement la loge maternelle, se trouve réduit à la souche, $s$, dont le noyau lâche et rempli de chromosomes volumineux prépare peut-être une nouvelle génération d'individus gemmipares.

La présence d'inclusions, de Diamylon nivale, $D$, en particulier, fait penser que la souche a conservé la faculté d'absorber des aliments solides, ou bien qu'il lui en est resté une réserve dont l'absorption remonte à une phase antérieure de l'évolution de l'organisme.

Les figures suivantes, I2 à I5, reproduisent, chez des spécimens de types différents, d'autres phases de la multiplication gemmipare dont il a déjà été question.

Planche IX, figure I2. L'organisme vient d'ètre amputé de l'individu porteur de la couronne orale antérieure. Les déchirures du protoplasme en font foi.

La couronne gemmaire, coj, parait apte à se mettre en extension dès que le corps actuellement déformé aura pu reprendre son équilibre normal. Les noyaux, $n$, au nombre de quatre ou cinq, y compris celui de la souche, paraissent en période de repos.

Planche IX, figure I3. Autre spécimen en voie de gemmiparité : co, péristome ou couronne orale maternelle; coj, couronne orale jeune vue obliquement; $n$, petit noyau 
strié, qu'il faut sans doute considérer comme l'analogue du micronucleus des autres infusoires. Des deux macronucleus visibles dans la figure, l'un, l'antérieur, appartiendra à l'individu supérieur; l'autre, l'inférieur, reviendra à l'individu gemme : $s$, souche; $D$, cellules de Diamylon avec d'autres inclusions.

Planche IX, figure I4. Phase évolutive analogue à la précédente, mais avec la couronne gemmaire - le péristome de nouvelle formation — vue de face, coj. Sur le flanc de l'individu mère un squelette de Distephanus speculum, $D$, s'observe au milieu d'autres inclusions, $i c$, dans le corps de l'animal. Plusieurs noyaux, $n$.

Remarque. - Nos échantillons ne nous ont pas fourni d'exemples d'enkystement avec ou sans division préalable du corps du Protozoaire, comme ceux que nous avons fait connaitre dans Cyttarocylis gigantea. Nous n'avons pas constaté non plus des indices de phénomènes de sporulation endogène.

Ne serait-ce pas seulement parce que les Ptychocylis rencontrés dans nos matériaux sont encore en période de division gemmipare active?

Planche IX, figures I5, I6, I7. Ces figures reproduisent des spécimens du type morphologique le plus répandu dans les hautes régions de la Mer de Barents et dans l'Océan Glacial. La membrane de la capsule est toujours très épaisse, résistante et nettement réticulée. La partie inférieure, harmonieusement arrondie, se prolonge, au delà d'un ressaut, en un bouton conique terminé en pointe obtuse.

La figure 16 en est une variante raccourcie et à membrane très épaisse, surtout à la base.

La figure i5 présente l'organisme atrophié, sur la souche duquel un péristome jeune, coj, se produit.

Dans les planches X, figures I à 6; XI, figures 20 et 2I; XII, figures 36 et 37, nous avons reproduit aussi fidèlement que possible d'autres formes de capsules trouvées tantôt ici, tantòt là, sans qu'il ait paru utile de repérer l'endroit précis. On serait mal fondé de vouloir urger de trop près les rapports de dépendance entre ces organismes flottants et l'endroit précis des mers où le filet planktonique les a fortuitement rencontrés, au hasard de l'itinéraire du bateau.

Rien ne prouve qu'on ne les aurait pas aussi bien trouvés ailleurs, si les circonstances l'avaient permis; rien ne prouve davantage que le brassage des eaux par le jeu des courants n'en modifie pas rapidement la distribution géographique dans les mers contiguës.

Il suffira de noter que dans la Mer de Kara dominent les petites formes à parois minces et peu structurées, tandis que dans la Mer de Barents prévalent les grandes formes à parois épaisses, plus ouvragées, parfois mème franchement alvéolaires.

Telles : la figure $\mathrm{I}$, planche $\mathrm{X}$, rencontrée seulement au large entre les còtes norvégiennes et le Détroit de Matotchkine. 
Les figures 2 et 5 , planche $X$, et 36-37, planche XII, pêchées seulement le long de la côte occidentale de l'ile sud de la Nouvelle-Zemble, nos i66 à i6g.

Les figures I5, I6, I7, planche IX, 20, 2I, planche XI, trouvées exclusivement dans l'Océan Glacial, nos 222 à 226.

Quelle est la valeur taxinomique de ces formes?

Faut-il y voir des espèces différentes, comme BRANDT (I896), ou des variétés d'une seule espèce, comme Jörgensex (IgOI)?

Sans vouloir prendre position entre les protagonistes de ces deux opinions, actuellement plus spéculatives qu'objectives, nous nous bornerons à quelques remarques.

D'abord, il est évident qu'on ne peut y voir des modalités dues seulement à l'âge de l'organisme, puisque toutes présentent des phénomènes évolutifs analogues malgré la diversité de structure de la loge.

En deuxième lieu, la forme ne paraît pas liée uniquement aux conditions d'ambiance locales, puisque des spécimens d'un module bien distinct se coudoient dans le mème milieu.

Enfin, si l'on ajoute aux formes ici figurées celles qu'ont fait connaitre d'autres planktonistes et celles plus nombreuses encore que nous avons pu observer nous-même dans d'autres mers, on se trouve en présence de variantes offrant de tels écarts de style, si l'on peut dire, qu'il ne parait réellement pas possible de les prendre pour de simples modalités d'un même type spécifique.

Ptychocylis amula (Claparède et Lachmann) Brandt est donc plutôt un type générique, évidemment très simple et partant uniforme, dont les variantes les mieux caractérisées peuvent être tenues provisoirement pour des types spécifiques, en attendant que la découverte des caractères physiologiques permette d'en compléter la diagnose, en confirmant ou en infirmant les vues systématiques fondées d'abord sur les seuls caractères morphologiques, à titre de premiers essais de classification.

Pratiquement, dans les travaux d'analyse planktoniques, il nous parait avantageux de s'en tenir à une nomenclature tenue provisoirement pour spécifique plutôt que d'encombrer la terminologie par la surcharge de noms de variétés que les moindres variantes d'aspect autoriseraient à créer et à multiplier dans des proportions exagérées, jusqu'à atteindre les simples variations individuelles.

Pour ces raisons nous extrayons de nos figures quelques formes qui pourraient vraisemblablement représenter des entités spécifiques.

Type A, figure IO, planche IX. Ptychocylis Drygalskii Brandt? (I).

Variantes : figures 9, I2, I3, I4, planches IX; figures 23, 26, 29, 30, planche VIII.

(1) Bibliothera Zoologica. Heft 20, Lfg. 2. Zoologische Ergebnisse IV, K. Brasit. Die Tintinnen. Stuttgart, I8g6. 
Type B, figure 3, planche X. Ptychocylis ventricosa sp. n.

Type C, figure 25, planche VIII. Ptychocylis cylindrica sp. n.

Type D, figure 24, planche VIII. Ptychocylis elongata sp. n.

Type E, figure I7, planche IX. Ptychocylis glacialis sp. n.

Variantes : figures I5, I6, planche IX; figure 27, planche VIII; figure 20, planche XI; figure 6 , planche X.

Cette dernière est remarquable par le développement exagéré de la membranule réticulée externe, à la base de la capsule, qui est, en outre, arrondie et non pointue comme dans le type.

Peut-être trouvera-t-on qu'il y a lieu d'identifier l'une ou l'autre de ces variantes avec Ptychocylis obtusa Brandt (I8g6), mais nous ne pouvons en accepter le nom, qui serait paradoxal en l'occurrence, vu que notre type a sa base terminée en pointe creuse. Le procédé de figuration mis en œuvre par cet auteur, en faisant abstraction de la transparence de l'objet, ne permet pas d'y contròler ce caractère, pas plus que celui de l'épaisseur de la membrane.

Le terme de glacialis rappelle ses origines : Océan Glacial.

Type F, figure I, planche X. Ptychocylis basicuriata sp. n.

Forme, en dé à coudre, de grandes dimensions, longuement cylindrique, arrondie à la base, sans ressaut ni étranglement. Membrane assez épaisse.

Type G, figure 2, planche X. Ptychoocylis diiplicata $\mathrm{sp} . \mathrm{n}$.

Forme grande, au profil tourmenté par plusieurs étranglements. Membrane assez mince, sauf sur la base, qui est obtuse ou même un peu ombiliquée en dedans.

Variante : figure 5, mème planche.

Type $\mathrm{H}$, figure 4 , planche $\mathrm{X}$. Ptychocylis media sp. $\mathrm{n}$.

Variante : figure II, planche IX.

Type I, figure 36, planche XII. Ptychocylis ampla sp. n.

La plus grande, à notre connaissance, des formes qui se réclament plus ou moins des caractères propres à la primitive urmula. La silhouette en est lourde, massive, obtuse. La membrane en est assez mince, sauf sur le renflement annulaire qui s'observe, à quelque distance de l'orifice, comme caractère commun à toutes les formes du mème groupe.

Type J, figure 37, planche XII. Ptychocylis elegans sp. n.

Forme assez grande, à membrane mince, au profil atténué du sommet à la base.

Type K, figure 2I, planche XI. Ptychocylis edentata sp. n.?

Forme étriquée, complètement édentée à l'orifice, douée d'une membrane double très épaisse. Il n'y a pas de rebord saillant au voisinage immédiat de l'orifice capsulaire. Observé rarement, à l'état vide. Serait-ce une déformation, post mortem, de l'un ou l'autre des types précédents? Nous ne savons, car nous n'avons pas la preuve d'une semblable déformation, mème après le passage dans l'organe digestif d'un autre animal. 
Remarque. - Le Ptychocylis acuta Brandt, que nous connaissons d'autres mers arctiques, ne se rencontre pas dans nos échantillons actuels. Il en est de même de nombreuses formes qui semblent faire la transition entre les plus caractéristiques des Ptychocylis et des Cyttarocylis, et qui rendent très indécises les limites entre ces deux genres.

N. B. - Bien que le lecteur en ait été prévenu, il n'est peut-être pas inutile de rappeler que toutes les figures relatives aux Ptychocylis, comme les autres du reste, ont été dessinées à la même échelle, c'est-à-dire au grossissement d'environ 500 diamètres.

\section{Genre Amphorella DADAY}

Nous tenons, avec Daday (i886-I887), le genre Amphorella comme un groupe de Tintinnides dont la coque à paroi mince est fermée à une extrémité et exempte à l'extérieur de granulations siliceuses.

Ce genre va nous fournir des difficultés de systématique analogues à celles que nous venons de rencontrer dans les genres Cyttarocylis et Ptychocylis; car, à côté de certaines formes dont la spécificité ne fait de doute pour personne, il s'en présente d'autres, au sujet desquelles on peut rester hésitant, avant d'être nxé par des considérations d'ordre plus intime, sur l'étendue possible des variations d'un même type spécifique.

\section{Amphorella vitrea (Brandt).}

Planche IX, figures $2 \mathrm{I}$ et 22 ; planche XIV, figures $I, 2$ et 3 .

Synonyme. - Tintinnus vitreus Brandt (I896); pour autant que la référence soit fondée, carl'auteur n'en figure que la capsule vide. Ses caractères de forme, de taille, de transparence semblent toutefois concorder avec ceux de l'objet que nous avons en vue ici et qui est commun dans la Mer de Kara.

Le spécimen que nous reproduisons figure 2I, planche IX, est complet, habité, surpris par la fixation dans un moment de pleine expansion et en travail d'une digestion peut-être laborieuse, vu la quantité d'objets absorbés, qui s'y observent à l'état d'inclusion, ic. Nous y voyons, entre autres, un Peridinium, $P$, des Diamylon nivale, $D$, des grains de fécule, etc.

La couronne orale ou péristome, co, est en épanouissement et laisse voir, en son milieu, la protubérance centrale et, à gauche de la figure, le prolongement œsophagien de la bouche marqué par l'accentuation des nervures du péristome strié et lobé. 
Un gros noyau, $n$, semble présenter un phénomène d'étranglement.

Des vacuoles nombreuses, $v$, se montrent dans la partie inférieure du corps.

La disparition éventuelle de ces vacuoles par l'action plasmolytique des réactifs fixateurs permet parfois une très notable réduction du corps, comme dans la figure 22 , où les mèmes désignations ont été reprises.

Les figures 2 et 3 de la planche XIV en montrent deux individus en voie de division, pris à l'aventure entre beaucoup d'autres.

La première (fig. 2) nous fait assister à l'ébauche du péristome jeune, coj, dont les dimensions grandiront rapidement, comme dans la figure 3 , pendant que se produira l'étranglement qui amènera la séparation des frères jumeaux.

A part des différences d'aspect qui tiennent surtout aux particularités morphologiques du corps, on voit que la division gemmipare suit ici la même marche que dans les autres Tintinnides. C'est chez tous le mème thème adapté à des variantes de forme et de rythme.

Figure 2. Spécimen en extension et gorgé d'inclusions alimentaires, in, dont un fragment de muscle d'un Crustacé, $m$, dans une grande vacuole digestive, $v d$.

Ces grands Tintinnides ont sans doute l'estomac complaisant et même indifférent à la nature du menu, car on y trouve occasionnellement de tout ce qui flotte dans le même milieu qu'eux et qu'on retrouve aussi dans les mêmes coups de filet.

Ces fragments de muscles, miettes tombées de la table de plus riches, reliefs de festins d'espèces mieux armées qui se font une guerre de cannibales, ne sont pas perdus, on le voit, dans ce milieu marin, où, plus rapidement que sur terre, la matière parcourt son cercle vital.

On remarquera à nouveau que la division du noyau, $n$ et $n$, a précédé l'apparition de la couronne gemmaire, coj, qui nait à la surface du corps, contre la paroi interne de la loge.

Dans la figure 3, qui reproduit une étape plus avancée du phénomène, les noyaux se divisent à nouveau. Chez l'inférieur, nd, le fait est déjà accompli; le supérieur, $n$, témoigne par sa forme allongée et étranglée d'une subdivision prochaine.

On remarquera les mouvements d'évagination (fig. 2) et d'invagination (fig. 3) de la protubérance orale, po, à l'intérieur du péristome qui porte la couronne ciliée.

La capsule, qui ne porte aucune dentelure sur son bord libre, semble susceptible de s'y accroitre longtemps suivant un mode qui se traduit, dans la zone d'accroissement, par une structure spiralée faiblement visible dans la figure 22 , planche $1 X$, très manifeste dans la figure I, planche XIV, où nous voyons un caractère de vieillesse.

\section{Amphorella fistula sp. n.}

Planche IX, figures $23,24,25$; planche XIV, figure 4 .

On serait assez porté à rattacher cette forme à la précédente qui n'en diffère à première vue que par un grand écart de calibre de la loge, si l'on était autorisé à n'y voir que des différences d'âge ou d'ambiance d'un même type. 
Ce n'est cependant pas notre avis, car rien ne nous démontre que ces organismes peurent s'accroitre en largeur aussi bien qu'en hauteur et, d'autre part, les phénomènes de division gemmipare (planche XIV, fig. 4), indices de maturité de l'organisme, se rencontrent aussi fréquemment dans les formes étroites de $A$. fistula que dans les formes larges de $A$. vitrea. Nous devons, en outre, considérer ici comme un caractère de vieillesse les plissements irréguliers qui se traduisent sur la capsule par une striation longitudinale un peu oblique (fig. 25, pl. IX).

Le processus d'accroissement y laisse aussi parfois son empreinte dans la structure spiralée de la zone orale principalement (fig. 24, pl. IX).

Les deux figures 23, planche IX, et 4, planche XIV, suffisent à illustrer le processus de multiplication du Protozoaire.

Dans l'une (fig. 23), la division du noyau, n, a déjà préludé au partage du corps entre les deux organismes jumeaux, qui existent déjà virtuellement dans l'autre (fig. 4), par le fait de la création d'une couronne gemmaire jeune, coj.

Autres désignations, comme plus haut.

\section{Amphorella tubularis sp. n.}

Planche IX, figures 26 et 27 ; planche $\mathrm{X}$, figure 18 .

Si nous pouvions avoir des doutes au sujet de la spécificité des deux formes vitrea et fistula, ils nous viendraient de l'existence constatée de formes telles que les spécimens reproduits dans les figures 26 et 27 de la planche IX qui, par leurs caractères de calibre et d'aspect, pourraient ètre regardés comme des jalons posés sur l'espace qui sépare ces deux types.

Cette opinion n'est pas sans fondement; mais, dans la pratique, il nous parait préférable de prêter à ces formes intermédiaires une entité spécifique provisoire pour guider les recherches dans l'analyse de ce groupe homogène, où la grande similitude cache peut-ètre une réelle diversité de nature.

En examinant attentivement la capsule, on y découvre presque toujours une structure spiralée, parfois très confuse (fig. 27), d'autres fois plus manifeste (fig. 26).

Exceptionnellement elle prend une expression très nette, commẹ dans la figure is de la planche XI; si bien qu'on n'hésiterait pas à prendre celle-ci pour la reproduction d'une espèce distincte, si le mélange de ces formes dans le mème milieu ne plaidait en faveur de leur rapprochement spécifique.

La connaissance de leurs caractères physiologiques intimes pourrait seule aider à trancher la question. 
Amphorella cochleata sp. n.

Planche $x$, figure 7 .

Faut-il voir dans cette forme celle d'un type spécifique distinct ou une simple modalité de l'un des précédents?

La structure spiralée, qui intéresse ici toute la capsule, se traduit en dépression vers la base, en saillie vers le haut, où l'évasement de l'orifice explique la genèse de la gouttière spirale qui orne la zone orale de la capsule.

Sur toute la surface de la paroi capsulaire s'observe un système de fins plissements obliques.

Des observations ultérieures dicteront la solution à intervenir au sujet de ses affinités réelles.

Distribution Géographioue. - Mer de Kara; rare.

\section{Amphorella subulata Daday.}

Planche $\mathrm{X}$, figures $\mathrm{I} 4$ et $\mathrm{I} 5$.

Srnonmune. - Tintimus subulatus Ehrenberg. Cfr. Bergens Museums Aarbog, I899, hefte I, no 2, Jörgensen, p. I6.

Cette espèce bien connue, dont la dispersion géographique est très étendue, ne se présente guère dans nos matériaux que dans les échantillons provenant de Tromsï, à une époque, fin juin, qui n'est pas celle de sa grande prolification, car nous n'y avons guère observé les phénomènes de division qui nous ont si souvent frappé dans d'autres circonstances : dans la Mer Flamande, par exemple, et qui suivent la même marche générale que dans les autres Tintinnides.

Les deux spécimens figurés présentent l'animal contracté dans des attitudes peu différentes. Nous ne l'avons pas vu enkysté, comme il nous est connu du bassin à flot de Nieuport.

Le noyau, $n$, est simple, en l'absence de phénomènes de division du corps.

La forme de la capsule nous a paru, dans d'autres circonstances, sujette à d'assez grandes variations, mais ce n'est pas le lieu, de toucher à cette question.

\section{Amphorella norvegica Daday.}

Planche $\mathrm{X}$, figures 20 à 25 ; planche XIV, figure 7. ?

Synomyn1E. - Tintinnus norvegicus Jörgensen; T. minutus Brandt; T. gracilis Brandt, I896; Cyttarocylis norvegica Jörgensen, I899.

La diversité des vues au sujet de cette intéressante petite forme n'est que la conséquence du défaut d'entente sur les bases mèmes de la classification des Tintinnides 
en général et en particulier sur les caractères respectifs des genres Tintinnus, Amphorella, Cyttarocylis et d'autres, qui n'ont jusqu'ici qu'une valeur conventionnelle, variable suivant les vues personnelles des auteurs.

Nos figures 22 à 25 la présentent, sous quelques variantes de forme, comme une Amphorella, s'il convient de réserver ce nom générique aux espèces dont la capsule fermée au bout postérieur est formée d'une membrane simple, exempte de structure visible et dépourvue de particules étrangères adhérentes.

S'il est vrai toutefois que Jörgensen y a vu une fine réticulation, ce qui lui en a fait faire un Cyttarocylis, il faut convenir que ce caractère est trop peu accusé généralement pour faire l'objet d'observations cursives et il perd ainsi sa valeur pratique.

Nous l'avons figurée hyaline comme elle apparait et c'est pourquoi nous en faisons une Amphorella.

La capsule tantòt cylindrique (fig. 22), tantôt un peu étranglée 'vers le haut (fig. 23), est ornée d'un cercle denté situé un peu en dessous de l'orifice libre.

La figure 26 en est une vue apicale montrant la disposition des denticules un peu recurvés à l'extérieur et distancés.

L'animal, à l'état normal, avec un seul noyau, $n$, dans les figures 22 et 24 , se montre en cours de division dans la figure 25, où l'on voit le noyau divisé et la couronne orale jeune, coj, en voie de formation.

A notre avis, il ne faut voir dans les formes des figures 20 et $2 \mathrm{I}$ qu'une simple variété apiculée de l'espèce, caractérisée par l'accentuation de la pointe basilaire.

Les caractères évolutifs $\mathrm{y}$ sont les mèmes : co, couronne orale; coj, couronne orale jeune; $n$, noyau unique avant la division, dédoublé pendant la division, mais caché derrière le péristome jeune dans l'individu de la figure $2 \mathrm{I}$.

Nous devons enfin attirer l'attention sur les caractères tant morphologiques que physiologiques de l'objet de la figure 7 , planche XIV, qui nous parait devoir ètre aussi identifié avec $A$. norvegica, malgré la longueur exceptionnelle de la logette.

Nous nous demandons si ce développement anormal n'est pas lié à la phase évolutive spéciale de l'individu, qui semble ètre en travail de production d'une spore quiescente, sp, aux dépens de la partie antérieure de son corps protoplasmatique. Cette partie, d'où la couronne orale a disparu, prend une forme globuleuse ou ovale autour d'un gros noyau, $n$, frère de celui qu'on observe dans la partie inférieure du corps, au fond de la capsule.

La division ultérieure de ce dernier préparera-t-elle la formation d'autres spores qui utiliseront le protoplasme somatique restant? Simple hypothèse que la rareté de l'objet et la nature inerte de nos matériaux ne nous ont pas permis de vérifier.

Distribution gŕographique. - Pas rare dans la Mer de Barents, surtout dans la partie nord. 


\section{Amphorella minutissima sp. n.}

Planche $\mathrm{X}$, figure 27 .

La transparence et la petitesse de cette forme la dissimulent aisément aux regards dans le fouillis des formes si diverses qui se pressent sous l'œil du micrographe.

Sa recherche exige un examen attentif et minutieux des préparations, où l'on peut croire qu'elle est moins rare qu'elle ne parait.

Sa coque hyaline est dépourvue de structure visible. Renflée un peu à la base, où elle porte une longue pointe acérée, elle se rétrécit insensiblement vers le sommet dont l'orifice oral est exempt d'ornements. Ce dernier caractère est à noter, car nous avons rencontré, dans d'autres milieux, des formes aussi petites que celle-ci, mais diversement, ornées de cercles et de denticules.

Distribution GÉgraphioue. - Mer de Barents.

\section{Amphorella annulata sp. n.}

Planche $\mathrm{X}$, figure 28 .

N'ayant observé ce petit organisme qu'à l'état de capsule vide, nous ne sommes pas certain qu'il s'agit d'un Tintinnide plutôt que d'un Vorticellide.

La logette, comme on voit, est longue, cylindrique, arrondie à la base, anneléc dans sa moitié antérieure. On n'y distingue ni sculptures, ni dentelures.

Distribution géographigul. - Mer de Kara; très rare.

\section{Genre Tintinnus (Schrank) Daday}

Suivant en cela DADAY et Jörgensen, nous ne maintenons dans ce genre que les Tintinnides à logrette chitineuse, simple, ouverte aux deux extrémités.

La présence ou l'absence de petits corps étrangers adhérents à la surface externe de la capsule nous parait être un caractère trop inconstant pour autoriser la reconnaissance du genre Leprotintinnls, que Jörgensen a proposé pour grouper celles de ces formes qui présentent des incrustations de particules minérales.

S'il y a lieu de scinder ce genre, comme nous sommes porté à le croire, on en trouvera la raison dans les caractères propres des Protozoaires eux-mèmes, dont la systématique actuelle fait trop généralement abstraction. C'est ainsi que Tintimms acuminatus Claparède et Lachmann, par exemple, nous parait égaré, sous cette dénomination, dans un groupe de formes avec lesquelles il semble réellement n'avoir que des rapports éloignés. 
Tintinnus acuminatus Claparède et Lachmann.

Planche $\mathrm{X}$, figures $\mathrm{i} 6$ à $\mathrm{ig.}$

Synovyme. - Tintimus secatus Brandt, I8g6.

La capsule est en forme de pointe de Paris, étroite, longuement cylindrique, évasée en pavillon en avant, conique en arrière, avec plissements obliques qui convergent vers une extrémité très étroite, tubulaire, ouverte au bout.

Cette forme si spéciale crée à l'espèce un signalement très net, que seules ont pu fausser de mauraises reproductions.

Des variantes s'observent dans la longueur relative de la partie cylindrique et dans la forme du pavillon antérieur, plus ou moins étalé, circulaire (fig. I6 et I7), ou plus ou moins déformé par la production de lobules anguleux (fig. I8 et surtout I9).

Le corps de l'animal, étroitement logé dans la capsule, a une allure vermiforme et présente un facies particulier qui nous fait mettre en question la nature des affinités réelles de cet organisme et la légitimité de la place qu'on lui fait occuper dans le genre Tintimus.

Nous y avons observé la présence parfois d'un noyau unique (fig. i8), parfois de plusieurs noyaux, dont les rapports avec d'autres éléments constitutifs du corps (fig. I6) ne nous sont pas connus, mais paraissent liẻs avec les phénomènes de division de ce dernier.

Distribution géograpingue. - Trouvé à l'état disséminé dans la Mer de Kara seulement.

\section{Tintinnus pellucidus Cleve.}

Planche XI, figures i a Io.

Loge longuement cylindrique, irrégulièrement rétrécie vers le bas et ouverte en évasement peu accentué à l'extrémité inférieure. Les anneaux qui se dessinent à distance régulière sur toute l'étendue de la membrane hyaline n'y forment ni dépression, ni saillie et ne sont rendus visibles que par une différence de réfringence.

L'organisme vivant est simple et normal dans la figure I, avec sa couronne orale, co, sa bouche, $b$, au fond du péristome; le conduit cesophagien, ces; un noyau unique, $n$; des inclusions, des enclaves, des vacuoles, etc., le tout quelque peu contracté par le milieu conservateur.

\section{SCISSiparité}

Dans la figure 2 , la division du noyau, $n, n$, a préludé au processus de scissiparité et la couronne orale jeune, coj, est déjà ébauchée et vue de face.

Figure 3. Phase un peu plus avancée du phénomène de division. La couronne orale jeune, coj, est présentée de profil. 
Figure 4. Autre étape analogue, avec couronne orale jeune, coj, presque achevée et présentée du còté de l'observateur, de mème que le conduit pharyngien, as, de l'organisme mère et les deux noyaux filles; $m$, membranule du corps détaché de la capsule; $f$, quelques petits grains de fécule.

Dans la figure 5, enfin, l'étranglement qui s'accuse, en $x$, entre les deux individus virtuellement formés, prépare leur séparation complète. Celle-ci sera suivie de l'exode de l'individu antérieur, héritier du péristome maternel, mais déshérité du logis familial.

Avec les variantes inhérentes aux caractères morphologiques de l'espèce, c'est donc la reproduction exacte des faits observés et signalés plus haut à propos d'autres espèces de Tintinnides. C'est le mème thème, àvec des modifications dans le rythme, ce qui plaide en faveur de l'homogénéité physiologique du groupe et de sa réelle valeur taxinomique.

\section{ENKYSTEMENT}

Nombreux sont aussi, dans certains de nos échantillons des còtes occidentales du nord de la Nouvelle-Zemble, - commencement de septembre - les exemples d'enkystement ou de formation de spores quiescentes.

Nous en reproduisons quelques-uns pour montrer comment, après la perte de l'appareil oral, l'animal se contracte et s'entoure d'une membrane pour prendre d'abord une forme ovoïde (fig. 6 et $7, s p$. ), et plus tard la forme caractéristique des figures 8 et 9 , qui nous parait définitive.

Le kyste ainsi produit reste serti dans la partie cylindrique de la capsule et vers son milieu. Il est bipolaire et présente au pòle inférieur un bouton, $a$, autour duquel la membrane plus épaisse offre une réfringence particulière, qui traduit une différence de nature et prépare sans doute la déhiscence de la coque, qui devra se produire après une période de repos.

Le spécimen dont nous avons reproduit seulement la capsule vide (fig. Io) présente de fines granulations siliceuses disséminées sur la surface, surtout dans la région inférieure. Ce sont des exemples de ce genre qui nous font émettre des doutes sur l'opportunité de la distinction qu'a voulu établir JöRGENSEN (I899) entre les genres Tintinnus et Leprotintinnus, en se basant sur l'absence ou la présence de particules minérales sur la paroi capsulaire.

A n'en pas douter cependant, ce speecimen, annelé comme les précédents, leur est spécifiquement identique, aussi bien du reste que celui que Jörgensen figure luimème - Bergens Muscums Aarbog, ISg9, no 6, pl. II, fig. I3 - sous le nom de Leprotintinnus botnicus, mais à tort, à notre avis. 
Cette particularité, inconstante dans les limites d'une espèce bien connue, ne saurait prendre la valeur d'un critérium de genre et légitimer la séparation d'organismes que la similitude de leurs principaux caractères doit faire considérer comme congénères.

Distribution Géographique. - Assez répandu sur les còtes occidentales dé la Nouvelle-Zemble, dans la Mer de Barents.

\section{Tintinnus botnicus Nordquist}

Planche XI, figures II et I2.

Synonymie. - Leprotintimus botnicus Jörgensen, i 899 .

Chez les formes que nous rapportons à cette espèce, la paroi capsulaire plus mince ne se montre pas annelée. Elle porte extérieurement des fragments siliceux plus ou moins ténus, plus ou moins distancés.

La figure II en reproduit un spécimen habité par le Protozoaire à l'état normal, avec un seul noyau, $n$, parce que éloigné de toute phase de division.

Dans le spécimen de la figure I2, on voit le résultat de l'enkystement du Protozoaire, sp.

La grande ressemblance du kyste avec celui de l'espèce précédente prouve l'étroite affinité des deux formes et s'oppose à leur attribution à des genres différents. Par contre, les petites différences qu'on y observe corroborent leur distinction spécifique.

L'accumulation plus grande des fragments siliceux sur une zone antérieure, comme en $t$ (fig. I2), - fait qui s'observe assez souvent - nous parait due à la richesse plus grande du milieu en particules de ce genre, pendant la période d'accroissement de cette partie de la loge.

Nous avons vu plusieurs fois le sporocyste localisé dans cette zone.

Distribution géographigue. - Mêmes parages que la précéclente, mais beaucoup plus rare.

\section{Genre Tintinnopsis STE1N}

Tintinnides à capsule mince, fermée en bas et chargée de granulations étrangères : des fragments de quartz, le plus souvent.

Autant ce caractère générique est net et peut ètre tenu pour suffisant dans la pratique, autant sont subtils et obscurs les caractères plus spéciaux des formes que l'on voudrait tenir pour spécifiquement distinctes, dans le but d'établir des coupures rationnelles dans cette série si homogène de variantes d'un type morphologique très simple. 
Personne, à notre connaissance, n'a encore tenté avec succès de mettre un peu d'ordre dans cet ensemble chaotique de formes, au sujet desquelles on a épuisé le vocabulaire des termes plus ou moins évocatifs d'une ressemblance avec des objets connus, après avoir fait de larges emprunts à des noms propres d'hommes et de lieux. Ceci, soit dit en passant, nous a toujours paru une détestable façon de qualifier des espèces organiques, car il serait désirable que le nom spécifique tout au moins fùt toujours évocatif d'une particularité saillante de l'espèce et facilitàt ainsi son identification.

C'est un abus qui dessert la science en servant peu la vanité des savants.

Il a du moins ici une excuse dans la multiplicité des formes à étiqueter. Voir Daday : Monographie der Familie der Tintinnideen - Mittheilungen aus der Zool. St. zu Neapel., vol. VII, I886-I887.

Comme on va le voir, nos observations nous forcent à apporter de nouveaux éléments de confusion dans ce petit monde, où l'on préférerait voir s'établir plus d'ordre intérieur plutôt que d'assister à de nouvelles immigrations.

L'homogénéité physiologique du groupe des Tintinnopsis n'est malheureusement pas moindre que la similitude morphologique, et nous avons vainement attendu de ce còté la base de certaines distinctions spécifiques trop mal étayées, à notre avis, sur des variantes dans le profil des capsules, si faibles, qu'il faudrait l'oil exercé du modeleur pour en saisir ce qui semble n'être plus que la touche d'un style ou d'une époque dans la conception esthétique de l'art du potier.

Quelle forme de vase ancien ou moderne l'imagination des artistes a-t-elle créée que la nature n'a pas réalisée, en miniature, dans les Tintinnides en général, dans les Tintimopsis en particulier?

Les personnes étrangères aux recherches microplanktoniques ne seraient-elles pas portées à voir clans nos planches des dessins relatifs à l'étude comparée du vase à travers les àges?

Et cependant, combien de formes étrangères aux parages explorés par la Belgica viendraient enrichir cette collection iconographique, si notre travail devait embrasser l'ensemble des Tintinnopsis dont les mers les plus diverses fournissent leur contingent!

Bref, il est évident que l'absence de caractères spécifiques bien fondés chez les Tintinnopsis et l'embarras qui en est résulté pour les micrographes a donné lieu à une synonymie aussi fàcheuse qu'encombrante; nouvelle difficulté ajoutée à celles inhérentes, par nature, à l'objet de cette étude.

Nous ne nous proposons pas d'en avoir raison; nous nous contenterons de présenter les formes observées dans nos matériaux, sans plus urger qu'il ne convient leur identification avec des espèces dont la légitimité reste problématique, mème dans les cas où elle parait le moins sujette à caution.

Il nous parait opportun de les subdiviser morphologiquement en quatre séries. 
Ire série. - LES TUBULEUSES

FORMES DON'T LA PORTION PRINCIPALE DU CORPS EST CYLINDRIQUE, QUEL QUIE SOIT L'ASPECT DES DEUX EXTRÉMITÉS

\section{Tintinnopsis major sp. n.}

Planche XII, figure I.

Très grande forme à capsule cylindrique dans sa partie médiane, spiralée dans la moitié antérieure, évasée en haut, conique en bas.

Elle n'est pas sans analogie avec T. cyathus Daday, var. anmulata Daday et T. Biitschlii Daday (I887) du golfe de Naples, mais les grands écarts géographiques d'habitat doivent rendre suspectes les simples similitudes de forme au point de vue de l'identification des espèces.

Dans notre figure, l'Infusoire contracté montre sa couronne orale, co, insérée sur le péristome; le prolongement œsophagien de la bouche, as; un noyau, $n$; une enclave ou inclusion, en.

Distribution Géographique. - Rencontré surtout dans l'échantillon no ${ }^{\circ}$, originaire de Tromsö.

\section{Tintinnopsis macropus sp. n.}

Planche XII, figures 2 , 3 et 4 .

Grande forme à capsule cylindrique en avant, dilatée en arrière, terminée à la base en còne très déprimé (fig. 2).

Dans la figure 3, un arrachement de la capsule laisse voir l'animal contracté pourvu d'un seul noyau, en dehors de toute phase de division. On le voit en division dans la figure 4, avec une couronne orale jeune, coj, sur le flanc, et deux noyaux, dont un seul, u, a pu être figuré, l'autre occupant le côté opposé.

Distribution géographique. - Associée à la précédente, dans le mème échantillon.

\section{Tintinnopsis Lobiancoi Daday? T. tubulosa Levander?}

Planche XII, figures 5 à 9 .

Malgré la forme de tube à essai de sa capsule grande, cylindrique, non évasée à l'orifice, arrondie en bas (fig. 5), cette espèce ne nous paraît pas pouvoir ètre rapportée, avec toute sécurité, à la forme ainsi dénommée du golfe de Naples par DaDAy. 
Celle-ci est plus longue, relativement à sa largeur, que la nôtre (I); mais indépendamment de ces petites différences morphologiques du test, la grande différence de milieu ne plaide-t-elle pas en faveur de la diversité spécifique d'organismes qui peuvent épouser une forme aussi simple sans être de même nature?

Ne serait-ce pas aussi bien $T$. tubulosa Levander, dont le nom tout au moins est plus évocatif de la forme?

Quoi qu'il en soit, nous ne voyons pas d'inconvénient à suivre les planktonistes, qui n'y ont pas regardé de si près, et à adopter provisoirement l'une ou l'autre de ces dénominations en attendant de meilleurs éléments d'appréciation.

Si l'on suppose que les spécimens reproduits (fig. 5 et 6) présentent la forme type, non spiralée, du test, il est difficile d'en séparer ceux reproduits dans les figures 7 et 8 , qui ne s'en distinguent autrement que par la structure spiralée de la partie antérieure de la capsule, circonstance qui conduit les auteurs à créer les variétés anmulata, nous ne saurions dire pour quelle bonne raison.

Le calibre de ces derniers nous a toujours paru un peu plus petit, plus étroit surtout; mais qui pourrait saisir ces faibles différences, s'il n'a sous les yeux les éléments de comparaison?

La figure 9 reproduit un spécimen anormal, présentant, en $x$, une déformation accidentelle, causée sans doute par un traumatisme remontant au jeune âge de l'organisme.

Le Protozoaire est figuré à l'état normal, arec un seul noyau, $n$, dans les figures 6 et 7 . Dans la figure 8 on le voit en division avec deux noyaux, $n$ et $n$, et une couronne orale jeune, coj, en roie de développement sur le flanc de l'individu. Chez tous la protubérance orale, po, s'observe très bien au sein du péristome que couronne l'organe ciliaire, co.

Distribution Géographigue. - Ces formes sont beaucoup plus répandues que les précédentes, elles s'étendent aux deux Mers de Barents et de Kara, mais toujours à l'état disséminé.

\section{Tintinnopsis lubulosoides sp. n.}

Planche XII, figures io et II.

Forme affine à la précédente, mais s'en distinguant par un léger évasemont de l'orifice et par la forme conique de la base. La partie cylindrique accuse une structure spiralée sur presque toute son étendue. 
Les deux Protozoaires figurés dans des attitudes différentes sont en voie de division et montrent la couronne orale jeune, coj, de profil (fig. ro) et de face (fig. II) entre les deux noyaux, $n$ et $n$, de formation récente.

Autres désignations comme plus haut.

Sans son évasement antérieur et l'absence de prolongement basilaire, cette forme rappellerait assez bien $T$. ammlata Daday.

Distribution Géographigue. - Mème distribution que la précédente, mais plus rare.

\section{Tintinnopsis cylindrica sp. n.}

Planche XII, figures $\mathrm{I}_{2}$ et $\mathrm{I} 3$.

Ces figures sont représentatives de formes beaucoup plus grêles, spiralées aussi, coniques à la base, plus manifestement évasées à l'orifice et garnies, sur le pourtour du pavillon antérieur, de nombreux fragments de quartz en saillie, s.

Distribution GÉographigue. - Elles sont plus spéciales aux parages septentrionaux de la Mer de Barents.

\section{Tintinnopsis beroidea Stein?}

Planche XII, figures it à 18 .

Il semble bien que l'on a abusé de cette étiquette pour l'épingler sur toutes sortes de formes qui n'ont guère de commun que leurs petites dimensions. C'est un abus que peut seule excuser la difficulté de préciser les caractères spécifiques de ces micro-organismes frustes, en dehors d'un travail d'étude monographique du groupe, étayé sur tous les éléments possibles d'appréciation.

Nous estimons ne devoir retenir ici sous ce nom que les petites formes tubuleuses, non évasées ni rétrécies à l'orifice, coniques ou légèrement pointues à la base, dont nos figures I4 à is fournissent quelques variantes d'aspect.

Si, comme c'est possible, il y a parmi elles des représentants d'espèces différentes, leur distinction n'est toutefois pas possible pratiquement, pas plus, du reste, que celle de variétés que l'on voudrait établir sur les différences de longueur de la capsule, légèrement spiralée ou non.

Distribution Géographique. - Spécimens assez rares, disséminés dans la Mer de Barents. 


\section{$2^{\mathrm{c}}$ série - LES ORTHOSTOMES}

FORMES NON CYLINDRIQUES, A OUVER'IURE DROITE, SANS ÉTRANGLEMENT

\section{Tintinnopsis lata sp. n.}

Planche XII, figures Ig à 22 .

Forme assez large, courte, renflée vers la base, légèrement atténuée vers le haut jusqu'à l'orifice qui ne présente ni évasement ni rétrécissement.

La capsule à paroi mince est peu chargée de fragments siliceux toujours très petits.

Nous avons figuré l'organisme dans des attitudes différentes, avant et pendant (fig. 2I) les circonstances physiologiques de la division. Mème légende que plus haut.

Distribution Géographique. - Còtes orientales de l'ile sud de la NouvelleZemble; Mer de Kara, nos IO4 $_{\text {à }}$ IO8.

\section{Tintinnopsis angusta sp. n.}

Planche XII, figures 23 et 24 .

Forme analogue à la précédente, mais beaucoup plus étroite, plus svelte, caractérisée aussi par un rétrécissement gradué de la capsule du bas vers le haut, jusqu'à l'orifice, qui est droit.

Distribution géographioue. - Plus rare; partie nord de la Mer de Barents.

\section{Tintinnopsis fusus sp. n.}

Planche XII, figure 25 et variantes : figures 20 à 28 .

Capsule renflée vers le milieu, fusiforme vers le bas, rétrécie et un peu étranglée vers le haut, où s'accuse souvent une structure spiralée (fig. 25); orifice tronqué, droit.

Dimensions assez variables; comparer les figures 25, 26 et 28.

La figure 27 est une coupe longitudinale optique montrant le propriétaire de la loge. Nous n'ignorons pas que des auteurs ont fait de cette forme un $T$. beroidea. Nous ne pouvons les suivre, car il y a certainement incompatibilité spécifique entre cette forme et celle décrite plus haut sous ce nom.

Distribution géociraphigue. - Toujours rare sauf, dans la Mer de Barents, les nos I66 ̀̀ I 68 . 


\section{Tintinnopsis rapa sp. n.}

Planche XII, figures 20 à 35, et, peut-être, planche XIII, figures It à I6?

Forme étroite fusiforme - en manière de rave ou de carotte - longuement atténuée vers le bas, brusquement tronquée vers le haut (fig. 30 et 32) ou prolongée de ce côté en un tube cylindrique (fig. 29 et 3I), qui parfois prend un très grand développement, $t$ (fig. 33), ce qui semble lié à une phase de sporulation de l'organisme, $s$. Celui-ci, après avoir perdu ses organes oraux, s'est ramassé en boule dans la partie dilatée de la capsule et s'y est subdivisé en un certain nombre de sphérules qui restent groupées.

Nous sommes porté à considérer la figure 35 comne un spécimen en voie d'enkystement. Le kyste oviforme, sp, formé à l'orifice de la capsule, est entouré d'une membrane fermée, garnie de fragments siliceux comme la capsule maternelle.

La position de ce kyste, fixé comme un bouchon sur l'orifice de la capsule, nous aurait semblé ètre un cas fortuit de juxtaposition de deux organismes indépendants, si nous ne l'arions observée plusieurs fois, ce qui réduit les probabilités d'un fait accidentel.

C'est ainsi que nous avons été amené à examiner de plus près ces kystes isolés que l'on rencontre assez rarement dans les préparations et que nous avons reproduits en vue stéréoscopique dans la figure I5, planche XIII, et en coupe optique dans la figure i6, même planche, où l'on peut voir le noyau, $n$, et le protoplasme granuleux, $p s$, , du contenu de la capsule, $c p$. Des particules de quartz, $s$, font saillie sur l'un des pôles.

Après réflexion, nous estimons que l'orientation de ces figures devrait être renversée, le haut mis en bas, car la figure if de la même planche XIII pourrait bien n'être qu'un kyste de ce genre incomplètement formé ou déjà ouvert, par déhiscence circulaire, pour constituer un spécimen rénové de l'espèce : terme initial un peu différent des autres d'une nouvelle série phylogénétique.

La figure 34 , planche XII, reproduit une phase physiologique de l'organisme, où il semble qu'il faille voir un phénomène de conjugaison. Deux individus rapprochés obliquement par leur sommet ont fusionné leur péristome et leur couronne orale, cof. L'organe résultant de cette soudure est ainsi dévié perpendiculairement à l'axe des deux organismes conjugués et dirigé vers l'ouverture que ménage, d'un còté, la position oblique des deux capsules.

Les cas de ce genre n'étaient pas très rares dans des échantillons provenant de Tromsö, d'où cette intéressante espèce nous est connue.

Nous n'avons pas pu nous attarder à rechercher les mouvements nucléaires dont cette conjugaison s'accompagne, ni contrôler leur concordance avec les phénomènes observés dans des circonstances analogues chez d'autres groupes de Protozoaires. 


\section{3e série. - LES STÉNOSTOMES}

FORMES NON CYLINDRIQUES, A ORIFICE RÉTRÉCI

\section{Tintinnopsis ventricosoides sp. n.}

Planche XIII, figures I à $S$.

Forme trapue, sensiblement isodiamétrale, présentant un maximum de dilatation vers le tiers inférieur, plus ou moins conique à la base, légèrement rétrécie à l'orifice (fig. 3).

Dans l'espèce ventricosa, gui lui est du reste fort ressemblante, le maximum de dilatation se trouve vers le tiers supérieur de la capsule, ce qui permet de l'en distinguer aisément. Cette dernière espèce, que nous connaissons de la Mer Flamande où elle est de loin la forme la plus commune du groupe avec $T$. campanula, semble faire défaut, aussi bien que celle-ci, dans les récoltes de la Belgica.

$T$. ventricosoides présente aussi toujours des dimensions plus grandes et sa capsule, cp, est chargée de gros fragments de quartz.

Dans les figures I, 2 et 5 le Protozoaire, faisant plus ou moins saillie en dehors de la capsule, montre la couronne orale, co, fixée sur le péristome, au milieu duquel s'élève la protubérance orale, po (fig. 5), que côtoie, d'un cỏté, l'assophage, as.

Le corps, en s'élevant en dehors de la capsule, relève avec lui les granulations siliceuses, ss (fig. I), qui, lorsque l'animal se contracte, forment, autour de l'orifice oral, une sorte d'opercule incomplet, ss (fig. 4, vue apicale d'un individu contracté).

Les figures 6 et 7 montrent respectivement de profil et de face le même individu en état d'extension complète et permettent de s'assurer de la structure lamellaire de la couronne orale divisée, par des sortes de nervures, en une bonne vingtaine de segments qui déterminent, à la périphérie, autant de lobules triangulaires arrondis, comme une corolle gamopétale multilobée.

La figure 8 en reproduit un dernier spécimen en état de contraction incomplète.

Distribution gÉographique. - Assez commune dans la Mer cle Barents, au voisinage de l'ile sud de la Nouvelle-Zemble.

\section{Tintinnopsis nivalis sp. $\mathrm{n}$.}

Planche XIII, figures 20 et 27 .

C'est la seule forme de Tintimnopsis trouvée communément dans nos échantillons de neige colorée, d'où elle a passé parfois dans les eaux marines sous-jacentes. C'est cette circonstance d'habitat qui nous lui fait donner le nom de nizalis, malgré ses étroites 
analogies de forme avec $T$. ventricosa, sous des dimensions plus petites. On remarquera, en effet, que le plus grand diamètre transversal se trouve vers le tiers supérieur de la capsule.

La grosseur des fragments de quartz qui revêtent la capsule est très variable, suivant l'état de ces particules dans les milieux fréquentés par l'animal.

Dans les individus provenant directement de la neige (fig. 27), les granules siliceux sont très ténus. Les rares spécimens provenant d'ailleurs (fig. 26) sont chargés de fragments de quartz sourent volumineux.

\section{Tintinnopsis oliva sp. n.}

Planche XIII, figures 9 à 13 , et planche XIV, figure 6 .

Forme cllipsoüde présentant généralement, près de l'orifice, un col étroit, c, exempt des granulations siliceuses qui couvrent tout le reste de la capsule, mais surmonté de granulations plus fines (fig. II et I3), mobiles avec les mouvements d'invagination et d'évagination du Protozoaire.

La figure II en montre une loge habitée, que le chloral a rendue suffisamment transparente pour y distinguer l'Infuṣoire à l'état normal, avec un seul noyau, $n$.

Dans la figure Io, l'animal, presque entièrement expulsé de sa loge par une cause mécanique, se montre organisé comme ses congénères.

La figure iz en montre la couronne orale, co, en demi-extension sur le péristome allongé et saillant.

La figure 9 le présente operculé par un amas de corpuscules quartzeux, ce qui nous le fait supposer en passe d'enkystement.

La figure 6, planche XIV, enfin, le montre en voie de division, avec deux noyaux, $n$, et deux couronnes orales, l'une terminale, l'ancienne, co, l'autre latérale, coj, de nouvelle formation. La capsule cp n'est figurée qu'en coupe optique.

Distribution GÉographigue. - Disséminée çà et là dans plusieurs échantillons des deux mers, mais moins rare dans les environs de Tromsö.

\section{$4^{\text {e }}$ série. - LES FAUCIFERES}

Formes Non Cylindriques, a orifice Évasé, aU-dessus d'Un Étranglement (fauces) PLUS OU MOINS MARQUÉ

Tintinnopsis patula sp. n.

Planche XIII, figures 18 et 19 .

Capsule à corps ellipsoïde surmontée, du côté antérieur, d'un large évasement en pavillon et incrustée sur toute son étendue de gros fragments quartzeux (fig. Ig). 
La figure IS en est un spécimen habité montrant, autour du péristome, des granulations siliceuses, gs, qui forment une sorte d'opercule au niveau du col rétréci de la capsule, quand l'animal est en contraction.

Distribution GÉographioue. - Assez rare; extrême nord de la Mer de Barents et Océan Glacial.

\section{Tintinnopsis sinuala Brandt}

Planche XIII, figure 20.

Capsule en forme de vase allongé, renflé vers le milieu, longuement atténué vers la base qui est obtuse, étranglé vers le haut, en dessous d'un évasement oral.

C'est sous cette forme exactement que BRANDT (Bibliotheca Zoologica, I8g6) figure son espèce simuata, dont il tire le nom, non de la forme de la capsule, mais de la disposition sinueuse des interstices entre les granulations siliceuses dont la paroi est incrustée. C'est là, ce nous semble, une particularité trop inconstante et trop individuelle pour être tenue pour caractéristique de l'espèce, car on sait que la grandeur, l'abondance et la disposition de ces corps étrangers dépendent des ressources minérales $\mathrm{du}$ milieu bien plus que de l'organisme lui-même.

Distribution géographique. - Très rare; nord de la Mer de Barents, no 2 í.

\section{Tinlinnopsis acuta sp. n.}

Planche XIII, figure 17 .

Capsule dilatée vers son milieu, terminée en pointe inférieurement, légèrement évasée en avant, au-dessus d'un ćtranglement.

Distribution GÉographioue. - Trouvée seulement dans le Détroit de Matotchline.

\section{Tintinnopsis lurnula sp. n.}

Planche XIII, figures $2 \mathrm{I}$ à 25 .

Formes ramassées comme $T$. ventricosa, mais légèrement évasées à l'orifice, au-dessus d'un faible étranglement. Nous en figurons plusieurs variantes entre lesquelles les écarts de forme sont trop faibles pour empêcher la confusion dans la pratique des recherches.

Le spécimen 24 est operculé par un amoncellement de fragments de quartz. Il y a lieu d'y voir sans doute un phénomène d'enkystement. 
Dans la figure 25, on voit la capsule normale surmontée d'un tube cylindrique, $t$, dù à une évolution exceptionnelle de l'organisme, dont la rareté du cas ne nous a pas permis de fixer la signification.

Distribution GÉographigue. - Disséminées, surtout dans la Mer de Kara.

\section{Tintinnopsis Undella sp. n.}

Planche XIII, figure 28 .

Forme présentant la remarquable particularité d'avoir sa membrane manifestement formée de deux lamelles dont l'écartement augmente progressivement vers le bas, $m d$. Par ce caractère elle se rapproche du genre Undella, dont on pourrait faire aussi bien une espèce caractérisée par la présence de particules siliceuses sur la surface externe.

Nous n'en avons vu que des exemplaires vides.

Distribution GÉographioue. - Très rare; Mer de Kara.

Tintinnopsis incurvala sp. n.

Planche XIII, figure 29 .

Forme aberrante, à orifice évasé, oblique sur le corps incurvé de la capsule.

Ne l'ayant pas rencontrée habitée, nous ne pouvons affirmer qu'elle soit bien d'un Tintinnopsis.

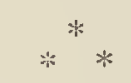

Les quelques microzoaires dont nous allons dire un mot ci-dessous, ne sont pas des Tintinnides. Ils n'en ont pas tous les caractères de famille, car ils manquent de la capsule dans laquelle ceux-là évoluent librement.

On trouvera sans doute qu'il y aura lieu de leur faire une place à part, quand on sera mieux fixé sur leurs caractères physiologiques.

\section{Genre Conocylis g. n.}

Nous nous proposons de désigner provisoirement sous ce nom générique des organismes de forme conique qui portent une couronne orale formée de lamelles fibrillaires, analogue à celle des Tintinnides, mais insérée directement, sans l'intermédiaire d'un péristome contractile, sur le corps même de l'organisme, qui nous a toujours paru soudé avec sá capsule.

Il en résulte, pour celui-là, l'impossibilité d'y ramener ses appendices oraux lorsqu'il les met en contraction. 
Conocylis helix sp. n.

Planche X, figures 32 et 33 et, peut-être, $3+$ et 35 .

Corps en forme de coquille conique et spiralée dont les tours de spire s'invaginent partiellement l'un dans l'autre (fig. 32) et sont décorés d'une striation longitudinale irrégulière.

Cytoplasme étroitement serti dans une coque qui semble chitineuse et avec laquelle il parait intimement soudé.

La coupe longitudinale optique, reproduite dans la figure 33, montre bien comment les tours de spire de la coque s'invaginent légèrement de haut en bas.

La paroi en est à double contour; elle présente une striation superficielle propre à chaque tour de spire.

La partie émergente du corps protoplasmatique nous a toujours paru divisée en deux lobules arrondis, sans que nous puissions dire s'il existe entre eux une sorte d'orifice buccal.

La portion logée dans la coque est très grossièrement granuleuse et présente un noyau.

La couronne lamellaire orale, $c o$, semble insérée au niveau du pourtour supérieur de la coque, dont l'accroissement est hélicoïde.

A côté des exemplaires du type décrit, que leurs grandes dimensions nous font considérer comme adultes, nous avons trouvé des formes beaucoup plus petites chez lesquelles la coque ne présente que deux à trois tours de spire. Ne pourrait-on les tenir provisoirement pour des formes jeunes, en attendant la connaissance de leur genèse et de leur évolution?

La figure $3_{4}$ en est une vue extérieure, la figure 35 une coupe optique longitudinale.

On saura plus tard s'il faut y voir des représentants d'une autre espèce analogue, mais beaucoup plus petite.

Distribution géographioue. - Nous tenons ces deux formes, grande et petite, de l'Océan Glacial, $\mathrm{n}^{\circ}$ 226, échantillon malheureusement très peu copieux.

D'autres échantillons nous avaient montré antérieurement des fragments de coque vide, dont nous avons figuré un exemplaire (pl. VII, fig. 28) avant d'en connaitre la nature.

\section{Conocylis constricta sp. n.}

Planche $\mathrm{X}$, figures 36 et 37 .

Forme conique présentant de nombreuses cannelures longitudinales et brusquement rétrécie à la base, où apparait un petit bouton turbiné séparé du corps de la capsule par un étranglement étroit. 
A l'état d'extension, le corps protoplasmatique, soudé ici aussi avec la coque chitineuse, forme, à son sommet, une émergence hémisphérique développée dans la couronne lamellaire orale, co, qui est très développée (fig. 36).

A l'état de contraction, cette protubérance est rétractée (fig. 37), mais la couronne orale, co, reste à l'extérieur de la coque, dans laquelle elle ne sait pas s'invaginer.

Distribution géographique. - Très rare, dans la Mer de Barents; no i66.

\section{Conocylis striata sp. n.}

Planche XI, figure 22, et planche XXIII, figure 13.

Corps conique, un peu irrégulier, orné de cannelures longitudinales plus accusées vers le milieu, peu prononcées vers la base, qui se termine en pointe obtuse arrondie.

Le nombre des cannelures apparaitra très variable si l'on compare les deux figures que nous consacrons à cette forme rare. On y verra aussi une grande différence de calibre.

Malgré ces écarts dont nous ne saurions actuellement apprécier la portée, nous les réunissons sous une même dénomination, afin de ne pas multiplier les distinctions spécifiques dans des objets si peu observés, sans doute, jusqu'ici et que nou's n'avons encore vu signaler nulle part.

Des observations ultérieures dicteront ce qu'il faut en penser.

\section{Genre Strombidium Claparède et LachManN}

\section{Strombidium longipes sp. n.}

Planche XIV, figure 8 .

L'embarras que nous éprouvons à trouver une place convenable pour l'organisme que nous figurons ici, nous vient autant de sa rareté dans nos échantillons que de l'impossibilité où nous nous sommes trouvé d'en noter les caractères physiologiques.

C'est néanmoins avec les Strombidium qu'il nous parait devoir recenser cette forme de Protozoaire oligotriche dépourvu de capsule, dont nous reproduisons les traits dans l'état où l'ont mise les réactifs fixateurs.

Le corps arrondi est prolongé inférieurement en une longue queue pointue. On y distingue un noyau, $n$, des inclusions, $i n$, et un corps, $x$, très réfringent, dont la section transversale a l'aspect d'un anneau et dont nous ignorons la nature.

Une couronne incomplète de cils d'inégale valeur, co, occupe le sommet du corps et $\mathrm{y}$ forme un organe qui n'est pas sans analogie avec la couronne orale des Tintinnides. Nous doutons toutefois que cet organe soit lamellaire plutôt que simplement cilié.

Distribution géographique. - Mer de Kara; très rare. 


\section{Strombidium sp.}

Planche XVIII, figure 20.

C'est aussi, nous semble-t-il, à ce genre qu'il y a lieu de rapporter ce petit Infusoire que nous n'avons pu observer qu'à l'état momifié dans le milieu conservateur de nos échantillons. Comme il ne présente en cet état aucun caractère morphologique spécial, il serait aussi téméraire de vouloir l'identifier avec une forme connue que de lui attribuer une spécificité nouvelle.

Renaroue. - On estimera peut-être que ces deux formes trouveraient aussi bien leur place dans le groupe des Infusoires non loriqués; nous n'en disconvenons pas. Cependant elles suggèrent l'idée de Tintinnides échappés de leur capsule et adaptés momentanément à la vie libre.

\section{Section II. - Vorticellides}

C'est aux Vorticellides capsulaires que nous croyons devoir rapporter de rares formes qui, eu égard aux échantillons dont ils proviennent, nous ont paru d'origine còtière et ainsi plus ou moins étrangères au plankton marin proprement dit.

Nos matériaux fixés ne nous ayant mis en présence que de spécimens contractés, il serait difficile d'en urger l'identification avec des espèces connues, qui tirent leurs caractères distinctifs bien plus du Protozoaire lui-même et spécialement de la structure de son appareil oral que de la forme de la capsule.

\section{Genre Cothurnia Ehrenberg}

Planche X, figure 3I. Forme droite à pédicelle bien distinct dans le prolongement, atténué à la base, de la capsule dont le corps est à peu près cylindrique.

Serait-ce C. imberbis Ehrenberg? Voir Saville Kent (I), page 720.

Planche XIV, figure 9. Forme droite à capsule, cp, ondulée transversalement, renflée à la base, atténuée vers l'orifice. Le pédicelle, pd, est assez court et dilaté au voisinage de la, capsule.

Serait-ce C. nodosa Claparède et Lachmann? Voir SArilde ÍEent, page 72 I. 
Planche XIV, figure Io. Capsule courbe, renflée à la base, très rétrécie vers l'orifice. Le pédicelle, $p d$, est presque aussi long que la capsule et incurvé comme elle.

Serait-ce C. curva Stein, malgré la moindre longueur du pédicelle donné à cette espèce?

\section{Genre Vaginicola Lanarck ou Thuricola Saville Kent}

En l'absence du Protozoaire, nous ne savons auquel de ces deux genres il convient de rattacher les deux petites capsules (pl. X, fig. 29 et 3o), sensiblement de mème forme incurvée, l'une lisse, l'autre finement réticulée.

\section{Section III. - Rhizopodes Amœbiens}

La capsule des Rhizopodes Amabiens ou Monothalames simule assez bien parfois celle de certains Infusoires loriqués, des Tintimnopsis les plus frustes spécialement.

Nous avons jugé bon de ne pas les séparer, afin d'en faciliter la comparaison, bien que nous ayons prévu un chapitre spécial pour les Rhizopodes.

Nous ne comptons rattacher aux Rhizopodes Amabiens, avec quelque certitude, qu'une forme très commune dans nos échantillons de neige colorée et qui semble affine aux Difflugiides, par sa capsule ouverte à une extrémité et incrustée de corpuscules étrangers.

\section{Difflugia nivalis sp. n.}

Planche XIII, figures 3i à to.

Petites formes très variables d'aspect et de dimensions, comme on s'en rendra compte par l'examen des figures qui s'y rapportent.

Tantôt minuscules (fig. 37), tantòt assez grandes (fig. 34 et 35), étroites (fig. 38), ou assez larges (fig. 33), les capsules sont fragiles, chargées de granulations étrangères et pourvues d'un orifice plus ou moins étranglé.

L'organisme, toujours contracté, ne révèle aucune structure, se présentant sous la forme d'un globule protoplasmatique souvent huileux, ph (fig. 40, coupe optique). On y découvre un noyau, $n$ (fig. 38 et 39 , coupe optique), quand on a pris soin d'éclaircir suffisamment l'objet par le chloral.

Distribution géographique. - Commun dans les neiges colorées de la Mer de Kara. 


\section{Di/flugia spharica sp. n.}

Planche XIII, figure 30.

Nous ne pouvons hésiter non plus à faire un Difflugia de l'organisme dont nous ne connaissons que la capsule sphérique trouvée en rares spécimens dans les eaux de la Mer de Kara.

Ses dimensions plus grandes, sa forme globuleuse, son orifice, o, très étroit relativement, son revêtement de gros fragments de quartz, son milieu spécial, etc., sont autant de circonstances qui nous la font considérer comme spécifiquement distincte de la précédente.

\section{Annexe aux Amobiens}

Bien que nous ne soyons pas fixé sur leur nature intime, c'est ici que nous devons faire mention de kystes de nature énigmatique, dont nos échantillons de la Mer de Kara nous ont fourni quelques exemples :

Io Planche XIII, figures 4I et 42. Organisme sphérique circonscrit par une capsule fermée de toutes parts et chargée de granules siliceux assez gros, si ce n'est autour du pôle antérieur, ca, où les granulations incrustées sont beaucoup plus ténues.

La figure $4 \mathrm{I}$ en est une vue de profil, la figure 42 une vue oblique montrant mieux le centre organique antérieur, ca. On s'attend à voir se produire là une déhiscence, dans des circonstances données.

$2^{\circ}$ Planche XIII, figures 43, 44, 45. Organisme turbiniforme à capsule fermée et chargée également de corpuscules quartzeux, plus gros sur la partie conique inférieure que sur la calotte supérieure.

La figure $4^{3}$ le montre en vue stéréoscopique, de profil.

La figure 44 en est une coupe longitudinale optique montrant le contenu protoplasmatique grossièrement granuleux et un volumineux noyau, $n$.

La figure 45 en est une vue apicale visant la calotte antérieure moins chargée de corpuscules étrangers.

\section{Groupe VII. - INFUSOIRES NON LORIQUÉS}

Certains échantillons de pèche de la Mer de Kara particulièrement nous ont mis en présence de Protozoaires ciliés de grande forme qui tiennent une place importante dans le plankton de cette mer.

Leur présence dans ces parages s'explique par la formation de nappes superficielles d'eau presque douce dues à la fonte des neiges et des glaces pendant la saison estivale. 
Ce sont, en effet, des pêches effectuées dans des sortes de petits lacs situés sur les glaces mêmes qui nous ont fourni les meilleurs contingents de cette faunule exceptionnelle.

Ces microzoaires y constituent une population étrange dont les représentants, livrés à une àpre lutte pour l'existence, nous ont fourni des documents très intéressants pour l'histoire de leur vie intime et de leurs rapports sociaux.

Nous ferons donc plus que d'en dresser l'inventaire.

Nous nous attacherons à faire connaitre celles de leurs phases évolutives qu'ils nous ont permis de surprendre. Nous appellerons aussi l'attention sur quelques épisodes de leur vie sociale qui traduisent d'une manière saisissante l'àpreté des rapports que la concurrence vitale établit entre eux.

\section{Section I. - Hypotrichides}

Les Hypotrichides rencontrés ici sont des Euplotes géants qui nous paraissent appartenir à deux espèces distinctes de celles décrites précédemment.

\section{Genre Euplotes Ehrenberg}

Euplotes caudata sp. n.

Planche XV, figures I à 5 ; planche $\mathrm{XX}$, figures 6 et 7 .

L'examen comparé des figures I à 4, planche XV, permettra au lecteur de s'orienter dans la structure compliquée de cet organisme, qu'une description littéraire serait impuissante à traduire d'une façon suffisamment précise pour éviter toute confusion avec ses congénères.

On remarquera la forme atténuée du corps du côté postérieur, circonstance qui suffit à lui constituer un caractère spécifique, auquel fait allusion le nom de caudata que nous lui attribuons.

La figure I en reproduit une vue ventrale. C'est le côté qui porte les cirres ou pinceaux de cils agglomérés qui servent d'organes de locomotion à l'animal : cls, cirres supérieurs ou frontaux; clv, cirres ventraux; cli, cirres inférieurs.

La figure 2 en est une vue dorsale.

La figure 3 en reproduit une vue latérale, gauche ou orale.

La figure 4 le montre en vue latérale droite.

La membrane extérieure, assez rigide, est cannelée longitudinalement sur toute son étendue. Le nombre des cannelures visibles du còté dorsal dépasse une douzaine. 
Le péristome garni de puissants cils oraux, co, s'étend sur toute la partie frontale et sur presque toute la longueur du còté gauche.

Le corps est généralement gorgé d'inclusions alimentaires, in (figg. 6 et 7 , pl. XX), parmi lesquelles Diamylon nivale manque rarement dans les parages où ce microphyte abonde.

Le noyau, $n$, est en forme de boyau long, sinueux, convexe du côté oral et incurvé aux deux extrémités. La figure 5 le montre isolé, avec sa membrane propre, mn, et sa structure filamenteuse grossière, en dehors des périodes de division.

Les figures 6 et 7 , planche $\mathrm{XX}$, en montrent un individu en division, sous ses deux aspects, ventral (fig. 6) et dorsal (fig. 7), avec étranglement médian, indice du processus de scissiparité.

Le péristome cilié y est déjà double : la partie ancienne, $b$, et la partie jeune, $b^{\prime}$.

Le noyau, $n$, accuse déjà une structure fibrillaire beaucoup plus fine, avant de se raccourcir pour subir la division.

Il y aurait beaucoup à dire tant sur les particularités de structure anatomique que sur les modalités du phénomène de division chez cette espèce; mais ce n'est le lieu de nous y attarder dans cette étude, où tant d'autres formes réclament notre attention. Nous en ferons plutòt l'objet d'une étude ultérieure, en y joignant sa congénère, non moins curieuse, que nous ne ferons guère que signaler ci-après.

\section{Euplotes truncala sp. n.}

Planche $\mathrm{XX}$, figures 8 à it.

La caractéristique de cette espèce, plus volumineuse encore que la précédente, nous parait être suffisamment traduite dans la forme tronquée du còté postérieur du corps, lequel paraît aussi beaucoup plus trapu.

La figure II en montre un individu normal, dụ còté ventral, avec ses cannelures longitudinales dans la membrane, son péristome très développé, sa membrane ondulante préorale qui, du còté frontal, constitue une sorte d'opercule, ma, sur le péristome; son noyau, $n$, ses inclusions alimentaires abondantes, $i n$, avec Diamylon, $D$, etc.

Les figures 8, 9 et Io en montrent trois individus en voie de division :

Figure 8 , en vue dorsale,

Figure 9, en vue ventrale,

Figure Io, en vue latérale gauche ou orale.

On y remarquera : $I^{\circ}$ des étapes variées de la formation du péristome jeune, $b^{\prime}$, en dessous et en arrière du péristome primitif, $b ; 2^{\circ}$ la forme contractée du noyau, $n$, qui se ramasse en prenant une structure fibrillaire plus fine pendant cette période critique de l'évolution de l'animal.

Les figures I2, I3 et I4, relatives au noyau seul, montrent quelques-unes des variantes de forme et d'aspect de cet organe pendant les divers états du Protozoaire. 
Dans les figures $\mathrm{I} 2$ et $\mathrm{I} 3$, on remarquera les étranglements, é, dont nous rechercherons ailleurs la signification.

La figure $\mathrm{r}_{4}$ donne une idée de la structure fibrillaire que prend l'élément chromatique du noyau, pendant qu'il se raccourcit et tend à prendre la forme ramassée et globuleuse qu'on lui voit dans les figures 8 et 9 , figuratives de phénomènes variés de la scissiparité.

\section{Section II. - Holotrichides}

Devant rencontrer ici plusieurs représentants de ce groupe d'Infusoires, nous devons accorder la priorité à celui d'entre eux qui, dans ce milieu spécial, affecte sa supériorité sur tous les autres par sa puissance, ses moyens d'attaque, sa voracité insatiable, tout ce qu'il faut pour en faire un monstre redouté vis-à-vis de la population microplanktonique que la nature met à sa portée.

\section{Genre Didinium Stein}

Genre d'Infusoires holotriches libres, caractérisé par la présence sur le corps, en grande partie nu, de deux couronnes de cils locomoteurs et par la structure de la bouche, qui est terminale, extrêmement dilatable et armée d'une sorte de nasse pharyngienne mobile.

De l'espèce nasutum Müller, qui est la seule connue jusqu'ici, que nous sachions, et qui est d'eau douce, nous arons à faire connaître une congénère marine chez laquelle les caractères si particuliers du genre ont une expression beaucoup plus tangible.

\section{Didinium Gargantua sp. n.}

Planche XV, figures 9 à 15 ; planche XVI, figures $1,3,5,6,7,8$, 9, Io, i ct 12 ; planche XVII, figures i à 5 : planche XVIII, figures $1,2,4,6,8$, Io et II; planche XIX, figures 14,10 , 17 et I8; planche XXIli, figure in.

\section{Morphologie de L'animal}

La plasticité du corps et l'élasticité de sa membrane tégumentaire lui permettent de revêtir des formes tellement diverses et inconstantes qu'il serait difficile de lui en attribuer une spéciale, susceptible d'ètre considérée comme normale et caractéristique. 
Tantòt sphérique (fig. I2, pl. XV), tantòt cylindro-sphérique (fig. I I, pl. XV), tantòt ellipsoïde (fig. 8, 9, r4, pl. XV), le corps peut aussi s'allonger et présenter un étranglement médian (fig. Io, pl. IV) ou enfin prendre des contours plus compliqués dont on verra des exemples dans les figures I et 2 de la planche XVI, particulièrement.

L'appareil tégumentaire présente toujours une membrane hyaline externe distincte de celle qui fait corps en quelque sorte avec l'ectoplasme : c'est une sorte de cuticule qui s'observe toujours plus distinctement au voisinage de la bouche, comme dans les figures II et I2, planche XV, par exemple.

La plus grande partie du corps est glabre, mais on y observe constamment deux ceintures ciliaires, $c c a$ et ccp, formées chacune d'une seule série de cils longs, serrés, d'égale valeur et semblablement orientés, par une sorte de solidarité qui ne parait pouvoir s'expliquer que par une certaine coalescence. Elles prennent ainsi la forme d'une collerette ou d'une membranelle annulaire à texture finement fibrillaire; l'antérieure, cca, toujours creusée en pavillon vers le haut, dans l'animal fixé; la postérieure, ccp, plus souvent étalée et parfois même réfléchie en sens inverse vers le póle anal du corps.

Ces ceintures dépriment légèrement le corps, au niveau de leur insertion, suivant deux cercles perpendiculaires à l'axe longitudinal. Elles divisent ainsi le corps en trois régions : la région postérieure ou anale, au milieu de laquelle s'ouvre l'anus, $a$, en rapport avec une grande vacuole anale, $v a$, entourée de beaucoup d'autres; la région médiane toujours la plus développée; enfin la région antérieure ou orale, où s'ouvre la bouche dans un prolongement conique dont'l'aspect varie, du reste, beaucoup avec les manifestations biologiques du Protozoaire.

L'aspect du sarcoplasme varie aussi énormément avec la nature des inclusions plus ou moins abondantes et en cours de digestion plus ou moins avancée.

Il est à noter, en outre, que, indépendamment des inclusions alimentaires souvent reconnaissables par leurs caractères propres, le sarcoplasme plus ou moins vacuoleux est souvent très riche en enclaves, produits de son activité physiologique.

Ce sont des corps protéiques, des globules de matières grasses et fréquemment aussi des grains de fécule, $f$ (fig. $\delta$ et 9 , pl. XV), dont la forme assez constante et l'état disséminé dans le protoplasme périphérique semblent témoigner d'une formation sur place.

Rien cependant, dans l'état de nos matériaux d'étude, n'autorise à penser que l'animal vivant soit pourvu d'une chromophylle quelconque.

Cette particularité se reproduisant dans d'autres Protozoaires habitant le mème milieu, on doit se demander quelles sont les conditions spéciales qui confèrent à ces organismes cette faculté que l'on considère d'ordinaire comme liée exclusivement à la fonction chlorophyllienne.

Le noyau, toujours volumineux, affecte aussi des formes variées qui peuvent se ramener à deux types qui correspoindent, sans doute, à des phases évolutives différentes de l'organisme. 
Nous distinguons : Io le type A (fig. I5, pl. XV); en forme de croissant présentant une troisième corne dans la partie concave; $2^{\circ}$ le type $\mathrm{B}$ (fig. 8, pl. XVI), plus volumineux et plus long, en forme de saucisson diversement recourbé.

Dans les deux cas, la structure de l'élément chromatique est manifestement fibrillaire, fn (fig. S, pl. XVI), à l'intérieur de la membrane propre du noyau, m.

La bouche, b, est terminale. C'est un orifice circulaire prolongé inférieurement en une sorte d'entonnoir pharyngien qui se prolonge, en se rétrécissant, dans le sarcoplasme où il se perd et que limitent des baguettes disposées en forme de goulot de nasse.

De cet orifice émerge habituellement une masse sarcodique, formant bouchon, d'où s'élancent de fortes soies orales, so, qui semblent revêtir plutôt les caractères de trichocystes buccaux, à destination d'armes offensives (fig. 8, 9, 10, II, I2, pl. XV).

Il semble qu'il y a identité de nature entre ces trichocystes désordonnés et les baguettes bien ordonnancées de la nasse pharyngienne. Ce sont des éléments interchangeables, qui peuvent se substituer les uns aux autres suivant les circonstances.

Leur nombre n'est du reste pas fixe. Il peut varier indéfiniment “au gré de l'animal qui, suivant les besoins du moment, en produit de nouveaux aux clépens de son sarcoplasme ou en réduit le nombre par une sorte de résorption. Certains cas figurés en fourniront des exemples et préciseront davantage les détails de structure de la bouche dans l'exercice de ses fonctions.

\section{Manifestations Biologioues de L'animal}

Il est bien regrettable qu'on n'ait pas songé à observer ce microzoaire pendant la vie, pour étudier ses mours. L'observateur aurait certainement joui d'assister à des scènes dramatiques dont les matériaux fixés ne nous permettent de saisir que des moments' d'action.

L'animal, anodin d'apparence, avec sa bouche étroite en temps ordinaire, doit en réalité être un véritable fléau pour la population microscopique que la nature met à sa portée.

Talonné, semble-t-il, par un appétit insatiable, il s'attaque à tout ce qui est à sa portée, mème ses semblables.

Armé de trichocystes buccaux, dont il use sans doute comme de traits empoisonnés, il en larde sa victime et la paralyse; puis, ouvrant une bouche dont l'extension n'a guère de limites, il aspire sa proie, en quelque sorte, dans un pharynx nassiforme qui aboutit à une vacuole sarcodique dilatable, où elle finit par passer, quelque soit son volume souvent supérieur à celui de l'agresseur.

L'attaque débute vraisemblablement par une projection en fusée des trichocystes buccaux, co (fig. I, pl. XVIII). Cette projection s'accompagne d'un rétrécissement du pòle oral, rétrécissement sans doute violent et subit qui cause lui-même la projection. 
Les trichocystes ainsi projetés sont maintenus en faisceau par une gangue plasmatique qui les retient au sarcoplasme et les empêche de se perdre. Le coup ayant porté sur la proie convoitée, celle-ci, mortellement atteinte ou seulement paralysée, peut être ramenée vers l'orifice buccal, dans lequel les trichocystes reprennent peu à peu leur ordonnance régulière, autour de l'entonnoir pharyngien.

Quand la proie est volumineuse, la bouche, dilatée à suffisance, s'y applique aussi étroitement que possible et la déglutition s'opère avec la lenteur qu'exige la difficulté de l'opération.

Quelques exemples choisis parmi des centaines de cas observés suffiront à illustrer ces procédés.

Planche XV, figure I4. L'animal est en train de "gober " un Ptychocylis, Pt, dont 'il dédaigne la caṕsule, pour s'en prendre uniquement au corps protoplasmatique du Tintinnide. La bouche, $b$, de Didinium Gargantua est dilatée à la mesure de la capsule du Ptjchocylis, dans laquelle elle s'introduit, pour ne pas perdre une miette de cette proie facile. Celle-ci passera tout entière dans l'entonnoir œsophagien, autour duquel les baguettes-trichocystes ont repris une disposition régulière, en nombre suffisant pour en revêtir toute la paroi, quoiqu'elles y soient très rapprochées les unes des autres. Ceci doit faire admettre que l'animal les a multipliées pour les besoins du moment, car on ne les voit pas si nombreuses dans l'organisme en dehors de ses exercices gastronomiques. C'est un fait qui, on le verra, se reproduit dans tous les cas similaires. Entretemps l'agresseur reste coiffé de la dépouille de sa victime. Il s'en débarrassera aisément plus tard, par le retrait de la bouche et son retour au calibre normal.

Le noyau, $n$, est ici en saucisson, du type B, peu volumineux.

Planche XVIII, figure 3. Didinizum Gargantua procède ici autrement vis-à-vis d'un Ptychocylis encore, de dimensions presque égales aux siennes. Il se dispose à l'ingérer tout entier, contenant et contenu, et on peut croire que ce n'est pas toute présomption de sa part, car nombreux sont les cas observés où l'opération s'est faite sans mécompte pour l'agresseur, sinon pour la victime.

Le noyau, $n$, est ici du type A.

Dans le Ptychocylis, il y a deux groupes de noyaux géminés, $n$ et $n$. Plusieurs cellules de Diamylon nivale, $D$, s'y observent au milieu d'autres inclusions, in. L'appareil oral du Protozoaire est déjà complètement détruit.

Planche XIX, figure I6. Cette figure nous met en présence d'une opération de ce genre menée à bonne fin. Le Ptychocylis, $P t$, gorgé lui-mème d'inclusions, parmi lesquelles on reconnait Diamylon nivale, a été englouti vivant.

L'ingestion a dù précéder de très peu la pèche et la fixation du produit de celle-ci par le formol, car, d'une part, le Ptychocylis ne présente encore trace de digestion 
et, d'autre part, la bouche, b, de Didinimm, bien que légèrement rétrécie déjà, n’a pas encore eu le temps de revenir aux dimensions étroites qu'elle présente à l'état d'inaction.

Le noyau, $n$, est du type $B ; r$ désigne des résidus de digestions antérieures; va est la vacuole anale.

Planche XVII, figures I et 2 soudées. L'animal agresseur (fig. I) s'en prend ici à un de ses congénères (fig. 2), dont les dimensions sont un peu plus petites.

La victime est saisie par le flanc. Serait-ce pour en éviter les trichocystes buccaux? Elle présente des caractères morbides, traduits par une déformation de la masse buccale, par la sortie d'une partie de son sarcoplasme en dehors de la bouche, par une sorte de plasmolyse commençante du corps, par le décollement de la cuticule externe du tégument, qui devient flottante, etc.

L'agresseur, de sor còté, semble mettre toute son énergie en œuvre pour dilater sa nasse buccale à la mesure de sa proie et aspirer celle-ci par une sorte de succion.

Peu avant son entreprise actuelle, il avait absorbé des Diamylon nivale, $D$, que l'on reconnait intacts au milieu d'autres granulations de la masse sarcodique. Celle-ci est très vacuoleuse, conséquence probable d'un jeùne prolongé.

Le noyau, $n$, est volumineux et du type B. La vacuole anale est dilatée; un amincissement de la membrane, en $a$, marque l'orifice anal.

L'individu capturé (fig. 2) a un noyau du type A. On remarquera les grains de fécule, $f$, disséminés dans le protoplasme cellulaire; ils nous font l'effet de produits de l'activité propre de l'organisme; ce sont des enclaves plutôt que des inclusions.

Planche XVI, figures I et 2 soudées. Notre Gargantıa (fig. I) s'attaque ici à un Cyclotrichinm karianum. C'est l'objet favori de ses poursuites, bien que ce curieux organisme, que nous décrirons plus loin, soit souvent plus gros que lui.

Dans le cas reproduit ici, le monstre a saisi sa proie par l'extrémité buccale, ce qui semble être sa méthode préférée quand il chasse ce gros gibier, aussi délicat sans doute que mal armé pour sa défense.

On doit se demander s'il parviendra à surmonter les difficultés de l'ingestion d'une proie si disproportionnée à sa taille. L'entreprise peut paraître téméraire, mais les nombreux cas de succès observés démontrent bien qu'elle ne dépasse pas ses moyens au service d'une patiente ténacité.

On remarquera la déformation du corps qui traduit les efforts énergiques exercés par l'appareil tégumentaire dans le travail de succion de la bouche largement ouverte en entonnoir. Celle-ci s'applique adéquatement sur la partie saisie de la victime et fonctionne vraisemblablement à la façon d'une ventouse.

Les matières sarcodiques du Cýclotrichium sont aspirées dans une vacuole digestive qui augmente de volume à mesure de son remplissage, en même temps que le corps lui-même qui est éminemment dilatable. 
Le noyau, $n$, est du type A et figuré en coupe optique. Les granulations qui s'y dessinent sont la coupe transversale des filaments chromatiques.

Planche XVI, figures 3 et 4 soudées. On voit ici la reproduction d'un cas analogue au précédent mais plus étonnant encore, eu égard à la disproportion de taille entre les deux organismes aux prises.

L'écoulement du sarcoplasme mou de la victime (fig. 4), vers l'entonnoir-ventouse de l'agresseur, est rendu tangible en quelque sorte par l'orientation que prennent les grains de fécule, $f$, qui s'y trouvent, à l'état d'enclaves, pensons-nous, et que l'iode colore en bleu sur fond jaune.

D'autre part, on assiste au mélange des produits ingérés par le Didininm avec le protoplasme de celui-ci, car les grains de fécule, $f$, qu'on y découvre, ont sensiblement le mème aspect que ceux signalés tout à l'heure et semblènt bien provenir directement de la vidange progressive du Cyclotrichizm.

Planche XVIII, figures 6 et 7 réunies. Ici l'attaque du gibier - encore un Cyclotrichium (fig. 7) - s'est faite par le flanc, ce qui nous a paru plus rare.

Son corps mou, plastique, faiblement tégumenté, est déjà déformé, sur l'aire d'attaque, pàr la succion de la ventouse de Didinizm (fig. 6); mais l'absorption proprement dite n'en a pas encore commencé. On peut voir que la membrane du Cyclotrichinm n'est pas percée en cet endroit, mais y est soumise sans doute à l'action de principes dissolvants. De plus, la grande vacuole centrale du corps de Didinium, $v$, est encore vide de tout bol alimentaire, occupée seulement, peut-on croire, par des sucs digestifs qui n'attendront plus longtemps leur utilisation. Les grandes dimensions de cette vacuole empêchent la nasse pharyngienne de prendre sa forme régulière; c'est pourquoi l'extrémité inférieure de celle-ci est déjetée sur le côté.

Nous sommes porté à y voir le résultat d'un jeùne prolongé : ce que confirme du reste l'aspect anémique du protoplasme vacuoleux, dépourvu de presque toute réserve alimentaire, sous forme d'inclusions ou d'enclaves.

C'est seulement dans l'ectoplasme ou la couche périphérique du cytoplasme que le noyau, $u$, du type A, trouve à se caser. C'est à cette circonstance qu'on doit de le voir si bien, dans tout son développement.

Planche XVII, figures 3 et 4 réunies. Cette figure nous met en présence d'un cas du mème genre, mais dans lequel l'ingestion de la proie (fig. 4) est en bonne voie. Le corps de la victime, à moitié ingéré sous forme d'inclusion, in, dans une grande vacuole digestive, présente, dans la partie encore émergente, des caractères frappants de dislocation. Cela semble dù en partie à l'action des sucs gastriques dégorgés par Didinimm, en vue de faciliter l'absorption de la proie.

La membrane de celle-ci, $m$, semble en effet morcelée par déchirure et par digestion; le protoplasme, $p r$, se désorganise et devient diffluent; le noyau, $n^{\prime}-n^{\prime}$, traduit 
l'étirement auquel il est soumis en passant par la filière du cône pharyngien. Autres indications comme plus haut; le noyau, $n$, est encore du type $\mathrm{A}$, en croissant.

Planche XIX, figures I4 et I 5 réunies. Voici encore le même Protozoaire (fig. I4) aux prises avec une proie (fig. I5) qu'il a déjà réduite à l'état passif, par son attaque meurtrière, et dont il a commencé l'ingestion dans des conditions de facilité qui n'exigent pas la mise en ceuvre de tous ses moyens.

On constatera que les baguettes de la nasse pharyngienne affectent ici une disposition spiralée en harmonie avec la forme tordue de cet organe.

La déformation morbide de la victime l'a rendue méconnaissable.

La présence d'un Peridinizm, $P$, dans le sarcoplasme témoigne d'un repas antérieur dont cet organisme a fait les frais.

La capsule indigeste en sera sans doute rejetée ultérieurement par l'ouverture orale, car l'orifice anal, en rapport avec la vacuole anale, $v a$, ne semble pas pouvoir se prêter à l'évacuation de corps excrémentiels quelque peu volumineux.

Planche XIX, figure I7. Cette figure fait assister à une phase plus avancée d'un de ces curieux phénomènes de déglutition d'un organisme vivant de grande forme par Didinium Gargantua.

L'opération touche à sa fin. Le Protozoaire indéterminé, $P$, se trouve complètement engagé dans l'orifice buccal, qui se confond maintenant avec la grande vacuole gastrique suffisante pour loger la volumineuse inclusion, qui y entre d'une pièce. Tout le menu d'un repas en une bouchée; mais quelle bouchée!

C'est un fait d'observation générale que la nasse pharyngienne fortement dilatée pendant l'ingestion d'un bol alimentaire de grandes dimensions met du temps à revenir à la mesure restreinte de l'organe inactif. La cause en est sans doute dans la lenteur du processus de transformation régressive des baguettes, trichocystiques.

Celles-ci, devenues très nombreuses pendant la période d'attaque et de déglutition, doivent rentrer peu à peu dans le cytoplasme normal, suivant un processus dont nos matériaux morts ne nous ont pas permis de saisir la nature intime.

Le lecteur n'aura pas manqué d'observer dans cette figure, comme dans toutes celles qui se rattachent aux mêmes phénomènes, que ces baguettes sont de deux sortes : les unes très fortes, qui paraissent plus permanentes; les autres plus nombreuses, plus délicates, encadrées dans les premières et plus fugaces probablement.

Leur résorption éventuelle dans le cytoplasme constituerait un intéressant sujet d'étude, que nous signalons aux micrographes qui auraient la bonne fortune de disposer de matériaux vivants.

Dans la figure I7, $n$ désigne le noyau du Didinizm qui est du type B; n' désigne le noyau de l'Infusoire ingéré; $r$ signale des résidus de digestion antérieure; va est la vacuole anale très développée. 
Planche XVIII, figures 4 et 5, en réunion. La voracité extrême de Didinium Gargantua l'entraine dans des aventures sans issue, dont il nous reste à donner des exemples.

C'est le cas ici. On voit notre animal en travail d'ingestion d'un Fusopsis umbracula (fig. 5), dont la grande longueur et la rigidité de la membrane peu digestible semblent devoir faire obstacle à une incorporation utile.

A voir la physionomie de l'organisme, qui a été reproduit avec toute la fidélité possible, ne dirait-on pas que, revenu de son erreur, il cherche à se débarrasser de cette prise encombrante?

Planche XVIII, figures 8 et 9 , en réunion. On ne peut rien augurer de bon non plus, pour l'organisme qui nous occupe, de sa tentative inconsidérée d'absorber un crottin de Crustacé (fig. 9), dont nous ne figurons qu'un fragment et qui, en réalité, est au moins quinze fois plus long que lui.

Exemple curieux de voracité aveugle qui pousse l'animal à jeter son dévolu sur tout ce qui flotte à sa portée et lui parait bon à prendre pour peu qu'il puisse commencer à l'appréhender. Nous l'avons vu se jeter sur des particules charbonneuses provenant évidemment du navire explorateur, ce qui témoigne d'un manque de goùt inconcevable.

L'observation actuelle n'est pas de nature à modifier l'opinion à cet égard, puisqu'il se montre merdigère, sans en paraître dégoùté.

Il est vrai que ce crottin témoigne d'un certain gaspillage de matières alimentaires de la part du Crustacé qui l'a commis, un gros Copépode sans doute, présomptueux lui aussi de ses facultés digestives, car il semble n'avoir fait aucun discernement entre les proies les plus disparates qui se présentaient à sa portée : Diamylon, D; Distephanus, Ds; Ptychocylis, Pt; Echimm, E; Amylax, A; Piropsis, X; œufs de Rotateur, o $R$; Diatomées naviculées, $N$, et mille autres organismes dont on reconnait aisément la présence dans ce boudin glaireux, où des résidus réfractaires à la digestion coudoient des formes organiques à peine entamées par les zymases gastriques.

Comme on s'en convaincra aisément par l'examen de la figure 9 , cette dernière observation s'applique tout particulièrement à Diamylon nivale, $D$.

Abondamment ingéré par le Crustacé, ce microphyte se retrouve presque intact dans le crottin, après avoir parcouru tout le tube digestif.

Faudrait-il en conclure que les Copépodes ne sécrètent pas d'amylase et n'ont pas, par le fait, la faculté de digérer la fécule? Cela paraît; à moins que la membrane de Diamylon ne soit telle qu'elle ne se laisse pas traverser par les sucs digestifs.

Pour en revenir à notre Didinimm, nous ne voyons pas comment il pourra se tirer d'affaire, si l'inutilité de ses efforts ne lui fait renoncer à une entreprise au-dessus de ses forces, malgré le parti que, absolument parlant, il pourrait tirer, au point de vue alimentaire, des résidus excrémentiels du Crustacé. 
Nous aimons à y voir, tout au moins, un exemple intéressant du fait bien connu que rien ne se perd dans la nature et que la rétrogradation des substances organiques ne se fait que par degrés, d'une façon parcimonieuse et suivant les lois d'une économie scrupuleuse des forces vives emmaganisées par l'organisme végétal.

Le noyau de l'individu qui nous occupe ici est du type A. Son cytoplasme vacuoleux semble refléter un long jeùne qui excuse son intempérante convoitise.

Planche XVIII, figure Io. On voit ici l'organisme repu, après l'ingestion d'une proie volumineuse. On en reconnait les éléments et, en particulier, les gros grains de fécule, incorporés à titre d'inclusion, $f$, in, dans une grande vacuole digestive qui occupe le centre du sarcoplasme de l'individu et dont on distingue suffisamment les limites.

Les dimensions de ce spécimen sont considérables; elles n'ont néanmoins rien d'excessif pour l'espèce, si on le compare avec d'autres dont nous n'avons pas entrepris la figuration, afin de ménager la surface de nos planches.

On remarquera la bouche, $b$, en voie de rétrécissement; le sarcoplasme riche en inclusions; le noyau, $n$, du type $B$, volumineux et parcouru par les innombrables anses des filaments chromatiques qui sont noueux; la vacuole anale, va, bien développée, etc.

\section{REPRODUCTION}

Il nous semblait étonnant que cet organisme, si abondant dans plusieurs échantillons de nos matériaux d'étude recueillis à une époque qui semblait devoir être celle de sa plus grande prolifération, ne nous eùt présenté d'abord aucun cas certain de multiplication, là où il était le plus richement représenté.

Nous étions disposé à signaler l'absence des phénomènes. de fissiparité, n'ayant $\mathrm{pu}$ en trouver un spécimen à cette phase intéressante de son évolution, pour le figurer en bonne place dans les planches que nous lui consacrons.

Ce n'est que tardivement, en étudiant plus soigneusement le no 48 , que nous avions négligé à ce sujet comme beaucoup moins riche que d'autres et encrassé de substances huileuses provenant des petits Crustacés qui y pullulent, que nous avons pu enfin saisir quelques spécimens en voie de division.

Nous en donnons un exemple (pl. XXIII, fig. II), à une échelle un peu plus réduite, par manque de place, où ce phénomène se signale par l'étranglement du corps, peu marqué encore, et par le dédoublement des ceintures ciliaires qui sont au nombre de quatre.

Le noyau, $n$, affecte la forme en saucisson recourbé, du type $B$, que nous avons signalé dans d'autres spécimens qui ne témoignaient pas autrement d'une divivision prochaine. 
Nous nous croyons fondé maintenant à dire que cette forme du noyau est significative d'une tendance à la fissiparité.

Nous ne pouvons rien dire du micronucleus, pas même affirmer ou nier son existence. Le caractère cursif de nos recherches actuelles ne nous a pas permis de nous livrer aux minuties d'une technique spéciale propre à nous faire une opinion à ce sujet.

La chose nous paraît néanmoins assez intéressante pour avoir le désir d'en faire ultérieurement l'objet d'une petite étude spéciale.

Il n'y a pas lieu de considérer comme similaire, par sa portée, le cas de la figure I8, planche XIX, où l'organisme se présente avec un noyau indivis, $n$, et deux cònes buccaux, $b$ et $b^{\prime}$, entourés, l'un et l'autre, d'une ceinture ciliaire, $c c a^{\prime}$. Ces deux cônes convergent inférieurement l'un vers l'autre, au centre de la masse sarcoplasmique toute pétrie de baguettes trichocystiques en désordre.

Il n'y a qu'une seule ceinture ciliaire postérieure, ccp. Cette dernière observation nous porte à croire qu'il s'agit plutôt d'un cas de malformation accidentelle, d'un monstre bicéphale, et non d'une phase de division.

\section{Conjugaison}

Par contre, nombreux sont les exemples de rapprochement des deux individus que nous prenons pour des cas de conjugaison.

Les sujets qui participent au phénomène sont toujours de petite taille. Ils s'appliquent étroitement bouche à bouche, après des modifications de cet organe qui en détruisent l'harmonie ordinaire. Celui-ci n'offre plus qu'une ébauche de nasse pharyngienne dont les éléments, diversement écartés et dispersés dans le cytoplasme, semblent traduire la participation de toute la masse de ce dernier au phénomène intime de la copulation, que fait supposer la position des individus géminés.

Nous n'avons cependant pas pu songer à interrompre notre travail de dépouillement des produits de pêche de la Belgica pour soumettre ces objets à une technique propre à nous laisser pénétrer les caractères intimes du phénomène.

Les quelques reproductions que nous en avons faites n'en montrent que les caractères extérieurs, avec de légères variantes dans la forme et la disposition des conjoints.

Comparez à la figure 5 de la planche XVII les figures IO, II, I2 de la planche XVI. Les mêmes indications ont été reproduites pour signaler les détails correspondants. Aussi bien, elles concordent avec celles dont il a été fait usage dans toutes les figures relatives au même organisme.

Nous prenons le spécimen reproduit dans la figure 9 de la même planche XVI pour un sujet nouvellement séparé de son conjoint, après la copulation. 
Les trichocystes oraux, co, sont en grand désordre et font, en partie, saillie en dehors de la bouche, tandis que ceux qui restent à l'intérieur délimitent un cône tronqué dont la direction est inverse de celle qu'offre normalement la nasse buccale.

Ce caractère, dont nous ne saisissons pas la signification, se traduit plus nettement encore, dans la figure 6 (même planche), chez un sujet qui affecte une forme sphérique et que nous serions tenté de considérer comme un jeune, si les observations relatives à sa genèse ne nous faisaient complètement défaut.

Le sujet reproduit dans la figure 7 nous parait aussi énigmatique, avec ses dimensions fort réduites, sa nasse renversée, sa bouche mal conformée, etc.

C'est, enfin, à titre documentaire et pour servir à l'histoire naturelle de cet organisme aussi étrange dans ses manifestations biologiques que dans ses modalités morphologiques, que nous avons reproduit des formes naines telles que les figures 4 et 5 de la planche XX. Elles paraitraient peut-ètre s'écarter assez du type pour être considérées comme étrangères à celui-ci, si l'on pouvait ètre mieux fixé sur les limites de ses variations.

La présence des deux ceintures ciliaires est du reste à peu près le seul caractère saillant qu'elles partagent avec les figures précédentes.

Dans la figure 5, la bouche, b, est comme fermée, au sommet de la nasse pharyngienne étroite.

Dans la figure 4, la bouche, $b$, parait fendue et garnie de très longs cils, co, qui n'ont pas tout à fait l'apparence des trichocystes observés ailleurs.

De plus, la nasse pharyngienne fait défaut, sans qu'on puisse affirmer que les cils oraux, $c o$, en représentent les baguettes sous une autre forme et avec d'autres fonctions.

Par leurs dimensions réduites et aussi quelque peu par leur aspect, ces petites formes, particulièrement la figure 5, rappellent Didinium nasutum, que nous connaissons des canaux d'eau douce ou à peine saumàtre du littoral belge. Mais ce n'est pas le lieu de nous livrer à la critique de ces espèces.

\section{Genre Cyclotrichium g. n.}

Nous proposons ce vocable pour désigner le gros Infusoire cilié que nous figurons, sous quelques-uns des aspects qu'il présente dans nos matériaux, aux différentes phases de son évolution et dans l'exercice de son activité physiologique.

Corps mou pourvu, suivant les cas, d'une ou de deux zones circulaires de cils locomoteurs. 


\section{Cyclotrichium cyclokaryon.}

Planche XVI, figures $2,4,13,14,15,16$ et 17 ; planche XVIl, figures 6 et 7 ; planche XVIII, figure 7

Sa forme la plus habituelle est celle d'un ellipsoïde plus au moins atténué en fuseau soit à l'une des extrémités polaires, soit aux deux, ce qui peut dépendre dans une certaine mesure de l'action des réactifs fixateurs et de l'attitude que l'organisme avait au moment de la fixation.

Sa membrane tégumentaire mince, souple, finement striée dans la direction du grand axe de la cellule, semble en effet devoir se prêter à toutes les modifications morphologiques qui témoignent de son irritabilité.

Elle ne porte de cils que sur la région médiane, souvent un peu antérieure, du corps, où ceux-là forment une épaisse ceinture qui semble constituer tout l'appareil locomoteur de l'animal.

Cette ceinture, $c c$, se dédouble longtemps avant toute autre manifestation de scissiparité de l'organisme. C'est ce qui explique que bon nombre d'individus observés présentent deux ceintures ciliaires, sans témoigner autrement d'une division prochaine, et pourraient être pris pour spécifiquement différents, si l'observation des cas de multiplication ne donnait la clef de ce dimorphisme apparent.

En arrière de la ceinture ciliaire simple ou dédoublée, la striation longitudinale est plus fortement accusée sur une zone de faible largeur, ce qui force à reconnaître une relation entre cette striation et les zones ciliaires.

La bouche, $b$, située au pôle antérieur, est difficile à observer, parce qu'elle ne porte aucun appendice et qu'elle se ferme sans l'action des réactifs fixateur's en prenant la forme d'une fente étoilée que l'on voit dans la figure i4 de la planche XVI, qui reproduit une vue apicale de l'organisme. On n'y voit rien qu'on puisse considérer comme un organe pharyngien.

Le noyau figuré isolément, figure I7, planche XVI, a la forme toute spéciale d'un anneau ouvert, développé sur un peu moins d'un tour complet. C'est cette particularité qui nous a fait attribuer à l'espèce le nom de cyclokaryon.

Il est placé perpendiculairement à l'axe polaire dans une ceinture de protoplasme plus dense, $c p$, qui le maintient dans l'ectoplasme cellulaire. Son aplatissement transversal le fait toujours paraitre plus étroit quand on l'observe perpendiculairement au plan dans lequel il se développe. Sa section transversale est, en effet, elliptique, comme il apparaît dans plusieurs figures : I3 et I6, par exemple, de la planche XVI.

Sa structure est finement fibrillaire, dans le sens de son plus grand axe, qui est circulaire. Il possède une membrane propre, mn (fig. I7), que l'on aperçoit très bien quand l'organe subit la plasmolyse.

Le cytoplasme est très vacuoleux, dans toute l'étendue du corps, en dehors toutefois de la ceinture à texture plus dense dans laquelle se trouve logé le noyau (fig. 2, 4, I3, I5, I6). 
On n'y voit pas d'inclusions, indices d'un régime carnassier, mais bien des enclaves amylacées, $f$ (fig. 4, I5, I6), et autres qui paraissent formées sur place, par l'activité propre du protoplasme.

Pour donner à ces notions générales une expression plus concrète, nous présenterons les différentes figures que nous avons consacrées à cet imposant représentant du monde des Protozoaires, en appelant l'attention du lecteur sur des particularités qui permettent de lier connaissance plus intime avec lui.

Planche XVI, figure I6. Forme fréquemment observée, avec cette particularité que le sarcoplasme semi-fluide, pé, s'épanche en quelque sorte et fait hernie en dehors de la bouche.

Dans cette circonstance il ne faut voir évidemment qu'un accident : un effet de la contraction du corps déterminée par le milieu fixateur. Mais cet accident fait comprendre et la faiblesse du sphincter oral et le peu de résistance de l'appareil tégumentaire. Il explique aussi pourquoi Didinium Gargantua en fait si volontiers ses délices, comme d'un morceau qui fond dans la bouche.

Le protoplasme vacuoleux, po, s'étend à peu près à toute la capacité du corps. On y voit presque toujours des grains de fécule, $f$, disséminés, mais généralement plus abondants du côté apical.

En arrière s'observe une vacuole plus grande qui a l'air d'une vacuole anale.

Le noyau annulaire, $n$, est logé dans une ceinture de protoplasme dense, $c p$, que nous avons reproduite arbitrairement en coupe optique pour en mieux laisser supposer la forme et la position immédiatement en arrière de la zone ciliaire, $c c$.

Celle-ci n'est pas une simple ceinture de cils rangés en une seule série circulaire, comme dans Didinium Gargantua; elle comporte un fouillis beaucoup plus dense de cils plus fins, dont il n'est pas possible de déterminer le nombre des rangées, à cause de leur extrême rapprochement.

La striation longitudinale visible sur toute la surface du tégument est notablement renforcée, comme il a été dit plus haut, immédiatement en arrière de la zone ciliaire.

La figure 2, mème planche, en montre un exemplaire capturé par son redoutable adversaire. On remarquera que la zone ciliaire, $c c a$, est située très en avant du corps, mais néanmoins à la limite antérieure de la ceinture protoplasmatique interne, cp, dans laquelle se trouve logé le noyau, $n$.

La figure 7, planche XVIII, donne aussi une bonne idée des caractères moyens de l'organisme, en forme de fuseau court, avec une seule ceinture ciliaire, dans l'attitude qu'il a sans doute pendant ses ébats auxquels a mis fin l'attaque brusque de son agresseur. La fermeture de la bouche, $b$, se traduit par un léger froncement du pòle antérieur, à droite de la figure. 
Chez le spécimen reproduit (fig. 4, pl. XVI) dans une situation aussi critique, la zone ciliaire s'est dédoublée en zone antérieure, $c c a$, et postérieure, ccp. La ceinture protoplasmatique intérieure s'est, de ce chef, élargie. Le noyau, $n$, est vu obliquement sur tout son développement, grâce à la position favorable du sujet. Le cytoplasme ordinaire, très vacuoleux, est riche en grains réniformes de fécule, $f$.

Bien entendu, il a fallu l'usage de réactifs éclaircissants pour obtenir cette transparence que n'ont plus naturellement les matériaux fixés.

Les figures I3, planche XVI (vue de profil) et I4 (vue apicale d'un même spécimen) le montrent sous une forme ellipsoïde plus ramassée, bien que la zone ciliaire y soit aussi déjà dédoublée. Outre les observations auxquelles les figures précédentes ont donné lieu, on remarquera combien peu s'accuse la structure de la bouche, $b$, sur la vue de profil (fig. I3). Il faut, pour en saisir quelque chose, réaliser une vue apicale de l'objet, comme en figure I4, ce qui ne s'obtient pas sans difficulté à cause de l'instabilité de l'organisme dans cette position.

\section{Multiplication}

Nos matériaux fournissent beaucoup de spécimens en voie de division, par exemple : figures 6 et 7 de la planche XVII.

Longtemps après le dédoublement de la ceinture, le corps s'est allongé et le noyau, $n$, s'est déroulé pour changer de position et se mettre dans la direction du grand axe.

Pendant qu'il s'y redresse incomplètement en orientant ses deux bouts vers les pôles de la cellule, celle-ci s'étrangle dans son milieu (fig. 6) jusqu'à amener la séparation des deux cellules-filles. Cet étranglement s'accompagne d'un phénomène de torsion visible dans la figure 7 .

La restauration consécutive des deux individus jumeaux se fait rapidement. On les reconnaît à la forme raccourcie et trapue qu'ils affectent d'abord.

Nous tenons pour tel l'individu reproduit figure I5, planche XVI, où l'on voit que le noyau, $n$, a déjà repris sa forme annulaire et sa position normale au sein d'une épaisse couche de protoplasme périphérique plus dense, $c p$. En avant les grains de fécule, $f$, abondent sous la bouche froncée, $b$. En arrière, une grande vacuole anale, $v a$, apparaît au milieu du cytoplasme, toujours très vacuoleux, de cette région $\mathrm{du}$ corps.

Dans la figure I6, planche XVIII, nous avons reproduit le plus petit individu observé, terme éloigné, sans doute, d'une longue série de générations scissipares, où la dégénérescence se traduit par le nanisme. 


\section{Cyclotrichium sp.?}

Planche XV, figure 7 ; planche XVII, figure 14 ; planche XX, figures 15 , I6 et 17 ; planche XXIII, figure 9 .

La présence d'une ceinture ciliaire située au milieu du corps, dans une dépression qui divise le corps en deux parties subsphériques, est le seul caractère commun saillant que nous trouvons entre un certain nombre de petites formes rencontrées trop rarement dans nos produits planktoniques pour avoir pu les soumettre à l'étude, en vue de rechercher leurs affinités réelles.

Le nom que nous leur attribuons provisoirement, avec réserves, n'a d'autre raison que de souligner le caractère extérieur qu'ils partagent avec l'organisme décrit ci-devant.

Qu'il suffise de les présenter rapidement, sans préjuger de leur nature vraie.

Planche XVII, figure I4. Corps allongé, étranglé vers le milieu, muni d'une ceinture ciliaire, $c c$, pourvu d'une bouche en fente, $b$, peu visible, au pôle antérieur, rempli d'un cytoplasme vacuoleux riche en enclaves et en inclusions, in, qui ne permettent pas de voir le noyau.

Planche XV, figure 7. Corps étranglé, gorgé d'enclaves uniformes d'aspect particulier, et porteur d'une ceinture ciliaire, $c c$, insérée dans l'étranglement médian.

Planche XX, figure I7. Forme analogue à la précédente, mais plus petite, gorgée d'enclaves qui bleuissent sous l'action de l'iode et semblent ainsi ètre de nature amylacée, $f$.

Planche XX, figures I5 et I6. Très petites formes, atténuées plus ou moins à la base ou même prolongées en un petit éperon (fig. I5), toujours gorgées aussi de corpuscules d'aspect particulier. Ces microzoaires seraient-ils affines aux Halteria?

Il faudrait des observations sur le vif pour en juger avec quelque sécurité.

Planche XXIIl, figure 9. Organisme rétréci en son milieu, entre deux renflements garnis chacun d'une ceinture ciliaire. On y distingue, par transparence, deux noyaux en forme d'haltère placés parallèlement l'un à l'autre suivant le grand axe du corps. Leur forme étirée, en rapport avec la silhouette extérieure, semble traduire un phénomène de division de la cellule binucléée. Observé une seule fois sous cette forme.

\section{Genre Proboscidium g. n.}

Nous avons à présenter sous cette étiquette un Protozoaire commun dans la Mer de Kara, qui ne semble pas pouvoir trouver sa place dans les cadres taxinomiques jusqu'ici reconnus de ce vaste groupe d'organismes inférieurs.

La production d'une trompe par l'animal en extension nous a semblé un caractère propre à évoquer le nom générique sous lequel nous le plaçons. 


\section{Proboscidium armatum}

Planche XVII, figures 8, 9, I0; planche XVIII, figures I2, I3, I+, I5; planche XIX, figures I, 2.

La forme qu'il prend le plus souvent dans nos matériaux fixés est celle d'un ovoïde (fig. I3, pl. XVIII). La membrane mince et souple présente une fine striation dans deux directions croisées. Des trichocystes défensifs nombreux, tri, sont répartis dans toute l'étendue de l'ectoplasme. Ils sont en grande partie projetés en dehors du corps, à cause de l'irritation produite par le réactif fixateur.

Le noyau, $n$, que nous avons figuré isolément (fig. I4 et I5, même pl.) affecte la forme bien spéciale d'un long boyau disposé en forme de cravate dont la partie médiane décrirait une courbe autour d'un cou fictif et dont les extrémités non nouées pendraient librement en décrivant des' sinuosités variables. Sa structure intime est fibrillaire.

Une autre particularité de l'organisme, c'est qu'il présente presque toujours dans son endoplasme un certain nombre de gros grains de fécule, $f$, trop semblables de forme et de dimension pour qu'il soit possible de les considérer comme objets d'inclusion glanés dans le milieu marin.

Disséminés au sein du corps ils n'affectent pas une distribution spéciale en rapport ni avec l'organe buccal, ni avec des vacuoles digestives, dans lesquelles ils devraient subir des transformations en vue de leur assimilation plus ou moins immédiate.

Ces grains lenticulaires seraient-ils plutòt un produit propre de l'activité du protoplasme?

La question nous semble plus difficile à trancher du fait que d'autres corps : cellules de Diamylon nivale, $D$, fragments de muscles de Crustacés, $m$, etc. (fig. 2, pl. XIX), s'y rencontrent aussi accidentellement dans les mèmes conditions.

Ceux-ci y sont évidemment des produits d'inclusion. S'il convenait d'attribuer la même origine étrangère aux grains de fécule susdits, il restẹait toutefois à expliquer l'aptitude si particulière de l'organisme à se les incorporer, après les avoir recherchés dans un milieu où ces productions végétales ne se trouvent à l'état libre et flottants que dans des circonstances rarement réalisées.

La bouche, $b$, est en fente excentrique, inclinée sur un còté du còne apical; mais comme cet organe prend un tout autre aspect quand la trompe est développée, on ne saurait se faire une bonne idée de sa structure sans dégager celle-ci de la comparaison de nombreux individus qui la présentent sous des aspects divers. C'est ce qui nous oblige à jeter un coup d'œil sur les quelques figures qu'il nous a paru utile de tracer du mème organisme, pour suppléer à l'absence de ses manifestations biologiques, dans des matériaux fixés.

Planche XंVIII, figure I3. T'ue latérale, montrant la striation croisée de la membrane et les nombreux trichocystes, tri, restés en place ou expulsés. Dans le 
cytoplasme des grains de fécule, $f$, de forme lenticulaire cachent en partie les extrémités libres du noyau, $n$, contourné en cravate. La bouche, $b$, est en fente latérale, à bords garnis de cils, les seuls qui s'observent sur le corps de l'animal.

Planche XVIII, figure I2. Vue apicale d'un autre spécimen très analogue au précédent. On y remarque la fente buccale, $b$, ciliée. La courbure décrite du côté antérieur par la partie médiane du noyau, $n$, est ici d'une observation facile. Les extrémités ondulées de cet organe se profilent dans la profondeur du cytoplasme.

Planche XIX, figure 2. La trompe, tr, développée ici sur le côté de la bouche, $b$, révèle l'existence de cet organe dont rien ne fait soupçonner l'existence dans l'animal contracté, tel que le représentent les figures précédentes.

Cette trompe parait se produire de toutes pièces, au gré de l'animal, aux dépens du cytoplasme qui subit une sorte d'évagination, en prenant une structure particulière.

Nous n'avons pas pu apprécier toute l'extension possible de cette trompe, car nous devons croire que son extrême sensibilité ne permet pas aux réactifs de la fixer en son plein épanouissement.

On ne saurait y voir sans doute qu'un organe de tact protractile et rétractile au gré de l'Infusoire; car la bouche, $b$, en fente, garde sa position pendant les modifications de cet appendice.

Le noyau, $n$, est ici bizarrement contourné. Outre les gros grains de fécule, $f$, on aperçoit, dans le sarcoplasme, des cellules de Diamylon nivale, $D$, dans une grande vacuole digestive, un fragment de muscle, $m$, de Crustacé et des résidus, $r$, de digestions antérieures, dans des vacuoles excrémentielles.

Les trichocystes n'ont pas été figurés, afin de ne pas obscurcir la figure, au détriment du contenu cellulaire.

Planche XIX, figure I. Autre aspect du Protozoaire, dont la trompe, tr, est en voie de rétraction et ne forme plus qu'un court appendice de structure spéciale, sur le côté de la bouche, $b$, dont on voit la bordure ciliée, co. On remarquera, en outre, le noyau tordu, $n$, et les grains de fécule habituels, $f$.

Dans l'ectoplasme se voient des trichocystes en place. Nous n'avons pas jugé opportun de reproduire en outre ceux dont l'action irritante des liquides fixateurs a produit la projection en dehors et que l'on retrouve engagés dans des mucosités dues à la même cause.

Planche XVII, figure 8. Ce spécimen montre l'appendice buccal, $a b$, plus réduit encore sur le côté de la bouche, $b$. Le noyau, $n$, a la forme typique. Des grains de fécule, $f$, et des inclusions variées, in, s'observent dans le sarcoplasme. En arrière, une grande vacuole anale, va. Des trichocystes, tri, dans l'ectoplasme et en dehors. 


\section{Multiplication}

La multiplication se fait par étranglement. Nombreux ont été les cas observés dont les figures 9 et ıo, planche XVII, suffisent à donner une idée.

Planche XVII, figure 9. Commencement d'étranglement du corps, vers le milieu, en vue de la scissiparité. Le noyau, $n$, s'est beaucoup raccourci et s'est placé longitudinalement pour pouvoir subir, lui aussi, la sténose qui en fera un partage égal entre les deux portions de la cellule-mère. La contraction de la masse nucléaire due aux réactifs facilite l'observation de sa membrane propre et de la structure fibrillaire de son contenu chromatique.

En $b$, se voit une dépression qui marque la position de la bouche, que l'attitude du Protozoaire ne permet pas de voir directement.

Planche XVII, figure Io. La sténose est presque achevée. Les deux cellules-filles vont se séparer; $b$, bouche; $n$, noyau; $f$, fécule; tri, trichocystes expulsés.

\section{Genre Prorodon Ehrenberg}

Caractères du genre. - Cfr. Saville Kent, page $49 \mathrm{I}$.

\section{Prorodon karianus sp. n.}

Planche XIX, figures $3,4,5$.

Corps ellipsoïde, rigide, peu déformable, finement strié d'un pòle à l'autre et entièrement couvert de nombreux cils courts, tous semblables, $c p$.

La bouche, $b$, s'ouvre au pòle antérieur, sous la forme d'un cercle dilatable, sans y déterminer aucune protubérance. Elle se prolonge en un long pharynx conique garni de très nombreuses baguettes analogues à celles de Didinium, mais qui ne nous ont pas paru déplaçables comme chez ce dernier.

Au pòle opposé s'observe une vacuole anale, va.

Le noyau, $n$, volumineux et grossièrement granuleux, affecte soit une forme globuleuse (fig. 3), soit, plus fréquemment, une forme allongée et plus ou moins recourbée en $U$ (fig. 4 et 5).

Un second noyau, $n^{\prime}$, plus petit et d'aspect strié s'observe parfois à quelque distance du premier, simple ou divisé (fig. 3). Peut-être n'est-ce que l'abondance des grosses enclaves qui empêche de l'observer toujours, comme un organe constant. 
Le cytoplasme présente, en effet, de nombreux corps très réfringents qui nous ont paru ètre de nature graisseuse, $g$. Ces corps sphériques ou de forme irrégulière sont un excellent caractère permettant de reconnaître, en toutes circonstances, ce Prorodon des autres Protozoaires qui l'accompagnent généralement dans les mêmes produits de pêches planktoniques.

La figure 3 en reproduit un spécimen à bouche assez étroite; vue de profil.

La figure 5 en reproduit, aussi de profil, un autre individu dont le cône pharyngien dilaté est dévié inférieurement.

La figure 4, enfin, en présente un troisième, en vue apicale oblique, avec la bouche, $b$, fortement dilatée; $i c$, inclusions indéterminées; $i g$, inclusions graisseuses.

Assez rare dans la Mer de Kara, comme ceux qui vont suivre.

\section{Genre Cephalotrichium g. n.}

Les caractères du genre sont, provisoirement, ceux de l'espèce suivante :

\section{Cephalotrichium tonsuratum sp. n.}

Planche XIX, figures 7 à 9 et ro à i2.

Sous ce nom, nous voulons désigner un Infusoire holotriche, en forme de poire asymétrique, qui porte sur le gros bout - le bout antérieur - des cils longs et nombreux disposés en séries radiales, $s c$, sur une zone qui n'occupe guère qu'un quart de la longueur du corps.

Les cils sont très rapprochés et comme soudés en lamelle dans chacune des séries; mais celles-ci sont assez espacées.

La zone ciliée ménage vers le sommet ou centre apical, c (fig. 9, Io, I2), une petite aire circulaire, comme une tonsure, exempte de cils. Ceux-ci font également défaut sur les deux tiers inférieurs du corps.

Le sarcoplasme est dense, grossier, peu pénétrable à la vue et revêtu d'un cytoderme mince. On y voit, après éclaircissement, un gros noyau, $n$, sphéroïde, à texture fort grossière.

La bouche, $b$ (fig. 7, 9, Io), est excentrique, rejetée du. côté du corps qui est déprimé.

Elle affecte la forme d'une fente incurvée et cachée sous des cils spéciaux, qui lui font un péristome.

La forme asymétrique du corps en rend l'intelligence difficile, soit qu'il s'agisse de l'étudier, soit qu'on veuille le décrire. C'est ce qui nous en a fait figurer deux spécimens dans diverses attitudes, les plus propres à en faire saisir l'ordonnance des parties. 
Les figures, 7, 8, 9 se rapportent à un même individu. Vu du còté bucco-ventral (fig. 7), il laisse voir la fente orale, en $b$, et les séries radiales de cils, sc, qui couvrent la zone céphalique, à l'exception de la tonsure apicale.

La figure $\delta$ en est une vue dorsale, diamétralement opposée à la précédente. Elle montre plus nettement encore les rangées de cils longs et dressés, sc, qui garnissent la zone péripolaire du còté antérieur. Des cellules de Diamylon nivale, $D$, se trouvent dans le sarcoplasme, à titre d'inclusions.

La figure 9 en est une vue apicale oblique, montrant presque toute l'aire polaire supérieure, avec la tonsure apicale dont le centre est en $c$.

Un autre spécimen, d'un facies un peu différent, est figuré dans des poses différentes dans les figures io à I2. Figure Io, vue du còté ventral; $c$, aire apicale dépourvue de soies; $s c$, séries ciliaires rayonnantes; $b$, bouche; $n$, noyau. Figure I I, vue de profil droit. Figure I2, vue de profil gauche, un peu oblique. Mêmes désignations.

\section{Genre Zonotrichium g. n.}

Les caractères du genre sont, provisoirement, ceux de l'espèce ci-après :

\section{Zonotrichium discoïdes sp. n.}

Les trois figures $\mathrm{I}, 2,3$ de la planche $\mathrm{XX}$ sont nécessaires à l'intelligence de la forme de ce curieux Infusoire holotriche, qui ne présente des cils que sur une zone annulaire, autour d'une sorte de ventouse circulaire qui occupe l'un des còtés du corps.

Celui-ci a la forme d'une lentille biconvexe, irrégulière, dont un còté est nu, tandis que l'autre porte des cils longs et nombreux autour de la dépression circulaire à allure de ventouse, $v$, qui en occupe le centre. On s'en rendra compte par l'examen de la figure 2, qui en reproduit une vue de profil.

La figure I en est une vue que nous dirons apicale, parce que c'est de ce côté que se trouvent la ventouse et la zone ciliée. Le centre de la ventouse présente une petite aire circulaire plus claire.

La figure 3 nous montre la vue antapicale, le côté nu.

La bouche, $b$, s'ouvre sur le bord du disque lenticulaire et s'y traduit par des replis contournés de la membrane.

Dans le protoplasme central s'observent plusieurs tubes étroits, $x$, distancés et orientés parallèlement à l'axe du corps lenticulaire. Les vues apicale et antapicale n'en montrent que les sections transversales, avec leur épaisse paroi. 
A quelque distance du centre s'observent de nombreux corps, $f_{n}$, de structure grossière, de forme anguleuse, et sensibles aux réactifs colorants de la nucléine. Disposés en cercle autour d'une partie différenciée du sarcoplasme central, ils semblent représenter les parties d'un noyau fragmenté.

Les particularités si remarquables de la structure de ce curieux organisme semblent résulter d'une adaptation au parasitisme ou au commensalisme. Toutefois, ne l'ayant jamais trouvé qu'à l'état libre, en très rares exemplaires, nous ne saurions dire quelle espèce animale lui sert d'hòte ou de commensal.

La nature de nos matériaux momifiés par le. formol ne nous a pas permis de sortir des caractères morphologiques, insuffisants le plus souvent à traduire les affinités réelles d'organismes simples, dont on trouverait mieux l'expression propre dans les manifestations vitales.

L'observation de celles-ci nous est malheureusement interdite.

Chacun sait, en outre, quelle difficulté il y a même à identifier les figures données d'une même espèce par les auteurs qui ont le mieux fait connaître le petit monde si varié des Protozoaires.

C'est pourquoi nous demandons l'indulgence du lecteur pour les tentatives de rapprochement que nous allons être amené à faire, pour quelques formes moins vigoureusement structurées, de caractères morphologiques moins précis et dans lesquels l'action des réactifs a pu déterminer des déformations dont nous ne saurions toujours déterminer sûrement la portée, à cause du trop petit nombre d'exemplaires observés.

\section{Genre Condylostoma DujARDin?}

Caractères du genre. - Cfr. Stein. Der Organismus der Infusionsthiere, II Abtheilung, page I 7 I.

\section{Condylostoma patens Dujardin?}

Planche XV, figure 6 .

Corps longuement lancéolé, aplati, entièrement cilié, présentant dans le protoplasme, du côté antérieur, une forte striation oblique qui semble indiquer la présence d'une bouche latérale, $b$, dont l'ouverture n'est pas visible.

Cet organe parait en connexion assez vague avec un tube longitudinal, ta, qui rappelle le canal aquifère de certains Protozoaires hétérotriches. 
Le cytoderme est très délicat, finement strié longitudinalement, couvert de cils courts très serrés, cp, qui nous ont paru d'une seule sorte.

Le cytoplasme vacuoleux est exempt d'inclusions et d'enclaves reconnaissables.

Le noyau, $n$, en chapelet, est formé de douze renflements ellipsoïdes rattachés entre eux par de très fins prolongements polaires.

\section{Condylostoma circumpedatum sp. n.}

Planche XXIII, figures 5,6 et 7 .

Organisme qui nous paraît du mème type, eu égard à la structure du corps et à la position latérale de ce que nous devons prendre pour la bouche, $b$.

Il est asymétrique aussi, de forme lancéolée, aplatie. Il présente une face ventrale déprimée (fig. 5) et une face dorsale convexe (fig. 7). La figure 6, qui le présente de profil, permet mieux d'en saisir le caractère dorso-ventral.

Le noyau, $n$, est aussi moniliforme, avec huit nodosités sphériques.

Mais, ce qui frappe ici, à première vue, c'est la présence, sur le pourtour en lame de couteau qui sépare les deux faces dorsale et ventrale, de protubérances ciliées, $p$, qui semblent avoir pu jouer le ròle d'un appareil locomoteur spécial.

Ces protubérances, diversement défléchies sur le côté ventral dans nos spécimens fixés, portent, à leur extrémité, quelques soies plus fortes que les cils qui revêtent tout le corps et qui sont en relation avec la striation longitudinale du cytoderme. Il faudrait pouvoir suivre cet Infusoire pendant ses ébats, sur le vif, pour s'éclairer au sujet de la signification de ces organes, qui font défaut au type précédemment décrit. On estimera peut-ètre que c'est là une raison suffisante pour ne pas les considérer comme congénères; nous n'en disconvenons pas.

\section{Genre Climacostomum STEIn?}

\section{Caractères du genre. - Cfr. Stein, loc. cit., page 208.}

\section{Climacostomum gigas sp. n.}

Planche XVII, figure Ir.

Corps piriforme, à l'état plus ou moins contracté sans doute, strié longitudinalement et uniformément cilié, avec une bouche, $b$, située latéralement à quelque distance du gros bout. Cette bouche, contournée à son ouverture, se creuse en pharynx hélicoïde qui se prolonge plus profondément dans le sarcoplasme en un tube œesophagien, as, également spiralé. 
L'ouverture buccale est garnie de soies plus fortes, qu'on voit aussi revêtir toute la paroi interne du pharynx.

Le cytoplasme est homogène, avec enclaves globuleuses.

Le noyau, $n$, très volumineux, a une structure fibrillaire. Sa forme ramassée et légèrement étranglée vers le milieu semble indiquer que l'animal se préparait à se diviser quand la fixation l'a surpris.

Cet organisme n'est pas sans analogie avec les Stentor, dont le polymorphisme exagéré peut parfois faire douter de leur nature. Il n'est pas impossible que ce soit le cas et nous n'hésiterions pas à considérer notre organisme comme un Stentor contracté sous l'influence du formol, si nous avions pu saisir une trace de la zone adorale de cils forts, autour du champ frontal antérieur, qui caractérise les Stentor en état d'extension.

Quant au noyau, on sait que la forme en varie beaucoup pendant les phases de division et on peut croire que celui de notre organisme, seul spécimen observé, se serait montré moniliforme dans d'autres circonstances.

\section{Genre Trachelius Claparède et Lachmann?}

\section{Trachelius sp.}

Planche XIX, figure 6 .

Ce genre, qui n'est coṇnu que par des espèces d'eau douce, pourrait bien avoir un représentant marin dans l'organisme que nous avons reproduit ici, sous l'aspect que lui a fait prendre le milieu conservateur de nos échantillons.

La striation du cytoderme est croisée en quinconce; les cils périphériques, $c p$, s'étendent sur toute la surface du corps; l'orifice buccal, $b$, est latéral, orné de soies plus fortes; le noyau, $n$, présente une structure fibrillaire; il est grêle, très long, replié sur lui-même dans le sens de la longueur du corps.

Celui-ci est longuement piriforme, même dans son état contracté. La partie antérieure plus rétrécie se serait sans doute montrée extensible sur le vif, comme la trompe des Dileptus.

Il y aurait peut-être des rapprochements aussi plausibles à faire avec d'autres genres du groupe des Trachélides, mais nous voulons nous garder d'hypothèses hasardeuses dans ce domaine des Infusoires, où la critique moderne ne s'est pas encore suffisamment exercée à débrouiller les caractères génériques indépendants des caractères purement morphologiques qui ne se traduisent bien, dans leurs modalités fugitives, que pendant la vie et qu'il est généralement presque aussi difficile de traduire en figures qu'en texte.

Distribution Géographique. - Mer de Kara, très rare. 


\section{Genre Lacrymaria Ehrenterg?}

Lacrymaria marina sp. n.

Planche XVIII, figures 17 et 18 .

C'est la position de la bouche, $b$, qui est située au sommet du pôle antérieur et entourée d'une couronne de cils un peu plus forts que les cils périphériques, $c p$, qui nous fait considérer l'organisme dont nous donnons ici deux variantes, comme une espèce de Lacrymaria, genre d'Holotrichides connu seulement d'eau douce.

Le corps allongé, lagéniforme et incurvé d'un côté, se montre fortement strié longitudinalement et garni de cils sur ces stries distancées.

Une vacuole anale, va, occupe le pôle postérieur du corps.

Le noyau, $n$, est contourné en forme du chiffre 6. Les inclusions font défaut.

\section{Genre Enchelys Ehrenberg?}

\section{Enchelys sp.}

Planche XVIII, figure I9.

Nous n'avons aucune peine à reconnaître l'embarras que nous éprouvons à donner à cet organisme une place en harmonie avec les caractères frustes que nous lui avons reconnus sur cadavre.

Corps fusiforme, garni de longs cils, $c p$, insérés sur une striation longitudinale. Protubérance buccale antérieure ornée de cils spiralés, co.

\section{INFUSOIRES incertee sedis}

Planche XVII, figure I2. Organisme piriforme à cytoderme strié dans deux directions, longitudinale et transversale, et dépourvu de toute trace de cils, dans nos matériaux.

Bouche latérale, $b$, bordée d'un côté d'une ailette membraneuse, lo, doublement striée comme le corps et exempte de cils vibratiles.

Le protoplasme est vacuoleux; on $y$ voit un Péridinien, $P$, seule inclusion reconnaissable au milieu d'autres. 
Planche XVII, figure i5. Corps ramassé, piriforme, présentant du côté antérieur, effilé en pointe molle, une bouche latérale, $b$, en fente, dépourvue de cils comme le corps lui-même.

Le noyau, $n$, très volumineux est transversalement strié.

Dans le cytoplasme on aperçoit des inclusions, parmi lesquelles Diamylon nivale, $D$.

Planche XVI, figure I3. Corps nu, en forme de manche d'alène, coiffé antérieurement d'un dôme hémisphérique hyalin sur le côté duquel s'ouvre une bouche, $b$, en forme de nasse, qui communique inférieurement avec une grande vacuole. Celle-ci épouse la forme ellipsoïde de la moitié postérieure de l'organisme.

Le cytoplasme est partout très vacuoleux; il renferme des inclusions, in, et un noyau allongé, $n$, dont la structure est fibrillaire.

Planche XIX, figure I3. Forme ellipsoïde dans laquelle nous n'avons pas pu observer d'orifice buccal.

Le cytoderme est nu; le cytoplasme clair et vacuoleux.

Le noyau, $n$, est bilobé comme s'il résultait de la soudure de deux parties symétriques renfermant chacune de petits corps denses et réfringents, m, qui doivent ètre des nucléoles. La structure du noyau géminé est fibrillaire. L'orientation transversale des filaments nucléaires semble devoir écarter l'idée d'une division par sténose de cet organe, au niveau de son étranglement.

On peut émettre des doutes sur l'individualité de ces cellules que nous n'avons rencontrées qu'un petit nombre de fois, dans les pêches planktoniques de la Mer de Kara. Il serait toutefois difficile, nous semble-t-il, de les considérer comme des organes de reproduction : ceufs, spores ou kystes.

Planche XXIII, figure Io. Corps ellipsoïde, allongé, surmonté d'un couronnement multilobé, $b$, dont les trois ou quatre lobes inférieurs en supportent un dernier qui est terminal.

Les fins cils qui les recouvrent et l'épaisseur notable de la membrane molle qui les limite forcent à les considérer comme constituant un organe stable et non comme un produit d'épanchement du cytoplasme au pòle apical.

On n'y reconnait pas un orifice oral distinct.

Le cytoderme est strié longitudinalement, en fuseau, et couvert de menus cils, $c p$.

Le noyau, $n$, est sphérique et central.

Le cytoplasme, assez transparent, présente des granulations espacées.

Planche XXII, figure I8. Corps globuleux, excentrique, un peu réniforme, légèrement incurvé d'un còté vers lequel se ramènent les deux pôles organiques.

L'antérieur présente une couronne de soies courbes, co, formant péristome autour d'un orifice oral étroit. 
Le cytoderme mince présente des stries incurvées, dirigées d'un pôle à l'autre où elles convergent.

Des cils somatiques très fins sont insérés sur ces stries.

Dans le cytoplasme, qui est peu dense, on observe, du côté convexe du corps et parallèlement à sa courbure, une série de granules de même valeur et également distancés, qu'il serait peut-ètre hasardeux de considérer comme des noyaux multiples bien qu'on n'aperçoive aucun autre organe auquel on doive réserver ce nom.

Planche XXIII, figures 20, 2I, 22. Nous ne ferons que signaler en passant des kystes chitineux, hérissés de piquants (fig. 20 et $2 \mathrm{I}$ ) ou ornés d'épaississements réticulés (fig. 22), qui se rencontrent ici et là dans les matériaux de provenances diverses et qui semblent se rattacher aux Infusoires.

On sait que ces formes d'enkystement sont très variées et on n'ignore pas non plus combien il est difficile de les rapporter à des espèces déterminées quand on n'a pas assisté, sur le vif, à la mue de l'organisme lui-même.

\section{Genre Stappersia g. n.}

Les caractères génériques se confondent provisoirement avec ceux de l'espèce ci-après :

Stappersia fusus sp. n.

Planche XVIII, figure 2 I.

Petit organisme énigmatique que nous avons fréquemment rencontré de-ci de-là, non seulement dans les produits planktoniques de la Belgica, mais aussi dans des matériaux des mers du Nord, de provenance diverse.

Le corps est longuement fusiforme, tronqué en avant, pointu en arrière. La surface est quadrillée, par un double système de stries rigides longitudinales et transversales, et porte des bavures dans lesquelles il serait difficile de reconnaitre des cils déformés plutôt que de petites expansions du cytoplasme.

Le contenu semble divisé en sphérules que leur structure interne ferait prendre soit pour des noyaux, soit pour des sporules, mais dont il serait hasardeux de préjuger la nature avant d'ètre fixé sur les affinités de l'organisme lui-même et sur ses caractères évolutifs.

D'autres planktonistes n'ont pas manqué de l'observer aussi, comme nous l'avons appris, mais sans prendre la peine de le signaler, pour des raisons que nous ignorons.

Mieux vaut, nous semble-t-il, le tirer de son anonymat pour le signaler plus expressément à l'attention des observateurs. 
C'est pourquoi nous proposons d'en faire l'espèce fusus, à cause de sa forme, d'un genre nouveau Stappersia, du nom de M. L. Stappers, le naturaliste de la Belgica pendant la campagne arctique de I907.

Peut-être lui trouverait-on des rapports assez étroits avec le genre Coleps, pour lui faire une place à còté de celui-ci dans les Infusoires holotrichides si, outre sa forme rigide, on consentait à voir dans la sorte de capsule grillagée qu'il présente une certaine analogie avec la carapace articulée de Coleps.

Distribution Géographique. - Se rencontre un peu partout, mais est toujours très rare.

\section{Genre Gymnozoum g. n.}

Nous proposons ce nom générique pour un Infusoire nu, qui se montre aussi intéressant par son polymorphisme que par la variété de ses manifestations physiologiques et qui ne semble pas pouvoir trouver sa place dans les cadres taxinomiques actuels.

Ses caractères. ressortiront de l'étude comparée des figures par lesquelles nous avons cherché à fixer les principales attitudes et les curieuses phases évolutives dont il nous a fourni de nombreux exemples.

\section{Gymnozoum viviparum sp. n.}

Planche XX. figures 18 à 27 ; planche XXI, figures I à 24 .

Nous interpréterons d'abord les figures qui s'y rapportent; nous résumerons ensuite les principaux faits morphologiques, physiologiques et évolutifs qui s'en dégageront d'eux-mêmes.

Planche XX, figure I8. Individu jeune, en forme d'olive. Le cytoderme très mince est renforcé par des stries élastiques orientées d'un pôle à l'autre et également distancées. Il est complètement exempt de cils.

La bouche, b, ouverte à l'un des pôles, se prolonge intérieurement en une sorte de pharynx en forme de nasse, na, qui, rétrécie en arrière de la bouche, se renfle vers son extrémité postérieure pour s'ouvrir finalement dans le cytoplasme.

Les fibrilles qui la forment sont en nombre limité. Elles sont en relation étroite avec les stries élastiques du cytoderme dont elles ne sont, en réalité, que le prolongement replié dans le sarcoplasme de l'animal.

Le sarcoplasme est très vacuoleux, ce qui lui permet de devenir très transparent par suite de l'action des réactifs éclaircissants, le chloral particulièrement. 
C'est à ce traitement que nous avons eu recours pour préparer les objets que nous avons dù étudier pour les figurer.

Le noyau, $n$, est ici relativement petit, peu développé et mal situé du reste, derrière le pharynx, pour faire l'objet d'un examen utile. D'autres exemples suppléeront.

Planche XX, figure I9. Individu encore relativement jeune, mais traduisant l'aspect piriforme qui semble le plus communément adopté par les individus adultes, chez lesquels l'orifice oral s'ouvre habituellement au sommet d'un còne apical peu développé. Outre des détails qui reproduisent ceux de la figure précédente, avec de simples variantes individuelles, on remarquera ici le noyạu, $n$, sous l'un des nombreux aspects qu'il présente dans la série des phases évolutives de l'organisme. Cet organe, figuré en coupe optique, est formé de deux parties un peu inégales séparées par un changement de structure plutôt que par une cloison (I). Chacune d'elles présente un caryoplasme à structure rayonnante autour de granulations centrales. Au-dessous de la membrane nucléaire se présentent, sur une seule couche, de nombreux petits corps irréguliers de forme et fortement réfringents.

Planche XX, figure 20. Le spécimen ici reproduit vient de s'incorporer une inclusion volumineuse, consistant en une chainette de quatre individus d'Amylax catenata nobis, $A$. Comment s'y est-il pris pour absorber une proie si disproportionnée au calibre de sa bouche, dont l'ouverture, visible en $b$, est actuellement si étroite? C'est que cet organe est dilatable dans une mesure qui dépasse réellement toute vraisemblance et qui paraîtrait impossible si d'autres attitudes de l'organisme ne donnaient la clef du mystère, en montrant par quel mécanisme l'animal parvient à saisir des objets hors de proportion avec les siennes et à les déglutir d'une pièce. Comme nous le verrons tout à l'heure, le processus opératoire est ici tout autre que celui mis en œuvre par Didinimm Gargantua nobis, mais il ne lui est pas inférieur en efficacité.

En $r$ se voient les résidus de digestion d'un bol alimentaire antérieurement absorbé.

Le noyau, $n$, a sensiblement le même aspect que dans la figure ig.

Il est partiellement caché par la nasse, na, qui est rejetée sur le côté par la vacuole digestive développée autour de la chainette d'Amylax. Celle-ci représente, en effet, le dernier bol alimentaire absorbé par l'organisme avant sa fixation.

Planche XX, figure 2I. Ce sont ici des organismes beaucoup plus petits qui ont fait les frais du dernier repas de notre Gymmozoum. On les voit groupés dans une vacuole digestive, $v d$, située à peu de distance de l'extrémité de la nasse, na, qui parait pharyngienne, mais qui est plutòt un organe de préhension évaginable et extensible, comme nous en aurons la preuve dans d'autres exemples.

(r) “Noyau à cloison " ou " noyau à fente " des auteurs, suivant l'impression optique produite par la séparation équatoriale entre les deux moitiés hémisphériques. 
Le bol alimentaire comporte trois cellules de Diamylon nivale nobis (I), gonflées par l'action des sucs digestifs, mais peu déformées encore gràce à l'indigestibilité relative de ce protophyte constatée dans d'autres cas; une cellule d'Echinum micraster nobis, E, que sa membrane réfractaire rend aussi facilement reconnaissable et un résidu, $r$, reste méconnaissable d'un petit organisme indéterminable que le suc gastrique a déjà partiellement dissous.

Le noyau, $n$, présente ici une structure toute particulière. Il est simple et de forme ellipsoïde. Au centre se trouve un nucléole sphérique entouré d'un caryoplasme hyalin, à structure rayonnante, dans lequel apparaissent trois zones concentriques équidistantes de granulations réfringentes, que nous n'avons pu figurer, cela va sans dire, qu'en coupe optique.

C'est un des nombreux aspects que présente cet organe au cours de ses évolutions.

Nous appellerons immédiatement l'attention sur deux autres de ces aspects :

Io Figure 26, où l'on voit le nucléole central, mu, entouré d'une seule zone de corpuscules réfringents. Le caryoplasme a une structure réticulée assez grossière. Chargé de gros granules vers l'un des deux pòles, il est exempt de ces corps vers l'autre. Il témoigne ainsi d'un caractère de bipolarité marquée, que nous avons déjà signalée et qui se traduit plus manifestement dans la figure suivante, qu'il convient de rapprocher de celle-ci.

$2^{\circ}$ Figure 27, où l'on voit le nucléole, $m$, relégué vers l'un des deux pòles, au sein d'un caryoplasme très hyalin et finement radié, tandis que l'autre moitié présente des sortes de chromosomes nombreux disséminés dans le protoplasme nucléaire plus grossier.

Ne conviendrait-il pas de considérer cette moitié comme le macronucleus et l'autre comme le micronucleus, dont les éléments, plus ou moins mélangés et confondus dans certaines circonstances, reprendraient une certaine individualité, après épuration respective, à l'approche des phénomènes de division dont nous verrons plus loin des exemples? Il ressortira de ceux-ci que le ròle de micronucleus revient au seul corps que nous appelons ici nucléole, mu, car c'est lui seul qui se divise par mitose. On peut donc dire que le micronucleus est l'hòte du macronucleus. Le caryoplasme de celui-ci gravite autour de celui-là comme autour d'un centre d'attraction et traduit cette influence mystérieuse par sa structure rayonnante.

(I) Nous avons dit ailleurs quel est l'habitat de Diumylon nivale et expliqué sa présence dans les eaux de la Ier de Kara ou la fusion des neiges et des glaces l'a fait passer et où tant d'organismes divers, Protozoaires et petits Crustacés, en font une consommation qui semble désigner cette petite Algue comme un aliment de choix dont la succulence tenterait la gourmandise de la plupart des représentants du monde microscopique de ces parages.

D'autre part, si nous l'avons pu reconnaitre dans le cytoplasme des Protozoaires, après des durées d'ingrestion variables, et même dans les crottins évacuès après avoir parcouru tout le tube dirrestif de petits Crustacés, sans altération sensible, n'est.ce pas la preuve que cet aliment présente des caractères d'indigestibilité qui sont en opposition avec l'appétence unanime dont il parait c̀tre l'objet?

Cela peut paraitre paradoxal, mais les faits sont tels. 
Planche XX, figure 22. Gymnozomm a avalé un Péridinien, $P$, que l'on voit engagé dans le fond du sarcoplasme, au sein d'une vacuole digestive, vd. Dans le voisinage immédiat se voit une autre vacuole renfermant les restes méconnaissables, $r$, d'une autre proie antérieurement absorbée et en grande partie digérée.

Le noyau, n, qui est au repos, se montre biparti, avec l'aspect d'un noyau dit noyau à fente, à cause de l'espace lenticulaire hyalin qui sépare les deux hémisphères. L'une des deux moitiés est plus riche en corps analogues aux chromosomes; l'autre, moins chargée de ces corps, plus hyaline, renferme une sorte de nucléole central : le micronucleus, dans l'hypothèse formulée tout à l'heure.

Sur le còne apical, on voit l'orifice oral, $b$, au fond d'une légère dépression en entonnoir.

L'objet a été figuré à dessein dans une pose un peu oblique pour permettre de saisir cette particularité.

Planche XX, figure 23. Autre aspect de notre organisme, orienté de façon à montrer la nasse dans une position favorable pour en apprécier la forme suivant son plan de symétrie.

On remarquera la configuration un peu exceptionnelle de la bouche, $b$, qui est plus protubérante que de coutume, au sommet du cône apical.

Dans la vacuole digestive, $v d$, on aperçoit, en $r$, un assez petit Infusoire, que le suc gastrique a déjà défiguré en y provoquant la plasmolyse et en soustrayant les substances les plus facilement assimilables. A côté, vers le pôle apical, se trouvent les résidus d'un autre bol alimentaire moins copieux.

Le noyau volumineux, $n$, peut être considéré comme type du noyau à l'état quiescent. Il est biparti, à fente médiane, et présente, dans ses deux moitiés, les différences de structure et d'aspect déjà signalées.

Planche XX, figure 24. La nasse, na, est évaginée et se dresse en dehors du cône apical à la façon d'une trompe. On voit manifestement que les fibrilles qui la constituent, et qui ont leur extrémité libre, ne sont que le prolongement des stries élastiques qui sillonnent le corps de l'organisme d'un pòle à l'autre.

Cette évagination est évidemment physiologique et non traumatique ou accidentelle, car aucune cause mécanique n'aurait pu la produire sans laisser des traces de lésions sur le corps.

Ainsi évaginée au gré de l'animal, cette nasse doit servir d'organe préhensile susceptible de s'ouvrir en large pavillon à son sommet et d'arrimer solidement des proies même très volumineuses, avant d'en opérer la déglutition. Il faut admettre pour cela que l'organe soit éminemment dilatable sur toute sa longueur et particulièrement à sa base, où il est cependant également apte à s'étrangler en sphincter pour fermer l'orifice buccal, après sa réinvagination. 
On peut présumer que l'extrémité des fibrilles de la nasse évaginée peut agir à la façon des trichocystes d'autres Infusoires, pour paralyser les victimes qu'elles atteignent ou même en provoquer la mort, comme le serpent fait de sa proie avant de se l'incorporer.

Une question se pose ici. La déglutition du bol alimentaire se fait-elle dans le parcours de la nasse pendant qu'elle reste en érection, comme dans la figure 24, par des mouvements de cet organe, qui rappelleraient les mouvements péristaltiques de l'intestin des animaux supérieurs, ou bien la proie saisie est-elle amenée à l'intérieur du sarcoplasme par un mouvement propre de réinvagination de la nasse elle-mème?

Dans cette dernière hypothèse, l'opération se ferait en un temps : la rétraction de la nasse ou sa réinvagination, si l'on veut, s'accompagnant de la déglutition de la proie y incluse. L'opération se ferait en deux temps dans la première hypothèse : la déglutition de la proie, par mouvements péristaltiques de l'organe, précédant la réinvagination de celui-ci, à la manière d'un doigt de gant qu'on retourne.

Bien que nous ayons observé des centaines d'individus, fixés, bien entendu, dont la plupart témoignent, par la diversité de leurs inclusions, d'une grande activité physiologique et d'une grande ardeur à la chasse au moment de leur capture et de leur momification par le formol, nous n'avons pu rencontrer un seul spécimen en travail de déglutition d'une proie. Faut-il en conclure ou bien que le phénomène se passe très rapidement, ou bien que le sentiment de défiance de l'animal, mis en éveil par les circonstances, lui a fait làcher sa proie ou en précipiter l'absorption?

Appliquons nos doutes au cas de la figure 24, où l'on voit, dans le sarcoplasme, un petit spécimen de Didinium Gargantua nobis, récemment incorporé au sein d'une vacuole digestive, $v d$. Notez son appareil pharyngien, $b$, bien reconnaissable, son noyau incurvé, $n$, son cytoplasme riche en réserve amylacée, $f$, la striation de sa membrane et jusqu'à ses deux ceintures ciliaires dont la figuration n'a pas été possible à cause de sa position étriquée.

La nasse ou trompe du Gymnozoum est évaginée, en pleine érection.

Comment faut-il interpréter l'attitude de l'organisme que les réactifs ont figé à ce moment précis de ses manifestations biologiques?

Faut-il penser que le Didinium vient d'être incorporé en parcourant tout le conduit de la trompe restée en érection? Faut-il croire au contraire que l'animal, inassouvi par l'absorption d'une proie qui n'est cependant pas sans importance, a évaginé à nouveau son organe préhensile à la recherche d'une nouvelle victime?

Cette seconde hypothèse n'a rien d'invraisemblable quand on connaît la voracité de l'organisme dont nous donnerons plus loin d'autres preuves.

On remarquera que Didinizm Gargantua nobis, carnassier redoutable dans le monde des Protozoaires, peut devenir gibier lui-même pour Gymnozoum, mieux armé encore pour la chasse. 
Planche XX, figure 25. Cette reproduction nous met en présence d'un autre phénomène physiologique de l'animal, sa fissiparité.

Le corps allongé s'est étranglé dans la région médiane, après formation, sur le còté de la moitié inférieure, d'un second appareil buccal, b, pourvu d'une nasse encore assez courte.

La nasse primitive, très longue, est en voie de transformation et montre une dislocation partielle des fibrilles pharyngiennes, près de l'orifice oral, $b$, qui occupe le sommet du còne apical. On prévoit que les progrès de l'étranglement médian produiront la subdivision de l'individu-mère en deux rejetons rajeunis, suivant un processus tout simple de division transversale dont la plupart des Protozoaires fournissent des exemples bien connus.

L'Infusoire avait ingéré préalablement une chaînette de quatre individus d'Amylax catenata, $A$, que l'on voit logés dans une vacuole digestive, $v d$, du sarcoplasme où, gràce à l'indigestibilité de leur capsule, ils ont conservé toute la netteté de leurs caractères morphologiques.

Chose remarquable : le noyau, $n$, de Gymnozoum se présente encore avec la structure bipartite qui le caractérise à l'état de repos, restant ainsi en retard vis-à-vis de la division somatique au lieu de la précéder pour lui donner l'impulsion initiale.

Non loin du noyau se montre un corps, de structure spiralée et d'aspect très réfringent, que nous avons $s^{\circ}$ indiqué à tort sous la rubrique in, comme une inclusion, mais qui nous parait bien plutòt être l'expression rudimentaire d'un organe que nous verrons sous des aspects différents dans d'autres individus où nous l'avons désigné par la lettre $x$, signe de notre incertitude vis-à-vis de sa véritable nature. Il n'y a pas lieu de s'en occuper présentement.

Planche XXI, figure I. On voit ici, logé dans une vacuole digestive, vd, une coque disloquée de Dinophysis rotundata Claparède et Lachmann, que la digestion a vidée.

Les deux valves, grâce à leur nature cellulosique, ont résisté à l'action des zymases, mais le ciment qui les unissait a été dissous et elles se sont séparées.

Des masses résiduaires, $r$ et $r$, d'autres bols alimentaires s'observent aussi dans le cytoplasme.

Le noyau, $n$, est à l'état quiescent et présente, dans les deux moitiés polaires, la diversité de composition sur laquelle nous avons déjà appelé l'attention.

Planche XXI, figure 2. L'intérèt que présente ce spécimen résulte de la présence de nombreux grains de fécule, $f$, que leur dispersion régulière dans le cytoplasme doit faire considérer plutòt comme des enclaves produites par l'activité propre de la cellule que comme des produits d'inclusion empruntés tels quels au milieu extérieur. C'est un fait digne de remarque que l'aptitude de ce Protozoaire omnivore à produire des substances amylacées qu'il peut mettre en réserve, sous forme figurée, à la façon des organismes pourvus de chromophylle. 
On se rappelle que nous avons signalé la même particularité chez d'autres Infusoires habitant le même milieu.

A noter : $b a$, bol alimentaire en voie de digestion dans une vacuole, $v d$; $r$, résidu de digestion. La nasse, la bouche, $b$, et le noyau, $n$, n'offrent rien de particulier.

Planche XXI, figure 3. Le corps énigmatique, $x$, sur lequel nous devrons appeler l'attention dans d'autres figures encore, se présente ici sous des dimensions très grandes, avec l'aspect d'un gros noyau à structure fibrillaire entouré d'une substance de caractère très particulier. Cette production n'est pas isolée dans une vacuole comme un produit d'ingestion, mais fait corps avec le cytoplasme comme un organe propre de la cellule.

Faut-il y voir une sorte de noyau spécial, dont l'apparition, le développement et les évolutions sont liés aux phénomènes de viviparité dont Gymnozoum fournit des exemples qui seront présentés plus loin? Nous le pensons, bien que nos recherches purement analytiques ne nous aient pas permis d'en préciser le ròle et de reconnaitre l'ortre naturel de ses phases évolutives en rapport avec celles de la reproduction.

Planche XXI, figure 4. Autre aspect du corps, $x$, plus petit, à allure de noyau renfermant lui-mème un noyau de dimensions moindres et de structure fibrillaire.

Pour le reste, mêmes désignations que dans les autres figures similaires.

La structure bipolaire du noyau, $n$, est manifeste : d'un côté, les granulations grossières du macronucleus, de l'autre, son caryoplasme hyalin qui recèle, en son milieu, l'apparence de nucléole que nous croyons représenter le micronucleus.

Planche XXI, figure 5. Le corps énigmatique, $x$, présente une sorte de nucléole central, entouré de deux sphérules concentriques formées d'une substance très réfringente. Sa partie périphérique révèle une structure rayonnante. Il est figuré en coupe optique, seule façon de laisser voir les sphérules concentriques qui englobent le nucléole. Le noyau ordinaire, caché derrière ce corps, n'a pu ètre figuré.

La nasse, $n a$, présente au voisinage de la bouche, $b$, des preuves de dislocation partielle.

Dans la vacuole digestive, $v d$, on roit plusieurs grains de fécule groupés, $f a$, qui sont ici évidemment alimentaires : produits d'inclusion empruntés au milieu, peut-être avec l'organisme qui les contenait à titre d'enclaves et que les sucs gastriques ont déjà dissous; $r$, résidu de digestion.

Planche XXI, figure 6. La complication grandit. Deux noyaux normaux, $n$ et $n^{\prime}$, sont en présence, doués des mêmes caractères : indices d'une tendance à la division. Mais quel serait le but de cette subdivision, la cellule étant déjà binucléée?

Il existe aussi deux corps énigmatiques, $x$ et $x$, à des phases différentes de développement. Chez l'un, le plus petit, le nucléole central est homogène; chez l'autre, il affecte une structure fibrillaire très nette. 
Le sarcoplasme renferme deux bols alimentaires, constitués, l'un par un Infusoire globuleux à trichocystes périphériques, in, l'autre, par une chainette de quatre coques vides d'Amylax catenata nobis, $A$, vue de profil et légèrement incurvée dans la vacuole digestive, $v d$, où elle reste à l'état de résidu réfractaire à la digestion.

Planche XXI, figure 7. Voici le plus curieux tour de force que nous ayons vu réalisé par notre Gymnozoum. C'est un Rotateur tout entier et vivant, Ro, que l'animal vient d'ingérer, malgré ses dimensions qui dépassent notablement les siennes. L'ingestion en est récente, car les sucs gastriques de la vacuole digestive, dans laquelle il est étroitement logé, $v d$, ne l'ont pas encore entamé ni défiguré. Il est intact de la tète, $t$, à la queue, $q$, laquelle a dù se recourber pour s'adapter aux dimensions du corps du monstre qui l'a englouti.

L'absorption d'un gibier aussi volumineux ne va pas d'autre part sans inconvénient pour le chasseur. La dilatabilité du cytoderme semble exercée jusqu'à ses dernières limites. Le volume du corps est presque doublé; sa forme est profondément modifiée. La bouche, $b$, et sa trompe rétractile sont reléguées sur le côté.

Dans une autre vacuole voisine de la queue fourchue du Rotateur, on voit la coque vide d'un Péridinien, $P$, un Protoceratium reticulatum Bütschli, déjà digéré dans ce qu'il a de digestible, le protoplasme sarcodique.

L'encombrement du corps par deux proies aussi volumineuses n'a pas empêché l'animal de s'ingérer encore une inclusion, $i n$, dont on ne reconnait que des grains de fécule groupés dans un liquide hyalin, à l'extrémité de la nasse ou trompe, na.

Dans le noyau, $n$, la partie plus claire renfermant le nucléole central - le micronucleus, dans l'hypothèse émise plus haut, — s'est amplifiée aux dépens de la partie granuleuse et donne des signes avant-coureurs d'une division prochaine.

Planche XXI, figure 8. Nouveau cas de division transversale de l'organisme surpris au moment mème de la division du noyau, nd, en deux parties symétriques qui reprennent la forme sphérique. Le caryoplasme est clair et ne présente de granulations qu'au voisinage de ce qui représentait les deux pòles du noyau-mère, avant sa bipartition. Nous n'y avons pas saisi les produits de subdivision du nucléole, le micronucleus hypothétique. L'action prolongée du chloral l'a sans doute rendu trop hyalin.

Entre la nasse jeune, $n a^{\prime}$, et l'ancienne, $n a$, on voit des fibrilles disloquées et brisées qui témoignent d'un travail de réorganisation de cette dernière. Elle subit une réduction de longueur.

L'étranglement du corps est encore peu prononcé, bien que, si l'on a égard à la division déjà accomplie du noyau normal, le phénomène de fissiparité doive ètre considéré comme plus avancé que dans l'individu de la figure 25, planche XX.

Des produits résiduaires de digestion incomplète d'inclusions, $r$ et $r$, se voient au sein du sarcoplasme dans des vacuoles gastriques. 
Planche XXI, figure 9. L'organisme est ici en gestation d'un jeune, $j$, qui, sous des dimensions moindres, présente tous les organes de l'adulte : forme ellipsoïde, avec des stries dans le cytoderme qui en partagent la surface comme les douves d'un tonneau; bouche, bj, située à l'un des pòles et en rapport avec une nasse pharyngienne, développée dans toute la longueur du corps; noyau, $n j$, déjà bipolaire, mais de petites dimensions et de structure assez compacte.

Il est mobile, mais à l'étroit dans le cytoplasme maternel, où il a pris naissance, semble-t-il, aux dépens ou du moins par le concours d'un de ces corps énigmatiques, $x$, sur lesquels nous avons déjà appelé l'attention à plusieurs reprises.

La mère n'en est pas modifiée, ni dans son aspect piriforme, ni dans son appareil buccal, ni dans son noyau, $n$, dont la physionomie témoigne d'un état de repos.

C'est bien, comme on le voit, un cas de reproduction vivipare, que nous reproduisons ici, sous son aspect le plus simple. Il servira à faire comprendre d'autres cas plus compliqués, dont il nous a paru utile de figurer quelques exemples.

Planche XXI, figure IO. Le jeune individu est ici beaucoup plus grand, il approche des dimensions de la mère. On y reconnaîtra aisément tous les organes essentiels, dans leur situation normale : bouche, $b j$; nasse pharyngienne, naj; noyau bipolaire, $n j$; et, accessoirement, un résidu de digestion, $r$.

Le cytoplasme maternel est réduit à une assez mince couche périphérique, dans laquelle le noyau, $n$, ne peut trouver place qu'en se déformant.

La nasse, $n a$, est évaginée en trompe préhensile, en position d'attaque pour la chasse. On remarquera une fois de plus l'extrémité libre des fibrilles auxquelles on doit vraisemblablement attribuer un rôle de trichocystes offensifs. A rapprocher de la figure 25 , planche $\mathrm{XX}$.

Planche XXI, figure II. Dans ce spécimen reproduit en vue apicale un peu oblique, pour montrer la configuration un peu spéciale de la bouche, $b$, on voit un jeune, $j$, à l'état encore embryonnaire, flottant dans une vacuole incubatrice, $v i$. Avec un peu d'attention, on y reconnaitra les stries longitudinales du cytoderme, la bouche, bj, la nasse et le noyau, $n j$, d'apparence homogène et encore très petit.

Dans le cytoplasme maternel on voit le noyau, $n$, qui traduit, par son aspect hyalin et sa structure rayonnante dans l'une de ses moitiés, des phénomènes avantcoureurs de la division. On remarque, en outre, deux corps, $x$ et $x$, d'aspect différent et renfermant des masses très réfringentes, diversement agencées. Leur présence ici semble préluder à la procréation de nouveaux rejetons.

Planche XXI, figures I2, I3, I4. L'embryon vivipare, $j$, a été surpris, dans l'individu reproduit figure I2, à une étape plus rapprochée de la genèse. Il a la forme d'un tonnelet allongé. Il flotte dans la vacuole incubatrice, vi. 
Une autre grande vacuole, digestive celle-ci, $v d$, renferme un bol alimentaire, $b a$; un petit Infusoire, sans doute, en voie de digestion dans le suc gastrique de la mère, dont on reconnait la bouche, $b$, et la nasse invaginée, $n a$.

Mais l'intérêt de ce spécimen git dans le noyau, $n$, que nous avons figuré en place, vu en coupe longitudinale optique. La forme de calebasse qu'il a prise lui a permis, par son extrémité amincie, de percer le cytoderme du Protozoaire et de développer, en dehors de celui-ci, une sorte de panache, $p$, à structure fibrillaire et rayonnante, en forme de coupe très évasée.

Pour aider à l'intelligence de la structure du noyau pendant cette phase aussi curieuse qu'inattendue de son évolution, nous l'avons reproduit isolément : figure I3, en coupe longitudinale optique, et figure I4, en vue polaire supérieure, un peu oblique.

La partie renflée de l'organe présente, autour du nucléole, mu (fig. I3), un caryoplasme hyalin et strié radialement, circonscrit par trois zones concentriques de granulations analogues d'aspect avec celles qui occupent toute la partie rétrécie de la calebasse (I).

Ces granulations doivent représenter sous une autre forme celles qu'on observe dans l'hémisphère granuleux du noyau quiescent.

Quant au panache fibrillaire, $p$, on voit, dans la figure I4, qu'il forme une auréole régulière autour du sommet rétréci de l'organe.

Quelle peut ètre la signification de cette production étrange que nous avons observée plusieurs fois dans des spécimens parfaitement fixés, ce qui exclut l'idée d'une déformation accidentelle?

A quoi tend la saillie en dehors du cytoderme de l'extrémité amincie du noyau et de son couronnement si évidemment adapté à un ròle défini?

Ne serait-on pas tenté d'y voir une manifestation de sexualité dont les Protozoaires actuellement connus ne fournissent pas d'exemples analogues?

Nous serions mal fondé toutefois à soutenir cette opinion, sans avoir puisé des renseignements dans d'autres objets d'étude que les seuls matériaux morts que nous possédons. Ceux-ci, naturellement, ne nous ont pas permis de scruter le rôle de cette curieuse formation et d'en suivre la destinée, comme on pourrait l'espérer d'organismes étudiés sur le vif. Mais, tel quel, le fait est intéressant à relever, en attendant que l'on trouve la solution de l'énigme à la faveur d'observations faites in vivo.

Planche XXI, figure I5. Bien qu'aussi volumineux que beaucoup d'individus adultes et libérés, le jeune vivipare, $j$, est encore inclus ici dans le sarcoplasme maternel. Il se présente en vue apicale, tandis que la mère, en gestation de son rejeton encombrant, est vue de profil, dans la position que nous avons donnée à l'objet, pour le reproduire de la façon la plus propre à traduire l'intérêt qu'il offre.

(I) Comparez cette structure du noyau avec celle de l'organe similaire de la figure 2I, planche Xx. On ne peut se défendre de voir, dans ce dernier, un acheminement vers la forme mystérieuse qui trouve ici sa parfaite expression. 
L'individu maternel montre sa nasse orale, $b$, sous un aspect particulier qui est dù à ce que l'organe a été surpris en voie d'évagination ou d'invagination, nous ne savons, car ces deux mouvements inverses s'exécutent sans doute suivant le même rythme.

Le noyau, n, a l'aspect des noyaux dits " à fente " des auteurs.

On ne pourrait dire qu'il est à l'état quiescent, car ses deux moitiés assez semblables présentent chacune une sorte de petite sphérule claire à contenu granuleux, dont la position symétrique, aux deux côtés de l'espace lenticulaire médian, ne paraît pas fortuite, mais semble plutôt liée à une manifestation d'ordre intime de l'activité physiologique de cet organe polymorphe.

En $D$, se voit une cellule de Diamylon nivale, en inclusion.

L'individu jeune, $j$, mobile dans sa vacuole incubatrice, présente sa bouche, bj, de face, ce qui permet de voir les stries cytodermiques qui y convergent.

Son noyau, $n j$, est bipolaire et au repos. On y observe, enfin, deux bols alimentaires de petit volume, $b a$ et $b a$, dont l'un est en voie de digestion, l'autre à l'état résiduaire.

Planche XXI, figure i6. Spécimen analogue au précédent, lesté d'un embryon volumineux, $j$, vu de profil, dont on voit la bouche, $b j$, le noyau, $n j$, bipolaire et de grosseur à peu près normale et la nasse pharyngienne, naj, bien développée.

L'individu-mère a son noyau dans la couche périphérique du cytoplasme, du còté de l'observateur, où cet organe se profile optiquement sur une portion du jeune. Son aspect semble révéler un mouvement intérieur, une sorte de division interne, dirait-on, à une phase un peu antérieure à celle que présente le même organe dans la figure i 5 .

La nasse est physiologiquement déformée par des replis produits dans les fibrilles qui la constituent, fait qui en montre la souplesse et l'élasticité, en même temps qu'il éclaire la façon dont peut s'opérer le retroussement de l'organe.

Planche XXI, figure I7. D’après ce qui a déjà été dit de la structure si particulière de Gymnozoum vivipanum, on interprétera facilement le cas présent qui renchérit encore un peu sur la complexité déjà entrevue de ce bizarre organisme.

L'individu adulte a absorbé un Péridinien, $P$, dont on voit la capsule indigeste dans une vacuole digestive, $v d$. Le noyau, $n$, est presque revenu à l'état normal, au repos, après une de ces crises intimes dont nous avons vu des manifestations assez peu explicites dans les figures 15 et $\mathbf{1} 6$.

En $x$, se voit un corps dont les relations avec la fonction vivipare nous paraissent très probables, bien que nous n'en ayons pas la preuve évidente. Jusqu'à plus ample information, nous le tenons pour un germe embryonnaire.

Le fait nouveau à noter ici, c'est que l'embryon formé, $j$, dont le noyau, $n j$, encore réduit, témoigne de son évolution relativement peu avancée, recèle déjà dans son cytoplasme propre un Péridinien, $P$. 
Ce fait mème révèle son aptitude à s'incorporer, pendant qu'il est dans le sein de sa mère, des inclusions même volumineuses, que celle-ci a dù préalablement ingérer elle-même.

C'est une déglutition en partie double : de la part de la mère d'abord, ce qui est naturel, de la part du rejeton utérin ensuite, ce qui semble inédit.

Cette façon de la mère de se décharger sur son rejeton intra-utérin du soin de s'alimenter lui-même en aliments solides ne manque pas d'imprévu, non moins que l'aptitude du rejeton à exempter sa mère du soin d'en opérer d'abord la digestion.

Cela ne va pas sans difficultés apparentes et ce n'est possible évidemment, facile même probablement, que par la mise en exercice de l'organe pharyngien de l'embryon : opération qui semblerait malaisée, vu la longueur de l'organe, à l'état de trompe préhensile, et l'étroitesse de son champ d'opération, si l'on ne connaissait la souplesse du corps sarcodique de la plupart des Infusoires.

Planche XXI, figure I8. L'intérêt de cette figure réside dans le noyau, $n$, de l'individu adulte. Reproduit en coupe optique, il montre une structure rayonnante autour d'un pseudo-nucléole central, m. Nous avons fait remarquer que celui-ci n'était sans doute que le micronucleus hébergé par le macronucleus. Des granules réfringents forment une seule couche immédiatement en dessous de la membrane nucléaire. C'est une phase préparatoire à la division de cet organe.

Le cytoplasme ne présente pas d'autre inclusion qu'une cellule de Diamylon mivale, $D$, mais un embryon y a pris naissance et sa situation du còté de l'observateur permet de le bien dévisager tel qu'il se montre à cette période de son développement : en façon de petit tonnelet, avec de légers froncements transversaux autour de la bouche, bj, qui font l'effet de cercles rapprochés autour des douves figurées par la striation longitudinale du cytoderme.

Le noyau, $и j$, est encore fort petit et non visiblement différencié.

Planche XXI, figure Ig. Le cas actuel se complique de la présence simultanée de deux individus jeunes, $j^{A}$ et $j^{B}$, dans le sarcoplasme de la mère, dont on voit la bouche, $b$, et le noyau, $n$.

La nasse très longue, $n a$, est en partie cachée dans le dessin et par le noyau, $n$, et par les deux embryons qui se touchent presque, bien qu'ils soient confinés chacun dans une vacuole incubatrice propre, vi.

Ceux-ci ne sont pas au mème degré de développement; ce ne sont pas des jumeaux, car ils ne sont pas du mème àge; ce ne sont pas non plus des frères utérins à proprement parler, - si l'on veut bien nous passer cette expression évidemment figurée, à propos d'organismes où cette expression n'a pas d'objectivité réelle - car ils occupent deux vacuoles incubatrices distinctes.

Le plus petit, $j^{A}$, ne renferme pas d'inclusions reconnaissables. Son noyau allongé, $n j^{A}$, présente déjà une différence de structure vers les deux pòles. On remarquera sa bouche, $b j^{i}$, et sa nasse pharyngienne, $n a j^{A}$. 
Son frère ainé, $j^{B}$, lui, s'est incorporé une chaìnette de quatre individus d'Amylax catenata, $A$, espèce de Péridinien commune dans les eaux où vit Gymmozoum.

La nasse, $n a j^{B}$, qui a dù opérer la déglutition difficile de ce gros morceau, n'a pas encore repris sa structure régulière. On en voit les fibrilles plissées dans le tiers inférieur. La bouche, $b j^{B}$, est rétrécie en sphincter contracté. Le noyau bipolaire, $n j^{B}$, présente les caractères de cet organe adulte, mais sous des dimensions plus réduites.

Planche XXI, figure 20 (I). Nouvelle complication due à la présence simultanée de trois générations de Gymnozoum en un même individu.

La mère est en gestation de deux filles, $2 j^{1}$, de première génération, $j^{A}$ et $j^{B}$, mais la fille, $j^{B}$, a déjà donné naissance à un embryon, $j^{2}$, de deuxième génération, qui est conséquemment la petite-fille de la mère commune, son aïeule.

C'est là un cas réel d'emboîtement de germes. Il démontre que la faculté vivipare n'est pas l'apanage de l'adulte et a d'autres causes déterminantes que la maturité du sujet.

Le corps, $x$, en forme de croissant qui présente, vers son extrémité libre, une sorte de noyau et qui se dégage de l'embryon, $j^{A}$, est une nouvelle énigme à ajouter aux autres particularités exceptionnelles de cet organisme, auquel ses caractères tant morphologiques que physiologiques semblent devoir faire attribuer une place à part dans le groupe déjà si varié des Infusoires.

Nous derons faire remarquer que la cellule de Diamylon nivale, $D$, qui figure dans le dessin, est une inclusion du cytoplașme de la grand'mère, vue en projection sur le jeune, $j^{A}$.

Le noyau, $n$, de l'adulte est au repos; il est situé près de la bouche, comme il arrive le plus souvent quand le sarcoplasme est encombré par d'autres productions d'origine interne ou externe.

Planche XXI, figure 2I. Nous voici devant le fait non moins imprévu de la division scissipare d'un embryon, $j$, encore inclus dans le sein maternel.

Son corps, légèrement étranglé vers le milieu, présente déjà deux bouches armées chacune d'une nasse, l'une, longue, primitive, $b^{\prime} j$, l'autre, courte, secondaire, $b^{\prime \prime} j$.

Le noyau, $n j d$, figuré en coupe optique, témoigne d'une division cinétique dont nous traduisons l'aspect, sans pouvoir préciser les détails intimes du phénomène, à défaut de recherches spéciales sur ce sujet. Il semble bien toutefois que ce qui fournit la figure caryocinétique, c'est uniquement le nucléole signalé plus haut à plusieurs reprises, le micronucleus, dans l'interprétation donnée de ce corps.

(I) Toutes les figures relatives à cet organisme étrange, celle-ci plus spécialement, ne manquent pas de complication et les détails n'en sautent pas aux yeux. Leur examen demande, de la part du lecteur, autant d'attention que nous avons dû en apporter nous-même dans l'étude des objets et dans la reproduction, par le burin, des traits principaux de leur étonnante coinplexité.

Il faut les examiner de près, avec des yeux de myope, à défaut de quoi l'usage d'une loupe est tout indiqué. 
La mère vivipare, en gestation d'une fille prématurément prolifère à son tour, présente, en inclusion, des Diamylon mivale, $D$.

Son noyau bipolaire, $n$, est à l'état quiescent.

Sa nasse pharyngienne, na, a été fixée en cours d'évagination ou d'invagination, nous ne savons, mais la structure actuelle permet de se rendre compte de la façon dont s'accomplit ce double mouvement.

Planche XXI, figure 22. On assiste ici à la mise en liberté d'un embryon, $j$, qui semble prendre lui-même l'initiative de sa libération en forçant les parois de sa prison, le cytoderme maternel.

Le fait est surpris au moment où la partie antérieure qui porte la bouche, bj, est déjà libérée; le reste suivra et la lésion produite par cet exode violent se réparera par une cicatrisation rapide comme les Acinètes vivipares, que nous avons fréquemment observés ailleurs, nous en ont fourni des exemples sur le vif.

On remarquera une fois de plus la structure caractéristique du noyau, $n$, de l'organisme adulte.

Planche XXI, figure 23. Cette figure reproduit l'un des plus petits produits de viviparité que nous ayons eu l'occasion d'observer à l'état libre. Le lecteur y reconnaitra, en petit, tous les organes de l'adulte : la bouche, $b$, avec sa nasse pharyngienne, le noyau bipolaire, $n$, le cytoplasme vacuoleux, etc.

Planche XXI, figure 24. L'individu encore très jeune, mais libre, se montre déjà en état de grossesse vivipare, état dans lequel il était sans doute avant sa mise en liberté, comme nous savons que la chose est possible par l'exemple de la figure 20.

Signalons sa bouche, $b$; son noyau bipolaire un peu aplati, $n$; sa nasse, $n a$.

L'embryon qu'il renferme a sa bouche en $b j$, sa nasse en $n a j$, son noyau encore peu différencié et globuleux en $n j$.

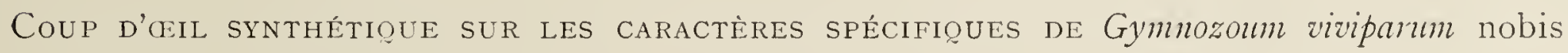

Après avoir observé un très grand nombre de ces curieux organismes dont nous avons présenté au lecteur un choix de spécimens propres à l'édifier sur les modalités biologiques de l'espèce, il semble utile de résumer en quelques propositions les faits saillants de son histoire naturelle.

I. Le corps, plus ou moins contracté, sans doute par le liquide conservateur à base d'alcool et de formaldéhyde, se montre globuleux, ramassé, sphéroïde, ellipsoïde ou piriforme. Il est plastique, mou, extensible et déformable.

II. Le cytoderme est mince et nu, c'est-à-dire dépourvu de cils apparents, mais soutenu par des fibrilles élastiques orientées d'un pôle à l'autre et en continuité 
avec les éléments figurés d'une nasse pharyngienne, qui n'en sont que les prolongements repliés dans le cytoplasme quand l'animal n'est pas en exercice de chasse.

III. Le cytoplasme est très vacuoleux, généralement exempt d'enclaves, parfois pourvu de grains de fécule disséminés (fig. 2, pl. XXI).

IV. La bouche, qui est terminale, est située au pòle aminci du corps; elle se ferme incomplètement, en manière de sphincter.

V. La nasse pharyngienne, formée de fibrilles durables et solidaires dans leurs mouvements, est habituellement invaginée dans le sarcoplasme où elle plonge librement et où elle est mobile. Elle peut s'évaginer et se mettre en érection, sous forme de trompe préhensile, pour la chasse (fig. 24, pl. XX, et fig. Io, pl. XXI). Elle est suffisamment dilatable pour permettre la préhension et la déglutition de proies volumineuses.

VI. L'animal chasse les organismes les plus variés qui vivent dans son milieu : Diamylon, Infusoires, Péridiniens, Rotateurs, etc., qu'il maitrise, quand il est besoin, par les propriétés meurtrières dont sont douées, sans doute, les extrémités des fibrilles de la trompe préhensile.

VII. Le noyau, au repos, présente des caractères de bipolarité traduits par une structure différente dans les deux moitiés. Il semble représenter à la fois le macronucleus et le micronucleus, ce dernier inclus dans le premier. Il présente des phénomènes de cinèse, lors de la division fissipare de l'organisme. Il offre des exemples de production d'un organe externe à la cellule, dont les relations possibles avec des phénomènes de sexualité demanderaient confirmation (fig. I2, I3, I4, pl. XXI).

VII. L'animal se reproduit par fissiparité (fig. 25, pl. XX, et fig. 8, pl. XXI).

IX. Il se reproduit aussi par viviparité; la production interne de l'embryon semblant liée à l'apparition dans le cytoplasme maternel de corps énigmatiques, germes? - soumis à des phases évolutives variées.

X. Les embryons peuvent devenir vivipares à leur tour, avant leur sortie.du corps maternel (fig. 2O, pl. XXI).

X1. Ils peuvent s'y diviser transversalement aussi (fig. 2I, pl. XXI).

XlI. Ils sont aptes à s'incorporer des inclusions, sous forme de bols alimentaires, avant leur mise en liberté.

XII. Celle-ci se fait par déchirure du cytoderme maternel.

\section{Section III. - Infusoires tentaculifères ou suceurs}

Nos matériaux ne nous ont fourni que de très rares représentants des Infusoires suceurs. Encore ceux-ci n'ont-ils été trouvés que dans des produits de pèche côtière, dans les environs de Tromsï. 
Nous n'en avons guère que trois formes à présenter dont deux sont des Podophrya, Acinétides nus à tentacules semblables, fixés sur un pédicelle rigide; la troisième, qui est à la fois loriquée et pédicellée, doit se rapporter au genre Acineta.

\section{Podophrya macropus sp. n.}

Planche XIV, figure II.

Corps sphérique, nu, pourvu de pseudopodes tentaculiformes, ps, répartis uniformément sur tout l'hémisphère apical. La brièveté des tentacules suceurs est sans doute le fait d'une contraction due à l'action des réactifs conservateurs.

Le noyau, $n$, est central et globuleux.

Le pédicelle, $p d$, large à son insertion sur le corps, se rétrécit en cône allongé et plissé longitudinalement pour se prolonger enfin en un long cordon de calibre uniforme et de structure striée dans trois directions perpendiculaires, à la façon du sarcoplasme d'une cellule musculaire.

Distribution géographique. - Environs de Tromsö; très rare.

\section{Podophrya conipes Mereschkowsky?}

Planche XIII, figure 46 .

Corps sphérique, porteur sur toute sa surface de nombreux pseudopodes tentaculiformes, ps, qui paraissent avoir été capités pendant la vie de l'organisme.

Le noyau, $n$, est central, globuleux, à structure fibrillaire.

Le pédicelle ou pied, $p$, est longuement conique, incurvé et strié transversalement.

Distribution géographique. - Sud de la Mer de Barents; très rare.

\section{Acineta inclusa sp. n.}

Planche XIII, figure 47 .

L'insuffisance des observations faites au sujet de l'organisme dont nous avons figuré l'unique spécimen rencontré nous défend de nous appuyer sur ses caractères, peut-être trop individuels, pour rechercher ses affinités avec ses congénères.

Il vit dans une capsule chitineuse assez haute, dilatée vers le bas, rétrécie en tube cylindrique vers le haut où elle s'ouvre circulairement. D'étroits replis transversaux en garnissent toute la surface. Elle se rattache à un pédicelle étroit par l'intermédiaire d'un renflement hyalin très finement strié dans les deux directions longitudinale et transversale. 
L'animal, déprimé et contracté au fond de la loge, laisse voir, sur une sorte de bouton apical, quelques pseudopodes capités, $p s$, qui nous le font rapporter aux Infusoires suceurs.

Distribution géographique. - Sud de la Mer de Barents.

\section{Groupe VIII. - RHIZOPODES}

\section{Section I. - Radiolaires}

Nos matériaux ne sont pas riches en Radiolaires. Seuls les échantillons 3o à II5 de la Mer de Kara, 2r6 à 22I (de la station 22) et 226 de l'Océan Glacial, en présentent des spécimens rares.

Ceux-ci sont peu variés de genres et d'espèces : une dizaine de formes appartenant aux Acanthaires et aux Monopylaires.

La plupart sont assez petits, ce qui n'est pas regrettable pour le micrographe; mais leur dissémination au milieu d'un fouillis d'autres espèces planktoniques, enchevètrement de Chétocérées particulièrement, en rend l'observation difficile.

Leur rôle dans l'objet qui nous occupe est assez effacé. Ce n'est vraiment pas le lieu de prendre contact avec les formes somptueuses que E. HæCKEL a magnifiquement reproduites dans les admirables planches qui illustrent la publication des résultats scientifiques de l'Expédition du Challenger. Ces merveilles microbiologiques sont particulièrement des productions des mers plus chaudes; mais d'autres produits de pêche des mers boréales nous ont néanmoins mis en présence de formes qui ne manquent pas d'intérêt morphologique.

Ce n'est pas le cas ici et il n'y aura pas lieu de nous arrêter longtemps à décrire les spécimens figurés dans les planches XXII et XXIII. Nous disons décrire seulement, car, dans ce monde d'organismes d'une structure plus complexe et partant plus variable, il serait peu scientifique d'accorder plus d'importance qu'il ne convient à des modalités de forme qu'un mème type spécifique peut présenter dans ses représentants très dispersés, différents par l'àge, par l'état de fixation et par l'intégrité relative de leurs parties constitutives. D'autre part, il serait téméraire de tenter la reconstitution du type spécifique d'après des documents fragmentaires dont rien ne garantit, parfois, ni la sincérité, ni la pertinence, ni la suffisance à constituer un dossier complet de l'espèce.

Il ne faut pas chercher ailleurs la cause des discordances synonymiques dont les listes planktoniques fournissent trop d'exemples, quand les auteurs n'évitent pas cet écueil en laissant ces organismes dans l'ombre du silence, ce qui est une façon de tourner la difficulté mais non de la résoudre. 
De fait, beaucoup de Radiolaires donnent peu de prise à l'observation cursive des matériaux planktoniques. La fragilité des pièces délicates clu squelette siliceux ou acanthineux, suivant les cas, la mauvaise tenue du sarcoplasme que la plupart des milieux conservateurs contractent et rendent opaque, l'indiscernement qui en résulte pour les parties profondes, - la capsule centrale, par exemple, quand elle existe l'agglutination facile de ces organismes mous, gélatineux ou hérissés de spicules et de pseudopodes avec d'autres organismes ou aussi avec des corps détritiques de toute nature réunis dans un même coup de filet, sont autant de circonstances qui expliquent les légitimes hésitations des auteurs vis-à-vis de certaines formes aux caractères peu tranchés.

Dans ce domaine surtout, les observations sur le vif sont d'autant plus désirables qu'elles sont plus rarement réalisables.

\section{A. Ordre des Monopylaires (I)}

C'est aux Monopylaires évidemment, aux Cyrtö̈des en particulier, que se rattachent les formes qui suivent.

\section{Botryopyle setosa Cleve?}

Planche XXII, figures + et 6 .

Cette forme de Dicyrtoïde, la moins rare dans nos matériaux, n'est pas sans analogie, pour le corps du squelette du moins, avec l'objet figuré par CLEve (2) sous le nom de Botryopyle setosa (pl. I, fig. Io) et aussi, mais avec une approximation moindre, avec celui de la figure 83, planche XVI, de Jörgensen (3), sous le nom de Lithomelissa setosa, ou encore avec Acanthocory's umbellifera Hreckel? figuré par le même auteur (pl. XVIII, fig. I07).

Dans ces deux derniers, la tête de l'organisme est simple et non lobée comme elle l'est dans celui de Cleve.

L'objet qui nous occupe présente toujours ce caractère d'une façon bien accusée.

La tête irrégulièrement lobée a sensiblement la même largeur que le thorax, dont elle est séparée par un étranglement cervical peu prononcé. Au niveau de celui-ci, il existe, à l'intérieur, un septum à claire-voie formé de gros trabécules contournés,

(I) Voir Delage et Hérouard : Traité de Zoologie concrète, t. Ier, Paris, isg6.

(2) Cleve : Plankton collected by the Swedish Expedition to Spitzbergen in I8gs, Kong. Sv. Vet. Akad. Handlingar, Bd 32. Stockholm, 1899 .

(3) Jörgensen : The Protist Plankton and the Diatoms in Bottom Samples, in Nordgand : Biolog. and Hydrogr. Investigations in Norwegian Fjords. Bergens Muscum, I905. 
ramifiés et anastomosés, dont notre figure 6 montre la vue du côté antérieur, abstraction faite du thorax qui est supposé amputé. Ces trabécules sont en relation avec les lobules de la tète et en consolident les attaches.

Le thorax a un développement varié en hauteur depuis moins d'une fois jusqu'à deux fois la longueur de la tête. Légèrement évasé à la bouche, il porte sur le bord libre de celle-ci des soies rigides très longues, qui s'épanouissent à leur base sous forme de côtes peu saillantes sur le thorax, entre les mailles irrégulières du squelette grillagé.

De nombreuses soies, plus ou moins anastomosées et de valeur inégale, hérissent la tête dans toutes les directions; les flancs du thorax en présentent aussi quelquesunes dirigées obliquement vers l'avant, c'est-à-dire du côté opposé à la tête (r).

\section{Botryopyle stenostoma sp. n.}

Planche XXII, figure 5.

Forme étroitement apparentée à la précédente, avec laquelle on la confondrait aisément à première vue, mais qui s'en distingue par le rétrécissement de la bouche. Celle-ci ne porte sur son pourtour que des soies spiculaires moins fortes et sans prolongement basilaire sous forme de côtes saillantes entre les mailles inégales de la capsule grillagée du thorax. Elle est tantòt libre et ouverte, tantòt diaphragmée d'un léger septum réticulé, plan ou concave, comme le spécimen figuré.

Il faudrait être mieux fixé sur l'étendue des variations possibles d'un même type, suivant ses étapes ontogéniques et ses états physiologiques, pour être à même de juger si cette forme n'est pas spécifiquement référable à l'autre. Le lecteur mieux documenté appréciera.

Distribution géographique. - Ces deux formes se rencontrent dans la Mer de Kara et dans la Mer de Barents, partie nord; la première moins rare que la seconde, l'une et l'autre en spécimens disséminés dans un enchevêtrement de grandes formes de Chétocérées.

\section{Peridium sp.?}

Planche XXII, figure 8.

Petite forme de Monocyrtoïde, dont la partie la plus dense du squelette réticulé est réduite à la tète globuleuse simple ou bilobée, comme dans le spécimen reproduit.

(I) Nous employons ici, sans l'approuver, cette terminologie bizarre empruntée au vocabulaire des entomologistes. Appliquée aux Monopylaires, elle manque de justesse, par dẻfaut d'analogie mème éloignée entre les parties du corps des Arthropodes et des Rhizopodes et elle conduit, chez les formes compliquẻes pour lesquelles on en use le plus, à cette conséquence paradoxale de rejeter la bouche aux antipodes de la tête, au delà du thorax et de l'abdomen, là où l'usage a préralu d'appeler d'un tout autre nom l'exutoire qui s'y trouve. 
Le thorax n'est représenté que par des trabécules arqués qui prennent attache sur la tète, comme. des contreforts, pour soutenir les trois prolongements spiculaires principaux qui ornent l'orifice buccal et qui représentent le pied.

Il n'est guère possible de rencontrer deux spécimens identiques pour l'aspect et l'ordonnance des détails. Nous avons clù nous borner à faire choix d'un exemplaire d'une structure moins indécise et d'une conservation plus intégrale.

Distribution Géographique. - Mer de Kara principalement. Rencontré aussi accidentellement dans des échantillons d'autre provenance, mais toujours rare.

\section{Peridium sp.? Pharmacantha sp.?}

Planche XXII, figure 7 .

S'agit-il d'une forme représentative de Peridium hystrix ou Pharmacantha hystrix Jörgensen? Nous posons la question, ne pouvant la résoudre, tant sont variables d'aspect les organismes rencontrés ci et là, à l'état de squelette d'une structure indécise, donnant peu de prise à la constatation d'un caractère morphologique commun qui puisse servir de base à la référence.

La forme tétraédrique du squelette est marquée par des trabécules plus accentués qui soulignent confusément les arêtes du polyèdre. Ces trabécules sont reliés entre eux par d'autres moins forts qui dessinent un réseau à mailles irrégulières d'où émergent des soies spiculaires très ténues qui irradient dans toutes les directions. Mais ces notes un peu caractéristiques ne s'observent, même obscurément, que dans des spécimens moins frustes et bien orientés sur le slide.

Il est toujours désagréable d'avoir affaire à des formes d'organismes qui, bien qu'ayant une réalité spécifique objective, manquent, dans l'état où on les rencontre, d'attributs nettement définissables dont on puisse faire état pour leur dresser un signalement propre.

C'est le cas pour l'objet qui nous occupe ici. Les spécialistes en la matière diront si la forme que nous figurons est susceptible du rapprochement que nous en faisons plus haut.

Distribution géographioue. - Très rare dans les matériaux de la Mer de Kara.

Setopilium Hæckel, sp.

Planche XXIII, figure 4 .

Forme de Dicyrtoïde assez simple, analogue à Dictyophymus Ehrenberg, mais dépourvue de corne sur la tête.

Le squelette vigoureusement structuré est grillagé et percé de mailles inégales. 
La tête est arrondie et dépourvue de corne apicale et de toute autre expansion spiculaire.

Le thorax est évasé et triangulaire. Les angles en sont rehaussés de côtes saillantes qui se prolongent, au delà du squelette thoracique, en trois expansions libres qui forment le pied. Le manque de place nous a fait tronquer ces prolongements pédiaux, dans la figure susdite, mais cette mutilation n'en modifie guère le caractère.

Distribution géographique. - Nous tenons cette forme, rarissime dans nos matériaux, de la Mer de Kara. L'examen minutieux de centaines de préparations de produits planktoniques de cette provenance ne nous a permis d'en observer que deux exemplaires vides.

\section{B. Ordre des Acanthaires}

\section{Asirolophus sp.?}

Planche XXII, figure 13.

Forme d'Actinélide, sans doute, caractérisée par un nombre indéterminé de spicules qui divergent radialement du centre du sarcoplasme, où ils sont étroitement unis par une sorte d'épaississement en forme de fer de lance losangique.

Ces spicules très longs, écourtés à dessein dans la figure I3, par défaut de place, sont d'importance inégale et formés, les plus forts, de deux fines lamelles d'acanthine croisées à angle droit, suivant leur axe longitudinal.

Sans être distribués rigoureusement dans le mème plan, les spicules ne s'écartent guère de celui de la figure. La forme du corps est, en effet, lenticulaire aplatie.

Le sarcoplasme, assez homogène du centre à la périphérie, présente, dans toute son étendue, une structure vacuolaire dans laquelle chaque vacuole semble avoir la signification d'une cellule, avec noyau et cytoplasme distincts.

Nous l'avons toujours vue dépourvue de myophriscas.

Distribution géographique. - Mer de Barents; rare.

Aslrolophus sp.?

Planche XXII, figure I2.

Serait-ce une forme très jeune de la précédente ou une forme autonome de dimensions très réduites? Nous ne savons, n’ayant pas pu, vu la rareté des spécimens observés, nous faire une opinion à ce sujet. 
L'organisme est astroïde, très petit, soutenu par huit spicules qui rayonnent du centre. Ces spicules délicats, très minces, sont également distancés au sein d'un sarcoplasme vacuolaire qui rappelle l'aspect du précédent. Ils ont été écourtés à dessein dans la figure.

Nous ne trouvons rien dans la littérature qui nous permette d'établir un rapprochement entre cette forme minuscule et celles décrites jusqu'ici par nos devanciers.

Distribution Géographique. - Hautes latitudes de la Mer de Barents et Océan Glacial. Très rare.

\section{Litholophus sp.?}

Planche XXII, figure II.

Autre Actinélide coniforme, figurant une ombrelle à moitié fermée et dépourvue de manche. Les spicules nombreux sont très longs et formés de deux minces lamelles d'acanthine croisées suivant leur axe longitudinal. Ils vont s'élargissant à partir d'un centre commun où ils viennent en contact, grâce à une légère dilatation terminale, en forme de losange allongé.

Nous n'avons figuré que ceux qui se trouvent sur le còté visible de l'organisme supposé couché sous le couvre-objet.

Le sarcoplasme, rétracté par la fixation, garde des adhérences avec les spicules, mais s'isole plus ou moins des myophriscas, $x$ (I), qui y restent attachés un peu plus haut. Il accuse une structure vacuolaire plus claire vers la base du cône, plus dense vers le sommet où s'observent des corps ayant l'apparence de noyaux.

Distribution géographigue. - Partie nord de la Mer de Barents; rare.

\section{Litholophus sp.?}

Planche XXIII, figure $\mathrm{r}$.

Encore un Actinélide dont le corps affecte aussi la forme d'un còne, que notre figure reproduit vu un peu obliquement de la base. Les caractères du sarcoplasme et des spicules sont les mèmes que dans la forme décrite ci-devant. La seule différence sensible consiste ici dans l'absence des myophriscas; mais il ne nous parait pas que cette circonstance suffise à distinguer spécifiquement les deux formes qui ont du reste la même distribution géographique.

(I) D'après DELAGE et HEROUARD, loc. cit., ces myophriscas n'existeraient que chez les Acanthonides. Nous ne voyons cependant pas le moyen de verser cette forme dans ce groupe. 
Rentarque. - Les deux formes que nous allons présenter ci-après n'ont pas de parties squelettiques rigides. Sont-ce des Acanthonides? Cela nous parait; mais, dans l'état de nos connaissances, nous ne saurions les rattacher avec quelque sécurité à aucun des genres décrits.

Il faut reconnaître que, à part quelques espèces classiques, les formes à corps entièrement mou ont été plus négligées que celles dont le squelette, plus ou moins résistant, fournit des éléments de diagnose non seulement plus faciles à saisir et à consigner, mais encore plus réfractaires à toutes les causes d'altération, de déformation ou même de destruction complète dans les milieux conservateurs.

La charpente rigide plus ou moins symétrique et plus ou moins ouvragée de ces organismes a plus souvent fixé l'attention des planktonistes que lés organismes euxmêmes. Ceux qui en sont naturellement dépourvus ont eu plus rarement la faveur d'une description; souvent même ils ont dì être sacrifiés complètement clans les manipulations préliminaires à l'étude des formes mieux loties sous ce rapport.

Planche XXIII, figure 2. Le corps est lenticulaire, à contours polygonaux, dans l'état où l'ont mis les liquides conservateurs. Le protoplasme sarcodique, plus dense vers le centre, où se montrent des globules réfringents et d'autres granulations elliptiques plus petites, se montre plus clair vers la périphérie, où la structure devient plus vacuolaire, pour devenir enfin réticulée dans une sorte de couche tégumentaire.

Des rayons nombreux, flexibles, protoplasmatiques en grande partie, irradient dans des plans peu différents de celui de la figure et ont des attaches fibrillaires dans la zone vacuolaire. Des corps, qui ne sont sans doute que des Zooxantelles, s'observent surtout dans la couche tégumentaire, où ils paraissent en rapport assez constant avec la base libre des prolongements radiaires.

Distribution Géographique. - Nord de la Mer de Barents et Océan Glacial.

Planche XXIII, figure 3. Le corps est lenticulaire aussi et de dimensions généralement plus grandes que le spécimen figuré qui a été choisi parmi les plus petits observés.

Nous n'en n'avons reproduit qu'une moitié qui suffit à donner une idée de l'ensemble.

Le sarcoplasme s'est évidemment contracté pendant la fixation, particulièrement dans les intervalles entre les expansions radiaires que des fibrilles, acanthineuses sans doute, maintiennent mieux en place.

Le centre est occupé par de grosses sphérules amoncelées. Le protoplasme est vacuolaire et rétracté de la fine membrane gélatineuse, $g$, à laquelle il devait adhérer pendant la vie.

Les expansions radiaires les plus fortes portent des myophriscas, my; les plus faibles en sont dépourvues.

Distribution Géographigue. - Assez rare; dans la Mer de Kara seulement. 


\section{Formes incerte sedis}

Planche XXII, figures 9 et Io. C'est encore aux Radiolaires que nous semble devoir se rapporter l'organisme représenté sous deux orientations différentes dans les figures 9 et io de la planche XXII.

Le corps est sensiblement isodiamétral et sa forme polyédrique résulte de la structure particulière de la faible charpente extérieure qui soutient le sarcoplasme. Cette charpente est formée de fins trabécules spiculaires, tangents au corps et se soudant vers leurs extrémités par trois ou quatre, pour former de grandes mailles polygonales peu nombreuses, dont la distribution symétrique, par rapport à l'axe principal de l'organisme, n'apparait que lorsque l'objet est vu dans le plan de cet axe, position dans laquelle nous avons oublié de le présenter.

Le sarcoplasme présente un centre hyalin, entouré d'une zone de corpuscules sombres recouverte elle-mème d'une couche périphérique plus claire mais de texture grossière (fig. 9).

A l'un des pòles, que l'on peut observer en faisant rouler l'objet sur le slide pour l'orienter convenablement, il existe une légère protubérance conique arrondie que la figure io montre de face. La même figure montre en outre la structure, finement réticulo-ponctuée, de la couche superficielle qui est sous-tendue par les trabécules aciculaires du squelette.

L'extrême rareté des spécimens observés - deux seulement, ne nous a pas permis d'observer cet organisme aussi complètement que nous l'aurions désiré pour nous fixer sur sa nature réelle et ses affinités probables.

Nous n'avons non plus rien trouvé dans la littérature scientifique qui nous permît de nous documenter autrement à son sujet, en bénéficiant d'observations de nos devanciers.

Distribution Géographique. - Observé seulement dans la Mer de Kara.

Planche XXIII, figure I9. Petite forme globuleuse, avec une sphérule centrale nucléiforme entourée d'une zone hyaline limitée elle-mème par des sortes de spicules nombreux, très petits, irrégulièrement incurvés et tangents à la surface.

Serait-ce quelque chose d'analogue à Raphidiophry's marina Ostenfeld (I), que cet auteur considère comme un Héliozoaire marin?

Distribution géographique. - Très rare; cà et là clans les produits de la Mer de Kara.

(I) Ostesteld : On two new marine species of Heliozoa, etc., dans .11eddelelser fru Komissionen for Hąundersögelser. Série : Plankton, B. I. Copenhague, igo.t. 


\section{Section II. - Foraminifères}

La rareté des Foraminifères dans nos matériaux d'étude nous dispense d'en parler, si ce n'est pour faire à leur sujet cette observation négative.

En dehors des Rhizopodes thécamœbiens ou Monothalames dont nous avons dit un mot plus haut en les recensant à la suite des Protozoaires loriqués, à cause de la ressemblance de leur capsule avec celle de certains Tintinnides, nous n'avons trouvé dans les produits de pêche au filet fin qu'une seule forme de Polythalame, un Reophax apparemment, que nous avons reproduit planche XXII, figure 23.

Des échantillons de boues glaciaires nous l'ont aussi représenté, en même temps que des fragments peu reconnaissables d'autres espèces qu'il nous a paru inutile de figurer.

\section{Section III. - Taxopodes H. FOL?}

Le Sticholonche Zanclea R. Hertwig, pour lequel H. Fol a créé un nouvel ordre de Rhizopodes intermédiaires aux Héliozoaires et aux Radiolaires, est le seul organisme qui, à notre connaissance, ait des affinités morphologiques avec un microzoaire aussi singulier que lui, trouvé dans plusieurs produits de pêches planktoniques provenant de la Mer de Kara.

Il nous paraît rationnel d'en faire provisoirement un congénère de cette espèce bien que nous ne puissions le décrire que d'après ses seuls caractères morphologiques tels que le formol les a fixés.

\section{Genre Sticholonche Hertwig}

Sticholonche ventricosa sp. n.

Planche XXII, figures $I, 2,3$.

La figure I en donne une vue stéréoscopique de profil longitudinal ou suivant l'axe de plus grande symétrie.

Le corps est piriforme, ventru en bas, un peu rétréci vers le haut où existe une sorte de tète séparée du ventre par un léger étranglement, au niveau duquel sont insérés des bouquets de spicules divergents. 
Ces bouquets nous ont paru au nombre de six, bien qu'il soit difficile de s'en assurer à cause des mucosités étrangères dans lesquelles ils sont communément engagés, à cause aussi de la difficulté de faire rouler l'organisme sous le couvre-objet dans le but de l'examiner successivement sous toutes ses faces, ce qui tient à la présence des spicules.

Ceux-ci sont tous du mème type, en forme de sabre, droits ou un peu courbés, pointus au bout, plus larges vers le milieu, rétrécis vers la base où ils présentent une nouvelle dilatation qui semble les souder entre eux. Ils sont creux et quelque peu flexibles.

La partie ventrale du corps présente un aspect granuleux autour d'une grande cavité ventrale, $v$ (fig. 2, coupe longitudinale optique). Les granulations qui y sont disposées en plusieurs couches s'éclaircissent dans le chloral et se colorent en brun dans l'iode. Celles qui forment l'assise extérieure ne semblent pas de nature protoplasmatique; elles sont toutefois insolubles dans les acicles forts. La tête est creusée d'une cavité réniforme dans laquelle se trouve une masse brunâtre, dense, que l'on prendrait pour un noyau, $n$ (fig. 2), si la structure aberrante de l'objet ne rendait toute assimilation d'organe sujette à caution. Les parois de cette cavité, bien délimitée à l'intérieur, présentent une structure rayonnante et une épaisseur variable qui en rend la surface externe très irrégulière. Il semble qu'on peut voir dans cet organe, qui est ici externe et terminal, l'analogue de la capsule réniforme qui est interne dans Sticholonche Zanclea.

La figure 3 est une vue apicale de l'organisme, où la tète, avec sa capsule et son noyau?, $n$, inclus, vu par transparence, se profile sur la partie ventrale plus dilatée

- du corps. Cette figure permet d'observer que le corps est un peu écrasé latéralement, ce qui rendrait la coupe transversale elliptique, et non circulaire comme les deux autres figures le laisseraient supposer.

C'est aussi la seule vue qui permette de se renseigner sùrement sur le nombre et la disposition des bouquets de spicules.

Distribution GÉographique. - Mer de Kara exclusivement; rare. 
$\bullet$ 


\title{
SECONDE PARTIE
}

\author{
Groupe IX. - DIATOMACÉES
}

GÉNÉRALITÉS

Nous avons dit, dans l'introduction à ce mémoire, les raisons pour lesquelles nous avons relégué à la fin l'examen des Diatomacées.

A certains égards, c'est un désordre car, par leur caractère végétal éminemment plasmodome, par, leur extension à tous les milieux, par leur rôle alimentaire vis-à-vis des espèces plasmophages, c'est elles qui ont le plus de titres à se trouver à la base d'une étude microplanktonique.

Quelle que soit la provenance des pêches au filet fin, il n'en est guère, il n'en est pas, peut-on dire, dont les produits soient complètement dépourvus de représentants de ce groupe d'organismes aussi vaste que varié et adapté aux stations les plus diverses. Souvent ils en constituent l'élément dominant, parfois même l'élément exclusif.

Cela est vrai; mais ce qui ne l'est pas moins, c'est la difficulté de leur étude systématique, si l'on se préoccupe en mème temps quelque peu du caractère synthétique qu'il convient de donner à l'exposé des résultats d'une longue étude analytique portant sur des matériaux nombreux, variés, complexes.

C'est que les Diatomacées elles-mèmes, pour homogènes qu'elles soient comme groupe naturel de Phéophycées à membrane bivalve silicifiée, n'en sont pas moins extrêmement différentes sous le rapport des caractères distinctifs saisissables et partant des procédés d'étude dont elles sont susceptibles.

Les unes, comme beaucoup de Naviculées, par exemple, libres, fortement silicifiées et délicatement ciselées sur leur face valvaire, n'ont pas de meilleurs caractères que ces sculptures, mais les détails en sont souvent impossibles à résoudre en présence du contenu cellulaire qui en contrarie la perception nette. L'élimination du cytoplasme s'impose dans ce cas, non toutefois avant d'avoir épuisé tous les autres moyens de renseignement, et bien qu'elle ne puisse être obtenue que par des procédés violents 
- macération ou incinération - les formes bien silicifiées n'en pâtissent pas suffisamment pour discréditer complètement ces pratiques, un peu barbares, qui réduisent l'organisme à son squelette.

Beaucoup de diatomistes n'ont pas eu d'autre objectif et se sont cantonnés dans l'étude patiente et aride de ces résidus minéraux et fragmentaires où le biologiste ne trouve plus son compte.

Appliquées aux espèces fixées, stipitées, agrégées, sériées, groupées d'une façon quelconque en colonies : fascicules, rubans, chainettes, frondes, etc., de pareilles manœuvres sont destructives des excellents caractères spécifiques que fournissent leur mode de fixation et d'agrégation, les particularités de leur coléoderme, leurs rapports symbiotiques et parasitaires, leurs groupements naturels, etc., etc.

Quant aux espèces plus délicates, peu silicifiées, comme beaucoup de Chétocérées, par exemple, elle sont irrémédiablement perdues si on leur impose ces procédés.

C'est pour l'avoir fait constamment, par routine ou par principe, que des diatomistes éminents se sont privés de la connaissance des espèces les plus fragiles, les plus délicates en même temps que les plus planktoniques, tant marines que d'eau douce.

Le regretté docteur Henri Van Heurck, qui a si bien illustré les Diatomacées de la Belgique, n'a recensé que deux espèces reconnaissables de Chatoceros, alors que notre florule algologique en possède une cinquantaine dont un bon nombre, cependant, se sont trouvées dans les matériaux d'étude de cet excellent diatomiste. D'autres genres non moins intéressants ont été complètement omis, aussi bien dans son classique Traité des Diatomées que dans son précieux Synopsis des Diatomées de Belgique.

Il y a plus : ces pratiques appliquées a priori, par système, avant tout autre examen, sont exclusives de toute recherche des phénomènes physiologiques, sans lesquels les organismes perdent le còté le plus intéressant de leur histoire naturelle.

Les phénomènes de multiplication, de sporulation et d'enkystement, les mouvements du cytoplasme et de l'endochrome, la production et l'utilisation des enclaves, la cytodiérèse, les accidents traumatiques, l'influence des causes physico-chimiques du milieu naturel ou artificiel, les productions tératologiques, les cas de parasitisme, de symbiose, de commensalisme, les associations des espèces, le caractère des florules locales ou saisonnières, etc., etc., tous ces sujets d'intérêt sont perdus, tous les horizons sont fermés. C'est comme si l'observateur se privait délibérément du spectacle animé et fécond en surprises de la nature vivante pour ne s'attacher qu'à des collections de momies ou de fossiles rangées derrière les vitrines d'un musée.

Certes, les matériaux fixés et conservés en milieu liquide ne valent pas les matériaux vivants; mais cependant, si la fixation en a été bien faite et si le milieu conservateur a été bien choisi, exempt d'acides surtout, on peut en tirer grand parti, si l'on a soin de ne procéder à leur étude qu'en leur appliquant une série graduelle de procédés de recherche dont la succession méthodique permet la mise en lumière de caractères isolés dont l'acquisition n'est pas préjudiciable à d'autres complémentaires qui 
seront ultérieurement relevés par d'autres voies et dont l'ensemble, mis en ordre logique, constituera l'histoire naturelle plus ou moins complète de chaque espèce.

Les résultats définitifs d'une étude ainsi conduite d'une façon fragmentaire, si l'on peut dire, se font attendre car ils comportent l'obligation de passer plusieurs fois en revue tous les matériaux, en les soumettant à une technique toujours renouvelée; mais ils procurent la satisfaction de prendre contact intime avec ces organisines non moins curieux par leurs manifestations biologiques que par leurs formes gracieuses et décoratives.

Le charme de ces formes d'un modelé toujours impeccable en a, de tout temps, constitué le principal attrait pour les micrographes sensibles au caractère esthétique des productions de la nature.

Mais quelle distance entre ces objets à l'état naturel et les reproductions qu'on tente d'en faire par des procédés graphiques ou mème photographiques!

Comme ces images sont ternes, effacées, mortes, insignifiantes, quand elles manquent de l'éclat, du coloris que la lumière transmise communique aux originaux en subissant des phénomènes de réfraction dans les mille détails de structure microscopiques et ultra-microscopiques de certains tests merveilleux, certains Coscinodiscus par exemple, dont rien n'égale l'éclat lumineux et la splendeur des irisations spectrales!

L'observateur direct est seul à avoir la jouissance de ces joyaux et, à moins d'être par trop égoïste, il souffre de ne pouvoir la faire partager à d'autres, car s'il essaie de traduire par le burin ce qui a fait l'objet de son admiration, il a honte de son impuissance et se détourne de son œuvre avec dépit et dégoùt. C'est bien là notre sentiment personnel vis-à-vis des planches qui accompagnent ce mémoire et qui ne peuvent avoir d'autre prétention que de schématiser des détails d'observation susceptibles d'être notés graphiquement, mais rebelles à une reproduction adéquate.

Les figures en ont été gravées toutes à la mème échelle de 500 diamètres; la plupart d'après des matériaux à l'état naturel, simplement éclairés par des réactifs appropriés à la délicatesse relative des objets; quelques-unes après macération de ceux-ci, soit par ébullition dans un mélange de chlorate de potasse et d'acide nitrique, soit, d'une manière plus douce et plus facile à graduer, dans l'eau de javelle.

L'examen à sec des frustules macérés nous a paru le seul pratique, parce que très expéditif au regard de l'examen dans le baume - liquidambar ou styrax — qui suppose des manipulations préalables, longues, méticuleuses, peu compatibles arec des recherches devant porter rapidement sur un grand nombre d'espèces.

\section{Division}

Plusieurs auteurs, H. VAN HEURCK entre autres, subdivisent les Diatomacées en trois sous-familles:

I. Les Raphidées, qui ont un vrai raphé, au moins sur l'une des deux valves. 
II. Les Pseudo-Raphidées, qui n'ont qu'un faux raphé, c'est-à-dire un espace clair simulant un raphé.

III. Les Crypto-Raphidées, ou plutòt les Araphidées, faudrait-il dire, qui n'ont pas de raphé du tout, ni vrai, ni faux.

Pour nos besoins présents, cette classification nous parait aussi bonne que toute autre, bien que la base en soit purement morphologique et conventionnelle. Nous la suivrons, mais en en renversant les termes pour donner aux Araphidées la préséance que leur vaut la place prépondérante qu'elles occupent dans la florule pélagique, objet plus spécial de nos recherches actuelles.

C'est dans leurs rangs, en effet, que l'on trouve le plus grand nombre de formes vraiment planktoniques, les mieux adaptées à la vie flottante et par leurs groupements en colonies linéaires ou dendritiques et par les appendices souvent très longs qui les ornent et en agrandissent d'une façon aussi simple qu'efficace la surface de résistance au milieu liquide et à la pesanteur.

C'est un sujet bien digne de remarque d'ailleurs que les ressources merveilleuses mises en œuvre par la nature pour augmenter la flottabilité de ces organismes plasmodomes infimes et en assurer la dissémination abondante dans les couches océaniques peu profondes, là où les organismes plasmophages de petite taille trouvent réalisées, eux aussi, les conditions les plus favorables à l'exercice de leurs manifestations biologiques.

Dans ce milieu mobile, les Araphidées, qui correspondent assez bien aux Centrica de Schütt (I), sont mieux adaptées à la vie errante et libre que leur imposent les mouvements perpétuels des courants, des marées, des vagues.

Les Raphidées et les Pseudo-Raphidées, mieux faites généralement pour la vie sédentaire, se cantonnent davantage dans les eaux douces et saumàtres et n'ont guère pour représentants dans les eaux pélagiques que de rares espèces exceptionnellement douées, au point de vue de la flottaison, gràce à la solidarité des individus maintenus en colonie. Ce sont les Pcmate de Schütr. Nous aurons à en recenser un assez grand nombre, parce que nos matériaux d'étude ne sont pas exclusivement planktoniques, mais se rattachent en partie à la florule des glaces.

\section{DESCRIPTION DES ESPËCES}

Sous-famille I. - Araphidées (Centrice de certains auteurs)

Cette section a été subdivisée, comme on sait, en Discoïdes et Biddulphiö̈des.

Bien que cette subdivision soit fondée, nous n'en tiendrons pas compte dans notre exposé analytique.

(1) F. Schütt : Bacillariaceae, dans ENGLer ET Pravti : Die Natïrlichen Pflanzenfamilien, Isy6. 
Il nous a paru plus commode pour nous et plus utile pour le lecteur de présenter les genres rencontrés dans nos matériaux dans l'ordre d'importance relative de leur rôle planktonique, celui aussi suivant lequel nous avons dù orienter nos recherches et ordonnancer nos procédés techniques.

A cet égard, les Chétocérées, qui sont des Biddulphoïdes, doivent avoir le pas sur toutes les autres. Le genre Chatoceros, avec ses nombreuses espèces, y détient la place distinguée que l'on sait. Nous ne pouvons lui refuser la préséance qui lui revient du chef de son importance numérique et de ses attributs planktoniques.

\section{Genre Chætoceros Ehrenberg (I)}

Caractères du genre. - Consulter Nordisches Plankton. Dritte Lieferung, XIX, H. Gran : Diatomeen, page 58 .

Nous renverrons fréquemment à cet utile ouvrage synthétique dans lequel se trouvent condensées et méthodiquement classées les connaissances acquises sur les Diatomacées planktoniques signalées dans les mers du nord de l'Europe jusqu'en igo5, époque de sa publication.

Rien de plus essentiellement planktonique que les représentants variés de ce genre qui ne s'est révélé dans son ampleur qu'à mesure de l'avancement des recherches océanographiques entreprises avec méthode depuis quelques années seulement.

C'est à ce titre que nous le mettons en vedette, car, à lui seul, par le nombre de ses espèces et la profusion des individus, il tient en balance toutes les autres espèces de Diatomacées dans la plupart des produits de pêche au filet fin.

Rien de plus homogène non plus, à première vue, que ce vaste genre où il semblerait que la nature s'est ingéniée à moduler, sur le thème très simple d'un frustule porteur de quatre soies, toutes les variations imaginables.

Les variantes réalisées en si grand nombre dans des limites si étroites ne sont pas évidemment sans présenter plus d'analogie que de dissemblance. Il ne faut pas s'étonner conséquemment de la difficulté qu'ont éprouvée les premiers planktonistes à fixer les notes distinctives des espèces et les suivants à identifier les formes précédemment décrites.

Celles-ci n'ont malheureusement pas toujours été figurées avec toute la rigueur de précision que réclame ce difficile sujet d'étude, ni avec l'expression fidèle des variantes dont une même espèce est susceptible, suivant son milieu et ses phases évolutives. Il en est résulté bien des erreurs d'appréciation qui ont eu leur répercussion dans une fâcheuse et encombrante synonymie.

(1) EnRenberg écrit Chactoceros et non Chactoceras, comme le font plusieurs auteurs molernes, Gran entre autres. 
L'extraordinaire étendue du genre Chcetoceros y a nécessité des découpures dont il faut louer Ostenfeld (I) d'avoir pris l'initiative et qui introduisent un peu d'ordre dans ce qui n'était d'abord qu'un ramassis de formes indisciplinées dont plusieurs bénéficiaient d'un état civil usurpé.

\section{Sous-genre Phæoceras GRAN}

Chromatophores petits, très nombreux, répandus non seulement dans le corps du frustule, mais jusque dans les soies, dont le calibre est toujours assez fort.

Spores quiescentes inconnues.

\section{Section I. - Atlantica Ostenfeld}

Soies maintenues toutes dans le plan sagittal, les terminales un peu différentes des autres par leur courbure; une petite épine au milieu des valves; foramen (2) assez large.

\section{Chatoceros atlanticum Cleve.}

Planche XXIV, figures $1,2,3$.

Synonyme et littérature. - Cfr. Nordisches Plankton, XIX, page 64.

C'est, dans nos échantillons, le seul représentant de la section des Atlantica.

Colonies en chaînettes droites, peu longues généralement, différenciées aux deux extrémités, formées d'un petit nombre de frustules - 3, 4, 5, 6 souvent - dont la hauteur est inversement proportionnelle à la largeur.

Remarque. - Ce dernier caractère n'a rien de spécifique, car il est d'application générale non seulement dans les Chcetoceros mais encore dans toutes les Diatomacées. L'allongement du frustule va de pair avec son rétrécissement au fur et à mesure du développement de la série généalogique; ce qui tend à la conservation d'un volume constant. C'est assez dire que les distinctions d'espèces ou de variétés fondées sur le seul caractère de la longueur relative des axes des frustules manquent complètement de valeur taxinomique, parce qu'elles ne marquent que des étapes phylogéniques plus ou

(I) Ostenieli) : Phytoplankton from the sea around the Faröer. Copenhague, I9o3.

(2) Yous désignerons par ce terme lespace libre entre les faces valvaires de deux frustules voisins. 
moins éloignées du frustule initial. Celui-ci passe, comme on sait, par degrés, au cours des divisions répétées qu'il subit, de ses dimensions les plus grandes, suivant l'axe dit apical, à d'autres de plus en plus petites, jusqu'à ce qu'une restauration s'effectue par voie d'auxospore.

Celle-ci, quand elle est connue, ne cloit pas être distraite de sa descendance sous un nom spécifique différent, ni tenue pour une varieté ou une forme de l'espèce, mais seulement pour ce qu'elle est en réalité : la cellule régénératrice, souche d'une nouvelle lignée généalogique.

Pour les mêmes raisons, nous nous abstiendrons des données métriques qui sont sujettes à trop de variations pour qu'il soit possible d'en tirer un parti utile et qui d'ailleurs parlent peu à l'imagination. Nous préférerons généralement reproduire quelques spécimens choisis, susceptibles de donner une idée de l'amplitude des variations de l'espèce, tout en en complétant le signalement.

Si l'on considère la portion de chainette de la figure I, qui reproduit assez bien le type morphologique moyen de l'espèce, on la voit formée de frustules à section rectangulaire un peu plus longs que larges, dans le plan sagittal qui est celui de la figure.

Les soies, un peu épatées à leur base, s’insèrent légèrement en retrait sur le bord des frustules et se soudent, en se croisant, avec celles du frustule voisin exactement au niveau du bord latéral de la chainette. Immédiatement au-dessus du croisement, elles offrent, en $d$, un renflement caractéristique et divergent ensuite, mais sans s'écarter sensiblement du plan sagittal. Au delà du renflement, $d$, les soies sont finement barbelées et se terminent en pointe émoussée, après s'être quelque peu amincies progressivement.

Les soies terminales ne diffèrent guère des autres que par un peu plus de force dans leur calibre, dans l'épaisseur de leur membrane et dans les denticules qui les ornent. Elles se rapprochent plus que les autres de la direction de l'axe longitudinal de la colonie.

Les unes et les autres ont sensiblement la même longueur. Celle-ci n'est jamais bien considérable, comme on peut en juger par l'examen des figures I, 2, 3, où seules les soies terminales ont pu être figurées en entier, grâce à leur direction favorable, tandis que les autres ont dû être tronquées par économie de place.

Tous les frustules portent, au milieu de chacune de leurs deux faces valvaires, une petite épine, $p$, qui se profile dans le foramen, $f$, au sommet d'un très léger renflement de la valve, lequel s'observe toujours dans les spécimens un peu larges (fig. I et 2), mais fait défaut, par manque de place, entre la base des deux soies, dans les spécimens étroits (fig. 3).

Dans la figure 2, qui reproduit une chainette, à trois frustules, d'un type plutôt large, la cellule médiane, dessinée en coupe optique, montre un noyau central, n, 
entouré d'un cytoplasme assez clair, mais parsemé de nombreux chromatophores, ch, de petite taille, qui s'épanchent jusque dans les soies.

La figure 3 est d'un type plus étroit, plus avancé donc dans la série phylogénique. Nous n'avons pas rencontré de frustules isolés.

Distribution GÉographique. - Espèce constante, mais assez peu représentée dans nos échantillons de la Mer de Kara et du Détroit de Matotchkine; absente de la Mer de Barents, si ce n'est tout au nord, vers l'Océan Glacial où elle est représentée par des spécimens rares mais amples.

\section{Section II. - Boreales Ostenfeld}

Valves sans épines; soies divergentes en dehors du plan sagittal; les terminales peu différentes des autres; foramen assez étroit.

\section{Chatoceros boreale Bailey.}

Planche XXIV, figures $+, 5,6,7$.

Litrérature. - Cfr. Nordisches Plankton, page 73.

Il y a généralement accord entre les auteurs au sujet de cette espèce commune dans les mers du nord. Prenons comme type moyen la figure 5.

Frustules toujours de grandes dimensions, exempts de ceinture ou n'en présentant qu'une très étroite, peu distincte en l'absence de sillons limites. Faces valvaires relevées par la base élargie des soies et par une légère protubérance médiane.

Soies brusquement géniculées à angle droit, à très peu de distance dé leur base, et soudées latéralement à leurs voisines, sans les croiser, car, en divergeant les unes des autres au delà de leur point de soudure, elles se maintiennent toutes sensiblement dans des plans perpendiculaires à l'axe de la chaìne, les unes restant dans le plan sagittal, qui est celui de la figure, les autres prenant une direction presque perpendiculaire à ce plan. On se rendra compte de ce fait par l'examen de la figure 6, qui reproduit l'orientation de la base des soies sur la face valvaire présentée en coupe optique, bien que les chainettes de l'espèce, volumineuses et hérissées de soies fortes et très longues, dans deux plans croisés, se placent rarement sur le slide dans une position nettement farorable à cette observation.

Ces soies très longues, trop longues pour que nous ayons pu en figurer quel'qu'une dans tout son développement, sont d'abord légèrement rétrécies au delà de la partie géniculée; elles s'élargissent plus loin pour se rétrécir encore vers leur extré- 
mité où elles se terminent en pointe mousse. Sur toute leur longueur, elles sont ornées de denticules qui prennent, surtout vers l'extrémité libre, la valeur de véritables spinules de plus en plus dégagées et acérées.

Les soies terminales ne se distinguent guère des autres et gardent la même orientation.

On jugera de la forme du foramen, $f$, par l'examen des figures 5 et 7 , car il est à la fois plus aisé et plus utile de la montrer que de la décrire.

Dans la figure 7 , qui reproduit un fragment de chainette plus large, les frustules sont plus courts, suivant la loi connue. L'un des frustules, figuré en coupe sagittale optique, montre le contenu cellulaire éclairci par le chloral, avec le noyau, n, et les chromatophores, ch, particulièrement petits et nombreux dans le cas présent.

En fait, l'aspect en varie de colonie à colonie suivant les circonstances d'état physiologique et suivant l'action des réactifs, employés pour en déceler les détails ou pour les enrober simplement; il serait vain d'en vouloir noter toutes les particularités.

La figure 4 représente, selon nous, une cellule isolée de la même espèce, chose très commune dans nos échantillons, parmi les spécimens en chaînettes longues et courtes. A quoi bon lui donner un nom spécial, celui de f. solitaria Cleve, par exemple, s'il est avéré, comme nous le pensons, que, dans cette espèce, les frustules se libèrent avec une grande facilité à cause de la faible soudure et de l'absence de croisement des soies?

Dans les cellules isolées, le coude formé à la base des soies s'atténue parfois au point de s'effacer complètement. Les deux soies d'une même valve se mettent ainsi dans le prolongement l'une de l'autre, au niveau mème de la valve, et forment avec leurs symétriques de la valve opposée un couple parallèle.

Distribution GÉographique. - Il est curieux de constater que, dans nos matériaux, cette espèce ne s'observe en quantité que là où sa congénère, Ch. atlanticum, fait presque radicalement défaut, c'est-à-dire, dans la Mer de Barents, tout le long des côtes occidentales de la Nouvelle-Zemble.

Gran dit qu'elle est propre à toutes les mers. Nous n'y contredirons pas, mais il ne sera pas inutile peut-être de faire remarquer que, à notre connaissance, elle ne se rencontre dans la Mer Flamande qu'à titre absolument accidentel, en spécimens rarissimes et divagants.

\section{Chatoceros criophilum Castracane.}

Planche XXIV, figures 8 à i 4 .

Littérature. - Voir Nordisches Plankton, page 7I.

Cette forme, actuellement bien dégagée de sa similaire moins boréale, Ch. perntvianum, ne saurait plus, dorénavant, donner lieu à des discordances de vues entre les planktonistes. 
Rattachons-en les notes distinctives à la figure ıo, qui en traduit les caractères spécifiques moyens.

Colonies en chainettes droites; frustules à ceinture nulle ou rudimentaire et peu discernable; valves dissymétriques : l'une bombée, l'autre plate. De la valve bombée se dégagent les bases très rapprochées de deux soies qui s'incurvent dès leur origine en demi-cercle, pour se diriger ensuite obliquement vers l'extrémité opposée de l'axe de la chainette.

De la valve aplatie émergent également deux soies épatées à leur base qui prennent, presque sans courbure, la direction des premières et s'y soudent latéralement, sans les croiser, le plus souvent. Leur insertion sur la valve est fort en retrait sur les bords de celle-ci.

Les séries alternantes de soies des deux sortes se tiennent dans des plans obliques sur le plan sagittal, comme le montre la figure I2, qui traduit, en projection, l'orientation des quatre soies solidaires de deux frustules contigus, vus en coupe apicale optique.

Les soies, rétrécies vers leur point de soudure, dilatées plus loin et très longuement développées, sont armées de spinules longues, libres, acérées et se terminent en pointe émoussée, armée elle aussi de spinules qui y atteignent leur maximum de développement.

La figure II reproduit un fragment de colonie à frustules plus étroits et partant plus longs, avec les différences d'aspect du foramen, $f$, qui résultent de l'étroitesse des valves et du rapprochement des soies.

Dans les frustules isolés (fig.' 8) dont certains auteurs ont fait une espèce distincte, Ch. volans Schütt, Ch. currens Cleve, les soies de la valve plate, que nous dirons inférieure, tendent à se rapprocher dans la direction de l'axe longitudinal ou transvalvaire, tandis que les deux supérieures, celles de la valve bombée, se rapprochent davantage à leur base, s'y soudent même souvent et s'écartent ensuite de cet axe pour tendre à prendre une direction qui lui est perpendiculaire.

La figure 9 les montre en projection sur la vue de face de la valve supérieure, dirigées dans le prolongement l'une de l'autre.

Des déformations tératologiques de l'espèce s'observent très fréquemment dans nos matériaux.

Dans le spécimen de la figure I3, les trustules qui se sont divisés, en $x$, n'ont pas développé leurs soies et sont restés en contact immédiat, comme des cellules de Conferva.

Le spécimen de la figure I4 montre un frustule qui a développé ses soies dans le squelette de sa sœur jumelle, morte au moment de la cytodiérèse qui devait lui donner naissance.

Ces soies, emprisonnées dans le cytoderme maternel resté indivis, s'y sont contournées en pelote, visible en $x$. Nous aurions pu noter beaucoup d'autres anomalies mais il faut se borner. 
Distribution GÉographique. - Espèce constante, mais peu abondante, dans les produits de pêche provenant du Détroit de Matotchkine et de son voisinage, dans la Mer de Kara; partout ailleurs elle ne s'observe qu'accidentellement, en spécimens clairsemés.

\section{Chretoceros densum Cleve.}

Planche XXIV, figures 15 et 16.

Synonyme et littérature. - Voir Nordisches Plankton, page 67.

Chaines longues ou mème très longues, le plus souvent droites mais parfois légèrement tordues sur leur axe longitudinal. Frustules très rapprochés, ne laissant entre eux qu'un foramen, $f$, très étroit, en forme de fente étroitement lancéolée, difficile à percevoir pour peu que la vue ne soit pas normale au plan sagittal de la colonie. Ceinture toujours visible, d'un tiers environ de la hauteur du frustule. Valves semblables, peu bombées, d'où se détachent, assez loin des bords, des soies d'un calibre uniforme, longues, fortes, ornées de denticules peu saillants et terminées en pointe émoussée.

Ces soies se croisent dès leur naissance, à l'intérieur de la ligne latérale de la colonie.

Dans le milieu des chainettes, elles se dirigent plus ou moins perpendiculairement à l'axe longitudinal, tandis que vers les extrémités elles s'incurvent de plus en plus vers le bout le plus rapproché et que les terminales tendent à prendre une direction tout à fait parallèle entre elles et parallèle aussi à l'axe longitudinal de la colonie.

La figuration d'une portion de chainette suffisante pour traduire l'allure des soies, dans tout leur développement, à l'échelle adoptée, aurait pris trop de place.

Notre figure I5, empruntée au corps d'une chainette, ne montre aucun frustule terminal.

Le cytoplasme est toujours très dense, plus dense que ne le laisse supposer la cellule figurée en coupe optique, après éclaircissement par le chloral, pour en laisser voir plus distinctement le noyau, $n$, toujours central et les chromatophores, ch, nombreux. Cette densité remarquable du cytoplasme se maintient jusque dans les soies, sur toute leur longueur.

La figure i6 montre, en vue valvaire, les rapports et la direction des quatre soies solidaires de deux frustules contigus.

Distribution Géographique. - Rencontrée seulement tout au nord de la Mer de Barents; absente dans nos matériaux de la Mer de Kara et du Détroit de Matotchkine, si ce n'est à son entrée occidentale. Cette grande espèce est loin de prendre ici l'importance que nous lui connaissons dans le microplankton de la Mer Flamande. 


\section{Chatoceros convolutum Castracane.}

Planche XXIV, figures I7, I8, I9.

Synonyme et littérature. - Cfr. Nordisches Plankton, page 69.

Chaînes longues, rencontrées beaucoup plus souvent en fragments qu'à l'état complet, avec soies terminales distinctes des autres par leur aspect ou leur direction.

Frustules à ceinture visible, de la hauteur d'environ un tiers de celle de la cellule.

Valves semblables - caractère contrôlé expressément, parce que Castracane lúimême et Gran, à sa suite, disent le contraire - d'où émergent, en retrait sur les bords, deux soies, qui non seulement se soudent avec leurs voisines du frustule contigu mais se tordent entre elles, en s'accrochant : caractère qui a inspiré à Castracane (I), créateur de l'espèce, le nom de convolutum qu'il lui attribue, comme il le dit formellement, bien que la figure qui accompagne son texte ne soit pas dessinée avec un soin suffisant pour traduire cette particularité. On la verra plus fidèlement traduite dans nos figures i7 et i8.

Le rapprochement des soies, à leur naissance, ne laisse place qu'à un foramen, $f$, assez petit, de forme à peu près circulaire.

Grâce à la torsion que les soies subissent à leur base, elles reviennent en direction vers le frustule auquel elles appartiennent, en s'écartant plus ou moins du plan sagittal.

La figure I9, qui reproduit une vue valvaire, montre, sous un autre aspect, la torsion caractéristique que les soies subissent à leur base.

A cet égard, les figures de Gran, aussi bien dans Nordisches Plankton, loc. cit., que dans Famna Arctica - Die Diatomeen der arktischen Meere. Iena, I904, page 53I, figure I, - sont fautives, car elles revêtent un faux air de Ch. criophilım, qui ne répond pas à l'aspect vrai de Ch. convolutum. Elles en dénaturent le caractère principal, en ne tenant pas compte de la torsion réciproque des soies connexes.

La dissemblance des valves d'un même frustule, affirmée par Castracane et confirmée par GRAN, nous parait être une erreur d'observation résultant de l'obliquité de l'objet vis-à-vis de l'axe optique, erreur dont nous avons dù nous défendre nousmème, en nous astreignant à disposer les chaînettes observées dans une position rigoureusement perpendiculaire à cet axe.

Les colonies sont généralement plus grêles que celles de Ch. densım, avec lesquelles elles ont d'ailleurs beaucoup de traits communs; les soies longues, d'un calibre uniforme, comme celles de cette dernière, sont plus grêles mais ornées, comme elles, de denticules faiblement marqués et bien visibles seulement à sec.

(1) Castracane : Report on the Scientific results of the Voyage of H. M. S. Challenger. Botany, vol. II. London, I886. 
Les caractères du protoplasme et des chromatophores sont ceux du groupe des Boreales : $n$, noyau; ch, chromatophores, dans un frustule de la figure I 8 représenté en coupe longitudinale optique.

Distribution GÉographique. -- Trouvée seulement dans' les échantillons 200 à 206 provenant de la côte occidentale de l'île nord de la Nouvelle-Zemble et 225 et 226 de l’Océan Glacial.

\section{Sous-genre Hyalochæte Gran}

Soies plus minces, dépourvues de chromatophores; ceux-ci pariétaux, en nombre limité, souvent mème très réduit. Spores quiescentes généralement connues.

Ostenfeld, nous l'avons dit plus haut, a subdivisé ce sous-genre en un certain nombre de sections que Gran a reprises, en en modifiant quelque peu l'arrangement.

C'est une façon louable de chercher à mettre un peu d'ordre dans cet ensemble caléidoscopique, en rapprochant les espèces qui témoignent, à certains égards, de caractères communs.

Cet arrangement artificiel n'est toutefois que provisoire, car il manque de l'unité de principe qui devrait se trouver à sa base.

Pour nos besoins actuels, nous nous bornerons à pratiquer dans les Hyalochate une seule coupure, pour les partager en deux grands groupes, d'après le nombre et l'individualisation des chromatophores, c'est-à-dire des chromoleucites ou simplement des leucites.

Groupe A. Les Phanerolencita, qui ont un certain nombre - supérieur à deux - de leucites distincts et individualisés dans le cytoplasme.

Groupe B. Les Cryptolencita, qui n'ont qu'un ou deux leucites diffus, indistincts du cytoplasme dense et confondus arec lui.

\section{Groupe A. Phanerolencite}

Plusieurs chromatophores assez gros, individualisés et distincts du reste du cytoplasme.

\section{Chatoceros decipiens Cleve.}

Planche XXV, figures I2 à I7; planche XXVII, figure 45 ; planche XXVIII, tgure I

Synonyme et littérature. - Cfr. Nordisches Plankton, page 74.

Cette grande espèce, vraiment classique, est trop répandue et trop bien connue pour qu'il soit utile d'insister sur ses notes spécifiques. Il y a lieu toutefois d'en 
préciser les détails plus qu'on ne l'a fait jusqu'ici. Nous les rencontrerons, avec leurs variantes, dans quelques figures nécessaires pour les atteindre séparément.

Chaînes droites, courtes, longues ou même parfois très longues, douées de soies différenciées aux deux bouts. Leur caractère le plus saillant consiste dans les rapports des soies géminées qui se soudent longitudinalement dès leur origine, sur l'angle même des frustules, et ne se séparent qu'après un assez long parcours commun, pour diverger ensuite en se croisant sous un angle aigu, mais en restant sensiblement dans un même plan, le plan sagittal (fig. I4). On peut regretter que le nom donné à l'espèce ne soit pas évocatif de cette remarquable particularité.

La figure I5, qui est une vue transversale optique de la chaînette voisine (fig. I4), au niveau du plan de séparation de deux frustules contigus, montre l'allure des soies qui s'écartent peu du plan sagittal et traduit en même temps la forme particulièrement écrasée du frustule, suivant son axe transversal le plus court.

La solidité de soudure des frustules rend impossible leur dissociation accidentelle et l'émiettement des colonies, même par les pratiques de macération artificielle.

Les frustules ont toujours une ceinture bien visible, d'un tiers environ de leur hauteur.

Les valves ont une section plus ou moins concave et ménagent conséquemment des foramens, $f$, de forme oblongue lancéolée et plus ou moins ouverts.

Les soies tant terminales qu'intercalaires sont très finement structurées; mais ce caractère ne s'accuse qu'avec l'âge et n'y est souvent bien visible qu'à sec dans les faibles colonies et spécialement dans les soies latérales jeunes de celles-ci.

Il faut noter que les détails de cette structure sont internes et ne se traduisent pas à l'extérieur, comme chez beaucoup d'autres espèces, sous la forme de denticules saillants.

A l'échelle trop faible de nos figures, la reproduction en $s$, dans quelques soies seulement, en est très difficile et peu distincte.

Les chromatophores sont en nombre variable, mais jamais bien considérable : souvent quatre dans les cellules jeunes, huit dans les cellules adultes, avant la cytodiérèse. Ils ont la forme de plaquettes assez grosses, mal délimitées, plus denses sur leur pourtour, plus claires au centre.

L'examen de quelques figures empruntées à des spécimens variés fournira des documents complémentaires pour l'histoire de l'espèce.

Figure I2. Fragment de colonie à frustules larges mais courts, en voie de division active; $d$, division d'un frustule figuré avec son contenu cellulaire; $n d$, noyau divisé; ch, chromatophores au nombre de huit que les deux cellules-filles se partagent par moitié; $f$, foramen naissant, avant l'apparition des soies sur les valves de formation nouvelle.

Des soies plus faibles que d'autres traduisent ainsi leur caractère de jeunesse relative.

Toutes les soies ont été tronquées intentionnellement, pour ménager l'espace.

Figure I3. Fragment d'une colonie du type moyen, à foramens larges. 
Deux frustules, dont l'un est en voie de cytodiérèse, sont figurés avec l'aspect normal de leur contenu cellulaire. Le nombre des chromatophores, ch, est ici supérieur à huit. Même légende que plus haut.

En $m$, se prépare la division physiologique de la colonie. Les soies de deux frustules contigus ont brisé leur longue adhérence, ou bien n'ont pas contracté originairement cette adhérence, pour se croiser brièvement dès leur base et s'incurver de suite en s'orientant suivant l'axe longitudinal de la chainette et en prenant les caractères propres aux soies terminales, st.

C'est grâce à cette fragmentation naturelle, plus ou moins souvent répétée, que les chainettes ne prennent pas une longueur démesurée et laissent voir, à leurs deux extrémités, des soies qui sont différenciées dans leur direction plus droite, leur calibre plus fort, leur structure intime plus accusée.

Figure I4. Fragment d'une colonie à foramens étroits, ceux-ci ayant la forme d'une fente étroite, linéaire. Les spécimens de cette sorte, sans ètre aussi communs que ceux à foramens larges, ne sont cependant pas d'une rareté exceptionnelle dans nos matériaux d'étude et, comme ils sont en mélange avec d'autres présentant tous les degrés d'ouverture du foramen dans les produits d'un mème coup de filet, il serait difficile d'y voir, comme Gran le dit, un caractère saisonnier, hivernal.

On remarquera que dans le frustule médian de la figure, qui est en division, en $d$, la cellule-mère présente à ce moment, comme celle de la figure I2, qui est au même stade physiologique, huit chromatophores, ch, qui se répartissent par moitié entre les deux cellules-filles.

Figure i6. Fragment de colonie plus grêle, dans laquelle les frustules plus étroits se montrent aussi plus longs, suivant la loi de constance de volume. Les foramens $y$ ont une dilatation moyenne.

L'un des frustules y est figuré avec son noyau, $n$, son cytoplasme clair et ses chromatophores, ch, au nombre de huit encore.

Un autre est parasité. Il est complètement envahi par un Olpidium, ol, sans doute, dont le thalle, qui parait granuleux, témoigne d'un commencement de sporulation.

Remaroue. - Les exemples d'un parasitisme de ce genre sont très communs dans nos matériaux, non seulement dans Ch. decipiens et ses congénères, mais aussi dans les Diatomacées d'autres genres. C'est pourquoi nous ne nous attarderons pas à exposer ici les phases évolutives du parasite, ce qui nous entraînerait à un trop grand nombre de figures.

Mais nous ne négligerons pas de signaler à l'occasion certaines phases de son évolution à propos d'autres espèces parasitées et de l'ensemble des notes graphiques glanées çà et là à son sujet — car tout fait croire qu'il s'agit toujours du même parasite - résulteront les éléments d'une étude complète de cet organisme fàcheux. 
C'est lui, sans doute, que nous avons déjà signalé, après Gran, dans Lauderia borealis Cleve, sous le nom de Olpidium Lauderice et, en outre, dans Ditylum Brightwelli Grunow, sous le nom de Olpidium Dityli.

Si, comme nous le pensons, c'est toujours le même organisme parasitaire, peu difficile sur le choix de ses hòtes, il serait plus logique de le dénommer dans tous les cas de la même façon, Olpidium phycophagum, par exemple, plutôt que de lui attribuer une spécificité différente chaque fois, suivant la nature de son hôte.

C'est ce que nous ferons.

Les pêches pratiquées dans l'extrême nord de la Mer de Barents et dans l'Océan Glacial nous ont fourni des spécimens de cette belle espèce intéressants par leur rapprochement de l'auxospore, qui, croyons-nous, est encore inconnue.

Telle, la forme reproduite dans la figure I de la planche XXVIII : forme unicellulaire extrêmement large, mais très courte proportionnellement, pourvue de quatre soies très différenciées, assez courtes relativement, renflées de la base vers le sommet, arrondi et obtus, et ornées de vigoureux détails de structure qui, ici, font saillie à l'extérieur sous la forme de denticules courts mais bien prononcés.

Les valves présentent une faible convexité vers leur centre, comme on peut en juger sur leur profil, dans la figure en question. Elles sont réunies par une ceinture qui a environ la même hauteur qu'elles-mèmes.

On remarquera que les quatre soies, situées dans le plan sagittal, ont leur direction principale suivant l'axe latéral du frustule. Ceci empêche toute tentative de conciliation entre cette forme, que nous considérons comme tête de lignée, comme auxospore, en d'autres termes, et la forme singularis présentée par Gras, dans Fauna arctica (loc. cit., pl. XVII, fig. 7).

Cette figure est d'un dessin fruste, vaguement schématique, insuffisamment précis, peu détaillé : genre dans lequel l'auteur se complait. En y suppléant par l'imagination, ne fút-ce que ce qui lui manque pour y reconnaître sûrement un frustule de Ch. decipiens, il semble bien que la forme visée ne saurait être qu'une cellule détachée isolément d'une chainette, suivant le processus décrit plus haut, à propos de notre figure I3, planche XXV.

Si l'on jugeait qu'il fût utile d'affubler la nôtre d'un nom spécial, nous lui proposerions le nom de $f$. princeps, pour marquer son caractère de cellule initiale de toute une lignée phylogénique. A cela il n'y a pas de besoin certainement, mais de l'utilité peut-être, pour souligner son identification avec les formes ordinaires de l'espèce decipiens, malgré l'impression contraire qu'elle pourrait produire sur des esprits non prévenus.

Que cette forme soit bien ce que nous en pensons, malgré ce que ses caractères pourraient sembler avoir d'exagéré, nous en fournissons la preuve dans la figure 45 de la planche XXVII, qui reproduit un spécimen un peu plus petit mais du même 
type morphologique et phylogénique, chez lequel la première cytodiérèse vient de s'accomplir, avec production de soies latérales qui traduisent le caractère essentiel de l'espèce decipiens, aussi bien que les chromoleucites, ch, la ceinture et le foramen, $f$. Les soies terminales, fortement différenciées, sont ici disposées en croix de Saint-Georges et manifestent déjà, relativement à l'auxospore, ou $f$. princeps, une tendance à se rapprocher de l'axe longitudinal de la future colonie qui prendra peu à peu les caractères habituels décrits plus haut.

Distribution Géographioue. - Cette espèce très répandue ne manque, peut-on dire, à aucun de nos échantillons vraiment planktoniques. Elle est toutefois plus abondante dans ceux qui proviennent de la Mer de Barents, le long des còtes de la Nouvelle-Zemble.

\section{Chatoceros mitra Bailey.}

Planche XXV, figures I à 7 .

Synonymie et littérature. - Cfr. Nordisches Plankton, page 75.

Malgré l'analogie de la forme des frustules, en vue sagittale, de leurs dimensions, de leur contenu cellulaire, y compris les chromatophores, des chainettes larges, longues et droites de cette belle et grande espèce avec la précédente, Ch. decipiens, elle s'en distingue à première vue par plusieurs caractères. Les soies contiguës ne font que se croiser à leur naissance, sur la ligne latérale de la colonie (fig. I); les soies divergent dans des plans très obliques sur le plan sagittal - plan de la figure - ce qui s'observe mieux sur une vue transversale optique (fig. 2); enfin la section transversale des frustules est oblongue lancéolée (mème figure).

La présence fréquente de spores quiescentes ou d'endocystes et la forme si caractéristique de celles-ci fournissent un caractère d'une non moindre valeur pour la distinguer de toute autre espèce (I).

Certaines notes biologiques plus spéciales ont été fixées dans les figures suivantes:

Figure I, planche XXV. Fragment de chainette large à frustules courts, à foramens, $f$, larges et lancéolés oblongs, présentant, à l'extrémité supérieure, des soies terminales, st, qui ont été tronquées, par défaut de place. Ces soies, arquées à leur base, prennent plus loin une direction droite, suivant le grand axe de la colonie. Elles sont d'ailleurs peu différentes des soies latérales, assez minces, longues, exemptes de structure reconnaissable, même à sec.

La ceinture des frustules est assez large et est limitée des deux côtés par un sillon.

(I) Ces spores se rapprochent cependant un peu, par leur valve primaire bicornée, le celles d'une espèce inédite que nous connaissons de la Mer Flanande et que nous comptons faire prochainement connaitre, en décrivant le facies propre du microplankton particulièrement riche de ce coin privilégié de la Mer du Ninrd. 
Les deux frustules figurés intégralement, en vue stéréoscopique, en montrent le noyau central, $n$, et les chromatophores, $c h$, en plaques volumineuses, ourlées sur les bords, plus claires au milieu. Leur nombre, facile à compter dans chaque cas particulier, varie naturellement avec l'âge du frustule, jusqu'au moment de la cytodiérèse qui en opère le partage numérique entre les cellules-filles.

Figure 3, planche XXV. Fragment de chainette plus étroite, à frustules plus longs, dont un figuré en vue stéréoscopique, avec les mêmes éléments indiqués par les mêmes lettres que ci-dessus.

Les chromatophores sont pariétaux et rattachés entre eux par des cordons du périplasme qui en rendent les contours irréguliers, indépendamment du polymorphisme qu'ils doivent à leurs phases de division par sténose.

Les foramens, $f$, sont largement ouverts, exempts d'étranglement médian.

Microspores. - Figure 4. Fragment de colonie en gestation de microspores ou de spores d'essaimage, spe, dont nous n'avons pu suivre l'évolution ultérieure, mais qu'il importe de signaler à l'attention des chercheurs.

Bon nombre de spécimens de nos matériaux d'étude traduisent cette phase physiologique remarquable, dont l'examen minutieux nous aurait détourné trop longtemps de ce travail analytique.

En quelques mots, voici le fait dans ses grands traits.

Le noyau subit des subdivisions répétées et les chromatophores aussi.

Les noyaux et les chromatophores fragmentaires, ainsi obtenus successivement, se groupent dans des compartiments de la cellule et ceux-ci s'isolent d'abord par la production de membranes intracellulaires, $m$; plus tard, par d'autres, $m$ ', incurvées autour de groupements secondaires et enfin, sans doute, car les étapes observées ne vont pas plus loin, par de vagues membranes qui ont été figurées, mais non désignées, lesquelles circonscrivent les produits de troisième subdivision. Ceux-ci semblent destinés à constituer des spores d'essaimage, spe.

Chacun d'eux comporte un petit noyau et un certain nombre de chromatophores fragmentaires, dont il est difficile d'apprécier le nombre exact à cause de leur rapprochement dans un espace restreint.

Sans vouloir outrepasser ces faits d'observation positive, nous croyons pouvoir affirmer qu'il y a là quelque chose d'analogue à ce que Gran a vu se produire dans Ch. decipiens et qu'il a décrit, avec les particularités que cette espèce comporte, dans les figures I à 6 de la planche XVII déjà citée de Famna Arctica.

Cette formation parallèle, mutatis mutandis, dans deux espèces analogues à d'autres égards encore, est assurément remarquable et propre à confirmer le rapprochement deviné des deux espèces et à légitimer leur réunion dans une section commune : les Dicladice. 
Enkrstement. - Figure 5. Fragment de colonie très ample, à frustules sensiblement aussi hauts que larges, dans lesquels on aperçoit des endocystes ou spores quiescentes, sp, en voie de formation.

La valve primaire, seule formée jusque-là, est rehaussée de deux fortes protubérances coniques qui lui donnent la forme d'une mitre et légitiment le nom judicieusement donné à l'espèce. Ces protubérances doivent être surmontées d'un appendice terminal, $x$, qui n'est encore ici qu'ébauché. Le cytoplasme n'a pas été figuré, pour ne pas surcharger le dessin et laisser voir l'anneau qui est à la base de la valve primaire de l'endocyste.

On remarquera, en outre, la grande extension de la ceinture des frustules sporifères.

La figure 6, empruntée à un spécimen de largeur plutôt faible, montre les spores quiescentes, sp, complètement formées. Celles-ci ont leur valve primaire en forme de mitre et leurs deux lobules sont surmontés d'un appendice, $x$, que couronne un bouquet de ramifications dichotomiques de plus en plus ténues aboutissant à la paroi de la matricule.

La valve secondaire est peu bombée, sertie dans l'anneau de la valve primaire et exempte de tout prolongement ou ornement quelconque.

On remarquera que les éléments cytologiques du frustule fertile, le noyau, $n$, le cytoplasme et les chromatophores, ch, se sont docilement laissé inclure dans l'endocyste. La formation de celui-ci est donc le résultat d'une rénovation cellulaire effectuée en deux temps, dont les moments correspondent à la production successive des deux valves. Elle a évidemment pour but la condensation des éléments actifs de la cellule végétative et leur adaptation à une phase de repos physiologique, sous une livrée nouvelle, mieux appropriée à l'état d'isolement qui attend la spore après sa libération de sa matricule.

Comment s'effectuera la déhiscence de celle-ci, dans quelles circonstances de milieu l'endocyste passera-t-il sa période de sommeil saisonnier, quelles conditions présideront à son réveil, par quelles métamorphoses reviendra-t-il à la forme végétative, etc.?

Ce sont là autant de questions auxquelles la science actuelle n'a encore rien à répondre, pas plus au sujet de Ch. mitra que des nombreuses autres espèces dont on s'estime déjà heureux de connaître les spores.

Il y a là, comme on voit, un vaste champ encore ouvert aux recherches de l'avenir. Il y a là aussi la constatation de l'état encore rudimentaire de l'histoire naturelle des Chcetoceros. L'étude de ces intéressants microphytes est à peine ébauchée; nous en fournirons plus loin d'autres preuves. Leur complexité, pressentie et entrevue par H. VAN HEURCK, lui faisait dire que les Chétocérées ne sont pas des Diatomées, suivant la conception qu'il se faisait de celles-ci, mais des microphytes d'un autre ordre, dont il fallait aborder l'étude par des procédés différents de ceux qui araient été suivis de son temps. 
Figure 7. Les spores constituent le meilleur caractère distinctif de l'espèce. Heureusement elles ne sont pas rares. Nous en présentons ici une autre vue, de profil celle-ci, pour en parfaire le signalement.

La figure 6 présente en outre, dans sa partie inférieure, deux frustules jeunes provenant d'une division récente. Les noyaux-filles, $n d$, sont encore très rapprochés des valves nouvelles qui ne laissent entre elles qu'un étroit foramen.

Cela nous donne l'occasion de faire remarquer que la production des spores n'est pas, comme on pourrait le croire, l'indice d'une fin de végétation, car on la voit se réaliser dans des colonies en plein exercice de multiplication cellulaire.

Distribution géographique. - Assez commune dans nos matériaux de surface provenant des côtes occidentales de la Nouvelle-Zemble, nos I66 à I99. Elle n'est guère en sporulation, toutefois, que dans les échantillons Ig3 et I94, recueillis au voisinage immédiat de la presqu'île de l'Amirauté, par $73^{\circ}$ de lat. N.

\section{Chatoceros teres Cleve.}

Planche XXV, figures $8,9,10,1 \mathrm{I}$.

Littérature. - Voir Nordisches Plankton, page 76.

C'est une grande et bonne espèce dont les figures 8 et 9 suffisent à préciser les caractères morphologiques.

La coupe valvaire optique de la figure 9 en montre, avec la grande divergence des soies dès leur naissance, la forme cylindrique, à peu près, des frustules; caractère qui a porté Ostenfeld à ranger cette espèce dans sa section des Cylindrica.

La figure 8 reproduit l'extrémité d'une chainette avec soies terminales peu ou pas différenciées, mais dirigées parallèlement au grand axe de la colonie. La ceinture des frustules est indistincte. Les foramens, $f$, sont en fente très étroite. Les soies latérales, longues, s'insèrent sur l'extrême bord des frustules et se soudent strictement sur la ligne latérale de la colonie.

Dans les cellules bien fixées, les chromatophores, ch, sont distribués dans la couche pariétale du cytoplasme. Ils sont en forme de plaquettes irrégulières, assez grandes. Leur nombre est variable.

Enkystement. - Les spores se produisent au milieu des frustules. La figure io fait assister à la production de la valve primaire, sp, sous laquelle le noyau, $n$, et les chromatophores, ch, se ramassent. Cette valve est simplement bombée et dépourvue d'appendices et d'ornements.

Dans la figure rr, on voit les spores mùres dans leur matricule, représentées l'une vide, l'autre à l'état naturel, avec ses chromatophores. La valve secondaire est 
moins bombée que la primaire, ce qui est d'ailleurs d'application générale dans les endocystes de tous les Chatoceros, comme on sait.

On n'ignore pas non plus, sans doute, que le processus d'enkystement intéresse généralement à la fois deux frustules frères issus d'une même cytodiérèse; que l'apparition des valves kystiques y est parallèle, mais que la position de celles-ci y est inverse et symétrịque, conséquemment, au plan transversal mené entre les frustules jumeaux.

Les deux sporocystes d'un même couple génétique sont en opposition par leur valve secondaire dont l'àge relatif est ainsi en concordance avec celui des valves matriculaires qui leur sont contiguës et qui sont aussi les plus jeunes.

Les deux spores de la figure 6 , qui a trait à l'espèce précédente, sont ainsi orientées et constituent un couple naturel.

On se rappellera que $C h$. teres Cleve ne se distingue guère avec sécurité de sa congénère Ch. Weissflogii Schütt que par ses spores. Nous croyons pouvoir affirmer que cette dernière n'est pas représentée dans nos matériaux.

Distribution Géographique. - Rencontrée seulement dans la Mer de Barents; assez peu abondante, si ce n'est au roisinage de Tromsö où elle se montre en sporulation.

\section{Chatoceros contortum Schütt.}

Planche XXV, figures I7 à 27 , et planche XXVI, figure I.

Sinoninie et littérature. - Cfr. Nordisches Plankton, page 78 .

C'est l'espèce type de la section des Compressa Ostenfeld, qu'elle est sans doute seule à représenter, s'il se confirme, comme cela paraìt établi déjà, que Ch. compressum Cleve, Ch. subcompressum Schröder, et Ch. medium Schütt n'en sont que des modalités.

Colonies longues, 'illimitées, droites, mais tordues sur leur axe longitudinal et présentant, par intervalle, des frustules géminés porteurs de soies contiguës très différenciées. Chromatophores nombreux, de dimensions moyennes.

La figure $\mathrm{I} 8$ en traduit les caractères morphologiques, sous les dimensions les plus habituelles.

Elle présente entre d'autres frustules ordinaires figurés les uns vides, les autres pleins, un couple de ces éléments qui ne diffèrent pas autrement des autres, mais dont les quatre soies contiguës, so, sont très différenciées vis-à-vis des autres, beaucoup plus fortes, généralement plus courtes, incurvées dans la même direction mais dans des plans différents, sinueuses sur tout leur parcours et atténuées en pointe à leur extrémité.

Elles ne sont pas destinées, comme on pourrait le supposer, à devenir terminales par leur décollement et la segmentation consécutive de la colonie. Leur soudure est stable et résiste même à la macération. 
Elles sont insérées en retrait sur les bords des valves, comme les soies ordinaires.

Celles-ci, beaucoup plus grêles, se soudent à une certaine distance de leur base, au niveau ou même un peu à l'intérieur des bords latéraux de la colonie, en ménageant des foramens, $f$, plus ou moins larges, et divergent ensuite sans s'écarter beaucoup d'un plan normal à l'axe de la colonie.

Les soies terminales, qui s'observent rarement et dont nous donnons une reproduction incomplète dans le haut de la figure 27, ont les caractères des soies ordinaires et n'en diffèrent que par leur direction droite, au delà d'une courbure de leur base.

La figure Ig - vue valvaire optique - montre la forme largement elliptique de la section des frustules et l'orientation des soies ordinaires.

Endocystes. - Les figures 20, $2 \mathrm{I}$ et 22 sont destinées à illustrer la production et les caractères des spores quiescentes.

Dans la première (fig. 20) la valve primaire est seule formée, vers le milieu des frustules, et les chromatophores, ch, se concentrent en dessous d'elle avec le cytoplasme et le noyau.

Dans la deuxième (fig. 2I) les spores se sont complétées par l'adjonction d'une valve secondaire, moins bombée encore que la primaire. Celle-ci est surmontée d'une sorte d'anneau appliqué contre la paroi interne de la matricule, où sa présence se signale par un épaississement de cet organe, mais reste néanmoins un sujet d'observation difficile.

Pour rester dans la note vraie, nous n'avons pas pu le dessiner avec plus d'évidence que nous ne l'avons fait dans les deux figures 20 et $2 \mathrm{I}$. C'est le seul ornement des spores.

La figure 22 montre que les sporocystes, sp, peuvent aussi bien se produire dans des colonies réduites à leurs plus faibles dimensions transversales. L'observation de l'anneau caractéristique qui surmonte ces productions alors fort petites devient pratiquement impossible.

Auxospores. - Les figures 23 à 26 nous mettent en présence d'une autre manifestation physiologique : la production d'auxospores. Celles-ci (fig. 24) apparaissent latéralement, sur des frustules arrivés au dernier degré de la réduction, sous la forme d'un épanchement du protoplasme, $a$, qui, perçant la membrane du frustule, $b$, fait hernie à l'extérieur. Il en résulte une protubérance dans laquelle passe tout le contenu de la cellule génératrice : noyau, cytoplasme et chromatophores. Cette protubérance, d'abord ovoïde, n'a qu'une très faible membrane extensible, ce qui lui permet de s'accroitre sous la poussée du protoplasme et de revêtir bientôt la forme d'un frustule initial agrandi, mais formé d'une seule pièce; c'est l'auxospore.

Celle-ci reste implantée perpendiculairement, par sa base rétrécie, sur sa génératrice, b, qui s'est complètement vidée. Nous la représentons en vue valvaire, $a$, dans la figure 25, dès que les premières soies terminales ont fait leur apparition. 
Dans la figure 26 elle a déjà subi sa première cytodiérèse. Les frustules ainsì obtenus sont, par le fait, pourvus de deux valves silicifiées.

Les divisions ultérieures donneront naissance à une colonie amplifiée (fig. 23) qui se tord sur son axe, conformément au caractère de l'espèce. Les frustules en sont larges, courts, séparés par des foramens étroits. Elle peut rester longtemps adhérente à la colonie souche, $b$, dont le calibre très étroit contraste avec celui de la formation nouvelle. Dans celle-ci, nous avons figuré, en $d$, un frustule en division, dans lequel le foramen naissant, $f$, précède l'apparition des soies sur les deux valves jeunes.

La figure I7 montre, à son tour, un fragment de colonie régénérée dont les frustules ont la taille maxima, ce qui permet de mieux apprécier leur caractère morphologique propre et spécialement la légère convexité des valves qui se traduit dans la forme rétrécie des foramens, $f$, vers leur milieu. On y reconnaîtra peut-être la modalité dont SchÜтT a fait son Ch. medium.

Microspores. - Outre les endocystes et les auxospores, nos matériaux nous offrent, dans la même espèce toujours, des exemples de production de spores d'essaimage ou de microspores, dont nous reproduisons une étape dans la figure I de la planche XXVI.

C'est bien, comme on voit, un fragment de colonie tordue de cette intéressante espèce, dont les frustules sont le siège d'un phénomène de division interne qui doit aboutir à la formation de spores endogènes. Les deux moitiés du protoplasme résultant d'une première division se sont subdivisées à leur tour, pour donner, dans chaque frustule, deux groupes de deux petites cellules géminées, spe, dépourvues de membrane visible.

D'autres subdivisions doivent-elles survenir avant la constitution définitive des microspores qui seraient alors plus nombreuses, 8 ou I6, par cellules? Nous ne savons, car nos observations ne vont pas plus loin.

Parasitisme. - Une dernière observation au sujet de Ch. contortum. C'est le parasitisme dont ses frustules sont souvent atteints de la part d'un Olpidimn, ol. On l'y rencontre sous les aspects variés qui correspondent à ses diverses phases évolutives.

Suivant le programme que nous nous sommes tracé plus haut (I) à son sujet, nous nous bornons à le représenter ici, dans la figure 27 , en état d'enkystement, à l'intérieur d'une membrane épaisse et réfringente. Nous considérons les granulations extérieures au kyste comme des produits résiduaires du protoplasme du frustule parasité. 
Distribution Géographique. - Très rare dans la Mer de Kara et dans le Détroit de Matotchkine; commune sur les còtes ouest de la Nouvelle-Zemble, dans la Mer de Barents, jusqu'au n 203 de la station I9; absente dans l'Océan Glacial.

\section{Groupe B. Cryptoleucita}

Espèces à chromatophores diffus, confondus avec la partie dense du cytoplasme.

\section{Chatoceros ingolfianum Ostenfeld.}

Planche XXVI, figures $2 \grave{a} 14$.

Littérature. - Cfr. Nordisches Plankton, page 9o, où Gran a versé cette espèce dans sa section des Brevicatenatce, dont la valeur taxinomique nous parait bien discutable.

C'est une espèce bien curieuse, qui ne s'éloigne pas seulement de ses congénères par la structure de ses spores, dans lesquelles on a cru reconnaitre un microorganisme connu d'ailleurs et décrit sous le nom de Xanthiopyxis, mais aussi par les particularités dont nous allons donner une idée d'après quelques figures empruntées à nos matériaux d'études.

Une des choses qui d'abord frappent le plus, c'est la gélification partielle de la membrane des frustules, vers leurs extrémités, en dehors de la partie occupée par l'endocyste, sp, comme en $g$, dans les figures I I et I2 de la planche XXVI.

Cette gélification, qui semble préparer l'isolement des spores, sp, est la cause évidente de l'émiettement des colonies, dont presque tous les frustules sont sporifères.

Cette circonstance fait qu'il est bien difficile de trouver des fragments quelque peu importants de colonies propres à en étudier les caractères végétatifs.

La figure 2 en reproduit un. On y voit, en $a$, un frustule stérile, dont le noyau, $n$, est entouré d'un protoplasme dense dans lequel se montrent plusieurs petits chromatophores peu distincts, il est vrai, mais assez cependant pour nous faire douter de la légitimité de la place que nous faisons à cette espèce dans notre groupe des Cryptoleucite.

Deux autres frustules, $b$, sont en voie de sporification et montrent l'ébauche des valves primaires. Celles-ci sont hémisphériques, brièvement spinuleuses encore, étroitement serties d'abord dans le frustule, vers son milieu, pendant que le cytoplasme s'y rassemble progressivement. Les chromatophores deviennent à ce moment plus distincts, ce qui nous parait ètre d’une application générale. Dans les espèces où les chromatophores sont comme diffus dans le cytoplasme des frustules stériles, ces éléments cytologiques s'individualisent, en effet, plus nettement dans les spores quiescentes. 
La cellule médiane de la même figure montre, en c, la valve primaire plus achevée, devenue spinuleuse sur toute sa surface et logée dans une partie renflée du frustule, qui semble s'ètre distendue localement sous la poussée interne des spinules.

On remarquera, dans cette figure comme dans les suivantes, que la ceinture est large mais peu distincte, que les foramens sont très étroits, que les soies latérales sont soudées dès leur origine, sur les bords mêmes de la colonie.

Dans la figure 3, qui est dans le plan sagittal, et dans la figure 4, qui est oblique sur ce plan, on voit les spores, sp, achevées, pourvues de leur deuxième valve, moins bombée mais spinuleuse comme la première, et logées dans un renflement caractéristique des frustules.

Dans la figure 5, les spores, sp, réduites encore à leur valve primaire, montrent leur partie arrondie en dôme, hérissée de longues spinules, tandis que leur anneau cylindrique n'en porte encore que de beaucoup plus courtes. Au sommet des longues spinules, on voit se dessiner une membranule, $x$, qui couronnera plus tard l'endocyste, de ce côté, quand il se libérera de son frustule matricule, à la suite d'une gélification plus ou moins complète de celui-ci.

La figure 6 reproduit deux frustules contigus, plus larges, vus un peu obliquement, dans lesquels les sporocystes ne possèdent encore non plus que leur valve primaire; la membranule, $x$, visible dans la figure 5, ne s'aperçoit pas ici.

Dans la figure 7 , cette membranule, $x$, est déjà marquée, à grande distance au-dessus des valves primaires des spores, bien que les spinules qui hérissent la partie hémisphérique de celles-ci soient encore très courtes. Leur partie cylindrique est exempte de ces appendices et les frustules ne semblent pas devoir jamais présenter le renflement local que l'on observe chez d'autres, à la même étape de développement.

La figure 8 reproduit deux frustules de taille maxima, dont les sommets ont déjà été éliminés par gélification, jusqu'au niveau de la membranule, $x$, bien que les kystes ne soient encore en possession que de leur valve primaire; celle-ci est chargée sur toute sa surface de spinules de longueur très inégale.

Figrule 9. La spore univalvaire, incomplète, s'est déjà dégagée, du côté supérieur, de la paroi du frustule. Celle-ci est remplacée de ce còté par la membranule, $x$, qui s'est raccordée à la portion primitivement renflée de la matricule.

Figure ro. Vue valvaire d'une spore isolée. On en déduira la forme de la section transversale des frustules, qui est largement elliptique.

La figure II montre la façon dont se traduit souvent la gélification dans les extrémités des frustules, aux deux côtés des kystes, sp, alors que ceux-ci ne sont encore en possession que de leur valve primaire. Les endroits gélifiés, comme en $g$, deviennent mous, flasques et se déforment complètement avant leur dissolution complète. Cette gélification n'atteint pas les soies.

Figure I2. Phénomène analogue dans une colonie à frustules de très petit calibre; mêmes indications. 
On remarquera que dans les objets des deux figures II et I2, aucun renflement ne s'est produit dans les matricules, au niveau des spores inachevées.

Figure I3. Autre aspect d'une spore réduite encore à sa valve primaire. Celle-ci a sa partie arrondie relevée en dòme beaucoup plus haut. Elle est logée dans un renflement de la matricule qui en épouse la forme. Elle est brièvement spinuleuse sur tout son pourtour.

La figure I4, enfin, montre, à còté d'un frustule sporifère non gélifié, avec sa spore, sp, et sa membranule, $x$, un second frustule envahi par un Olpidium, ol, dont le thalle ramassé s'est partagé en un grand nombre de sporules qui n'attendent plus que leur mise en liberté et leur essaimage, dès qu'une porte de sortie leur sera ouverte.

L'objet est emprunté à une chainette dont on aperçoit les soies terminales; ce qui est un sujet d'observation très rare dans nos matériaux, bien que l'espèce soit même commune dans bon nombre d'échantillons. Ces soies, largement courbées à leur base, ont une tendance à se diriger, plus loin, parallèlement au grand axe de la colonie.

Elles ont été tronquées à dessein, par défaut de place.

De cette série de gravures reproduites de spécimens qui se coudoient souvent sur un mème slide et qui, sans conteste, sont afférents à la même espèce, il résulte une grande difficulté de résumer les caractères de celle-ci, en la forme d'une diagnose à la fois brève et précise.

Le lecteur en gardera, ce qui vaut mieux, des éléments d'appréciation personnelle.

La constatation du polymorphisme des endocystes en particulier le mettra en garde contre l'idée éventuelle d'attribuer à des espèces différentes celles de leurs modalités qui pourraient se rencontrer isolément.

Distribution géographique. - Commune le long des còtes occidentales de l'île nord de la Nouvelle-Zemble. Elle est sporifère sur tout ce long parcours, entre les nos de pêche I83 et 203. Souvent mangée par des espèces animales, des Crustacés surtout, les débris s'en retrouvent abondants dans leurs crottins.

\section{Chatoceros diadema Ehrenberg.}

Planche XXVI, figures 15 à 23 .

Synonymie ét littérature. - Voir Nordisches Plankton, page 84.

Espèce accablée d'une synonymie compliquée, à laquelle ses caractères si francs, si distingués auraient dù la soustraire si la diagnose en avait été mieux précisée dès l'abord.

Maintenant qu'elle s'est dégagée de toute compromission avec d'autres espèces également mieux appréciées, les planktonistes la connaissent suffisamment pour qu'il soit utile de faire ici autre chose que d'en figurer le facies particulier dans nos matériaux. 
La figure I5 en note les caractères moyens. Ceinture bien marquée; valves très légèrement renflées dans leur partie médiane, donnant aux foramens, toujours bien ouverts, une forme oblongue un peu déprimée dans le milieu; soies latérales insérées un peu en retrait sur le bord arrondi des valves et divergentes dans des directions variées, après leur brusque soudure à quelque distance de leur base; soies terminales courbées à leur base, droites plus loin et dirigées de manière à former entre elles un angle peu ouvert, comme dans la figure ig, où elles ont été figurées dans leur entier développement.

La figure I6 montre, en même temps que la forme elliptique peu large de la section transversale des frustules, la divergence des soies latérales, au delà de leur point de croisement.

Endocystes. - Les spores sont bien connues, avec leur valve primaire peu bombée, surmontée de nombreux prolongements, $x$, qui portent à leur extrémité des ramifications dichotomiques de plus en plus ténues, et leur valve secondaire, d'une courbe différente, dépourvue de tout ornement. On en voit des aspects variables avec leur état d'avancement, dans les diverses figures relatives à l'espèce.

L'examen de quelques figures fournira une documentation complémentaire.

Figure i8. Fragment stérile d'une chainette large, dont deux frustules représentés intégralement, contenant et contenu, montrent leur noyau petit et le cytoplasme ramassé contre les deux valves, mais non différencié en chromatophores distincts.

Figure Ig. Fragment d'une chainette étroite dont on peut voir les soies terminales, qui diffèrent des autres par leur direction et souvent aussi par leur calibre un peu plus fort. Une cellule figurée en coupe sagittale optique montre mieux que la figure précédente le noyau, $n$, au milieu des deux masses cytoplasmatiques refoulées sous les deux valves.

Figure I7. Colonie plus étroite encore, mais à frustules plus longs, dans l'un desquels on peut voir, sous l'aspect qu'elle revêt dans cette circonstance, la valve primaire d'un sporocyste.

-Figure 20. Fragment de colonie large dont les frustules élaborent la valve primaire de leur endocyste, sp. Dans les deux frustules supérieurs, qui forment un couple physiologique, le cytoplasme se concentre sous ces organes, en prenant un aspect plus granuleux dans lequel on peut voir une tendance à l'individualisation de plusieurs chromatophores. Les valves kystiques suivent, dans le couple, un développement parallèle et les appendices, $x$, qui les surmontent ne sont encore que des épines courtes et simples.

Dans le frustule inférieur, qui appartient à un autre couple, ces appendices, $x^{\prime}$, commencent à produire leurs ramifications dichotomiques.

Figure 2I. Ici les spores sont mûres. Elles montrent dans leur cytoplasme des chromatophores assez gros, bien délimités, parmi lesquels il semble qu'il y a aussi des 
matières de réserve, également sous forme globuleuse. Les appendices dichotomiques des valves primaires ont ici leur épanouissement complet et définitif.

La figure 22 est une vue de coté d'un fragment de chainette dont deux frustules fertiles sont munies de sporocystes mûrs, sous la forme étroite qu'ils présentent dans cette position de profil.

Avec un peu d'attention on verra bien ici que les soies ne se soudent qu'en un point de contact et divergent ensuite sans se croiser réellement.

Parasitisne. - Le petit champignon phycophage que nous avons déjà signalé plus haut, sous le nom d'Olpidium phycophagum, est ici extrêmement répandu et infeste souvent des portions entières de colonies, comme dans la figure 23. Nous croyons d'autant plus utile d'insister sur ce fait intéressant que nos devanciers se sont plus complètément dispensés d'en relever le caractère général.

Dans la figure 23, choisie à cause de sa portée instructive, le parasite, ol, se montre sous des aspects variés, à des phases diverses de son développement.

En $a$, il a envahi toute l'étendue d'un frustule, sous la forme végétative, en thalle mobile, d'aspect granuleux et riche en enclaves. En $b$, il est en voie de sporulation. En $c$, il se montre enkysté, à l'abri d'une membrane qui lui est propre. En $d$, enfin, sa présence dans un frustule exceptionnellement long, sous l'aspect homogène et la forme irrégulière que nous reproduisons, a contrarié la division de son hôte ou en a provoqué l'accroissement anormal.

C'est un fait d'observation fréquente, en effet, que l'hypertrophie des cellules parasitées; phénomène dù évidemment à l'irritation que le parasite y détermine. Cette déformation ne se traduit cependant pas toujours, car le parasite s'observe parfois dans des frustules restés normaux au point de vue de leurs dimensions. Exemples : c, au bas de la figure 23; ol, au bas des figures I9 et 22, dans lesquels on voit des kystes d'Olpidium.

Dans un frustule du bas de la figure i5, l'essaimage des sporules du parasite, ol, s'est effectué, à la faveur d'une petite tubulure qui a percé la paroi du frustule et qui a donné issue aux zoospores par le petit orifice extérieur, o. Le sporange vidé, mais parfaitement visible à l'intérieur de la cellule infestée, montre aussi bien la façon dont la déhiscence s'est effectuée que si l'on y avait assisté.

Remarquons enfin que toutes ces productions mycologiques présentent, sous l'action de l'iode, une teinte très vive, d'un beau brun d'acajou, qui révèle leur richesse en matières albuminoïdes et sert à en signaler la présence.

Distribution géographioue. - Fréquente au voisinage de Tromsö et tout le long des côtes occidentales de la Nouvelle-Zemble. Plus rare dans le Détroit de Matotchkine et à proximité de ce détroit dans la Mer de Kara. Presque absente dans cette dernière Mer, dont, à vrai dire, nous arons peu d'échantillons pélagiques de surface. 


\section{Chatoceros laciniosum Schütt.}

Planche XXVI, figures 24 à 31 .

Synonymie ét littérature. - Cfr. Nordisches Plankton, page 82. Gran y ramène les formes Ch. distans Cleve et Ch. commutatum Cleve. Il y a lieu, selon nous, d'y adjoindre aussi $\mathrm{Ch}$. breve Schütt, $\mathrm{Ch}$. hicmale Cleve et peut-ètre Ch. pelagicum Cleve, qui a aussi été appelé Ch. Ostcnfoldii Cleve.

Il ne faut voir sans doute dans cet imbroglio synonymique que la traduction des impressions personnelles des auteurs qui se sont trouvés en présence d'une espèce commune et fort répandue, dont le polymorphisme, plus apparent que réel, a été exagéré soit par les méthodes de fixation, soit par le peu de précision des figures destinées à en vulgariser les caractères. Ces formes ne sont ainsi probablement que des variantes d'aspect et d'interprétation dont l'espèce laciniosum Schütt est susceptible. Elles ne jouissent que d'un titre spécifique mal acquis, dont il faut les dépouiller.

Chaines généralement longues et souvent terminées par des soies, st, différenciées, dans leur direction, sinon toujours dans leur calibre et leur aspect. Comparer leur allure dans les figures 24 et 28 , où elles sont développées en projection sur le plan sagittal, avec la figure 25, - vue latérale d'une colonie - où elles se profilent sur un plan à peu près perpendiculaire à celui-là, mais parallèle aussi, évidemment, à l'axe longitudinal de la colonie.

Les soies ordinaires émergent d'un prolongement latéral des valves, plus large et un peu en retrait sur le bord du frustule. Après un parcours assez long généralement, suivant la ligne latérale de la chainette (fig. 24 et 28 ), elles se soudent brièvement et se déjettent ensuite brusquement, en dessinant des courbes divergentes, conformément à la figure 26 , qui en montre la direction en vue valvaire, soit en coupe transversale de la colonie.

Les foramens, $f$, sont, par le fait, exceptionnellement spacieux, un peu rétrécis au milieu, dans les colonies larges, par le léger renflement médian des valves.

Les frustules, aux dimensions inversement proportionnelles, - comparer les différentes figures - ont une ceinture visible qui n'est cependant pas limitée par des sillons apparents.

Le noyau est central dans les cellules au repos, $n$ (fig. 24 et $3 \mathrm{I}$, en haut). Le cytoplasme est alors plus ramassé près des deux valves, sous la forme des deux chromatophores des auteurs.

Dans les frustules qui viennent de subir la cytodiérèse, ce qui se reconnait à la position excentrique des deux noyaux-filles, nd (fig. 3I, au milieu), ceux-ci sont en regard l'un de l'autre dans les deux cellules sours, et le cytoplasme momentanément refoulé du còté opposé, en une seule masse, y simule le chromatophore unique des auteurs. 
L'unicité ou la dualité des chromatophores, qui n'ont du reste ici aucune objectivité réelle comme éléments individualisés du cytoplasme, est donc simplement l'expression d'une phase végétative des frustules.

La figure 27 reproduit un spécimen extrêmement étroit, réduit à ses dimensions minima : $n$, noyau; $f$, foramen.

Endocystes. - L'espèce est très sporifère. Les sporocystes, sp, diversement bombés des deux côtés, ont leurs deux valves lisses, exemptes de tout appendice. Leur forme varie naturellement avec les dimensions des frustules qui leur servent de matricules. Larges et plats dans les grandes colonies, ils sont plus étroits mais plus rebondis dans les petites.

La figure 30 en présente un couple génétique, où les valves primaires sont en voie d'élaboration, pendant que le noyau et le cytoplasme, devenu granuleux, s'y concentrent.

La figure 24 montre, dans le frustule supérieur, une étape analogue d'un sporocyste qui n'a pas de symétrique dans le frustule voisin.

La figure $3 \mathrm{I}$ en montre, dans sa partie inférieure, un couple génétique où l'on a fait abstraction du contenu cellulaire, pour n'en figurer que les squelettes capsulaires, en vue sagittale.

La figure 29 en présente un autre en vue latérale, sous des dimensions beaucoup plus grandes.

Dans les figures 28 et 25 enfin, les kystes mûrs sont figurés respectivement en vue sagittale et en vue latérale, avec leur contenu cellulaire dans lequel on distingue des corps isolés, que nous considérons comme des chromatophores différenciés dont l'individualisation s'est opérée pendant la maturation de ces organes de reproduction.

Parasitisme. - Olpidium, ol, n'est pas rare ici non plus; nous l'avons figuré, à titre d'exemples, sous forme enkystée, dans les frustules inférieurs des figures 25 et 28 .

Dans ce dernier, il a laissé persister autour de lui un résidu granuleux qu'il ne s'est pas assimilé.

Distribution GÉographigue. - Même extension et même abondance que sa congénère $C h$. diadema, dans les échantillons de surface, surtout de la Mer de Barents et du Détroit de Matotchkine. Presque étrangère à nos produits de pêche de la Mer de Kara, de la Mer Mourmane et de l'Océan Glacial.

\section{Chcetoceros Schüttii Cleve.}

Planche XXVI, figures 32 à 35 .

Synonyme et littérature. - Cfr. Nordisches Plankton, page 8i.

L'espèce que nous reproduisons ici sous les trois aspects classiques : sagittal (fig. 32), latéral (fig. 33) et valvaire (fig. 34), nous laisse perplexe à cause de l'absence, 
dans les spécimens de nos matériaux actuels, de ces belles soies terminales, fortes, élégamment courbées et très écartées que nous lui connaissons dans des produits de la Mer Flamande et dans d'autres.

Ici ces soies, assez rarement observables, sont plus longues, moins fortes, moins symétriquement courbées, comme on peut le voir dans la figure 32 où l'une des deux est développée et l'autre écourtée par manque de place.

Les soies ordinaires se soudent dès leur origine sur le bord même des frustules et ne laissent conséquemment entre ceux-ci que des foramens, $f$, très étroits.

Elles divergent ensuite brusquement, presque à angle droit, suivant la figure 34, qui montre, en outre, la forme brièvement elliptique de la section transversale des frustules.

Ceux-ci ont une ceinture étroite, bien délimitée, en vue sagittale, par des sillons (fig. 32) et en vue latérale, par une atténuation du calibre des valves qui s'arrondissent vers leur sommet (fig. 33).

Le cytoplasme habituellement étalé sur une des faces sagittales du frustule donne l'apparence d'un seul chromatophore pariétal diffus, sur lequel se profile le noyau médian, n (fig. 32, dans un frustule figuré en coupe sagittale optique).

La figure 35 reproduit un spécimen très étroit du mème type, avec les caractères externes et internes des frustules. La petitesse des colonies peut en rendre l'identification difficile.

Endocystes. - Dans nos échantillons, les endocystes assez rares ne sont encore représentés que par leur valve primaire, sp (fig. 32), spinuleuse sur leur dôme arrondi. Nous nous défendons de les figurer tels que nous les connaissons, à l'état parfait, d'après des matériaux étrangers aux récoltes de la Belgica, pour conserver à celles-ci leur physionomie propre.

Nos matériaux actuels ne se prètent pas non plus à l'étude comparée de cette espèce et de certaines de ses congénères dont les différences spécifiques paraissent bien subtiles.

Nous aurons l'occasion de formuler notre avis à leur sujet dans une autre publication.

Distribution géographique. - Très rare, dans des produits còtiers de la Mer de Kara; assez constante, mais clairsemée tout le long des còtes ouest de la NouvelleZemble; non rencontrée dans le Détroit de Matotchkine.

\section{Chatoceros sp.}

Planche XXVlí, rigures $ı$ à 6 .

Il s'agit ici d'une assez petite forme qui nous a surtout frappé par la présence très fréquente dans ses frustules d'une Chytridiacée, parasite que l'action de l'iode met en particulière évidence. 
Dans nos matériaux les colonies en sont droites et toujours fragmentaires, c'est-à-dire sans soies terminales. Les éléments en sont allongés, cylindriques à peu près, pourvus de soies raides qui se soudent sur l'angle même des valves et ne laissent place qu'à des foramens, $f$, en forme de fente étroite.

La ceinture est large, peu visible, parfois cependant limitée d'un côté par un sillon.

Les soies divergent à angle droit et en ligne droite dès leur soudure (fig. 6, vue valvaire optique) et s'écartent peu du plan transversal des chaînettes.

Les frustules ont un noyau central, $n$ (fig. 4), et deux chromatophores diffus, ch, rapprochés des extrémités valvaires et souvent confondus par leur extension jusqu'autour du noyau; mais le parasitisme en modifie trop constamment l'état naturel pour en saisir les caractères cytologiques normaux.

Enkystement. - Les endocystes occupent le centre des matricules. Nous n'en connaissons que la valve primaire, $s p$ (fig. I et 5), ornée sur son dôme hémisphérique de spinules courtes.

Auxospores. - Nous tenons pour telles les hernies latérales, ax (fig. 2 et 5), de forme sphérique d'abord (fig. 2), de forme ovale ensuite (fig. 5), qui résultent de l'épanchement du contenu cellulaire d'un frustule générateur. En même temps que le calibre s'en accroît et devient bien supérieur à celui de la cellule génératrice, le cytoplasme s'enrichit d'enclaves et devient grossièrement granuleux.

Nous regrettons que la rareté de l'espèce et son peu d'extension ne nous aient pas permis de rechercher les phases, ultérieures de ces productions, qui sans doute rappelleraient celles observées et décrites plus haut dans Ch. contortum.

Parasitisme. - La plupart des individus sont envahis par un Olpidium, ol, circonstance qui rend difficile l'observation de leurs caractères normaux. Quelques exemples permettront de faire connaître les principales variantes d'aspect des frustules parasités.

Figure I. La cellule marquée, $a$, s'est hypertrophiée, par la présence du parasite, ol, qui apparait sous l'aspect d'une masse protoplasmatique de structure grossière et qui est sans doute seul à occuper toute l'étendue du frustule, après s'en être assimilé le propriétaire. La vue du fragment de chaînette figuré est ici latérale.

Figure 4. Les cellules marquées, $a$, montrent le parasite enkysté dans une membrane propre qui épouse à peu près la forme du frustule hospitalier. Le contenu en est grossier, irrégulièrement granuleux.

Dans la cellule marquée, $b$, le parasite, ol, est en voie de sporulation. Le thalle s'y est, en effet, transformé en un grand nombre de sphérules pourvues chacune d'un point central réfringent. Ce ne peuvent être que des sporules dont l'essaimage se produira bientòt. 
Figure 3. Olpidium, ol, sous forme de kyste limité à une partie du frustule parasité, dans lequel il reste des résidus mortifiés du cytoplasme.

Figure 2. La cellule inférieure, marquée $a$, renferme un kyste d'Olpidium, ol, de forme ellipsoïde. Dans la cellule supérieure, $b$, l'ingérence du parasite a contrarié la formation de l'endocyste dont on aperçoit inférieurement l'ébauche de la valve primaire encore dépourvue de spinules et restée difforme.

L'état pathologique de l'espèce est trop généralisé pour qu'il soit possible de bien faire la part de son influence sur ses caractères propres et de se faire une opinion fondée sur ceux-ci. C'est pourquoi nous nous abstenons de la juger au point de vue taxinomique.

Distribution Géographique. - Trouvée seulement, en mélange avec d'autres nombreuses espèces, dans les échantillons i97, ig8 et r99 pêchés au nord de la presqu'ile de l'Amirauté.

\section{Chatoceros sp.}

Planche XXVII, figures 7 à Io.

Nous consacrons quelques figures à une forme assez rarement rencontrée qui, par la forme des foramens, par l'aspect du noyau et du cytoplasme condensé sous les valves en forme de deux chromatophores diffus, n'est pas sans analogie, à première vue, avec Ch. diadema. Mais l'absence de ceinture bien délimitée et la disposition irrégulière et dévariquée de la partie libre des soies, qui se soudent d'ailleurs un peu en dehors de la ligne latérale et s'insèrent moins en retrait sur les bords de valves, leur communiquent un air tout autre. Or, la physionomie des Chatoceros en est bien le meilleur caractère pour l'observateur.

La figure 7 en reproduit, en vue sagittale, un fragment de colonie large, dont la figure 8 en est la vue valvaire, en coupe optique, et la figure ro une vue latérale.

La figure 8 en montre un autre fragment plus étroit, en vue sagittale. On remarquera la forte divergence des soies qui leur fait souvent traverser le plan sagittal, en projection.

Nous n'y avons pas observé de sporocystes. A défaut de ceux-ci, nous nous abstenons de la déterminer spécifiquement.

Distribution gÉographigue. - En mélange avec beaucoup d'autres formes dans les produits de pèche de surface de la Mer de Barents et du Détroit de Matotchkine. Peut-ètre moins rare qu'elle ne parait, à cause de ses analogies d'aspect soit avec Ch. diadema, soit avec Ch. contortum, si, comme il arrive dans des observations cursives, on ne prend pas soin de reconnaitre la torsion des chainettes de cutte dernière espèce, ses chromatophores multiples et, généralement, les caractères précis des formes qui s'en rapprochent par quelque còté. 
Chatoceros furcellalum Bailey.

Planche XXVII, figures II à I6, et planche XXVIII, figures 2 et 3 .

Littérature. - Voir Nordisches Plankton, page 95.

Cette petite espèce est on ne peut mieux connue sous la forme d'endocystes.

Ceux-ci sont géminés, renflés sur leur valve primaire, aplatis sur l'autre, étroitement accolés et porteurs de soies différenciées, fortes, raides, un peu tordues, soudées longuement à leur base avec leur voisine et très peu divergentes dans leur partie libre (sp. fig. I3.)

Souvent libérées, dans nos matériaux d'études, et engluées dans des matières muqueuses, les spores s'y présentent en très grand nombre sous les aspects des figures I4 et I6, à l'état géminé, ou sous l'aspect de la figure I5, à l'état dissocié. Ce n'est que plus rarement que l'on peut les observer en place, dans leur matricule, comme dans la figure i3.

Il n'en est pas de même, pour autant que nous sachions, de la forme des frustules et de leurs rapports, quand ils se trouvent réunis en colonies. Celles-ci doivent être fort sujettes à l'émiettement, car on se trouve rarement en présence de chaînettes sùrement identifiables.

Nos matériaux, peut-être exceptionnellement défavorables à cet égard, ne nous en ont fourni que de rares sujets d'observation, d'une interprétation plutôt difficile, dont nous reproduisons quelques exemples.

Dans la figure I3, les deux couples de frustules sporifères ne portent pas de soies ordinaires sur leur valve libre.

Dans la figure II, on observe la même particularité entre les deux groupes de soies différenciées, $a$ et $b$, qui ont déjà revêtu le caractère de celles des endocystes, bien que ceux-ci ne soient pas encore formés. Là aussi il existe des valves frustulaires, et même un frustule entier, dépourvus de soies. Il en résulte que la soudure des frustules en colonie se fait ici par les valves elles-mèmes et que celles-ci ne ménagent entre elles aucun foramen. C'est une anomalie qui, à notre connaissance, n'a pas été relevée par nos devanciers. Il y a là peut-être une explication de l'émiettement si général des colonies.

Le cytoplasme des frustules est diffus, mais comporte des granulations simulant des chromatophores assez nombreux qui nous porteraient à transférer l'espèce en question dans notre groupe des Phaneroleucita, si nous avions pu observer la même chose dans des frustules de caractère franchement végétatif. Observées ici, ces granulations peuvent n'être qu'une adaptation à l'enkystement, prochain auquel prélude la différenciation des soies. Dans les sporocystes mûrs de la figure I3, on observe, en effet, d'assez nombreux corps individualisés qu'on peut prendre pour des chromatophores, conformément à ce que nous avons signalé à propos d'autres espèces. 
Notons, en passant, la figure I2, qui est une vue valvaire de la chainette voisine (fig. II), en coupe optique, au niveau des soies différenciées. Elle en complète le signalement morphologique.

La singularité de l'espèce ne s'arrête pas là.

Dans la figure 2 de la planché XXVIII, toutes les valves de la chainette portent des soies : les unes, $a$, différenciées, exclusives de foramen entre elles; les autres, $b$, plus minces, divergentes dès leur point de soudure et productrices d'un foramen, $f$, assez ouvert. On voit que le protoplasme, pr, devenu granuleux, des frustules, s'est condensé aux endroits où doivent naitre les endocystes.

La figure 3, planche XXVIII, représente un thalle dont tous les frustules sont encore stériles - objet d'observation fort rare - et porteurs de soies ordinaires, qui déterminent toutes, avant leur soudure locale, un foramen assez ouvert. En a, toutefois, on voit le foramen, $f$, se réduire, en mème temps que les soies destinées à se différencier, lors de la production prochaine d'un couple d'endocystes en cet endroit, se mettent déjà dans une position plus parallèle, qui permettra leur soudure plus tard, conformément à ce qu'on voit réalisé, en $a$, dans la figure voisine, 2.

$\mathrm{Si}$ ces faits sont bien interprétés, nous devons en conclure que la différenciation des soies kystiques, dans leur calibre, leur direction, leur rapprochement et leur soudure, n'est pas primitive. Elle ne se produit que lorsque les frustules se disposent à l'enkystement; mais elle précède la formation des endocystes eux-mêmes.

On s'étonnera, sans doute, des grandes différences entre les notes ici résumées et celles que Gran applique à la même espèce dans Nordisches Plankton. Qu'on nous dispense d'en rechercher la cause.

Distribution géographigue. - Assez abondante, à l'état d'endocystes surtout, dans les nos de pêche 216 et 217 de la station 22, dans l'Océan Glacial. Exceptionnelle dans d'autres échantillons des mêmes parages, dans des crottins de Crustacés; rare dans les environs de Tromsö; inobservée sur les autres parties de l'itinéraire de la Belgica.

\section{Chatoceros sp.}

Planche XXVII, figure 17 .

Spécimen unique d'un fragment de chaînette trouvé dans un crottin de Crustacé.

Les caractères du thalle sont tout à fait ceux de Ch. laciniosum, avec des foramens, $f$, très ouverts. Mais la valve primaire, seule présente, des endocystes est hérissée de spinules; ce qui empêche toute tentative d'identification avec cette espèce dont les spores sont lisses sur les deux valves. 


\section{Chatoceros subtile Cleve.}

Planche XXVII, figure 18 .

\section{Littérature. - Cfr. Nordisches Plankton, page 88.}

Très petite forme à thalle étroit, sans foramens, à soies latérales très minces toutes dirigées vers la même extrémité de la colonie, qui porte des soies différenciées, plús longues, plus fortes, presque parallèles.

Dans nos échantillons, le cytoplasme des frustules est grossièrement granuleux.

Nous n'y avons pas observé d'endocystes, mais les caractères végétatifs de l'espèce, qui s'identifie sans doute avec Ch. longisetum du même auteur - variante plus large suffisent à la reconnaitre.

Distribution GÉographique. - En spécimens très rares, perdus dans un fouillis d'autres espèces, vers le nord de la Mer de Barents; no igo. Peut-ètre ses caractères délicats la dissimulent-elles dans les produits pèche où ses congénères abondent.

\section{Chatoceros debile Cleve.}

Planche XXVII, figures ig à 23

Synonyme et Littérature. - Voir Nordisches Plankton, page 92.

C'est une bonne espèce, bien connue, mais assez difficile à distinguer à première vue de Ch. curvisetum quand elle n'a pas d'endocystes, comme c'est le cas dans nos matériaux de la Belgica.

Les colonies forment des chainettes enroulées en spirale, en façon de ressort à boudin (fig. 22).

Les soies ordinaires, soudées dès leur naissance, presque sur le bord même des frustules, s'incurvent toutes régulièrement du côté convexe de la spirale décrite par la chaînette, comme le montre la coupe valvaire optique de la figure 20 et mieux encore la vue latérale de la figure 23.

La figure 2I en montre un fragment de colonie de petit calibre, supposé vu dans le plan sagittal, abstraction faite de sa courbure.

La figure Ig reproduit un fragment, présenté de la même façon, d'une colonie plus forte.

Les frustules qui occupent la partie inférieure de la figure ont leur aspect normal, avec leur contenu, dont un petit noyau, central quand il est au repos, latéral quand cet organe jeune se reconstitue à l'issue d'une cytodiérèse.

Dans cette figure, comme dans la précédente (fig. 2I), le cytoplasme figure un seul chromatophore diffus, ou deux, suivant l'état physiologique de la cellule et le résultat de la fixation. 
Dans les spécimens reproduits, les foramens, $f$, sont assez étroits, légèrement étranglés vers leur milieu dans les chaînettes larges.

La cellule supérieure de la même figure ig montre un frustule hypertrophié par la présence d'un Olpidium parasite, ol, dont les sporules ont déjà été mises en liberté par un orifice en rapport avec la paroi de la capsule sporangiale encore visible.

Distribution géographidue. - En spécimens rares dans quelques échantillons de la Mer de Barents, au sud de la presqu'ile de l'Amirauté et aussi dans l'échantillon $n^{\circ} 4, \mathrm{du}$ voisinage de Tromsö, particulièrement riche en Chétocérées. Cette espèce nous est beaucoup mieux connue de la Mer Flamande, où elle est extraordinairement commune presque en toutes saisons et souvent en sporification.

\section{Chatoceros crinitum Schütt?}

Planche XXVII, figures 24 et 25.

Lirtérature. - Cfr. Nordisches Plankton, page 89.

La forme que nous avons en vue est en chaînettes droites, mais peu rigides. Les frustules ont une section rectangulaire en vue sagittale, leurs valves se touchent presque et ne laissent entre elles que des foramens, $f$, extrêmement étroits, souvent même inapparents.

Des angles des valves s'échappent des soies faibles qui se soudent, dès leur origine, sur la ligne latérale et divergent ensuite dans des directions très diverses, ce qui peut tenir à la grande flexibilité de ces organes dont l'ordonnance naturelle se dérange facilement dans les préparations.

Le cytoplasme ne forme qu'une masse pariétale continue que l'on peut prendre, si l'on veut, pour un chromatophore diffus. Le noyau est central dans les cellules au repos, latéral dans les jeunes produits de division. La ceinture n'est guère observable et ne fournit aucun bon caractère morphologique. Les endocystes font défaut.

Faut-il voir dans ces caractères ceux de Ch. crinitum Schütt : caractères frustes et figurés de façons inconciliables, par SchütT lui-même, I895 (fig. I2 $2^{\mathrm{ae}}$ ); par Gran, I897 (fig. 5i) et par Ostenfeld, igol (fig. io) : cette dernière reprise telle quelle dans Nordisches Plankton, loc. cit.?

Dans la figure de SchürT, I2 $2^{b}$, les soies sont en retrait prononcé sur les bords des valves et se croisent bien en dehors de la ligne latérale. Celle de Gran n'est qu'une vue latérale insignifiante. Celle d’Ostenfeld montre les soies insérées et soudées sur les bords mêmes des valves. Il faut en conclure ou bien que ces auteurs n'ont pas eu en vue la même forme de Chatoceros ou bien qu'ils n'ont pas apporté un soin suffisant à en fixer au moins les traits essentiels. 
Nos observations antérieures ne nous ayant pas fourni l'occasion de nous familiariser personnellement avec cette forme équivoque, nous ne croyons pas opportun de nous baser sur nos matériaux d'étude actuels, trop douteux eux-mèmes, parce qu'ils manquent d'endocystes, pour prendre position relativement à la légitimité de l'espèce et au rapprochement proposé dubitativement plus haut. La question demande contrôle.

Distribution GÉographioue. - Rarement observée sur quelques points distancés de l'itinéraire de la Belgica.

\section{Chcetoceros Wighami Brightwell.}

Planche XXVII, figure 26 .

Synonyme et littérature. - Cfr. Nordisches Plankton, page 88.

Petite forme en chaînettes droites, courtes, à frustules biconcaves, porteurs de soies toutes délicates, soudées à leur origine sur le bord même du thalle et divergentes de leur voisine, sans s'écarter toutefois beaucoup du plan sagittal, si des causes mécaniques n'en ont pas dérangé la position naturelle.

Les foramens, $f$, y sont plus ou moins ouverts, en forme de boutonnière en lancette.

Les sporocystes font défaut dans nos matériaux.

Distribution géographique. - Très rare, dans la Mer de Kara, au voisinage du Détroit de Matotchkine, no 34; plus rare encore dans l'Océan Glacial, n 226.

\section{Chatoceros fragile sp. $\mathrm{n}$.}

Planche XXVII, figures $27,28,29$.

Forme assez petite, délicate, en colonies fragiles, formées de frustules à section sagittale rectangulaire. Ceux-ci portent, sur leurs angles un peu émoussés, des soies courtes, très fines, flexibles, qui se soudent à leur contiguë sur la ligne latérale, en ménageant des foramens étroits, mais toujours visibles, et divergent ensuite dans des directions quelconques. Le cytoplasme granuleux simule un chromatophore pariétal diffus.

La figure 27 en reproduit, en vue sagittale, un spécimen moyen, que les moindres heurts ou pressions ont suffi à disloquer. On n'en voit guère, dans les préparations, qui aient pu garder leurs éléments dans l'axe rectiligne de leur agencement naturel.

C'est ce caractère de fragilité des soudures des soies qui nous suggère le nom spécifique que nous lui attribuons. (Qu'on se garde de confondre cette espèce avec Ch. anastomosans, chez qui la soudure des soies se fait loin en dehors de la ligne latérale, par l'intermédiaire d'un petit trabécule transverse.) 
La figure 28 en représente un spécimen de taille maxima. On n'y observe pas de soies terminales différenciées, ce qui pourrait s'expliquer par l'extrême fragilité des colonies.

Une autre particularité de l'espèce c'est la propriété qu'ont les soies, très peu silicifiées sans doute, de s'accoler entre elles par l'intermédiaire d'une sorte de mucilage qui fait gaine autour de la colonie et qui se charge aisément des fines particules de sable qui flottent dans le même milieu.

La figure 29 traduit cette singularité dans la vue latérale d'une chainette dont la gaine susdite, $g$, est dessinée en coupe seulement, pour ne pas cacher le reste, car les grains de silice, $s$, qui y sont englués la rendent parfois peu transparente.

Les endocystes ne nous sont pas connus.

Distribution Géographique. - Assez commune dans certains échantillons du Détroit de Matotchkine et de ses abords dans les deux Mers de Kara et de Barents.

Associée souvent à d'autres petites formes sociales : Ch. sociale et radians, dans le fouillis desquelles il est parfois difficile de la discerner.

\section{Chatoceros simile Cleve.}

Planche XXVII, figure 37 , et planche XXVIII, figure 4.

Littérature. - Voir Nordisches Plankton, page 87.

Bonne petite espèce en colonies courtes, droites, caractérisées à première vue par la direction rectiligne des soies au delà de leur soudure réalisée par un croisement très oblique, à une notable distance en dehors de la ligne latérale (fig. 4, pl. XXVIII).

Les soies homologues des différents membres de lạ colonie sont parallèles entre elles, dans des plans qui s'écartent peu du plan sagittal.

Les valves portent une protubérance médiane suffisante pour que leur rapprochement, entre deux frustules contigus, soit complet ou à peu près et coupe le foramen, $f$, en deux parties étroites et symétriques.

La ceinture est étroite, mais ne s'observe bien que dans les spécimens robustes.

La figure 37 de la planche XXVII en est un frustule isolé, comme on en voit beaucoup, car la dissociation du thalle semble se faire aisément. Leur discernement dans les slides encombrés d'autres formes microplanktoniques est alors malaisé.

Le cytoplasme est ramassé sous les deux valves ou plus ou moins régulièrement réparti dans toute la cellule, particulièrement quand celle-ci est courte.

On en connaît bien les spores spinuleuses sur les deux valves; mais elles nous font ici défaut.

Distribution géographigue. - En colonies clairsemées au voisinage de Tromsö, $n^{\circ}$ I; en frustules épars, difficilement visibles et paraissant rares dans plusieurs échantillons de pêche des còtes occidentales de la Nouvelle-Zemble. 


\section{Chatoceros karianum Grunow.}

Planche XXVIII, figures 5 à 12 .

Littérature. - Gran, dans Nordisches Plankton, page 98, met en doute la réalité objective de cette espèce connue seulement jusqu'ici par un mauvais dessin de Cleve et Grunow : Beiträge zur Kenntniss der Arctischen Diatomeen, planche VII, figure I35, dans Kong. Sv. Vet. Akad. Handl, vol. XVII, no 3. Stockholm, I880.

Cette reproduction d'un spécimen incinéré est certainement d'une exécution défectueuse et peu interprétative. Elle nous a également toujours paru faussée ou mème irréelle, jusqu'au moment où nos matériaux de l'Océan Glacial nous ont mis en présence de l'objet lui-mème.

On conviendra, après examen des figures que nous en donnons d'après nature, qu'aucune confusion n'est possible entre cette curieuse espèce et Ch. Wighami.

C'est au contraire une très bonne espèce, parce qu'elle jouit d'une physionomie singulière qui la met à l'abri de toute confusion avec ses congénères.

Colonies droites, courtes, à soies terminales différenciées dans leur force et surtout dans leur courbure. Celle-ci est tout à fait caractéristique. Les foramens, $f$ et $f^{\prime}$, affectent deux formes différentes, suivant l'âge des valves qui les déterminent.

Les valves provenant d'une cytodiérèse récente sont planes et ne laissent d'abord entre elles qu'un foramen, $f$, très étroit, d'où s'échappent des soies latérales faibles, perpendiculaires à l'axe longitudinal de la colonie.

En vieillissant, les valves deviennent concaves et ménagent entre elles un large foramen, $f^{\prime}$, de forme lenticulaire, d'où les soies, un peu fortifiées en mème temps, s'échappent en épousant la courbure concave de leur valve respective. Après leur soudure, sur la ligne latérale, au sommet aigu des valves, elles continuent leur courbe pour dessiner un cercle à peu près complet et diverger obliquement ensuite, après une nouvelle courbure assez brusque, en sens inverse.

Cette courbure caractéristique prépare la séparation physiologique des frustules qui sont pourvus de soies différenciées et empêche les colonies de devenir ou de rester longtemps multicellulaires.

L'examen détaillé de quelques figures empruntées à des spécimens d'aspect différent permettra au lecteur de se pénétrer de la physionomie spéciale et des caractères propres de l'espèce.

Figure 5. Colonie large, formée de six frustules dont quatre doivent leur individualisation à deux cytodiérèses récentes, produites au niveau des soies rectilignes, si, ou des foramens étroits, $f$, ce qui revient au mème.

Figure 6. Tue en coupe valvaire optique d'une colonie, au niveau d'une division récente, là où les soies, si, sont encore rectilignes. 
Figure 7. Vue en coupe valvaire optique d'un frustule terminal, avec les deux soies différenciées y afférentes, st, projetées sur le plan perpendiculaire optique, pour montrer leur divergence sous cette incidence.

Figure 8. Colonie plus étroite, à cinq frustules pourvus alternativement de valves jeunes et vieilles et, corrélativement, de soies les unes rectilignes, les autres courbes.

Figure 9. Colonie large, formée de trois frustules seulement, figurés en coupe sagittale optique, pour montrer les deux noyaux-filles, $n d$, de la cytodiérèse qui vient de se produire dans le frustule inférieur, au niveau du foramen étroit, $f$, formé par les valves jeunes, terminées maintenant par des soies rectilignes minces.

Le frustule supérieur, plus àgé, a ses deux valves concaves. Il montre, à l'intérieur, un petit noyau au sein d'un chromatophore pariétal diffus.

Figure io. Variante d'aspect d'une colonie moyenne formée de frustules d'âge différent, que l'on reconnait aussi bien à la largeur des foramens, $f$ et $f^{\prime}$, qu'à la direction des soies, si et st.

Figure II. Deux frustules géminés provenant de la division d'une cellule-mère dont les valves conservées sont concaves et terminées par des soies courbes, st, tandis que les valves nouvelles sont planes, rapprochées et ornées de soies intermédiaires rectilignes, si.

Figure I2. Même cas dans un spécimen dont la faible largeur des frustules est compensée par une plus grande longueur.

Remarque. - Que cette forme soit spécifiquement distincte, ce n'est pas douteux.

Que ce soit là l'espèce visée par la figure indéterminable et assurément vicieuse de Cleve et Grunow, on peut l'admettre, en y mettant une dose suffisante de bonne volonté. C'est ce qui nous porte à en maintenir le nom, bien que celui-ci ne trouve aucun fondement dans nos observations : nos matériaux de la Mer de Kara ne nous en ont pas fourni le moindre exemple.

Distribution GÉographioue. - Nous tenons cette forme de l'Océan Glacial, exclusivement.

\section{Chatoceros radians Schütt.}

Planche XXVII, figure 32.

Littérature. - Cfr. Nordisches Plankton, page 97.

Remarque. - Peu d'espèces ont été aussi mal décrites et iconographiées que $C h$. radians Schüt et sa similaire Ch. sociale Lauder. C'est pourquoi leur distinction, bien que réelle et fondée, a dù rester et reste encore une énigme bibliographique pour 
ceux qui n'entrant en contact arec elles qu'en passant, n'ont pas pris la peine de se libérer, par l'étude personnelle comparée des deux espèces, du souci de mettre d'accord les savants publicistes et l'objet de leurs publications.

Que de temps perdu à chercher la conciliation entre ce que l'on voit sur un slide et les figures que l'on trouve dans les livres, figures que l'on présume ingénument devoir ètre des portraits ressemblants et qui ne sont parfois que des caricatures : chose qu'on constate trop tard!

On peut regretter que Gran lui-mème, dans son travail de compilation de Nordisches Plankton, œuvre de synthèse d'une grande valeur, n'ait pas voulu ajouter à celle-ci le mérite d'un contrôle personnel des espèces qu'il décrit et d'une figuration meilleure, mieux en harmonie avec la science planktonique actuelle, qui a plus clairement pris connaissance de son objet.

On comprend que, par souci d'impartialité, Gran ait voulu reproduire les figures originales des auteurs, si archaïques ou si insuffisantes fussent-elles; mais comme celles-ci sont presque toujours défectueuses par quelque côté, incorrection de dessin ou d'interprétation, leur réédition est de nature à entretenir des équivoques et à désorienter les chercheurs présents et à venir. A quoi bon consacrer des erreurs? Pourquoi négliger de les redresser? C'est retarder de vingt ans les progrès de la science microplanktonique.

Caractères morphologiques. - Ch. radians est en colonies longues, grêles, peu cohérentes, fragiles, gélatineuses, courbées naturellement dans le plan sagittal, mais diversement contournées en outre dans les préparations, par le fait du tassement et des pressions exercées entre les deux verres, dans les sens les plus variés.

Les frustules, plus ou moins rapprochés, laissent entre eux des foramens un peu étranglés dans leur milieu. Le cytoplasme simule un seul chromatophore diffus.

Les soies, très faibles, se soudent près de leur naissance, sur la ligne latérale; mais, tandis que l'une des quatre contiguës entre deux frustules voisins prend une direction radiale à l'intérieur de la courbure déterminée par la colonie, les trois autres prennent une direction inverse, soit, sans incurvation préalable, pour les deux externes; soit, pour l'interne restante, après une courbure de ISO ${ }^{\circ}$ nécessaire pour lui permettre de prendre enfin la même direction centrifuge que les deux externes.

C'est par leur soie radiale centripète surtout que les frustules, alors même qu'ils sont dissociés, restent néanmoins groupés; mais c'est grâce aussi aux faibles adhérences mucilagineuses qu'ils gardent entre eux.

C'est plus long à dire qu'à voir. Un coup d'œil sur le groupe de la figure 32, dont un frustule isolé, $a$, est représenté en vue valvaire, supplée avantageusement à ce que la description pourrait avoir d'obscur. Dans cette figure, qui est synthétique, nous avons groupé les différents aspects des frustules, dans les colonies souvent brisées et disloquées dont ils font partie. 
Endocystes. - Dans nos matériaux actuels, les spores, sp, en voie de formation, ne sont encore représentées que par leur valve primaire. Celle-ci est actuellement peu bombée et dépourvue de spinules, contrairement à ce que ScHütt lui-mème dit des spores mùres.

Distribution géographique. - Très répandue dans beaucoup d'échantillons de provenance variée, mais extrèmement abondante surtout dans les nos I7I et I72 de l'entrée occidentale du Détroit de Matotchkine. Elle s'y trouve intimement mélangée, dans une même gangue mucilagineuse, à sa similaire $C h$. sociale, dont nous reproduisons également un paquet dans la figure 33, en regard de la figure 32, pour faciliter la comparaison des deux espèces que Ostenfeld a réunies dans sa section des Sociales. Si l'on voulait tenir compte d'un caractère commun plus suggestif, le nom de Radiantes leur conviendrait encore mieux.

\section{Chatoceros sociale Lauder.}

Planche XXVII, figures $33,34,35$ et 36.

Synonyme ext littérature..- Cfr. Nordisches Plankton, page 96.

Par plusieurs caractères extérieurs, les colonies ou plutòt les paquets gluants de Ch. sociale ressemblent à ceux de Ch. radians. Le besoin d'un caractère distinctif facile à sàisir est d'autant plus impérieux. Nous le voyons, en dehors des endocystes, sur la présence desquels on peut rarement compter, comme c'est le cas dans nos matériaux actuels, dans la forme des frustules moins larges, plus cylindriques, plus petits, à section sagittale plus rectangulaire, à valves un peu concaves, d'où résultent des foramens diversement ouverts, mais exempts d'étranglement dans leur milieu.

Dans les petites espèces, comme celle-ci, on ne peut tirer aucun caractère utile du cytoplasme qui est pariétal, granuleux, simulant un chromatophore diffus.

Les soies filiformes, toutes semblables dans les frustules en colonie, affectent, peut-on dire, la même orientation que dans l'espèce précédente, comme en témoigne la figure 33, qui synthétise les aspects variés des fragments de chaînettes et cles cellules isolées, $a$, qui s'entremèlent et se présentent sous toutes les orientations dans les paquets de cette petite forme.

C'est cette mème espèce qui constitue ces curieuses colonies de frustules dissociés, mais maintenus groupés et disposés en éventail par la confluence d'une de leurs soies vers un centre commun (fig. 36, b), que l'on rencontre exceptionnellement dans les produits de la Bélgica et qui sont parfois si communes sur les còtes belges, où l'espèce ne se rencontre guère que sous cet aspect singulier.

Les soies radiantes sont alors particulièrement longues. 
Sous cette forme, que l'on pourrait éventuellement désigner d'un nom plus significatif : forma flabelliformis, par exemple, l'espèce est remarquable par la longueur exceptionnelle de ses soies radiantes et par la convergence plus manifeste de celles-ci vers un centre commun, au voisinage duquel elles s'agglutinent parfois en un cordon indissoluble.

La même espèce encore existe en outre souvent - plus souvent que les observations en milieu liquide ne le laisseraient supposer - sous la forme de frustules tout à fait libres : forma solitaria, si l'on veut. Dans ce cas, la disposition des soies les oblige à se présenter sur le slide en vue valvaire, suivant leur position d'équilibre stable, comme dans les figures 34 et 35 .

Les quatre soies d'un même frustule prennent alors la même orientation, gràce à des courbures harmonisées, non pas dans la direction du petit axe valvaire des frustules, comme dans Ch. debile (fig. 20, pl. XXVII), mais bien dans la direction du grand axe valvaire, comme le montrent les figures 34 et 35 afférentes à Ch. sociale, f. solitaria.

Dans ce cas, les quatre soies gardent sensiblement la même longueur, la soie que nous avons appelée radiante plus haut se dépouillant de sa singularité vis-à-vis des autres et prenant la mème courbure que sa symétrique placée à l'autre extrémité $\mathrm{du}$ frustule. Mais tandis que celles du couple externe - celles qui subissent une courbure de près de $180^{\circ}$ - restent plus minces et simplement curvilignes, celles du couple interne, peu divergentes entre elles, se montrent habituellement un peu plus fortes et revètent parfois une forme sinueuse, comme en témoigne l'exemple de la figure 35. Inutile de faire remarquer que ces soies, dont nous faisons ici le couple interne, ne sont pas autres que celles qui ont une direction centrifuge dans les colonies radiantes ou flabelliformes.

Cette petite forme parait souvent faire défaut dans des préparations examinées en milieu liquide, alors qu'on la voit extrêmement abondante dans les mèmes matériaux étudiés à sec. Tant il est vrai qu'il faut varier les procédés de technique et d'examen des matériaux microplanktoniques si l'on ne veut se résigner à en ignorer les éléments les plus délicats!

Distribution gÉographique. - Mêmes observations que ci-dessus, à propos de Ch. radians.

Entre les deux espèces sociale et radians, qui sont souvent mélangées dans les mêmes produits de pêche, l'équivoque est facile et provient de ce qu'elles sont toutes deux sociales et radiantes. L'observateur non prévenu prendrait aisément l'une pour l'autre.

Nous avons cherché à empêcher cette confusion; moins toutefois que nous n'aurions pu le faire en empruntant à d'autres matériaux des renseignements complémentaires, relatifs aux sporocystes spécialement, si nous n'avions craint de sortir du cadre naturel de ce mémoire. 


\section{Chatoceros glaciale sp. n.}

Planche XXVII, figures 38 à 44 .

Il ne s'agit pas ici de Ch. gracile Schütt - espèce à frustules isolés, pourvus de deux chromatophores fixés latéralement à droite et à gauche des frustules, sur les petits côtés de la ceinture; - voir Nordisches Plankton, page 97 - mais d'une autre espèce que nous croyons propre à la florule des glaces et des neiges.

On en saisira les caractères particuliers en examinant les figures qui s'y rapportent et qui en traduisent les principales variàntes morphologiques, à défaut des sporocystes qui nous restent inconnus.

Frustules toujours isolés, à valves concaves, d'où émergent, un peu en retrait sur leurs bords, des soies qui gardent d'abord une direction parallèle entre elles sur un certain parcours, avant de diverger en s'enroulant irrégulièrement en spirale, comme dans la figure 38, qui en reproduit un type large. Les autres figures, 39 à 44, en retracent d'autres modalités, différentes sous le rapport du volume et des dimensions relatives de longueur et de largeur des individus, mais identiques par l'allure des soies flexibles dont nous avons cherché à donner une idée par la multiplicité des exemples.

Distribution géographique. - Nous tenons cette espèce des neiges colorées - neiges jaunes, neiges verdàtres - et des glaces, où elle est souvent abondante, mais assez difficile à observer, si l'on ne soumet les slides à l'examen à sec. Elle est très rare dans les produits planktoniques proprement dits, où des spécimens ont pu passer accidentellement par fusion des neiges et des glaces.

\section{Chatoceros baculites sp. n.}

Planche XXVII, figure 30.

A défaut de référence possible actuellement à aucune des espèces signalées dans les mers septentrionales, nous signalons, sous un nom spécifique propre, cette très petite forme qui passerait facilement inaperçue si l'on n'épuisait toutes les ressources de la technique micrographique dans l'examen des produits planktoniques.

Les frustules disposés en colonies rectilignes sont très étroits, laissent entre eux des foramens elliptiques assez ouverts et portent des soies grêles qui divergent dans des plans perpendiculaires à l'axe longitudinal de la colonie. Le cytoplasme est central, disposé sur les parois, aux deux còtés du noyau qui est à peine perceptible.

Distribution GÉOgRaPhique. - Assez abondante mais difficile à observer au milieu des espèces sociale et radians, dans les produits de pèche du Détroit de Matotchkine. 


\section{Chatoceros filiforme sp. n.}

Planche XXVII, figure $3 \mathrm{I}$.

Espèce filiforme, très étroite, en colonies droites, illimitées, dépourvues de foramens visibles, garnies de soies grêles et courtes qui ne deviennent bien visibles qu'à sec. Le cytoplasme, d'une structure très fine, est pariétal et s'étend à toute la surface du frustule.

Nous ne saurions y reconnaitre les caractères de Ch. perpusillum Cleve, qui est en colonies courtes constituées de quelques éléments seulement, avec des soies terminales un peu différenciées.

Distribution Géographique. - Observée surtout dans les produits de pêche du nord de la Mer de Barents.

Remarque. - Beaucoup d'espèces, connues d'ailleurs et même tout spécialement des régions boréales, n'ont pas de représentants dans les récoltes de la Belgica. C'est ce qui explique notre silence à leur sujet. Il se peut toutefois que l'une ou l'autre nous ait échappé; tant il est vrai que ce petit monde des Chatoceros est plein d'embûches dont il faut attribuer une part aux influences saisonnières et aux conditions de l'ambiance, vis-à-vis de ces organismes si polymorphes par eux-mêmes et si semblables d'un autre côté par les caractères fondamentaux des espèces les mieux authentiquées.

On comprend par là combien il est difficile de maintenir les espèces les plus voisines dans les limites qui leur sont réellement propres. A cette fin, les descriptions littéraires sont absolument inopérantes; car, quelles que soient leur minutie et leur précision, elles laissent dans l'esprit du lecteur trop de marge à l'interprétation personnelle de celui-ci.

Les figures descriptives demandent de leur côté beaucoup d'exactitude.

Non seulement il faut en fouiller les détails d'une manière méticuleuse, d'après la conception type de l'espèce, mais il n'importe guère moins d'en repérer les principales modalités d'adaptation aux circonstances les plus diverses. Il faut pouvoir mesurer en quelque sorte l'amplitude de leurs variations.

Malgré tout cela leur étude restera un problème difficile. Elle demande de la part de ceux qui s'y livrent une sorte de flair acquis par la connaissance devenue familière d'un grand nombre d'espèces. 


\section{Genre Rhizosolenia}

Caractères du Genre. - Cfr. Nordisches Plankton, page 46.

Rhizosolenia setigera Brightwell.

Planche XXVIII, figure 13.

Synonyme et littérature. - Voir Nordisches Plankton, page 53.

Espèce commune et bien connue. Frustules droits, cylindriques, longuement atténués obliquement au sommet, d'où s'échappe une soie longue, filiforme, flexible caractère particulier des spécimens de nos matériaux, car, dans d'autres circonstances, nous l'avons vue avec une soie plus courte et plus robuste, parfois mème un peu dilatée sur une partie de son' parcours, en façon d'alène plate de bourrelier.

Notre figure i3 n'en reproduit qu'un fragment. Le noyau, $n$, est pariétal, comme les chromatophores. Ceux-ci sont petits, nombreux, orientés en séries spiralées vers le noyau comme vers un centre d'influence.

Endocystes. - Nous n'en avons pas figuré les endocystes dont nos matériaux ne fournissent que de très rares exemples, mais que nous connaissons depuis longtemps des còtes belges. Ils ont, du reste, à peu près les mêmes caractères que ceux de Rh. styliformis de la figure I9, mème planche.

Les auxospores, que nous connaissons aussi d'ailleurs, font ici défaut.

Distribution géographique. - Trouvée communément, en spécimens clairsemés au milieu des Chatoceros, sur tout l'itinéraire de la Belgica, à l'exclusion, toutefois de la Mer de Kara, où nous ne l'avons pas observée, et de l'Océan Glacial, où elle est très rare.

\section{Rhizosolenia hebetata Bailey.}

Planche XXVIII, figures $\mathrm{I}_{4}, 20,2 \mathrm{I}$, et planche XXX, figure 50.

Synonyme et littérature. - Cfr. Nordisches Plankton, page 55.

Ces formes désignées d'abord sous deux noms différents et tenues longtemps pour spécifiquement distinctes, comme le faisait supposer la terminaison si différente des frustules, ont été reconnues par Gran, en I904, pour appartenir à une mème espèce, après la découverte de spécimens, synthétiques en quelque sorte, qui présentent à la fois, à leurs deux bouts, les particularités prises jusque-là comme caractéristiques des deux espèces hebctata Bailey et semispina Hensen. 
Nos matériaux nous ont fourni l'occasion de refaire la mème observation et de confirmer ainsi l'assertion de notre devancier.

La figure 5o de la planche XXX reproduit un de ces spécimens dimorphes, qui lèvent tout doute sur la légitimité de la fusion des deux formes.

Cette forme heterothrix n'est guère plus rare dans nos produits de pêche que les autres.

A l'une des extrémités, $a$, l'appendice terminal est court, en forme de lancette arrondie au sommet et entaillée à la base d'une ouverture longuement triangulaire. A l'autre, b, l'appendice terminal est aciculaire et présente à sa base un petit orifice, 0 , qui complète son analogie avec une aiguille pourvue de son chas.

Dans la forme semispina, reproduite dans les figures 20 et 2I, d'après deux spécimens de calibre très différent, les deux extrémités sont surmontées l'une et l'autre d'une soie aciculaire étroitement perforée à la base. On peut négliger les caractères de structure de la paroi du frustule, bien que nous les ayons marqués dans la figure 20 , à l'exclusion du cytoplasme qui ne présente, du reste, non plus rien de particulier.

Dans les frustules très étroits comme celui de la figure 2I, ces détails ne sont pas visibles en milieu liquide, ils sont même peu perceptibles à sec.

La forme hebetata proprement dite, dont la figure I4 reproduit un spécimen complet, contenant et contenu, les appendices terminaux sont en forme de curette entaillée d'une fente longitudinale, o. Le noyau, $n$, et les chromatophores sont pariétaux. Ces derniers sont petits, très nombreux, plus serrés autour du noyau, alignés plus loin en séries dichotomiques, variables d'aspect d'ailleurs avec l'état physiologique de la cellule. Les stries en zigzag, qui subdivisent la membrane en petites aires polygonales disposées en chicane, sont généralement assez accusées pour être perceptibles en milieu liquide.

GRAN en fait sa forme hiemalis, la considérant sans doute comme une variété saisonnière. Nous ne saurions dire que c'est avec raison, car nous la trouvons en mélange avec les autres dans les mêmes récoltes effectuées pendant les mois d'été.

Distribution GÉographique. - Assez rare; rencontrée à l'entrée occidentale du Détroit de Matotchkine et dans l'Océan Glacial surtout.

\section{Rhizosolenia styliformis Brightwell.}

Planche XXVIII, figures 18 et 19.

Littérature. - Cfr. Nordisches Plankton, page 54.

Forme bien connue et heureusement dénommée, dont les extrémités coupées en biseau sont surmontées d'un appendice en forme de stylet assez court, p, ajouré à la base et flanqué de deux ailettes latérales, $a$, qui intéressent à la fois la base élargie du stylet et le sommet atténué du frustule. 
La figure is en montre l'extrémité d'une colonie rectiligne, dont un frustule complet présentant de face ses stylets et son contenu cellulaire : noyau, $n$, et chromatophores, ch, petits, rrès nombreux, alignés et pariétaux, comme le noyau.

Dans la figure Ig, qui reproduit aussi un fragment de colonie, les frustules sont vus latéralement, de manière à présenter leur stylet terminal de profil. Sous cette incidence cet organe se prête mal à l'observation, car ses ailettes, $a$, vues ici en projection sur la partie creuse du stylet ne s'y dessinent que sous la forme d'un trait mince.

D'autre part, cette figure montre très bien comment les frustules se soudent intimement sur toute leur partie biseautée et y gardent leur empreinte mutuelle après le décollement. Les partitions de la membrane sont les seuls détails de structure visibles en milieu liquide.

Endocystes. - Dans le spécimen du haut de la même figure I9, il s'est produit une rénovation cellulaire, pour donner naissance à un kyste court, sp, dont le corps cylindrique, arrondi aux deux bouts mais inégalement, reste étroitement serti dans le cytoderme du frustule matricule. Le cytoplasme s'y est modifié profondément en se condensant, car on n'y perçoit plus qu'un petit nombre de chromatophores beaucoup plus volumineux, ch. La production similaire du frustule inférieur a été figurée vide, pour permettre d'en dessiner plus nettement la forme.

Nos matériaux ne nous ont pas mis d'auxospores sous les yeux.

Distribution GÉographique. - Très rare dans nos matériaux. Rencontrée seulement au voisinage de Tromsö, dans le $n^{\circ} 2$.

Rhizosolenia oblusa Hensen et Rh. alata Brightwell.

Planche XXVIII, figures I5, I6, I7 et 22.

Synonymie et littérature. - Cfr. Nordisches Plankton, page 56.

Frustules cylindriques, droits, rétrécis vers leur extrémité, tronqués au bout et dépourvus de tout prolongement épicytaire.

La figure I7 en reproduit un spécimen de gros calibre, dont un frustule entier qui a une extrémité libre et l'autre encore soudée avec celle de son voisin dans la colonie rectiligne.

On y voit les détails de la paroi cylindrique qui sont visibles en milieu liquide.

La figure i6 en est un frustule isolé vu latéralement, dans une position qui permet de saisir, en profil, la forme de l'encoche pratiquée à la base du rétrécissement terminal par le sommet du frustule contigu dans la colonie. Nous y avons figuré, en outre, le noyau, $n$; les chromatophores petits et nombreux et la partition en quinconce du cytoderme, tous détails analogues à ceux des espèces précédentes. 
La figure I5 en reproduit un spécimen isolé, très étroit et vide, présentant de face l'encoche dont il vient d'être question. Certains y verraient peut-être la forme gracillima Cleve.

La figure 22 enfin traduit le facies un peu particulier d'un thalle à frustules plus longuement atténués au sommet. On y voit, sous l'incidence la plus favorable, le mode d'attache de deux éléments contigus.

Tous ces spécimens se réclament assurément d'une parenté étroite et leurs caractères individuels tiennent le milieu, peut-on dire, entre les caractères plus expressifs attribués parfois comme spécifiques aux deux formes mises ci-dessus en vedette : obtusa et alata. Entre ces deux les différences sont spécieuses et pourraient bien ne tenir qu'au calibre très inégal des frustules et à l'influence de l'ambiance, car si l'on tenait compte des variantes observées dans d'autres milieux, il semble qu'il serait bien difficile d'établir entre elles une ligne de démarcation suffisamment nette pour les rattacher à deux souches distinctes.

Dans les limites de nos observations actuelles, nous ne voyons pas le besoin de faire la part des deux espèces, si toutefois il y a un fondement réel à leur distinction.

Distribution GÉographique. - Rencontrée en spécimens très dispersés dans l'Océan Glacial; nos 2 I 6 et 226.

\section{Rhizosolenia Shrubsolei Cleve.}

Planche XXVIII, figures 23 et 24 .

Synonymie et littérature. - Cfr. Nordisches Plankton, page 52.

Forme aussi rare dans les récoltes de la Belgica qu'elle est commune sur nos côtes belges pendant les mois d'été.

La figure 23 en montre un bout de thalle rectiligne, dont un frustule complet, dans ses rapports de soudure avec son voisin. Les détails de structure du cytoderme y sont figurés tels qu'on peut les saisir à sec. L'extrémité brièvement biseautée du frustule se prolonge en une sorte de petite bulle, o, surmontée d'une pointe courte, aiguë.

La figure 24 en représente un autre exemplaire, en vue un peu oblique, avec le contenu cellulaire : $n$, noyau; $c h$, chromatophores petits, nombreux, pariétaux comme le noyau.

Distributiox GÉographique. - Exceptionnelle; dans les environs de Tromsö seulement. 


\section{Rhizosolenia delicatula Cleve.}

Planche XXVIII, figures 25,26 et 27.

Littérature. - Voir Nordisches Plankton, page 48.

Frustules cylindriques, courts, tronqués transversalement, surmontés d'une toute petite pointe latérale, $p$, qui, dans les colonies en chaînettes rectilignes (fig. 25), confine à l'organe similaire du frustule voisin.

Les chromatophores, ch, sont volumineux, mais peu nombreux.

Le noyau, $n$, est central ou pariétal, suivant les cas et suivant l'action des réactifs fixateurs.

La figure 26 en montre deux frustules vides, un peu séparés artificiellement l'un de l'autre pour mieux traduire les rapports de position des petites pointes, $p$.

La gélification facile de la membrane peu silicifiée des frustules, vers leur milieu, en $g$ (fig. 27), y détermine une déformation fréquente qui se traduit par une courbure ou même par une dislocation des membres de la colonie.

C'est un caractère accidentel que l'espèce revèt aussi bien dans la Mer Flamande où elle est parfois très abondante.

Distribution Géographigue. - Très rare. Ne paraît guère que dans le no $2 \mathrm{r}$, au débouché du Détroit de Matotchkine dans la Mer de Kara.

\section{Rhizosolenia Stolterfothii Peragallo.}

Planche XXVIII, figure 28.

Synonyme et littérature. - Cfr. Nordisches Plankton, page 49.

Les frustules sont courbes et associés en colonies souvent très longues et contournées en forme de ressort à boudin. Ils sont en rapport de soudure par des surfaces planes perpendiculaires à l'axe circulaire de la colonie, mais à aire réduite par une sorte de chanfrein circulaire qui rabat l'extrémité des individus associés.

Les petites pointes, $p$, bien visibles à l'extrémité libre des frustules; dont ils occupent toujours le côté convexe, le sont beaucoup moins entre deux membres accolés, où ils sont contigus.

Le cytoderme est annelé, comme on le voit dans la partie inférieure de la figure 28.

Le frustule supérieur de la mème figure montre la disposition habituelle du noyau, $n$, et des chromatophores, ch, fixés sur la paroi interne de la membrane.

Distribution géographique. - Seuls les produits de pèche du sud de la Mer de Barents nous ont présenté quelques rares spécimens de cette espèce si commune dans la Mer du Nord, sur les còtes belges spécialement. 


\section{Genre Eucampia Ehrenberg}

Caractères du Genre. - Consulter Nordisches Plankton, page 98.

\section{Eucampia groenlandica Cleve.}

Planche XXVIII, figures 29 et 30 .

Colonies courbes et spiralées dont les membres sont soudés par deux protubérances valvaires qui ménagent entre eux un foramen, $f$, dont la largeur varie avec l'âge des valves.

Les deux fragments figurés, de calibre différent, en montrent les détails morphologiques dans leur expression naturelle, en mème temps que des phases physiologiques de la cytodiérèse, avec les noyaux-filles; nd, séparés nouvellement par des fentes intervalvaires qui deviendront les foramens.

Les noyaux au repos, $n$, occupent le milieu des frustules; les chromatophores, ch, assez gros et en nombre variable, sont en rapport avec les cordons cytoplasmatiques qui irradient autour des noyaux.

La loi de constance de volume trouve son application ici comme dans les Diatomacées en général. Il en résulte des différences d'aspect, dues aux dimensions relatives des frustules, qu'il faut se garder d'ériger en variétés, comme certains ont une tendance à le faire.

Distribution GÉographique. - Rencontrée en spécimens assez rares dans la Mer de Barents et dans le Détroit de Matotchkine.

\section{Genre Leptocylindrus Cleve}

Caractìres DU GenRe. - Voir Nordisches Plankton, page 24.

\section{Leptocylindrus danicus Cleve.}

Planche XXVIII, figures 3 I et 32 .

Littérature. - Voir Nordisches Plankton, page 24.

Espèce commune et bien connue dont les deux figures ci-dessus suffisent à traduire le facies dans nos matériaux.

Frustules cylindriques solidement soudés, par leur base circulaire, en thalles rectilignes illimités. Le noyau, $n$, et les chromatophores, ch, plus ou moins nombreux 
sont pariétaux, quand la fixation a réussi à les maintenir en place dans leur position naturelle. Le cytoderme ne révèle aucune structure, pas même à sec; il ne porte non plus aucun appendice.

Distribution géographique. - Voisinage de Tromsö, dans les nos $\mathrm{I}$ et 2 , et Océan Glacial, dans les échantillons 216 et 217, particulièrement riches en Chétocérées.

\section{Genre Skeletonema Greville}

Caractères du Genre. - Voir Nordisches Plankton, page r 5.

\section{Skeletonema costatum (Greville).}

Planche XXVIII, figures 33 à 36.

Nos observations au sujet de cette espèce bien connue ne nous ont rien offert de particulier. Les quelques fragments de colonies figurés suffiront à en traduire les différences de calibre, depuis les plus étroits, à frustules très allongés (fig. 33 et 34), jusqu'aux plus larges, à frustules plus courts (fig. 35 et 36), dont quelques-uns en division, $d$.

On sait que les frustules cylindriques, bombés aux deux bouts, sont dépourvus de sculptures, mais sont surmontés, sur leurs deux valves, d'une couronne cylindrique de fins trabécules droits, qui se rattachent à ceux des éléments contigus dans la colonie, non toutefois dans leur prolongement direct, mais en alternant entre eux; ce qui n'est bien visible que dans les exemplaires larges (fig. 36).

Distribution Géographigue. - Rencontrée seulement près de Tromsö et près de la presqu'ile de l'Amirauté; toujours très peu abondante.

\section{Genre Thalassiosira CLeve}

Caractères du Genre. - Nordisches Plankton, page í.

\section{Thalassiosira gravida Cleve.}

Planche XXIX, figures I à 3 I.

Littérature. - Voir Nordisches Plankton, page is.

Certains de nos échantillons particulièrement riches en représentants de cette espèce nous ont entraîné à en présenter les principales variantes, pour en préciser les 
caractères morphologiques, les phases physiologiques de l'élaboration des endocystes et les cas de parasitisme dont elle est souvent atteinte de la part d'une Chytridiacée, un Olpidinm, sans doute.

Caractères morphologiques. - Les frustules sont réunis en colonies souvent très longues par un solide funicule, $f$. De calibre différent, variant du simple au double en diamètre (fig. I et 5), ils sont plus ou moins longuement cylindriques et présentent une ceinture toujours bien visible entre les deux valves. Celles-ci sont planes, mais brièvement arrondies sur leur bord circulaire. Sur ce bord s'implantent en grand nombre des soies gommeuses, s, qui vont obliquement à l'encontre de celles du frustule voisin et les croisent quand elles sont assez longues. Leur base est évidemment en rapport avec les fines ponctuations que l'on observe sur les valves examinées à plat et à sec, après avoir été vidées. Ces ponctuations plus ou moins serrées et abondantes (fig. 3I), parfois peu visibles ou presque inexistantes (fig. 29), ne se traduisent que sur la lisière arrondie des valves et non sur la partie centrale qui est plane. Elles ne se traduisent par aucun relief, même sur la coupe transversale optique des valves, quand les soies gommeuses paraissent ou sont réellement absentes.

Celles-ci ne se montrent en effet pas toujours avec la mème évidence, mais l'action de l'iode en solution alcoolique en révèle souvent l'existence là où l'examen en milieu quelque peu éclaircissant n'en laissait pas percevoir d'abord.

Ce caractère nous a surtout paru prononcé dans les spécimens originaires de la Mer de Kara, comme ceux des figures 7 et 8, où l'on observe parfois des frustules très longs, $a$ (fig. 7), encerclés d'une auréole de soies, en leur milieu, avant même que la division ne s'y soit produite.

Le noyau, $n$, toujours central, est logé dans un cylindre de protoplasme dense qui traverse la cavité cellulaire suivant l'axe de symétrie du frustule et s'étale ensuite en cordons sur toute la paroi interne où sont disséminés les chromatophores assez nombreux, bien délimités, en plaquettes de forme variable.

Les figures I, 3, 5 montrent ces détails en vue stéréoscopique, en $a$, et en coupe optique, en $b$, dans des frustules ordinaires; en $c$, et $d$, dans des frustules en voie de division plus ou moins avancée.

Les figures 2, 4, 6 en sont des vues valvaires correspondantes, avec ou sans soies périphériques.

Endocystes. - Comme les frustules sont en colonie et que beaucoup sont en voie de rénovation cellulaire pour la production de spores quiescentes, il est facile d'en suivre les phases évolutives dans leur succession naturelle.

Nous les suivrons d'abord dans les figures I5 à $2 \mathrm{I}$, à travers une série de couples de frustules solidarisés en quelque sorte pendant la sporification. Pour plus de clarté, les éléments sont figurés en coupe longitudinale optique. 
Figure I5. Le phénomène débute par une cytodiérèse un peu inégale qui, à première vue, paraîtrait ordinaire, mais qui n'est pas précédée d'une caryocinèse.

Le noyau indivis reste dans l'une des deux moitiés, la plus grande, au sein du cordon protoplasmatique axial que la cytudiérèse a coupé en deux parties inégales. C'est donc une réduction cellulaire plutòt qu'une véritable cytodiérèse. Aussi bien. comme nous allons le voir, cette division purement cytoplasmatique n'est pas suivie de l'élaboration de deux valves symétriques, jeunes, clestinées à compléter des cellulesfilles normales, mais seulement de fines membranes azotées propres à chacune des deux moitiés.

Figure I6. La partie nucléée, $a$, se renfle ensuite en développant une courbure de plus en plus prononcée dans la partie stérile, $b$, et la mème chose se produit dans la seconde cellule du même couple physiologique, mais en ordre inverse, de manière à présenter entre elles deux une disposition symétrique par rapport à un plan mené entre elles perpendiculairement à leur axe longitudinal.

Il semble bien que la partie nucléée se nourrit aux dépens de la partie stérile, car le cytoplasme de celle-ci subit une réduction progressive pendant que l'autre s'amplifie et gagne en densité.

Figure I7. La convexité de la partie nucléée a refoulé la membrane de la partie stérile. Celle-ci, devenue maintenant très concave, semble avoir été aidée dans son mouvement de refoulement par la rétraction du culot protoplasmatique axial, désigné comme résidu par la lettre $r$, qui y reste adhérent en son milieu.

Entre les deux membranes internes, dont l'une, celle de la partie stérile, est devenue plus épaisse, tout en restant homogène et réfringente, on aperçoit un faible intervalle un peu plus large au centre que sur les bords. C'est ce que nous devons considérer comme le moule, $m$, dans lequel prendra naissance la valve primaire de l'endocyste.

On remarquera que l'évolution est parallèle et symétrique dans les deux frustules du mème couple.

Figure IS. Le moule, $m$, décrit dans la figure précédente, est ici occupé par la valve primaire, $v$, de l'endocyste. Le culot protoplasmatique, $r$, subsiste toujours, maintenant en place la matricule concave de la valve; mais le cytoplasme, avec les chromatophores y inclus, s'atrophie de plus en plus en se résorbant sans doute au profit de la partie nucléée.

Figure I9. La partie nucléée subit une nouvelle réduction qui se produit cette fois du côté opposé. Son cytoplasme se rassemble partiellement autour du noyau et s'entoure d'une membrane nouvelle, $m^{\prime}$. Celle-ci prend une courbure caractéristique et laisse en dehors d'elle une partie du cordon axial, $r^{\prime}$, en mème temps que d'autres fragments non utilisés du cytoplasme et mème certains chromatophores. Elle doit constituer la matrice de la valve secondarre, $v^{\prime}$, du kiyste et sera, à cette fin, maintenue dans la forme voulue par le culot protoplasmatique, $r^{\prime}$. 
Figure 20. On assiste ici au moulage, peut-on dire, de la valve secondaire, $v^{\prime}$, dans sa matrice, $\boldsymbol{m}^{\prime}$. En effet, celle-ci, en se dédoublant pour isoler la portion nucléée de la partie nouvellement sacrifiée, a ménagé un intervalle étroit dans lequel on voit se mouler la valve secondaire. Celle-ci s'adapte circulairement à la primaire, pour compléter la coque de l'endocyste en lui donnant sa forme lenticulaire.

En mème temps le premier culot, $r$, qui maintenait en place la matrice, $m$, de la valve primaire, est devenu inutile et se résorbe plus ou moins complètement.

Le culot opposé, $r$, encore utile dans sa fonction vis-à-vis de la seconde matrice, $m^{\prime}$, persiste jusqu'à la consolidation de la valve secondaire, $v^{\prime}$.

Figure 2I. C'est la dernière étape pendant laquelle les valves se consolident et se silicifient au sein de leur matricule, en mème temps que disparaissent les derniers vestiges du cytoplasme résiduaire.

Le phénomène a pris fin avec l'individualisation complète du kyste, vers le milieu du frustule dont il semble utiliser la ceinture étroite.

On peut dire qu'il consiste en une rénovation cellulaire opérée en deux temps, avec perte répétée d'une partie du cytoplasme, qui concourt néanmoins d'une façon curieuse au succès de l'opération, par la résorption dont il est l'objet.

Les figures 9 à II en retracent les principales phases dans des frustules de grand calibre.

Couple de la figure 9. Production de la valve primaire, $v$, sous sa matrice, $m$, un peu écartée par l'action des réactifs fixateurs et maintenue par son culot, $r$.

Couple de la figure Io. Constitution de la matrice, $m^{\prime}$, de la valve secondaire, $v^{\prime}$, avec son culot, $r^{\prime}$.

Couple de la figure i I. Formation de la valve secondaire, $v^{\prime}$, et dissolution du culot, $r^{\prime}$.

Les figures I2 à I4 en montrent le facies particulier dans les spécimens étroits.

Couple de la figure I2. Etablissement de la matrice, m, maintenue en sa forme par le culot, $r$.

Couple de la figure I3. Genèse de la valve primaire, $v$, sous sa matrice, $m$, et production de la matrice, $m^{\prime}$, génératrice de la valve secondaire, avec son culot, $r^{\prime}$.

Couple de la figure I4. Création de la valve secondaire, $v^{\prime}$, disparition des culots et autres parties résiduaires et constitution définitive de la spore.

Dans ces figures 9 à I4, nous nous sommes abstenu de représenter les déchets des divisions fragmentaires du cytoplasme, pour ne pas obscurcir les détails de l'élaboration des valves, à cause de la petitesse de l'objet.

Enfin, les figures stéréoscopiques des couples 26, 27 et 28 donneront une idée de l'aspect extérieur des frustules sporifères, abstraction faite du cytoplasme.

Couple de la figure 26. Valve primaire de l'endocyste sous sa matrice fixée par le culot, $r$. 
Couple de la figure 27. Achèvement de la spore par la production de la valve secondaire, $v^{\prime}$, dont la matrice est encore tenue en place par le culot, $r^{\prime}$. Le culot, $r$, de la matrice primaire est désaffecté et en voie de résolution.

Couple de la figure 28. Spores définitivement constituées au sein de leur matricule; celle-ci vidée de tous les produits accessoires.

Les spores ont, comme on le voit, la forme d'une lentille légèrement dissymétrique, dont un côté - celui de la valve primaire - est un peu plus convexe et d'une courbe un peu différente.

Il reste à faire remarquer la structure intime des valves de l'endocyste.

Vues de face, dans les figures 30 et 32 , elles révèlent une texture très finement alvéolaire à pores arrondis, très serrés, un peu moins étroits vers le centre que vers la périphérie, sans orientation radiale marquée.

En coupe transversale optique (fig. Is à $2 \mathrm{I}$, par exemple), les valves, un peu plus épaisses au centre qu'à la périphérie, sont manifestement marquées d'une striation très fine qui correspond aux parois des pores alvéolaires.

Parasitisme. - Les frustules de cette intéressante espèce sont très souvent infestés d'Olpidium, particulièrement dans la Mer de Kara, où les représentants traduisent moins d'activité physiologique et sont beaucoup moins sporifères.

Quelques exemples suffiront à en montrer le caractère.

Dans la figure 22, la Chytridiacée, ol, a formé ses sporules en un sporange sphérique.

La déhiscence d'un sporange plus avancé se prépare dans la figure 23, par la formation d'un prolongement herniaire que perforera bientôt la membrane du frustule parasité, par le moyen d'une diastase spécifique capable de dissoudre la silice.

Ce résultat est obtenu dans la figure 24 , où la membrane de l'hôte présente deux orifices, o, qui ont donné issue aux sporules de deux sporanges d'Olpidium logés sans doute d'abord côte à côte dans le même frustule.

La ceinture est un endroit d'élection pour cette perforation, à cause probablement de la moindre résistance et de la moindre silicification de cette partie du frustule.

La déformation corrélative que l'on observe dans ce dernier semble indiquer que l'action de la zymase s'est exercée également, mais à un moindre degré, sur une aire plus étendue et a assoupli la membrane en la rendant plus extensible.

Il n'est pas rare de voir des frustules ainsi attaqués se disloquer en deux pièces, à la suite d'une gélification plus ou moins complète de la ceinture.

Distribution géographioue. - Assez répandue dans nos échantillons de surface des deux Mers de Barents et de Kara. Abondante surtout dans l'Océan Glacial où elle sporifie largement. 


\section{Thalassiosira rotula sp. $\mathrm{n}$.}

Planche XXIX, figures 67 à 70 .

Nous croyons utile de présenter ici, pour la mettre en parallèle avec Th. gravida Cleve, une espèce que nous étions loin de supposer, sinon inconnue, du moins non signalée par les planktonistes du nord, tant elle nous est familière de par nos recherches sur le microplankton de la Mer Flamande, poursuivies depuis igoz.

C'est une de nos plus vieilles et aussi de nos plus fidèles connaissances dans ce singulier petit monde de Diatomacées pélagiques flottantes.

Comment croire que cette forme si commune sur nos côtes belges pouvait ne pas ètre ce qu'une fausse indication nous avait fait prendre pour Th. gravida, espèce sıgnalée comme répandue partout dans le domaine où s'exerce l'activité de la Commission Internationale instituée pour l'exploration de la Mer.

Il fallait la publication du travail synthétique de Gran sur les Diatomacées observées et décrites dans ce vaste ensemble de recherches, pour nous désabuser, en nous démontrant que l'espèce que nous avons ailleurs signalée sous le non Th. gravida n'est pas celle qui répond à ce nom et dont il s'est agi ci-devant, si l'on s'en réfère aux caractères attribués à celle-ci par Cleve : Diatoms from Baffins Bay and Davis Strait. Stockholm, Igo6, dans Bihang t. k. Sv. Vet. Ak. Handl., Bd 22, Afd. III, no 4, pl. II, fig. I4 à I6.

Nous confessons notre erreur; erreur complète, radicale, car il ne s'agit pas d'une simple confusion de deux formes insuffisamment distinguées et réunies sous une dénomination commune. En effet, Th. gravida Cleve n'existe pas sur les còtes belges, si ce n'est peut-ètre dans des conditions d'excessive rareté, qui, éventuellement, nous l'ont fait ranger, comme forme douteuse, sous la rubrique peu compromettante de Thalassiosira sp.

Celle qui y abonde est celle que nous devons présenter ici sous une rubrique nouvelle, parce qu'elle ne figure pas parmi les espèces recensées par Gran, dans Nordisches Plankton.

Nos matériaux de la Belgica ne nous en ont présenté que l'un ou l'autre spécimen, dont l'excessive rareté nous fait douter qu'ils soient bien originaires des parages de la Nouvelle-Zemble et n'aient pas été plutòt introduits accidentellement dans nos préparations par l'intermédiaire d'une pipette qui n'aurait pas été suffisamment lavée, après avoir servi à manipuler des produits de la Mer Flamande.

Caractères morphologigues. - Colonies dont les membres sont maintenus a distance régulière par un funicule, $f$, plus épais et plus rigide que dans aucune autre espèce. Frustules cylindriques en forme de disques aplatis, formés de deux valves circulaires planes, un peu défléchies seulement sur leur extrème bord, et relevés, sur la face suturale cylindrique, d'un ou deux annean x épais qui simulent des bandages étroits appliqués sur la jante trop large d'une roue, "rotula ». 
Ces anneaux constituent le trait saillant de la physionomie de l'espèce.

Très visibles, en $o$, sur les frustules vus de champ, comme dans la figure 67 , $a$ et $b$, ils ne forcent pas moins l'attention en vue valvaire et s'imposent encore plus quand on les voit isolés, après macération voulue ou après destruction accidentelle des frustules, comme en o, figure 70. Ces anneaux, excentriquement développés, sont toujours plus épais d'un còté que de l'autre. Ils sont les premiers à appeler l'attention sur la présence de l'espèce dans les slides où elle est peu représentée.

Le noyau est central et logé dans un gros cordon axial de protoplasme dense (fig. $67, c$, qui reproduit deux frustules en coupe optique).

Les chromatophores, $c h$, sont pariétaux et surtout abondants sur les faces valvaires des frustules (fig. 68). Ceux-ci, vidés et examinés à sec, laissent voir toute la surface de leurs faces valvaires couverte de ponctuations assez régulièrement distancées mais sans ordre apparent (fig. 69). Ces valves présentent ainsi beaucoup d'analogie d'aspect avec celles de Lauderia glacialis (Grunow) (fig. 66, même planche), dont il sera question plus loin.

Bien que nous ayons eu cette franche espèce sous les yeux, plusieurs années durant, surtout pendant les mois d'hiver, époque de sa plus grande fréquence sur nos còtes belges, nous n'avons pas souvenir de l'avoir vue en sporification.

Distribution GÉographique. - Il est probable, comme nous l'avons suggéré tout à l'heure, que cette espèce n'est pas boréale.

\section{Thalassiosira Nordenskiöldii Cleve.}

Planche XXIX, figures 33 à +4 .

\section{Littérature. - Cfr. Nordisches Plankton, page i6.}

Jolie petite forme, bien connue, très répandue et toujours aisément reconnaissable avec un peu d'attention, malgré les modalités diverses qu'elle peut revètir.

La figure 38 la montre, en vue stéréoscopique, sous des dimensions très faibles, en colonie formée de frustules diversement écartés, $a, b, c$, d'après l'époque relative de leur individualisation. Ceux-ci sont souvent chargés, comme en $d$, de soies gommeuses, fugaces, qui sont en relation avec les spinules qui garnissent la zone tronquée en chanfrein de la périphérie des valves. La figure 39 en est une vue valvaire.

La figure 40 la montre sous un autre calibre; à frustules plus larges mais moins hauts, distancés, en $a$, par le funicule; encore soudés, en $b$, à la suite d'une cytodiérèse récente.

Les mêmes sont reproduits, en coupe optique, en $a, b, c$, de la figure 4 , à différentes phases de leur division, de manière à montrer la petite dépression qui occupe le centre des valves et dans le fond de laquelle le funicule prend son point d'attache. 
La figure 42 en est une vue valvaire montrant la forme et la disposition des chromatophores, qui sont habituellement pariétaux.

Dans la figure 43, on voit les frustules diversement développés d'une colonie, $a, b, c$, reliés entre eux non seulement par le funicule axial, mais en outre par une gaine gommeuse, $g$, qui a ses attaches sur les spinules valvaires, $p$, vues de profil.

Celles-ci sont figurées en projection sur le bord chanfreiné d'une valve vue de face, dans la figure 44 .

Les différentes phases de la cytodiérèse sont représentées, en coupe optique, dans les éléments $a, b, c$, de la figure 33, empruntée à un thalle de calibre moyen.

Endocrstes. - La sporification est très rare dans nos matériaux actuels. Ceux que nous possédons de la Mer Flamande, où l'espèce est commune, se prêteraient mieux à l'étude de ce phénomène; mais nous ne voulons pas nous en servir ici, pour ne pas sortir du cadre restreint que nous nous sommes imposé dans ce travail.

Les figures 34 et 35 reproduisent les seules étapes observées ici; c'est-à-dire la formation de la valve primaire, $v$, de l'endocyste, présentée en coupe optique, avec contenu cellulaire, dans la figure 34 ; en vue stéréoscopique, abstraction faite du cytoplasme, dans la figure 35.

La figure 36 en reproduit les détails de structure, en vue valvaire. Cette structure est celle qui résulterait de stries menées dans trois directions, sous une incidence respective de $60^{\circ}$, déterminant conséquemment des alvéoles hexagonaux orientés comme ceux de Coscinodiscus lineatus, mais en beaucoup plus petit. Des denticules nombreux en ornent le pourtour. On constatera, par comparaison avec la valve de la matricule mise en regard figure 37, la différence entre celle-ci et celle de l'endocyste.

Distribution géographigue. - Assez abondante dans le Détroit de Matotchkine et à son voisinage dans la Mer de Kara; rencontrée aussi dans l'Océan Glacial.

\section{Thalassiosira bioculata (Grunow).}

Planche XXIX, figures $4^{5}$ à 62 .

Synonmme et littérature. - Cfr. Nordisches Plankton, page ig.

Frustules de grande taille, cylindriques, faiblement reliés en colonie par un funicule, $f$, fragile et inconstant, pourvus de chromatophores pariétaux, ch (fig. 45 et 49), et d'un cordon cytoplasmatique axial, dans lequel se trouve blotti le noyau, n, au repos dans la figure 45; en division, $n d$, dans la figure 49.

Dans nos matériaux, les parois suturales des frustules sont le plus souvent exemptes de toute trace de cercles visibles (fig. 47 et 5I); plus rarement on en distingue sur une zone plus ou moins large (fig. 56 et 57 ). 
Les faces valvaires circulaires sont planes dans leur milieu et ont leur pourtour faiblement rabattu. C'est là que sont portés des denticules très peu saillants, de simples ponctuations, $p$, que l'on peut voir de profil dans la figure 47 , mais qui apparaissent beaucoup mieux, à sec, sur la vue valvaire de la figure 48 , où on les voit disposés en cercle, tout près du bord, sur une seule rangée, au nombre de vingt-cinq à trente, équidistants.

Vers le centre s'observent deux ponctuations un peu excentriques, d'où l'espèce tire son nom.

Elles sont en relation avec le funicule dont elles sont les points d'attache.

Endocystes. - La sporification n'est pas rare dans nos matériaux de l'Océan Glacial; mais la dislocation habituelle des colonies en individus épars et le morcellement facile de ceux-ci rendent assez difficile la reconstitution des phases successives du phénomène. Celui-ci ne suit du reste pas toujours la mème voie.

a Valve primaire. - La division interne par laquelle il débute (fig. 49) n'est souvent suivie de l'apparition que d'une seule valve primaire, $v$ (fig. 5o). Dans ce cas, le frustule ne produira qu'un seul endocyste, aux dépens de la cellule-fille qui l'a formée; l'autre cellule-fille restera stérile et, bien qu'elle ait un noyau, $m$, elle ira s'atrophiant de plus en plus, en servant de nourrice, en quelque sorte, à sa sceur jumelle. Le frustule de la figure 5i, dont le développement est symétrique à celui de la figure 5o, qui lui est contigu dans la colonie, montre la même phase, abstraction faite $\mathrm{du}$ contenu cellulaire.

Cette atrophie, visible dans la figure 5o, est plus avancée déjà dans la figure 46 , où l'on voit les chromatophores en voie de régression, comme aussi le culot, r, résidu du cordon cytoplasmatique axial de la partie stérile. La figure 47, qui lui est similaire, fait abstraction du cytoplasme de toute la matricule.

D'autres fois (fig. 53), la division interne de la cellule est égale, et les deux cellules-filles, avec leur part équivalente du noyau, nd, et du cytoplasme, se constituent chacune une membrane convexe, $m$, qui deviendra la matrice d'une valve primaire.

Dans la figure 55, on voit, en coupe optique, les deux valves primaires, $v$, ainsi produites, opposées dos à dos. Ces valves manquent d'adhérence avec la paroi du frustule matricule. Cela explique leur glissement facile à l'intérieur de celui-ci et la position souvent oblique (fig. 54) que la contraction du cordon axial, pc, leur fait prendre en les écartant sous l'action des réactifs fixateurs.

La figure 57 montre un frustule vide, arrivé à cette étape. Souvent il se produit alors une rupture médiane de la matricule; chaque cellule-fille emportant avec elle, à l'une de ses extrémités, la valve primaire, v, de son endocyste (fig. 58).

\& Valve secondaire. - La figure 60 montre comment se forme la valve secondaire destinée à compléter le kryste. 
On voit qu'une partie seulement du cytoplasme de la cellule fertile se ramasse sous la concarité de la valve primaire, $v$, et $y$ prend d'abord une forme hémisphérique en s'entourant d'une mince membrane azotée, m', qui sera la matrice de la valve secondaire. Une partie des chromatophores, chr , et du cytoplasme axial, $r^{\prime}$, est laissée en dehors et ne pourra servir éventuellement clu'à nourrir l'endocyste par endosmose, après digestion.

$\gamma$ Maturation de L'endocyste. - Dans la figure 6i, la dernière étape est franchie et la spore, constituée de ses deux valves symétriques et peu bombées, $v$ et $v^{\prime}$, reste logée dans la matricule complètement vidée, dont elle n'est qu'un produit de rénovation, avec abandon momentané d'une partie du cytoplasme, qui est néanmoins utilisée plus tard indirectement.

La figure 62, qui montre le kyste à l'état isolé et de profil, laisse voir comment les deux valves se raccordent par l'intermédiaire d'un anneau dépourvu de structure et limité par un étroit sillon, déjà noté, en $s$, dans la figure 58.

En vue valvaire (fig. 59), la spore révèle une fine structure analogue à celle de Coscinodiscus excentricus, résultant de trois systèmes de stries qui se coupent sous des angles de $60^{\circ}$. Elle ne porte pas de ponctuations périphériques; bien que cela paraisse toutefois lorsque, comme dans la figure 52, la valve de la matricule se projette sur la spore encore incluse.

Distribution géographique. - Assez rare dans le Détroit de Matotchkine et au voisinage de celui-ci dans les deux Mers de Kara et de Barents; plus répandue dans l'Océan Glacial, où elle est sporifère.

\section{Thalassiosira fallax sp. n.}

Planche XXX, figures I à 4 .

Nous désignons ainsi une petite forme, que nous aurions facilement confondue avec Th. gravida, si l'étude du phénomène de sporification ne nous avait appris à l'en distinguer.

Le thalle végétatif en chaînette (fig. I) est formé de frustules cylindriques, à peine un peu rabattus sur le pourtour des valves; plus courts en $a$, plus longs en $b$, réunis par un funicule axial intercalaire assez long ou rapprochés souvent, comme en $c$, en petits groupes de quelques individus, porteurs ou non de soies gommeuses insérées sur le bord circulaire des valves.

Endocystes. - La sporification débute (fig. 2, vue en coupe optique) par une division le plus souvent droite, comme en $d$, parfois oblique, comme en $c$, suivie directement de la production de deux matrices convexes, placées dos à dos, comme en $f$, 
génératrices de deux valves primaires, comme en $g$, qui sont des indices certains de la formation de deux spores jumelles, au sein d'une même matricule. Par ce fait seul déjà cette petite espèce diffère essentiellement de Th. gravida.

L'évolution ultérieure (fig. 3, vues stéréoscopiques, sans figuration du cytoplasme) nous apprend que la matricule, bipare en $h$, se clive ensuite, pour isoler les deux moitiés symétriques, $i$ et $i$, qui élaborent individuellement leur valve secondaire et montrent, en $j$, leur endocyste complet, dans leurs relations génétiques avec les demifrustules dont on voit les restes vidés.

La spore ainsi réalisée est de forme lenticulaire. Ses deux valves présentent à peu près la même convexité, elles sont assez épaisses. Vues de face (fig. 4), elles témoignent d'une structure très finement alvéolaire et portent une garniture périphérique de fins denticules, visibles au moins sur la valve primaire.

Distribution GÉographique. - Assez rare, dans le Détroit de Matotchkine, et facile à confondre avec les petits spécimens de Ch. gravida, à l'état végétatif.

\section{Genre Lauderia Cleve}

\section{Caractères du Genre. - Voir Nordisches Plankton, page 22.}

\section{Lauderia glacialis (Grunow).}

Planche XXIX, figures 63 à 66 , et planche XXX, figures 5 à 9.

Synonyme et littérature. - Cfr. Nordisches Plankton, page 23.

Frustules assez grands, cylindriques, légèrement renflés sur les valves circulaires qui présentent toutefois, vers leur centre, une dépression ombilicoïde signalée, en om (fig. 64).

La figure 63 en reproduit un élément en vue stéréoscopique pour en montrer le cordon cytoplasmatique axial et les nombreux chromatophores ramenés presque tous sous les faces valvaires.

Dans la Mer de Kara, il n'est pas rare de les voir réunis en colonies linéaires, fragiles, où ils n'ont pas d'autres traits d'union que de nombreuses soies gommeuses, $s$, insérées surtout sur le pourtour des valves et dirigées soit obliquement, pour se croiser avec celles du membre voisin, soit même dans une position renversée.

Vues de face et à sec (fig. 66), les valves se montrent couvertes de ponctuations distancées, semées sans ordre sur toute la surface, mais beaucoup plus denses vers la périphérie. Ces ponctuations sont les empreintes évidentes de fins filaments mucilagineux que leur délicatesse empêche de saisir et que leur fragilité rend fugaces.

On ne manquera pas de remarquer la similitude frappante de ces valves avec celles de Thalassiosira rotula nobis (fig. 69). Nous en avons à dessein rapproché les 
figures au bas de la planche XXIX. Si l'on n'en connaissait d'ailleurs l'origine différente, on pourrait hésiter à les distinguer, car l'écart de grandeur ne constituerait pas toujours un critérium suffisant.

Sporification. - Les figures 5 à 9 de la planche XXX se rapportent à la production des endocystes.

Figure 5. Frustule ordinaire dont les chromatophores, ch, ont quitté, en partie, les parois valvaires pour se disposer sur le cytoplasme axial.

Figure 7. Division intérieure, en $d$, d'un frustule qui deviendra sporifère et qui montre une disposition analogue des chromatophores.

Figure 6. Le même présenté en vue valvaire. On y voit la disposition rayonnante de cordons protoplasmatiques qui en garnissent la paroi interne, autour des chromatophores, ch, ramenés au milieu.

Figure 8. L'une des deux cellules-filles issues d'une cytodiérèse récente se transforme seule en spore, $s p$, dont la valve primaire, $v$, semble résulter de la modification directe du disque externe préalablement bombé. Le retrait du cytoplasme sous cette valve prépare l'apparition de la valve secondaire.

La spore sera donc ici le fruit d'une rénovation cellulaire, sans autre perte de substance que la paroi cylindrique de la matricule.

Figure 9. Valve primaire du kyste, vue de face. La structure en est très finement ponctuée; de nombreux denticules, également distancés sur une seule rangée, en garnissent le pourtour.

Nous devons faire remarquer que nous n'avons jamais vu la spore se former de toutes pièces au milieu d'une cellule génératrice, comme le figure Gran, loco citato.

Le processus varierait-il avec les conditions d'ambiance?

Distribution géographioue. - Assez répandue aux environs de Tromsö et dans la Mer de Kara, très rare ailleurs; en sporification exceptionnellement dans l'Océan Glacial.

\section{Genre Bacterosira GRAN}

Caractères du Genre. - Consulter Nordisches Plankton, page $2 \mathrm{I}$.

Bacterosira fragilis (Gran).

Planche XXX, figures io à r 5 .

Synonyme et littérature. - Cfr. Nordisches Plankton, page 2 I.

Frustules cylindriques, un peu rodés sur les bords circulaires des valves, étroitement unis en chaines indéfinies, tout en ménageant entre eux un étroit foramen, $f$ (fig. II), dont l'observation demande un peu d'attention. 
La mobilité bien connue des chromoleucites et leurs migrations dans la cellule, sous l'influence des variations de la lumière principalement, expliquent les différences d'aspect entre les frustules de la figure rI, où ces éléments, ch, tapissent la paroi cylindrique, et ceux de la figure I2, où ils ont déserté cette paroi pour se grouper sous les faces valvaires.

La figure r3 reproduit la vue extérieure de frustules vides, pour mortrer la disposition variable des anneaux de la ceinture et noter, en $x$, le cloisonnement assez souvent oblique qui préside à leur multiplication.

Endocystes. - Nos matériaux ne nous fournissent pas des sujets d'étude du processus de sporification; mais ils nous présentent les spores, sp, toutes faites, au milieu de leurs matricules restées en chainette, comme dans la figure I4.

Ces spores, de forme lenticulaire, résultent de la juxtaposition de deux valves modérément bombées, dont la figure i5 donne une vue de face.

La structure en est finement alvéolaire, difficile à saisir à ce faible grossissement; ses bords ne sont pas garnis de ponctuations ou de denticules, ce qui permet de distinguer les valves isolées de $B$. fragilis de celles, aussi petites et de même aspect à première vue, de Thalassiosira fallax nobis (fig. 4, même planche).

Parasitisme. - La Chytridiacée phycophage, espèce d'Olpidium déjà citée plus haut, exerce ici ses ravages fréquemment. La figure to en montre deux thalles à des phases évolutives différentes, $a$ et $b$, dans un même frustule.

Distribution géographique. - Détroit de Matotchkine et nord de la Mer de Barents, dans des numéros de pèche riches en Chétocérées.

\section{Genre Melosira Agardh}

Caractères du genre. - Voir Nordisches Plankton, page ir.

\section{Melosira hyperborea (Grunow).}

Planche XXX, figures 16 à $2 \mathrm{r}$.

Synonymie et littérature. - Cfr. Nordisches Plankton, page i3.

Frustules cylindro-sphériques, à parois assez minces, dépourvus de structure visible, rattachés en colonie par une sorte de joint annulaire fixé au sommet très convexe des valves. 
Cet anneau, qui n'est guère que la délimitation circulaire de l'aire de contact des frustules voisins, ne s'observe bien que sur la vue valvaire, comme dans la figure ig.

Les membres d'une même colonie, différents par l'âge, diffèrent aussi par le développement en largeur de la ceinture cylindrique, à l'abri de laquelle les divisions et subdivisions se répètent (fig. I6, I7 et I8 d'une même colonie de calibre moyen).

Dans les colonies d'un moindre calibre (fig. 20), les jeunes frustules revêtent la forme d'un ellipsoïde plus ou moins allongé. Pour un calibre supérieur (fig. 2I), les frustules jeunes se rapprochent de la forme sphérique.

Les chromatophores, ch, sont assez gros. Nous les avons figurés dans leur position naturelle dans plusieurs éléments des figures I6, I7 et 2 I.

Distribution géographique. - Rencontrée surtout dans les numéros 30 et 34 de la Mer de Kara; assez rare et exceptionnelle ailleurs.

\section{Melosira Borreri Greville.}

Planche XXX, figures 22,23 et 24 .

Littérature. - Cfr. Nordisches Plankton, page i 2.

Espèce bien connue, dont il suffira de reproduire ici l'aspect en précisant les seuls détails visibles, au grossissement employé.

Frustules cylindriques, à valves circulaires un peu convexes, à membrane épaisse, couverte de ponctuations fines et serrées, à ceinture striée circulairement (fig. 22, vue stéréoscopique, à sec; fig. 23, vue en milieu liquide).

La figure 24 en montre, vue à sec et de face, la valve, au milieu de laquelle on aperçoit l'aire de contact plus légèrement ponctuée.

Distribution Géographique. - Très rare; à Tromsö et sur les glaces de la Mer de Kara.

\section{Melosira Jurgensii Agardh.}

Planche XXX, figure 25.

Litrérature. - Voir Nordisches Plankton, page i 2.

Frustules cylindro-sphériques dont la paroi des valves va s'amincissant vers les bords de suture. Ceinture plus ou moins large, suivant l'àge des éléments de la colonie.

Nous en avons figuré l'aspect en milieu liquide. A sec, les valves se montreraient finement ponctuées.

Distribution géographique. - Rare, dans certains échantillons du Détroit de Matotchkine. 
Melosira varians Agardh.

Planche Xxx, figure 26 .

Littérature. - Cfr. H. Tan Heurck, Traité des Diatomées, page 44I.

Frustules cylindriques, à valves presque planes, présentant une grande surface de contact entre les éléments voisins et favorisant l'adhérence de ceux-ci en colonies linéaires.

La figure 26 en retrace les caractères, en milieu liquide, d'après un des rares spécimens égarés dans nos échantillons marins. On sait, en effet, que l'espèce est d'eau douce.

Distribution céographique. - Rencontrée sur les glaces de la Mer de Kara; assez rare.

\section{Melosira nummuloüdes (Dillwyn).}

Planche XXX, figure 27.

Synonyme et littérature. - Cfr. Nordisches Plankton, page i2.

Frustules souvent géminés dans la ceinture de leur cellule-mère, plus ou moins allongés et partant ellipsoïdes ou sphéroïdes. Vers le milieu de la partie courbe des deux valves on observe un anneau saillant qui se rattache circulairement à la ceinture cylindrique de la matricule, avec une convexité inverse de celle de la valve.

La figure 27 reproduit un fragment de colonie moniliforme, en vue stéréoscopique, en milieu liquide; circonstance qui ne permet pas d'apercevoir les fines stries ponctuées qui décorent les valves.

Distribution géographique. - Environs de Tromsö; espèce littorale.

\section{Melosira granulata Ehrenberg.}

Planche XXX, figure 28 .

Littérature. - Voir H. Van Heurck, Traité des Diatomées, page 444.

Petite forme à frustules cylindriques, ornés de lignnes droites ou obliques de ponctuations assez écartées.

Distribution géographique. - Neiges colorées; sur les glaces de la Mer de Kara. 


\section{Genre Paralia Heiberg}

Les caractères du genre sont ceux de l'unique espèce ci-après :

Paralia sulcata Ehrenberg.

Planche XXX, figures 29 à 32 .

Synonyme et littérature. - Cfr. Nordisches Plankton, page i4.

Frustules brièvement cylindriques, associés en colonies rigides et résistantes, ce qu'ils doivent à l'épaisseur et à la structure particulière de leur membrane.

La vue de quelques spécimens de calibre différent, figurés en vue stéréoscopique, suppléera avantageusement à toute description littéraire qui manquerait assurément son but; car s'il est vrai que cette espèce est facile à identifier, les détails de structure en sont aussi difficiles à saisir qu'à figurer et à décrire (fig. 29, 3I et 32). La figure 30 en est une vue valvaire optique.

Distribution GÉographique. - Remarquablement rare, ici et là, dans les récoltes de la Belgica.

\section{Genre Coscinodiscus Ehrenberg}

Caractères du genre. - Cfr. Nordisches Plankton, page 27, et H. Van Heurck, Traité des Diatomées, page 525.

Coscinodiscus lacustris Grunow.

Planche XXX, figures 33 et 34 .

Sinonymie et mittérature. - Cfr. H. Van Heurck, loc. cit., page 533.

Forme assez petite de Coscinodiscus dont les valves circulaires, vues de face (fig. 33), sont couvertes de ponctuations très fines disposées en séries radiantes fort rapprochées.

Ces valves, au lieu d'être planes ou plus ou moins convexes, comme il arrive dans d'autres espèces, présentent des ondulations qui se reproduisent en sens inverse des deux còtés du frustule et donnent à l'objet, vu de profil, l'aspect de la figure 34 
qui le montre tordu, dans cette position, bien que la ceinture ou zone connectire ne participe pas à la torsion.

Il importe de placer l'organisme bien de champ pour percevoir ce 'caractère particulier.

H. VAN Heurck range cette espèce dans son groupe des Ponctuées.

Distribution géographigue. - Espèce réputée d'eau douce. Très rare sur les glaces de la Mer de Kara.

\section{Coscinodiscus oculus iridis Ehrenberg.}

Planche XXX, figures 35,36 et 37 .

La littérature relative à cette belle forme planktonique est fort embrouillée.

Réunie par certains auteurs, VAN HEURCK, par exemple, à C. radiatus; démembrée elle-même, par d'autres, en sous-espèces, C. oculus iridis a fini par perdre sa spécificité taxinomique. A notre avis, il y a lieu de la lui rendre, car peu de formes ont autant de titres qu'elle au rang d'espèce.

Frustules grands ou même très grands, libres, en forme de boîte ronde dont les bords cylindriques s'engagent l'un dans l'autre, comme dans la plupart des Diatomées naturellement, mais avec une évidence qui force l'attention, à cause de l'épaisseur des deux pièces.

La figure 37 , qui en reproduit de profil un échantillon plutôt petit, montre ce caractère si net sur la zone connective. Celle-ci présente, en outre, des cercles dont le nombre et la disposition, variables avec l'àge et l'état physiologique des individus, ne sauraient guère fournir un caractère de quelque valeur.

Les valves orbiculaires sont bombées et offrent une structure alvéolaire manifeste dans tous les milieux. Les alvéoles sont hexagonaux, grands, disposés en séries radiales et si bien harmonisés, dans leurs rapports latéraux sur l'ensemble de la valve, qu'ils y dessinent, dans toutes les directions, des courbes hélicoïdes d'un brillant effet.

La gravure à main levée est presque impuissante à traduire cette impeccable régularité qui est le trait le plus caractéristique de l'espèce. Notre figure 35 n'en donne qu'une bien faible idée, et celles qui ont été obtenues par des procédés photographiques, la figure 63, planche XXIII, du Traité et la figure 5, planche CXXIX, du Synopsis de VAN Heurck, par exemple, ne sont elles-mêmes qu'approximatives.

La difficulté provient en partie de la nécessité qu'il y a de figurer les alvéoles en projection sur une surface plane, tandis qu'en réalité ils se développent sur une surface convexe.

Dans l'exemplaire encore assez petit, figuré ici, il existe une aire centrale occupée par des alvéoles plus grands disposés en étoile. Des auteurs ont fait état de cette particularité pour en faire une variété asteromphalus. 
Ce caractère est inconstant. Il ne se retrouve pas, par exemple, dans l'exemplaire de la figure 36, choisi parmi les plus petits, par économie de place dans nos planches.

Y a-t-il lieu de faire état de ces différences accidentelles et d'autres plus subtiles encore et plus inconstantes sans doute, pour démembrer le type en variétés plus ou moins nombreuses et assurément dépourvues de valeur pratique? C'est bien discutable quand on voit les auteurs hésiter entre deux formes bien autrement distinctes pourtant, comme celle qui nous occupe et $C$. radiatus, par exemple.

Ce n'est pas, du reste, d'après des figures notoirement insuffisantes de beaucoup de publications qu'on pourrait en juger. Il faudrait une étude comparée sur le naturel, en tenant compte de l'influence éventuelle de l'àge et des conditions de l'ambiance sur les modalités de ces formes affines.

Distribution géographique. - C'est l'espèce de Coscinodiscus la plus répandue et la plus constante dans les produits de pêche de la Belgica. On peut dire qu'elle ne manque à aucun échantillon de surface, si ce n'est à ceux de provenance des côtes occidentales de la Nouvelle-Zemble, jusqu'à la latitude de la presqu'ile de l'Amirauté.

\section{Coscinodiscus radiatus Ehrenberg.}

Planche XXX, figures 38,39 et fo. $^{\circ}$

Littérature. - Nous entendons dégager cette forme spécifique de toutes celles que VAN HEURCK et d'autres auteurs y ont rapportées dans un esprit de synthèse à outrance qui fausse la réalité objective. Voir, à ce sujet, VAN Heurck, Traité des Diatomées, page 53o, où l'auteur s'abstient de toute allusion directe, aussi bien dans son texte que dans ses planches, à la forme précisément que nous avons ici en vue.

Frustules discoïdes, en forme de boìte ronde à fond et à couvercle plats, libres ou géminés, à la suite d'une division récente, variables de dimensions, sans être jamais bien grands. Valves vigoureusement sculptées, à structure alvéolaire dessinant des mailles hexagonales, larges, disposées radialement et un peu plus petites vers l'extrême bord périphérique.

Les figures 38 et 40 en sont deux vues valvaires empruntées à des spécimens de calibre différent, dans les limites de grandeur les plus habituelles.

La figure 39 en reproduit deux frustules jumeaux, fruits d'une cytodiérèse récente, $d$.

On remarquera qu'aucune émergence ne fait saillie sur la ligne parfaitement droite du profil des valves. Celles-ci sont rigoureusement planes et leur arête circulaire est à peine légèrement rodée.

La comparaison de cette figure avec la figure correspondante 37 de C. oculus iridis permettra de mesurer la distance entre les deux espèces. Les particularités de structure 
du cytoplasme et du processus de la cytodiérèse auraient accusé davantage leur éloignement si nous n'avions craint de leur accorder trop de place en les retraçant.

Distribution Géographiøue. - Extrêmement rare dans les pêches de la Belgica; circonstance qui nous a d'autant plus frappé que cette espèce est abondante et constante, en toutes saisons, dans la Mer Flamande, alors que C. oculus iridis ne s'y rencontre qu'à titre exceptionnel.

\section{Coscinodiscus excentricus Ehrenberg.}

Planche XXX, figures $4 \mathrm{I}, 42,43$.

Synonyme et littérature. - Voir Nordisches Plankton, page 29.

Frustules isolés ou géminés, cylindriques, discoïdes, à valves plates, un peu chanfreinées sur leur pourtour et ornées, sur le bord interne du chanfrein, d'une couronne de spinules (fig. 42) souvent assez accusées pour être perceptibles dans tous les milieux; mais parfois aussi beaucoup moins prononcées et peu saisissables, même de profil.

Les sculptures de la valve sont vigoureuses et se traduisent en une structure alvéolaire dessinant de petites mailles polygonales égales, engendrées par plusieurs systèmes de lignes plus ou moins courbes et excentriques qui se croisent sous des incidences variables mais rapprochées de $60^{\circ}$. Cependant on y observe presque toujours, sur un secteur ou l'autre, une certaine irrégularité naturelle dans la maillure, ce que la figure $4 \mathrm{I}$ rend assez fidèlement.

Les figures $4^{\mathrm{I}}$ et 42 se rapportent au même exemplaire, vu de face et de profil. Dans cette dernière position, la zone connective montre la disposition des cercles et les fines striations qui les soulignent dans les spécimens de forte taille.

La figure $4^{3}$ est la vue valvaire d'un exemplaire du module moyen le plus répandu.

Remarque. - La structure valvaire de C. cxcentricus est captieuse et on doit se garder d'y borner son attention, car elle est partagée, avec de faibles variantes, par plusieurs autres formes - à l'état de frustules végétatifs ou de sporocystes qui n'en sont que les sosies, mais dont il est parfois malaisë de démasquer la véritable nature.

Distribution géographique. - Cette espèce, si commune en toutes saisons dans la Mer du Nord, est d'une rareté remarquable dans les matériaux qui nous occupent ici. Ce n'est que dans les produits de pèche de l'Océan Glacial que nous avons pu la rencontrer à l'état très disséminé. 


\section{Coscinodiscus Kützingii A. Schmidt.}

Planche XXXI, figure 1 .

Synonymie ét littérature. - Cfr. Nordisches Plankton, page 36.

La vue valvaire (fig. I, pl. XXXI) présente assez d'analogie avec C. excentricus pour que la distinction n'en saute pas aux yeux. A un examen attentif, elle se reconnait : Io à l'absence d'une couronne de spinules sur la zone périphérique; $2^{\circ}$ à la structure particulière $\mathrm{du}$ bord chanfreiné de la valve, qui est un peu bombée vers la périphérie; $3^{\circ}$ à la disposition des alvéoles polygonaux qui couvrent le disque valvaire en y dessinant une douzaine environ de secteurs confusément distincts, grâce au parallélisme des stries radiales développées dans chacun d'eux.

La figure que nous en donnons facilitera l'intelligence de ces détails trop subtils pour pouvoir être utilement traduits par un texte concis.

Nous l'avons placée en regard d'un dessin de Coscinosira polychorda (Gran), que nous rencontrerons plus loin, afin d'en faciliter la comparaison.

Distribution géographique. - Très rare, en spécimens épars, dans des matériaux provenant de régions diverses.

Coscinodiscus concinnus IV. Smith.

Planche XXX, figure 4 .

Littérature. - Cfr. Nordisches Plankton, page 33.

Espèce géante, à valves orbiculaires bombées, délicates, fragiles, très grandes, remarquables par la finesse de leur structure alvéolaire. Celle-ci se traduit par un réseau serré de petites mailles disposées radialement, suivant des secteurs très étroits, délimités vers la périphérie par une couronne de petites spinules submarginales.

Le centre est occupé par quelques mailles plus grandes, disposées en étoile, plus ou moins accusées et parfois chargées de fines ponctuations.

La partie figurée suffit à reconstituer la valve entière, dont les grandes dimensions auraient pris trop de place dans nos planches.

Distribution géographigue. - Cette belle espèce, très commune ailleurs, dans la Mer Flamande en particulier, est remarquablement rare dans les produits de pêche de la Belgica. Nous ne l'avons guère rencontrée que dans le $n^{\circ}$ I, au voisinage immédiat de Tromsö. 


\section{Coscinodiscus nobilis Grunow.}

Planche 'XXX, figure 45 .

Littérature. - Cfr. Van Heurch, Traité des Diatomées, page 530.

Espèce géante aussi, assortie à la précédente pour la forme et l'aspect, mais en différant par l'aire hyaline étoilée qui règne au centre de la valve et dont les rayons diversement dichotomisés se poursuivent, sous la forme de lignes radiantes plus claires, jusque vers la périphérie, où ces lignes aboutissent à des spinules submarginales fort rapprochées.

Le souci de ménager la place dans nos planches nous en a fait limiter la reproduction à un fragment suffisant néanmoins pour en traduire le caractère propre.

On voit que la maillure de la valve revêt le caractère de très petites perforations radiées, qui ont fait placer cette grande espèce, par H. VAx Heurck, dans son groupe des Perforées, plutòt que dans celui des Alvéolées, comme C. concinnus.

Distribution GÉographique. - Cette belle et remarquable espèce n'est pas signalée par Grax dans Nordisches Plankton. Elle est rare dans nos matériaux d'étude.

Elle est, du reste, réputée rare généralement. Nous l'avons cependant observée en quantité dans un échantillon de pêche planktonique des environs de l'île Helgoland, que le regretté $\mathrm{H}$. VAN Heurck a bien voulu nous communiquer.

\section{Coscinodiscus curvatulus Grunow.}

Planche XXX, figures 46 et 47 , et planche XXXI, figure 30 .

Synonyme et littérature. - Cfr. Nordisches Plankton, page 35.

Frustules cylindriques, assez délicats, à valves planes, orbiculaires, à chromatophores, ch, pariétaux, cruciformes, dans nos beaux spécimens (fig. 47, vue suturale). Noyau, n, central, rattaché à l'ectoplasme par des cordons cytoplasmatiques déliés.

Les valves (fig. 46), bien que finement sculptées, laissent voir, mème en milieu liquide, une structure alvéolaire divisée en une vingtaine de secteurs radiaux, chez lesquelles les mailles uniformes sont disposées en séries parallèles à l'un des côtés.

La limite des secteurs est soulignée, à la périphérie, par d'assez grosses ponctuations marginales qui ne se traduisent pourtant pas en relief, mème sur la vue de profil.

Dans nos échantillons grands et petits, les lignes radiantes, qui délimitent les secteurs, sont rectilignes et non pas curvilignes, comme le ferait supposer le nom spécifique curvatulus, qui semble tiré d'une particularité de cette sorte. Dans les dessins frustes et grossiers de Gran (Nordisches Plankton, fig. $37^{\mathrm{a}}$ et $37^{\mathrm{b}}, \mathrm{p} .36$ ), ces lignes sont courbes, mais le caractère d'ébauche de ces reproductions ne permet pas de juger s'il y a là l'indication voulue d'une particularité objective plutôt qu'une défectuosité du dessin. 
Dans l'exemplaire reproduit dans notre figure 46 , on observe un pseudo-nodule visible vers le haut, à droite, au milieu d'un secteur. Est-ce là ce qui a paru légitimer le transfert de la forme dans le genre Actinocyclus? (Actinocyclus curvatulus, var. subocellata Grunow; Actinocyclus subocellatus (Grunow) Rattray - voir Jörgensen : Protist-Plankton, Bergen, igo5.)

Dans les échantillons plus petits, dont nous avons figuré un exemplaire dans la planche XXXI, figure 3o, la structure est bien la même, mais le pseudo-nodule ne s'observe pas.

Faut-il attacher beaucoup d'importance à ce détail qui semble n'être qu'accidentel et inconstant? Nous ne le pensons pas; car dans les recherches cursives tout au moins on ne peut s'y arrèter.

Distribution géographique. - Rencontrée, en grands exemplaires, au voisinage de Tromsö seulement; en exemplaires plus petits, dans la Mer de Kara, no 34, et dans la Mer de Barents, au nord, vers l'Océan Glacial.

\section{Genre Stephanodiscus (Ehrenberg) Grunow}

Caractìres du genre. - Cfr. H. Van Heurck, Traité des Diatomées, page 520.

\section{Stephanodiscus densus sp. n.}

Planche XXXI, figure 27.

Forme beaucoup plus grande que $S$. hantschianus Grunow, couverte, sur les valves orbiculaires, de ponctuations radiantes très serrées, qui ne laissent guère d'espace libre entre les séries.

La périphérie porte une couronne de petites épines saillantes et peu distancées. Nous ne saurions l'identifier avec aucune autre espèce décrite.

Distribution géographique. - Trouvée dans les boues glaciaires, en spécimens épars.

\section{Genre Coscinosira GRAN}

Caractères du Genre. - Voir Nordisches Plankton, page 20.

Coscinosira polychorda (Gran).

Planche XXXI, figures 2 à 6 .

Synonyme et littérature. - Cfr. Nordisches Plankton, page 20.

Les frustules sont cylindriques (fig. 6) et laissent voir, sur la zone connective, des anneaux en nombre et en disposition variables suivant l'âge des individus. 
Les valves sont planes, à peine un peu rodées sur le bord, où l'on distingue de fines spinules marginales très serrées.

Des funicules, $f$, au nombre de cinq à huit dans nos échantillons, relient les frustules entre eux, comme le funicule unique des Thalassiosira. Ils manquent souvent de parallélisme avec l'axe de la colonir.

La figure 5 en reproduit de profil un grand spécimen, dont la figure 2 est la reproduction de la vue valvaire. La surface de la valve y est divisée en huit secteurs, sur chacun desquels se dessine un fin réseau de mailles hexagonales cléterminé par le croisement à $60^{\circ}$ de trois séries de stries dont les unes sont parallèles à la ligne radiante médiane de chaque secteur. C'est au milieu de ces secteurs que s'insèrent les funicules, $f$, dont la trace est marquée d'une façon indélébile dans la structure même de la valve. On remarquera que celle-ci est ornée, comme nous l'avons dit, d'une couronne marginale de denticules rapprochés.

La figure 3 en est une autre, de calibre plus petit, mais d'aspect analogue, parce que le nombre des secteurs étant ici de sept, leur distinction est encore possible.

Dans la figure 4, au contraire, l'aspect est tout autre, parce que le nombre est ici réduit à six, ce dont témoigne le nombre égal des empreintes funiculaires.

Il en résulte que les stries des divers secteurs, inclinées qu'elles sont à $60^{\circ}$ entre elles, se mettent mathématiquement dans le prolongement les unes des autres.

Les secteurs deviennent conséquemment indistincts et le dessin de la valve devient celui d'un Coscinodiscus anguste-lineatus. S'il arrive alors qu'on néglige de remarquer les empreintes d'insertion des funicules et les denticules marginaux, on peut très bien confondre les deux espèces ou encore se croire en présence d'une énigme.

On voit par cet exemple et d'autres déjà signalés, combien peuvent ètre déroutantes des diagnoses hàtives, basées sur des observations restreintes.

Distribution Géographique. - Peu répandue dans les produits de pêche de la Belgica; observée en petit nombre dans l'Océan Glacial.

\section{Genre Phacodiscus g. n.}

Nous proposons ce nom pour désigner des espèces de Mélosirées dont le caractère le plus saillant est la forme lenticulaire (ọxós, lentille) du frustule et dont les valves discoïdes bombées sont dépourvues d'un ombilic central différencié, dont les Hyalodiscus devraient garder le monopole, à titre de caractère générique.

Cette proposition nous est suggérée par l'impression pénible que nous laisse la littérature équivoque, hésitante, qui concerne certaines formes ballottées avec une égale incertitude entre les genres Coscinodiscus, Podosira, Melosira, Hyalodiscus, etc., parce que les caractères conventionnellement attribués à ceux-ci ne sont pas adéquats à toutes 
les réalités objectives pour lesquelles ces genres ont été créés et ne peuvent ètre étendus à certaines d'entre elles sans des concessions qui ruinent leur diagnose propre.

\section{Phacodiscus punctulatus (Gregory)?}

Planche XXX, figures 48 et 49 .

Synonyme et littérature. - i857. Coscinodiscus punctulatus Gregory. Cfr. H. Van Heurck, Traité des Diatomées, page 533, et planche XXXIV, figure go4.

Nous n'avons rencontré l'objet figuré ici que sous la forme lenticulaire, à deux valves convexes soudées directement par leur bord, sans l'intermédiaire d'une zone connective cylindrique (fig. 49).

Les valves présentent une très fine réticulation alvéolaire traduite surtout par une striation radiale, difficilement visible en milieu liquide, évidente à sec et relevée de ponctuations distancées, éparses sur toute la surface (fig. 48).

Notre figure ne présente pas l'aire hyaline centrale que l'on voit dans celle de H. Van Heurck; c'est ce qui nous fait douter de l'identité de l'objet.

Distribution géographigue. - Très rare; dans le sud de la Mer de Barents.

\section{Genre Hyalodiscus EHREnBERG}

- Caractères du Genre. - Voir Nordisches Plankton, page 26.

\section{Hyalodiscus stelliger Bailey.}

Planche XXXI, figures 28 et 29 .

Frustules lenticulaires libres ou plus souvent géminés, à la faveur d'une gaine cylindrique, - libres seulement dans nos échantillons.

Valves à ombilic central différencié, visible aussi bien de face (fig. 28) que de profil (fig. 29).

La zone périphérique est divisée en une quinzaine de compartiments sur chacun desquels se dessine une structure alvéolaire très fine résultant du croisement à $60^{\circ}$ de trois séries de stries dont l'une est radiale.

Distribution Géographique. - Espèce très rare dans nos matériaux. Nous n'en avons rencontré que quelques échantillons, tous de petite taille. Cette rareté fait contraste avec sa fréquence, en grands spécimens, et sa constance en toutes saisons dans la Mer Flamande. 


\section{ENDOCYSTE INDÉTERMINÉ}

Planche XXXI, figures 3 i et 32 .

Nous croyons devoir considérer comme un endocyste l'objet figuré de face (fig. 3I) et de profil (fig. 32) que nous avons rencontré, assez abondant, dans la florule des neiges et des glaces, toujours isolé de l'organisme qui a dù le produire.

Il a la forme d'une lentille plus convexe d'un còté que de l'autre et résulte de la soudure, suivant une bordure hyaline, de deux valves discoïdes, inégalement bombées, plus épaisses au centre qu'à la périphérie.

Vues de face, les valves présentent une structure finement alvéolaire dont le dessin rappelle quelque chose d'intermédiaire entre celui de Coscinodiscus cxcentricus et de C. lineatus.

On pourrait lui trouver une analogie aussi avec celui de l'endocyste de Thalassiosira bioculata, si on ne portait pas suffisamment son attention sur le facies particulier du bord marginal de l'objet ici décrit.

C'est une preuve de plus qu'on ne peut pas tabler exclusivement sur la structure classique de Coscinodiscus excentricus, lineatus et anguste-lineatus, pour asseoir une diagnose fondée des formes observées.

De plus en plus nombreuses deviennent les formes connues qui en simulent l'aspect.

On y verra sans doute la cause de beaucoup de confusions et de l'obscurité qui règne dans la nomenclature des Diatomacées à valves orbiculaires, dont un trop grand nombre n'ont été étudiées que sur des slides montés dans le baume.

\section{Genre Isthmia AGARDH}

Frustules volumineux, comprimés, trapézoïdaux, munis d'une zone connective très large, pourvus, sur l'une des valves, d'un prolongement creux, irrégulièrement cohérents entre eux.

\section{Isthmia enervis Ehrenberg.}

Planche XXXI, figures 7 et 9 .

Valves et ceinture, c, sculptées de larges mailles ovalaires, plus petites sur la ceinture et disposées là en séries longitudinales, tandis que sur les valves les rangées confluent vers le centre de celles-ci.

Inutile d'ajouter quoi que ce soit à la figure 7, qui reproduit un spécimen assez étroit de l'espèce, sous l'aspect qu'il offre dans sa position d'équilibre stable. 
La figure 9, grarée exceptionnellement à une échelle moindre (I50 diamètres seulement), montre la façon dont les frustules restent cohérents en colonie, gràce à un grumeau de substance gommeuse qui sert de soudure, $s$, entre les individus issus d'une souche.

Il y aurait lieu de s'arrêter sur la manière un peu spéciale dont la division s'opère ici, en vue de la production de deux valves dissymétriques et de la soudure subséquente des produits de la division; on pourrait aussi exposer comment il se fait que, dans les trois frustules associés, la ceinture, $c$, déborde sur la valve exempte de prolongement adhésif; mais l'exposé même succinct de ces particularités nous détournerait de notre sujet, surtout si l'on considère que cette espèce n'est guère planktonique.

Distribution géographioue. - Voisinage de Tromsö; en colonies et en frustules isolés.

\section{Isthmia nervosa Kützing.}

Planche XXXI, figure 8.

Forme assez pareille à la précédente, mais s'en distinguant néanmoins à première vue par les nervures qui ornent les valves, en soulignant les doubles rangées des mailles qui sont ici polygonales. La ceinture porte, en outre, une lisière de mailles beaucoup plus amples que les autres.

L'exemplaire figuré, choisi à dessein parmi les plus petits et les plus étroits observés, suffira à donner une idée du type, sans qu'il soit utile d'en reproduire la vue valvaire, qui serait peu interprétative et qui, d'ailleurs, ne se prête guère à l'observation.

Distribution géographique. - En mélange avec sa congénère, aux environs de Tromsö, mais parait un peu plus rare.

\section{Genre Biddulphia GRAY}

Les caractères du genre sont bien connus; on pourra les trouver toutefois dans Nordisches Plankton, avec la clef des espèces.

Biddulphia arctica (Brightwell) Boyer.

Planche XXXI, figures io à $\mathrm{I} 3$, et planche XXXII, figures 16 à i 8 .

Synonyme ext littérature. - Cfr. Nordisches Plankton, p. iog.

Cette espèce est représentée par deux formes douées de caractères propres suffisamment éloignés, pour qu'il soit utile d'en maintenir la distinction dans la pratique des recherches planktoniques. 
Nous les présenterons successivement telles que nous les avons observées.

I $F$. balana. Planche XXXI, figures io et II.

Synonyme. - Zygoceros balana Ehrenberg, I854, et Biddulphia balana Brightwell, 1859 .

Frustules très grands, formés de deux valves elliptiques, très légèrement relevées aux deux extrémités du grand axe et bombées sur tout leur pourtour.

La structure en est alvéolaire, bien accusée, visible en tous milieux. Les alvéoles hexagonaux irradient du centre, où ils sont plus petits, en séries dichotomiques qui s'étendent à toute la surface des valves, sauf sur les extrémités, où elles sont remplacées par de très fines ponctuations sérielles (fig. Io). Vers le centre on aperçoit quelques ponctuations éparses, qui ne se traduisent pas en spinules saillantes. On n'y observe pas des soies longues comme dans beaucoup d'autres espèces.

L.es valves se rattachent, sans étranglement marqué, à la zone connective plus ou moins large (fig. II, vue de profil), qui est décorée d'un fin quadrillé.

Les quatre angles des frustules sont à peine relevés et représentent, sous une forme très atténuée, les cornes saillantes qui s'observent chez les congénères.

Distribution GÉographioue. - Assez rare dans la Mer de Kara; pas rencontrée ailleurs.

$2^{\circ}$ F. typica. Planche XXXI, figures I2 et 13 , et planche XXXII, figures I6, I7 et IS.

Synonyme. - Triceratium arcticum Brightwell, I853, et Trigomum arcticum Cleve, I864.

Frustules très grands, formés de deux valves triangulaires aux angles arrondis et légèrement relevés. Ceux-ci sont décorés de fines ponctuations sérielles. Le reste de la valve est occupé par une trame alvéolaire radiante. La vue de profil de la figure i3 montre le quadrillé qui orne la zone connective.

Les spécimens observés nous y ont révélé deux facies différents :

$A$. Celui de la figure I2, planche XXXI, dont les côtés du frustule triangulaire sont convexes et dont les mailles de la trame alvéolaire sont au moins aussi grandes au centre qu'à la périphérie.

$B$. Celui de la figure i6, planche XXXII, dont les còtés du trigone sont plutôt concaves et dont le dessin réticulé est beaucoup plus fin au centre des valves. Il en résulte une radiation plus marquée des mailles et celles-ci sont plus tétragonales.

La figure I7 de la mème planche en reproduit, en coupe optique, la distribution du contenu cellulaire.

Le noyau, $n$, est central. L'endoplasme forme une masse centrale périnucléaire d'où se détachent de nombreux cordons qui irradient vers l'ectoplasme pariétal. 
Les chromatophores, $c h$, sont nombreux, arrondis, éparpillés, dans le spécimen figuré.

Les frustules des figures I6 et 17 sont reliés entre eux par un petit grumeau de substance adhésive, c, suffisant pour maintenir en colonie des membres parfois nombreux, conformément à la figure I8, qui en reproduit un fragment à un grossissement d'une centaine de diamètres seulement.

Dans ces groupements en zigzag de forme variée, les frustules sont soudés par un ou plusieurs angles et peuvent même se trouver dans des plans différents.

Distribution géographigue. - Environs de Tromsö et Mer de Kara; un peu moins rare que la forme balana.

\section{Biddulphia aurita (Lyngbye) Brébisson.}

Planche XXXI, figures it à 19 .

Synonyme et littérature. - Voir Nordisches Plankton, page io5.

Il serait fastidieux de décrire dans ce texte les détails de forme, de position et de structure tant des pièces constitutives des frustules - valves et ceinture - que de leurs appendices - cornes et soies - et des rapports réciproques des frustules dans une même colonie.

Le lecteur trouvera mieux son compte dans l'examen comparé de quelques figures empruntées à des colonies de calibre différent, qui nous ont paru indispensables pour donner une idée synthétique de la physionomie variable de cette élégante espèce, même sans sortir de nos matériaux des parages de la Nouvelle-Zemble.

Les frustules, réunis en thalle, forment habituellement de très longues chaînes droites, dont nous avons dû nous borner à figurer quelques chaìnons détachés de la série, mais néanmoins soudés encore avec un de leurs pareils, pour permettre d'en saisir les rapports.

La figure I4 est empruntée à une colonie de grandes dimensions. Les deux frustules jumeaux ont encore leur valve jeune protégée par la ceinture de la matricule, bien que leur ceinture propre se soit déjà fort développée.

La figure I5 montre l'espèce sous ses dimensions maximales suivant l'axe latéral, ce qui entraîne, comme on voit, la réduction corrélative de l'axe longitudinal ou transvalvaire.

La vue valvaire de la figure i6 complète le signalement de ces formes amples, en en détaillant l'ornementation et la position respective des cornes et des soies, figurées en projection sur la coupe optique de la ceinture qui a la forme d'une ellipse allongée.

La figure I7 retrace les traits d'un spécimen de calibre moyen, à frustules beaucoup plus longs, mais moins larges. Dans l'un des frustules, b, nous avons figuré 
le contenu cellulaire : noyau, $n$; chromatophores, $c h$, éparpillés dans le cytoplasme pariétal. Dans un autre, $a$, on remarquera une phase de la division, soulignée par l'apparition du foramen, $f$, entre l'ébauche des deux valves en voie d'élaboration.

Le fragment de colonie de la figure i 8 montre, dans sa position latérale, l'aspect de la chainette ainsi orientée, tant dans les membres pris individuellement que dans les rapports qui existent entre eux par l'intermédiaire des cornes et des soies.

Les spécimens de petit calibre, enfin, ont leur expression traduite dans la figure ig.

Les rapports des dimensions axiales sont ici complètement intervertis au profit de l'axe transvalvaire et le facies en est profondément modifié.

Le frustule d'en haut, $a$, porte sa décoration extérieure; le frustule d'en bas, $b$, qui est en division, montre les rapports actuels des noyaux-filles, $n d$, et la silhouette des jeunes valves déjà séparées par le foramen, $f$, mais non encore pourvues de leurs soies.

Distribution géographlque. - Ne s'observe nulle part avec une certaine abondance, mais se rencontre accidentellement sur les points les plus éloignés de l'itinéraire de la Belgica.

\section{Biddulphia granulata Roper.}

Planche XXXI, figures 20 à 25.

Synonyme et littérature. - Cfr. Nordisches Plankton, page io7.

Les quelques figures destinées à illustrer cette belle espèce nous dispenseront de nous arrêter longtemps à en décrire les détails.

Les frustules en sont généralement grands, isolés ou géminés, à la suite d'une division récente, comme dans la figure 23, où l'on voit persister la ceinture de la matricule, décorée d'un triple système de stries qui se coupent sous un angle de $60^{\circ}$, l'un d'eux étant parallèle à l'axe transvalvaire.

C'est aussi la même décoration qui se continue sur les valves proprement dites; mais elle est ici relevée de nombreuses spinules peu saillantes, semées sans ordre apparent jusque sur les cornes. Celles-ci subissent une torsion qui les écarte du plan sagittal et dans des directions opposées sur une même valve : caractère traduit dans la figure 22 , qui est une vue valvaire, en projection sur la ceinture, $c$, dont on peut ainsi apprécier la forme, en coupe optique. On y remarquera la place d'insertion des deux longues soies terminales que les autres figures montrent dans leur développement, sous d'autres incidences.

Nous ne dirons rien de la forme spécifique des frustules raduite de face, dans la figure 20 , et de profil, dans la figure $2 \mathrm{I}$, d'après un individu de grande taille dont la zone connective est fort développée. Il suffit d'en scruter les détails d'exécution qui sont tous objectifs. 
Nous regrettons seulement qu'on ne puisse traduire autrement qu'en traits noirs et ternes qui assombrissent l'objet, les détails de sculpture que la nature réalise dans le cristal le plus transparent et que la lumière transmise colore des teintes les plus riches du spectre solaire.

Distribution géographigue. - Limitée, dans nos matériaux, à certains échantillons de la Mer de Kara.

Biddulphia rhombus (Ehrenberg) IV. Smith.

Planche XXXI, figures 24,25 et 26.

Syomynie et littérature. - Voir Nordisches Plankton, page io8.

Forme massive, à frustules libres ou maintenus en colonie irrégulière par une de leurs cornes seulement.

Très rare dans nos matériaux actuels, elle est loin d'y revêtir les variantes de forme et de physionomie avec lesquelles l'étude du microplankton de la Mer Flamande nous a familiarisé.

Nous nous bornerons donc à en reproduire ici un même spécimen dans trois positions classiques: en vue connective (fig. 24), en vue valvaire (fig. 25), en profil latéral (fig. 26), pour en faire voir le facies spécial, dans une région qui paraît peu propice à l'espèce.

Les sculptures des parois y sont sensément du même style que dans $B$. gramulata, mais la facture en est plus grossière. Les valves porttent un certain nombre de spinules éparpillées et peu saillantes, mais non des soies longues, comparables à celles des deux espèces précédentes.

La figure 25 montre que l'extrémité des cornes, peu saillantes aussi, dévie peu du plan sagittal.

Distribution géographique. - Extrêmement rare, au point que nous avons pensé tout un temps qu'elle était complètement étrangère à nos matériaux, comme d'autres de ses congénères : $B$. mobilensis, B. sinensis, B. fauns, B. alternans, B. Smithii, etc., dont l'aire d'habitation paraît cependant très étendue.

\section{Sous-familles II et III. - Pseudo-Raphidées et Raphidées}

(Pennate d'autres auteurs)

Nous ne voyons pas le besoin, dans cet exposé analytique de nos recherches sur des matériaux limités dans le temps et dans l'espace, d'urger la distinction entre les Pseudo-Raphidées et les Raphidées, d'autant moins que cette distinction des deux 
sous-familles est parfois assez spécieuse et pourrait nous entraîner, dans certains cas critiques, à des digressions dont il n'y a pas lieu d'encombrer ces pages.

Dans une certaine mesure néanmoins, nous présenterons d'abord les PseudoRaphidées pour finir par les Raphidées les plus authentiques : les Naviculées.

\section{Genre Amphiprora Ehrenterg}

Caractères du Genre. - Consulter Nordisches Plankton, page I27, et H. VAN Heurck, Traité des Diatomées, page $26 \mathrm{I}$.

\section{Amphiprora hyperborea (Grunow). \\ Planche XxxII, figure $\mathrm{I}$.}

Synonyme et littérature. - Cfr. Nordisches Plankton, page 127.

Espèce franchement planktonique, gràce à ses colonies rubanées, dans lesquelles les frustules restent associés par les flancs croisés de leur carène sigmö̈de, qui se placent alternativement au-dessus et en dessous de la partie similaire du frustule contigu.

La figure I en montre clairement la disposition curieuse dans un fragment de colonie.

Certains frustules, $a$, sont figurés vicles; d'autres, $b$, montrent leur protoplasme, pr, contracté par le liquide fixateur, sous la forme de deux vastes chromatophores diffus, encadrant le noyau, $n$, qui est central. L'un des frustules, $c$, qui est en voie de cytodiérèse, fait assister à l'élaboration des jeunes valves, v', en mème temps qu'à la reconstitution des deux cellules jumelles au sein de leur matricule dont elles se partagent les valves, $\%$

Les valves nous ont toujours paru exemptes de sculptures. perceptibles même à sec, au contraire de la striation de la zone connective, qui est toujours bien apparente.

Distribution Géographique. - Espèce assez commune dans l'Océan Glacial.

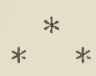

Remaroue. - La florule glaciaire principalement et les pêches planktoniques accessoirement nous ont mis en présence de quelques formes qu'il n'est pas commode de comparer avec celles dont la littérature ne possède souvent qu'une figure, dans laquelle l'auteur n'a pas toujours eu le souci de reproduire tous les détails avec une fidélité suffisante pour mettre l'espèce envisagée à l'abri de toute confusion avec d'autres acquisitions faites ou à faire dans le même groupe organique. 
Les reproductions faites d'après des frustules incinérés sont généralement faussées dans le principe, par les déformations qu'entraine l'incinération d'objets trop peu silicifiés pour supporter ce traitement sans préjudice.

D'autre part, l'emploi de trop forts grossissements obtenus par des objectifs à immersion, d'un usage courant peu pratique, modifie la physionomie des espèces au point d'en rendre les dessins méconnaissables quand on les met en regard des objets eux-mêmes examinés sous des objectifs moins puissants, mais plus usuels.

On sait encore qu'un objet à facies hétérogène ne saurait être suffisamment signalé par une seule vue, à moins qu'il ne soit assez bien connu d'ailleurs pour que la présentation d'un aspect soit évocatrice des autres et que l'imagination instruite y supplée.

Ce n'est assurément pas le cas pour des formes qui ne sont que rarement observées et auxquelles leur dissymétrie fait prendre des aspects variés, sans qu'il soit toujours possible de les revoir sous l'angle sous lequel il a plu à un observateur d'occasion d'en profiler la silhouette.

Ces raisons nous commandent beaucoup de circonspection dans les rapprochements que nous tenterons d'établir entre les organismes figurés tels que nous les avons vus et ceux, autrement vus peut-être, avec lesquels on peut essayer éventuellement de les identifier.

Ce sera le cas non seulement pour les Amphiprora, mais aussi pour plusieurs autres genres de ceux dont il nous reste à parler, particulièrement ceux dont les représentants appartiennent à la florule glaciaire.

Amphiprora formosa sp. n.

Planche XXXII, figures 2 et 3 .

Grande et belle forme, abondante sur les glaces de la Mer de Kara.

Les frustules toujours isolés sont formés de deux valves carénées, peu sigmoïdes, dont la figure 3 reproduit la vue valvaire, peu facile à prendre, à cause de l'instabilité de l'organisme dans la position figurée. La figure 2 en est la vue connective, d'observation plus courante.

La structure intime de la carène est très nettement différente de celle de la valve proprement dite. Celle-ci n'est que finement striée perpendiculairement à son grand axe; celle-là présente une double striation oblique, qui ne se révèle que dans les meilleures conditions d'observation : à sec, par exemple.

La zone connective est fortement sillonnée de côtes orientées en sens inverse sur les deux faces suturales opposées, suivant le développement dissymétrique de ces dernières.

Distribution géographique. - Assez commune dans les neiges et sur les glaces de la Mer de Kara, en mélange avec plusieurs des formes mentionnées plus loin. On en observe aussi accidentellement des échantillons disséminés dans les pêches planktoniques des mêmes parages. 


\section{Amphiprora Kariana Grunow?}

Planche XXXII, figure 5.

Littérature. - Consulter Cleve et Grunow, Beitrigue zur Kenntuiss der Arctischen Diatomeen, I880.

Forme que nous croyons pouvoir rapporter à l'espèce Kariana Grunow, à cause de la striation beaucoup plus forte et plus espacée sur la carène que sur le reste de la valve. Nous en reproduisons, en vue suturale, un spécimen de taille ordinaire, assez petit, comme on voit.

Même distribution que la précédente.

\section{Amphiprora Kjellmanii Cleve?}

Planche XXXII, figures 6 et 7 et peut-être aussi 4 .

Littérature. - Consulter Cieve et Gruxow, loc. cit.

Frustules à carène bien distincte, comme dans les précédentes et manifestement sigmoïde, comme le montre la figure 7 . La note différentielle de notre forme nous parait consister dans l'uniformité de la striation qui se continue de la carène à la valve, sans changer de caractère.

Les deux vues, de face (fig. 6) et de profil (fig. 7) en complètent le signalement.

La forme plus trapue, renflée, carénée, fortement sigmoïde à striation uniforme de la figure 4, n'en serait-elle pas un spécimen un peu exceptionnel, une variante?

Elle ressemble toutefois à $A$. alata Kützing.

Même origine que plus haut, de même que la suivante.

\section{Amphiprora paludosa IV. Smith?}

Planche XXXII, figure ro.

Petite forme à frustule plus tordu, plus sigmoïde que la reproduction de face suturale (fig. Io) ne permet de le montrer. Striation délicate sur les valves, visible à sec seulement.

\section{Genre Orthotropis CLEvE}

Valves carénées, à carène non sigmoïde. Face suturale fortement contractée.

\section{Orthotropis lepidoptera (Gregory) Cleve.}

Planche XXXII, figures 8 et 9 .

Frustules très peu tordus, lancéolés, rétrécis au milieu (fig. S, vue suturale), dépourvus d'ailette saillante sur la carène non sigmoïde des valves (fig. 9, vue valvaire). 
Les valves sont finement striées transversalement; la zone connective est simple, sans replis et accuse tout au plus une très fine striation longitudinale.

Distribution géographigue. - Assez rare, dans la florule des glaces.

\section{Orthotropis maxima (Gregory) Cleve.}

Planche XXXVI, figure 88 .

Frustules à valves grandes, longuement lancéolées, atténuées, aiguës aux extrémités, déprimées à la partie médiane, carénées, surmontées d'une ailette, striées transversalement, sauf au voisinage du nodule médian, où on observe une aire hyaline étroite, stauronéiforme.

Nous n'en avons figuré que la vue valvaire, seule observée.

Distribution géographique. - Très rare, dans les pêches planktoniques du Détroit de Matotchkine.

\section{Genre Donkinia Ralfs}

Caractères du gentere. - Voir Van Heurck, Traité des Diatomées, page 248.

Donkinia recta (Donkin) Grunow?

Planche XXXII, figures ir, i2 et $\mathrm{i} 3$.

Syconymie. - Pleurosigma rectum Donkin. Voir Van Heurck, page 248.

Frustules contractés à la partie médiane, à zone connective presque droite (fig. II, vue suturale), à valves carénées, mais non munies d'ailette saillante. Carène très sigmoïde, marquée d'un nodule médian (fig. I2, vue valvaire).

Les valves portent une très fine striation, difficile à percevoir, que nous n'avons pu saisir que dans le sens transversal.

La figure I3 montre les rapports latéraux des frustules associés en colonie rubanée, fragile.

Distribution Géographique. - Rare, dans la florule des glaces.

\section{Genre Campylodiscus Ehrenberg}

Frustules courbés en selle, peu épais, formés de deux valves circulaires croisées entre elles par rapport à leur ligne médiane et à leur trame décorative. 


\section{Campylodiscus Thuretii Brébisson.}

Planche XXXII, figures i9 et 20

Littérature. - Voir Van Heurck, Traité des Diatomées, page 378.

Nous figurons les deux valves d'un même frustule, que nous avons réussi à séparer sous le couvre-objet, après macération suffisante pour préparer cette sorte de dissection.

C'est ce qui nous permet d'affirmer que les deux pièces présentent entre elles de légères différences.

La comparaison des deux figures les fera mieux saisir que toute explication.

Notre spécimen, malgré sa forme ensellée, est bien circulaire, quand on prend soin de le disposer normalement à l'axe optique. Il se peut que la forme irrégulière donnée à la figure de Vax Heurck, Traité des Diatomées, planche XIV, figure 378, soit due à une vue un peu oblique.

Distribution géographique. - Très rare, dans la Mer de Kara.

\section{Campylodiscus angularis Gregory?}

Planche XXXII, figure 2I.

Valve circulaire, ensellée, présentant une aire centrale, lancéolée, hyaline, entourée d'une large zone marginale de côtes radiantes, fortes, entre lesquelles d'autres plus fines se profilent et se perdent, à quelque distance du pourtour.

Distribution géographique. - Très rare, aux environs de Tromsö.

\section{Genre Surirella TurPIN}

\section{Surirella ovalis Brébisson.}

Planche XXXII, figures 22 et 23.

Très petite forme, à valves ovales, garnies sur leur pourtour de côtes délicates radiantes qui ménagent une aire hyaline centrale (fig. 23). La vue connective est cunéiforme (fig. 22).

Il n'y a pas lieu, sans doute, d'y voir autre chose qu'une des nombreuses variétés de l'espèce polymorphe à laquelle nous rattachons l'unique forme du genre Surirella trouvée dans nos matériaux.

Distributiox géographlque. - Rencontrée surtout sur les glaces. Peut-être assez commune, mais difficile à observer en milieu liquide, à cause de ses petites dimensions et de la faible accentuation de ses caractères. 


\section{Genre Cocconeis Grunow}

Valves largement elliptiques ou discoïdes, dissemblables entre elles : l'une ayant un véritable raphé, avec un nodule central; l'autre n'ayant qu'un pseudo-raphé. Cette diversité des valves d'une même espèce crée une difficulté réelle pour l'identification des types spécifiques connus, quand on ne dispose que de très rares spécimens rencontrés fortuitement, comme c'est le cas dans nos matériaux.

Pour ces raisons nous nous contenterons de signaler, sans commentaire, les valves que nous avons observées et dont les figures ont été réunies dans la planche XXXII.

Figure 24. Valve elliptique bordée d'une zone marginale hyaline très étroite et sillonnée d'un raphé sigmoïde traversé lui-même par une ligne médiane, stauronéiforme, droite, qui ne s'étend pas à toute la largeur de la face. Celle-ci parait hyaline en milieu liquide. A sec, on y découvre une très fine striation croisée dont l'orientation est parallèle aux deux traits réfringents du raphé et de son croisement.

Figure 25. Valve elliptique, à zone marginale hyaline, étroite, à raphé droit, coupé par un nodule médian. Ce n'est qu'à sec qu'on peut y entrevoir une faible striation croisée, suivant les deux axes de la valve.

Ne parait pas éloignée de $C$. dimpta Gregory.

Figure 26. Valve elliptique assez grande, à zone marginale hyaline assez étroite, à raphé sigmoïde interrompu au milieu, où règne une aire hyaline circulaire, dépourvue de la fine striation transversale qui couvre le reste de la surface valvaire.

Figure 27. Valve elliptique lancéolée, à zone marginale large sur laquelle se profilent aussi bien que sur la partie centrale, mais avec une accentuation plus grande et avec de fines stries interposées, des séries transversales un peu rayonnantes de ponctuations visibles en tous les milieux, qui atteignent le raphé. Celui-ci est droit et dépourvu de nodule médian apparent.

Figure 2S. Valve largement lancéolée, entourée d'une zone hyaline très large, pourvue d'un raphé droit renforcé au milieu par un nodule diffus et couverte d'une striation croisée fortement imprimée et visible même en milieu liquide.

Figure 29. Valve elliptique striée sur la zone marginale, striée-ponctuée en courbes divergentes sur les deux còtés du raphé peu accusé. Présente beaucoup d'analogie avec C. scutellum Ehrenberg.

Figures 30 et 3I. Valves elliptiques, très petites, quadrillées en milieu liquide (fig. 2I), striées-ponctuées à sec (fig. 30). 


\section{Genre Raphoneis Ehrenberg}

Valves lancéolées, elliptiques, rhombiques, striées grossièrement dans deux directions, en milieu liquide; parsemées, à sec, de séries transversales et peu radiantes de grosses perles; à pseudo-raphé plus ou moins large, sans nodules ni médian ni terminaux. Vue suturale linéaire.

Remarque. - Les diatomistes n'ignorent pas que la structure des valves change d'aspect d'après la mise à point au microscope et, tout autant, d'après la réfringence du milieu dans lequel elles sont observées.

C'est l'usage, nous dirions volontiers l'abus, d'enrober les frustules - préalablement débarrassés de toute matière organique - dans des baumes de réfringence plus ou moins élevée, quï a fait intervertir les détails de leur structure, en faisant prendre pour des perles saillantes ce qui n'est en réalité que des trous ou, du moins, des parties moins épaissies de la membrane siliceuse. On a ainsi trop généralement substitué un simple effet d'optique à la réalité.

Celle-ci se traduit plus sincèrement en milieux aqueux; mais nous convenons que dans ces conditions elle peut rester peu distincte ou mème indiscernable quand les détails de structure sont très délicats.

C'est ce qui a lieu pour beaucoup d'espèces boréales, qui semblent avoir été trop parcimonieusement rationnées en silice. Nous en avons déjà fait l'observation au sujet de la florule des neiges et des glaces. C'est aussi une vérité banale en ce qui concerne les Diatomacées pélagiques : les Chétocérées, sensu lato, par exemple.

\section{Raphoneis amphiceros Ehrenberg.}

Planche XXXII, figures $3+$ et 35 et aussi, sans doute, 32 et 33.

Frustules fortement silicifiés, à pseudo-raphé très étroit, à structure quadrillée, un peu rayonnante, si on les observe en milieu liquide, comme nous l'avons fait pour les originaux de nos figures.

L'étude des variantes de forme et de dimension, observées dans les représentants de cette espèce polymorphe dans la Mer Flamande, nous a enclin à tenir peu compte, au point de vue taxinomique, des modalités diverses qu'elle peut affecter.

La figure 34 en reproduit de face un petit spécimen lancéolé, subcapité. Nous en avons dessiné, en vue connective, deux frustules associés, dans la figure 35, pour montrer comment les éléments se maintiennent en colonie rubanée, parfois assez longue.

La figure 33 en est un beaucoup plus grand, de forme lancéolée; nous n'en séparerons pas celui de la figure 32, qui affecte la forme d'un rhombe aux angles émoussés. 


\section{Raphoneis Surirella Grunow.}

Planche XXXII, figure 36.

Espèce à pseudo-raphé assez large, rétréci au milieu, dilaté vers les deux extrémités de la valve, qui est lancéolée et légèrement subcapitée.

Distrizution géographique. - Ces deux espèces littorales ne se rencontrent qu'accidentellement dans nos matériaux, attachées, comme on sait, à titre de commensales, à d'autres espèces planktoniques qui les véhiculent parfois au large.

\section{Genre Cymbella AgardH}

Caractères du gexre. - Cfr. Van Heurck, Traité des Diatomées, page ifo. d'eau douce.

Nos matériaux sont très pauvres en représentants de ce genre, qui est plutôt

Nous n'avons à signaler que les deux espèces suivantes de la florule des glaces de la Mer de Kara :

\section{Cymbella recurva sp. n.}

Planche XXXII, figure 40.

Valve longuement lancéolée, excentrique, à extrémités diminuées, rostrées et recourbées en sens inverse de la courbure du côté le plus convexe ou dorsal. 'Le bord opposé ou ventral est convexe dans sa partie médiane, concave vers les extrémités, en deçà des deux rostres récurvés à leur extrémité.

Raphé arqué, récurvé aussi aux extrémités. Striation transversale assez serrée, atténuée vers le raphé et aussi autour du nodule central.

\section{Cymbella amphicephala Nægeli?}

Planche XXXV, figures 63,64 et 65.

Nous croyons reconnaitre ici une variante de cette espèce, qui confine elle-même de si près à l'espèce cuspidata Kützing.

Forme assez petite, trapue, bicéphalée, très convexe du côté dorsal, renflée, aplatie du côté ventral; à raphé presque droit, peu dégagé de la striation transversale, comme le nodule médian.

Nous la présentons sous ses trois aspects classiques : figure 64 , en vue valvaire; figure 63, en vue connective dorsale; figure 65 , en vue connective ventrale. 


\section{Genre Encyonema Kützing}

Caractères du genre. - Voir Van Heurck, loc. cit., page I 49.

\section{Encyonema sp.}

Planche XXXV, figures 66 et 67 .

Deux très petites formes d'Encyonema analogues soit à E. ventricosum Kützing, soit à E. caspitosum Kützing, qui, de l'avis de Cleve, pourraient bien n'être que des modalités d'un seul type, sujet à de nombreuses variations d'une distinction subtile.

Ces formes tiennent, du reste, très peu de place dans nos matériaux, où nous ne les avons rencontrées que dans les boues glaciaires.

\section{Genre Amphora Ehrenberg}

Caractères idu Genre. - Cfr. Van Heurck, Traité des Diatomées, page i25, et Cleve, Synopsis of the Naviculoid Diatoms, dans Kong. Sv. Vet. Akad. Handl., Band XYVII, n० 3, i 895 .

Nous tenons de Tromsö, du Détroit de Matotchkine et des glaces de la Mer de Kara, quelques espèces de ce genre à la fois très vaste et très compliqué. Les spécimens en sont toujours rares et tiennent peu de place dans les récoltes où on les rencontre.

Remarque. - Nous nous trouvons ici en présence d'un groupe naturel d'organismes, dont il nous semble que la science n'a pas encore parfaitement pris connaissance, à cause du manque de contròle de beaucoup de formes dont il faudrait discuter les titres, avant d'homologuer leur spécificité.

Nos sujets d'observation sont eux-mêmes trop rares pour avoir pu en mener l'étude à fond et discuter leurs analogies plus ou moins fondées avec d'autres formes déjà dénommées et décrites, mais qui n'ont souvent été figurées que sous l'un ou l'autre de leurs aspects. Or, on sait que le signalement de ces organismes excentriques requiert au moins trois vues complémentaires l'une de l'autre : une ventrale, une dorsale et une latérale ou valvaire. Cette dernière a été souvent négligée, à cause de la difficulté plus grande de l'obtenir; elle est pourtant révélatrice de caractères auxquels les autres ne peuvent pas suppléer et son absence est toujours regrettable. 
Amphora sp.

Planche XXXIl, figures 4 I, 42 et 43 .

Trois vues classiques d'un même spécimen de grande taille.

Figure 4I. Tue ventrale du frustule large, à extrémités arrondies, subtronquées, montrant les raphés fortement incurvés et flanqués d'un gros nodule. Du còté interne, on observe une rangée de mailles qui vont grandissant du centre vers les bouts.

Figure 43. Vue dorsale du frustule, avec la striation de la large bande connective et la striation un peu rayonnante des valves, dans leur partie assez large visible de ce côté.

Figure 42. Vue latérale du frustule. C'est, en réalité, une vue de la valve, mais elle n'en atteint que la partie extérieure au raphé, à cause de l'excentricité considérable de ce dernier. Sous cet aspect, le frustule est arqué, concave du côté ventral, arrondi aux deux bouts et fortement strié transversalement, par des rangées de petites mailles trop rapprochées pour qu'il soit possible de les figurer distinctement, à la faible échelle de nos clessins.

\section{Amphora sp.}

Planche XXXIII, figures $7 \overline{0}$ et 77 .

Forme de frustule plus longuement elliptique, à valves marquées, du còté interne du raphé, de deux rangs de mailles (fig. 76 , vue ventrale).

La vue dorsale du mème (fig. 77) montre l'aire suturale, elliptique, hyaline, bordée par la striation transversale de la partie externe des valves.

\section{Amphora sp.}

Planche XXXV, figures 50,5 I et 52 .

Trois vues classiques d'un frustule un peu plus petit, qui a ses extrémités plus tronquées et qui porte sur la face connective dorsale (fig. 52) des séries longitudinales de mailles séparées par des bandes hyalines ou striées de même direction.

La vue valvaire (fig. 5o) est assez large, peu arquée, obtuse-arrondie aux deux bouts, fortement sculptée de séries transversales de petites mailles.

\section{Amphora ovalis Küutzing.}

Planche XXXV, figures 59 et 6 .

Frustules elliptiques à extrémités tronquées, à aire ventrale étranglée par l'incurvation du raphé (fig. 59).

La vue latérale semi-valvaire est peu arquée, presque droite du côté ventral; atténuée-obtuse aux deux bouts et vigoureusement sculptée de séries transversales, un peu rayonnantes, d'alvéoles étroits. 
Amphora sp.

Planche XXXII, figures $+t$ et $t^{5}$.

Frustule lancéolé, brusquement rétréci vers les extrémités et tronqué aux deux bouts. Aire ventrale étranglée au milieu par la courbure des raphés. Valves exemptes de nodule apparent, dépourvues de structure spéciale du còté interne, faiblement striées seulement du còté externe (fig. 45).

La vue dorsale (fig. 44) ne montre que des bandelettes hyalines, peu convergentes, que nous avons figurées par une fine striation longitudinale.

\section{Amphora sp.}

Planche XXXII, figures $4^{6}$ et 47 .

Frustules allongés, à bords parallèles au milieu, arrondis aux deux extrémités. Aire ventrale rétrécie au milieu par une courbure assez douce des raphés. Nodules apparents. Partie externe des valves, visible de ce còté, peu large, striée transversalement. Partie interne assez large, striée longitudinalement. Bande connective droite (fig. 47).

Sur la vue dorsale (fig. 46) la zone connective est droite également, peu large, bordée par les stries transversales qui ornent les valves vues sur une assez grande largeur dans cette position.

\section{Amphora sp.}

Planche XXXII, figure 48 .

Nous ne figurons ici qu'en vue ventrale, pour la mettre en opposition avec la précédente, une forme de dimensions à peu près pareilles, dont le frustule est tronqué aux extrémités et rétréci au milieu.

L'aire ventrale, peu large, est rétrécie au milieu par une assez forte inflexion des raphés soulignés d'un nodule médian. La partie externe visible des valves est assez large et couverte d'une fine striation transversale serrée. La partie interne ne présente que des stries longitudinales, comme la zone connective, qui est sinueuse.

\section{Amphora sp.}

Planche XXXIII, figures 79 , so et $8 \mathbf{I}$.

Grande forme, à frustules largement elliptiques, tronqués aux deux bouts.

Aire ventrale rétrécie au milieu par la courbure des raphés. Les valves, largement développées du còté externe, s'y montrent hyalines, exemptes de striation, mais 
pourvues d'un renforcement stauronéiforme réfringent. Sur le còté interne le raphé est souligné d'une rangée de ponctuations dont le calibre diminue rapidement du centre vers les extrémités (fig. 78 ).

Le côté dorsal (fig. 79) ne laisse voir que des bandelettes longitudinales nombreuses, caractérisées. plutòt par une réfringence plus grande que par une striation véritable, comme celle à laquelle nous avons dù avoir recours pour en traduire graphiquement la différence d'aspect d'avec les lignes plus claires qui les séparent.

La vue latérale de la figure 80 est particulièrement intéressante, car elle fournit, en révélant le modelé tout particulier des frustules, un élément de diagnose que les deux autres ne pourraient faire présumer.

Amphora sp.

Planche XXXIII, figures 8 I et 82 .

Grande espèce à frustule subquadrangulaire, tronqué aux bouts, arrondi sur les angles, à aire ventrale (fig. 8I), très large, à peine déprimée au milieu par une très faible inflexion des raphés et occupée tout entière par une striation longitudinale peu prononcée. Les raphés sont pourvus d'un nodule médian. La partie visible des valves est très étroite et ne révèle aucune structure perceptible en milieu liquide.

La vue latérale (fig. 82) droite, longuement fusiforme, laisse voir le raphé peu arqué et le nodule qui le flanque du còté dorsal ou externe.

\section{Amphora sp.}

Planche XXXV, figures 53 et 54 .

Frustule petit, linéaire, oblong, un peu arrondi aux extrémités.

La vue ventrale (fig. 53) ne laisse voir qu'une faible partie des valves du còté extérieur aux raphés. Ceux-ci sont infléchis et rétrécissent au milieu l'aire connective ventrale.

La vue latérale ou valvaire de la figure 54 montre l'allure du raphé et l'extension de l'espèce de stauros qui intéresse tout le côté externe de la valve. Celle-ci est ornée d'une fine striation transversale, qui n'est bien visible qu'à sec.

\section{Amphora sp.}

Planche XXXV, figure 6I.

Frustule court, large, quadratique, à peine renflé vers le milieu, arrondi sur les angles.

L'aire ventrale, seule figurée, est très large et couverte de bandelettes longitudinales, parallèles, plus réfringentes que les lignes qui les séparent. 
Le raphé des valves est peu incurvé et ne laisse voir latéralement qu'une portion très étroite de surface valvaire hyaline, mais coupée d'un stauros.

Cette forme est probablement identique à Amphora complanata Grunow, que H. Van Heurck reproduit dans son Traité des Diatomées, planche XXVII, figure 785 , et qu'il décrit, page 235, sous le nom de Navicula complanata (Grunow).

Nous n'avons pas pu contrôler personnellement la légitimité du transfert de cet organisme dans le genre Navicula.

\section{Amphora sp.}

Planche XXXV, figure 62.

Frustule quadratique encore, court, large, arrondi aux angles.

Les valves présentent un raphé fortement incurvé; leur partie extérieure est hyaline mais traversée par un stauros. L'aire suturale ventrale est marquée de stries longitudinales qui épousent la courbure des raphés; ceux-ci en rétrécissent le champ vers le milieu.

\section{Amphora sp.}

Planche XXXV, figures 57 et 58 .

Très petite forme lancéolée, tronquée aux bouts, déprimée vers les extrémités.

Aire ventrale (fig. 57) très rétrécie surtout au milieu. Valves et aires connectives dorsale (fig. 58) et ventrale marquées seulement d'une fine striation longitudinale visible à sec.

\section{Genre Epithemia BRÉBISSON}

Caractères du genre. - Voir Van Heurck, loc. cit., page 294.

Les formes référables à ce genre, que nous avons observées en spécimens rares, sont toutes étrangères à nos connaissances des eaux douces de nos régions tempérées.

Manquant des moyens propres à les identifier, nous nous bornerons à en donner le signalement.

\section{Epithemia sp.}

Planche XXXV, figures 55 et 56 .

Frustule lancéolé, atténué, tronqué aux deux bouts, à aire suturale dorsale droite, striée longitudinalement (fig. 55). 
Valves peu arquées, à extrémités rostro-capitées, à bord ventral courbe, à bord dorsal presque droit. Striation valvaire transversale formée de rangées d'alvéoles étroits séparées par des trabécules transversaux bien accusés (fig. 56).

\section{Epithemia sp.}

Planche XXXIV, figures 59 et 60 .

Grande forme à frustule étroit, allongé, arrondi aux bouts, renflé vers le milieu.

La zone connective dorsale est linéaire et bordée d'une partie visible des valves qui accuse une forte striation transversale (fig. 59).

La vue ventrale (fig. 6o) montre que la zone connective est très resserrée de ce côté, vers le milieu, où elle ne se traduit plus que par une fente, mais va en s'élargissant de là vers les extrémités.

Les valves très dissymétriques ne présentent, du còté ventral, qu'une étroite bordure fortement rayée, séparée de l'autre moitié valvaire par une dépression presque hyaline.

L'autre partie a un développement large non seulement sur le côté ventral du frustule, mais aussi, comme on le voit dans la figure 59 , sur le côté dorsal.

\section{Epithemia sp.}

Planche XXXII, figures 62,63 et 64 .

Cette forme plus courte, plus largement lancéolée présente des caractères plus explicites que la précédente et peut servir à en faciliter l'intelligence.

La figure 62 en est la vue dorsale, avec l'aire connective un peu dilatée au milieu.

La figure $6_{4}$ en est la vue latérale ou valvaire, avec un pseudo-raphé large, de forme particulière, qui divise la valve en deux parties très inégales et dissemblablement structurées.

La figure 63 enfin en est la vue ventrale, montrant le rétrécissement médian de la zone connective, la zone marginale rayée des valves et l'aire hyaline qui tient lieu de raphé en quelque sorte et qui est coupée d'un pseudo-stauros.

\section{Epithemia sp.}

Planche XXXIV, figure 61 .

Forme plus petite dont nous n'arons pu saisir qu'une vue.

Les valves sont pourvues d'une sorte de raphé, en forme de sillon longitudinal, et présentent, sur les bourrelets parallèles que ce sillon détermine, des rayures transversales très accusées. 
Les valves laissent entre elles, au milieu du frustule, une aire hyaline en forme de fente, au delà de laquelle elles entrent en contact, pour s'écarter à nouveau vers les deux bouts du frustule.

\section{Genre Grammatophora}

Caractères du genre. - Voir Van Heurck, loc. cit., page 353.

Nous tenons quelques espèces de ce genre de certaines récoltes côtières et surtout de la rivière Matotchka, dans l'ile sud de la Nouvelle-Zemble.

Grammatophora islandica Ehrenberg.

Planche XXXII, figures 55, 56 et 57 .

Frustules à vue suturale quadratique, aux angles émoussés, disposés en chaînettes linéaires ou en zigzag, et se présentant naturellement, sur les slides, dans cette position qui est la plus stable et aussi la plus caractéristique de l'objet (fig. 55).

Cloisons internes serpentineuses dessinant trois ondulations terminées par un crochet recourbé.

La vue valvaire (fig. 57) est étroitement lancéolée, arrondie aux deux bouts, transversalement striée, sauf aux extrémités, où règnent de petites aires hyalines que l'on peut voir aussi bien sur la vue suturale.

La figure 56 en est une coupe optique, en profondeur, passant par les cloisons internes.

\section{Grammatophora serpentina Ehrenberg.}

Planche XXXIV, figures 55 et 56 .

Frustules rectangulaires, en vue suturale (fig. 55), munis de cloisons internes serpentineuses, de cinq à six ondulations, terminées par un crochet étroit. Ce crochet est souvent figuré plus large; il se peut que la forme en soit très variable.

La figure 56 montre la vue de la valve, linéaire, lancéolée, aux bouts arrondis et hyalins, tandis qu'elle présente ailleurs une fine striation transversale.

Dans cette figure, nous avons reproduit en mème temps la projection des cloisons internes. 


\section{Grammatophora arctica Cleve.}

Planche XXXIV, figures 57 et 58.

Frustules de grandes dimensions, quadratiques en vue connective, ornés, sous cet aspect, de cloisons internes faiblement sinueuses, renflées à leur bout libre, et de séries de ponctuations sur la face suturale, entre les couples de ces cloisons (fig. 57).

La vue valvaire (fig. 58) est linéaire, un peu renflée au milieu et aux extrémités, où s'observent de petites aires hyalines que n'atteint pas la striation transversale.

Cette forme curieuse n'est pas rare dans la rivière Matotchka. Nous aurions voulu pouvoir disposer de plus de place dans nos planches pour en traduire non seulement les modalités morphologiques mais aussi les particularités du cytoplasme qui nous a paru présenter des phénomènes curieux.

\section{Genre Plagiogramma Grevilli:}

Caracteres du genre. - Cfr. Van Heurck, loc. cit., page 337.

\section{Plagiogramma Gregorianum Greville.}

Planche XXXII, figures 58 et 59 .

Frustules associés en colonie rubanée par contact valvaire, comme dans la figure 58, qui reproduit trois éléments en vue suturale. Ainsi vus les frustules ont une section rectangulaire. En vue valvaire (fig. 59), ils sont oblongs, lancéolés.

Les valves ont leur partie médiane occupée par un pseudo-ocellus allongé transversalement et leurs bouts par un espace lisse, tandis que le reste est couvert de stries transversales résultant de séries d'alvéoles étroits séparées par des trabécules plus robustes.

Origine. - Rarement rencontrée, dans des produits de pêche côtière.

\section{Genre Rhabdonema KÜtzING}

Caractères du genre. - Voir Van Heurck, loc. cit., page 360.

\section{Rhabdonema arcuatum (Agardh) Kützing.}

Planche XXXII, figures 60,61 et 62.

Frustules grands ou même très grands, rectangulaires, disposés en série rubanée ou zigzaguée, suivant le développement de leur face connective, qui répond à la position la plus naturelle des Rhabdonema (fig. 60 et 6I). 
Cette vue connective présente, à l'intérieur, de nombreuses fausses cloisons parallèles dont les intervalles sont occupés par des trabécules superficiels transverses, qui sont beaucoup plus apparents et mieux discernables en milieu liquide qu'à sec, car dans ce dernier état les frustules deviennent opacues.

Les valves, vues latéralement dans cette position, se montrent striées transversalement, à l'exception des deux extrémités qui sont un peu protubérantes et lisses.

C'est par elles que se rattachent, à la faveur d'un grumeau d'une substance cimentaire, $c$, les frustules parfois nombreux d'une colonie, comme dans la figure $6 \mathrm{I}$.

La figure 60 en reproduit, dans la même position, un spécimen de très grande taille.

La vue valvaire (fig. 62), difficile à observer dans les objets naturels et difficile à identifier après macération et dislocation des frustules, apparait lancéolée, à extrémités obtuses et lisses, à pseudo-raphé bien marqué, à structure rayée transversalement.

\section{Rhabdonema minutum Kützing.}

Planche XXXII, figures 63,64 et 65.

Frustules plus petits, rectangulaires en vue connective, un peu arrondis aux angles, disposés en colonie linéaire (fig. 64) ou zigzaguée' (fig. 63). La différence d'avec la précédente gît surtout dans l'aspect et la disposition des fausses cloisons, qui présentent ici une sorte d'alternance entre leur point d'attache et leur terminaison. Les travées qui les soulignent sur la vue suturale sont reliées par des trabécules dont l'impression de grosseur varie avec la nature du milieu dans lequel se fait l'observation.

La valve, vue de face (fig. 65), se montre lancéolée et courerte de stries transversales coupées au milieu par un pseudo-raphé droit.

\section{Rhabdonema hyalinum sp. n.}

Planche XXXIV, figures 53 et 54 .

Frustules rectangulaires en vue connective (fig. 53) et réunis en colonie droite ou brisée par une substance cimentaire, c. La valve, vue de face dans la figure 54, se montre fortement élargie en son milieu, entre deux brusques rétrécissements terminaux. Elle est couverte de stries transversales ténues qui ne nous ont pas paru coupées par un pseudo-raphé.

La vue connective de la figure 53 montre, à l'intérieur des frustules, la présence de fortes cloisons incomplètes qui prennent leur point d'attache alternatirement à gauche et à droite et s'atténuent avant d'atteindre le còté opposé à celui de leur insertion. Cette dernière se traduit par un épaississement réfringent de la membrane. La face connective est dépourvue des striations qui donnent aux espèces précédentes une physionomie si spéciale.

Cette forme se rapporterait aussi bien au genre Tabellaria Ehrenberg.

Distribution géographinge. - Nous tenons ces trois formes de la partie sud de la Mer de Barents, dans des produits de pêche plus riches en petits Crustacés qu'en Protistes. 


\section{Genre Striatella AGARDH}

Caractètres du genre. - Voir Van Heurck, loc. cit., page 362.

\section{Striatella unipunctata Agardh.}

Planche XXXII, figures 61,62 et 63.

Frustules en forme de rectangle aux angles brièvement tronqués, parcourus intérieurement par de fausses cloisons nombreuses et délicates entre lesquelles se dessine une très fine striation transverse (fig. 6r). C'est naturellement sous cet aspect que l'objet se présente en position stable.

La figure 62 donne la forme de la valve, qui est parcourue par un pseudo-raphé, mais qui ne laisse roir aucune sculpture en milieu liquide.

La figure 63 est une coupe transversale optique du frustule passant par une des fausses cloisons. Celle-ci se profile sous la forme d'une ellipse plus courte inscrite à l'intérieur de celle que détermine la section du frustule.

Distribution GÉographinue. - Très rarement observée dans la Mer de Barents.

\section{Genre Denticula KüTzING}

Caractères du genre. - Voir Tan Heurck, loc. cit., page 35i.

Denticula sp.

Planche XXXIl, figures 66 et 67 .

Frustules isolés ou en chaìnette, à face connective quadrangulaire, montrant de chaque còté l'extrémité capitée de sept còtes assez courtes et inégales, dont on ne voit ainsi que la section optique (fig. 66).

La face valvaire est lancéolée (fig. 67) et montre les cloisons dans leur position transverse vis-à-vis du frustule.

Distribution géogRApHIQle. - Trouvée rarement dans les boues glaciaires.

\section{Genre Meridion Agardh}

Meridion circulare Agardh.

Planche Xxxll, figures is et $6 \mathrm{n}$.

Nous avons rencontré, clans les boues glaciaires, des frustules isolés de cette espèce, dont on connait mieux les colonies circulaires telles qu'elles se présentent si communément clans nos eaux douces. 
La figure 68 en reproduit une vue connective cunéiforme; la figure 69 , une rue valvaire également cunéiforme, montrant les còtes transversales assez distinctes : seuls détails de structure visibles en milieu liquide.

\section{Genre Diatoma de Candolle}

Caractères du Genize. - Voir Vai Heurck, loc. cit., page 348.

\section{Diatoma vulgare Bory.}

Planche XXXII, figure jo.

Frustules petits, étroits, linéaires, disposés en zigzag, bien connus partout dans les eaux douces.

Nous n'en figurons que la vue connective, avec l'endochrome, en $a$; sans l'endochrome, en $b$, pour mieux voir les còtes transversales des valves.

Origine. - Rivière Matotchka.

\section{Genre Gomphonema AGARdH}

Caractères du genre. - Cfr. Van Heurck, loc. cit., page 268.

\section{Gomphonema geminatum Agardh.}

Planche XXXili, figures 64 et 65 .

Belle et grande forme répandue dans la rivière Matotchka, d'où nous la tenons.

Frustule stipité sur pédicelle épais, $p$, présenté en vue valvaire dans la figure 64 , et en vue connective dans la figure 65.

Valve grande, renflée au milieu et inégalement renflée-capitée aux deux extrémités.

Raphé droit, médian, renforcé par un nodule hyalin porteur de quelques ponctuations en creux placées du mème côté et visibles même en milieu liquide. Stries transversales très rapprochées, bien accusées, radiantes autour du nodule central et des deux nodules terminaux, sauf à l'extrémité où s'insère le pédicelle.

Les frustules sont souvent géminés, soudés par leurs faces valvaires naturellement.

\section{Gomphonema sp.}

Planche XXXIII, figures $7^{\circ}$ et $7^{1}$.

Petite espèce répandue sur les glaces et dans les boues glaciaires, dont l'examen en milieu liquide est peu propre à la faire distinguer des nombreuses petites formes congénères que les diatomistes ont décrites. 
Gomphonema sp.

Planche XXXIII, figures 66 et 67 .

Forme très commune aussi dans les amas de Diatomacées nichés dans de petites dépressions creusées dans la partie immergée des glaçons de la Mer de Kara.

Elle y forme des amas compacts d'individus agrégés dans une sorte de gangue mucilagineuse.

La figure 67 en reproduit des vues valvaires. La striation y est difficile à saisir, en milieu liquide.

Elle parait transversale, aux deux còtés du raphé, sans auréole autour du nodule médian, qui est lui-mème peu visible.

La figure 66 montre la vue connective des frustules, qui sont cunéiformes.

\section{Gomphonema sp.}

Planche XXXIII, figures 68 et 69 .

Forme un peu plus grande, mais plus rare, qui parait affine à G. acuminatum Ehrenberg.

Striation très délicate, difficile à voir en milieu liquide.

Elle appartient aussi à la florule des glaces.

\section{Genre Licmophora AgARDH}

Caractemes du genre. - Cfr. Van Heurck, loc. cit, page 3 fi.

\section{Licmophora Lyngbyei (Kützing) Grunow.}

Planche XXXV, figures 68,69 et 70.

Frustules cunéiformes de grandeur variable, laissant voir, sur le còté connectif, de nombreuses stries sinueuses dont deux beaucoup plus marquées, en mème temps que des cloisons internes (fig. 68 et 69). Celles-ci se traduisent, sous un autre aspect naturellement, sur la vue valvaire, qui est lancéolée, claviforme (fig. 70).

()Rigine. - Cette espèce n'est pas rare clans le Détroit de Matotchkine, où elle est sans doute mélangée à d'autres de ses congénères, qu'il n'est guère possible d'étudier en milieu liquide et qu'on ne parvient pas d'ailleurs à isoler du fouillis de Chétocérées dans lequel elles sont engagées. 


\section{Genre Eunotia Ehrenberg}

Caractères du genre. - Voir Van Heurck, loc. cit., page 298.

\section{Eunotia dolium sp. n.}

Planche XXXIII, figures 72 et 73 .

Frustules longs, arqués, élargis au milieu comme des douves et déterminant, par leur groupement latéral régulier, des colonies en forme de tonneau, comme dans la figure 73 , qui n'en reproduit qu'un fragment de trois individus.

La valve (fig. 72) est arquée, pourvue d'un léger renflement au milieu du côté interne, réfléchie-rostrée aux deux extrémités et couverte d'une fine striation transversale.

Cette forme a un faux air de Ceratoncis arcus Kützing; mais le signalement n'en est pas concordant.

Origine. - Elle n'est pas rare dans la rivière Mlatotchka où elle semble être commensale d'autres Algues, sur lesquelles elle est fixée.

Eunotia arcus Ehrenberg.

Planche XXXIII, figures 74 et 75 .

Les figures 74, vue connective, et 75 , vue valvaire, suffisent à rappeler cette espèce d'eau douce, dont le spécimen reproduit n'est qu'une de ses variantes.

Origine. - Sur les glaces et dans les boues glaciaires.

\section{Genre Thalassiothrix Cleve et Grunow}

Caractères du Genre. - Voir Nordisches Plankton, page if6.

\section{Thalassiothrix longissima Cleve et Grunow.}

Planche XXXII, figure +9 .

Frustules linéaires, extrèmement longs, à section étroite, quadrangulaire, à arètes scabres, à faces couvertes d'une très fine striation transversale, marquée surtout sur les angles.

Notre figure n'en reproduit qu'un fragment terminal, sous l'aspect assez fruste qu'il présente au faible grossissement employé.

Distributiox GÉographique. - Cette espèce septentrionale est remarquablement rare dans nos matériaux. Nous n'en avons trouvé que de rares spécimens disséminés dans la Mer de Barents. 


\section{Genre Synedra EnREnberg}

Caracteres du genre. - Cfr. Tan Heurck, loc. cit., page 307.

Formes non planktoniques, mais assez fréquemment ramenées par le filet de pèche dans des régions còtières où les courants les ont entrainés. On les rencontre aussi communément dans la florule des glaces.

Synedra ulna Ehrenberg.

Planche XXXII, figures 50, 51, 52, et planche XXXlIl, figures 56 et 57.

Ces différentes formes nous paraissent se rapporter à diverses variétés connues de S. ulna.

Les figures 5o, vue connective, et 5I, vue valvaire, sont d'un même individu, remarquable par ses dimensions et son élargissement médian, observable sur les deux vues, de face et de profil.

La figure 52 en est un spécimen encore plus long, linéaire, un peu atténué vers les bouts et légèrement capité aux sommets, en vue connective.

Les figures 56 et 57 , d'un mème individu vu sous ses deux faces, le montrent plus rigoureusement linéaire encore ct subcapité, en vue valvaire.

\section{Synedra pulchella Kützing.}

Planche Xxxil, figure 53.

Fragment d'une colonie en éventail. Les frustules y sont naturellement représentés en vue connective, la plus interprétative des caractères de l'espèce.

\section{Synedra affinis liützing.}

Planche XXXII, figure 54 , en bas de la planche.

Talve lancéolée, linéaire, à extrémités amincies, faiblement rostro-capitées, à stries marginales laissant un pseudo-raphé lancéolé de largeur notable.

\section{Synedra acus Grunow.}

Planche XXXlII, figure 58.

Valve étroite, lancéolée, amincie vers les bouts, très faiblement capitée, couverte de stries transversales qui ne semblent pas laisser ni de pseudo-raphé ni de nodule, comme dans la variété delicatissima Grunow. 


\section{Synedra Gallionii Ehrenberg.}

Planche XXXIII, figures $5+$ et 55.

Valves linéaires aux extrémités arrondies, à pseudo-raphé distinct, mais sans nodule hyalin central (fig. 55, vue valvaire; fig. 54, vue connective).

La striation, bien visible sur les deux vues, apparait assez grossière à sec, comme en $a$, beaucoup plus délicate, en milieu liquide, comme en $b$.

\section{OBSERVATION}

Après avoir cléblayé notre exposé d'un certain nombre de genres peu ou non planktoniques, auxquels nous arons dù faire une place sur nos planches au petit bonheur de la rencontre opportune de leurs représentants, nous allons nous arrêter un moment sur les organismes que nous avons plus spécialement groupés dans les planches XXXIII et XXXIV.

Gràce à leurs groupements en colonies, ils peuvent vivre de la vie pélagique.

Leurs thalles, d'aspects divers, forment parfois l'élément dominant de certains produits de pèche au filet fin et leur distinction ne manque pas de difficulté, tant à cause des aspects variés de leur cytoplasme plus ou moins modifié sous l'action des réactifs, que de leurs phases évolutives et de leur faible silicification, qui ne permet guère de les étudier à sec et moins encore après certaines pratiques de macération qui n'en laisseraient rien de discernable.

Il s'agit d'espèces de Fragilaria, Achnanthes, Stanroncis, Navicula, Nitzschia, etc., que les diatomistes ont généralement sacrifiées dans leurs procédés d'incinération appliqués à la mise en évidence des minutieux caractères de structure d'autres espèces plus réfractaires à cette technique violente, chère aux fabricants de "Typenplatte ", clont le mérite est d'ailleurs incontestable et l'habileté au-dessus de tout éloge.

\section{Genre Fragilaria Lringite}

Caractères du genre. - Cfr. Van Heurck, loc. cit., page 323, et Nordisches Plankton, page i 13 .

\section{Fragilaria islandica Grunow.}

Planche XxxIll, figures i à 6 .

Littérature. - Voir Nordisches Plankton, page il4.

Caractères. - La figure I en reproduit un fragment de colonie rubanée, très large. Les frustules se touchent sur toute leur face valvaire. Ils sont généralement 
étroits, longuement linéaires en vue connective, et ne laissent pas percevoir des détails de structure en milieu liquide.

Le noyau est petit, central; le cytoplasme, très clair vers les extrémités, mais accumulé sur les deux faces valvaires, y forme deux chromatophores diffus, ou mieux un endochrome partagé en deux portions valvaires, dans les cellules au repos, comme en a (fig. I).

Lors de la cytodiérèse (en b, fig. I), les cellules-filles emportent chacune une des deux moitiés de l'endochrome maternel. Ce n'est que plus tard, pendant la consolidation des valves jeunes, que le dédoublement de l'endochrome s'opère à son tour et que la cellule reprend son aspect ordinaire.

Ces phénomènes apparaissent beaucoup mieux dans la figure 4 empruntée à une colônie beaucoup moins large et conséquemment formée d'individus à axe transvalvaire plus développé. Le premier frustule, à gauche, est adulte et montre son noyau en repos physiologique, entre les deux chromatophores pariétaux.

Son voisin, marqué $b$, présente les premiers symptòmes de la division, qui se poursuit à des phases diverses, dans les frustules marqués $d$ et $e$. Ceux qui se trouvent sous la lettre $a$, enfin, sont des jeunes en voie de reconstitution.

La figure 5 reproduit la vue latérale, la vue de champ, si l'on veut, du même fragment de colonie.

Les figures 2 et 6 , où l'on a fait abstraction du protoplasme, montrent les membranes sous l'aspect qu'elles présentent en milieu aqueux. On voit que la membrane connective, qui est très délicate, accuse de légers plissements visibles surtout aux extrémités des frustules.

La figure 3, qui est la coupe optique d'un frustule, parallèle au plan valvaire, montre l'épaisseur de la membrane plus accusée sur les flancs qu'aux extrémités.

Parasitisme. - Cette espèce est très sourent parasitée par Olpidizm, ol. Nous en avons figuré plusieurs kystes vidés, qui se sont développés dans des frustules dont ils ont provoqué l'accroissement hypertrophique.

Dans la figure 2, on voit, en ol', un kyste qui a envahi deux frustules contigus.

La figure I montre deux kystes, ol, développés dans le même frustule.

L'orifice de sortie, o, des sporules d'essaimage peut se produire à n'importe quel endroit de la zone connective.

Distribution gÉgraphique. - Espèce assez abondante aux environs de Tromsö et dans la Mer de Kara, près du Détroit de Matotchkine.

\section{Fragilaria mollis sp. 11 .}

Planche XxxIII, figures $7,8,9$.

Caractéres. - Nous devons faire une place à part pour une forme beaucoup plus petite qui, à défaut d'un examen attentif, pourrait ètre confondue avec les plus petits représentants de l'espèce ci-dessus. 
Les frustules forment des colonies le plus souvent incurvées en portion de spire (fig. 7).

Ils ont des parois minces; leur zone connective parait sillonnée de plissements longitudinaux dont l'accentuation croit vers les extrémités; leur contenu cellulaire forme encore deux chromatophores valvaires, mais ceux-ci paraissent diffus et irréguliers; la coupe optique transversale, reproduite à droite de la figure 8 , est largement lancéolée, ventrue au milieu, arrondie aux sommets.

Nous tenons ce dernier caractère surtout comme spécifique, indépendamment de la mollesse des frustules, qui nous a suggéré le nom que nous proposons.

Parasitisme. - C'est une espèce de la florule glaciaire tout particulièremęnt sujette au parasitisme de la part d'Olpidium, qui envahit souvent tous les sujets d'une colonie.

Figure 8. Fragment parasité présentant, en $a$, le phycophage, ol, en voie d'envahissement; en $b$, en voie de sporulation; en $c$, à l'état de kyste vidé, après la sortie des sporules par l'orifice 0 .

La figure 9 présente d'autres phases du parasite que l'on voit, en a, à l'état végétatif dans un frustule qu'il envahit entièrement; en $b$, en voie d'enkystement; en $c$, en période de sporulation; en $d$, après vidange du zoosporange.

Distribution géographioue. - Abondante sur les glaces de la Mer de Kara et exceptionnellement dans les eaux sous-jacentes.

\section{Fragilaria oceanica Cleve.}

Planche XXXIII, figures it à $2 .+$.

Caractères. - Colonies rubanées droites ou diversement contournées, d'aspect très variable suivant l'état de fixation du cytoplasme, la diversité des phases évolutives des frustules et aussi la nature du milieu.

Pour ces raisons, la connaissance de l'espèce réclame un examen comparé d'un grand nombre de spécimens aussi rapprochés que possible de l'état naturel.

C'est sous un aspect analogue à celui de notre figure If qu'elle a fait son entrée dans la science en 1873 ( 1 ).

Cette vue suffirait en effet à caractériser l'espèce, si elle était toujours en sporification, ce qui n'est évidemment pas le cas. Elle a donc besoin d'un signalement plus complet. Celui-ci ressortira des figures que nous lui consacrons. 
Dans notre figure I4, outre les spores, sp, représentées vides, en c, comme dans la figure de CLEIE, on voit, vers la gauche, en $a$, le protoplasme de deux cellules contiguës se ramasser près de la paroi qui les sépare et prendre la forme d'un couple de spores, que l'on voit, en $b$, pourvues d'une membrane propre.

Vers la droite, on a la vue connective de frustules non sporifères dont la striation valvaire se profile sur les bords, tandis qu'elle est représentée de face sur une valve isolée reproduite dans la figure I5. On remarquera que la forme de celle-ci est lancéolée-elliptique.

La figure I6 reproduit, en coupe latérale optique, une partie de l'objet de la figure I $_{4}$ et permet de se rendre compte de la forme des spores géminées et de leurs rapports avec leurs matricules.

Mais tel n'est plus l'aspect des frustules végétatifs (fig. I7). Ceux-ci sont souvent remarquables par la position latérale de leur noyau rapproché du noyau également latéral de la cellule-sœur voisine. C'est que les cytodiérèses répétées créent des couples génétiques de frustules, dans lesquels les noyaux-filles restent rapprochés tout un temps, pendant que les chromatophores, ch, deux placés obliquement dans chaque frustule jeune $y$ affectent entre eux la disposition d'une croix de Saint-André.

La plus ou moins bonne fixation de ces éléments délicats peut leur donner des aspects dont la même figure montre une série de dégradations, de la gauche vers la droite, où ils sont réduits à de petits grumeaux sphériques.

Au moment de la division (fig. I8) l'aspect change; car le noyau, d'abord latéral, dans les frustules situés à gauche de la figure, devient central, en $a$, pendant que les chromatophores se divisent latéralement et que les produits de cette division s'individualisent, en $b$, et en $c$, pour ètre séparés ensuite, en $d$, par une cloison qui départage le domaine de deux cellules-filles, au moment de la division nucléaire.

Nous avons dit et laissé supposer conséquemment que chaque frustule ne comportait, pendant le jeune àge, que deux chromatophores; c'est que la seule rue de face des colonies rubanées fait croire qu'il en est bien ainsi. En réalité, il n'en est rien, car les chromatophores visibles dans cette position en cachent d'autres symétriques situés derrière.

Pour se rendre compte du fait, il faut examiner les colonies dans une position de champ, comme il a été fait pour l'objet de la figure IS, dont l'aspect, sous cette autre incidence, est reproduit dans la figure Ig.

Mais dans cette position encore, les chromatophores supérieurs cachent les inférieurs et on ne peut s'assurer de la présence de ces derniers qu'en abaissant l'objectif, pour les mettre au point à leur tour.

On roit par là comme il serait aisé de confondre ces vnes de champ de colonies larges arec cles vues de face de colonies étroites. Pour les distinguer, il importe de culbuter les sujets d'observation sous le couvre-objet, à l'effet de juger des dimensions relatives de leurs axes. 
Dans la figure 20 nous reproduisons un autre aspect, souvent observé, que présentent les thalles vus de champ : aspect en zigzag, dans lequel l'action des réactifs fixateurs doit ètre pour quelque chose, car, sur les angles saillants, on voit se profiler de petites masses protoplasmatiques, $s$, qui ont fait hernie au travers de la membrane connective et qu'on ne peut interpréter que comme des manifestations d'un traumatisme physicochimique.

Cette déformation accidentelle n'empèche pas la constatation de la présence, dans chaque frustule jeune, d'un nombre de chromatophores, ch, double de ce que ferait supposer la simple vue sagittale.

Ces facies divers qui, à première vue sembleraient inconciliables, seraient bien faits pour dérouter l'observateur, s'il n'avait sous les yeux des éléments suffisants de contròle ou s'il en négligeait l'étude minutieuse.

Les figures If à 20, dont il vient d'être question, se rapportent à des spécimens de la Mer de Kara.

Il faut un peu d'attention pour les assimiler à d'autres empruntées à des matériaux d'origine plus septentrionale, surpris dans d'autres conditions physiologiques propres ou sous l'influence d'une ambiance suffisamment différente pour leur imprimer une touche particulière. Nous leur consacrons les figures 2 I ̀̀ 24.

Figure 2I. Fragment de colonie de frustules peu larges, mais très développés suivant l'axe transvalvaire. Cette circonstance permet aux chromatophores, ch, de prendre un grand développement en vue valvaire et de réaliser, entre deux frustules jumeaux, des sortes de croix de Saint-André, dont les bras prennent parfois des courbures élégantes, comme on le voit à gauche de la figure. Il se peut que l'effet contractif du liquide conservateur soit pour quelque chose dans ce résultat.

Figure 22. Autre aspect d'une colonie similaire dont les chromatophores ont leur plein épanouissement et que nous avons figurée pour en noter les phénomènes de cytodiérèse.

Le frustule, a, a divisé ses chromatophores, qui sont actuellement au nombre de quatre, visibles du cóté de l'observateur. Comme nous l'avons dit, ils en cachent quatre autres qui leur sont symétriques, du còté opposé. Le frustule, b, laisse voir son noyau en voie de division, pendant que s'ébauche la plaque cellulaire. Dans le frustule, c, la division du noyau, $n$, est chose faite virtuellement, mais non encore suivic de la cytodiérèse. Celle-ci est accomplie dans le frustule, $d$, par la formation de deux valves nouvelles (jui, par la minceur et l'absence de striation, contrastent arec les valves anciennes, plus fortes et striées.

La figure 23 en reproduit encore une autre physionomie, qui est due à la forme contractée et arrondic des chromatophores, dans des frustules à petit développement transvalvaire. 
Sporification. - Quand elle se produit, la sporification s'étend d'habitude à un grand nombre d'éléments dans la même colonie. Nous en retraçons l'étape la plus intéressante dans la figure 24 dont l'intelligence réclame un peu d'attention.

On y observera que les spores accouplées, sp, proviennent de frustules jumeaux séparés par des valves jeunes qui ne se sont guère différenciées et qu'ils s'y sont formés, non pas par une simple concentration du protoplasme contre ces valves jeunes, mais bien à la suite d'une nouvelle division, qui a pour résultat le sacrifice d'une partie de la cellule génératrice de chacun d'eux. La partie ainsi sacrifiée reste blottie contre la valve ancienne, marquée de la lettre $x$, où nous la signalons par la lettre $r$.

Ce résidu est une véritable cellule avec noyau, cytoplasme et chromatophores, dont les matériaux seront utilisés pour la nutrition de la spore voisine et finiront par être résorbés et disparaitre ainsi complètement.

La dernière division dont nous venons de parler n'est pas suivie de la formation de deux valves nouvelles, mais d'une seule, qui est élaborée par le cytoplasme de la spore et qui prend une forme convexe.

On ne manquera pas de rapprocher ces phénomènes de ceux que nous arons mis en relief, à propos de la production des spores de Thalassiosira gravida Cleve, où l'on sait qu'une portion de la matricule est aussi sacrifiée au profit de la spore.

Dans l'espèce qui nous occupe ici, nous n'avons jamais vu le processus de sporification se compliquer davantage. Il aboutit naturellement à la production de spores géminées, que nous avons représentées à l'état squelettique seulement, en $c$, dans la figure I4.

Si tel est bien l'aboutissement réel et définitif du phénomène, cette forme doit être prise pour spécifiquement distincte d'une autre que nous tenons des parages du Grönland et qui produit des spores isolées.

Nous en présentons les principaux traits ci-après.

\section{Fragilaria groenlandica sp. n.}

Planche XXXIII, figures io à 13.

Caractères. - La figure io en montre un fragment de colonie rubanće dont les frustules, à l'état végétatif, rappellent singulièrement l'aspect de ceux de l'espéce précédente, malgré la différence d'extension des chromatophores; ce qui pourrait bien tenir, en partie du moins, au mode de fixation des objets.

Les cellules figurées présentent une série complète des phases de la division du noyau, $n$, et des chromatophores, $c h$, que le lecteur repérera aisément, avec un peu d'attention, lans l'ordre des lettres $a, b, c, d, e$, qui en marque la succession.

Sporification. - La figure it, reproduite d'un échantillon choisi pour sa valeur interprétative, nous aidera à faire saisir la série des phénomènes de sporification. Le 
processus aboutit ici à la production des spores individualisées dans autant de matricules distinctes, suivant une marche progressive qui va de gauche à droite dans la figure.

On ne pourra se défendre d'y reconnaitre une analogie remarquable, mutatis mutandis, avec les phénomènes similaires qui se produisent dans Thalassiosira gravida.

Le processus intéresse le plus souvent à la fois quatre frustules voisins, dérivés eux-mèmes de deux divisions successives d'une même souche, comme ceux marqués de la lettre $a$ dans la figure.

De ces quatre frustules, qui sont ainsi rendus solidaires en quelque sorte, les deux externes ont déjà subi une nouvelle division, inégale celle-ci, destinée à sacrifier la petite moitié, $r$, tandis que l'autre s'élabore une valve convexe qui sera la valve primaire d'un endocyste.

La mème chose se réalise un peu plus tard, mais en sens inverse, dans les deux cellules internes, qui sont contiguës. Un peu d'attention permettra de relever le début de l'opération dans celle de gauche qui est un peu plus avancée.

Dans la tétrade suivante, marquée $b$, la division inégale des cellules internes touche à sa fin, et l'on assiste à la formation des valves primaires convexes de deux nouvelles spores symétriquement placées vis-à-vis des premières, avec lesquelles elles forment ainsi deux groupes géminés.

L'abandon de produits résiduaires, $r$, qui s'était effectué aux còtés de la cloison $x$, qui sépare les tétrades, se renouvelle aux còtés de la cloison $x^{\prime}$, qui sépare les demi-tétrades.

Dans les deux tétrades suivantes, marquées $c$, on voit les spores, qui ne sont encore ici qu'ébauchées, présenter la disposition par couples qu'on leur connait dans $F$. oceanica, et qu'on a vues reproduites, sous des aspects divers, dans les figures I4 et 24 de la mème planche.

Mais ce qui est, dans cette dernière, la forme définitive de ces organes, s'il faut en croire les auteurs, n'est encore, dans $F$. grocnlandica, qu'un acheminement vers une évolution ultérieure.

En effet, dans la tétrade marquée $d$, on voit les prétendues sporeśs géminées détacher leur cytoplasme de la cloison mitoyenne et s'élaborer aussi de ce còté une valve nouvelle convexe, une valve secondaire, $v^{\prime}$, symétrique à la valve primaire, $v$, déjà acquise antérieurement, sans (qu'i] intervienne un nouveau phénomène de division pour présider à cette dernière étape du développement.

Par l'acquisition de cette valve secondaire, les spores deviennent indépendantes l'une de l'autre et mème du cytoderme de leur matricule respective, au milieu de laquelle elles se fixent, comme on le voit d'abord en $c$, et plus tard en $f$, où le processus prend fin.

Chaque frustule fertile est alors pourvu de sa spore propre, à la suite d'une rénovation cellulaire partielle, dont. les produits résiduaires sont cependant repris, par osmose sans doute, pour l'alimentation des spores, car ils disparaissent plus ou moins rapidement et ne laissent plus de traces quand la sporification a pris fin. 
La figure I2 reproduit, à l'état squelettique, une spore, sp, incluse dans sa matricule, détachée elle-mème d'une colonie, par macération, et examinée à sec.

La figure 13 montre la même, en vue valvaire. On peut voir ici que la spore, sp, est d'un calibre plus petit que sa matricule, aussi bien en vue valvaire qu'en vue connective.

Remargue. - Après cet exposé, le lecteur comprendra la raison et la portée des réserves formulées plus haut au sujet de ces intéressants organismes.

Si, comme nous le disions, il reste avéré, par des observations positives et péremptoires, que $F$. oceanica Cleve a réellement ses spores géminées à l'état de maturité complète, on conviendra qu'il y a lieu de tenir pour spécifiquement distincte $F$. groenlandica nobis, dont les spores sont isolées.

Si, au contraire, on découvre que la gémination des spores attribuée à $F$. oceanica ne représente qu'une phase évolutive de la sporification de cette espèce et non la phase ultime qui, éventuellement, devrait survenir pour l'individualisation des spores, comme nous l'avons montré dans $F$. groenlandica, la distinction entre ces deux formes tombera d'elle-même, par défaut de fondement réel; mais il restera démontré, une fois de plus, que la connaissance des micro-organismes planktoniques aurait tout à gagner d'une étude plus physiologique des matériaux recueillis.

\section{Fragilaria virescens Ralfs?}

Planche XXXIII, figure 25.

Espèce beaucoup mieux silicifiée dont les frustules longuement rectangulaires, en vue connective, laissent voir, même en milieu liquide, la striation des valves qui se profile sur les bords des individus soudés en colonie rubanée. Nous ne lui consacrons que la figure 25, qui suffit à la faire distinguer de ses congénères des hautes latitudes boréales.

Elle est d'eau douce; nous la tenons, en très rares spécimens, de la rivière Matotchka et de quelques échantillons de boues glaciaires, où elle n'est représentée que par des frustules vides.

\section{Genre Stauropsis g. n.}

Nous avons à rencontrer ici des Diatomacées vraiment planktoniques dont les frustules forment des colonies rubanées flottantes qui ont beaucoup d'analogie extérieure avec les Fragilaria, mais qui s'en distinguent manifestement par ce que l'on pourrait appeler un raphé double, à la fois longitudinal et transversal, qui dessine une croix bien marquée dont les bras inégaux sont centrés sur le milieu des valves. Celles-ci portent, en outre, une fine striation perpendiculaire au raphé longitudinal, c'est-à-dire aux longs bras de la croix. 
Ces espèces sont généralement très peu silicifiées, autre caractère des formes marines véritablement planktoniques.

Nous visons ici des organismes récemment entrés dans la science par les traraux de quelques planktonistes du nord et ballottés par eux, avec un égal insuccès, entre les genres Navicula et Stauroncis, dont ils n'ont, à vrai dire, les caractères ni de l'un ni de l'autre.

C'est le respect louable des cadres taxinomiques établis qui, pensons-nous, est la cause de l'embarras dans lequel se sont trouvés les auteurs en question et que nous éprouverions nous-mème, si nous ne prenions le parti d'en sortir en proposant un genre nouveau pour grouper ces espèces, qui n'ont pas de congénères notoires dans les formes décrites d'eau douce.

En fait, ce sont ces dernières qui ont presque exclusivement fait l'objet des recherches patientes des habiles diatomistes qui ont poussé leurs études morphologiques au plus haut degré de précision. Il n'est donc pas étonnant que des espèces, qui leur sont restées inconnues et qui, du reste, n'auraient pas pu subir leur technique sans courir les risques d'une destruction complète, ne trouvent pas une place réservée à leur intention clans les cadres prétablis.

Pour leur en faire une, nous tenons compte de leur caractère à la fois le plus apparent et le plus constant, croyons-nous : le croisement, au milieu des valves, de deux lignes renforcées, qui ne sont pas soulignées d'une aire hyaline, comme dans les Stauroncis Ehrenberg, en général, ni comme dans les Plcurostaurum Rabenhorst, en particulier, qui sont d'ailleurs disposés eux aussi en colonies rubanées.

Par là, elles se distinguent aussi des Navicula, dont les représentants autorisés sont déjà assez divergents et assez disparates pour qu'il ne soit pas désirable d'introduire dans leurs rangs des formes qui fausseraient davantage les caractères déjà nébuleux qui leur restent comme attribut commun.

Quant aux caractères plus intimes, ceux de l'endochrome spécialement, nous constaterons qu'ils sont assez discordants pour légitimer les objections que l'on pourrait formuler contre leur affinité réelle; mais il nous paraitrait inopportun d'en tenir compte actuellement pour pousser plus loin une tentative de classification relativement à des objets dont l'étude n'est encore qu'ébauchée.

Pour procéder de ce qui est mieux connu à ce qui l'est moins, nous dérogerons à l'ordre de nos figures de la planche XXXII, pour présenter d'abord :

\section{Stanropsis membranacea (Cleve).}

Planche XXXIII, figures 37 à to.

Syanymil: éT litTtérature. - Navicula membranacea Cleve, is97 (I). Désignation reproduite depuis par les planktonistes en général et par nous-mème, dans les listes

(1) A treatise on the phytoplankton of the Allantic and its Tributaries. Upsala, 1). 24. 11. II, fig. 25 à 28. 
planktoniques des recherches océanographiques internationales de la Mer du Nord. Mais, après plus ample information, nous aimons mieux revenir sur nos pas que de persévérer dans une voie qui nous paraît fautive.

Caractìres. - Cette intéressante espèce a été mal figurée par Cleve, d'après des spécimens avariés ou insuffisamment fixés, sans doute.

Nous en reproduisons, dans la figure 37, un fragment de colonie rubanée, pour en préciser les caractères internes, à l'état naturel, qui seuls fixent couramment l'attention de l'observateur sur cette belle espèce, très répandue particulièrement dans les pêches planktoniques des côtes belges.

La vue connective des frustules est rectangulaire, avec des angles brièvement arrondis.

La vue valvaire est elliptique, lancéolée, subaiguë aux extrémités (fig. 38).

Le noyau est central dans les frustules qui ne sont pas en période de division. Il est maintenu dans cette position par un cylindre de cytoplasme dense disposé perpendiculairement au plan sagittal de la colonie, suivant le petit axe des frustules, comme il apparait dans la coupe valvaire optique de la figure 38. D'autres cordons plasmatiques plus ténus parcourent la cavité cellulaire, au sein d'un suc liquide abondant.

Les chromatophores, ch, en forme de rubans étroits, plissés et méandriques, $y$ sont au nombre de quatre, fixés contre les parois connectives du fioustule et disposés par couples de chaque côté du plan sagittal. C'est ainsi que les deux rubans visibles dans chaque frustule au repos, du côté de l'observateur, ont leurs symétriques sur le còté opposé.

Vers les angles des frustules, en $x$, on aperçoit un réseau réfringent, à très petites mailles, d'une substance qui parait de même nature que la membrane et qui résiste comme celle-ci aux agents dissolvants du cytoplasme.

Pendant que la cytodiérèse se prépare, les chromatophores développent de nouveaux méandres et des ramifications, jusqu'à doubler de longueur, et se soudent, par deux de leurs bouts libres, pour permettre, au moment de la division (en $b$, fig. 37), leur partage entre les deux cellules-filles, de manière à ce que celles-ci soient loties chacune de deux rubans - dont un seul visible dans la figure - qui sont continus d'un bout à l'autre de la zone connective et dont les méandres sont ordonnés symétriquement vis-à-vis du plan de division. Plus tard, chaque ruban se coupe en deux vers son milieu, pendant que le noyau reprend sa position centrale, et la cellule adulte se rapproche de l'aspect normal qu'on lui voit dans les frustules qui occupent la gauche de la figure 37 .

Ces mouvements de quadrille des chromatophores seraient bien intéressants à suivre sur le vif. A défaut de matériaux vivants, il est facile d'en repérer les étapes, par la comparaison des individus d'âge différent d'une colonie convenablement fixée par l'emploi de réactifs efficaces, avant la dénaturation du contenu cellulaire. 
Les frustules vidés et examinés à sec se présentent sous l'aspect des figures 39, en vue connective, et 40 , en vue valvaire. Celle-ci montre, outre la croix réfringente, qui est bien visible en milieu liquide, la très fine striation transversale, dont on ne peut que soupçonner la présence dans ces conditions.

La travée transversale de la croix se voit aussi bien sur la face connective de la figure 39 et s'observe même aisément sur les frustules complets et disposés en colonie de la figure 37 , où elle se traduit par un trait transversal, aux còtés d'un léger écartement, en forme de boutonnière étroite, que l'on saisit entre les frustules, au milieu de la colonie.

Distribution géographique. - Cette belle espèce, parfois très commune dans la Mer Flamande, pendant les mois d'hiver, se montre fort rare dans les produits de pêche de la Belgica dans la Mer de Barents.

\section{Stauropsis Granii (Jörgensen).}

Planche XXXIII, figures 26 et 27,33 et 34,35 et 36 .

Synonymie et littérature. - Stauroneis Granii Jörgensen, igo5, page io7, planche VII, figure 25, Navicula Granii Gran, Igo5, Nordisches Plankton, XIX, page I24, figure I68.

Caractères. - Colonies rubanées de frustules en forme de rectangle allongé, brièvement arrondi aux angles et légèrement déprimé au milieu, où les individus disposés en série laissent entre eux une fente étroite, no, figure 26.

Vus en milieu liquide, les frustules ne traduisent guère d'autres détails que ceux de leur contenu cellulaire comprenant un noyau central, $n$, flanqué de chaque còté d'un large chromatophore lamellaire, ch, garni dans son milieu d'une sorte de gros pyrénoïde granuleux, $g$. Le rapprochement des jeunes noyaux, dans deux cellules jumelles, montre assez comment s'est opérée la division des chromatophores.

La figure 27 , en montrant l'objet en coupe valvaire optique, concourt à l'intelligence de la forme des frustules, qui sont ici particulièrement étroits.

Vus à sec, après vidange du cytoplasme, les frustules montrent leurs valves décorées d'une croix centrée et d'une fine striation parallèle à la travée transversale qui simule un stauros (fig. 34 et 36 ).

Les figures 33 et 35 en reproduisent des rues connectives, dont les différences permettent d'apprécier les variantes de l'espèce dans nos matériaux.

On remarquera que les vues valvaires $27,3_{4}$ et 36 sont d'un dessin différent. Nous croyons que ces différences sont dues à la délicatesse de l'objet, trop peu silicifié pour résister sans avaries aux manipulations techniques et mème à la simple dessiccation sur le slide. Il y a, de ce chef, une réelle difficulté d'étude de l'espèce.

Distribution géographique. - Assez rare, dans la Mer de Kara, non loin du Détroit de Matotchkine. 
Stauropsis septentrionalis (Grunow).

Planche XXXIII, figures 28 à 32 .

Synonimie et littérature. - Stauroneis septentrionalis Grunow i884; Navicula septentrionalis Gran, I905; etc. Cfr. Nordisches Plankton, page I24.

Caractìres. - Colonies rubanées dans lesquelles les frustules contigus sont en contact sur toute leur face valvaire.

En vue connective, ces frustules sont rectangulaires et renferment un noyau central vers lequel convergent les quatre partitions plus ou moins individualisées d'un chromatophore quadrilobé, en forme de la lettre X (fig. 28).

Quand les frustules se divisent (fig. 32), les produits immédiats de la division ne présentent qu'un chromatophore bilobé représentatif d'une moitié rectifiée du chromatophore maternel, comme on le voit à gauche de la figure, sous la lettre $a$.

Ce n'est que plus tard, pendant que la cellule devient adulte, que les deux lobes se subdivisent en lobules qui s'écartent légèrement et reprennent l'état habituel, comme dans les individus de la partie droite de la figure, sous la lettre $b$.

En coupe valvaire optique (figure 29), les frustules se montrent étranglés au milieu et atténués-arrondis aux deux extrémités. Sous cet aspect encore, les quatre lobules du chromatophore affectent entre eux une disposition croisée en $\mathrm{X}$, mais plus étroitement.

A sec, les frustules préalablement vidés se montrent très finement striés sur des zones assez larges de la face connective (figure 30) et laissent percevoir le nodule central du raphé, ou mieux le croisement de celui-ci avec le faux stauros transversal.

Celui-ci se traduit néanmoins beaucoup mieux sur la valve examinée dans les mêmes conditions (fig. 3i).

Distribution géographioue. - Rencontrée rarement dans les Mers de Kara et de Barents et comparée, pour l'étude, avec des matériaux des parages du Grönland, dans lesquels cette forme n'est pas rare.

\section{Stauropsis Vanhöffenii (Gran).}

Planche XXXIII, figure 46 .

Synonimie et littérature. - Navicula Vanhöffenii Gran, etc., i897. Chr. Nordisches Plankton, page I24.

Caractíres. - Nous n'avons pas d'aussi bonnes raisons pour prendre une attitude franche vis-à-vis de cette forme, parce que sa rareté exceptionnelle dans nos matériaux ne nous a pas permis d'en relever autre chose que l'aspect en colonie, dans 
un milieu liquide, celui du matériel d'étude (fig. 46). Nous n'avons pu en prendre une vue valvaire, encore moins en examiner des frustules à sec.

Ce sont les analogies seules qui nous guident dans le rapprochement que nous faisons plutòt d'instinct et qui nous font supposer même une identité de nature entre cette forme et $S$. Granii analysée plus haut.

Les frustules ont sensiblement le même modelé, sous des dimensions un peu moindres; le noyau, $n$, a le même aspect; les chromatophores, ch, ont la même disposition lamellaire et sont aussi renforcés dans leur milieu par des globules granuleux qui simulent des pyrénoïdes, etc.

La seule différence bien apparente semble consister dans l'écartement des frustules, qu'une substance mucilagineuse invisible doit maintenir en union latérale.

Mais ce caractère peut-il ètre tenu pour spécifique, quand on sait que les frustules de S. Granii, intimement soudés parfois, comme dans les figures 26 et 33 (même planche), peuvent aussi se montrer distinctement séparés, comme dans lá figure 35?

Ne peut-on pas supposer qu'ils peuvent encore s'écarter davantage, dans des conditions propres à exagérer ce qui pourrait bien n'être qu'un symptòme de dégradation?

C'est ce qui nous parait et c'est l'impression que nous ont laissée les rares spécimens que nous avons pu observer.

Si ces vues se confirmaient, il faudrait rayer des listes planktoniques l'espèce Granii, dont la création ne remonte qu'à Igo5.

\section{Stauropsis pelagica (Cleve).}

Planche XXXIII, figure 48 .

Synonyme et Littérature. - Navicula pelagica Cleve, I8g6; et peut-être aussi Navicula frigida Grunow, 1894-1895. Cfr. Nordisches Plankton, page 125.

Caractíres. - Colonies tordues de tout petits frustules, à face valvaire elliptique un peu convexe, qui ne sont guère en contact que par leur milieu (fig. 48), mais qui sont néanmoins maintenus en série par une gangue gommeuse, d'où émergent de fines soies, s, de même nature, qui ne sont qu'exceptionnellement visibles dans le liquide conservateur.

Dans ce milieu, les membranes nous ont paru hyalines et le cytoplasme s'est montré granuleux et trop uniforme pour qu'il nous ait paru utile de le figurer sous cet aspect évidemment artificiel.

En dehors de l'aspect bien particulier de ses colonies, les caractères frustes de cette espèce petite et fragile se prètent mal à une diagnose précise. Cela explique les réserves formulées à son sujet par Gran, loco citato, et les doutes qu'il émet sur sa distinction objective de Navicula frigida Grunow, espèce reprise par Jörgensen dans 
son mémoire de Igo5. C'est bien le mème organisme, sans doute, sous des aspects différents, dans des conditions d'observation diverses, avec ou sans soies gommeuses dont on sait l'inconstance.

Distribution gégraphique. - Très rare, dans la Mer de Kara, dans le no 34 spécialement, où des impuretés de toutes sortes qui s'y accolent en rendent l'examen difficile.

Stauropsis sp.?

Planche XXXIII, figure $t^{5}$.

Il s'agit encore ici d'un organisme planktonique agrégé en colonies rubanées, dont les frustules, vus du còté connectif, sont en contact sur la plus grande partie de leur face valvaire, parce qu'ils sont dépourvus d'une dépression médiane; ce n'est que vers les extrémités qu'ils arrondissent leurs angles et s'y écartent notablement (fig. $45, b$ ).

En milieu liquide, la membrane parait hyaline et nous n'y avons pas constaté positivement la croix valvaire dont nous avons fait la caractéristique de notre genre Stauropsis.

En coupe optique (fig. $45, a$ ), on y reconnait un noyau central flanqué, vers les extrémités de la cellule, de deux masses cytoplasmatiques granuleuses qui simulent des chromatophores diffus.

La rareté des spécimens observés, au milieu d'un fouillis d'autres formes rubanées d'espèces variées, ne nous a pas permis de les soumettre individuellement à des manipulations propres à en préciser davantage les caractères. Leur fragilité est du reste extrême et il y aurait lieu de se défier des déformations qui ne manqueraient pas de résulter, soit de l'action des acides minéraux forts, soit de l'incinération, soit même d'une simple dessiccation.

Origine. - Mer de Kara; très rare.

$$
* * *
$$

La florule des neiges et des glaces polaires nous a aussi fourni un petit contingent cle formes qui traduisent dans leur valve ces détails de structure, inconciliables avec ceux des Navicula et des Stauroneis vrais, qui nous ont fait proposer le genre Stauropsis pour faire une place à part aux Diatomacées qui les présentent et qui, pour cette raison, sont mal accueillies même dans les genres avec lesquels elles ont le plus d'affinité.

Toutefois, les conditions spéciales des récoltes dans lesquelles nous les avons rencontrées et les manipulations auxquelles nous avons dù soumettre ces produits ne nous ont pas permis de nous renseigner complètement sur leur état naturel ni de nous assurer de leur disposition éventuelle en colonies rubanées, comme leurs congénères probables présentées plus haut. 
Stauropsis acuta sp. n.

Planclie XXXIII, figure 5o.

Valve étroite, longue, lancéolée, aiguë aux deux bouts, marquée d'une croix centrée dont les deux travées, le raphé longitudinal et la branche transversale, ont la mème valeur optique. La très fine striation transversale qui reste partout parallèle au faux stauros ne peut pas ètre perçue en milieu liquide; on ne peut même que difficilement la saisir à sec, avec un bon éclairage.

La vue connective, que nous n'avons pas figurée, est étroite, linéaire et démontre que les valves sont presque planes.

Distribution GÉographique. - Forme assez commune; la plus commune peut-être des Diatomacées de la neige jaune. Elle est plus rare dans la florule des glaces proprement dite.

\section{Stauropsis amphicephala sp. $\mathrm{n}$.}

Planche XXXIII, figure 5 r.

Valve assez grande, large au milieu, longuement elliptique et céphalée aux deux bouts. La travée transverse, le faux stauros, nous a toujours paru ici n'atteindre pas les bords de la valve; le raphé est fendu longitudinalement. La fine striation transversale, que nous avons figurée sur la valve, n'est non plus visible qu'à sec, dans de bonnes conditions d'observation.

Distribution Géographique. - Beaucoup plus rare que la précédente, dans les mêmes matériaux.

\section{Stauropsis majuscula $\mathrm{sp} . \mathrm{n}$.}

Planche XXXIII, figures 52 et 53 , et aussi, sans doute, planche XXXV, figures $47,48,49$.

Ces diverses vues, les unes valvaires, les autres connectives, se rapportent évidemment à des individus de même espèce, qui ne diffèrent que par de faibles écarts de calibre.

La valve est linéaire, brièvement atténuée vers les bouts qui sont arrondis ou subaigus. Elle porte un faux stauros, qui coupe le raphé à angle droit, sans renforcement nodulaire au point de croisement; elle traduit, en outre, une striation transversale serrée, mais très évidente à sec.

En vue suturale, les frustules sont rectangulaires, arrondis aux angles, pourvus d'une zone connective large.

Origine. - Matériaux variés de la Mer de Kara. 


\section{Genre Achnanthes Bory}

Caracteres du Genre. - Consulter Nordisches Plankton, XIX, page I2I, et H. Van Heurck, Traité des Diatomées, page 277.

\section{Achnanthes taeniata Grunow.}

Planche XXXIII, figures $4 \mathrm{I}$ à 44 .

Synonyme et littérature. - Achnanthes hyperborea Grunow, repris par Jörgensen, Igo5. Cfr. Nordisches Plankton, page I2I.

L'aspect différent de cet organisme, suivant qu'il est à l'état végétatif ou en sporification, est sans doute l'unique fondement de la distinction spécifique que des auteurs ont cru devoir faire entre $A$. hyperborea (forme végétative) et $A$. taeniata (forme sporifère).

Caractères. - Les frustules forment de longues séries rubanées, ce qui leur permet d'être franchement planktoniques.

La figure $4 \mathrm{I}$ les présente, sous la lettre $a$, à l'état végétatif, avec leur forme courbée caractéristique, en vue connective. On y voit un noyau central, au sein d'un chromatophore lamellaire, mal délimité, qui se segmente dans le plan de la division du frustule, lorsque celle-ci se produit; ce qui lui fait prendre alors la forme de la lettre $H$.

Sous cet aspect, on n'observe que difficilement le nodule médian qui est propre à l'une des deux valves seulement et que l'on saisit mieux sur cette valve vue de face, comme elle est reproduite dans la figure 42 , avec sa forme lancéolée obtuse, son raphé longitudinal et sa fine striation transversale.

Sporification. - En $b$ et en $c$, dans la figure 4I, on voit les spores produites par couples, à la suite d'une dernière division de leur matricule, à l'intérieur de laquelle elles prennent la forme qui leur est propre, par un phénomène de rénovation cellulaire. Celle-ci entraine l'abandon du cytoderme maternel, dans lequel les spores restent néanmoins prisonnières.

En $b$, le cytoplasme a encore la mème physionomie que dans les cellules végétatives; en c, phase plus avancée, le contenu cellulaire est devenu plus pariétal.

Entre ces couples existent des frustules végétatifs morts et vides, qui en laissent mieux saisir la forme et les rapports mutuels.

Souvent la sporification s'étend presque simultanément à la plupart des frustules d'une colonie, comme dans le fragment reproduit dans la figure 43, où les spores sont figurées à l'état naturel, en coupe optique, sous la lettre $a$, et vides, sous la lettre $b$. 
La figure 44 en reproduit, en coupe optique, une vue de profil latéral. On y voit distinctement que les spores, sp, groupées par couples dans leur matricule, sont d'un calibre plus étroit que celle-ci et ont une membrane complètement indépendante d'elle.

Parasitisme. -- Cette espèce est souvent parasitée par un Olpidium, ol, dont nous figurons (fig. 4I, 43, 44) plusieurs kystes ouverts que les sporules du Champignon ont déjà évacués.

L'orifice de sortie peut se produire à un endroit quelconque de la zone connective, à l'extrémité d'une tubulure qui sert de couloir.

On ne manquera pas de remarquer que les frustules parasités sont généralement plus grands que les autres, non seulement en largeur, comme on le voit dans les figures $4 \mathrm{I}$ et $4^{3}$, mais aussi en épaisseur, comme il apparait nettement dans la figure 44, où le parasite a été éliminé, par macération, des frustules marqués ol.

Distribution géographioue. - Assez largement répandu : à Tromsö, dans la Mer de Kara et dans l'Océan Glacial.

\section{Achnanthes brevipes Agardh.}

Planche XXXII, figure I4.

Cette espèce bien connue forme aussi des colonies rubanées, mais celles-ci ne sont pas libres ni planktoniques, à proprement parler, car elles sont fixées par une sorte d'amarre, $p$, soudée à une des extrémités valvaires d'un frustule chef de file (fig. I4).

On connait la forme coudée des frustules, en vue suturale, la striation dissymétrique des deux valves et la sculpture propre à la zone connective: détails reproduits dans les individus figurés en vue stéréoscopique, sous la lettre a. D'autres, sous la lettre $b$, sont figurés en coupe optique, pour en traduire le noyau central, n, d'où s'échappent de nombreux cordons protoplasmatiques, et l'aspect particulier des chromatophores, ch. Ceux-ci dessinent de chaque còté clu noyau, vers les deux extrémités du frustule, une couronne autour d'une masse granuleuse que l'on peut prendre pour un gros pyrénoïde.

Distribution Géographigue. - Bien que l'espèce ne soit pas planktonique, nous l'avons rencontrée plusieurs fois dans des produits de pêche de la Mer de Barents, où les courants avaient entraîné des fragments de colonies arrachées à leur lieu d'attache. 


\section{Genre Achnanthidium Grunow}

Caractères du genre. - Cfr. H. Van Heurch, Traité des Diatomées, page 276.

\section{Achnanthidium sp.}

Planche XXXII, figure 15.

Nous avons dù nous borner à figurer ici l'un des rares spécimens de cette forme que nous tenons de la florule glaciaire et que nous n'avons pu saisir qu'en vue valvaire.

Le raphé est sigmoïde, avec nodules central et terminaux. La striation valvaire est très fine, serrée, légèrement radiante.

\section{Genre Hantzschia Grunow}

Caractéres du genre. - Cfr. H. Van Heurck, Traité des Diatomées, page 380.

\section{Hantzschia marina (Donkin) Grunow?}

Planche XXXII, figures $37,38,39$.

Synonyme. - Epithemia marina Donkin. Voir H. Vax Heurck, loc. cit., page 382.

Nous présentons, en regard l'une de l'autre, les trois vues nécessaires à l'intelligence de ces formes d'Hantzschia, qui n'ont que deux plans de symétrie, dont l'un passant par le milieu de la zone connective et l'autre transversal au frustule. On sait, en effet, que les carènes sont très excentriques et orientées toutes deux du mème còté du frustule, que nous dirons ventral, comme dans les Amphora.

La figure 37 en reproduit la vue valvaire. On remarquera que la valve n'est pas arquée comme dans la figure $489^{\mathrm{b}}$ de VAN Heurck, mais droite, linéaire, élargie au milieu, incurvée-rostrée aux deux extrémités déjetées du mème côté. La surface est toute couverte d'une vigoureuse striation transversale figurée telle qu'elle apparait en milieu liquide.

La figure 38 en est la vue ventrale rectangulaire, légèrement élargie parfois au milieu, sur laquelle se profilent les deux carènes valvaires infléchies symétriquement vers la zone connective.

La figure 39 la montre de dos, avec la zone connective droite sculptée, comme du còté ventral, d'un quadrillé très apparent et bordée de chaque còté des fortes stries transversales qui décorent les deux valves latérales symétriques.

Distribution géographigue. - Cette espèce est l'une des plus constantes dans les fines boues glaciaires, où elle n'existe, toutefois, qu'à l'état de frustules vides. 


\section{Hantzschia amphyoxis (Ehrenberg) Grunow?}

Planche XXXIV, figures I, $2,3$.

Il est regrettable que les auteurs n'aient pas toujours pris soin de figurer les espèces difficiles à identifier sous tous les aspects propres à en fixer la diagnose. Les seules vues valvaires sont souvent exclusives de certains traits caractéristiques qui peuvent n'avoir leur expression que sous d'autres aspects des frustules, surtout quand ces aspects sont l'objet d'une observation plus fréquente dans les matériaux à l'état naturel. C'est le cas pour les Hantzschia, qui, dans ces conditions, se montrent forcément en vue suturale, leur position d'équilibre stable; tandis que la vue valvaire ne peut s'observer qu'au prix de pratiques laborieuses pour les amener et les maintenir dans une position instable.

C'est ce qui nous fait douter de la légitimité de la référence que nous proposons ici.

La vue dorsale de notre forme, reproduite dans la figure I de la planche XXXIV, montre que les frustules sont rectangulaires, assez larges, arrondis sur les angles, quadrillés sur la zone connective, striés sur les côtés qui sont les parties visibles des valves dans cette position.

La figure 2 en reproduit la vue ventrale, le côté sur lequel se profilent les deux carènes incurvées ver's les extrémités et arquées l'une vers l'autre en leur milieu. Celles-ci sont ornćes d'entailles quadratiques inégalement distancées et interrompues au centre par une sorte de nodule. En dehors de ces détails plus apparents, la striation transversale des valves s'étend sur toute leur surface et est assez prononcée pour ètre aisément observable en milieu liquide.

En vue valvaire (fig. 3) le frustule se montre arqué, concave du còté ventral, le long duquel se profilent les entailles quadratiques bien délimitées de la carène, convexe de l'autre còté, le còté dorsal, sauf vers les extrémités où l'on observe une atténuation excentrique du frustule et une légère incurvation en sens opposé.

Sous cet aspect valvaire, notre figure ressemble assez bien à celles que les auteurs attribuent à $H$. amphyoxis. En vue suturale, l'accord n'existe plus. Voir Van Heurck, Synopsis, planche LVI.

Distribution géographique. - Trouvée dans la florule des glaces, plus rarement dans les boues glaciaires et parfois aussi dans les pèches planktoniques du Détroit de Matotchkine.

\section{Hantzschia virgata (Roper) Grunow?}

Planche XXXIV, figures $4,5,6$.

Les remarques formulées tout à l'heure trouvent à nouveau leur application ici : à savoir qu'il est difficile de juger de ces formes de Diatomacées par leur seule vue valvaire, la moins usuelle dans les recherches courantes. 
C'est ici la vue suturale ventrale (fig. 4) qui est la plus suggestive. Dans cette position le frustule est rectangulaire, brièvement arrondi aux angles. Les carènes valvaires visibles seulement de ce còté, sur toute leur longueur, sont soulignées de fortes travées qui s'en détachent, pour s'atténuer assez loin de leur origine, en dessinant des côtes, entre lesquelles se dessine une striation parallèle à elles, moins marquée assurément, mais néanmoins très manifeste, même en milieu liquide. La zone connective, élargie vers les extrémités, présente une structure grossièrement quadrillée.

En vue valvaire (fig. 5) le frustule se montre arqué et atténué-rostré aux deux bouts, à peu près comme dans l'espèce précédente; mais l'œil est frappé par le prolongement des côtes carénales sur la valve, où elles s'atténuent insensiblement et se perdent dans la forte striation transversale qui couvre toute la surface.

La vue dorsale, la moins interprétative (fig. 6), ne traduit que la forme du frustule et la vigueur des sculptures, aussi bien de la zone connective que de la partie visible des valves latérales.

\section{Genre Nitzschia Hassal}

Caractères du genre. -- Cfr. H. Van Heurck, Traité des Diatomées, page 382.

Ce genre, comme d'autres qui ont des représentants dans tous les milieux, a dù élargir ses cadres pour recevoir les nombreuses espèces pélagiques que les recherches océanographiques récentes ont mises au jour. Celles-ci ont généralement été décrites en tenant compte de leur état naturel et, gràce à cela, leur identification ne souffre généralement pas de difficulté. Il n'en est pas ainsi de beaucoup d'autres, des espèces de petites dimensions surtout, dont les caractères méticuleux, tirés d'observations sur des slides montés dans le baume, sont exclusifs d'autres que l'on aurait pu tirer avantageusement de leur mode d'agrégation et des circonstances physiologiques propres à éclairer leur histoire naturelle.

Dans les recherches planktoniques, le grand nombre des espèces à recenser ne permet pas de dépasser certain degré de minutie dans l'analyse des caractères les plus intimes, mais aussi les moins commodes à relever dans le milieu dans lequel il faut se contenter de les étudier. Nous estimons qu'il est pratiquement impossible d'identifier bon nombre de petites espèces, celles du groupe des Homaocladia, par exemple, qui virent dans des gaines muqueuses d'un facies spécifique, si on les dépouille de ce caractère qui est le plus important dans l'exercice cursif des observations. C'est cependant ce qu'ont fait d'excellents auteurs, que la difficulté du sujet et le souci de la précision ont entrainé, peut-ètre trop exclusivement, dans un genre de recherches dont on peut discuter l'utilité pratique sans infirmer la valeur scientifique. 
Nous présenterons d'abord les formes libres originaires des neiges et des glaces ou rencontrées exceptionnellement dans les eaux marines, sans en urger l'identification avec des espèces déjà connues sans doute, mais d'un contrôle difficile, tant à cause des mensurations micrométriques qu'il entrainerait, qu'à raison de l'absence de concordanće entre les points de vue sous lesquels les éléments d'appréciation, valves isolées ou frustules entiers, ont été figurés.

Nous passerons ensuite aux formes plus aisément reconnaissables, grâce aux caractères plus explicites qu'elles tirent soit de leur physionomie individuelle, soit de leur mode singulier de groupement.

Celles qui sécrètent un produit mucilagineux dans lequel elles vivent en famille seront réservées pour le genre Homaocladia, que son utilité pratique doit faire maintenir.

Nous les avons d'ailleurs toutes figurées dans la mème planche XXXIV, en vue d'en faciliter la comparaison entre elles et avec celles qui sont connues et décrites d'autres provenances.

Figures 7 et 8. Frustules assez grands, droits, linéaires, à extrémités arrondies aussi bien en vue valvaire (fig. 7) qu'en vue suturale (fig. 8). Les points carénaux sont très distancés et sont inégalement répartis, soit isolément, soit par petits groupes séparés par des espaces plus grands. Le milieu des valves présente un pseudo-nodule distinct des autres détails de la carène.

Origine. - Pêches littorales du Détroit de Matotchkine.

Figures 23 et 24. Frustules grêles, linéaires, droits, tronqués aux deux extrémités de la vue suturale (fig. 23), atténués aux bouts de la face valvaire (fig. 24). Points carénaux très distancés, mais uniformément répartis sur toute la longueur de l'organe.

Origine. - Florule des neiges et des glaces.

Figure 9. Forme assez grande, à face valvaire, la seule figurée, brusquement élargie en son milieu, en manière de losange, et atténuée vers les extrémités. Points carénaux fins, nombreux, très rapprochés, sans distinction d'un pseudo-nodule médian. La vue suturale a beaucoup d'analogie avec la figure II de l'espèce suivante.

Figures io et II. Forme assez grande, droite, linéaire, tronquée aux extrémités de la vue suturale (fig. II), atténuée vers les bouts de la face valvaire (fig. io). Celle-ci est étroite, linéaire, sans dilatation médiane. Les points carénaux sont très fins, serrés, également distancés. Nous en avons figuré l'endochrome, ch, également réparti des deux côtés du noyau central, $n$, sous la forme de lames déchiquetées, dont les segments étroits se profilent à la fois vers les extrémités du frustule et vers le cytoplasme périnucléaire avec lequel ils se tusionnent.

Origine. - Pèches planktoniques de la Mer de Kara. 
Figure I2. Forme assez grande, à valve atténuée-rostrée aux bouts, à carène droite au milieu, défléchie aux extrémités, marquée de gros points carénaux inégalement distancés.

Plus souvent que ses congénères, cette espèce assez répandue dans les neiges et les glaces se montre parasitée par Olpidium phycophagum, ol, dont on reconnaît aisément les kystes zoosporangiaux vidés, même dans les frustules dont la mort semble remonter assez loin, comme dans l'exemple figuré.

Nous rappelons, à cette occasion, l'observation que nous avons déjà faite, que les Diatomacées de la florule glaciaire sont tout spécialement sujettes à ce parasitisme.

Qu'est-ce qui leur vaut ce privilège à rebours? Serait-ce la faible salinité du milieu?

Figures i 3 et I4. Belle et grande forme à face suturale (fig. I4) large, linéaire, tronquée aux bouts et brièvement arrondie aux angles. La face valvaire (fig. i3) est large, droite, linéaire au milieu, brièvement atténuée aux extrémités. Les points carénaux ont un aspect rectangulaire, ils sont grands, distancés, séparés par des intervalles inégaux et surmontés d'une crête carénale hyaline qui se traduit par un liséré bien visible autour du frustule, lorsque celui-ci est bien présenté en vue connective.

Figure 15. Frustule contracté au milieu - en vue suturale, la seule figurée -.. brusquement atténué-arrondi aux extrémités, formé de deux valves à carène peu excentrique, que soulignent de gros points carénaux rectangulaires, inégalement distancés aux deux còtés d'un pseudo-nodule central.

Figure I9. Frustule plus grand que le précédent, déprimé aussi au milieu, atténué-arrondi aux bouts, tronqué sur la zone connective qui est assez large, marqué sur la carène peu excentrique de points rapprochés, prolongés quelque peu sur la valve en forme de côtes très courtes et fuyantes.

On doit considérer ces deux formes, I5 et I9, et aussi celle de la figure 4.9, qui est beaucoup plus petite, comme des expressions du type des Bilobatce de la monographie de Gruxow (Arctische Diatomeen, ISSo).

Figures 16 et 26. Formes de Nitzschia spatulata Brébisson, apparemment, très différentes l'une de l'autre par leurs dimensions, mais douées d'un caractère commun, qu'elles doivent à l'élargissement local de la crête carénale vers l'extrémité des valves.

Dans la figure $\mathbf{6} 6$, le frustule, figuré en vue suturale, est un peu élargi vers le milieu, les expansions terminales des crêtes carénales sont très accusées, les points carénaux sont vigoureusement sculptés et également distancés. La figure 26 traduit les mêmes caractères, mais en réduction.

Origine. - Mer de Kara.

Figures I7 et IS. Formes à rapprocher probablement de Nitzschia angularis W. Smith, si l'on tient compte de l'aspect des frustules, presque rectangulaires en vue suturale, du relèvement des carènes et de l'accentuation de celles-ci vers les extrémités. 
Dans l'objet de la figure i7 les points carénaux sont très distancés; dans celui de la figure i 8 ils sont beaucoup plus rapprochés et plus régulièrement espacés. Leur diversité spécifique ne parait pas douteuse.

Origine. - Neiges et glaces, comme les suivantes.

Figure 20. Forme très grande, présentée en vue valvaire. La valve est longuement linéaire, droite, atténuée-rostrée aux extrémités, rehaussée d’une carène ponctuée d'une manière uniforme et couverte d'une striation transversale assez prononcée pour être manifeste même en milieu liquide. Elle semble avoir beaucoup d'analogie avec Nitzschia spectabilis Ralfs.

Figures 21 et 22. Forme plus grande encore, dont nous avons dù nous borner à figurer l'extrémité d'un frustule en vue latérale (fig. 2I) et en vue valvaire (fig. 22).

La carène y est particulièrement accusée. Elle est traversée par de fortes côtes également distancées qui, vues en projection sur la valve, s'y prolongent au milieu de la striation transversale qui est aussi très bien marquée et visible en tous milieux.

Elle semble devoir trouver sa place dans le groupe des Scalares.

Figure 25. Forme longuement lancéolée, fusiforme, en vue suturale. Points carénaux ténus, rapprochés, partagés en deux séries par un espace hyalin central, dépourru de pseudo-nodule apparent.

Figure 27. Forme rhomboïdale très élargie au centre, atténuée-aiguë aux extrémités. Points carénaux bien marqués et distancés. Pas de pseudo-nodule.

Figure 32. Forme à zone connective très large, ce qui donne au frustule un aspect rectangulaire, en vue suturale. Elle semble devoir se rapporter aux variantes de Nitrschia lanceolata W. Smith, comme les deux précédentes.

Figure 33. Petite forme de N. tryblionella Hantzsch.

Figure 28. Grande forme à face, suturale sigmoïde, plus large au centre, notablement atténuée aux extrémités. Les points carénaux sont délicats et fort rapprochés.

A ranger parmi les variantes de $N$. sigma IV. Smith.

Figure 3I. Forme analogue à la précédente, mais beaucoup plus petite, plus sigmoïde, plus atténuée vers les extrémités.

Figure 29. Forme assez grande, sigmoïde, à face suturale sensiblement de même largeur sur toute la longueur. Points carénaux accentués, distancés, irrégulièrement espacés. Semble avoir beaucoup des caractères de $N$. sigmoidea (Ehrenberg) IV. Smith. 
Figure 3o. Forme beaucoup plus petite, sigmoïde aussi, référable sans doute également au groupe des Sigmoidea.

Les neiges, les glaces et les boues glaciaires nous ont offert un assez grand nombre d'autres formes généralement plus petites que celles dont nous venons de donner le signalement.

Nous n'avons pas cru utile de les reproduire, parce que leurs caractères spécifiques ne sont pas perceptibles à première vue et parce que nous ne pouvions songer à les soumettre à la technique patiente qu'elles réclament, ni à les reproduire à une échelle suffisamment grande pour en traduire les différences plus ou moins subtiles.

Qu'il suffise de rappeler la place considérable que tiennent les Nitzschiées dans cette florule non planktonique.

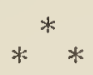

Nous accorderons ci-après plus d'attention aux quelques espèces qui, par leurs colonies flottantes, d'un facies caractéristique, sont susceptibles d'identification, sans qu'il soit nécessaire de les soumettre à des mensurations minutieuses des détails sculpturaux de leur valve.

\section{Nitzschia seriala Cleve.}

Planche XXXIV, figures 38,39 et 40 .

Synonyme et littérature. - Cfr. Nordisches Plankton, page i29.

Caractères. - Forme planktonique bien connue, dont les frustules bacillaires, en vue suturale (fig. 38 et 39 ), sont soudés latéralement par une partie de leur extrémité valvaire et toujours du mème còté, ce qui les met dans une position légèrement oblique ris-à-ris de l'axe longitudinal de la colonie.

Dans la figure 38, les frustules ont le maximum de longueur que nous leur avons trouvé exceptionnellement dans les eaux du Détroit de Matotchkine. Dans la figure 39, ils ont les dimensions qu'on leur voit le plus communément. Dans ces deux figures on remarquera que la face connective des frustules se montre tronquée aux sommets et non pas atténuée en pointe aiguë comme Gran les représente dans la figure I74, loc. cit.

C'est en effet suivant leur face valvaire que les frustules se soudent et, sous cet aspect, ils ont une forme longuement lancéolée, atténuée-aiguë aux extrémités. Dans cette position, on les voit se superposer partiellement, comme dans la figure 4o, suivant l'axe sagittal de la colonie. 
Dans la figure 38, les frustules ont été figurés vides, pour permettre de noter les très fines ponctuations carénales, qui ne seraient pas visibles autrement.

Dans la partie inférieure des figures 39 et 40 , ils ont été figurés avec leur contenu: noyau, $n$, et endochrome, $c h$, en lamelles, sous les deux aspects sutural et valvaire.

Parasitisme. - Nous avons d'autre part utilisé la partie supérieure de ces deux figures pour donner une idée de l'aspect qu'y revèt le parasite Olpidium phycophagum, ol.

Dans l'avant-dernier individu de la figure 40 , on le voit envahissant toute la cellule, sous forme végétative. Dans l'individu qui occupe le haut de la figure 39, on le voit transformé en zoospores globuleuses dans toute l'étendue du frustule, sans qu'il se soit formé une membrane kystique ou zoosporangiale propre, indépendante de celle de l'hòte. Celui-ci témoigne de dimensions hypertrophiques vis-à-vis de ses voisins restés indemnes et laisse voir l'exutoire tubulaire formé latéralement, par lequel les zoospores pourront s'échapper. Le frustule terminal, vers le haut de la figure 40, met l'observateur en présence du fait accompli de cette déhiscence, en lui montrant le frustule vide, en même temps que la tubulure latérale qui a servi à la vidange.

Distribution Géographigue. - Nitzschia seriata est assez fréquent dans la plupart de nos produits de pêche de surface. L'espèce ne manque pas non plus sur la glace; c'est là surtout qu'elle se montre sujette au parasitisme.

\section{Nitzschia frigida Grunow.}

Planche XXXIV, figure 36.

Littérature. - Cfr. Nordisches Plankton, page izg.

Caractères. - Forme planktonique dont les frustules sont disposés en colonies dendrö̈des, irrégulièrement ramifiées, gràce à une substance qui les rend coalescents par leur extrémité, sans qu'il y ait entre eux la moindre superposition. Dans ces colonies, à la fois zigzaguées et ramifiées, les frustules se montrent indifféremment en vue suturale ou en vue valvaire, ce qui doit tenir à la rotation facile du bout des frustules dans le grumeau gommeux qui les unit, en manière de charnière élastique. Il s'en suit que la colonie se développe dans toutes les directions et non pas dans un seul plan, comme l'examen de la figure pourrait le faire croire.

En vue valvaire, les frustules sont lancéolés, pointus et traversés longitudinalement par la carène finement ponctuée. En vue suturale, ils sont bacillaires, un peu atténués vers les bouts qui sont tronqués. Les carènes se profilent alors naturellement sur les còtés et traduisent, sous la forme de stries courtes, les ponctuations qui les ornent. L'un des frustules du haut du fragment de colonie figuré montre, en vue suturale, l'aspect du contenu cellullaire : noyau et endochrome. 
On ne manquera pas de remarquer la difficulté qu'il y aurait de distinguer les frustules de cette intéressante espèce d'autres similaires de petites dimensions, si l'on s'avisait, comme certains diatomistes le font a priori, de détruire ces objets délicats par des procédés de macération, avant d'en avoir reconnu le caractère d'association.

Distribution Géographioue. - Espèce très répandue dans toutes les régions explorées par la Belgrica.

\section{Nitzschia divaricata sp. n.}

Planche XXXIV, figure 37.

Caractères. - Nous tenons pour spécifiquement distincte de la précédente une forme plus rare qui est également disposée en colonies ramifiées par coalescence des frustules à leur extrémité, mais qui s'en distingue à première vue par les dimensions des éléments.

La figure 37 en reproduit un petit fragment du thalle, qui est parfois très étendu mais d'une grande fragilité.

Les frustules sont à la fois très longs et très étroits. En vue valvaire, ils sont aciculaires, à peine assez larges pour permettre d'y figurer, à l'échelle conventionnelle, la carène finement ponctuée qui les parcourt. C'est sous cet aspect que sont reproduits dans la figure ceux qui se trouvent dans l'axe longitudinal de la colonie, en $b$, par exemple.

En vue suturale, ils sont longuement linéaires, sans aucune dilatation vers le milieu, comme on peut s'en assurer par l'examen des deux éléments articulés sur les autres, à droite, en bas et en haut de la figure.

Celui du haut, marqué $a$, présente en outre son contenu cellulaire : noyau, $n$, et endochrome, ch.

Notons que la longueur des frustules peut rendre difficile l'observation de leurs rapports de soudure, quand on n'en est pas prévenu. Si on se rappelle en outre la fragilité des soudures et conséquemment du caractère spécifique du thalle, on comprendra qu'elle semble avoir pu garder l'incognito jusqu'ici.

Distribution Géographigue. - Cette espèce nous est connue surtout du Détroit de Matotchline.

\section{Genre Nitzschiella Rabenhorst}

Caractìres DU GenRe. - Valves à carène très excentrique et à extrémités longuement rostrées. Beaucoup d'auteurs ont délaissé ce genre pour en incorporer les espèces dans le genre Nitzschia. On ne peut pas dire que ce soit à tort, mais ce n'est pas avec profit; puisque ces formes longuement rostrées doivent quand même être 
séparées de leurs congénères présumées pour constituer un groupe, une section, bref un petit département à part dans le genre. N'est-il pas alors aussi avantageux de leur maintenir une étiquette générique distincte, directement évocatrice de leur singularité, quand on sait que dans le domaine des micro-organismes les découpures taxinomiques sont plus conventionnelles que réelles et que les meilleures sont celles qui éclairent le mieux la matière?

\section{Nitzschiella longissima (Brébisson) Rabenhorst.}

Planche XXXIV, figures $4 \mathrm{I}$ à 46 et 65 .

Frustules à valves lancéolées dont les rostres étroits égalent ou dépassent la longueur de la partie plus large. Des points carénaux ténus et rapprochés s'observent sur les grands spécimens; sur les petits ils sont pratiquement indistincts.

On sait qu'on en a décrit plusieurs variétés fondées sur la direction des rostres; simple façon, sans doute, de noter les différences d'aspect qui en résultent.

Nos matériaux nous en ont fourni des représentants: la variété type (fig. 43), la variété parva Van Heurck (fig. 4I), la variété reversa W. Smith (fig. 42), la variété Closterium W. Smith (fig. 44, 45, 46), dont les dimensions sont très variables.

Nous en ajouterons une autre, var. fasciculata (fig. 65), pour signaler la très petite forme que nous tenons de la Mer de Kara et dont les frustules droits et très délicats sont naturellement disposés en fascicules. Ceux-ci affectent la forme d'un cordon étroit, dans lequel les éléments sont tous orientés suivant l'axe longitudinal et alternent entre eux de manière à occuper tout l'espace. Ils doivent être soudés par une substance mucilagineuse peu abondante, qui n'est pas visible.

\section{Nitzschiella acicularis W. Smith.}

Planche XXXIV, figure 47 .

Frustules à valves lancéolées dont les rostres sont plus courts que la partie élargie. Cette très petite forme a surtout attiré notre attention par ses colonies faiblement agrégées (fig. 47) dont les individus se séparent aisément et se dispersent dans les préparations, où ils deviennent peu visibles, si ce n'est après dessiccation du slide.

\section{Genre Homoeocladia AgardH}

Pour les raisons déjà exposées, les Honnooladia, Nitzschiées agrégées en colonie à la faveur d'une gangue mucilagineuse enveloppante, perdent leur caractère planktonique principal entre les mains des diatomistes qui par besoin, par habitude ou par préférence n'ont guère attaché leur attention qu'à des slides montés avec art. 
On conçoit ainsi que H. VAN HEURCK ait déclaré formellement ne pas reconnaître ce genre, pas plus que le genre Schironema dans les Naviculées, sensu stricto.

C'est que l'ébullition dans les acides, dans les milieux oxydants, ou l'incinération simple des récoltes, détruit sans remède non seulement le caractère social commun de ces espèces, mais aussi celui qu'elles revêtent du chef de leur mode de groupement et que l'on peut, dans bien des cas, tenir pour spécifique.

Pour le microplanktoniste, le choix n'est pas douteux. Ce caractère doit avoir le pas sur les détails plus subtils de la structure valvaire, car, outre qu'il donne la raison de la vie pélagique de ces organismes, c'est lui que l'on saisit en premier lieu dans le dépouillement des produits de pêche.

Plusieurs des espèces auxquelles nous faisons allusion n'ayant pas été décrites, que nous sachions, du moins sous cet aspect, il serait difficile de retrouver dans la littérature les observations auxquelles il se peut qu'elles aient donné lieu, à un autre point de vue. Pour les désigner, au moins provisoirement, nous nous laisserons guider par l'impression qu'elles nous ont faite.

\section{Homoeocladia congesta sp. n.}

Planche XXXIV, figure 50.

Frustules grands, renflés, dilatés au milieu, brusquement rétrécis vers les extrémités, aussi bien en vue suturale qu'en vue valvaire, comme on le voit un peu obliquement, sous ces deux incidences, dans la figure 50 , la seule que nous consacrons à cette belle espèce, qui est assez commune dans la Mer de Kara.

La carène est peu excentrique et bien accusée, soulignée qu'elle est par des points très serrés.

Le noyau est central et le cytoplasme très peu abondant est disposé en deux plaques valvaires estompées et mal définies, dans une substance non miscible avec l'eau que l'on prendrait pour une matière grasse. De fait l'éther et le chloroforme l'éliminent rapidement; ce qui a été fait dans l'objet figuré.

Les frustules nous ont paru le plus souvent géminés dans un globule gommeux, $g$, dont le liquide conservateur contracte assez la substance élastique pour la détacher des frustules inclus. C'est la raison pour laquelle chacun d'eux parait flotter dans une chambre un peu plus large que lui, mais qui en épouse la forme.

L'enveloppe mucilagineuse qui réunit momentanément les frustules par couples ne tarde pas sans doute à se scinder pour les séparer, comme le fait pressentir l'étranglement de cette enveloppe, dont témoigne la figure 5o. De fait, on voit souvent les frustules entourés isolément d'une gaine propre; il est plus rare de les voir réunis en deux couples géminés dans une gaine commune, qui témoigne alors d'une subdivision retardée. 


\section{Homoeocladia glomerala sp. n.}

Planche XXXIV, figure $5 \mathrm{~s}$.

Espèce affine à la précédente, parce qu'elle se présente aussi communément en petits glomérules d'individus enveloppés en petit nombre, de un à huit, mais le plus souvent quatre, dans un globule mucilagineux, $g$.

Indépendamment de leurs dimensions beaucoup plus petites, les frustules se distinguent des précédents en ce qu'ils ne présentent pas, en vue suturale, le brusque retrait vers les extrémités, qui caractérise ces derniers.

On y observe la mème matière non miscible avec l'eau et très soluble dans les dissolvants des matières grasses. Son élimination préalable est nécessaire pour dégager le noyau et l'endochrome et en permettre la figuration.

Remaroue. - Quelle est cette substance réfringente dont beaucoup d'autres espèces de nos matériaux sont également farcies? Est-ce une enclave naturelle ou un produit d'absorption, post mortem, fourni par le milieu conservateur?

Dans d'autres circonstances, nous avons vu la plupart des espèces présenter un fait analogue après fixation dans un liquide où entrait l'alcool isobutylique à haute dose, si nos souvenirs sont exacts; mais nous ne pouvons pas dire que ce soit le cas ici.

Origine. - Mer de Kara et Détroit de Matotchkine.

\section{Homoocladia mucicola sp. n.}

Planche XXXIV, figure 48 .

Frustules assez grands encore, généralement disséminés par couples dans une enveloppe mucilagineuse, $g$, qui se prête à la production d'un thalle microscopique parfois élargi localement, mais le plus sourent filamenteux, dans lequel les couples de frustules s'alignent, à distance les uns des autres, suivant leur longueur. Ce sont ces deux modes d'arrangements que synthétise le fragment de colonie que reproduit la figure 48 .

Les frustules s'y présentent sous toutes les incidences et conséquemment sous des aspects variés, en vue valvaire et en vue suturale. Les valves sont assez longuement lancéolées et portent une carène peu excentrique, marquée de points nombreux et serrés. En vue connective, les frustules sont linéaires, un peu atténués vers les bouts qui sont tronqués.

Pour être figurés comme ils l'ont été, avec ou sans l'endochrome, il a fallu aussi les traiter par les dissolvants des matières grasses.

Origine. - Mer de Kara. 


\section{Homœocladia taniala sp. n.}

Planche XXXIV, figure 52.

Dans cette espèce, les frustules, moins grands que dans les précédentes, sont placés còte à côte, suivant leur face valvaire, en longues séries rubanées qu'une gaine mucilagineuse, g, maintient en bon ordre. Il en résulte qu'ils se présentent naturellement en vue suturale, comme dans le corps de la figure 52, où ils ont été dégorgés par l'éther de la substance réfringente qui les remplit plus ou moins complètement.

On observera qu'ils doivent leur origine à des subdivisions répétées dont certaines étapes restent reconnaissables, gràce à la position plus ou moins latérale du noyau et à l'excentricité variable de l'endochrome.

Pour ne pas multiplier les dessins figuratifs de cette espèce, nous avons reproduit, vers le haut de la même figure 52 , deux frustules dérangés de leur position naturelle et présentés en vue valvaire, l'un vide, l'autre en possession de son contenu, noyau et endochrome.

Plus haut encore, sous la lettre $b$, on voit quelques frustules figurés sous leur aspect naturel, avec la substance huileuse, $h$, dans laquelle se trouvent comme noyés le noyau et l'endochrome. Des vacuoles aqueuses plus claires y sont diversement localisées.

\section{Homœocladia lenuis sp. n.}

Planche XXXIV, figure 35.

Frustules beaucoup plus petits, plus grèles, mais de forme analogue à ceux de l'espèce précédente, associés en thalle mucilagineux de forme diffluente et souvent très étendue, dans lequel leur mode de division les présente le plus souvent géminés, mais orientés dans tous les sens.

On conçoit que si ces frustules étaient macérés, incinérés et fixés dans le baume, ils seraient peu révélateurs de caractères propres à les distinguer d'autres petites formes dont on trouve des spécimens isolés dans tous les milieux et dont il nous a semblè inutile de signaler la présence.

\section{Hom cocladia delicalissima (Cleve).}

Planche XXXIV, figure 34 .

Synonyme. - Nitzschia delicatissima Cleve, I897.

Frustules aciculaires, très petits, dont tous les planktonistes connaissent bien les rapports presque constants de commensalisme ou de symbiose avec les colonies mucilagineuses de Phcocystis. Si nous dérogeons aux habitudes reçues en présentant cette 
espèce dans le groupe des Homoocladia, c'est qu'il nous est arrivé plusieurs fois, dans l'examen des produits de pêche de la Belgica, de la rencontrer en dehors de toute solidarité avec sa commensale habituelle et néanmoins embarrassée dans une gangue mucilagineuse, qu'elle semble avoir dù sécréter elle-mème.

\section{Genre Schizonema AGARDH?}

Ce n'est qu'avec les réserves les plus expresses que nous allons signaler, sous le couvert de ce nom générique, deux espèces de Diatomacées dont les frustules naviculacés et faiblement silicifiés vivent dans une matière mucilagineuse qui les tient groupés en très petits thalles aptes à flotter au large.

Nous les avons fréquemment observées dans les produits de pêche de la Mer de Kara, mais dans des conditions qui ne nous ont permis d'en saisir autre chose que l'aspect naturel. C'est à titre d'indication seulement que nous les avons figurées sous cet aspect.

Planche XXXIII, figure 47. Frustules ramassés, brièvement elliptiques en vue valvaire, rectangulaires en vue suturale, gorgés d'une substance d'apparence huileuse, $h$, qui rend indistincts aussi bien les détails de structure des valves que la distribution normale du cytoplasme.

Placés còte à côte, suivant leur face valvaire, ils forment des séries linéaires au sein d'une substance mucilagineuse résistante, $g$, dans laquelle ils occupent chacun une logette spéciale dont les cloisons mitoyennes sont d'autant plus épaisses que l'indiridualisation des éléments est plus ancienne.

Planche XXXIII, figure 49. Frustules allongés, étroits, longuement lancéolés, noyés dans une gangue mucilagineuse, $g$, dans laquelle ils sont orientés dans toutes les directions, mais ne sont jamais en rapport entre eux que par leurs extrémités.

La substance mucilagineuse est plus dense au voisinage immédiat des membres de la colonie, qu'elle circonscrit isolément, en leur formant une coque propre dont les couches les plus externes deviennent progressivement plus molles et diffluentes.

Dans ces conditions défavorables d'observation, les frustules ne traduisent aucun détail de structure valvaire. Ils sont aussi toujours gorgés d'un produit d'aspect huileux, qui rend le contenu cellulaire homogène (voir celui qui est à gauche de la figure).

Le cytoplasme, pr, est peu abondant et ne devient distinct qu'après traitement de l'objet par l'éther ou par le chloroforme. 


\section{OBservation}

Il ne nous reste plus à signaler maintenant que des formes isolées dont le rôle planktonique est très restreint ou même nul et dont nous avons groupé presque pèle-mèle les reproductions dans les deux dernières planches, XXXV et XXXVI, pour faciliter autant que faire se peut les essais d'identification que d'autres, mieux documentés que nous, pourraient tenter.

Ce sont surtout des Pleurosigma et des Navicula, dont nous avons fixé les traits au hasard de leur rencontre et dans des conditions d'étude peu propres à en tirer les renseignements qu'une critique sévère serait en droit d'exiger.

Telles quelles, ces figures ajouteront néanmoins quelques documents à l'histoire des Diatomacées arctiques et rendront plus complet le tableau des récoltes de la Belgica.

C'est assez pour que nous n'ayons pas à regretter de les avoir exécutées, bien que nous ne soyons pas en état de les identifier toutes.

\section{Genre Pleurosigma W. Sмiтн}

Caractères du genre. - Voir H. Van Heurck, Traité des Diatomées, page 249.

Les diatomistes ont reconnu et dénommé une foule de Pleurosignna disséminés dans tous les milieux. L'analogie étroite de leur forme fournit peu de caractères différentiels et les détails de structure des valves sont trop délicats généralement pour être saisis, dans les observations usuelles, avec la précision qu'il faudrait pour y repérer les notes micrométriques que l'étude comparée et patiente des collections de types soigneusement triés a fait considérer comme spécifiques.

C'est particulièrement le cas pour les formes que nous avons figurées et que nous tenons des neiges et des glaces, où, nous l'avons déjà fait remarquer, la silicification des Diatomacées en général est particulièrement faible. Cette circonstance atténue singulièrement la striation des valves et la rend souvent indiscernable, même à sec.

La difficulté d'identification peut alors, se transformer en une réelle impossibilité, si l'on ne peut se rabattre sur d'autres caractères qui, malheureusement, sont d'ordre subjectif et dépendent alors trop de l'observateur pour ètre traduits d'une façon doctrinale.

Nous nous bornerons donc à appeler l'attention sur les caractères les plus saillants des spécimens reproduits tous dans la planche XXXV, en les ramenant à deux sections, d'après la direction des stries, conformément aux vues de Cleve et Grunow, dans Arctische Diatomeen. 


\section{I. - Formes a stries décussées}

Figures I, 6 et 7. Grande forme à valve lancéolée, sigmoïde, plus large au milieu, régulièrement atténuée vers les extrémités qui restent un peu obtuses-arrondies. Raphé sigmoïde. Trois séries de stries faibles, sensiblement de même valeur, se coupant sous des angles d'environ $60^{\circ}$ (fig. I).

Remarque sur L'endochrome. - C'est à un grand spécimen de la même espèce probablement que se rattachent les figures 6 et 7 destinées à montrer la disposition élégante des chromatophores cellulaires, ch.

Dans les frustules au repos, ces organes sont au nombre de deux, placés à gauche et à droite contre la zone connective. Chacun affecte la forme d'un long ruban plissé et sinueux dont les méandres sont symétriquement développés des deux côtés de l'anse centrale qui encadre le noyau, $n$; conformément à la figure 7 , qui ne reproduit que le chromatophore rapproché de la zone connective tournée vers l'observateur.

Le second, placé de l'autre côté de la zone connective, présente une disposition symétrique au premier, par rapport à un plan sigmoïde qui passerait par le raphé (fig. 6).

Il en résulte, comme on sait, que les deux moitiés valvaires de la cellule ont une structure différente, de même qu'elles sont aussi d'àge différent.

On sait aussi que les méandres de ces chromatophores rubanés s'accentuent avec la maturité physiologique de la cellule et que chacun d'eux acquiert, au moment de la cytodiérèse ultérieure, assez d'étoffe, peut-on dire, pour fournir aux cellules-filles un couple de chromatophores résultant de la subdivision des organes maternels homologues.

Le processus de dédoublement est toujours très curieux et présente des variantes suivant les espèces. Les caractères d'ordre physiologique qu'on pourrait en tirer vaudraient bien ceux d'ordre purement morphologique auquel l'usage a prévalu de s'en tenir exclusivement. Ils constitueraient en tout cas un appoint précieux, comme contròle et aussi comme guide dans la recherche de l'affinité naturelle des formes.

On remarquera que le noyau est central, fixé dans une masse de cytoplasme plus dense de forme cylindrique, qui fait axe entre les deux valves, au niveau des nodules médians.

De minces cordons cytoplasmatiques parcourent le suc cellulaire abondant, pour rattacher l'ectoplasme et les chromatophores qui y sont localisés à ce cylindre endoplasmatique axial. Celui-ci ne se montre dans son développement que sur la vue suturale (fig. 7); sur la vue valvaire (fig. 6) on n'en peut voir que la section transversale optique.

Figures 29, 30, 3I. Un autre exemple n'est pas de trop pour illustrer l'allure singulière de l'endochrome dans les représentants du genre Pletrosigma. Nous l'avons emprunté à une autre espèce dont nous avons négligé les caractères sculpturaux pour 
nous attacher à ses caractères internes. Ceux-ci sont tels qu'ils ne laissent aucun doute sur le caractère générique de l'objet, bien que la forme en soit régulièrement lancéolée, non sigmoïde du tout.

Dans la figure 3I, ce sont les anses méandriques médianes des chromatophores rubanés qui sont dirigées vers l'observateur, tandis que les autres circonvolutions se dirigent, dans la profondeur du frustule, vers la face opposée et s'orientent enfin vers les extrémités aiguës de la cellule. La figure 3o, qui n'est du reste que le même objet renversé, montre naturellement les anses médianes dans la profondeur, tandis que les circonvolutions moyennes viennent se développer sous la valve qui regarde l'observateur. Dans la figure 29, qui est une vue suturale, nous n'avons figuré que l'un des chromatophores, avec ses anses alternativement convexes et concaves vers les deux valves. L'autre, qui lui est symétrique, est placé en dessous, dans une situation où il n'aurait pas pu être figuré utilement sans nuire à l'évidence du premier.

On pourra noter ici aussi le cylindre de cytoplasme périnucléaire, $n$, qui traverse la cellule suivant l'axe transvalvaire.

Pleurosigma angulatum, var. quadratum W. Smith, qui est abondant sur les côtes belges, se prête très bien, gràce à sa grande largeur, à l'observation d'une disposition fort analogue des chromatophores; mais la touche en est autre : c'est comme le cachet de l'espèce.

Figure 2. Frustules à valves largement lancéolées et sigmoïdes, parcourues par un raphé droit dans son milieu, mais très courbé vers ses extrémités. Les stries obliques sont très marquées, au contraire des stries transversales qui ne sont guère saisissables et que pour cette raison nous n'avons pas indiquées dans la figure.

C'est l'une des rares espèces observées dont la striation apparaisse en milieu liquide. Les stries sont un peu courbes et sigmoïdes elles-mêmes, vers le milieu de la valve, où leurs extrémités sont comme entrainées dans la direction du raphé.

Figure t. Valve en forme de losange allongé et oblique, parcourue par un raphé dont les deux courbes sigmoïdes sont très rapprochées du nodule central et dont le reste du trajet est presque droit, jusqu'au voisinage des nodules terminaux. Les trois séries de stries y sont extrèmement faibles.

Figure 5. Valve largement lancéolée dont le caractère un peu sigmoïde n'apparait qu'aux extrèmes bouts. Par la forme du raphé, elle présente une certaine analogie avec la précédente dont elle partage la faible striation décussée.

Figure i5. Valve lancéolée, plus étroite, dont le caractère sigmoïde est plutòt sugggéré par la double courbure du raphé aux environs du nodule médian que par les bords de l'organe. 
Par la forme du raphé, ce spécimen se rapproche des deux précédents. Il semble qu'on aurait pu faire aussi utilement état de cette particularité que du nombre des stries par unité de mesure. Ce dernier détail, nous ne l'avons pas relevé; mais, l'eussions-nous fait, nous n'en serions pas moins perplexes, s'il s'agissait de les identifier avec des espèces sans doute décrites, mais qui ne nous sont pas autrement familières.

Figure II. Très petite forme, courte, trapue, largement lancéolée et sigmoïde, dont la valve, à striation décussée, est parcourue par un raphé très sigmoïde.

Figures I9 et 20. Deux spécimens d'assez petites dimensions qui par leur modelé et la striation décussée de leur valve rappellent, en petit, la variété quadratum de Pl. angulatum W. Smith.

Figure 23. Forme assez largememt lancéolée dont le caractère sigmoïde se traduit surtout par l'incurvation brusque des extrémités. Le raphé est très sigmoïde. La striation est décussée, peu apparente.

Figure 28. Espèce à valve lancéolée, sigmoïde, assez grande, parcourue par un raphé qui est presque droit et ne subit de faibles courbures sigmoïdes qu'au voisinage immédiat des nodules terminaux.

Le caractère particulier de cette forme a son expression plus franche dans un spécimen un peu plus petit, reproduit dans une figure non numérotée par oubli, entre les deux dessins $\mathrm{I} \delta$ et $2 \mathrm{I}$. Là, le raphé est tout à fait rectiligne jusque tout près des nodules terminaux. Striation décussée difficilement perceptible.

Figure 26. Très grande espèce à valves linéaires-sigmoïdes, obtuses-arrondies aux extrémités, parcourues par un raphé également sigmoïde qui tient sensiblement le milieu de l'organe.

Il serait téméraire de la rapporter à un des nombreux types décrits, d'aspect à peu près pareil, sans posséder une collection des slides originaux qui ont servi à en fixer la diagnose. La comparaison des objets donnerait la sécurité qui manque à la comparaison des figures et aux formules vagues des descriptions.

Figure 27. Par sa forme étroitement lancéolée, presque droite, obtuse-arrondie aux extrémités, peu ou pas sigmoïde, mais sillonnée par un raphé sigmoïde excentrique, le spécimen reproduit ici semble bien représenter les caractères morphologiques extérieurs de Pl. speciosum IV. Smith.

\section{II. - Formes a stries perpendiculaires}

Figures 8, 9 et Io. Dans cette forme à valve lancéolée-aiguë et non sigmoïde du tout, pas plus que le raphé, il semble bien que l'on doive reconnaitre $P l$. vitreum 
Cleve, dont le nom spécifique serait évocateur de l'aspect hyalin des valves, où la double striation longitudinale et transversale est à peine discernable dans les meilleures conditions d'observation. Dans la figure io, nous n'avons pu la traduire, même le plus légèrement possible, au diamant, sans en exagérer beaucoup l'expression.

Ce qui est beaucoup plus caractéristique pour nous dans les spécimens de cette espèce, qui est assez abondante dans les récoltes effectuées sur les glaces de la Mer de Kara, c'est la constance, dans les frustules, de grandes vacuoles occupées par une substance d'aspect huileux, $h$, qui refoulent le cytoplasme de la plus grande partie de la capacité cellulaire. Les figures $\delta$, vue suturale, et 9 , vue valvaire, sont destinées à traduire approximativement la physionomie spéciale que cette particularité leur communique. Si celle-ci est physiologique, comme il parait, et non pathologique et accidentelle, elle est incompatible avec la présence de chromatophores rubanés tels qu'on en observe dans les Pleurosigma vrais et il devient douteux que la forme en question appartienne à ce genre plutòt qu'au genre Navicula, dont elle a, du reste, d'autres caractères morphologiques.

C'est évidemment une espèce qui exige une étude critique plus appronfondie; mais ce n'est pas le moment pour nous de la faire.

Figures 3 et 13. Deux formes qui semblent se rapprocher du type de Pl. Fasciola IV. Smith, si l'on s'en tient, comme les diatomistes en ont peut-être trop l'habitude, aux caractères extérieurs. Nous n'avons pas de raisons pour penser autrement, bien (qu'il paraisse que l'arbitraire règne en maitre dans l'élévation des formes au rang d'espèce, ou leur déchéance au rôle secondaire de variété ou de simple forme.

C'est malheureusement encore une nécessité actuelle dans ce département obscur de la science biologique.

Mettons que l'un (fig. 3), par ses prolongements rostrés plus longs et peu courbés, rappelle davantage $\mathrm{Pl}$. temirostris Grunow et que l'autre (figure I3), par ses rostres plus courts et plus arqués, répond mieux au facies de Pl. Fasciola W. Smith.

Figure I2. Petite forme lancéolée, aiguë aux extrémités, à peine sigmoïde et sillonnée par un raphé presque clroit.

Figures 2 I et 22. Petites formes aiguës aussi aux extrémités, mais plus manifestement sigmoïdes et sillonnées par un raphé également sigmoïde, qui tient le milieu de la valve.

Figures I4, I7 et I8. Formes de dimensions diverses qui semblent partager la mème forme linéaire-sigmoïde, avec des extrémités obtuses et un raphé maintenu dans le milieu de la valve.

Figure i6. Forme d'un tout autre modelé, droite, dont les bords, parallèles sur presque toute leur longueur, sont seulement courbés en sens inverse aux deux extrémités. Le raphé est sigmoïde; la striation peu visible. 
Figure 24. Valve grande, longuement lancéolée, aiguë, sigmoïde. Le raphé est sigmoïde et central.

Figure 25. Valve grande, linéaire, sigmoïde, à extrémités arrondies.

Le raphé, beaucoup plus sigmoïde que la valve, est très excentrique sur celle-ci, vers les extrémités, et $y$ est fortement relevé en forme de carène.

\section{Genre Rhoicosigma Grunow}

Certains auteurs groupent sous ce nom générique des espèces que d'autres conservent dans le genre Plcurosigma. Elles en ont, en effet, la forme sigmoïde de la valve et du raphé, mais les frustules sont plus ou moins coudés, en vue suturale.

Nos matériaux ne nous en ont présenté qu'une espèce, dont nous avons reproduit quelques aspects dans les figures 32 à 35 de la planche XXXV.

La figure 33 en est une vue valvaire, de forme lancéolée, sigmoïde, à extrémités subaiguës, et parcourue par un raphé sigmoïde, très excentrique.

A l'intérieur on aperçoit les deux chromatophores rejetés latéralement contre la zone connective. Ils ont, comme dans les Pleurosigma auxquels nous avons fait allusion à ce sujet, la forme d'un ruban dessinant des méandres nombreux, dont les circonvolutions ont un développement symétrique vers les deux extrémités.

Les bords en sont frangés, déchiquetés, plus ou moins étirés par de minces cordons cytoplasmatiques, avec lesquels ils sont en rapport et par l'intermédiaire desquels ils sont rattachés à l'endoplasme périnucléaire.

La figure 35 permet d'en saisir le développement de face, suivant la vue suturale de l'objet, à un moment intéressant de leur évolution; car la cytodiérèse qui se prépare et qui est déjà indiquée par l"étirement du noyau, $n$, doit aussi en provoquer le dédoublement. Avec un peu d'attention, on peut reconnaitre que le partage est déjà préparé et que les deux cellules-filles futures seront pourvues de chromatophores semblables, dont les anses seront orientées symétriquement par rapport au plan de la division à intervenir.

La figure 32 est d'un frustule adulte vidé, dont la zone connective est très développée.

La figure 34 montre deux jeunes frustules encore inclus dans la zone connective de leur génératrice.

En milieu liquide les valves ne laissent voir aucun genre de sculpture. 


\section{Genre Scoliopleura Grunow}

Valves naviculacées très convexes, parcourues par un raphé sigmoïde; frustules un peu tordus sur la zone connective.

Planche XXXV, figures 36 et 37. Grande forme à frustule très large, en vue connective (fig. 37); ce qui tient à la convexité extraordinaire des valves, dont la plus grande surface se profile de ce côté. La zone connective, un peu tordue-sigmoïde, est relativement peu large.

La vue valvaire (fig. 36) est longuement elliptique, sillonnée obliquement par un raphé sigmoïde, coupé au milieu par un nodule. La striation est transversale et assez accusée pour ètre visible en milieu liquide. Ressemble à S. latestriata (Brébisson) Grunow.

Origine. - Détroit de Matotchkine; très rare.

Planche XXXVI, figures 86 et 87 . Forme beaucoup plus petite à valves linéaires, obtuses-arrondies aux extrémités. Raphé sigmoïde bordé d'un côté surtout d'une aire hyaline. Le reste est occupé par une grossière sculpture alvéolaire dont les mailles rectangulaires ont leur grand diamètre orienté transversalement à la valve et ne forment de chaque côté du raphé que deux ou trois rangées.

En vue suturale le frustule se montre étranglé en son milieu, arrondi-tronqué aux extrémités. Il est bien silicifié.

Origine. - Boues glaciaires.

\section{Genre Rhoiconeis Grunow}

Caracteres du genre. - Cfr. H. Tan Heurck, Traité des Diatomées, page 238.

Planche XXXIII, figures 59 et 60. Espèce achnanthitorme comme ses congénères, qui diffèrent des Achnanthes en ce que chacune des valves est munie d'un nodule.

Nous la tenons de la florule des glaces, où nous n'avons pu l'observer qu'à l'état de frustules isolés et vides. Ceux-ci, en vue suturale (fig. 6o), se montrent coudés ou plutôt courbés d'un seul côté, suivant un profil qui, à première vue, rappelle celui des Achnanthes, mais qui en diffère réellement. La zone connective paraît lisse ou finement striée longitudinalement. 
Les deux valves (fig. 59) sont identiques. Elles ont une forme lancéolée, un peu déjetée d'un còté; elles portent un raphé arqué, un nodule médian et une petite aire hyaline, ébauche d'un stauros, qui est loin d'atteindre les bords. Le reste est couvert d'une striation serrée, un peu radiante.

\section{Genre Navicula Bory}

\section{Caractères du genre. - Cfr. H. Van Heurck, loc. cit., page i62.}

Ce genre bien connu, le plus riche en espèces de toutes les Diatomacées, ne tient pas dans le plankton marin une place en rapport avec son extraordinaire diversité, surtout si l'on en distrait, comme nous l'avons fait, les espèces affines, que leur faculté de s'agréger en colonie rend plus aptes que d'autres à entrer en concurrence avec les Chétocérées et à s'immiscer dans leurs rangs, au milieu de la florule flottante des eaux pélagiques.

Celles dont il nous reste à dire un mot n'ont pas ce caractère. La plupart sont d'eau douce, soit que nous les tenions de la rivière Matotchka, ou des glaces de la Mer de Kara, ou des boues glaciaires dont l'origine terrestre ne semble pas douteuse. S'il en est quelques-unes qui ont pu se rencontrer dans des produits de pèche planktoniques, ce n'est guère qu'à la suite de circonstances fortuites : fonte des glaces, déversement des cours d'eau, courants marins còtiers, etc., qui en ont entrainé des spécimens isolés plus ou moins loin de leur habitat naturel.

Nous nous bornerons à extraire de la collection des formes reproduites, pour la plupart, dans la planche XXXVI celles dont les caractères morphologiques apparents donnent prise à une assimilation immédiate à des types connus, ou du moins à des groupes de formes qui constituent des découpures utiles du genre, comme celles de Van Heurck, dans son Traité des Diatomées.

Quant aux autres, nous n'avons pas pu les soumettre à la technique spéciale qu'exigerait leur étude approfondie, ni nous livrer aux recherches bibliographiques qui seraient nécessaires pour retrouver leur état civil légitime.

Figures 7, 8, 9 - IO, I I - 59, 60. N. glacialis Cleve. Il y a lieu de mentionner à part cette espèce, une des rares dont la présence soit assez régulière dans les pêches planktoniques de la Mer de Kara et du Détroit de Matotchkine.

Les figures $7,8,9$ se rapportent à de grands spécimens dont les valves, largement elliptiques et presque planes, présentent une fine trame alvéolaire, caractérisée en milieu liquide par des stries radiantes très rapprochées qui couvrent toute la surface (fig. 7) ou sont interrompues sur des plages plus ou moins étendues dans le voisinage du 
nodule central (fig. 9). Ces stries sont réunies par des trabécules transversaux très ténus qui délimitent de petites mailles. Celles-ci se traduiraient dans le baume sous la forme de perles qui seraient naturellement orientées en séries, comme les stries radiantes.

La figure 8 montre que ces grandes formes sont aplaties suivant la zone connective.

Les figures Io et I I sont les deux vues, valvaire et suturale, d'une forme beaucoup plus petite dans laquelle les faibles dimensions de la valve sont compensées par une largeur plus grande de la zone connective.

Il faut $y$ rattacher encore les figures 59 et 60 , qui en montrent la forme et la disposition de l'endochrome.

Celui-ci est formé de deux chromatophores valvaires symétriques qui, partant du centre de la valve, se subdivisent en un certain nombre de lamelles rayonnantes (fig. 6o), marquées irrégulièrement de nodosités plus sombres. Les extrémités amincies de ces lamelles se rattachent à leurs homologues de l'autre valve par des cordons cytoplasmatiques transversaux à la zone connective (fig. 59).

Le noyau, $n$, est central et logé dans un cylindre protoplasmatique transvalvaire.

Aux Didymées de Van Heurck se rattachent quelques-unes de ces formes au dessin compliqué, dont l'interprétation figurative est influencée beaucoup par le milieu d'enrobage, ce qui en rend l'identification difficile parfois.

Figure I. N. bomboides W. Smith. Tue valvaire. A part la différence d'amplification, c'est presque trait pour trait la figure I9, planche B (supplément de l'atlas du Synopsis).

Figures 53 et 54. N. chersonensis Grunow? Grand spécimen dont nous reproduisons deux vues qui ne diffèrent en réalité que par l'aspect que revêt l'objet soit en milieu liquide (fig. 54), soit à sec (fig. 53).

Dans la figure 54 on saisit mieux la structure alvéolaire vraie. Dans la figure 53 les alvéoles interposés aux còtes radiantes sont réduits à de petits pores qui, dans le baume, apparaitraient comme des perles alignées sur un seul rang. La valve laisse alors passer beaucoup moins les rayons lumineux et parait plus obscure.

La différence n'est donc évidemment qu'apparente, mais il nous a paru utile de montrer, par un exemple qui nous est personnel, combien les écarts de techniques et les modes de figuration adoptés peuvent rendre difficile la conciliation des notes bibliographiques des auteurs.

Planche XXXV, figures 38 et 39. Une des nombreuses formes qui semblent devoir se rattacher à $N$. crabro Ehrenberg. Notre reproduction ayant été faite d'après l'objet immergé dans l'eau, il serait difficile d'en urger la comparaison avec d'autres exécutées dans des conditions différentes.

On voit, par la vue suturale de la figure 39 , que le frustule se montre également étranglé en son milieu, sous cet aspect. 
Planche XXXVI, figure 22. A part une certaine dilatation des sillons qui côtoient le raphé, ce dessin ressemble étrangement à la figure 20, planche B (supplément de l'atlas du Synopsis de VAN HEurck), qui se rapporte à $N$. didyma Ehrenberg. Si n'étaient les assez grandes différences de grandeur attribuées aux objets, nous n'hésiterions pas à assimiler l'un à l'autre.

Les Ellipticées de Van Heurck, qui rentrent aussi avec les Didymées dans les Diploneis d'autres auteurs, ont également ici quelques représentants.

Figure 2r. N. Smithii Brébisson. Grande forme largement elliptique-arrondie; nodule très large, au milieu d'une aire hyaline qui se rétrécit vers les nodules terminaux et qui borde des deux côtés le raphé peu discernable; côtes robustes, radiantes, dont l'aspect en milieu liquide fait supposer des rangées de petites mailles interposées; sillons profonds, parallèles à l'aire hyaline centrale, un peu élargis vers le milieu de la valve.

Figures 23 et 24. N. elliptica Kützing. Forme plus petite, elliptique, à sillons plus étroits et à côtes radiantes moins distancées. Vue valvaire (fig. 23), vue connective (fig. 24).

Planche XXXV, figures 42 et 43 . Vues suturale et valvaire d'une forme analogue mais plus longuement elliptique-aiguë, à sillons plus élargis au milieu et à aire hyaline très étroite autour du raphé.

Planche XXXVI, figure 78. N. scutellum O'Meara. Petite forme à valve elliptique, suborbiculaire, que Van Heurck considère comme une variété de $N$. Smithii.

Figure 79. Forme affine aux précédentes par l'aspect, mais plus allongée, à bords presque parallèles, arrondie seulement aux extrémités. Peut-être trouverait-on rationnel d'en faire aussi une variété de $N$. Smithii, var. elongata, par exemple.

Figure 8I. N. littoralis Donkin. Petite forme à valve elliptique aiguë. Nodule allongé. Raphé bordé des deux còtés par des sillons étroits complètement droits. Striation transversale très légère et droite.

Figures 80 et 82. N. oculata Brébisson. Valve linéaire oblongue à extrémités arrondies. Nodule médian subquadrangulaire. Raphé bordé de sillons étroits, droits, légèrement incurvés près du nodule. Striation transversale droite très légèrement accusée. Les figures valvaires 80 et 82 nous en paraissent ètre des variantes de grandeur.

Aux Henned’ées, se rattache la seule espèce observée qui suit :

Figure 5o. N. Sandriana Grunow. Grande valve, de forme largement elliptique, analogue aux autres connues du groupe, mais s'en distinguant par les deux longs arcs étroits qui occupent, des deux côtés du raphé, l'aire mouchetée centrale de la valve.

Les Lyrées ont des représentants à la fois plus variés et plus nombreux.

Figure I6. N. lyra Ehrenberg. Variété de grand module, à valve elliptiquearrondie très large. 
Figure 15. N. lyra Ehrenberg. Autre variété à valve un peu étranglée au milieu et atténuée-aiguë vers les extrémités. Comme on sait, nous figurons par des stries les espaces qui séparent les séries de perles des auteurs qui reproduisent l'aspect dans le baume. Sans être aussi exact, c'est plus expéditif.

Figure 27. N. abrupta Gregory. Talve elliptique, présentant des sillons lisses lyrés qui convergent à leur extrémité vers le raphé et qui se terminent brusquement à une certaine distance des extrémités de l'organe.

Figures I3 et I4. N. abrupta Gregory. Vues valvaire et suturale d'une variété, à bords parallèles au milieu et à extrémités diminuées obtuses, qui présente néanmoins des sillons lyrés convergents vers leur sommet, à une certaine distance des extrémités de la valve.

Figures 25 et 26. N. forcipata Greville. Vues valvaire et suturale d'une forme longuement elliptique dont les sillons lyrés sont peu écartés du raphé et s'y fusionnent, en quelque sorte, vers les extrémités.

Figures 28 et 29. Vues valvaires d'autres modalités, différentes de grandeur et de forme, du même type de $N$. forcipata.

Nous n'avons à signaler qu'une forme des Palpébrales.

Figure 33. N. palpebralis Brébisson. Valve largement elliptique-lancéolée, à extrémités aiguës; stries un peu radiantes, marginales, laissant autour du raphé un large espace hyalin de forme lancéolée.

Aux Abréviées pourraient appartenir les formes suivantes :

Figure 43. N. brevis Gregory. Valve elliptique, à extrémités diminuées-rostrées, à rostre large. Raphé entouré d'une zone hyaline assez large, se dilatant autour du nodule médian en un espace hyalin lancéolé. Stries radiantes.

Figure 19. On pourrait peut-être y rattacher la petite forme plus obtusément rostrée que nous figurons ici et dont l'aire hyaline est arrondie et rend indistinct le nodule médian.

Les Perstrices n'ont sans doute dans nos matériaux qu'un représentant authentique.

Figure 44. N. Inmerosa Brébisson. Valve atténuée-rostrée de dimensions moyennes. Raphé bordé d'une aire hyaline dilatée transversalement autour du nodule médian. Stries radiantes, les médianes inégalement longues. Notre forme est étranglée au milieu; il y aurait lieu, sans doute, d'en faire une variété du type.

Il semble qu'il faille rapporter aux Crassinervices :

Figure 6r. N. cuspidata Küitzing. Valve longuement lancéolée, à extrémités rostrées, non céphalées, que couvre une striation fine, transversale, atteignant le raphé, sauf au voisinage du nodule médian, où elle devient indiscernable. 
Aux Stauronéidées se rapporteraient :

Figures 3 I et 32. N. pinmularia Cleve. Synonyme : N. quadratarea A. Schmidt. Vues suturale et valvaire d'une forme linéaire-oblongue, à extrémités arrondies; stries transversales, parallèles, atteignant le raphé et laissant, autour du nodule médian, un large espace hyalin, quadrangulaire, stauronéiforme.

Nous ne saurions dire s'il y a lieu d'y rattacher avec quelque fondement les formes reproduites dans les figures 65 et 64 qui, avec une forme plus courte, plus large et un peu atténuée-obtuse au sommet, présentent une aire hyaline stauronéiforme plus ou moins accusée. La striation en est transversale et assez ténue.

Les Aspérées n'ont guère d'autres représentants connus que :

Figures 2, 3, 4. N. aspera Ehrenberg. Valve elliptique-lancéolée à extrémités subaiguës (fig. 2). Raphé entouré d'une zone hyaline étroite qui, au niveau du nodule médian, prend une forme élargie, stauronéiforme, qui n'atteint pas les bords de la valve. Celle-ci présente une structure alvéolaire imbriquée, grossière. Les figures 2 et 3 sont d'un même spécimen présenté en vue valvaire et en vue suturale. La figure 4 est d'un spécimen un peu plus court, mais plus large en vue suturale.

Figures 46 et $47 . N$. Clepsydra Donkin. Il se peut que le spécimen ici figuré, en vue suturale et en vue valvaire, soit cette espèce marine, caractérisée par des stries transversales, peu rayonnantes, plus fines, qui soulignent une structure alvéolaire imbriquée moins apparente, par la forme circulaire de l'espace hyalin qui entoure le nodule central (fig. 47) et par l'étranglement médian du frustule, en vue suturale (fig. 46). L'examen en milieu liquide n'a sans doute pas permis de marquer davantage la structure alvéolaire imbriquée, entre les stries radiantes beaucoup plus visibles dans ces conditions spéciales d'observation.

Les Pinmulariées et les Radiosées ne sont guère représentées en dehors des échantillons de boues glaciaires, où on en rencontre quelques-unes à l'état de frustules vides le plus souvent.

Figures 67 et 68. $N$. viridis Kützing. Grande forme à valve linéaire-elliptique arrondie aux sommets. Les côtes transversales ménagent tout le long du raphé et mème autour des nodules terminaux une aire hyaline assez large.

Figures 39 et 40. Navicula sp. Grande forme du type des Pinnulariées, à valve lancéolée, décorée de grosses còtes radiantes qui n'atteignent pas le raphé et laissent une aire hyaline de forme elliptique autour du nodule médian.

Figures 70 et 7 I. N. tridens sp. n. Grande forme très analogue aux précédentes, mais s'en distinguant nettement par une aire hyaline centrale en forme de stauros, qui est due à ce que les còtes médianes, trois de chaque còté, sont moitié moins longues que leurs voisines. La valve est lancéolée (fig. 70) et très convexe, comme on peut le voir sur la vue suturale (fig. 7I). 
Figures 57-58 et 5I-52. N. cruciformis Donkin. Spécimens de dimensions différentes en vue valvaire (fig. $5 \mathrm{I}$ et 57 ) et en vue suturale (fig. 52 et 58).

Les frustules sont un peu étranglés au milieu; les valves sont linéaires, arrondies aux extrémités; les stries sont fines, flexueuses, radiantes au milieu de la valve, convergentes vers les bouts; le nodule médian est entouré d'un pseudo-stauros large, cunéiforme.

Figures 48 et 49. Navicula sp. Valve linéaire, large au milieu, atténuée-obtuse aux extrémités; stries transversales très serrées, courtes, laissant une aire hyaline large tout le long du raphé. Frustule légèrement étranglé au milieu (fig. 48).

Figures 37 et 38. $N$. cancellata Donkin. Vues suturale et valvaire d'une forme assez grande, à frustule contracté au milieu et à valve longuement lancéolée. C'est sans doute une des variétés connues du type.

Figures 5-6 et 4I-42. N. kariana sp. n. Une des formes les plus répandues sur les glaces de la Mer de Kara. Valve très longuement lancéolée-aiguë, couverte de fortes stries perpendiculaires au raphé et l'atteignant presque (fig. 5 et 42), vue suturale presque linéaire, témoignant de la faible convexité des valves (fig. 6 et 4I).

Figures 35 et 36. Navicula sp. Petite forme à valve lancéolée couverte de stries très rayonnantes au voisinage du nodule médian, où quelques-únes restent plus courtes sans néanmoins laisser place à une aire hyaline.

Figure 45. $N$. peregrina Kützing. Forme reconnaissable à la disposition spéciale des stries rayonnantes médianes qui sont de longueur inégale.

Figures 76 et 77 . Navicula sp. Forme de Radiosée dont les caractères sont trop frustes pour permettre une assimilation fondée.

Figure 74. Navicula sp. Valve largement lancéolée, aiguë aux extrémités, complètement courerte de stries rayonnantes, assez fortes.

Figure I7. $N$. lanceolata Kützing? Var. arenaria vraisemblablement.

Les figures 62, 63, 75, 55, 69 sont des reproductions de formes douteuses dont nous n'avons pu observer que des valves isolées dans les boues glaciaires.

Suivent quelques formes dont les valves paraissent hyaline en milieu liquide, tant sont délicates les stries transverses qui n'apparaissent un peu qu'à sec.

Figure I2. Forme élégante, à frustule allongé, bicéphalé, marqué au milieu d'une sorte de stauros qui se traduit par une réfringence spéciale.

Figure I8. Forme lancéolée à extrémités rostro-capitées, qui provient comme la précédente de la rivière Matotchlia. Parait être $N$. ambigua Ehrenberg.

Figure 20. Petite valve linéaire, arrondie aux extrémités, remarquable par la dilatation du raphé, entre les nodules médian et terminaux.

Figure 34. Forme lancéolée à extrémités subcapitées, à striation transversale atteignant le raphé.

Les figures 30, 72, 73, 83, 84, semblent devoir se rapporter au groupe des Fusiformes, remarquables par leurs valves longuement lancéolées et la ténuité extrême de la striation. Peut-être pourrait-on voir, au moins dans l'une d'elles (fig. 3o), N. fusiformis Grunow. 


\section{Genre Mastogloia Thwattes}

Caractères du genre. - Cfr. H. Tan Heurck, Traité des Diatomées, page r53.

Les figures 66 et 85 s'y rapportent vraisemblablement, bien que les caractères n'en soient pas aussi évidents que dans d'autres espèces bien connues des régions moins boréales.

Figure 66. Mastogloia apiculata W. Smith.

Figure 85. Mastogloia submarginata Cleve et Grunow.

Origine. - Ces deux espèces sont assez rares dans les matériaux de la Belgica; elles s'y rattachent à la florule des neiges et des glaces.

\section{NOTE FINALE}

Pour nous conformer à l'usage reçu dans les publications du genre de celle-ci, nous nous étions proposé d'abord de tracer, en annexe à ce Mémoire, le tableau synoptique de la répartition des espèces les plus fréquentes dans les divers produits de pèches microplanktoniques de la Belgica.

Il a été jugé préférable, dans la suite, de réserver ce supplément de documentation pour un autre travail, à concevoir sur un plan assez étendu pour atteindre en même temps les organismes non microplanktoniques qui ont fait l'objet d'autres études relatives à la mème Expédition Scientifique.

Ce travail permettra de répertorier tous les organismes rapportés des diverses stations de recherches et de souligner leurs rapports mutuels et leurs groupements harmoniques dans les mèmes conditions d'ambiance.

Il se peut qu'il y ait là des éléments d'étude d'un très vif intérèt. 




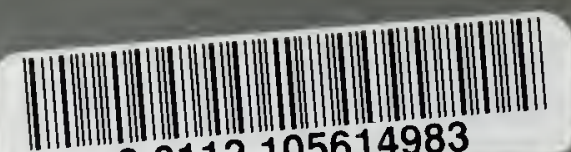

30112105614983 